\title{
Giant magneto resistance (GMR) sensors for non destructive testing
}

\author{
By
}

Joseph Bailey

A thesis submitted to the Victoria University of Wellington in fulfilment of the requirements for the degree of Masters of Engineering

Victoria University of Wellington

(2015) 


\section{Abstract}

The thesis investigates the use of giant magneto resistance sensors for eddy current testing in order to identify defects in steel pipes. An automated test rig which included the device under test, sensor array, excitation unit, electronic measurement equipment, mechanical setup and LabVIEW automation was designed and built. This was used to investigate the effect of excitation parameters such as current, frequency and distance to the pipe. Some preliminary algorithms to improve the signal were developed and tested. The effect of the shape and size of the defect and aluminum shield on the magnetic field was investigated. A qualitative model to describe the magnetic field, including measured defect signals, was developed. Minimum defect parameters and maximum distance values were evaluated in the context of signal to noise. 


\section{Acknowledgments}

I would like to thank everyone who helped me during my research project. My thanks go to my supervisors, Arvid Hunze and Gideon Gouws whose support made my project possible, thank you especially Arvid for your work in providing FEM modeling predictions. Thanks also to Simon Granville for measuring the permeability of the pipe samples and to Shaun Bredenkamp for manufacturing components for the test system. I would also like to express my thanks to Robinson Research Institute for providing financial support in the form of a scholarship which was greatly appreciated. To Phil Bondurant and Tony Mactutis from Qi2 and Kevin Stevens and Dan Drabble from Quest Integrity, my thanks for your insight into real world issues in the area of non-destructive testing. Finally my thanks go to my partner Jessie and my family for their support throughout my research, especially my mum Margaret for her many hours of patient proofreading. 


\section{Contents}

1 Introduction $\quad 6$

2 Background $\quad 8$

2.1 Non-destructive testing (NDT) . . . . . . . . . . . . . . . . . . . . . 8

2.2 Eddy current testing . . . . . . . . . . . . . . . . . . . . . . 10

2.2.1 Magnetic Sensors for eddy current testing . . . . . . . . . . . . . . . 12

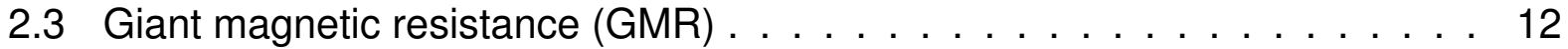

2.3.1 GMR effect . . . . . . . . . . . . . . . . . . . . 13

2.3.2 GMR sensor fabrication . . . . . . . . . . . . . . . . . . . . 16

2.4 GMR used in NDT applications . . . . . . . . . . . . . . . . . . . . . . . . . . . . 17

2.4.1 Corrosion under insulation detection . . . . . . . . . . . . . . . . . 19

2.4.2 Magnetic flux leakage measurements with GMR sensors . . . . . . . 20

2.4.3 Eddy current testing with GMR sensors . . . . . . . . . . . . . . . . 20

2.5 Qualitative Model of Eddy Current Generation . . . . . . . . . . . . . . . . . 20

2.5.1 Eddy current generation . . . . . . . . . . . . . . . . . . 21

2.5.2 Explanation of defect signals . . . . . . . . . . . . . . . . . 26

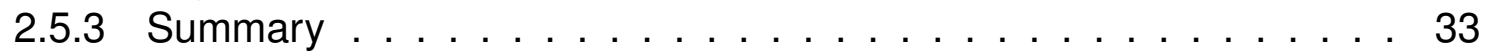

3 System Design 36

3.1 Mechanical setup . . . . . . . . . . . . . . . . . . . . . 37

3.2 Measurement system . . . . . . . . . . . . . . . . . . . . 38

3.2.1 Initial measurement system . . . . . . . . . . . . . . . . . . . . . . . . . . . . . . . . . . . .

3.2.2 Improvements to Measurement system . . . . . . . . . . . . . . . . 42

3.3 Excitation system . . . . . . . . . . . . . . . . . . . . . . . 51

3.3.1 Initial excitation system . . . . . . . . . . . . . . . . . 51

3.3.2 Improvements to excitation system . . . . . . . . . . . . . . . 53

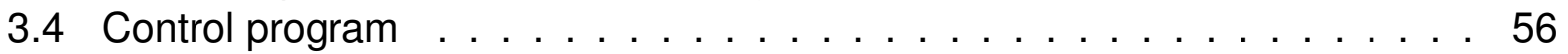

3.5 Data analysis algorithms . . . . . . . . . . . . . . . . . 58

4 Experimental Results: Measurement of standard pipe 62

4.1 Initial pipe parameters . . . . . . . . . . . . . . . . . . 63

4.2 Repeatability of measurements and data correction algorithms . . . . . . . 64

4.3 summary of repeatability and data correction . . . . . . . . . . . . . . . 75

4.4 Influence of excitation current . . . . . . . . . . . . . . . . . 78

4.4.1 Background level and total noise . . . . . . . . . . . . . 79 
4.4.2 Effects of excitation current on local variation . . . . . . . . . 84

4.5 Summary of current dependence . . . . . . . . . . . . . . . . . 91

4.5 .1 Magnitude . . . . . . . . . . . . . . . . . . . . . . 91

4.5 .2 Phase shift . . . . . . . . . . . . . . . . . . . . 92

4.5.3 Effects of excitation current on local variation . . . . . . . . . . 92

4.6 Influence of excitation frequency . . . . . . . . . . . . . . . . . . 94

4.6.1 Background signal level and total noise . . . . . . . . . . . . . . . 94

4.6.2 Effects of excitation frequency on local variation . . . . . . . . 97

4.7 Summary of frequency dependence . . . . . . . . . . . . . . . . . 105

4.8 Influence of excitation lift-off . . . . . . . . . . . . . . . . . . 110

4.8.1 Background signal level and total noise . . . . . . . . . . . . 110

4.8.2 Effects of excitation lift-off on local variation . . . . . . . . . . . 115

4.9 Summary of excitation lift-off dependence . . . . . . . . . . . . . 120

4.10 Effect of the Aluminum shield . . . . . . . . . . . . . . . . . . . . . . . 124

4.10.1 Effect of excitation frequency on features introduced by the shielding 126

4.11 Summary of effects of aluminum shield . . . . . . . . . . . 137

4.12 Effects of aluminum dents . . . . . . . . . . . . . . . . . . . 138

4.12.1 Effect of excitation frequency on features introduced by dents in

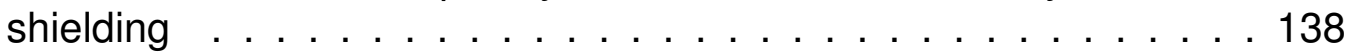

4.13 Summary of effects of aluminum dents . . . . . . . . . . . . . . 150

5 Experimental Results: Measurement regular defects 151

5.1 Defect parameters . . . . . . . . . . . . . . . . . . . . . . 151

5.2 Results . . . . . . . . . . . . . . . . . . . . . . . . . . . . 154

5.2.1 Influence of defect shape on defect signals . . . . . . . . . . . 154

5.2.2 Discussion: Influence of defect shape on defect signals . . . . . 170

5.2.3 Influence of excitation current on defect signals . . . . . . . . . 171

5.2.4 Discussion: Excitation current vs defect signals . . . . . . . . . . . . 178

5.2.5 Influence of excitation frequency on defect signals . . . . . . . 181

5.2.6 Discussion: Influence of excitation frequency on defect signals . . . 192

5.2.7 Influence of excitation lift-off on defect signals . . . . . . . . . . 198

5.2.8 Discussion: Influence of the excitation lift-off on defect signals . . . 210

6 Summary and conclusions 212

$\begin{array}{lll}\text { Appendix A NVE AA004 data sheet extract } & 215\end{array}$ 


\section{Chapter 1}

\section{Introduction}

Tools to detect corrosion are an important area of research as the annual maintenance related expenses for corrosion to just the petroleum refining industry in USA alone are 1.8 billion USD, reported by the National Association of Corrosion Engineers [1]. Insulated pipes are used extensively in this industry for steam transport. Figure 1.1 shows a view of a part of the BP Whiting Refinery with an extensive network of steam transport pipes. Currently the standard industry practice is to completely remove insulation and visually inspect the pipes. A tool that provides screening of pipes without removal of the insulation would be greatly beneficial as it would significantly reduce inspection time and cost.

Visual inspection of these pipes can be very costly as it requires the complete removal of weather jacketing and insulation, which has to be re-instated after inspection. A shutdown may also be necessary if the plant cannot operate with uninsulated pipes. The consequences of not inspecting for corrosion can be catastrophic since a steam pipe can rupture and result in the shutdown of a plant or process for an extended period. There are currently two NDT methods which are viable methods for detecting corrosion under insulation. These methods are guided wave ultrasonic inspection [3] and inspection with a meandering wire excitation system [4],[5],[6],[7]. However, both have limitations and there is not a widespread acceptance of these methods in industry. A tool that provides screening of pipes without removing the insulation and gives a rating on the level of corrosion of a pipe would be greatly beneficial to industry. It would provide prioritization of areas where insulation should be removed to check for corrosion.

The goal of the project is to develop a technology demonstrator to show that eddy current testing with GMR sensors is a viable method of detecting wall loss due to corrosion within a steel pipe without removing aluminum weather cladding or insulation. The parameter of interest to engineers determining whether a pipe needs to be replaced is the remaining wall thickness. The wall loss occurs when water ingresses into the insulation in a particular location and corrosion occurs. This corrosion typically starts at the point were the moisture is present and grows outwards and into the wall of the pipe. Areas of interest to inspectors are corrosion deeper than $2.5 \mathrm{~mm}$ which typically have surface an area of $2500 \mathrm{~mm}^{2}$ or larger [8].

A test system including a GMR sensor module and excitation unit was designed, customized, automated and then used to measure short lengths of pipe with insulation. Tests were completed for a range of excitation parameters on both a standard defect 


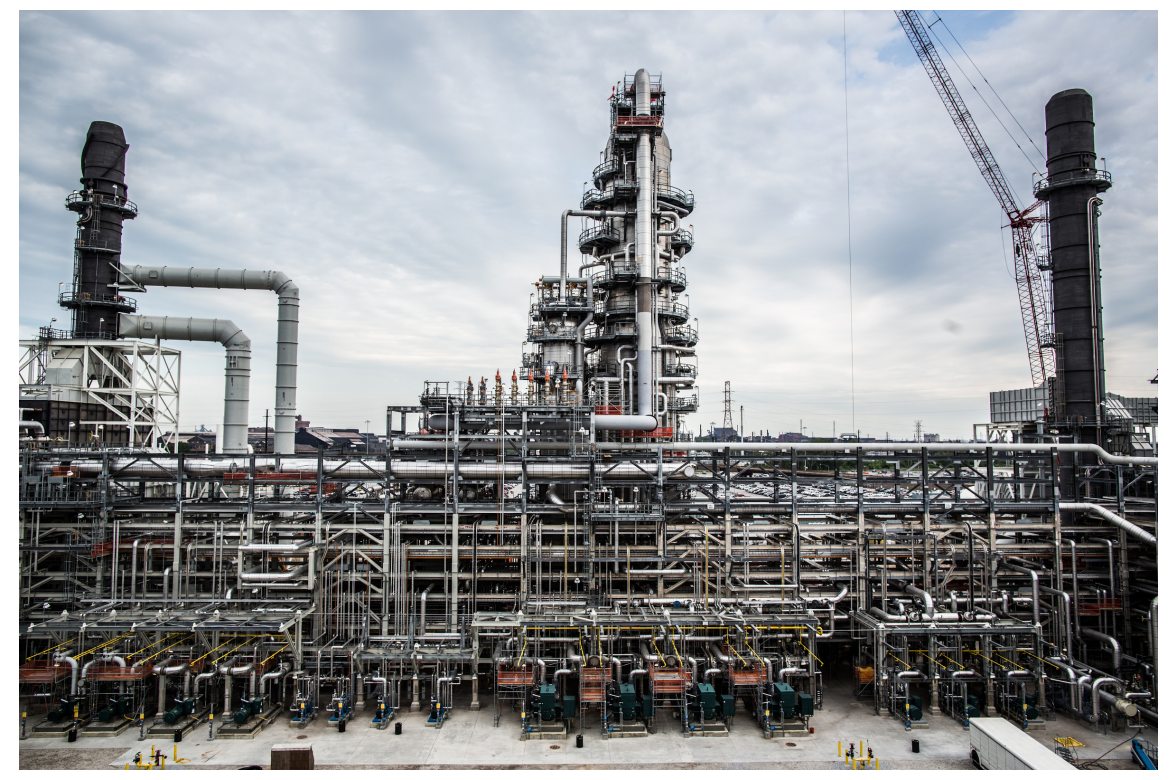

Figure 1.1: BP Whiting Refinery [2]

free pipe and a pipe with manufactured defects. The results obtained were analyzed to establish correlations between parameter changes and magnetic fields.

The eddy current method used in this thesis provides excitation via a coil with alternating current. This induces eddy currents in the test piece. By Lenz's Law this creates an opposing magnetic field which disturbs the excitation field. Discontinuities in the pipe will change the eddy current which changes the magnetic field above the pipe. This disturbance can be seen in the spatial map of the field above the pipe.

The measurement of the magnetic field was carried out with giant magneto resistance (GMR) sensors. GMR sensors were investigated due to their advantages over pickup coils, which are the standard magnetic sensors presently used in eddy current testing, because GMR sensors have a low $1 / f$ noise level. This allows for operation at a low frequency which is required to penetrate a high permeability material such as steel. The small size and good temperature stability also make GMR sensors a suitable choice for applications in harsh environments.

This thesis starts with background section in Chapter 2 giving a brief overview of NDT techniques that are used to test steel pipes. Then eddy current testing and GMR sensors are described in more detail. Then some specific solutions relevant to this thesis are reviewed. Finally a qualitative model of eddy currents in the pipe is described. Chapter 3 covers the test rig, including the design of the excitation system and sensor module. Chapter 4 presents and discusses the results from tests on a standard pipe. This helps to establish a baseline of the expected variation of the magnetic field and also shows how excitation parameters and the introduction of aluminum cladding change the magnetic field. Chapter 5 shows and discusses results for a range of defects. 


\section{Chapter 2}

\section{Background}

The application of GMR sensors for eddy current testing on steel pipes is a new area. The advantage of eddy current testing is that it does not require contact with the test object while the GMR sensor allows measurements at very low frequency. This low frequency is required to penetrate the steel and weather cladding made from conductive material. Until now methods for detecting defects within ferrous pipe, without removing the thick insulation and weather cladding that protects it, have been very limited. This work looks at providing a new option for detecting corrosion in steel pipes under insulation.

This chapter describes the most common NDT methods for testing ferrous pipes in section 2.1. The NDT method used in this thesis is eddy current testing, presented in section 2.2. A brief overview of how GMR sensors are fabricated and operated is discussed in section 2.3 and their current application is discussed in section 2.4. Finally a qualitative model is presented of the effect of excitation parameters and defects, that are present in the pipe, on eddy currents and their resulting magnetic fields.

\subsection{Non-destructive testing (NDT)}

NDT is used in many fields from medical applications, such as $x$-ray, to industrial applications, such as crack detection in aircraft wings. It is defined as any testing which does not negatively affect the object under test. Some of the main techniques of NDT in industrial context are ${ }^{1}$ :

\section{Visual inspection}

Visual inspection is the most widely used NDT technique. This process involves an experienced operator inspecting the site either unaided or with the help of cameras, lighting or microscopes. This is the most flexible technique which is why it is still widely used. However, it requires a highly skilled inspector with a lot of experience and still has a high chance of false negatives which can result in catastrophic failures. Furthermore it is a qualitative rather than quantitative method.

\footnotetext{
${ }^{1}$ A good summary of these techniques can be found in [9] and [10]
} 


\section{Radiographic inspection}

This technique uses X-rays to look at the relative density of test objects. Cracks and wall loss show up as areas of lower density. It has the advantage of easily penetrating though thick insulation since only dense steel distorts the X-rays. The drawbacks of this technique are the safety requirements and the angle dependence between source and detector making the setup very bulky.

\section{Ultrasonic inspection}

Ultrasonic inspection uses high frequency sound waves coupled into the test object. The echoes or reflections of the sound waves are measured by a transducer. This technique allows for the detection of defects deep within the object. The sound waves will travel a long way through the material only changing direction when a discontinuity is reached. It also allows for thickness measurements when there is only access to one side of the object as the sound waves will bounce off the inner wall. However, this technique needs good acoustical contact which requires surface preparation such as cleaning, removing paint or applying conductive gels. This is a major disadvantage if the pipe being tested has a weather-tight cladding which has to be removed which greatly increases the inspection effort.

\section{Guided wave ultrasonic}

Guided wave ultrasonic is a recent development in ultrasonic inspection. This involves sending an acoustical wave down a length of pipe. When a defect is encountered part of the wave is reflected and these reflections are measured by a transducer at the injection point. This allows for long lengths of pipes to be measured with only the removal of coating or insulation at the injection point. However, as the insulation is protected by a weather tight cladding, removal of this increases the risk of ingress of water making it a potential site for increased corrosion.

\section{Magnetic particle inspection}

Magnetic particle inspection is a method for detecting surface and slightly subsurface discontinuity in ferromagnetic materials. The test object is first magnetized either directly by passing a current through it or indirectly by applying a external magnetic field. Then magnetic particles are applied either dry or as wet particles suspended in liquid. Cracks in the test object create lines of magnetic flux leakage. The flux lines leaking out of the pipe attract the magnetic particles so clusters form in these cracks which can be recognized via visual inspection. This method is particularly good at finding very thin stress corrosion cracking that can be hard to identify with just standard visual inspection. The method's disadvantages are that it requires a lot of surface preparation and any insulation has to be removed. 


\section{Dye penetrant inspection}

Dye penetrant inspection involves cleaning the sample of all loose material, oil and paint. The test sample is then coated with dye which gets absorbed into surface flaws. After a certain time the dye is removed from the surface and a developer is applied which pulls the dye out of the cracks highlighting any flaws. Penetrant inspection has similar advantages and disadvantages to magnetic particle inspection as it requires a clean surface and is good at identifying thin cracking.

\section{Acoustic emission inspection}

Acoustic emission testing involves mechanically loading the test object. When defects are stressed they release energy in the form of stress waves. These stress waves are picked up by acoustic emission sensors placed on the object. This allows the testing of complex geometry but the process of stressing the object runs the risk of damaging it.

\section{Magnetic flux leakage}

Magnetic flux leakage requires direct magnetization of the sample. Defects cause the flux lines to leak out of the sample. These leakage flux lines are measured by magnetic sensors such as magneto resistive sensors or Hall sensors.

\section{Thermography}

Thermography images the thermal profile of the test object. It can show hot or cold spots which can indicate poor insulation areas. It only works when the medium within the test object is not at ambient temperature and it relies on damage to the insulation.

\section{Eddy current testing}

Eddy current testing involves bringing a coil with alternating current close to the test sample. This causes eddy currents to flow in the test sample. When a defect is present the eddy currents are disturbed and this causes the voltage across the coil to change. This is a non-contact method so it requires no surface preparation and can be done without touching the object. The main advantage of this technique is that the use of low excitation frequencies may make it possible to measure through a conductive weather shield that is often wrapped around steam pipes and has to be left in place to ensure the weather tightness of the system is not compromised.

\subsection{Eddy current testing}

Eddy current testing is used extensively in industry to identify cracking, pitting and corrosion. The basic principle of eddy current testing works by passing an alternating current through 
an excitation coil. The alternating current creates an alternating magnetic field around the coil following equation 2.1 (Ampere's law [11])

$$
\oint H . d l=n I
$$

where

$$
\begin{aligned}
H & =\text { magnetic field }(A / m) \\
n & =\text { number of turns in the coil } \\
I & =\text { total current }(A)
\end{aligned}
$$

The alternating field induces eddy currents in the test object according to Faraday's law of induction 2.2 [11].

$$
V=-\frac{d \phi_{B}}{d t}
$$

where

$$
\begin{aligned}
V & =\text { Electromotive force }(E M F)(V) \\
d \phi_{B} & =\text { change in magnetic flux }(W b / s)
\end{aligned}
$$

The eddy currents induced in the sample create an opposing magnetic field following Ampere's law 2.1 which disturbs the excitation field. The total resulting field can be measured by various types of magnetic sensors with a pick-up coil the most common method. Defects in the test piece cause the eddy current to be re-routed which further changes the measured magnetic field. This change in field is used to detect a defect.

The distribution of eddy currents in the test object depends on the permeability and conductivity of the test object. The current distribution into the test object from the surface follows an exponential decay curve described in 2.3. The skin depth is defined as the point at which the eddy current has decayed to $1 / e$ ( $37 \%$ [12] of the surface value and can be calculated with equation 2.4[12].

$$
J=J_{S} e^{\frac{-d}{\sigma}}
$$

where

$$
\begin{aligned}
J & =\text { current density }\left(A / m^{2}\right) \\
J_{S} & =\operatorname{surface} \text { current density }\left(A / m^{2}\right) \\
d & =\operatorname{depth}(m) \\
\delta & =\operatorname{skin} \text { depth }(m)
\end{aligned}
$$




$$
\delta=\sqrt{\frac{1}{\pi f \mu_{0} \mu_{r} \sigma}}
$$

where

$$
\begin{aligned}
\delta & =\text { skin depth }(m) \\
\sigma & =\operatorname{conductivity}(S / m) \\
f & =\text { frequency }(H z) \\
\mu_{r} & =\text { relative magnetic permeability } \\
\mu_{0} & =\text { magnetic permeability of free space }
\end{aligned}
$$

\subsubsection{Magnetic Sensors for eddy current testing}

In eddy current testing a range of magnetic sensors can be used to detect changes in the magnetic field. This thesis uses GMR sensors. Some other sensors that can measure magnetic field include but are not limited to:

Pick up coils: Pick up coils work according to Faraday's law of induction whereby the change in flux due to the eddy currents induces a voltage across the coil. Pickup coils are the most common sensor used in eddy current testing as they are a simple low cost passive device. The disadvantage is that they do not have good performance at low frequency due to high $1 / f$ noise which dominates at low frequencies. The $1 / f$ noise is a noise source which is inversely dependent on the frequency. To overcome this limitation they need to be physically large to get good signal levels which means their spatial resolution is low.

Hall sensors:Hall sensors use the Hall effect to detect magnetic fields. The advantage of the Hall sensor is that they can operate effectively all the way down to D.C. The disadvantage is that they have a lower sensitivity than magneto resistance based sensors and experience large drifts due to temperature sensitivity which requires compensation.

Anisotropic magneto resistive (AMR) sensors: AMR sensors are made of thin film permalloy. They are similar to GMR sensor in that they are also thin film devices which have an anisotropic response to applied magnetic fields. Their disadvantages compared to GMR sensors are that they have a lower measurement range and can be permanently changed by high magnetic fields. The advantage of the AMR sensor is that it is a very small device and can be cheaply manufactured, which gives the potential for low cost high resolution measurements. However, its sensitivity to magnetic fields is low with typical values of around $2 \%$ to $3 \%$ change in resistance for magnetic fields up to 10 Oersted [13].

\subsection{Giant magnetic resistance (GMR)}

The sensors used for eddy current detection in this thesis are GMR sensors. A GMR sensor is a thin film device and compared to pickup coils can be made much smaller which 


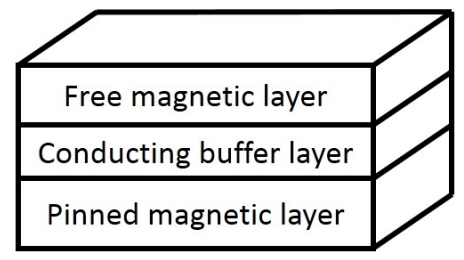

Figure 2.1: Basic GMR cell layer arrangement

allows for finer spatial resolution measurement as the sensors can be tightly packed. The other advantage of the GMR sensor is their low $1 / f$ noise level compared to pickup coils, this allows for DC and low frequency measurements. This low frequency measurement is required for this application as very low frequencies are used to penetrate through aluminum weather cladding and into high permeability material such as steel.

\subsubsection{GMR effect}

GMR sensors are multilayer thin film devices [14]. Their construction involves a stack of cells made of two thin ferromagnetic layers separated by a very thin conducting layer shown in figure 2.1. These unit cells have buffer layers to improve the GMR effect. A typical stack is presented in [15] shown in figure 2.2 with additional layers that improve the GMR effect.

The GMR multi layers are composed of three composite magnetic layers each composed of two $1.5 \mathrm{~nm}$ CoFe layers at the non-magnetic interfaces and with a single $2.0 \mathrm{~nm}$ $\mathrm{NiFeCo}$ layer between them. $1.5 \mathrm{~nm}$ layers of copper alloys are used as the non-magnetic interface layers.

The GMR effect is due to the transition between anti-parallel and parallel aligned magnetic material layers. Applying a magnetic field changes the resistance due to a change in electron scattering through the ferromagnetic layers. Figure 2.3 shows the electron scatter without an external field and figure 2.4 shows the electron scatter with a external field.

Electrons can have two different spins, parallel or anti-parallel to the magnetic field. When the spins of the electrons and the direction of magnetization of the ferromagnetic layer are parallel the electron scattering is low which leads to an decreased resistance to these electrons. When the spin of the electrons and the magnetization of the ferromagnetic layer are anti-parallel the scattering is high and so the resistance to these electrons is higher. The increase in scattering is due to higher probability of collision for a given length 


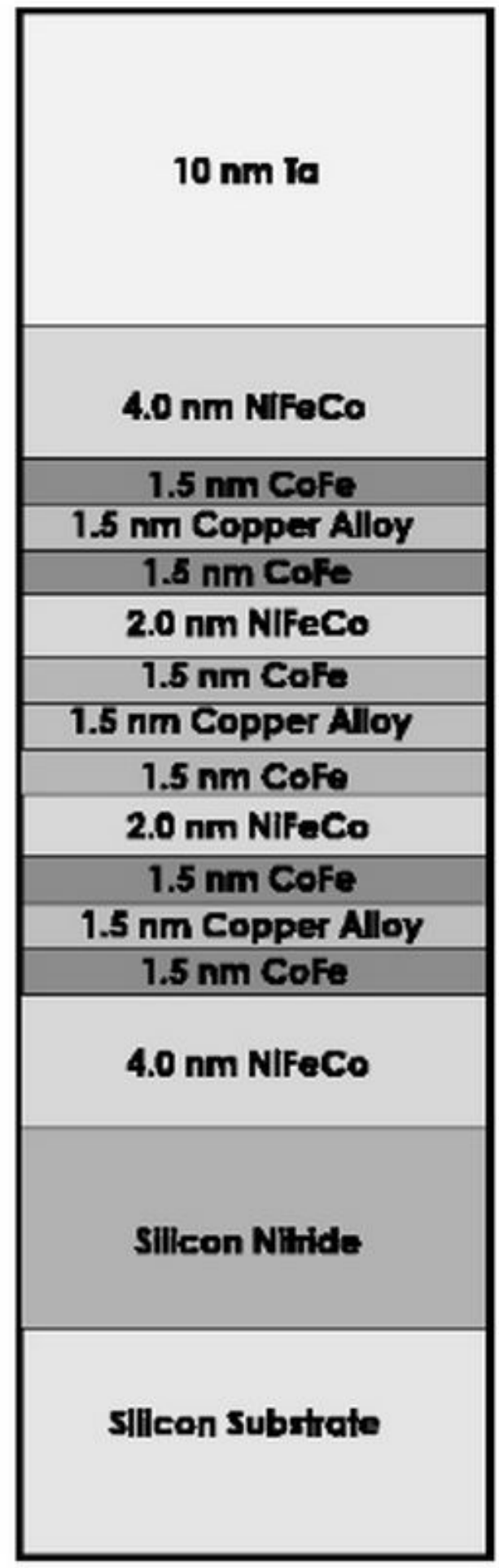

Figure 2.2: Typical multilayer GMR structure [15] 


\section{Spin UP Electrons Scatter at Interface with Spin DOWN Layer; Spin DOWN Electrons Scatter at Interface with Spin UP Layer Average Mean Free Path of the Electrons is Short}

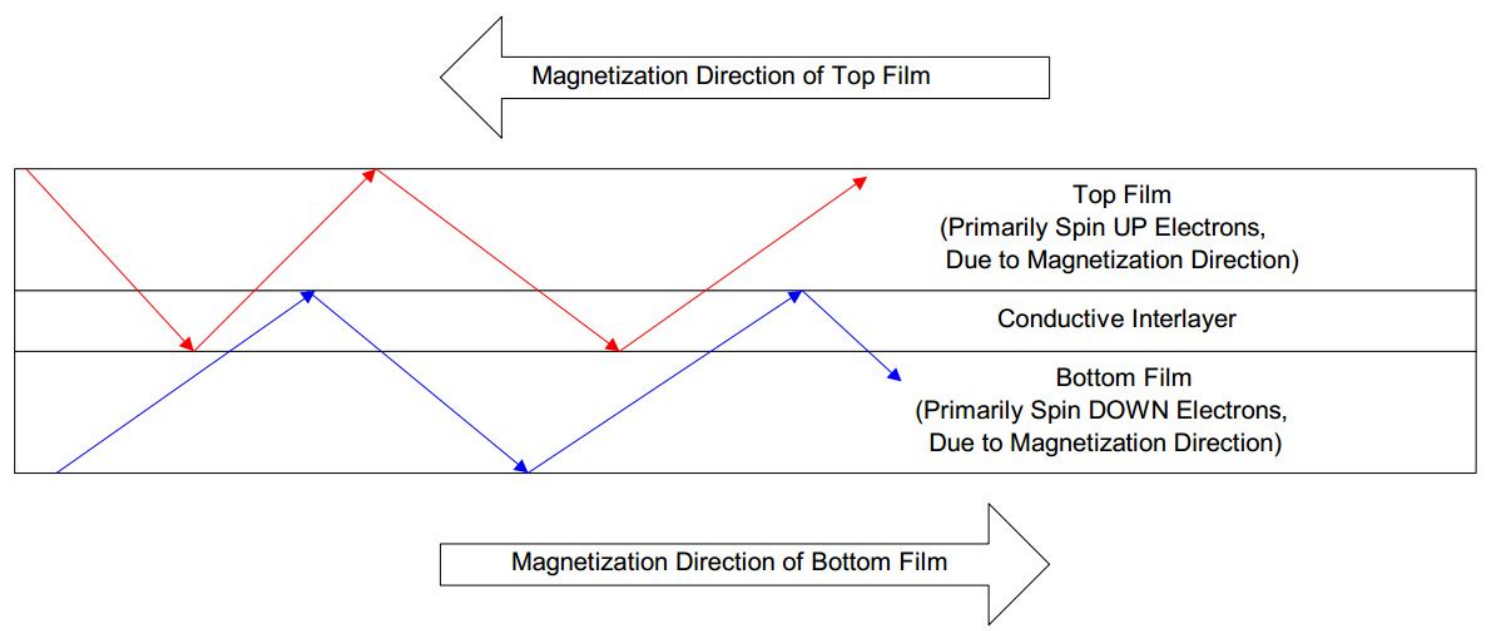

Figure 2.3: Electron scatter without external field [16]

Spin States of the Magnetic Layers are the same;

Electrons Travel More Readily Through Entire Stack of GMR Material.

Average Mean Free Path of the Electrons is Long

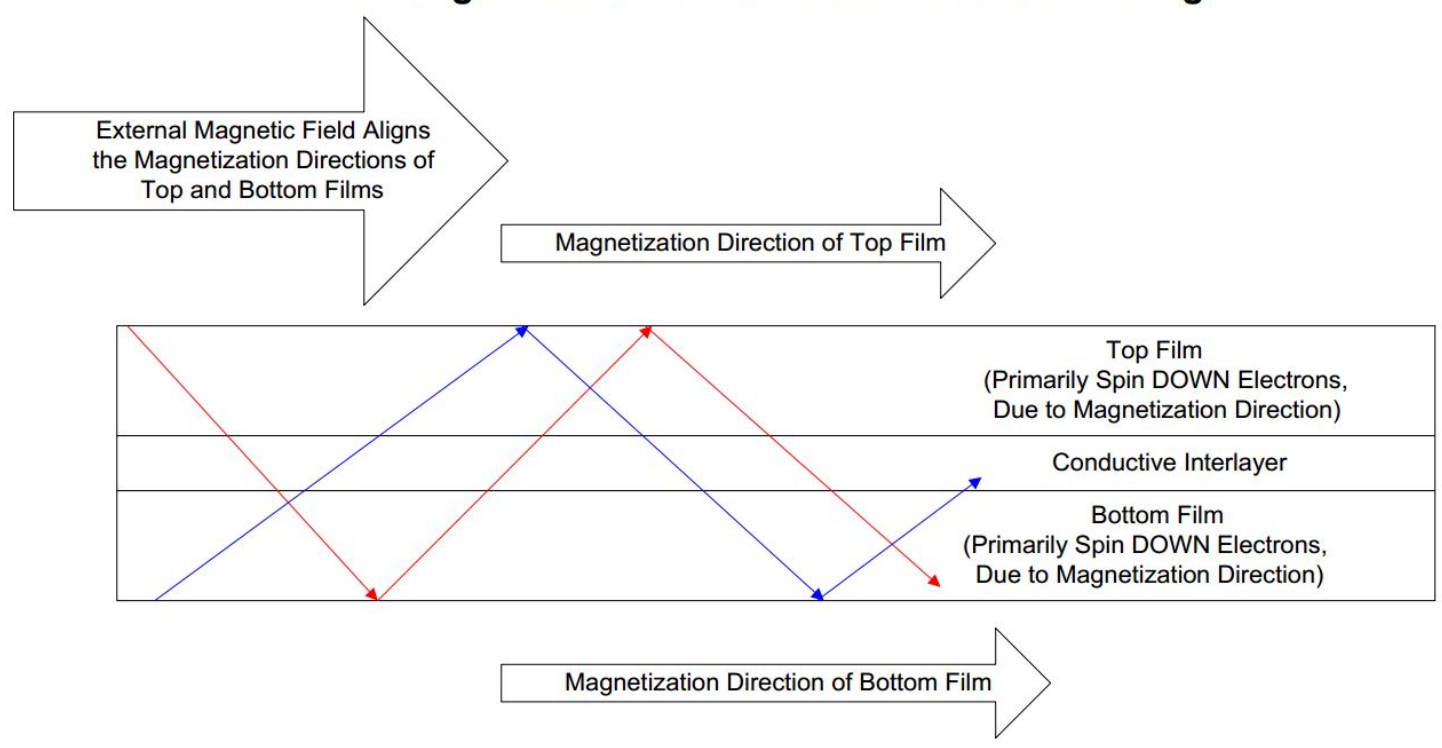

Figure 2.4: Electron scattering with external field [16] 


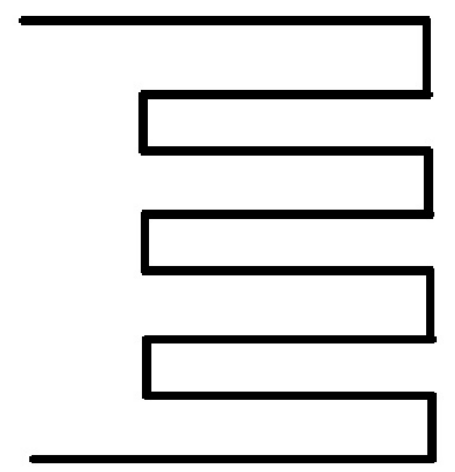

Figure 2.5: Serpentine path

of travel.

There are two types of magnetic layers in a GMR stack (shown in figure2.1). One layer is pinned and requires a large magnetic field to change the direction of magnetization. The other layer is free and its direction of magnetization follows the field applied. If no external field is present the free layer is anti-parallel to the fixed layer and the resistance is high. When an external magnetic field is applied the free layer magnetization changes its direction as the field strength increases and therefore resistance decreases. Individual GMR stacks can be combined to make sensors with increased sensitivity.

\subsubsection{GMR sensor fabrication}

The first step of creating accurate GMR sensors is to structure the GMR stack into a serpentine path (shown in figure 2.5). This is done to increase the path length the electrons have to flow through as long as possible so that the resistance is as high possible and thus the GMR effect is as high as possible. To build a typical GMR sensor that has a low temperature dependence and high sensitivity, four GMR stacks with a serpentine pattern are used in a Wheatstone bridge arrangement shown in figure 2.6. Two of the GMR stacks are shielded to provide a reference resistance. The Wheatstone bridge configuration provides temperature stabilization because changing temperature changes the resistance of all four GMR stacks. This means no voltage change is measured at the output. The shields additionally act as flux concentrators, increasing field strength at the unshielded GMR stacks.

A major limiting factor of the GMR sensors is that they do not operate in a bipolar mode, as shown by the response curve in figure 2.7. This issue can be overcome by applying a DC bias field to the sensor, this changes the response curve as shown in figure 2.8, allowing the sensor to be operated in a unipolar mode. However, this has the 


\section{Functional Block Diagram}

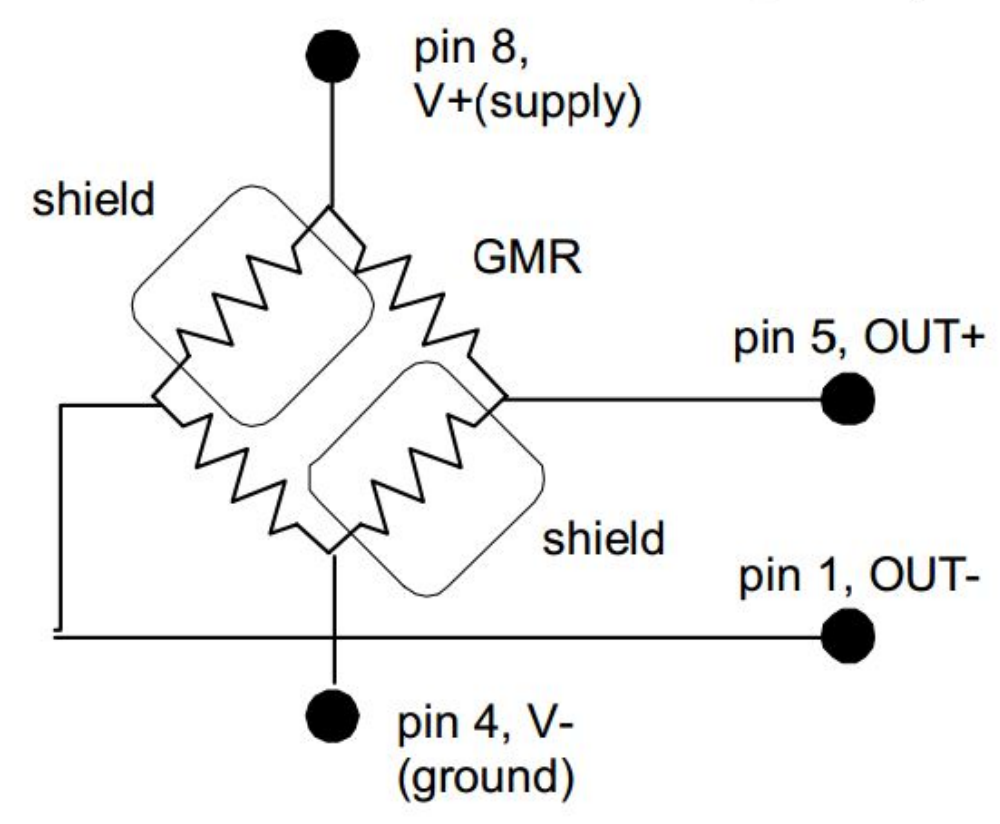

Figure 2.6: Wheatstone bridge layout of typical GMR sensor [17]

disadvantage of halving the measurement range. Some hysteresis effect is also present when operating in the uni-polar mode with the data-sheet value of $4 \%$ hysteresis when in unipolar operation (see appendix A).

The commercial sensor used throughout this project is the NVE AA004. The data sheet is provided in Appendix A. Figure 2.9 shows the die layout of the sensor. The majority of the die is occupied by the flux concentrators. This ensures that the field is increased approximately by an order of magnitude in the very small sensing area. In the sensing area between the two flux concentrators the two unshielded GMR stacks from opposing sides of the bridge are placed to give the maximum response. This technique of using flux concentrators to increase the field means that the average magnetic field over the total area of the flux concentrators is measured. The sensing area is small when compared to other magnetic field sensors, especially pickup coils, which allows for good spatial resolution in measurement of magnetic fields.

\subsection{GMR used in NDT applications}

GMR sensors show promise in a range of NDT applications. This section reviews a few specific applications including work done by JENTEK Sensor Inc [18],[19] on the specific problem addressed in this thesis. The problem addressed here is detecting wall loss from steel pipes that are wrapped in thick insulation and aluminum weather cladding, which 


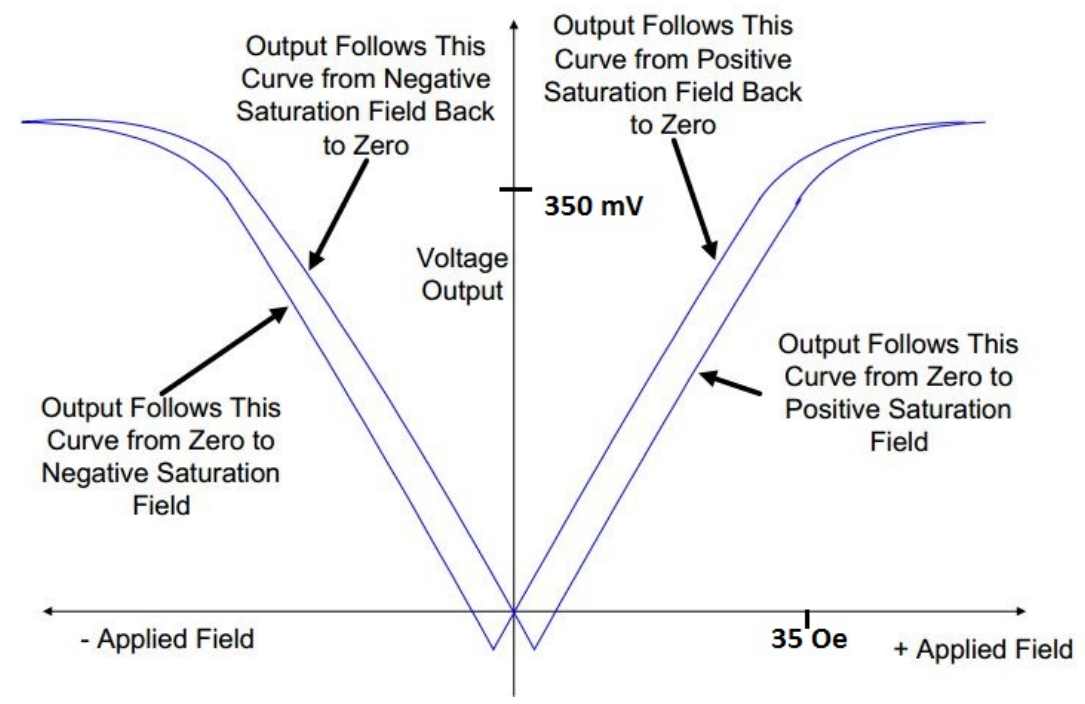

Figure 2.7: NVE GMR sensor's unipolar response to bipolar magnetic field [16]

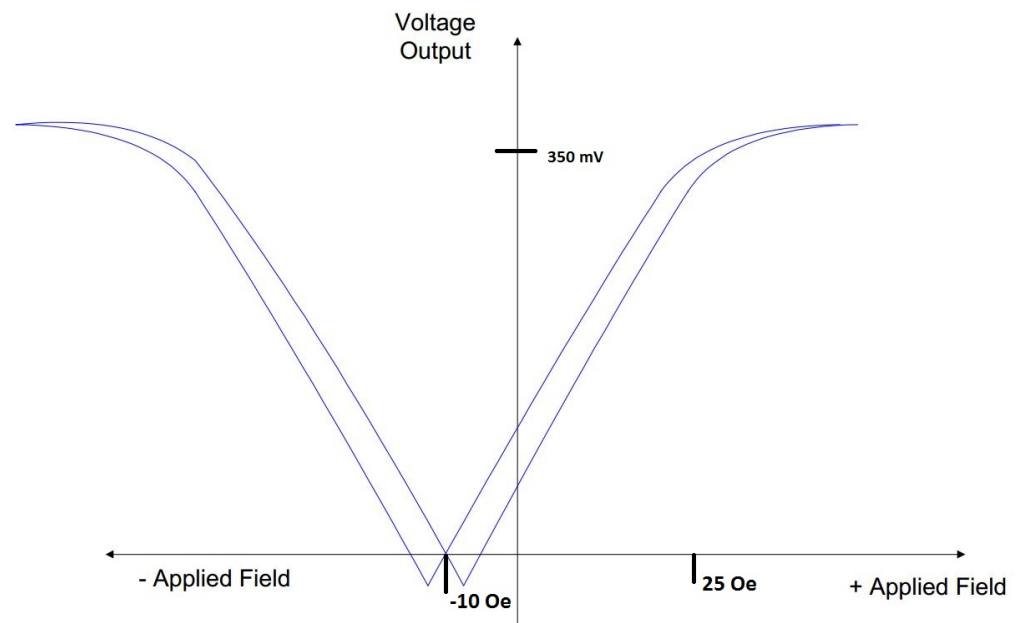

Figure 2.8: Sensor response after DC biasing

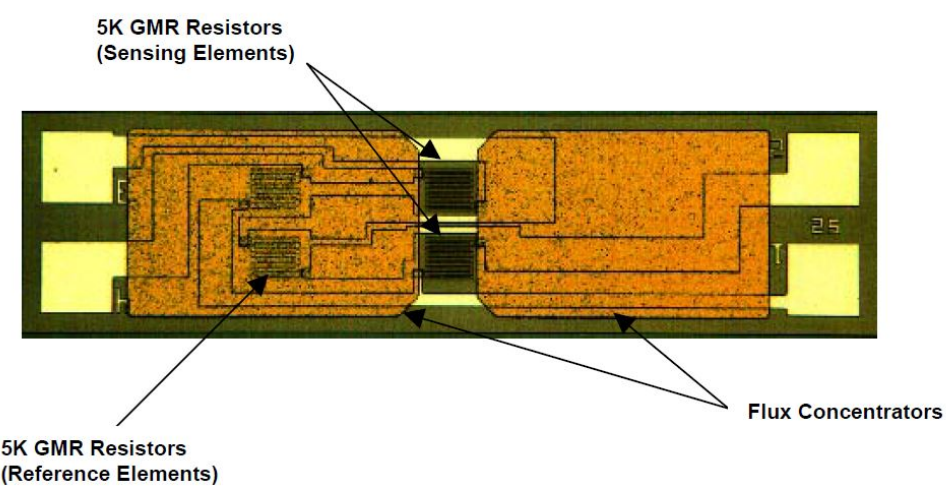

Figure 2.9: Die layout of NVE GMR sensor AA004 [17] 


\begin{tabular}{|c|c|c|c|c|}
\hline Product & Company & Price(USD) & Sensitivity & Range \\
\hline AA004 & NVE & 7.7 & $1.1 \mathrm{mV} / \mathrm{V} / \mathrm{Oe}$ & $5-35 \mathrm{Oe}$ \\
\hline AAH004 & NVE & 7.7 & $3.9 \mathrm{mV} / \mathrm{V} / \mathrm{Oe}$ & $1.5-7.5 \mathrm{Oe}$ \\
\hline AA002 & NVE & 13.85 & $3.6 \mathrm{mV} / \mathrm{V} / \mathrm{Oe}$ & $1.5-10.5 \mathrm{Oe}$ \\
\hline AAH002 & NVE & 13.85 & $\begin{array}{r}10.25 \\
\mathrm{mV} / \mathrm{V} / \mathrm{Oe}\end{array}$ & $0.6-3 \mathrm{Oe}$ \\
\hline GF705 & Sensitec & unknown & $10 \mathrm{mV} / \mathrm{V} / \mathrm{Oe}$ & $1.8-8 \mathrm{Oe}$ \\
\hline
\end{tabular}

Table 2.1: Commercially available GMR sensors

produces high lift-off to the sensor and excitation unit from the pipe surface. Several other applications are reviewed that involve testing of both ferrous steel structures and aluminum structures. A short review of commercially available GMR sensors is givin in table 2.1.

\subsubsection{Corrosion under insulation detection}

Most work done for detection of wall loss with a high lift-off is by JENTEK Sensors Inc. They use a meandering wire measurement technique described in [6],[7],[5],[4] . It Involves a meandering wire excitation which creates an excitation field of a certain spatial wave length at a fixed frequency. Sensor windings are then placed a quarter of the spatial wave length from the excitation winding. The limitation of this geometry is that when measuring with large lift-off a large spatial wavelength has to be used which reduces the spatial resolution of the measurements, as discussed in [4]. The phase and magnitude measured at the sensor coils is compared with pre-calculated values for a range of lift-off, permeability and conductivity values to determine the change in each parameter.

The spatial resolution issue can be solved by introducing multiple sensors for each excitation winding [4]. This moves the limitation to the excitation frequency required to penetrate deep into a steel pipe. Low frequency measurements are not possible with the coil type sensor as the noise increases with decreasing frequency to make measurements impossible. This was solved by using Hall or magneto-resistance sensors such as GMR sensors instead of coils to allow measurements at very low frequencies [4]. To compensate for conductive shields JENTEK uses high frequency excitation with coil sensors in addition to the low frequency excitation with magneto resistive sensors, which provides independent 
data for the shield and pipe [4]. Both the meandering wire technique and the use of the meandering wire to detect corrosion under insulation have been patented [18],[19].

\subsubsection{Magnetic flux leakage measurements with GMR sensors}

GMR sensors have been used as the sensors in magnetic flux leakage (MFL) measurements on steel pipes. MFL measurements involve magnetizing the sample with a large field and then using a magnetic sensor to search for areas where cracks cause the field lines to distort out of the pipe [20]. The use of GMR sensors in a plug mill that produces seamless steel pipe is described in [21]. The process takes advantage of the low noise level of GMR sensors to identify small cracks on the inside wall of the pipe where other sensing technologies have failed. This sensing method is patented in [22]. GMR sensors have also been used for MFL measurements in [23] and [24]. Their low cost and high sensitivity has made them the magnetic sensor of choice in these applications.

\subsubsection{Eddy current testing with GMR sensors}

Eddy current testing with GMR sensors has mainly been used for detecting flaws in aluminum such as aircraft structures. In this case the GMR sensor array is made with very small $200 \mu \mathrm{m}$ spacing between sensors which has allowed clean imaging of $0.75 \mathrm{~mm}$ diameter holes with $0.25 \mathrm{~mm}$ depth.

Pulsed eddy current inspection is discussed in [25] where the use of a pulsed excitation means a broad range of frequency responses can be sampled at once. This allows identification of corrosion at different depth values with the response at each frequency corresponding to a different depth position.

Crack detection with eddy current and GMR sensors in stainless steel pipes is investigated in [26] using a dense array of GMR sensors with a $500 \mathrm{~Hz}$ excitation field to detect buried and surface breaking defects. This paper investigates the use of two different excitation sources perpendicular to each other. The results obtained show a similarly shaped sensor response as measured in this thesis with sensors in one orientation producing two peaks and in the other orientation four peaks.

\subsection{Qualitative Model of Eddy Current Generation}

A qualitative model of the way eddy currents are generated and how they circulate is presented in this section. This will be used to explain some of the results obtained in Chapters 4 and 5 . The qualitative model describes how excitation currents generate a magnetic field, which creates eddy currents in a metallic object, and how these eddy currents are finally measured by a magnetic sensor. Section 2.5 .1 explains how the eddy currents are produced and how changes in the excitation parameters affect the excitation 

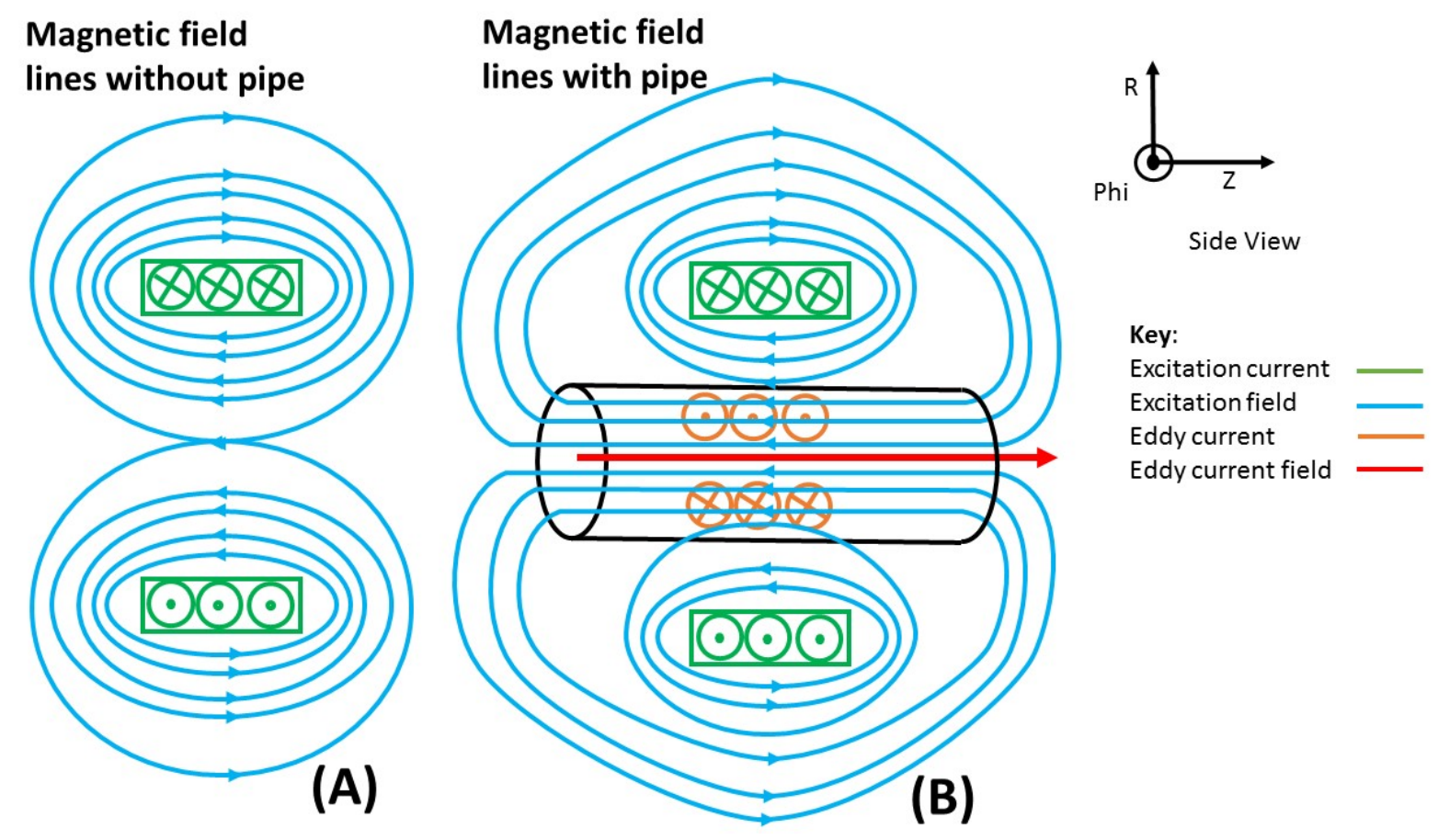

Figure 2.10: (A)Field produced by the excitation current (B)Field lines distorted by a steel pipe and induced eddy currents

field. Section 2.5.2 describes how the eddy current flow is distorted when a defect is present and how defect signals in the Z, R and Phi directions are generated.

\subsubsection{Eddy current generation}

To understand how a defect distorts the magnetic field it is important to understand how the excitation field interacts with the pipe. The basic concept explaining the generation of eddy currents is equation 2.2, Faraday's law, which shows that a changing magnetic flux produces an electromotive force (EMF). This EMF produces a current which opposes the current that produced the magnetic flux (Lenz's Law). The excitation method, as explained in Chapter 3, is produced by a copper excitation coil wrapped around the steel pipe. Exciting the coil produces a magnetic field which causes an eddy current to circulate in the pipe. This produces a magnetic field in the opposite direction of the generating field. In order to produce a change in flux, an oscillating excitation current is used. This results in an eddy current which is 180 degree phase shifted from the excitation current due to Lenz's law, which states the eddy current flow must oppose the excitation current. The generation of the eddy current in a pipe is shown in figure 2.10. If the pipe was a solid bar the eddy current strength would decay to zero as it approaches the center of the pipe. This Case is not discussed as it is not relevant to the problem presented in this thesis. Due to the high permeability of steel pipe, it is also found that the pipe will redirect the magnetic field lines to align with the pipe. 


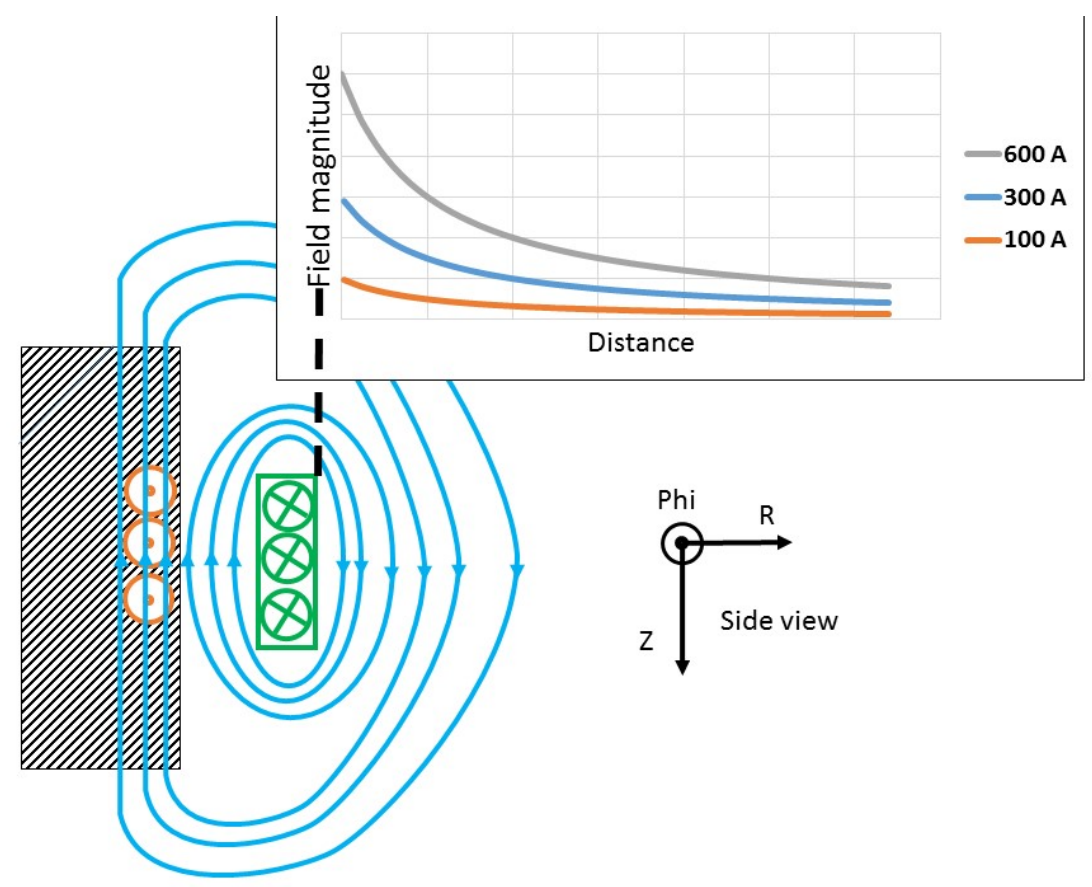

Figure 2.11: Effect of excitation current on excitation field plot shows field magnitude decay at increasing excitation current following equation 2.5[27](distance on inset plot shows the distance in the $\mathrm{R}$ direction from the coil)

\section{Excitation parameters}

An understanding of the influence of various parameters of the excitation field on the eddy current is important in order to be able to optimize the signals produced by the defects. Changes to the excitation field will change how the eddy currents flow, which will change the field that they produce. Changes in parameters discussed are: excitation current, excitation frequency and excitation lift-off, as these parameters correspond to the parameters analyzed throughout this thesis.

Excitation current:The influence of the excitation current on the excitation field is shown in figure 2.11. It shows a cross-section of the field in one wall of the pipe and a plot of the magnetic field decay at different excitation currents. The strength of the magnetic field at a given current and distance from the excitation coil can be approximated by equation 2.5[27].

$$
H=\frac{I}{2 \pi r}
$$




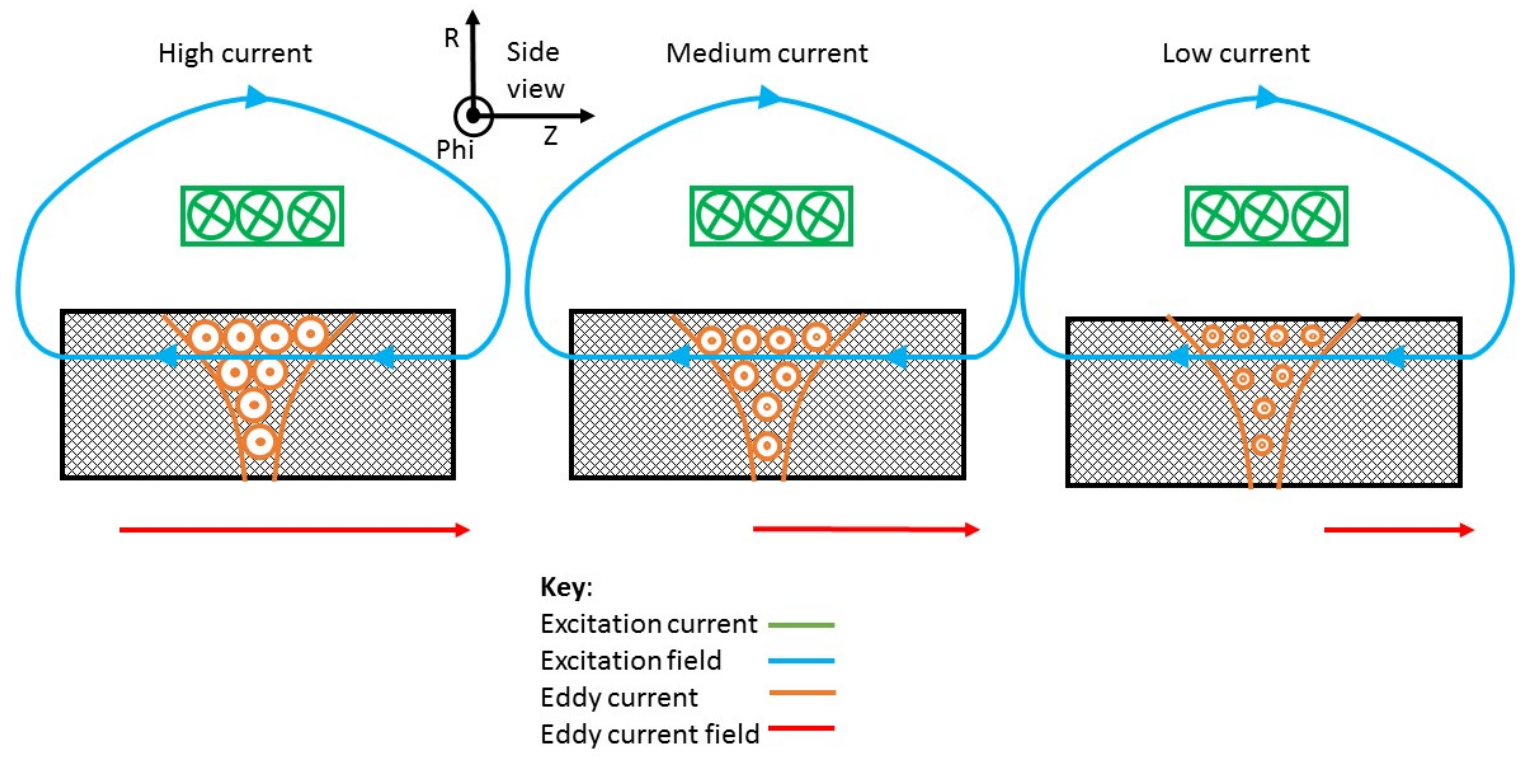

Figure 2.12: Effect of excitation current on eddy currents (The size of the orange circles represents the magnitude of eddy current)[28]

where

$$
\begin{aligned}
H & =\text { field strength }(A / m) \\
I & =\text { current in wire }(A) \\
r & =\text { distance from wire }(m)
\end{aligned}
$$

Increasing the current increases the magnetic field strength at the surface of the pipe, via Ampere's law (equation 2.1). The plot in figure 2.11 shows how the magnetic field decays with distance from the excitation coil. Close to the pipe, small changes in excitation current or position can have a large effect on the magnetic field. Large currents in the range of several 100 amps are considered in this thesis as the magnetic field of interest is created by eddy currents in the pipe. To produce signals large enough to be measured by commercially available sensors a large field needs to be present at the surface of the pipe to generate a large enough voltage to overcome the resistance of the steel. As a single turn coil was chosen to simplify the mechanical design, this required increased current to achieve the same amp turns to generate the required field.

The influence of the excitation current on the eddy currents is shown in figure 2.12. It shows how decreasing excitation current results in decreasing eddy currents. This drop in eddy currents is due to Faraday's law (equation 2.2), causing a drop in the opposing magnetic field that they generate.

Excitation frequency: The effect of changing the excitation frequency on eddy currents is shown in figure 2.13. The skin effect confines the eddy current close to the surface 


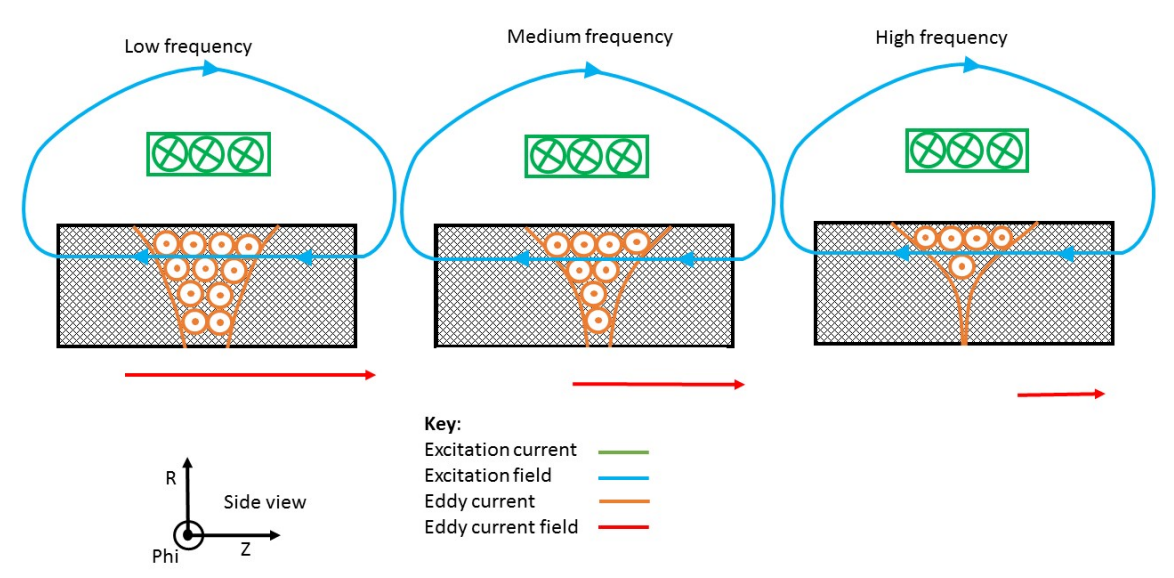

Figure 2.13: Effect of excitation frequency on eddy currents(The size of the orange circles represents the magnitude of eddy current)[28]

of the pipe as the excitation frequency increases, according to equation 2.4. The decay of the field becomes negligible when the penetration depth is roughly twice the wall thickness, as shown for the low frequency on the left side. Using equation 2.3 , only a $5 \%$ decay is seen in first $10 \%$ of the pipe. When the penetration depth reaches $10 \%$ of the wall thickness the eddy current in the surface becomes the dominating current. Using equation 2.3 a $95 \%$ decay is reached at $30 \%$ of the wall thickness. If the frequency values are in a medium range, then the eddy current in the upper part of the pipe wall is important. The pipe used in this thesis has a wall thickness of $9.1 \mathrm{~mm}$, lowest frequency used is 5 $\mathrm{Hz}$ (penetration depth $11 \mathrm{~mm}$ ) and highest frequency used is $200 \mathrm{~Hz}$ (penetration depth $1.5 \mathrm{~mm}$ ). Lower frequencies were not used due to size constraints on the size of the transformer and higher frequencies were not used due to drive voltage constraints from the amplifier.

To predict how changing the excitation frequency effects the excitation field, the changing current density profile in the excitation sheet has to be considered. The excitation coil is made up of a stack of very thin strips of copper which means, as the frequency increases, the current concentrates at the edge of the sheet due to lower opposition from back EMFs [29]. This changing distribution of current causes a change in the magnetic field. The current drops in the center of the sheet and increases at the edge with increasing frequency. This results in a decrease in the magnetic field in the center and an increase at the edge.

This prediction was further confirmed by finite element modeling with ANSYS Maxwell [30] of a $40 \mathrm{~mm}$ wide single turn excitation coil. Figure 2.14 shows the finite element model of the excitation coil. A 3D FEM model was used as it provided detailed information about the field near the coil, both inside and out, that could not be easily found with a analytical or 2D FEM approach, due to the coil having a rectangle cross-section. The concentration of current in the edge of the sheet at high frequencies is seen in figure 2.15. The concentration is symmetric as this simulation was done without the pipe.

Figure 2.16 shows a plot of the magnetic field strength vs the distance from the exci- 


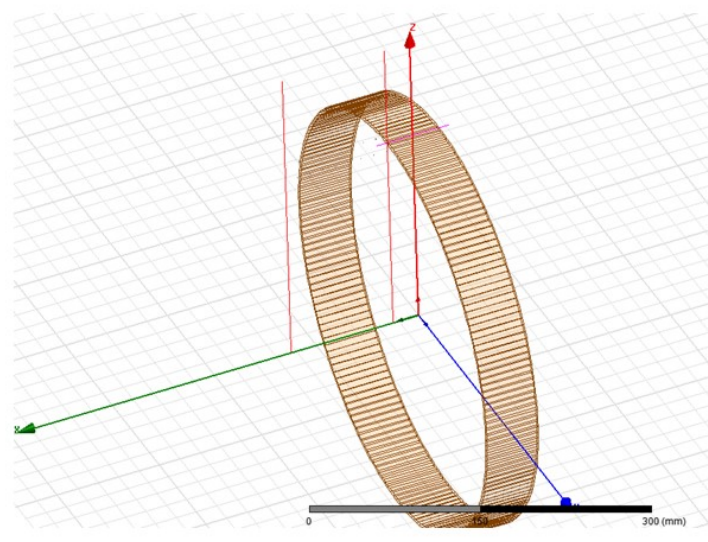

Figure 2.14: Finite element model of excitation coil [30]

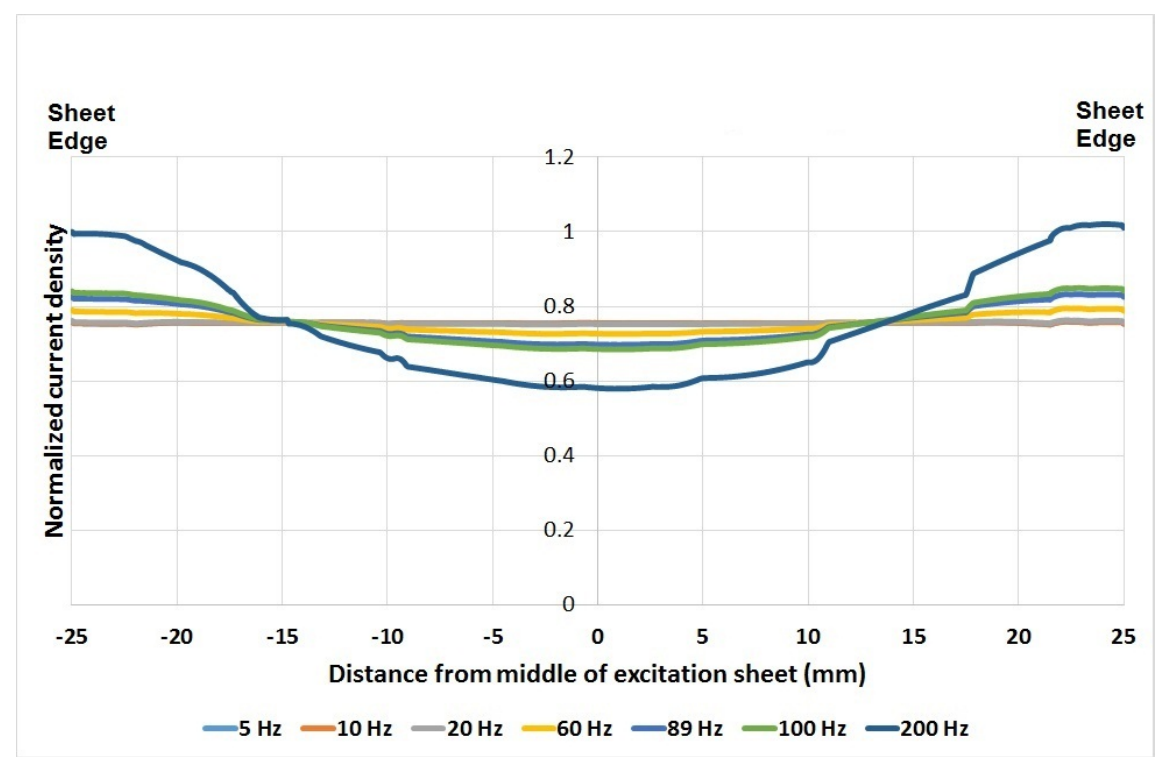

Figure 2.15: Normalized current density across excitation sheet change with excitation frequency [30] 


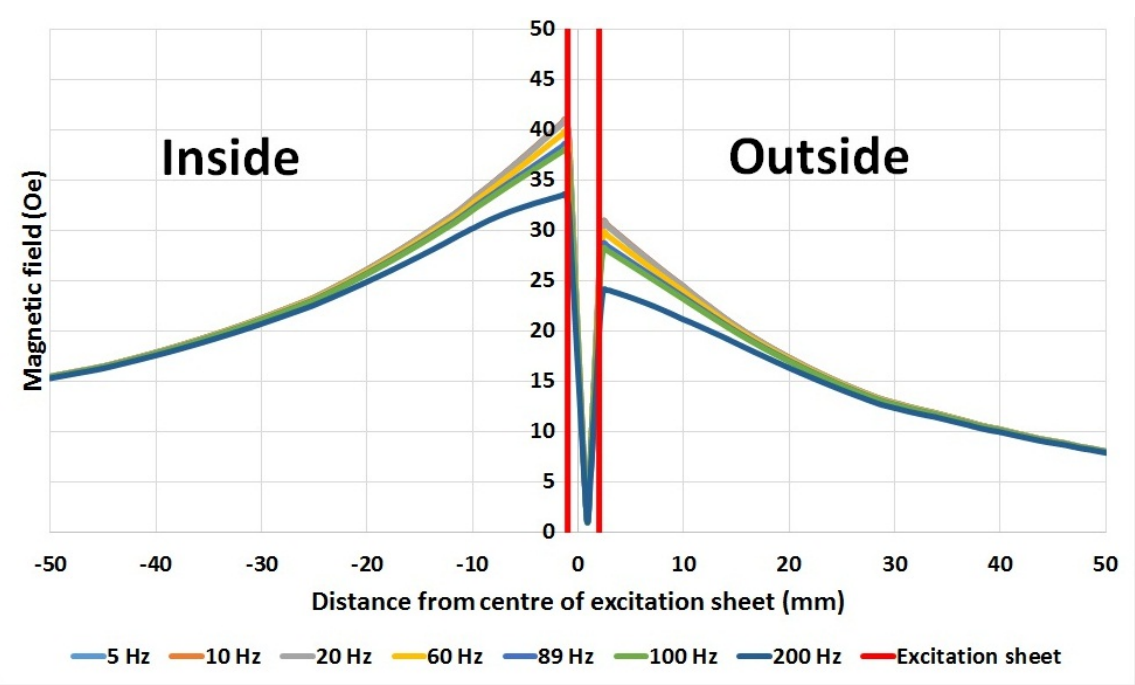

Figure 2.16: Magnetic field change with distance from excitation sheet, through the center of the excitation sheet [30]

tation coil, through the center excitation sheet. Figure 2.17 shows a plot of the magnetic field strength vs the distance from the excitation coil, through the edge of the excitation sheet. This shows the expected drop in field with increased frequency at the center and vice versa at the edge.

Excitation lift-off: The effect of changing the excitation lift-off on the excitation field is shown in figure 2.18. At small lift-off the field is distorted towards the pipe which compresses the field on the side opposite the pipe. The field above the pipe side spreads out so that it is almost constant between the pipe and the excitation coil. As the pipe moves away this asymmetry of the field around the conductor reduces until the pipe has moved sufficient distance and does not distort the field lines, as seen in figure 2.18. This means that if a sensor is placed outside the excitation coil, at a distance e.g position 2 from the pipe, the field strength will increase as the pipe is moved away and the field becomes symmetrical.

Since the magnetic field at the surface of the pipe increases with decreasing lift-off and the eddy current in the pipe is proportional to the magnetic field at the surface, increasing the lift-off will have the same effect as reducing the excitation current.

\subsubsection{Explanation of defect signals}

In a defect free pipe the eddy currents circulate counter-clockwise to the excitation current, solely in the circumferential direction. This produces the opposing field via Lenz's law. For a completely uniform pipe, with the sensor module mounted in the center of the excitation sheet, only a field in the longitudinal direction of the pipe would be measured.

When a defect is introduced into the pipe, such as a box defect shown in figure 2.19, the eddy current is rerouted to flow around the defect. It is this rerouting of eddy currents that produces a change in the magnetic field. The change in magnetic field is measured in 


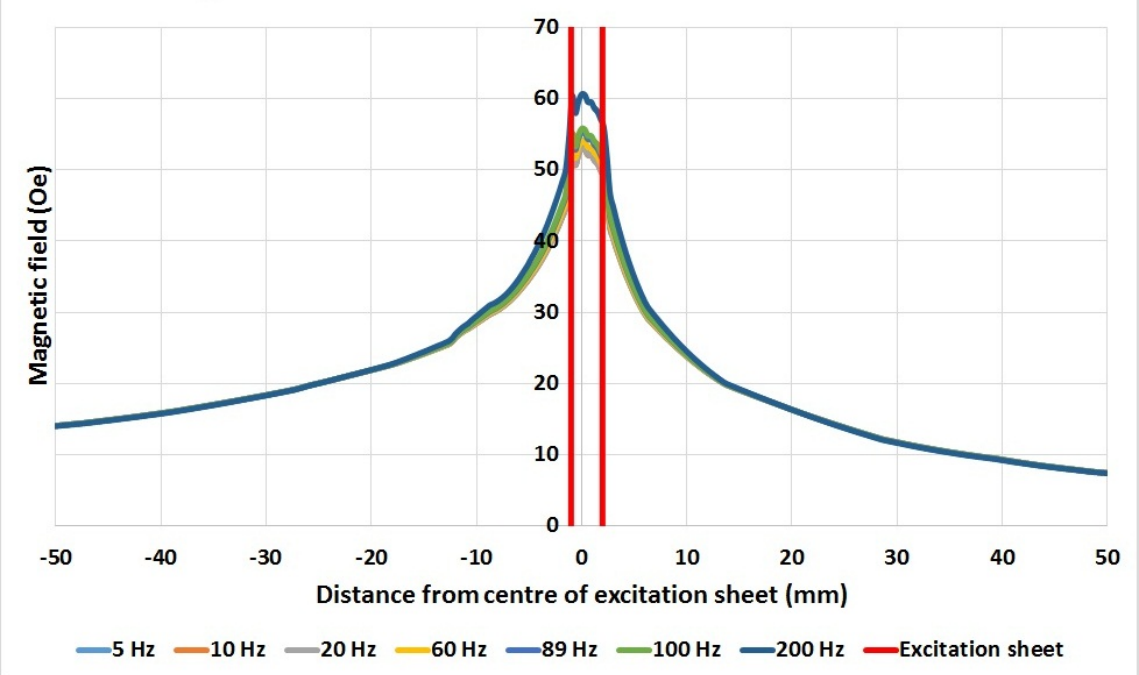

Figure 2.17: Magnetic field change with distance from excitation sheet, through the edge of the excitation sheet [30]

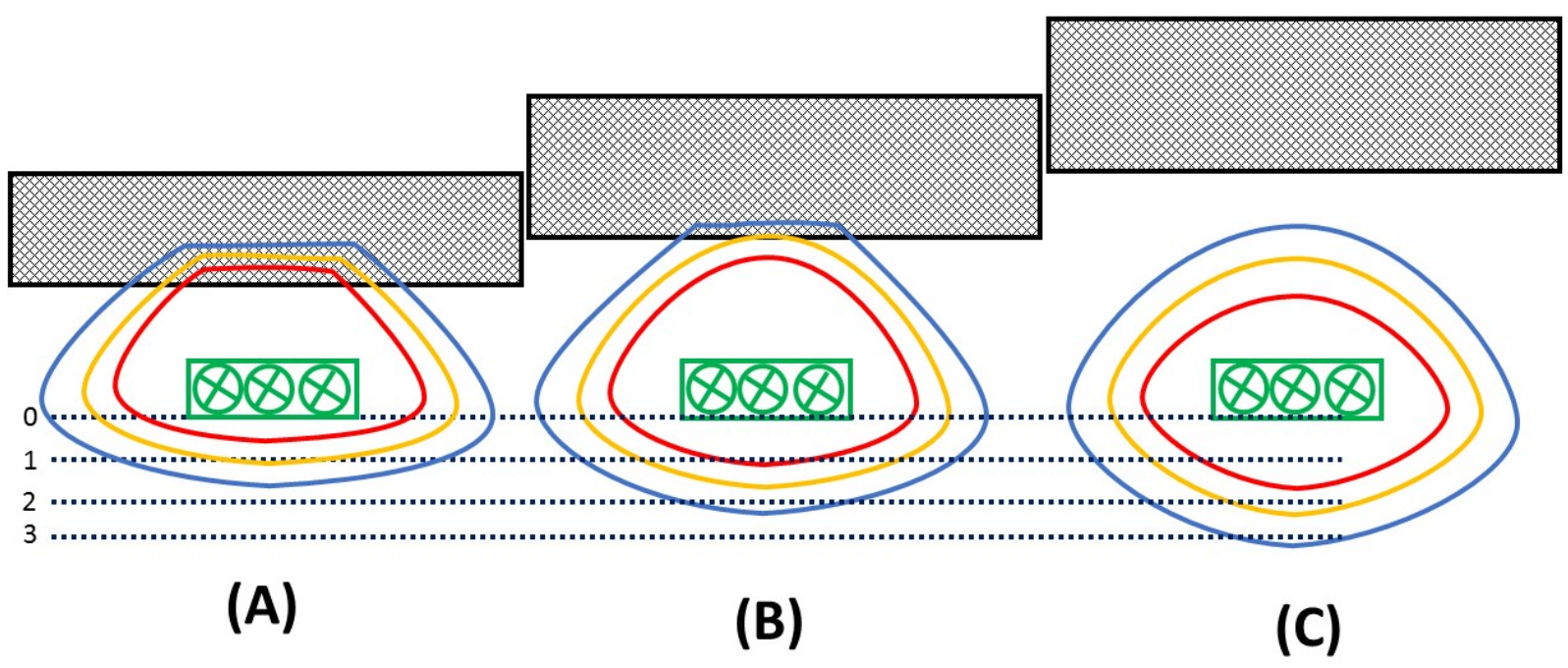

Figure 2.18: Effect of changing excitation lift-off on excitation field (A) low lift-off with high excitation field in pipe, (B) middle lift-off with moderate excitation field in pipe, (C) High lift-off with low excitation field in pipe(dashed lines show lines of constant distance from excitation coil) 

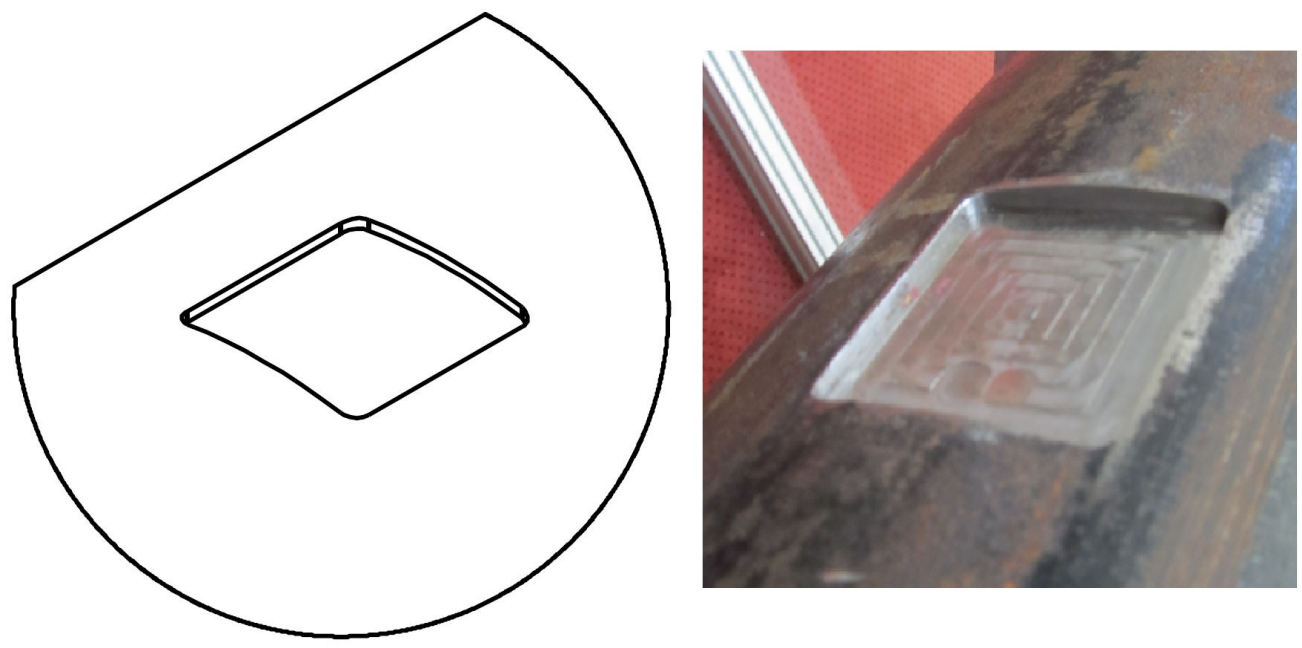

Figure 2.19: Box defect in pipe with insulation removed

a cylindrical coordinate system with the three axes being:

1. $Z$ axis which is parallel with the pipe axis.

2. R axis which points outward from the center of the pipe.

3. Phi axis which is tangential to the pipe surface

These three sensing directions are shown in figure 2.20 with a three sensor module shown above the pipe. To visualize how the eddy currents are distorted, 3 different views of the defect are used.

1. Bird's eye view looking down the $\mathrm{R}$ axis (figure 2.21)

2. Phi cross-section looking down the $Z$ axis (figure 2.23)

3. Z cross-section looking down the Phi axis (figure 2.22)

\section{$Z$ axis}

The defect distortion of the eddy currents around the $Z$ axis is shown in figure 2.24. The excitation current flows anti-clockwise around the pipe (green) causing the eddy currents to flow clockwise in the pipe wall (orange). When the eddy current approaches the defect it has to first bend clockwise then anti clockwise to flow around the defect. The bending of the current produces a field in the $Z$ direction, clockwise bending producing negative fields and anti-clockwise bending producing positive fields. As the sensor is moved in the Phi direction, a maximum field is expected in the middle of the defect because the curling at the bottom corners of the defect is anti-clockwise. At the edge of the defect, positive and negative fields from the eddy currents curling counter-clockwise to each other at the top and bottom of the defect start to cancel each other. The bird's eye view in figure 2.24 


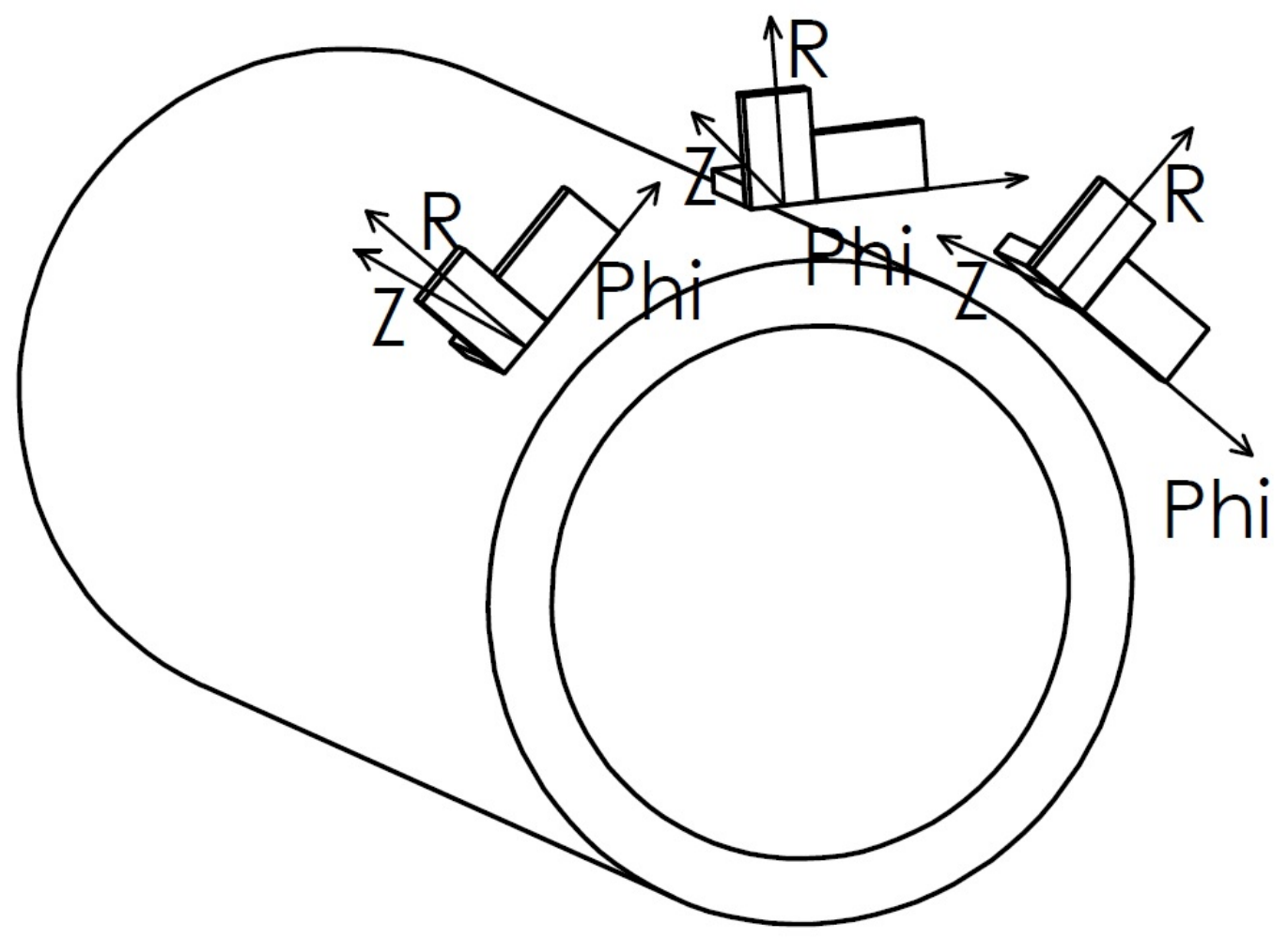

Figure 2.20: Sensor axis definition

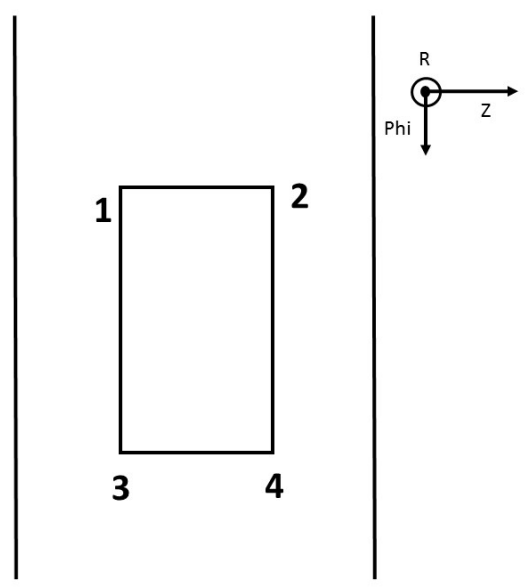

Figure 2.21: View along $\mathrm{R}$ axis 
ib.

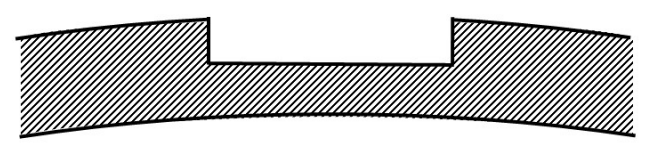

Figure 2.22: View along $Z$ axis
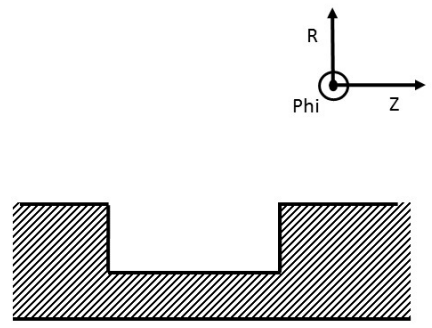

Figure 2.23: View of box defect along Phi axis 


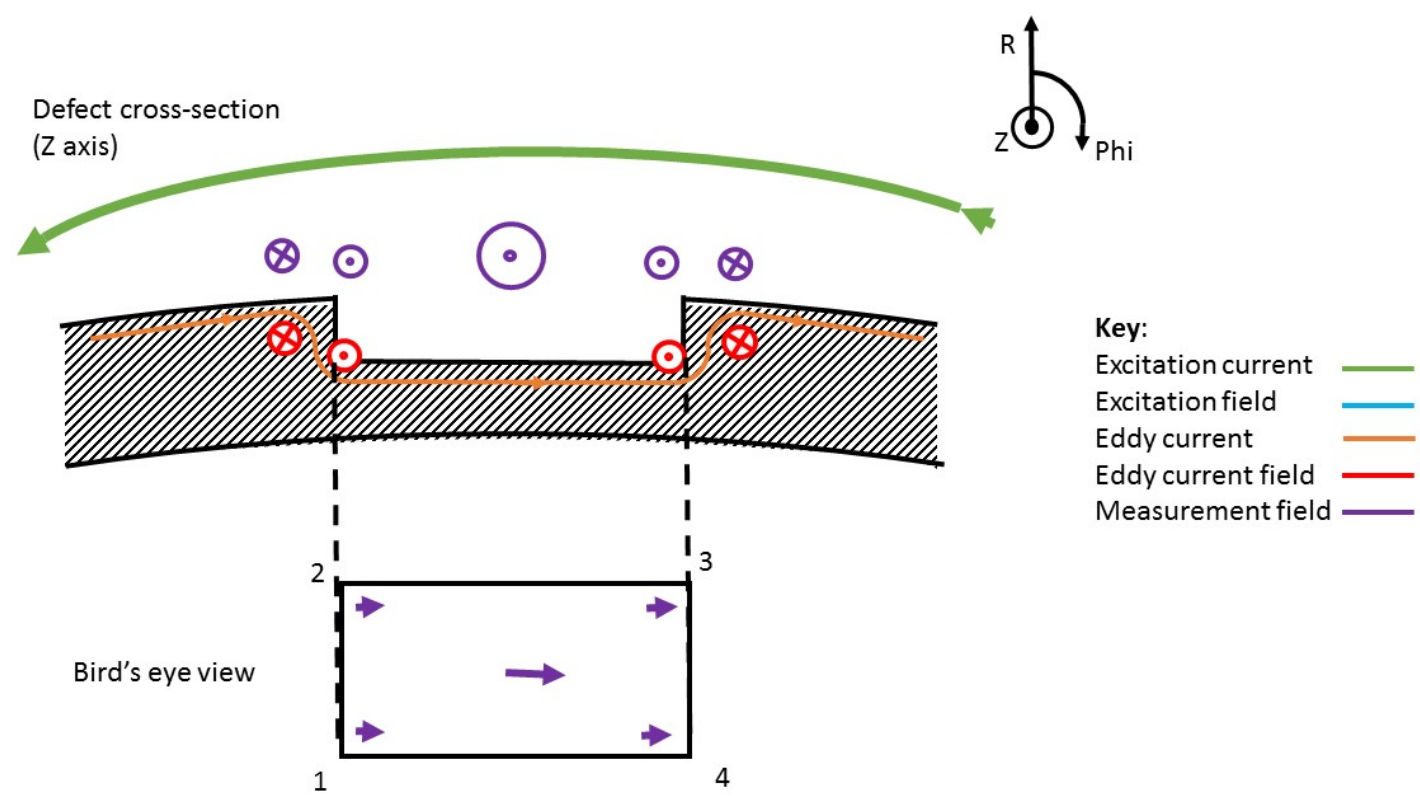

Figure 2.24: Effect of defect on eddy current in the $Z$ axis

shows how the field in the center of the defect must be a maximum as at this point the excitation sheet center is over the center of the defect and the maximum amount of eddy current is redirected under the defect.

\section{$R$ axis}

To show how the eddy currents are distorted around the $R$ axis a bird's eye view of the defect aligned with the $R$ axis is shown in figure 2.25 and the measured $R$ field over a defect is shown in figure 2.26. The figure 2.26 shows the eddy currents (orange) flowing in the opposite direction of the excitation current (green). When the eddy current approaches the defect it is re-routed around the defect, first it bending in one direction and then the other. This re-routing of the eddy current is emphasized in the drawing as 90 degree turns. When the current is bent anti-clockwise a field is generated that goes into the plane while, when the current bends clockwise, a field is generated coming out of the plane. In position A the excitation sheet is centered above the 1-2 edge of the defect and in position $B$ the excitation sheet is centered over the 3-4 edge of the defect. On the 1-2 edge a large positive field is expected in the middle (purple) as positive fields generated at each corners 1 and 2 are added together. As the sensor approaches either corner 1 or 2 , the field is expected to decay as the negative field generated on the 2-3 edge or 1-4 edge starts to nullify the field at the corner of the defect.

When the excitation sheet is moved to the 3-4 edge, the eddy currents are distorted in the opposite direction as this is now the shortest path around the defect. This means that the same value but opposite field is expected to be measured on the 3-4 edge. 


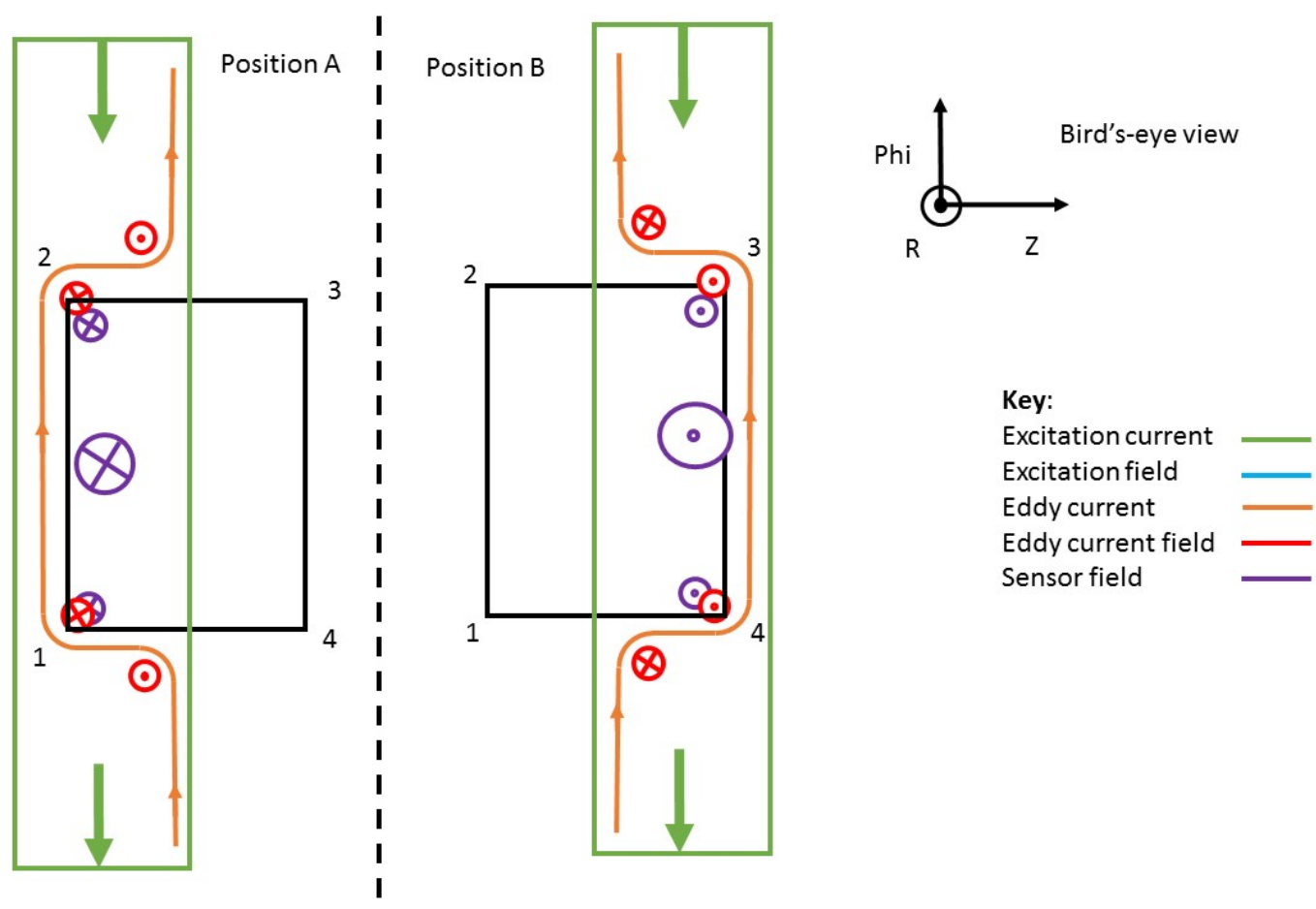

Figure 2.25: Eddy current distortion around $\mathrm{R}$ axis

When the excitation sheet is over the center of the defect, equal amounts of current can be expected to re-route either side of the defect. The measured field as a sum of both fields will be zero.

\section{Phi axis}

To understand how the field is created in the Phi direction the flow of the eddy current in all three directions must be considered. In figures 2.24 and 2.25 the eddy current redirection was presented as if it was only distorted in one plane. However, the eddy currents are redirected in all planes as it has to be redistributed around all the defect's edges. This range of distortions is shown in figure 2.27. Figure 2.28 shows the measured Phi field over a defect. The eddy currents (orange), when close to the edge of the defect, are mainly re-routed around the $R$ axis and, if close to the center, are rerouted around the $Z$ axis. In between the extremes of the edge of the defect and the center, the eddy current is distorted in a mix around the $\mathrm{R}$ and $\mathrm{Z}$ axes. This changing axis of distortion means a component of the eddy current is now distorted anti-clockwise around the Phi axis at corner 1, producing a positive field, and clockwise at corner 2 producing a negative field, shown in figure 2.27. Four different cases are shown in figure 2.27. Case A has the center of the excitation sheet over the 1-3 edge with the sensor over corner 1, Case B has the center of the excitation sheet over the 2-4 edge and the sensor over corner 2, Case $\mathrm{C}$ has the center of the excitation sheet over the 1-3 edge with the sensor above corner 3 , and Case $D$ has the center of the excitation sheet over the 2-4 edge with the sensor over corner 4. 


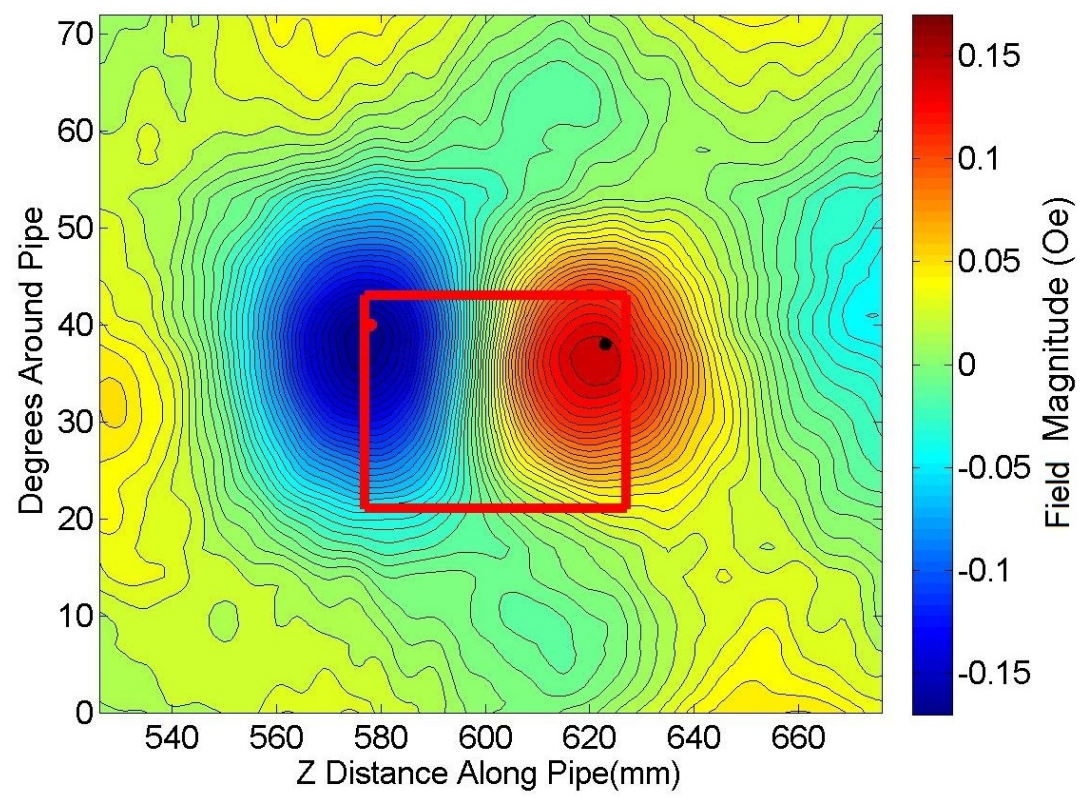

Figure 2.26: Measured magnetic field in the $\mathrm{R}$ direction over a defect

On the 1-2 edge of the defect the eddy currents (orange) are being distorted and are flowing towards the 1-3 and 2-4 edges of the defect. On the 3-4 edge the eddy currents are returning to their original distribution so the eddy currents are flowing away from the defect's 1-3 and 2-4 edges. This reversal of current direction from the 1-2 edge to the 3-4 edge means that the magnetic fields produced (red) switch polarity. The bird's eye projection in figure 2.27 shows how this affects the measured field (purple) at each corner of the defect with diagonally opposite corners having fields of the same polarity. Here the peaks are expected at the corners as this is the point where it is as far as possible from the neighboring corners whose field opposes the field of interest. This means that at the center of each defect edge the Phi field should be zero since the eddy currents at each corner bend counter clock-wise to each other.

\subsubsection{Summary}

There are two general sources of variation that will be observed during measurement.

- Changes in the excitation parameters will change the average magnetic values over the whole pipe. Specifically, increasing the excitation current will increase the average field, increasing the frequency will decrease the average field, and increasing lift-off will increase the average field.

- Change in the pipe such as conductivity variations, permeability variations or defects will cause variation in the magnetic field across the surface of the pipe. Specifically, in the $R$ field one pair of peaks of opposite polarity will be seen on each $Z$ edge of the region of change. In the Phi field, a set of four peaks on each corner of the region of change can be expected with the same polarity on diagonally opposite corners. 


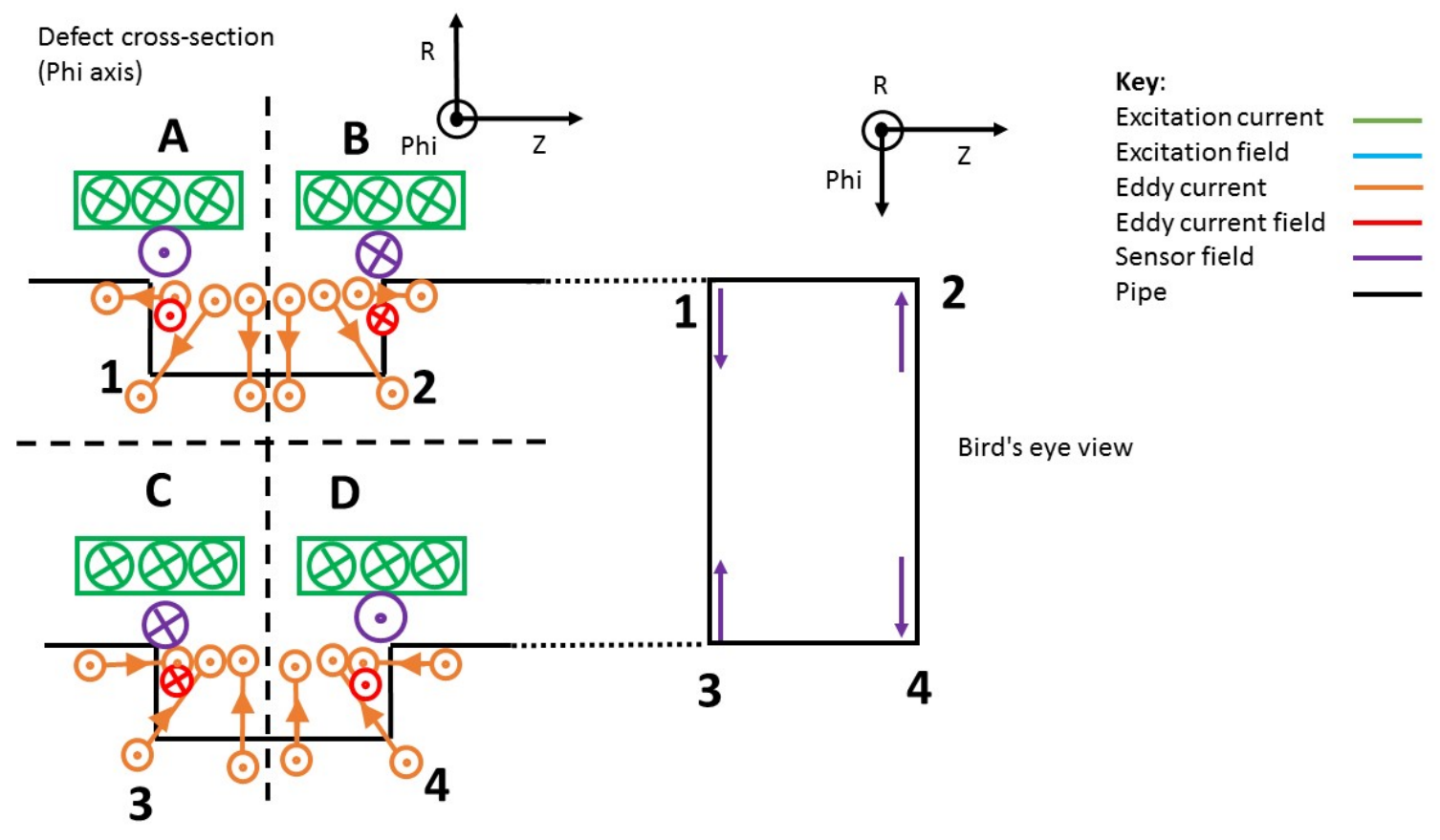

Figure 2.27: Eddy current distortion around Phi axis

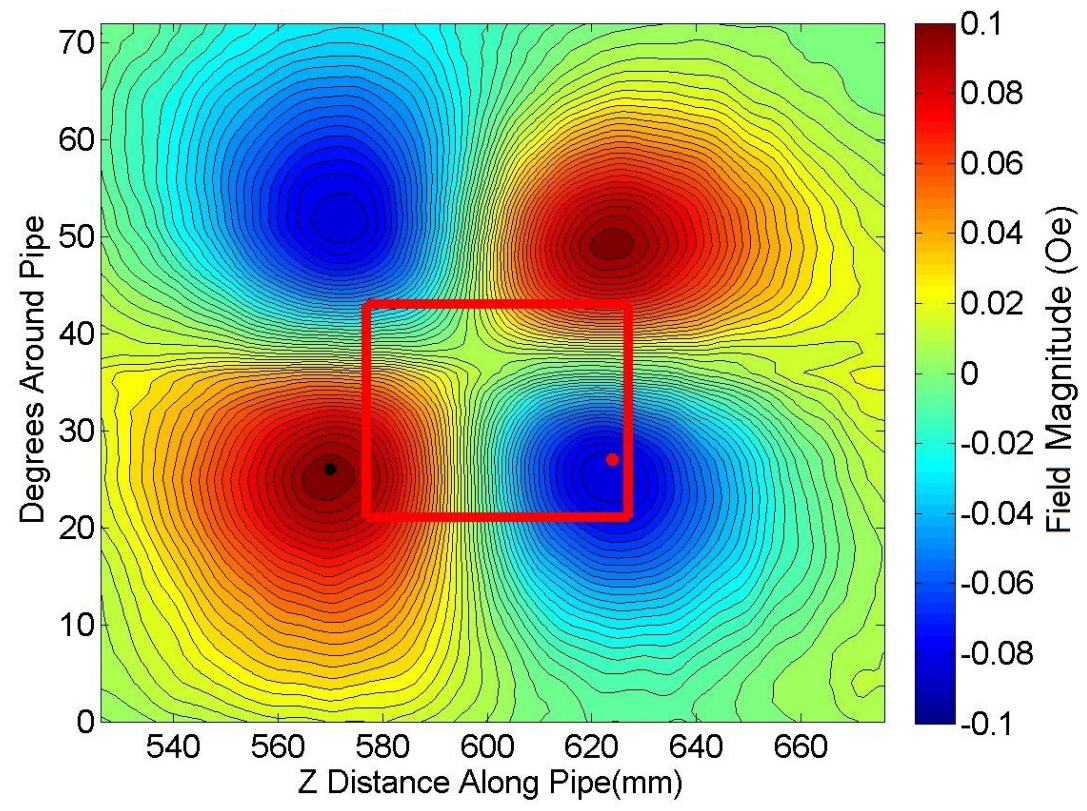

Figure 2.28: Measured magnetic field in the Phi direction over a defect 
The variations in the magnetic field are due to redistribution of eddy currents within the pipe wall. 


\section{Chapter 3}

\section{System Design}

This chapter describes the design of the whole measurement system as shown in figure 3.1. The description is broken down into four sections which are each covered in turn. These sections are:

- The mechanical setup which is compromised of three parts:

- The system for supporting and moving the pipe .

- The system for supporting and moving the excitation and sensor module.

- The system automation.

- The measurement system which is compromised of three parts:

- The sensor module which is mounted directly above the pipe and contains the GMR sensors.

- The support electronics which provide interfacing electronics between the sensor module and the off-the-shelf instrumentation.

- The off-the-shelf instrumentation that takes the magnetic field measurements and connects to the main LabVIEW program.

- The excitation system which provides the exciting magnetic field to the pipe and covers all hardware needed to deliver the large magnetic field with a high current excitation coil.

- The data analysis algorithms which take the raw sensor voltage data and convert it to meaningful field values and determine basic characteristics of the defect signals.

Note: The description of the measurement system and the excitation system both start with the initial design of the system and then a description of improvements to ensure that accurate data could be measured. 


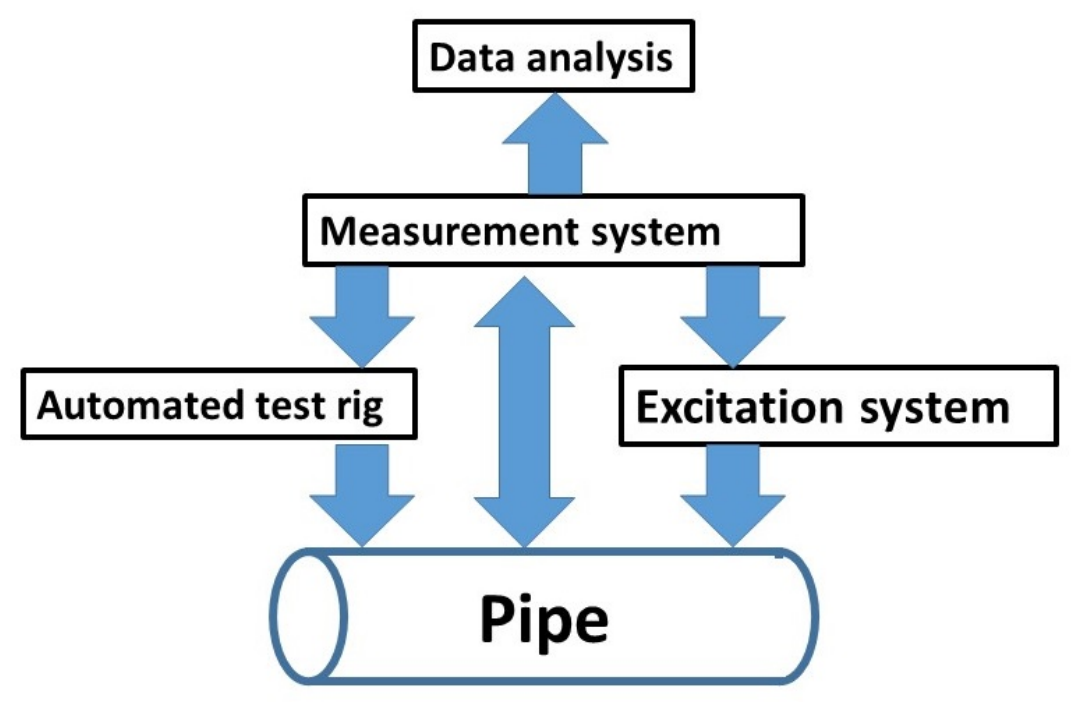

Figure 3.1: High level system diagram

\subsection{Mechanical setup}

This project started with a basic mechanical setup that could be used to mount and manipulate test pipes and sensor modules. This section describes the details of the mechanical setup including the pipe support and movement, sensor and excitation support and movement and lastly measurement automation.

Pipe support and movement: The basic set-up is pictured in figure 3.2. The mechanical setup consists of an adjustable frame with mounting system for a pipe up to $100 \mathrm{~kg}$, $1.3 \mathrm{~m}$ in length and $500 \mathrm{~mm}$ in diameter. The pipe mounting system fits machined plates into each end of the pipe with accurately machined center holes. Centering cones on the end of each of shafts are pushed into these holes in order ensure that the pipe is centered on the rotation axis. The rotation axis was driven by a servo motor and a 500:1 gearbox to allow high torque and fast, accurate positioning.

Sensor and excitation support and movement: The setup includes a linear stage parallel to the pipe that allows an excitation and measurement unit to be moved down the length of the pipe. The linear stage consists of two belt driven linear stages coupled by a shaft at one end. The shaft ensures that the two stages move in unison, thus stopping racking action of the excitation unit being moved down the length of the pipe. The linear stage is driven by a stepper motor through a 5:1 gear box to allow for sub-millimeter movement. The major drawback of this setup is that the rotational axis is not exactly parallel to the linear axes. This means that the excitation ring is not co-centric with the pipe for the full length of the pipe. This may lead to some small variations due to the sensor to pipe distance changing. 


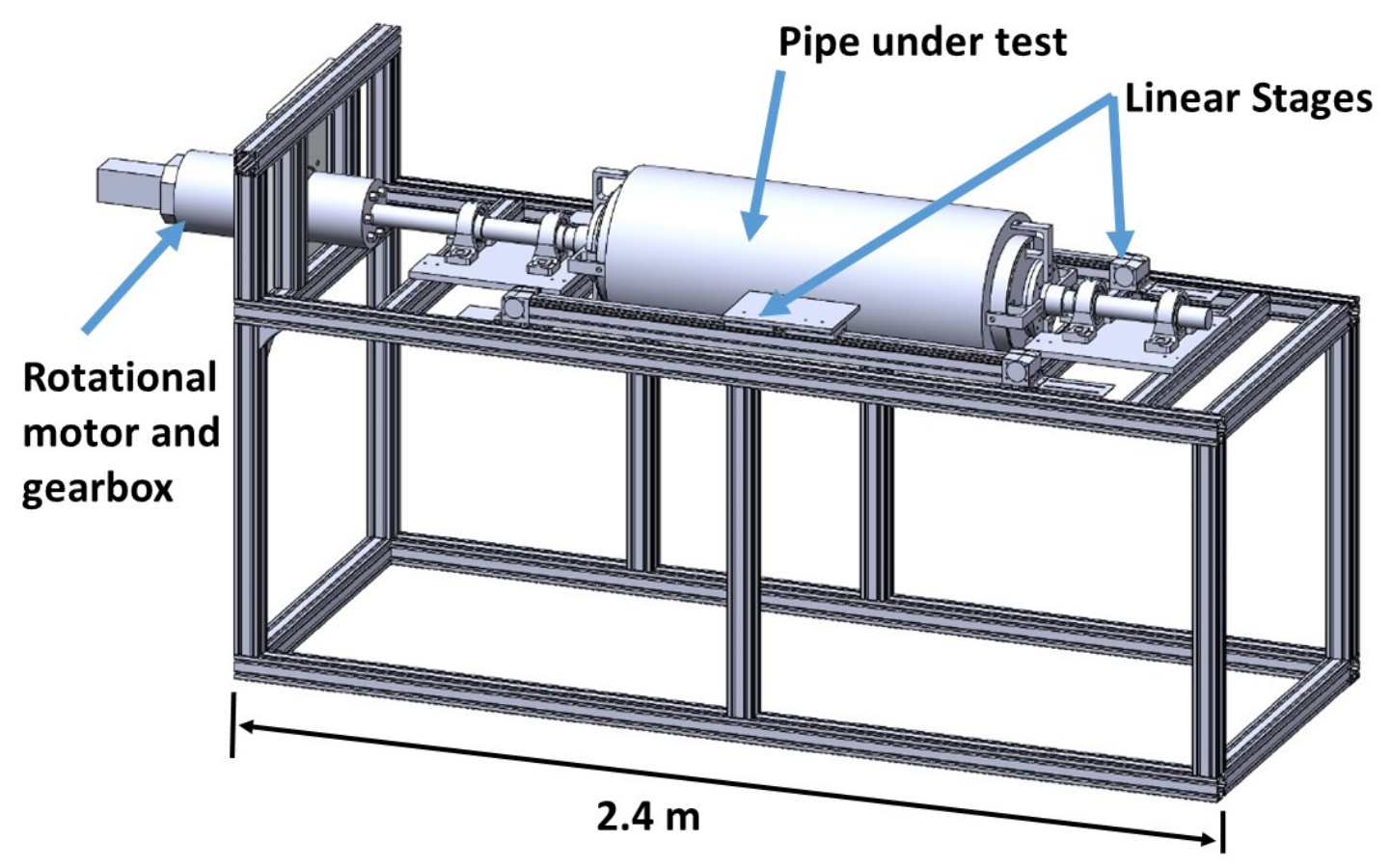

Figure 3.2: CAD drawing of test setup

Measurement automation: To allow a large number of measurements, the mechanical setup needed to be automated. The aim was to control the movement of both axes by a LabVIEW program that provided overall coordination of measurements. This was achieved by installing a Trio motion controller to command the two motor drivers for each of the axes and also to record the position of each axis. The Trio motion controller was controlled over an RS-232 link by a LabVIEW code which sends flags to execute different types of movements. It then waits, after sending a movement flag, for a confirmation signal to be sent to confirm movement completion. A simplified connection diagram of the automation hardware is shown in figure 3.3.

\subsection{Measurement system}

A block diagram of the measurement system is shown in figure 3.4. The initial aim of the measurement system was to measure the magnetic field magnitude and phase shift from excitation in three perpendicular axes. A cylindrical coordinate system was used and the three axes are:

1. $Z$ axis which is parallel with the pipe axis.

2. R axis which points outward from the center of the pipe.

3. Phi axis which is tangential to the pipe surface 


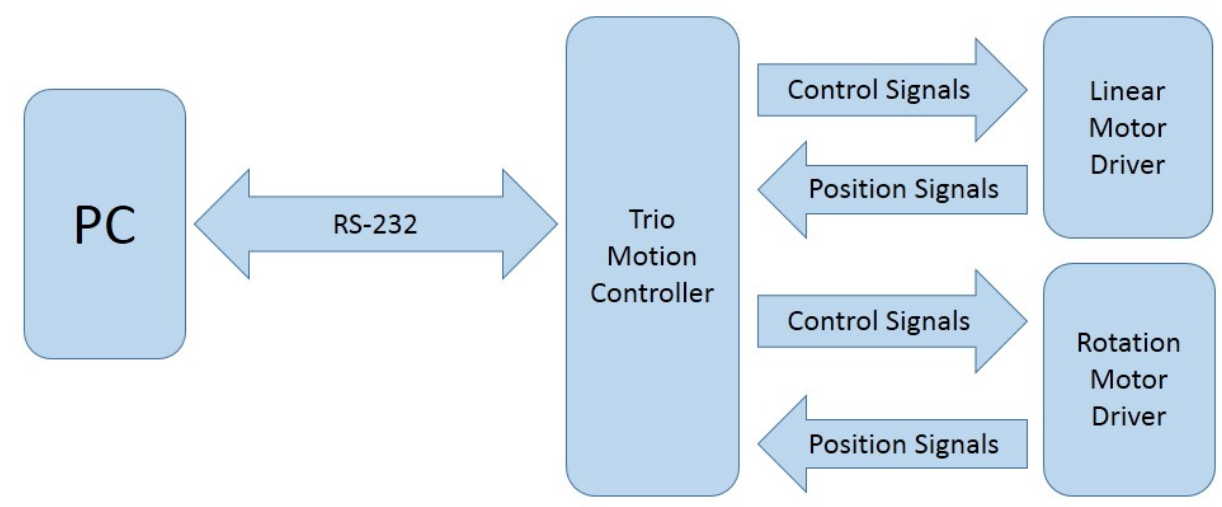

Figure 3.3: Simplified movement automation system

These three sensing directions are shown in figure 3.5 with a three sensor module shown above the pipe with the three sensing axes shown for each sensor.

The system can be broken down in to three general parts:

1. The sensor module which is mounted directly above the pipe and holds the magnetic sensor and any biasing hardware that is required to determine the output.

2. The support electronics including all electronics required for interfacing the sensor module to the of-the-shelf instrumentation.

3. The of-the-shelf instrumentation that takes the magnetic field measurements and interfaces with the main LabVIEW program.

The initial design of these parts is described in section 3.2.1 while the improvements that were made are covered in section 3.2.2.

\subsubsection{Initial measurement system}

Sensor module: The initial design of the sensor unit is pictured in figure 3.6. The sensor module is designed around three NVE AA004 sensors. The NVE AA004 sensors only measure the magnetic field in one axis, called the sensitivity axis, as shown in figure 3.7. To measure three perpendicular magnetic fields, so that a full 3D magnetic field vector can be evaluated, the three sensors are placed with their sensing axis perpendicular to each other. This is achieved by placing two of the sensors perpendicular to each other on one PCB while a second PCB has a single sensor and is mounted perpendicular to the first 


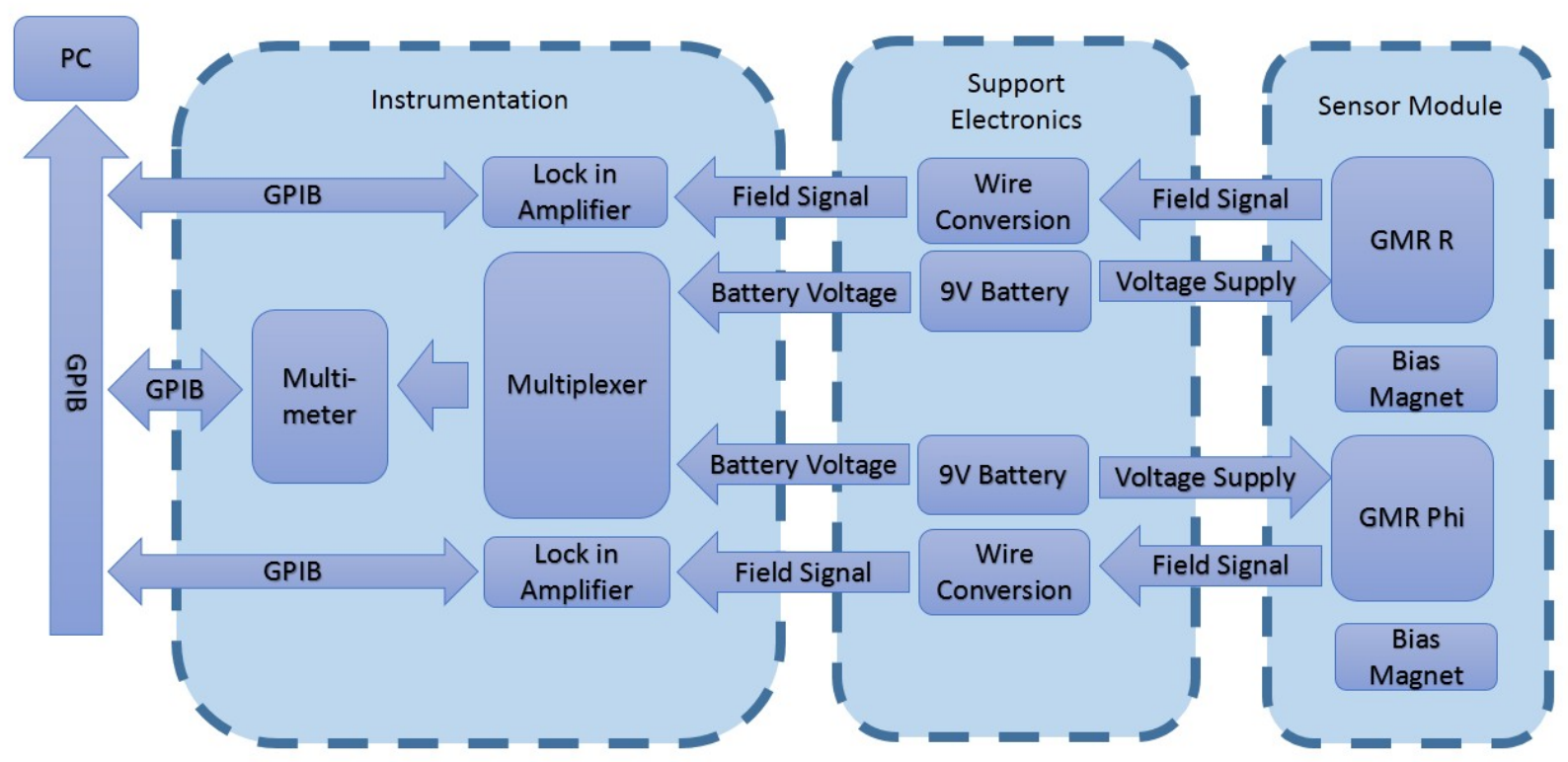

Figure 3.4: Measurement system design

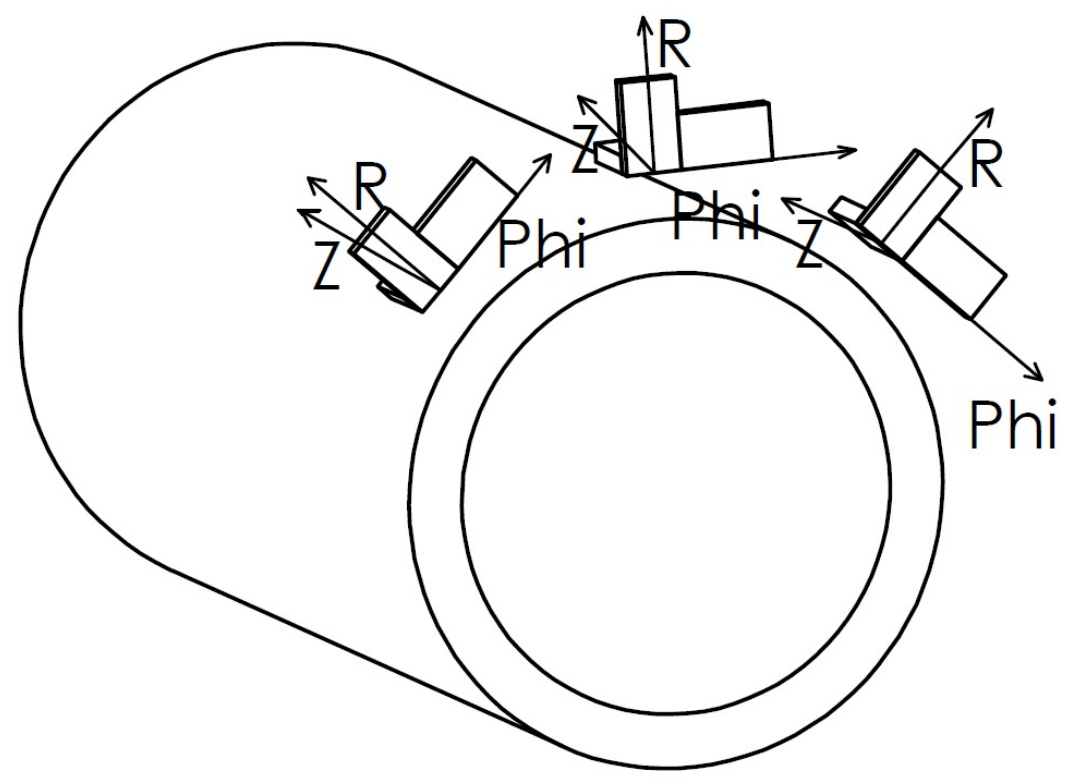

Figure 3.5: Sensor axis definition 


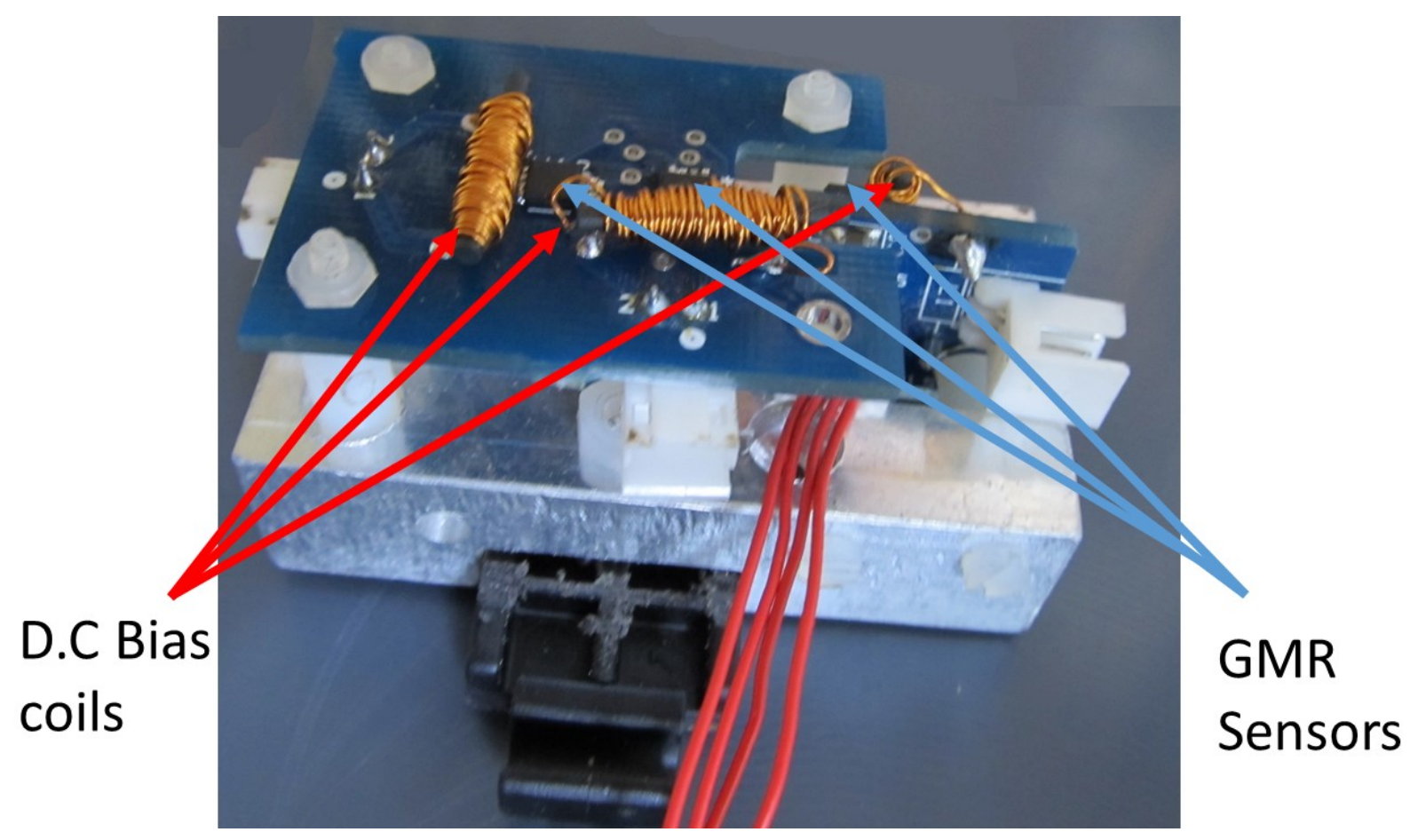

Figure 3.6: The first sensor module design

PCB. This configuration means that there is a small circumferential offset between each of the sensors, this will produce a small space related error in this axis. As the sensors are unipolar, as shown by the response curve in figure 3.8, the sensors require DC bias to operate effectively. The modified response curve when a 10 Oe bias field is applied is shown in figure 3.9.

The DC biasing was achieved by placing a small coil with an iron core near each sensor. These coils allowed the DC field offset to be tuned by adjusting the current in them $(0-0.8$ amps). The only other electronics on the sensor module were a $1 \mu \mathrm{F}$ capacitor between the power rails on the PCB to ensure a stable supply voltage to the sensors. A small connector on each PCB allows for attachment of wires to route the signal and power rails from a nearby interface board. The sensor module is mounted in a segment of a flexible cable chain providing a simple modular unit that could be added to and easily clipped around the pipe.

Support electronics: The support electronics were built onto an interface board pictured in figure 3.10. The interface board provided a power supply to the GMR sensor in the form of a $9 \mathrm{~V}$ battery. A $9 \mathrm{~V}$ battery was used to generate a steady power supply with no need for extra electronics. A tap was taken off the battery terminals and routed back to the instrumentation rack so that long term drift of the battery voltage could be recorded and corrected for. The interface board also included a constant current source for each bias coil in the sensor module. The constant current was made with a low dropout $1.2 \mathrm{~V}$ linear voltage regulator (NCV565ST12T3G) with a trim pot that allowed for current adjustment. Finally the interface board converted small unshielded signal wires from the sensor module 


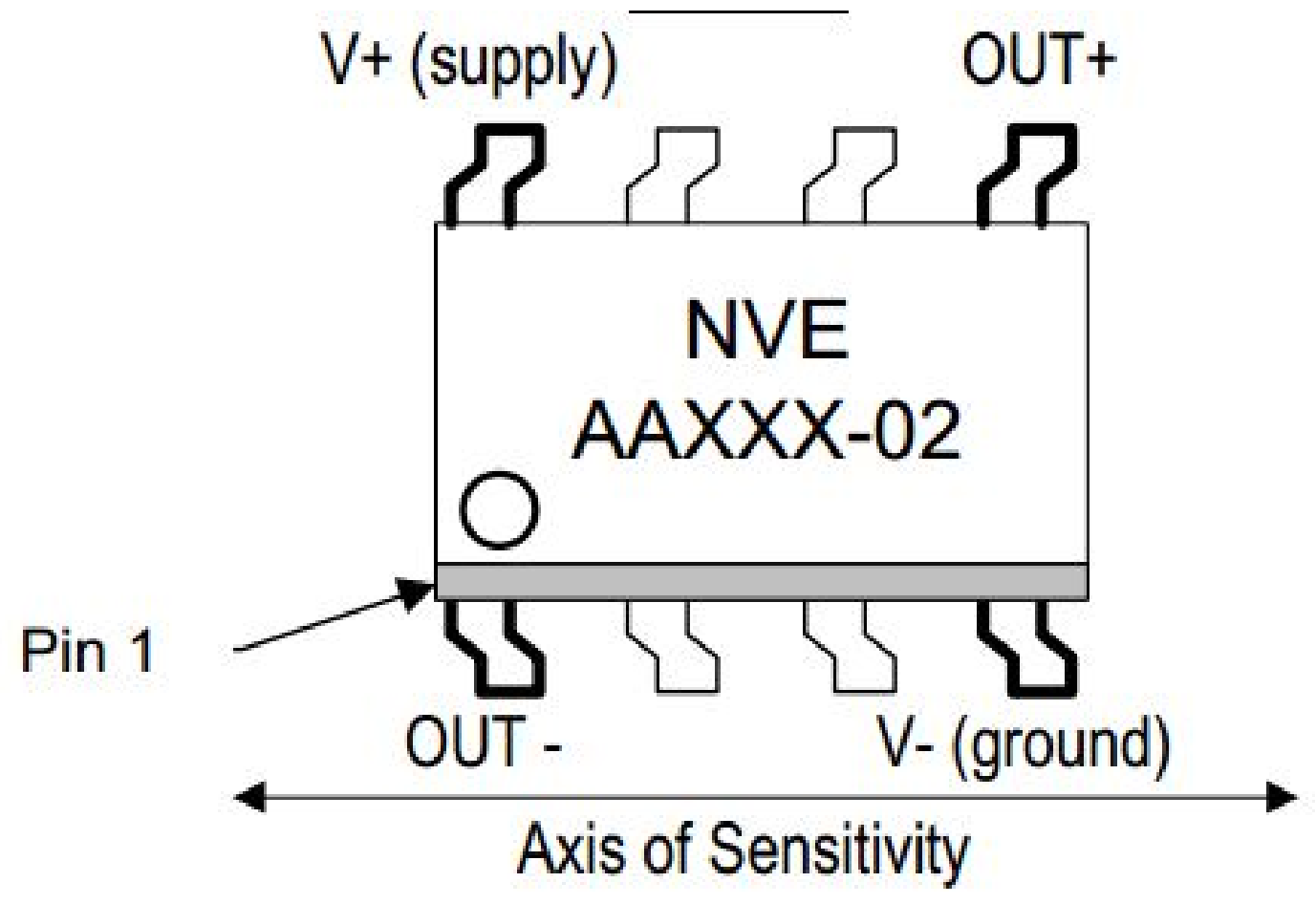

Figure 3.7: Sensitivity axis of NVE 004 (from NVE data-sheet [17])

to large shielded twisted pairs to route the signals back to the instrumentation rack.

Off-the-shelf instrumentation: The instrumentation system was all mounted in a 19 inch rack pictured in figure 3.11. The measurement of magnitude and phase of the magnetic field was done with a SR830 lock-in amplifier from Standford Research. The high accuracy of the lock-in amplifier is achieved by locking in on the excitation frequency of the system so that only signals in a very narrow bandwidth are measured. To allow for measurement of all three sensors, an Agilent 34970A multiplexer is used to switch the signal from each sensor to the lock-in amplifier inputs in turn. An HP 34410A multimeter was used to measure the battery voltage so the sensor voltage levels could be converted to a magnetic field via the sensitivity value for the AA004 sensor provided in the data-sheet in appendix A. All three instruments were connected over a GPIB bus to a PC which allows the LabVIEW program to read and record the measurements.

\subsubsection{Improvements to Measurement system}

Initial testing of the system revealed several issues that needed to be addressed to ensure accurate data. Each of the three sections of the measurement system are looked at in turn with improvements presented and their effects discussed.

Sensor module: The first problem identified with the sensor module was that the bias 


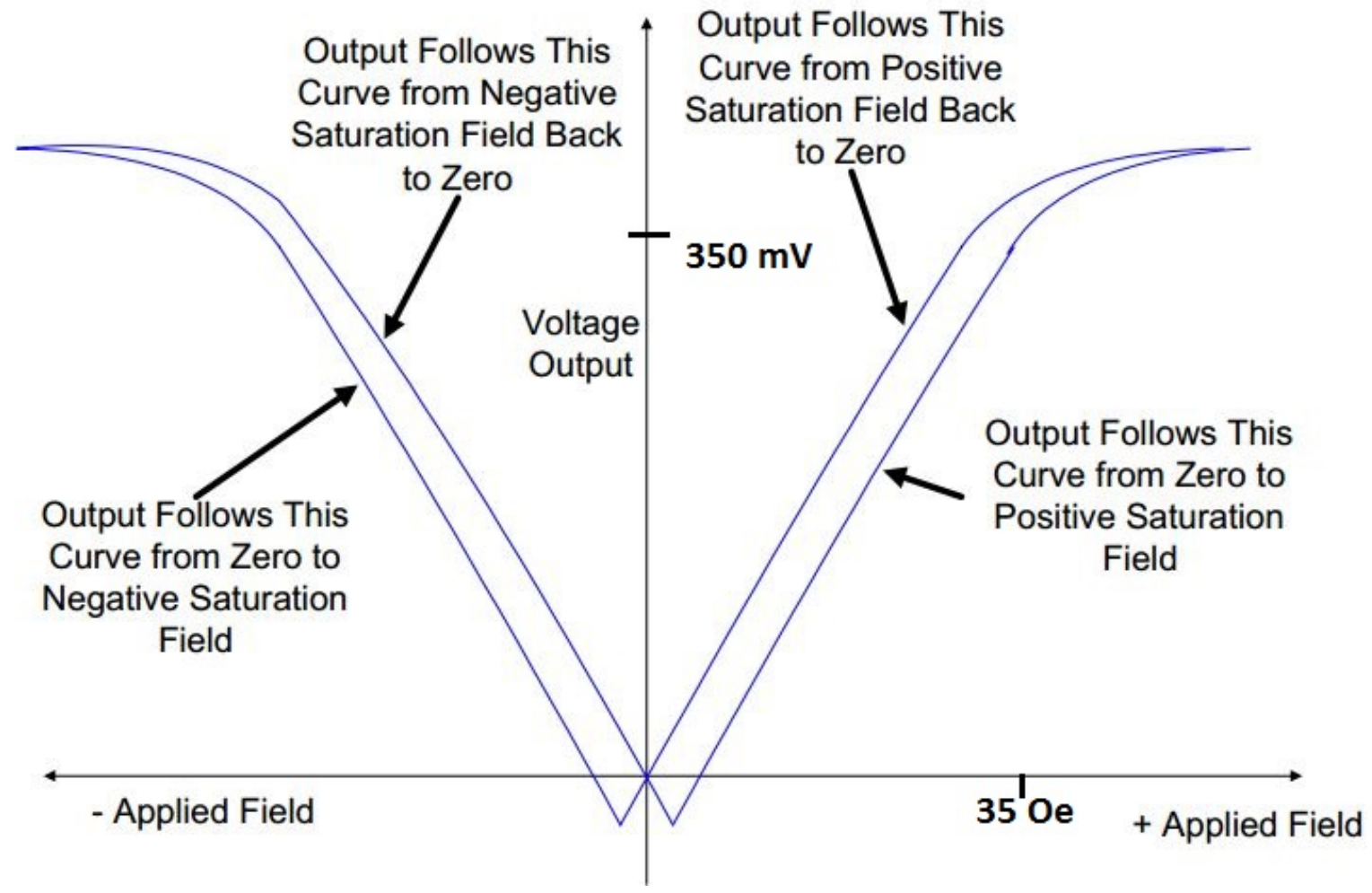

Figure 3.8: NVE GMR sensors unipolar response to bipolar magnetic field (from NVE GMR sensor application note [16])

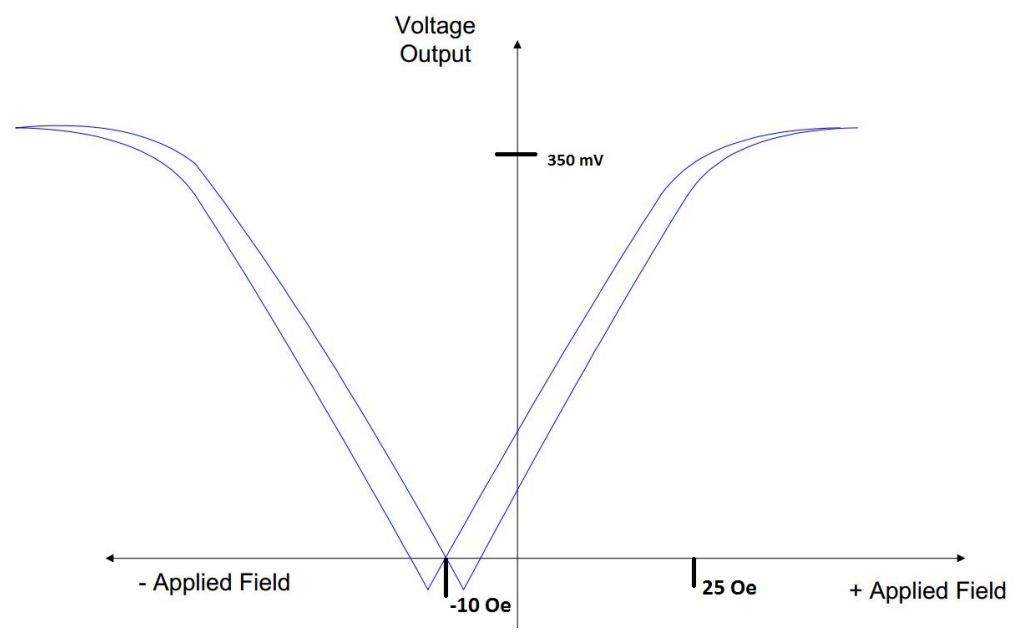

Figure 3.9: Sensor response with 10 Oe DC bias field applied [17] 


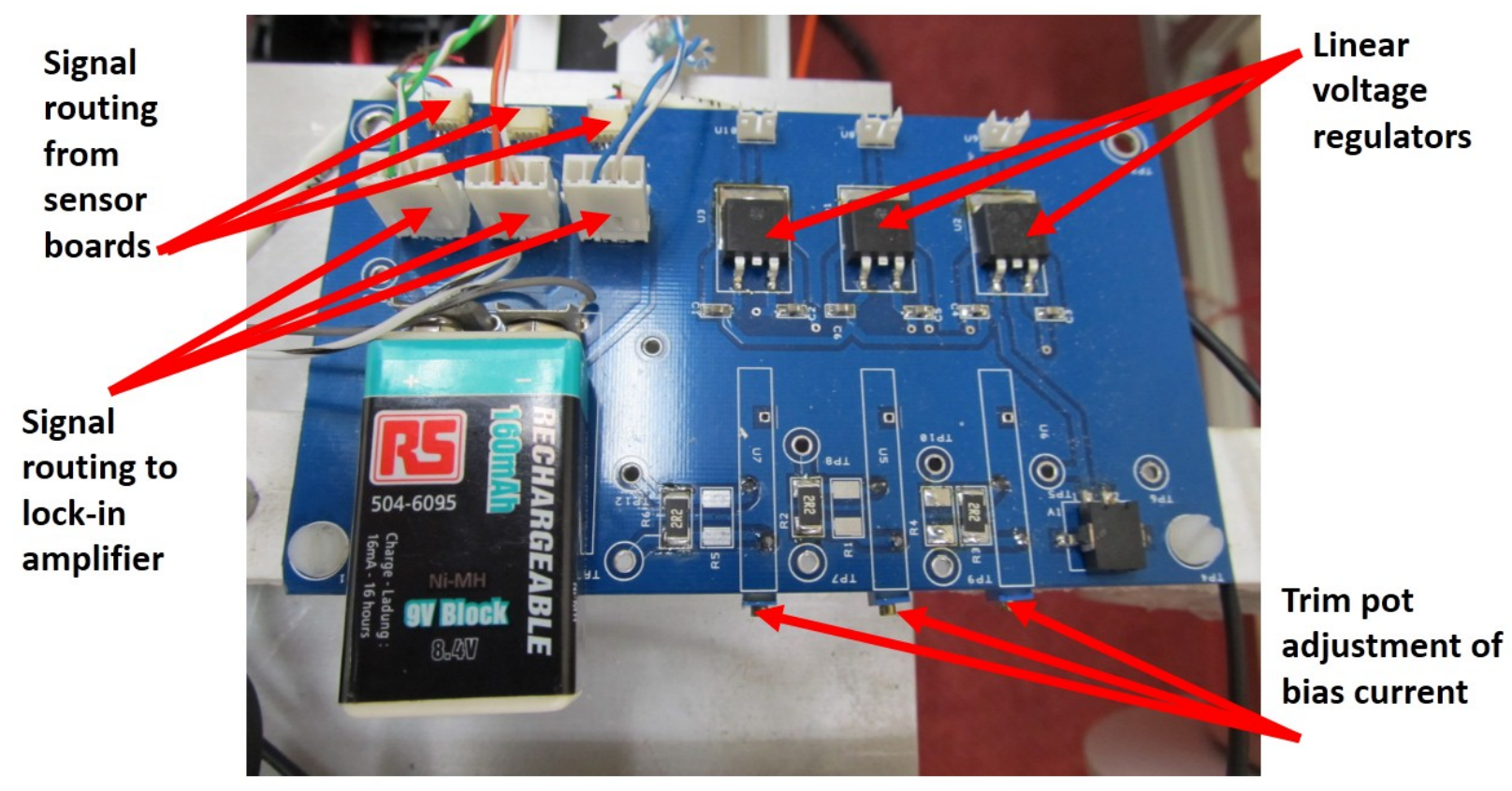

Figure 3.10: Sensor module interface board

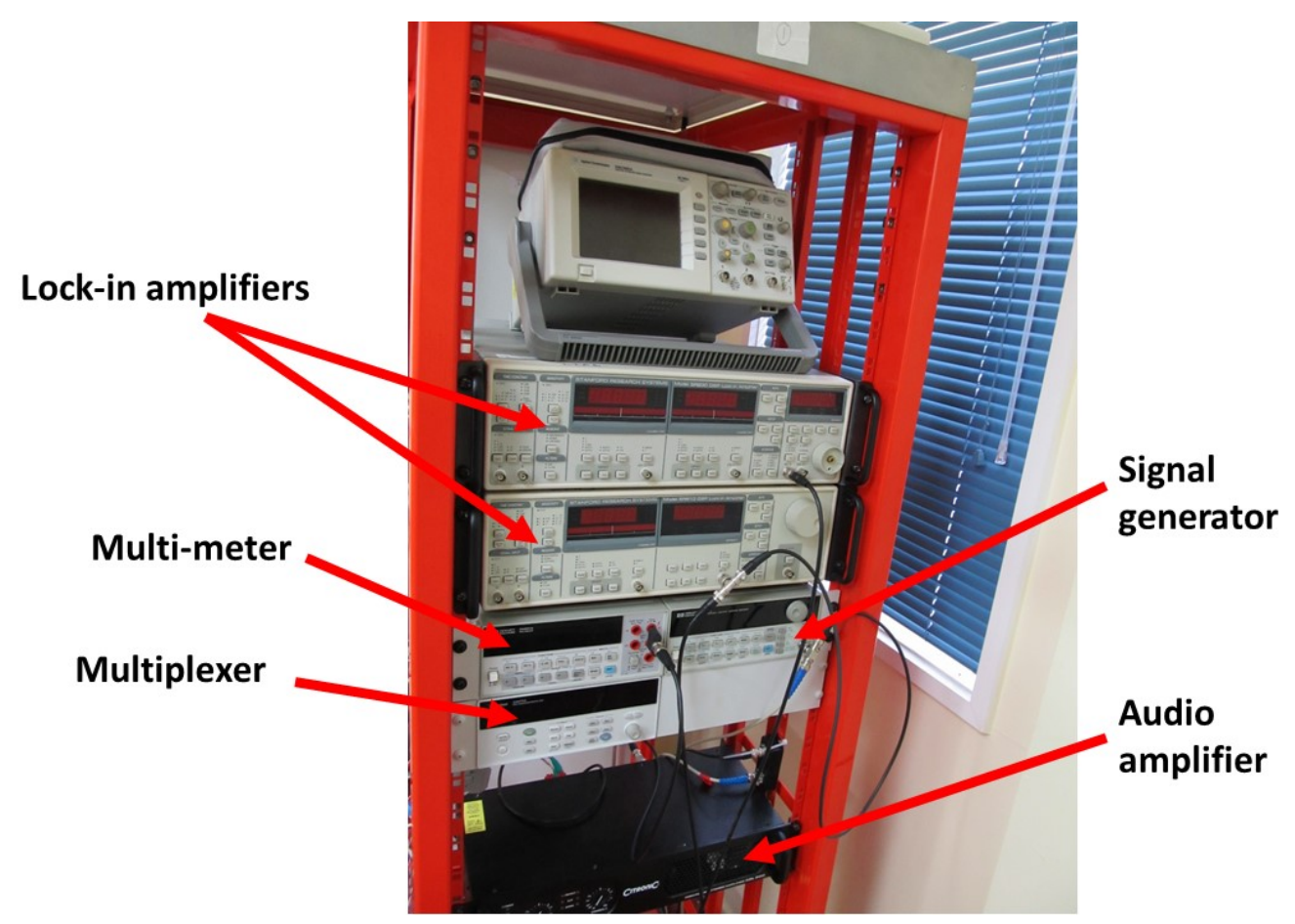

Figure 3.11: Instrumentation rack with all GPIB connected devices 


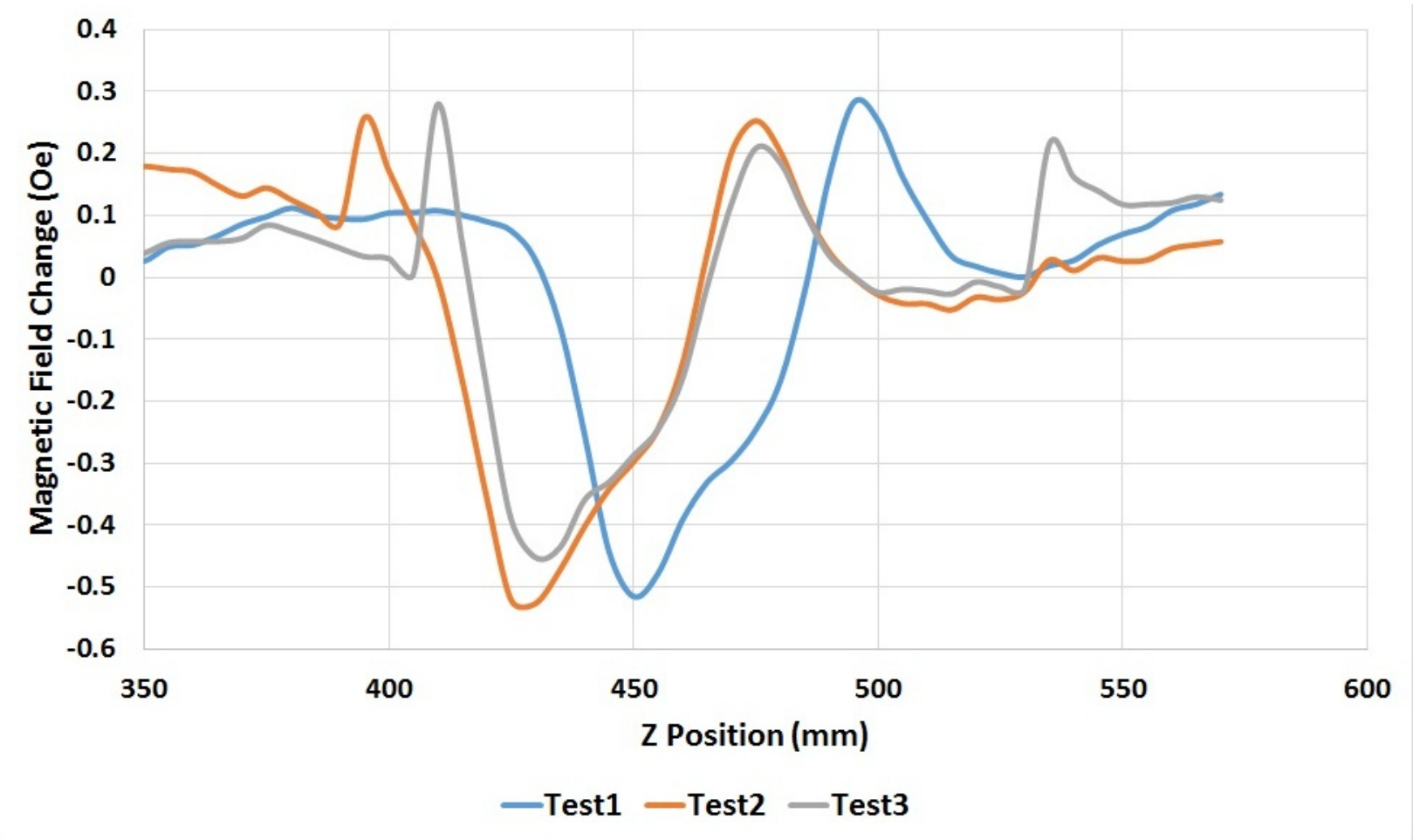

Figure 3.12: Original sensor module measurements of magnetic field change over a 50x50 $\mathrm{mm}$ segment defect for 3 tests

coil did not provide sufficient DC biasing field. The DC bias field at the sensor with 0.8 amps of current was from $7 \mathrm{Oe}$ to $10 \mathrm{Oe}$ dependent on the proximity to the sensor. This was not sufficient to ensure that the expected value for the magnetic field stayed within a linear measurement range. The ideal biasing field for the sensor is around 20 Oe [17]. A higher biasing field was achieved by placing neodymium magnets on the PCBs. After several measurements it was found that the mounting system for the sensor module did not provide a sufficiently stable platform. This is shown through some initial data in figure 3.12 which shows poor repeatability of the measurements. This meant that comparison of different measurement parameters were not possible because the variation in measurement was too large.

The other challenge of the mounting system was how accurately the sensors could be placed perpendicular to each other. This was a significant problem because of the large $Z$ direction field in the range of $40 \mathrm{Oe}$. The field strength perpendicular to this was 0.3 $\mathrm{Oe}$ in the Phi direction and $3 \mathrm{Oe}$ in the $\mathrm{R}$ direction. This meant that even a small rotation of the out-of-field sensor into the main field would result in a very large change in the field measured. The plot in figure 3.13 shows the field change in percentage for the $R$ and Phi sensors for small rotations according to equation 3.1. The magnetic field $(H)$, the resulting field, is the sum of two components. The first is the component from the main magnetic field $\left(H_{\text {main }}\right)$ which increases with rotation and second is the component from the perpendicular magnetic field $\left(H_{\text {perp }}\right)$ which reduces with rotation. These two vector components are shown in figure 3.14 with equation 3.1 showing the addition of these two 


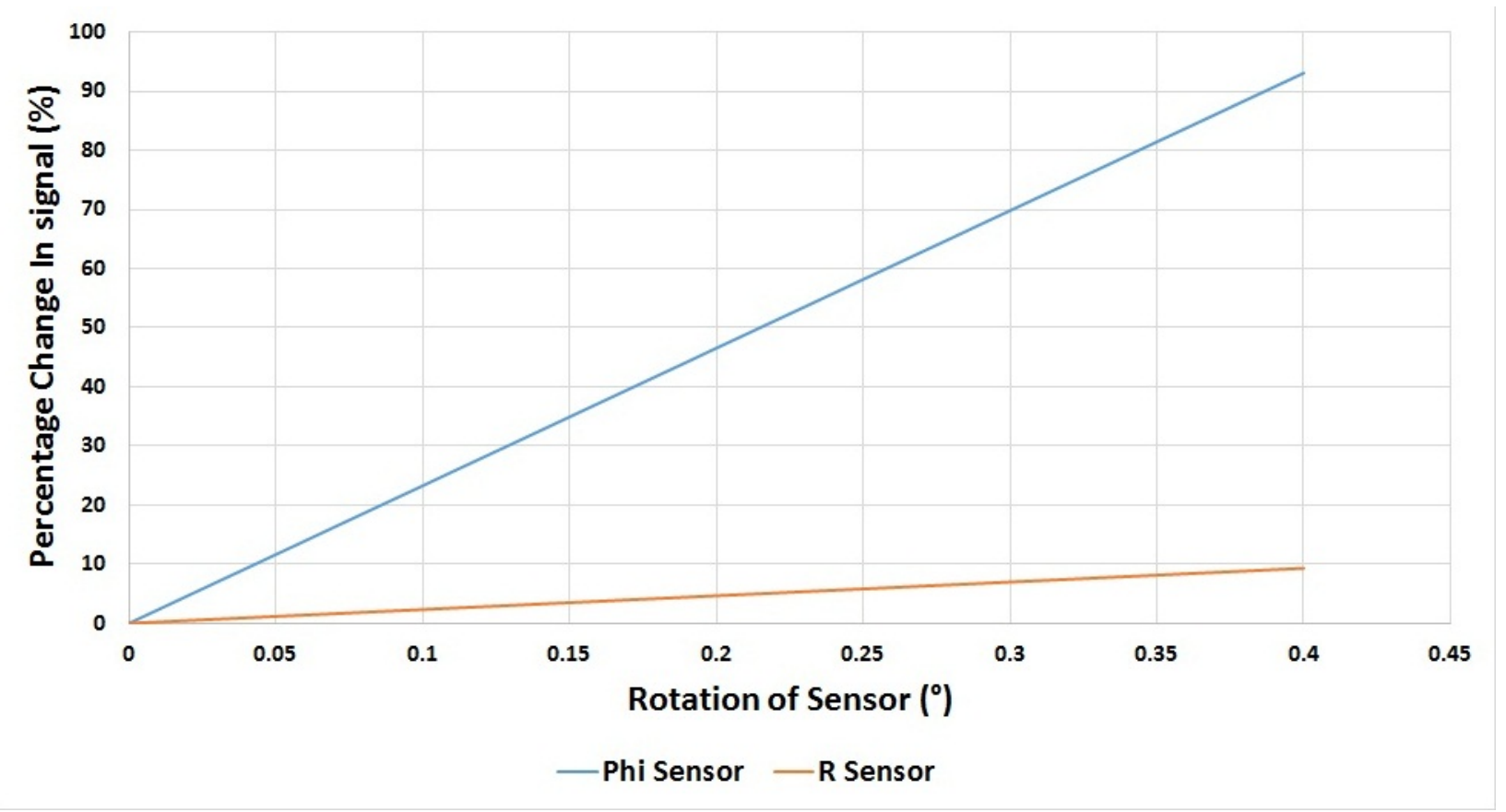

Figure 3.13: Change in measured magnetic field due to rotation of Phi and $\mathrm{R}$ sensors into the main magnetic field

vectors.

$$
H=\cos (90-\alpha) * H_{\text {main }}+\cos (\alpha) * H_{\text {perp }}
$$

To fix the mounting issues problem a solid ring, as shown in figure 3.15, was used in the final setup. This ring is slid over the pipe from one end and then the excitation sheet is wrapped around the ring in the large groove. The sensor PCBs are then mounted onto a machined plastic block with recesses carved out for components on the top side of the PCB. This allowed for a much firmer mounting of the sensor than the previous design. The PCBs were mounted against the faces of the plastic block which ensured that the PCBs were perpendicular to each other as shown in figure 3.16. The mounting block for the sensors was then sandwiched between the two edges of the mounting ring as pictured in figure 3.17. Nylon bolts were used to hold it all together to ensure there was no influence on the magnetic field measurements.

The final change to the sensor module was to remove the $Z$ sensor. This was done because, when operated with an excitation field below saturation of the sensor, only small variations in the $Z$ field over the largest defect were seen. Figure 3.18 shows a comparison between a $R$ magnitude signal with 300 amps excitation current and a $Z$ magnitude signal with a maximum 70 amps excitation current, recorded over a defect. This shows that any defect signal from the $Z$ sensor is going to be several orders of magnitude smaller than the Phi and R sensor. Not using this sensor means the measurement time is cut by a third, allowing more measurements to be done in the available time.

Support electronics: Changes to the support electronics were done to increase the 


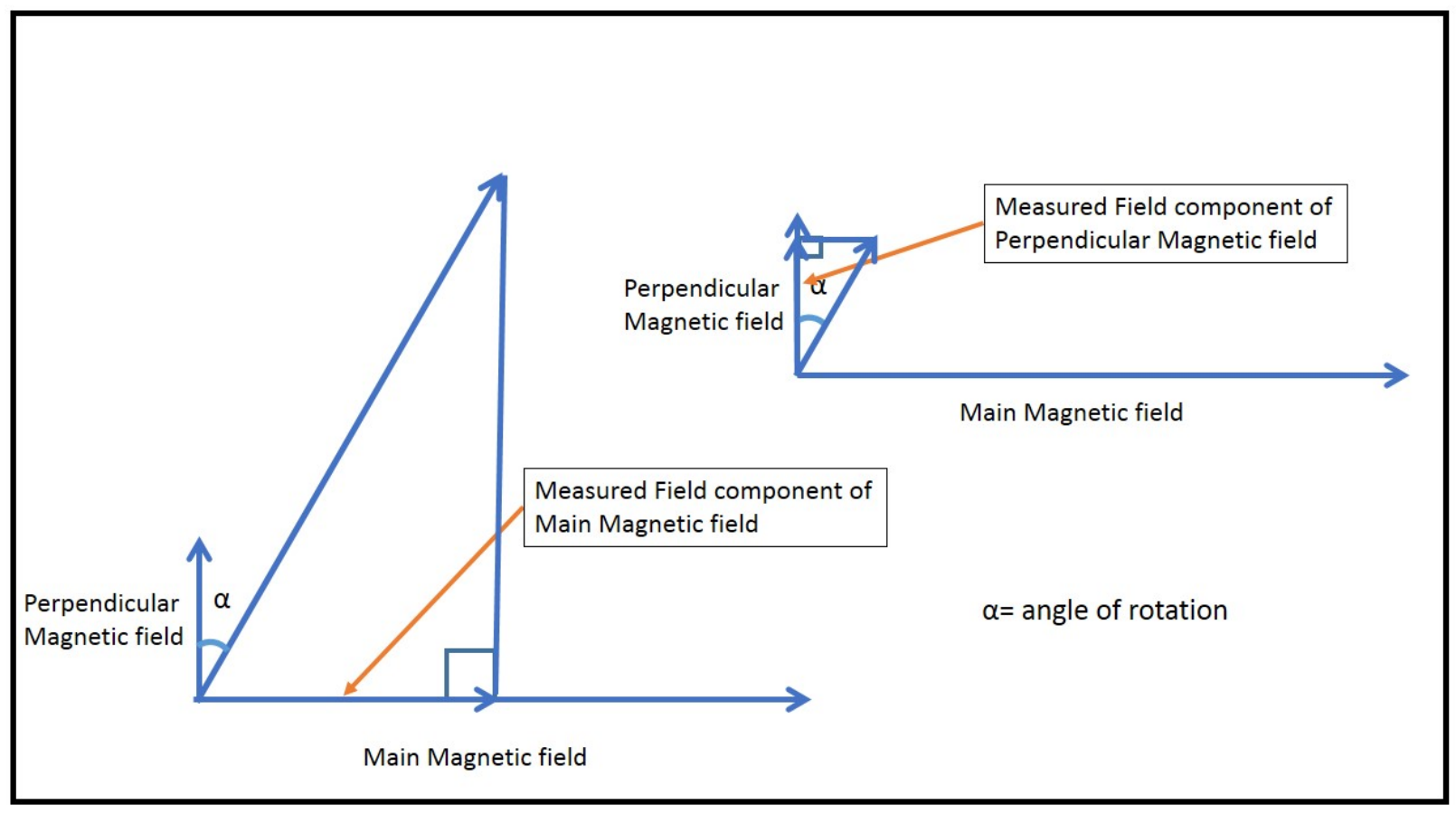

Figure 3.14: Vector components of measured magnetic field

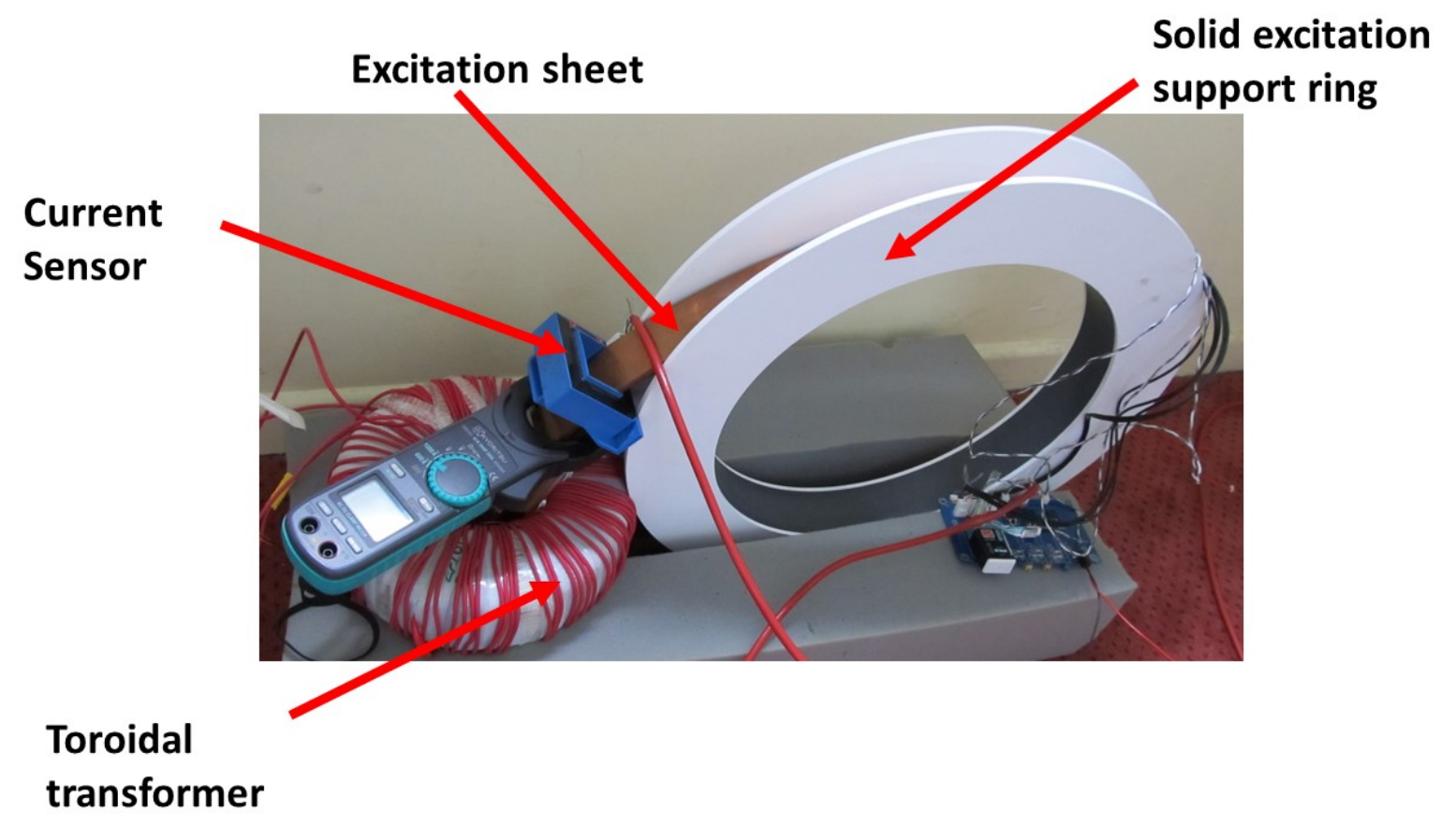

Figure 3.15: Photo of ring to support excitation 


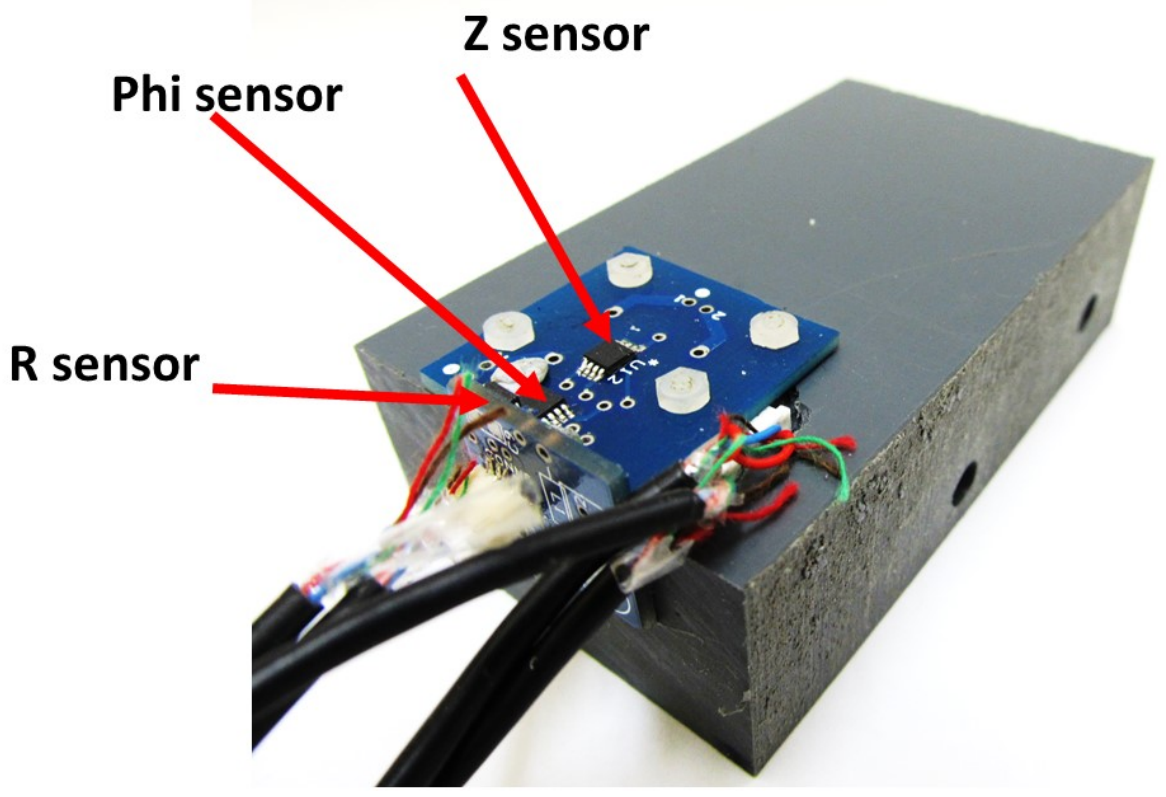

Figure 3.16: Final sensor module mounting system

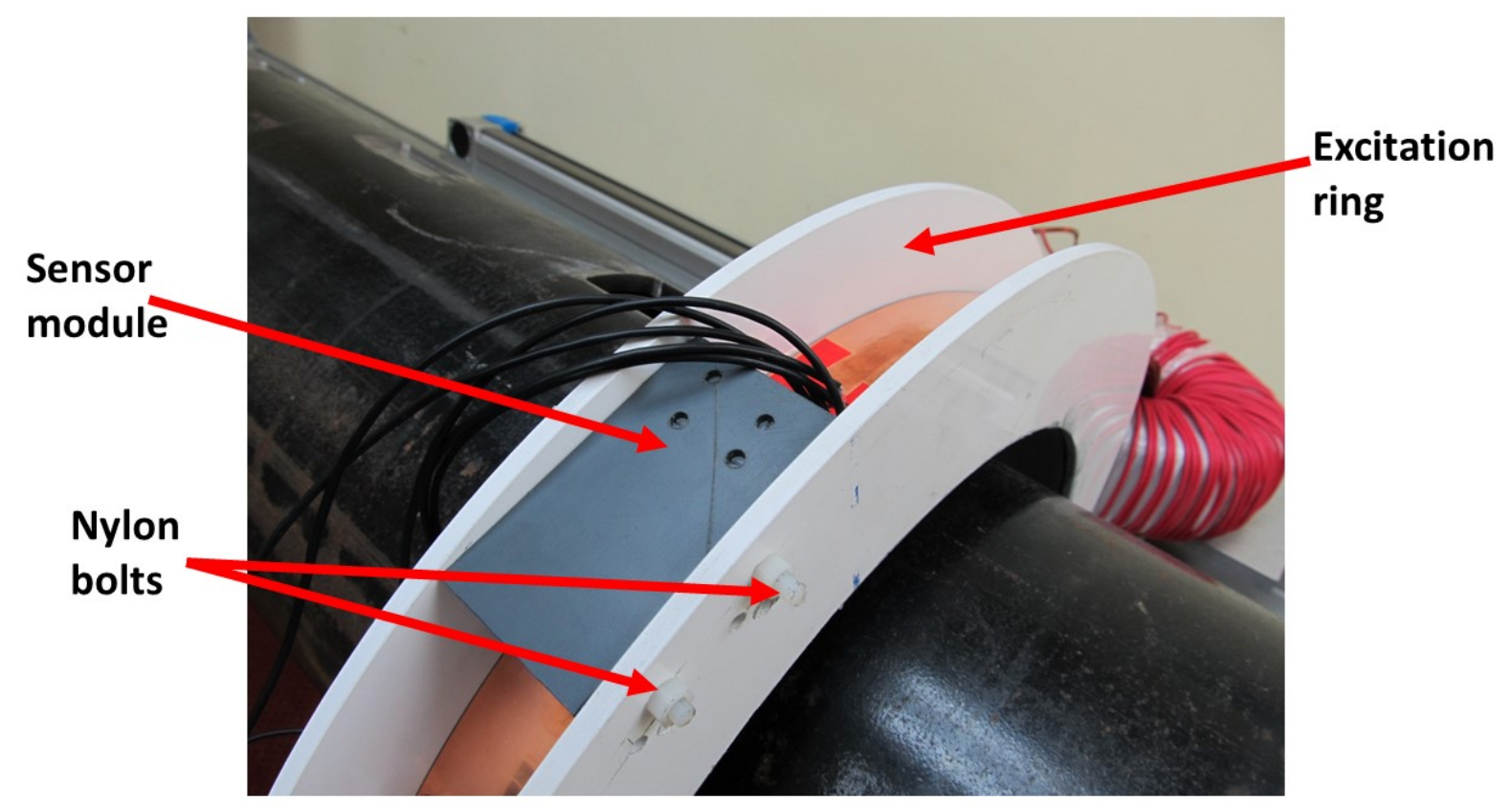

Figure 3.17: Sensor block sandwiched between side of excitation ring 


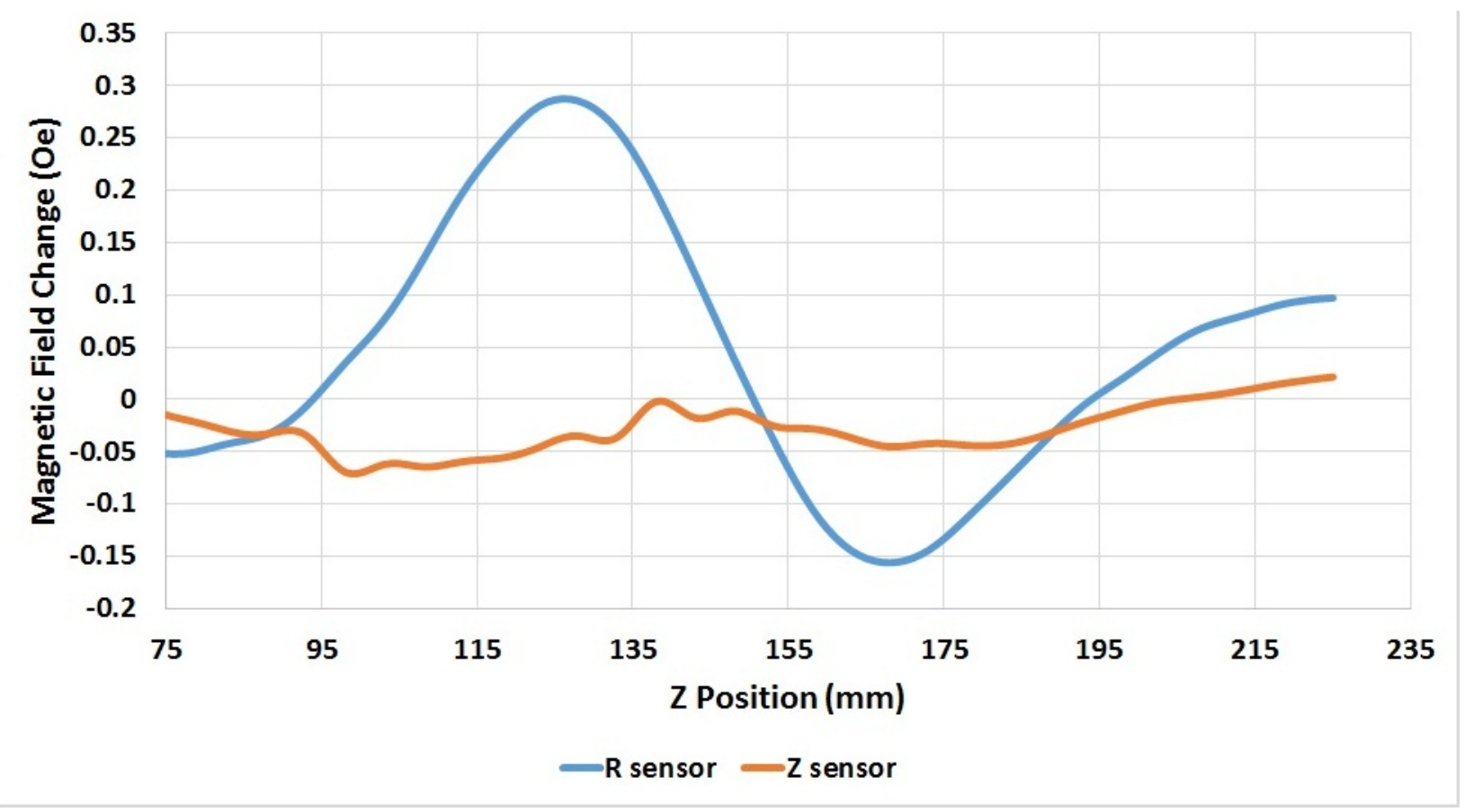

Figure 3.18: Comparison of $Z$ sensor and $R$ sensor defect signals over a $50 \times 50 \mathrm{~mm}$ through hole defect

measurement speed. Since the coils were replaced with permanent magnets there was no need for the constant current source to drive the bias coils, so these were left unused on the interface board. To measure two sensors simultaneously the sensor chain was duplicated and measurements done in parallel. Parallelization was achieved by adding a second lock-in amplifier. To avoid coupling between the sensor via the ground, as shown in the simplified circuit diagram in figure 3.19, a second power supply was used to decouple the sensors. This was done with a second battery, as shown in figure 3.20. With separate power rails for each sensor, the possibility of coupling between the sensors was excluded. This parallelization of the measurement reduced the measurement time to a third of the original time and two measurements of a whole pipe could be measured in $8 \mathrm{hrs}$.

Since the sensor measurements were parallelized, there was no longer any need to switch between the sensor signals. However, with two different supply rails, it was necessary to switch between the two supply rails to measure the battery voltage and convert the sensor signals to magnetic field values.

When monitoring the excitation current with a clamp meter, significant changes were found in the current. This was caused by changes in the temperature and location of the excitation coil relative to the end of the pipe. To allow for correction it was necessary to measure the excitation current during field measurements. This was achieved by putting the excitation sheet through a split core hall sensor current transducer. The differential output signal was routed into a NI 6009-USB DAQ and monitored and recorded via the main LabVIEW program. During initial testing the filter constant on the lock-in amplifier needed to be adjusted for lower excitation frequency. The nominal filter constant used was a 100 ms time constant. However, with frequencies below $10 \mathrm{~Hz}$ the signals started to 


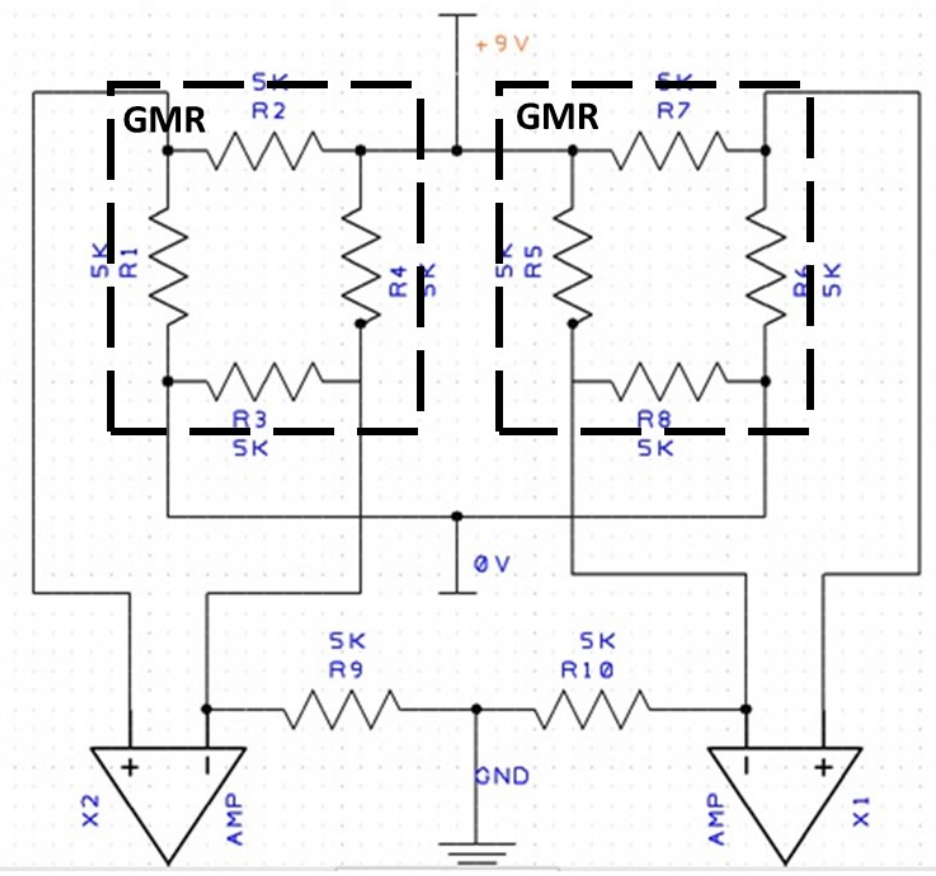

Figure 3.19: Circuit diagram of GMR and lock in amplifier with coupling through R9 and R10 and common $9 \mathrm{~V}$ rail

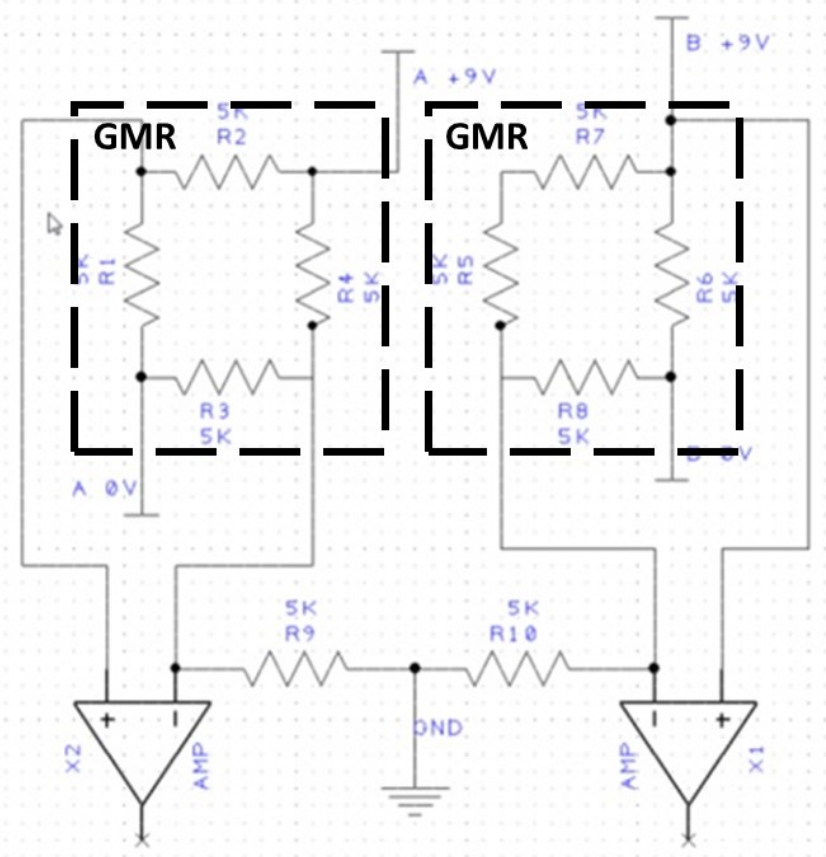

Figure 3.20: Circuit diagram of GMR and Lock in amplifiers with two $9 \mathrm{v}$ rails and no coupling 


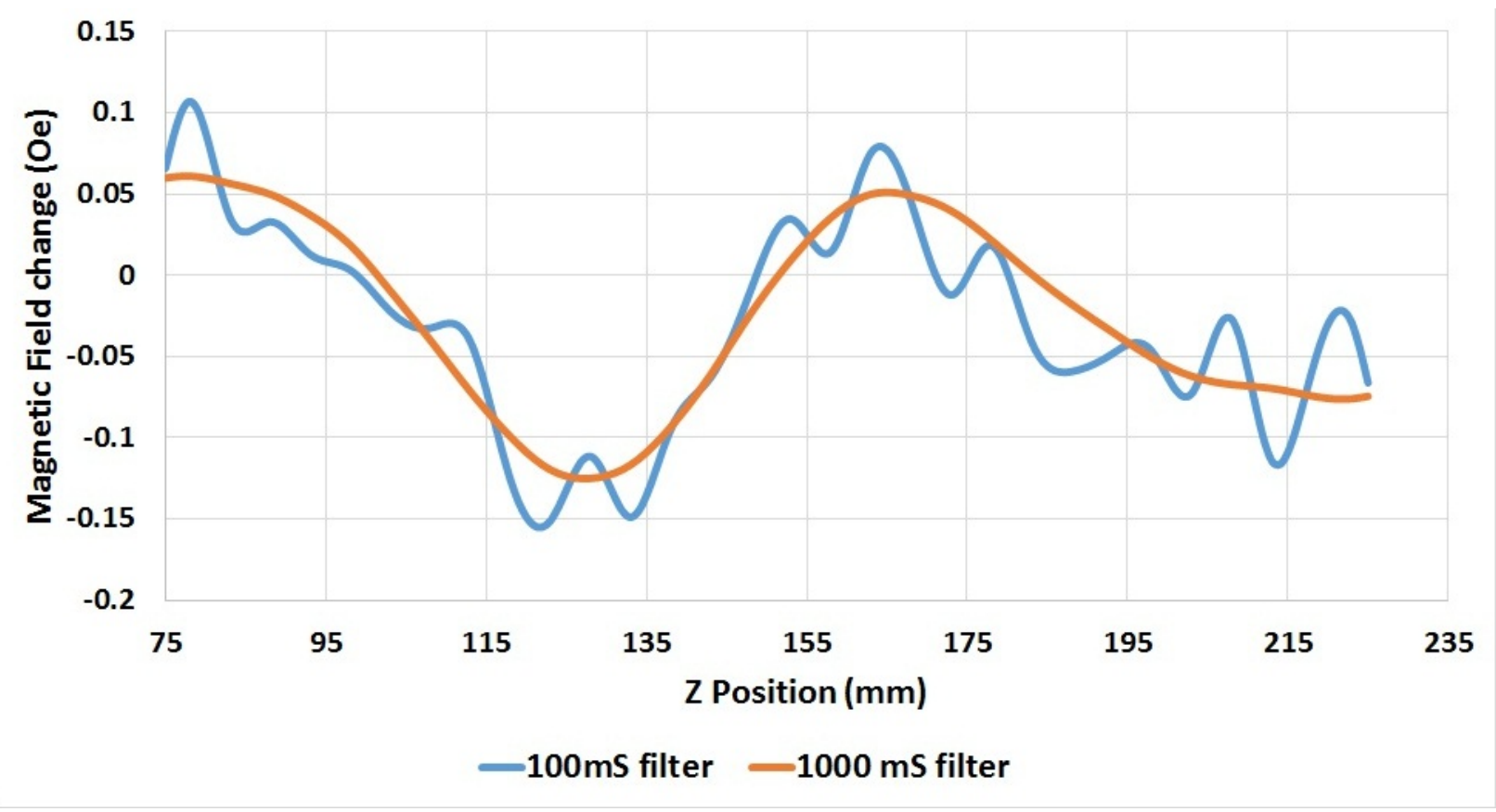

Figure 3.21: Effect of integration times on $5 \mathrm{~Hz}$ defect signal of $50 \times 50 \mathrm{~mm}$ through hole defect (field through center of defect)

alias as the lock-in frequency was lower than the filter constant. To correct for this when lower excitation frequencies were used, a $1000 \mathrm{~ms}$ time constant was used. Figure 3.21 shows a comparison between measurements taken at $5 \mathrm{~Hz}$ with a $100 \mathrm{~ms}$ and $1000 \mathrm{~ms}$ filter. It can be seen clearly that adjusting this filter constant is essential to obtain reliable results at low frequencies.

\subsection{Excitation system}

The flow diagram of the excitation system is shown in figure 3.22. The initial design parameters were to drive currents of up to 600 amps with frequency values from $5 \mathrm{~Hz}$ to $200 \mathrm{~Hz}$. The excitation system includes two parts: the drive electronics and the coil that generates the excitation field.

\subsubsection{Initial excitation system}

Drive electronics: The excitation drive electronics flow chart is shown in figure 3.22. To provide an input signal a 33120A HP signal generator, controlled over a GPIB bus from LabVIEW to output sin waves from sub $1 \mathrm{~Hz}$ to $15 \mathrm{MHz}$ with amplitude from 50 $\mathrm{mVpk}$-pk to 10 Vpk-pk, was used. This signal is routed through a voltage divider that limits the output voltage to $1.4 \mathrm{~V}$ so that the audio amplifier cannot be overdriven if a spike or mis-programing of the signal generator occurs. The output signal from the voltage divider 


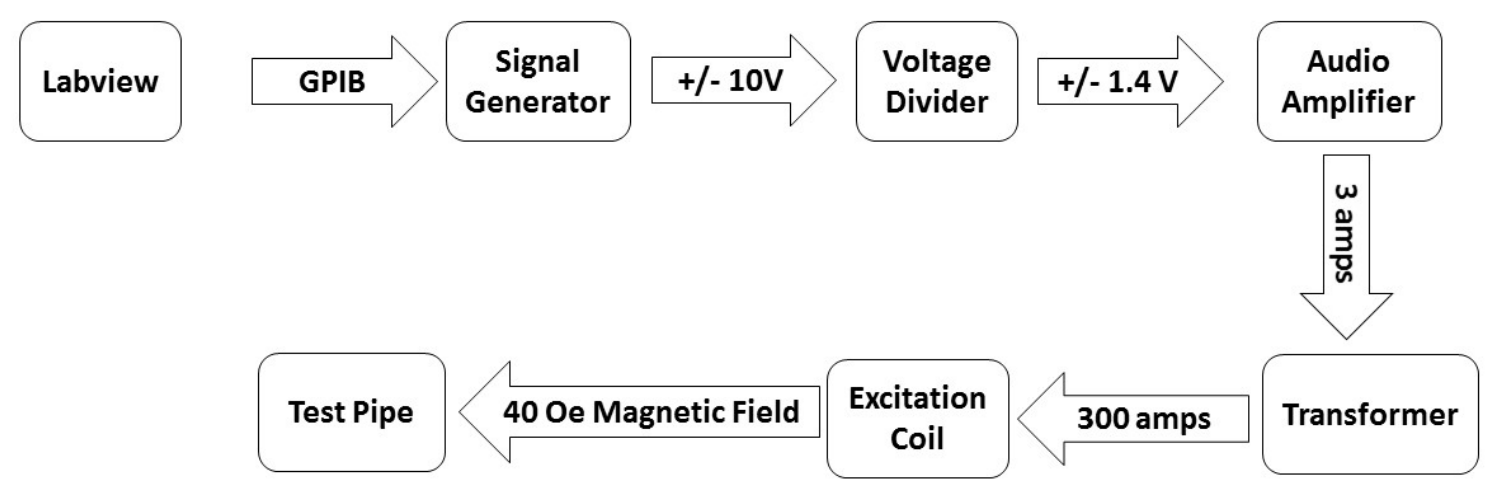

Figure 3.22: System diagram of excitation system

is routed into the input of a Citronic AEL600 audio amplifier which is capable of driving 300 watts into a 4 ohm load per channel. The output of the amplifier is protected with a fast blow in-line fuse that protects the output from current spikes that would occur if the transformer saturates. A varistor is placed across the output to clip voltage spikes that may appear from disconnection of the inductive load. This protected output drives a toroidal transformer with 106:1 turns ratio with the secondary being the excitation sheet. A transformer was used so that the low current-high voltage output of the audio amplifier could be transformed to a low voltage-high current in the excitation sheet. The turns ratio ensures that the impedance the audio amplifier had to drive was close to the rated 4 ohms. This was important as it ensured there was not excessive heating within the audio amplifier. The toroidal form factor was chosen as it contains the magnetic field better than standard rectangular transformers [31]. This ensured that the transformer field had as little as possible effect on the magnetic field measurements.

Excitation coil: An encircling coil geometry was chosen as this gave the best coupling efficiency, providing the highest ratio of input power to excitation field strength. It also provides a constant magnetic field for the entire circumference of the pipe to allow for future designs to use sensors all around the pipe to scan a larger area in one pass. This would be a significant advantage for a field application. The initial excitation design for the encircling coil used a stack of thin copper strips $0.15 \mathrm{~mm}$ thick and $40 \mathrm{~mm}$ wide. The stack sheet design was used to provide a large cross-sectional area to reduce resistance while remaining flexible. At either end of the stack, flat copper clamps with two $75 \mathrm{~mm}^{2}$ welding cables soldered on, were attached to a large automotive power plug rated for 700 


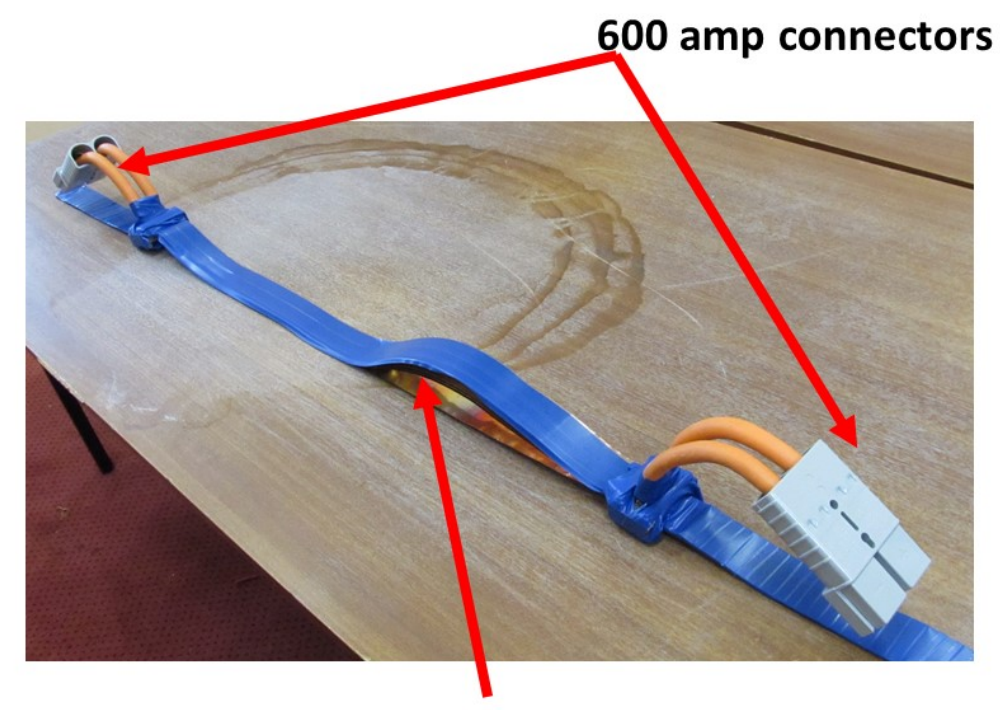

Stacked copper sheets

Figure 3.23: Initial excitation coil

amps (see figure 3.23). This plug design allowed for easy connection and disconnection. The strip could simply be wrapped around the pipe connecting the plugs through the transformer. The whole stack was supported in a cable chain. This provided support for the sensor and excitation coil while still allowing for any size of pipe to be wrapped around with the excitation sheet. The total resistance of this system was $0.72 \mathrm{~m} \Omega$. The highest current achieved with this system was 680 amps but the temperature quickly rose over 80 ${ }^{\circ} \mathrm{C}$ due to resistive heating and the system had to be shut down. Typically 400 amps was used, resulting in a temperature of $50^{\circ} \mathrm{C}$.

\subsubsection{Improvements to excitation system}

Drive electronics: The drive electronics worked as expected and were capable of driving the full range of excitation fields and frequencies required. The limiting factor to achieving higher frequency values over $200 \mathrm{~Hz}$ was the impedance increase since the amplifier was not able to drive currents greater than 100 amps. It was, however, capable of driving 300 amps up to $89 \mathrm{~Hz}$ and over $100 \mathrm{amp}$ at $200 \mathrm{~Hz}$. This was sufficient for the tests to be carried out.

Excitation coil: The sheet itself was not capable of dissipating all the heat generated from the resistive heating. By using equation 3.2, it can be shown a 600 amps peak current generates 260 watts. 


$$
P_{a v g}=I_{r m s}^{2} R
$$

where

$$
\begin{aligned}
I_{r m s} & =\text { RMS Current }(\text { Amps }) \\
R & =\text { Resistans }(\Omega) \\
P_{\text {avg }} & =\text { Real power }(W)
\end{aligned}
$$

To optimize the design of the excitation coil an equation was derived to predict the temperature rise from the ambient temperature. As the heat generated must match the heat dissipated to achieve a stable temperature, the equations predicting the heat generation were put on one side of the equation and the equation predicating the heat dissipation on the other.

To describe the heat generation equation 3.2, which gives the power generated for a given resistance, was combined with equation 3.3, which describing the resistance of the copper for a given temperature. An additional $R_{\text {joint }}$ term was added to the copper resistance to account for the resistance of joints in the excitation coil. From initial measurements of the excitation coil this was found to be $7 \mu \Omega$ per joint.

$$
R=\rho_{0}\left[1+\alpha\left(T-T_{0}\right)\right] \frac{l}{A_{c s}}+R_{\text {joint }}
$$

where

$$
\begin{aligned}
\rho_{0} & =1.72 * 10^{-8} \Omega . m[32] \\
\alpha & =0.004 K^{-1}[32] \\
T_{0} & =293 \mathrm{~K} \\
T & =\text { temperature of copper }(\mathrm{K}) \\
l & =\text { length of copper }(\mathrm{m}) \\
A_{c s} & =\text { Copper cross }-\operatorname{section}\left(\mathrm{m}^{2}\right) \\
R_{\text {joint }} & =\text { Resistance of joints }(7 \mu \Omega / \text { joint })
\end{aligned}
$$

To calculate the heat loss three methods are considered: Firstly, heat loss from free air convection follows equation 3.4 (Newton's law of cooling) with the surface area set as the surface area of two sides of the excitation sheet. A value of $h=7.5 \mathrm{w} / \mathrm{m}^{2} \mathrm{~K}$ was used for the heat transfer coefficient, which represents convection in still to slow moving air from [33]. This value was chosen instead of the still air value of $h=5 \mathrm{w} / \mathrm{m}^{2} \mathrm{~K}$ as the surface area of the heat transfer was underestimated where the coil passed through the transformer due to the layers separating and providing additional surface area for cooling.

$$
Q_{c v}=h A \theta
$$


where

$$
\begin{aligned}
Q_{c v} & =\text { Rate of heat transfer }(W) \\
h & =\text { heat transfer coefficient }\left(7.5 W m^{-2} K^{-1}\right) \\
A & =\text { Area of transfer } \operatorname{surface}\left(m^{2}\right) \\
\theta & =\text { Temperature difference }(K)
\end{aligned}
$$

Secondly, conduction through the plastic support ring follows equation 3.5. However, the conduction path to a surface that can transfer the heat through convection is approximately $200 \mathrm{~mm}$. Solving for typical values, between 1 and 2 watts of heat is lost through this process, so this is not used in the equation.

$$
q=\frac{\kappa A\left(T_{h o t}-T_{\text {cold }}\right)}{d}
$$

where

$$
\begin{aligned}
q & =\text { Rate oftransfer }(W \text { heat }) \\
\kappa & =\text { thermal conductivity } 0.19(W / m . K) \text { for } P V C \\
T_{\text {hot }} & =\text { copper temperature }(K) \\
T_{\text {cold }} & =\text { ambient temperature }(K) \\
d & =\text { thickness of barrier }(m)
\end{aligned}
$$

Lastly, radiation is described with equation 3.6 (Stefan-Boltzmann Law). Applying typical values to equation 3.6 it was found there was negligible effect from radiation cooling due to the relatively low temperature, so this term was not used.

$$
q=\sigma \theta^{4} A
$$

where

$$
\begin{aligned}
q & =\text { Rate of heat transfer }(W) \\
\sigma & =\text { Stefan }- \text { Boltzmann Constant } 5.670310-8\left(W / m^{2} / K^{4}\right) \\
\theta & =\text { absolute temperature }(K) \\
A & =\text { area of emitting body }\left(m^{2}\right)
\end{aligned}
$$

Combining the heat dissipation from convection with the heat generation, equation 3.7 was formed.

$$
I^{2}\left[\rho_{0}\left[1+\alpha\left(T-T_{0}\right)\right] \frac{l}{A_{c s}}+R_{\text {joint }}\right]=h A \theta
$$


where

$$
\begin{aligned}
I & =\text { Current }(\text { Amps }) \\
\rho_{0} & =1.68 * 10^{-8} \Omega . m \\
\alpha & =.003862 \mathrm{~K}^{-1} \\
T_{0} & =293 \mathrm{~K} \\
T & =\text { temperature of copper }(\mathrm{K}) \\
l & =\text { length of copper }(\mathrm{m}) \\
A_{c s} & =\text { Copper cross }- \text { section } \\
h & =\text { heat transfer coefficient }\left(20 \mathrm{Wm} \mathrm{m}^{-2} \mathrm{~K}^{-1}\right) \\
R_{\text {joint }} & =\text { Joint resistance }(\Omega) \\
A & =\text { Area of transfer surface }\left(\mathrm{m}^{2}\right)
\end{aligned}
$$

Equation 3.7 is solved for $\theta$, the temperature change from ambient of the copper $\left(T-T_{0}\right)$ in equation 3.8, which was used to plot the temperature rise with current for given excitation coil designs, this is shown in figure 3.24. The plot shows the resistance needed to be significantly reduced to achieve the excitation current required. The best method for achieving this resistance reduction was to reduce the number of joints from seven in the original design down to one in the final design. The number of copper sheets was also increased from 16 sheets to 28 sheets. The result of these modifications was that the resistance of the excitation coil was $126 \mu \Omega$ consisting of $7 \mu \Omega$ for the connection and $119 \mu \Omega$ for the copper sheets. Figure 3.24 indicates a 600 amps maximum for a stable temperature increase of $40^{\circ} \mathrm{C}$.

$$
\theta=\frac{I^{2} \rho_{0} \frac{l}{A_{c s}}+R_{\text {joint }}}{h A-\alpha \frac{l}{A_{c s}} \rho I^{2}}
$$

where

$$
\begin{aligned}
\theta & =\text { Temperature difference }(K)=\left(T-T_{0}\right) \\
\rho_{0} & =1.68 * 10^{-8} \Omega . m \\
l & =\text { length of copper }(m) \\
A_{c s} & =\text { Copper cross }- \text { section }\left(m^{2}\right) \\
R_{\text {joint }} & =\text { joint resistance }(\Omega) \\
I & =\text { Current }(\text { Amps }) \\
h & =\text { heat transfer coefficient }\left(20 W m^{-2} K^{-1}\right) \\
A & =\text { Area of transfer surface }\left(m^{2}\right)
\end{aligned}
$$

\subsection{Control program}

The system was controlled via a LabVIEW program. The operation of this program is summarized in the flow chart in figure 3.25. The program first initializes the systems via 


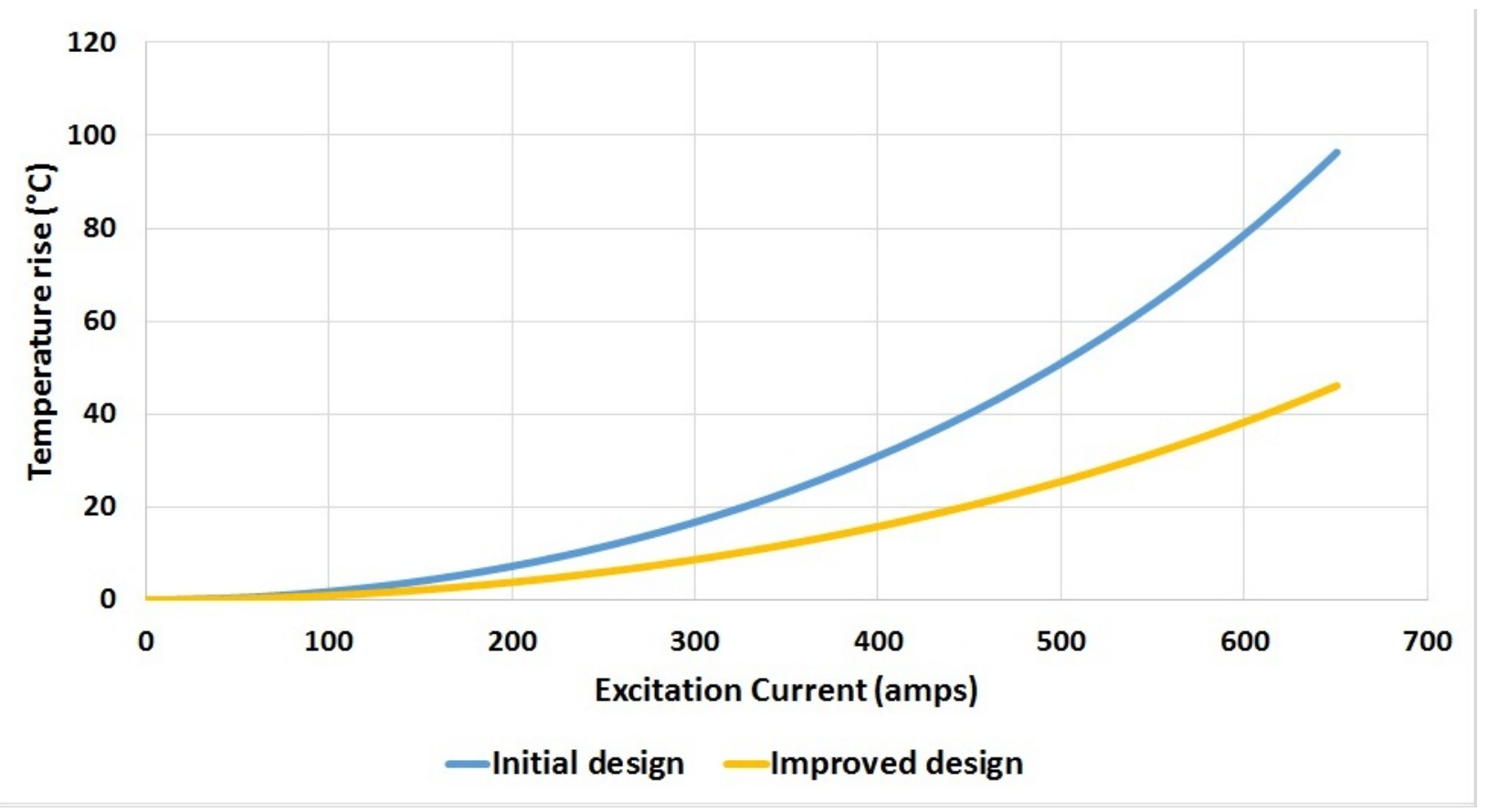

Figure 3.24: Excitation sheet temperature change with current for different designs

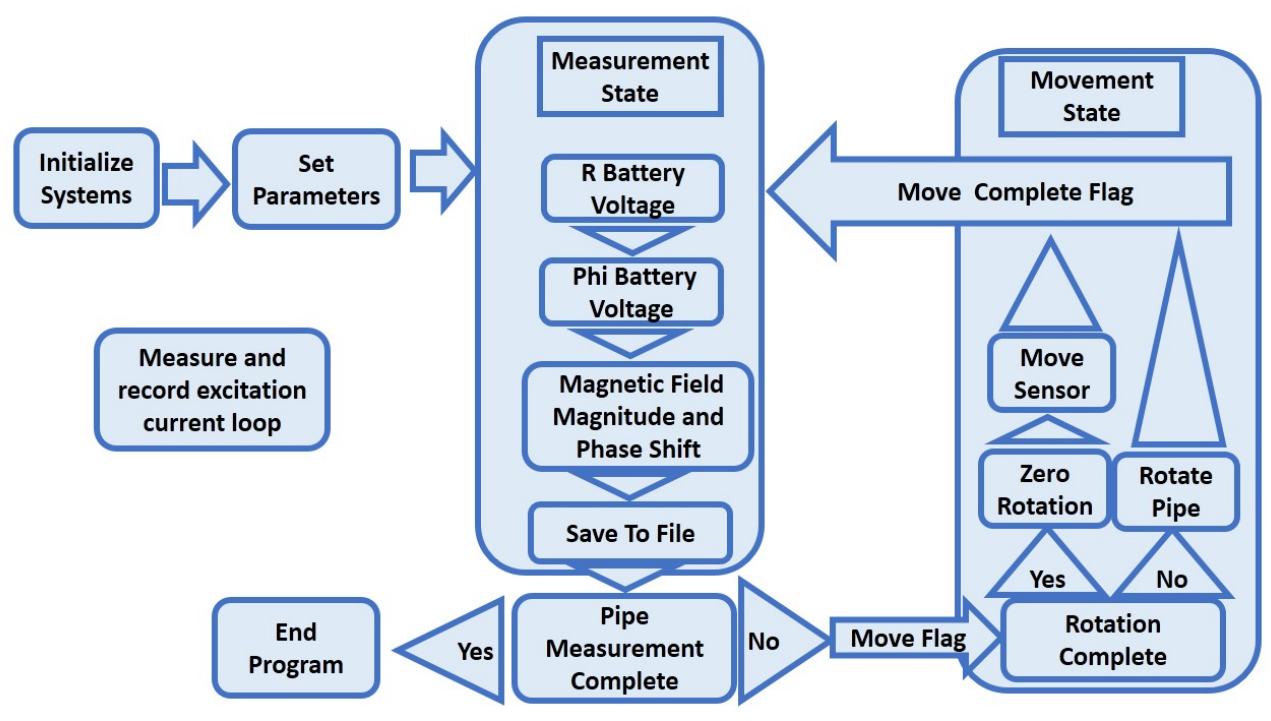

Figure 3.25: Main LabVIEW program flow chart 
GPIB bus and RS-232 communications. Then key values are initialized such as excitation frequency and level, step size and size of the scan area. The program enters its main loop which is a two-state, state machine consisting of the measuring state and the moving state. The measuring state first measures the battery voltages switching from one board to the other and then measures the phase and magnitude of both $\mathrm{R}$ and Phi sensors in parallel. These values are then recorded to a file at which point the move state is entered. The move state checks whether a full rotation is completed. If it has not been completed, it sends a command to the Trio to move one rotation step and then waits for the movement completed flag. Then it returns to the measurement state. If however the rotation has been completed, a Z move command is sent to the Trio. First the rotation axis re-datums with a magnetic reed switch to ensure cumulative error does not build up. Then the $Z$ stage is moved one step and the move complete flag is sent and the LabVIEW enters the measurement state again. During the commissioning of the system the maximum acceleration and speed of the rotation axis was tuned to achieve a fast $300 \mathrm{~ms}$ move time for a $5^{\circ}$ rotation. When a $5 \mathrm{~Hz}$ excitation current with a $1000 \mathrm{~ms}$ filter cut-off was used a lag was introduced to the measurement. The time between measurements was equal to the cut-off frequency of the filter so the measurements lagged by $5^{\circ}$. Finally the current was recorded in a second loop that runs independently of the main loop and takes measurements at $100 \mathrm{~ms}$ intervals, writing the position and current sensor value to a file after each measurement. This was done so that measuring the current did not further slow down the measurement of the magnetic field.

\subsection{Data analysis algorithms}

To assess the results quickly, a semi-automated data analysis process was developed. This was done with a MATLAB program and the basic program flow is shown in figure 3.26. First the data is loaded in Matlab and each set of values is arranged into a matrix that represents the pipe surface. Then voltage correction from the batteries is applied by multiplying each element in the voltage matrix by the corresponding value in the field matrix. The resulting matrix is multiplied by the sensitivity value for the AA004 sensor of $1.1 \mathrm{mV} / \mathrm{V} / \mathrm{Oe}$ from the data-sheet in appendix $A$. This results in a magnetic field output in Oersted.

Then current correction is applied using equation 3.9. The current correction is completed by setting a reference current level, then the difference between the reference current level and the actual measured current is calculated for each data point. Then this difference is multiplied by a correction factor that is a measure of field change per amp that can be expected and is found as described for each sensor in section 4.2.

$$
H_{\text {corrected }}=\left(I_{\text {ref }}-I_{\text {measured }}\right) * \alpha+H_{\text {measured }}
$$




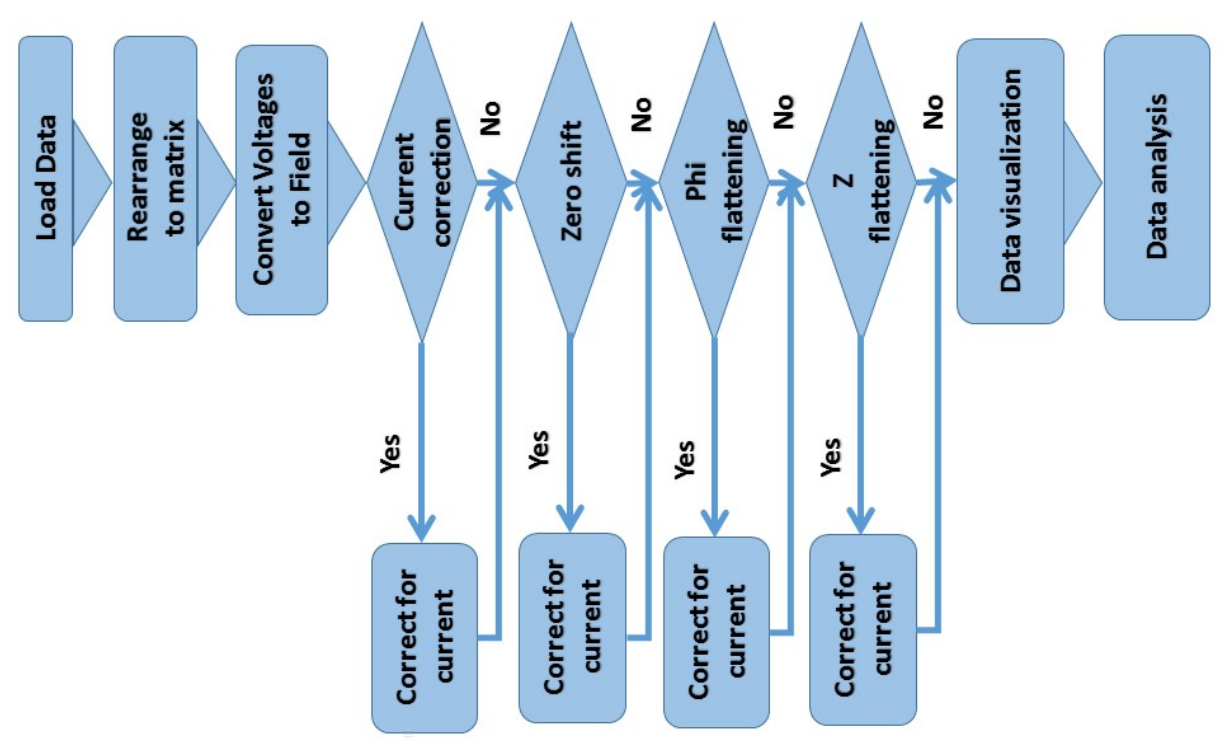

Figure 3.26: Flow chart of data analysis algorithms

where

$$
\begin{aligned}
H_{\text {corrected }} & =\text { Field at reference current }(O e) \\
I_{\text {ref }} & =\text { Reference current }(\text { amps }) \\
I_{\text {measured }} & =\text { Measured current }(\text { amps }) \\
\alpha & =\text { Current correction factor }(\text { Oe/amps }) \\
H_{\text {measured }} & =\text { Measured field }(\text { Oe })
\end{aligned}
$$

Normally only the change of the magnetic field is required to detect defects. Therefore the data is shifted to zero by finding the median value and subtracting from each point in the field matrix. Then the data is interpolated using a spline interpolation algorithm provided by MATLAB so that a 1 degree by $1 \mathrm{~mm}$ grid is achieved for better visualization. Finally the first 100 degrees of data is duplicated so that any defects near the start are shown in its entirety. Depending on the measurement, the $Z$ flattening algorithm and/or the Phi flattening algorithm is applied to the data. (see section 4.2)

Once the data manipulation steps are completed the data visualizations are carried out. Contour maps of the magnetic field magnitude and the magnetic field phase shift are plotted and red lines are then plotted on top of the contour maps representing the edges of defects. The edge locations are read from a file containing the location of all defects for the pipe. Then for each defect phase and magnitude contour plots are drawn just showing the defect itself.

Once the plotting is completed basic data analysis is performed for each defect. First the maximum and minimum points are found to define the defect signal Pk-Pk value. The 


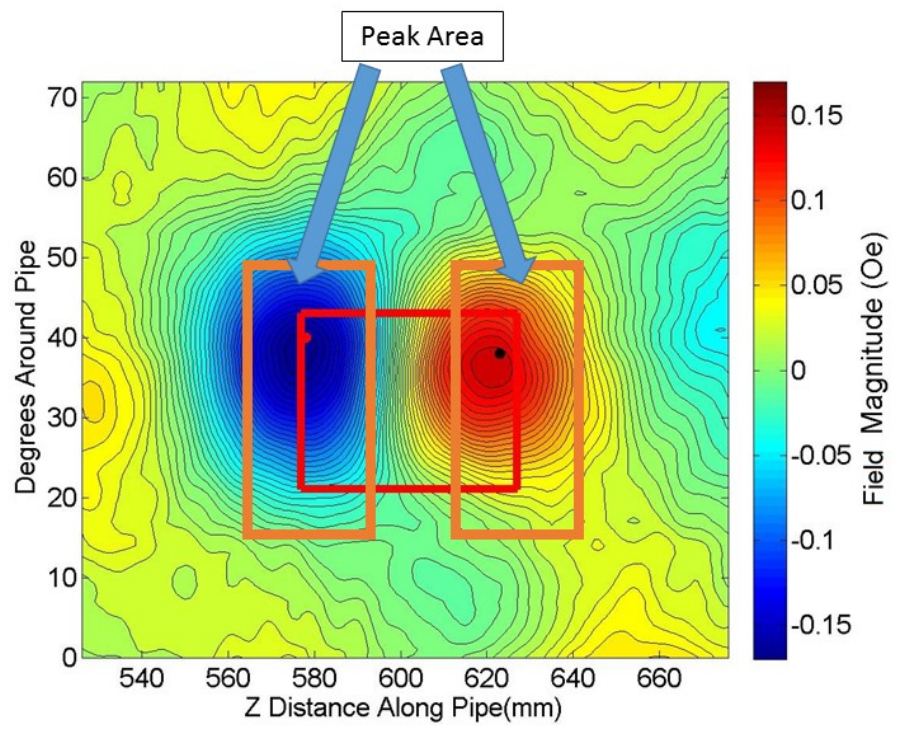

Figure 3.27: Area to determine the defect peaks for $\mathrm{R}$ sensor (Red box shows location of the defect, orange boxes show the search area for field extremes)

maximum and minimum are found for the $\mathrm{R}$ sensor by finding the maximum or minimum value within a box centered on the center of each of the $Z$ edges of the defect as shown in figure 3.27. For the Phi sensor four boxes are made, each centered on the corners of the defect as shown in figure 3.28. To decide if a maximum or minimum value should be found, an average of the values in each box is found. For the Phi sensor the boxes around the opposite corners on each edge are compared. The maximum value is found in the box with the highest average while the minimum value is found in the other box on the same Phi edge. 


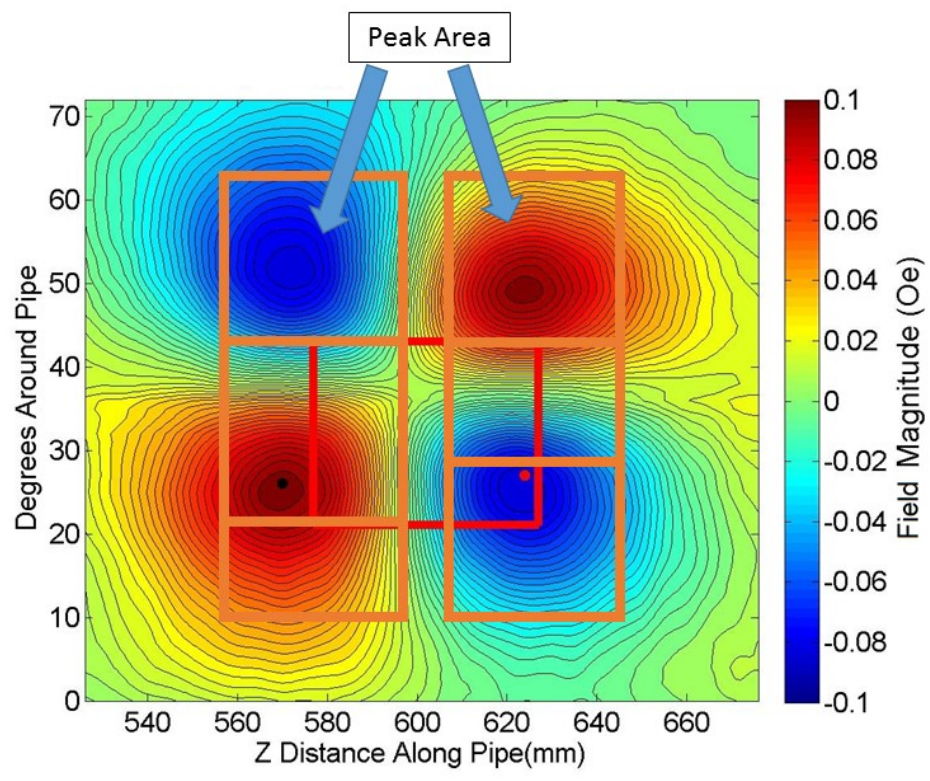

Figure 3.28: Area to determine the defect peaks for Phi sensor(Red box shows location of the defect, orange boxes show search area for field extremes) 


\section{Chapter 4}

\section{Experimental Results: Measurement of standard pipe}

Initial measurements were performed on a standard pipe to determine background levels and signal noise in the sensor. This standard pipe is a new $1.3 \mathrm{~m}$ pipe from NZ Pipes Ltd as delivered without any defects introduced. Of the $1.3 \mathrm{~m}$ of pipe a $700 \mathrm{~mm}$ section was tested shown in figure 4.1.

Tests were chosen to match all the tests that were done with regular defects. This allowed for a comparison of a defect free pipe to a pipe with manufactured defects in Chapter 5. These tests are also important to identify noise sources depending on excitation parameters by comparing the way in which the noise profiles change with the excitation parameters.

The chapter is broken down into a number of sections each covering the effect of changing a different excitation parameter or the effect of an aluminum shield. After each section a short summary section discussing the results is presented.

Section 4.2 Determines the repeatability of measurements. A set of five tests was completed at a nominal 300 Amps excitation current and $20 \mathrm{~Hz}$ excitation frequency. Measurements of the Phi and R sensor's phase and magnitude were taken for the $700 \mathrm{~mm}$ scan area.

Section 4.4 Determines the influence of the excitation current. A set of tests was completed at a fixed frequency of $20 \mathrm{~Hz}$ and with the excitation current varied from a nominal 100 Amps to a nominal 600 Amps. Measurements of the Phi and R sensor's phase and magnitude were taken for the $700 \mathrm{~mm}$ scan area.

Section 4.6 Determines the influence of the excitation frequency. A set of tests was completed at a fixed nominal current of 300 Amps and the frequency was varied from $5 \mathrm{~Hz}$ to $200 \mathrm{~Hz}$. Measurements of the Phi and R sensor's phase and magnitude were taken for the $700 \mathrm{~mm}$ scan area.

Section 4.8 Determines the influence of the excitation lift-off. A set of tests was completed at a fixed nominal current of 300 Amps and $200 \mathrm{~Hz}$ frequency with the lift-off varied from $25 \mathrm{~mm}$ to $41 \mathrm{~mm}$. Measurements of the Phi and R sensor's phase and magnitude 


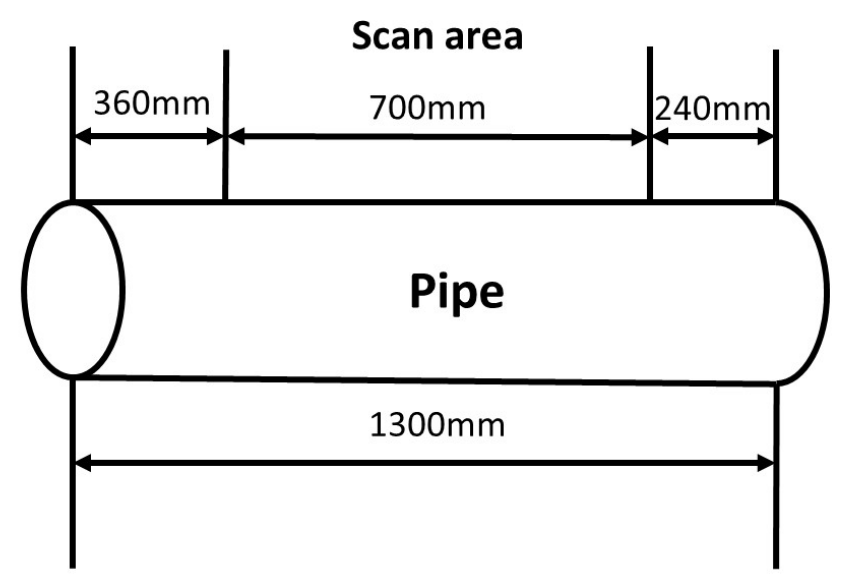

Figure 4.1: $700 \mathrm{~mm}$ scan area of pipe

were taken for the $700 \mathrm{~mm}$ scan area.

Section 4.10 Determines the influence of $0.5 \mathrm{~mm}$ aluminum weather shielding that would be present in a real measurement situation. A set of tests was completed with 25 $\mathrm{mm}$ thick insulation and $0.5 \mathrm{~mm}$ aluminum shield wrapped around the pipe. The lift-off was set at $25 \mathrm{~mm}$ so that the space between the excitation unit and the pipe was completely filled. The current was set to a nominal $300 \mathrm{Amps}$ and the frequency ranged from $5 \mathrm{~Hz}$ to $200 \mathrm{~Hz}$. Measurements of the Phi and R sensor's phase and magnitude were taken for the $700 \mathrm{~mm}$ scan area.

Section 4.12 Determines the influence of dents in the aluminum weather shield. Dents are often found in industry where insulated pipes are used for steam transport. A set of tests was completed after some dents had been introduced into the aluminum shield. Measurements were taken at a range of frequencies from $5 \mathrm{~Hz}$ to $200 \mathrm{~Hz}$. Measurements of the Phi and R sensor's phase and magnitude were taken for the $700 \mathrm{~mm}$ scan area.

\subsection{Initial pipe parameters}

The pipe used for standard pipe testing was a $1.3 \mathrm{~m}$ nominal 10 inch pipe schedule 40 electrical resistance welded pipe. The pipe was purchased from Pipes NZ Ltd as an offcut of a pipe typically used in New Zealand for steam transport. The actual outside diameter was $273 \mathrm{~mm}$ and wall thickness was $9.1 \mathrm{~mm}$ measured at each end, with less than 0.3 $\mathrm{mm}$ variation. When mounted in the test bed, the variation in distance from the sensor to the pipe surface varied by plus minus $2 \mathrm{~mm}$ along the length of the pipe. Permeability measurements were performed on pieces approximately $1 \mathrm{~mm}$ long and $60 \mu \mathrm{m}$ wide and 
Table 4.1: Permeability measurement on pipe samples

\begin{tabular}{|c|c|c|}
\hline Sample & Radial Position on pipe $\left(^{\circ}\right)$ & Relative permeability \\
\hline 1 & 263 & 20 \\
\hline 2 & 110 & 128 \\
\hline 3 & 170 & 23 \\
\hline 4 & 75 & 71 \\
\hline 5 & 4 & 82 \\
\hline 6 & 231 & 51 \\
\hline
\end{tabular}

$30 \mu \mathrm{m}$ thick. These showed an average relative permeability of $62.5^{1}$ with a standard deviation of 40. Six samples were taken from different locations around the pipe by drilling small holes to produce long lengths of swarf. The measurements were done in an applied field of $25 \mathrm{Oe}$, with the permeability calculated as the slope of the B-H curve at $25 \mathrm{Oe}$ field. The permeability of each of these samples is shown in table 4.1.

The resistivity of the pipe was measured as $17 \mu \Omega \mathrm{cm}$. The resistivity measurement was carried out on different pipe made of the same carbon steel. A $20 \mathrm{~mm}$ ring was cut from the end and then a small section cut out. Then a four point resistance measurement was performed and the resistivity calculated according to equation 4.1.

$$
\rho=R \frac{A}{l}
$$

where

$$
\begin{aligned}
\rho & =\text { resistivity }(\Omega m) \\
R & =\text { Resistance }(\Omega) \\
A & =\text { cross }- \text { sectional area }\left(m^{2}\right) \\
l & =\text { length of conductor }(m)
\end{aligned}
$$

\subsection{Repeatability of measurements and data correction algorithms}

The excitation current was set to $20 \mathrm{~Hz}$ and a nominal 300 amps with a lift-off of $25 \mathrm{~mm}$. The test was completed for a $700 \mathrm{~mm}$ section as shown in figure 4.1. During the test at each location, the $\mathrm{R}$ field and Phi field magnitude and phase shift were measured for a $5 \mathrm{~mm}$ by $5{ }^{\circ}$ grid. The test was carried out 5 times and the average values plotted as a contour map of the pipes surface. Different combinations of data correction algorithms were applied to improve measurement uniformity.

\section{R Field magnitude analysis}

The contour plot of the average field magnitude is shown in figure 4.2. The main feature

\footnotetext{
${ }^{1}$ The measurements were done by S. Granville with a physical property measurement system
} 


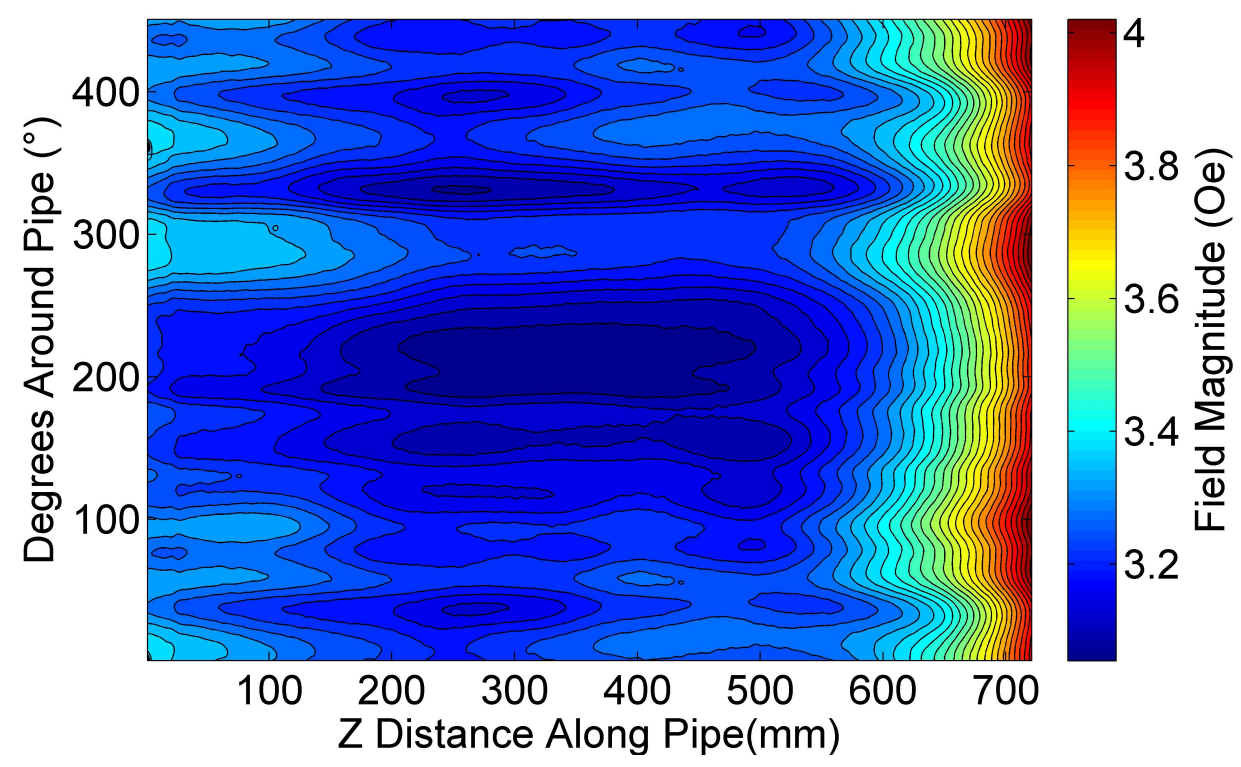

Figure 4.2: $R$ sensor field magnetic magnitude over standard pipe

in this plot is the rapid increase in the field strength at around $600 \mathrm{~mm}$. This can be attributed to two different effects. Firstly as the excitation ring nears the end of the pipe ${ }^{2}$, the impedance of the excitation ring drops because of the amount of steel that is acting as a core decreases. This decrease in impedance causes a corresponding increase in current, as the drive is a constant voltage. This increase in excitation current creates a larger magnetic field. Secondly, as the excitation ring nears the end of the pipe the magnetic field lines curl in the R sensor direction (seen figure 2.10). This also explains why the R sensor is affected by the end of pipe while the Phi sensor is largely unaffected.

To account for the change in current, a current correction algorithm has been developed. The current is measured at each measurement location and used to correct the measured field magnitude to a nominal $300 \mathrm{amp}$ level for each sensor. The current correction value was found by stepping the driving voltage from $0.1 \mathrm{~V}$ to $10 \mathrm{~V}$ in steps of $0.1 \mathrm{~V}$. At each point the current and field were measured and fitted with a linear function and this gave a correction value of $0.0024 \mathrm{Oe} / \mathrm{Amp}$ (see figure 4.3). The contour plot using the current correction algorithm is shown in figure 4.4 .

In comparing figures 4.2 and 4.4 there is a small reduction in field change at the end of the pipe. However, a significant increase in the field still needs to be removed. To account for the effect of the magnetic field curling at the end of the pipe, a second algorithm called "Z flattening" is applied. This algorithm first finds the median value for each line of points around the pipe at each $Z$ position on the pipe. The difference between this $Z$ position median and the median value for the whole pipe is calculated and defines the correction value. This correction value is added to all the data at this $Z$ position of the pipe. The effect of using this correction algorithm is shown in figure 4.5

This algorithm is more effective than the current correction algorithm, suggesting that

\footnotetext{
${ }^{2}$ The plot is not symmetrical as the scan area shown in figure 4.1 is not centered on the middle of the pipe.
} 


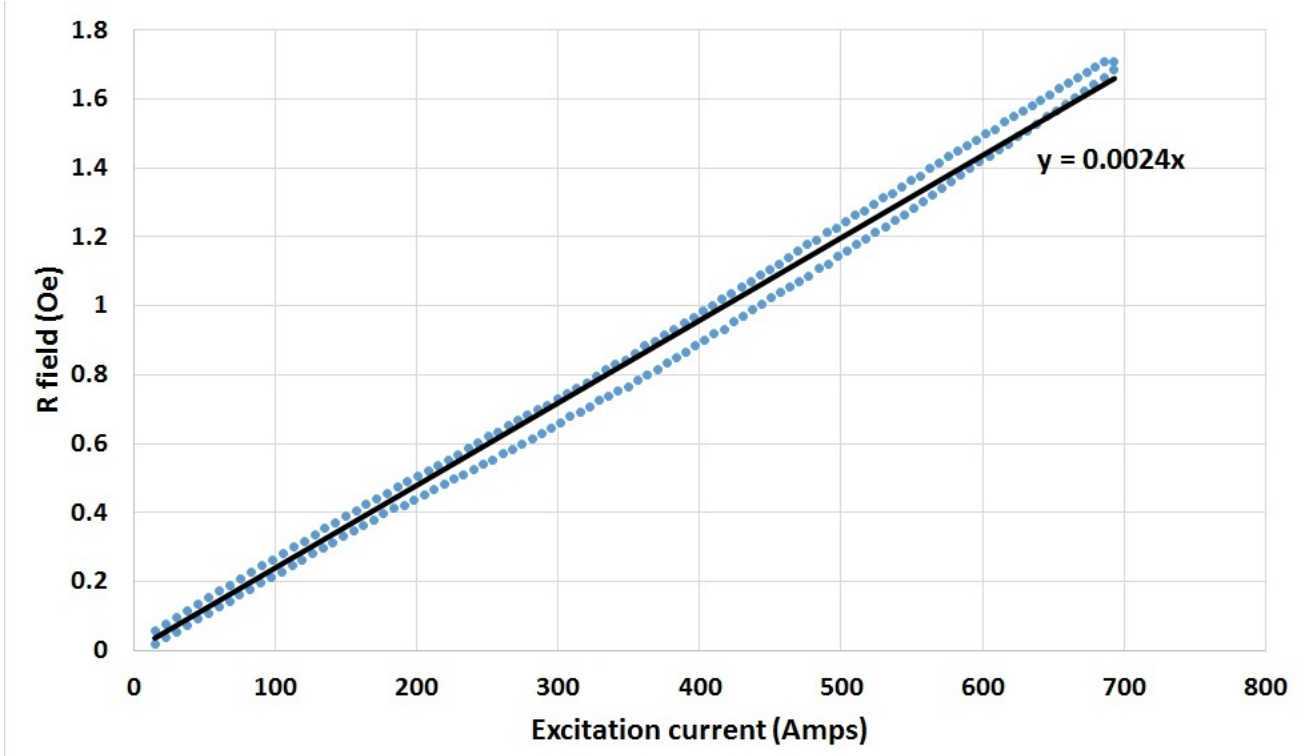

Figure 4.3: Calibration curve to correct excitation current drift for $\mathrm{R}$ sensor

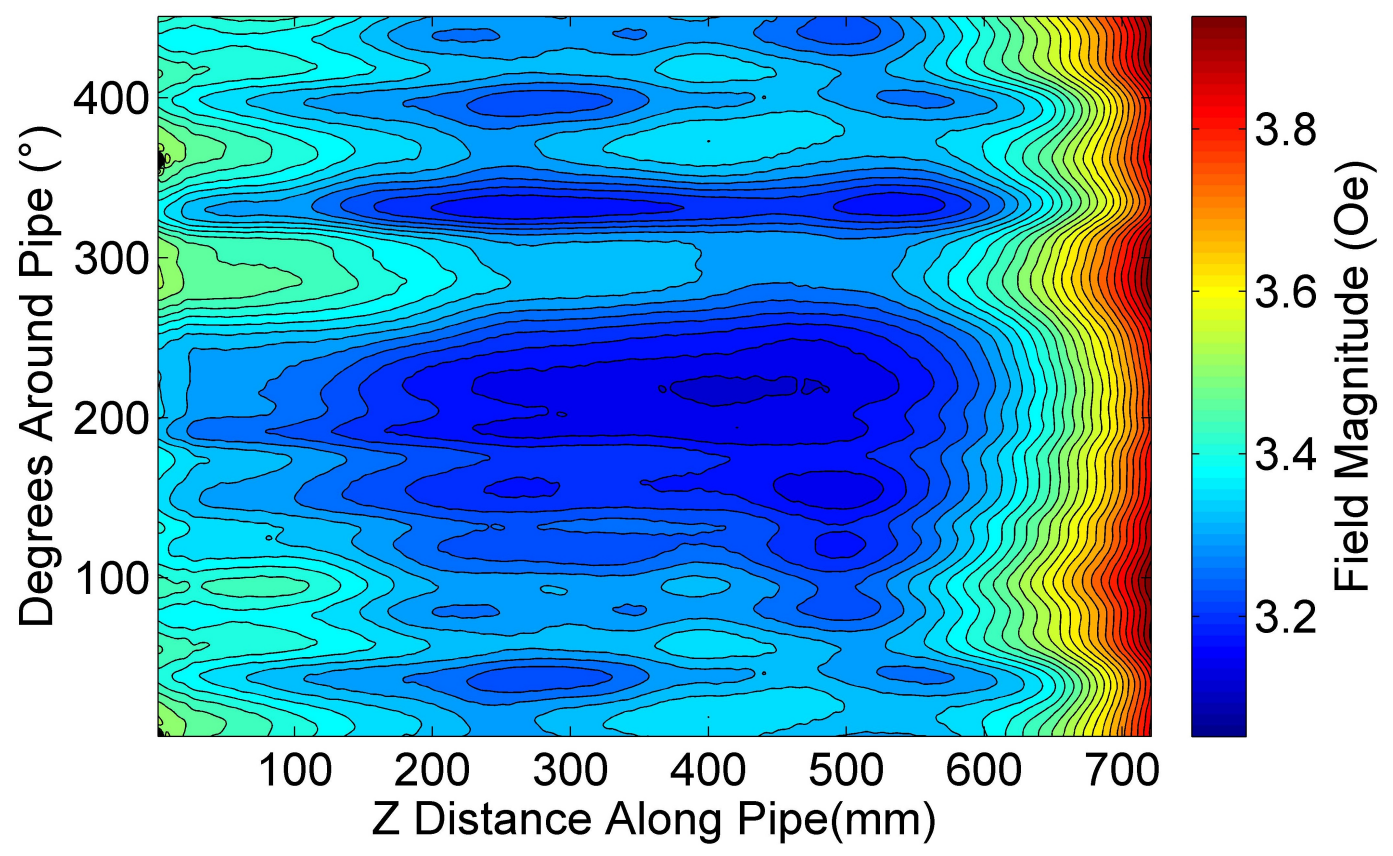

Figure 4.4: $\mathrm{R}$ sensor field magnitude over standard pipe with current correction algorithm applied 


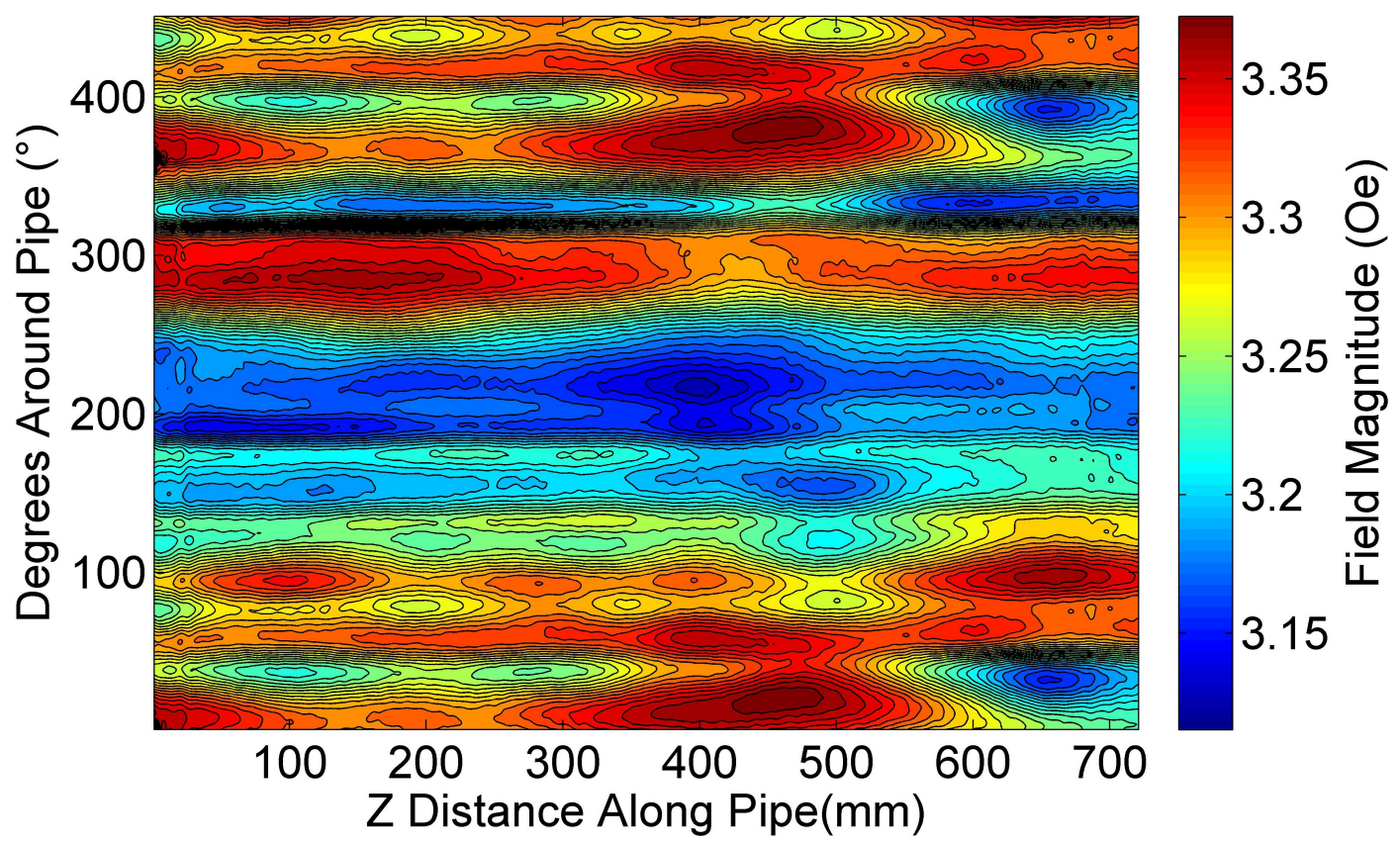

Figure 4.5: $\mathrm{R}$ sensor field magnitude over standard pipe with $\mathrm{Z}$ flattening algorithm and current correction applied

the dominant cause of the increase in field along the $Z$ axis is the effect of the fringe field rather than the drifting excitation current. Once the $Z$ axis increase is removed, the next major noise source is revealed. This can be seen as constant strips of higher or lower fields along the $Z$ axis. Since this plot is a mean value, this means that there is a change in magnetic field due to material properties in the pipe. The likely source of this variation is the permeability variation in the pipe. The dependence of the magnetic field magnitude in the $R$ direction on the permeability can be seen in figure 4.6. This plot takes the permeability measurements from table 4.1 and the corresponding magnetic field measured at the same locations and applies a linear fit. The linear fit is good once two outliers are removed. This shows that the permeability is the major cause of the variations in the field around the Phi axis of the pipe. However, the large outliers suggest that changes in the conductivity and wall thickness may also be playing a role in producing these field variations.

To correct for this variation in measurements, a third algorithm called "Phi axis flattening algorithm "was developed. It is based on the same idea as the $Z$ axis flattening algorithm but applied along the Phi axis. This means that the median value for all points along one angular position on the pipe is subtracted from the median for the total pipe to create the correction value. The correction value is then added to each data point at the angular position on the pipe. The effect of applying this Phi flattening algorithm after the $Z$ axis flattening algorithm is shown in figure 4.7.

By comparing figure 4.5 and figure 4.7 a further improvement can be seen in most areas. The variation has dropped significantly apart from two areas around 30 degrees 450 $\mathrm{mm}$ and 30 degrees $650 \mathrm{~mm}$. This is because in this area the assumption of a constant field along the $Z$ axis did not hold, most likely due to a variation in the pipe material 


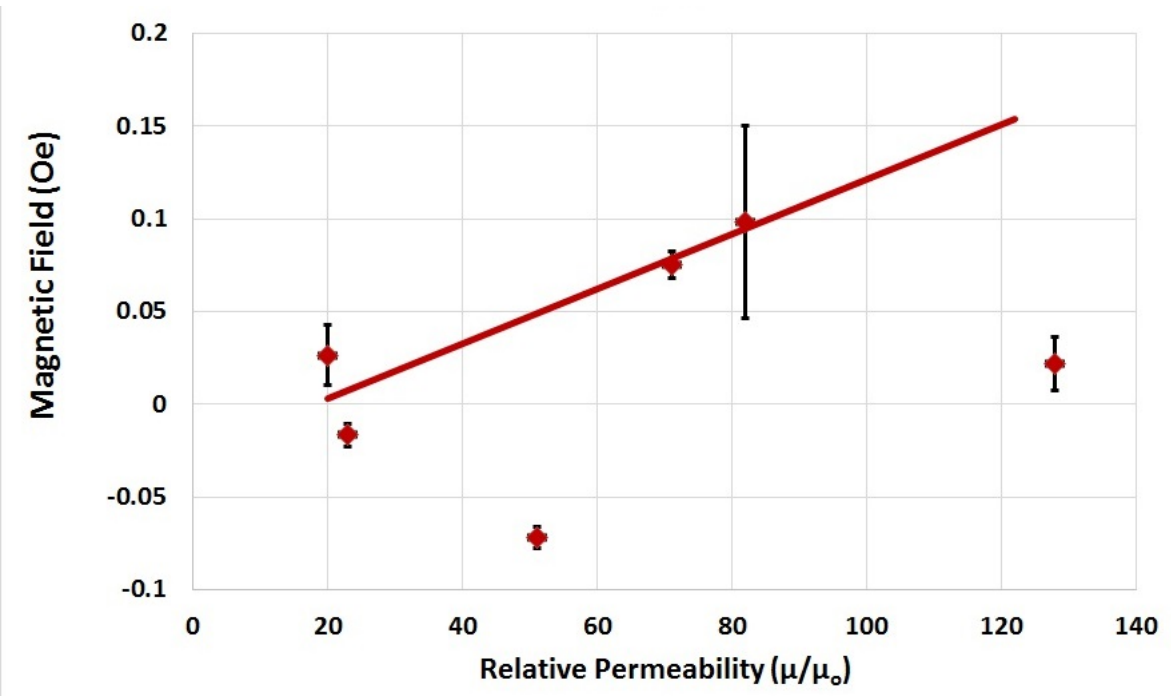

Figure 4.6: $\mathrm{R}$ field magnitude dependence on permeability

along the $Z$ axis (which does not occur elsewhere on the pipe). This means applying the algorithm to this area increases variation.

\section{R Field phase shift analysis}

The second measurement is the phase shift of the $R$ sensor measured as the phase shift of magnetic field from the excitation current. The average phase shift of the $\mathrm{R}$ sensor over the full pipe is shown in a contour plot in figure 4.8. The $\mathrm{R}$ sensor phase shift shows a clear decrease from one end of the pipe to the other.

In contrast to the amplitude, the phase shift does not show a linear trend with current. Therefore the same current correction algorithm cannot be used. However, as clearly seen, a correction algorithm for the $Z$ axis is necessary. The same $Z$ flattening algorithm as the field magnitude was used and the results are shown in figure 4.9.

Once the $Z$ axis curvature has been removed, lines of constant phase shift can be seen along the $Z$ axis of the pipe. Again this field is plotted with the permeability measurements in figure 4.10. This shows, unlike the magnitude, there is no dependence on the permeability for the phase shift. Applying the same Phi flattening algorithm as the field magnitude, flattens out these constant lines. The result of both algorithms is shown in figure 4.11.

Once both algorithms have been applied to the data the variations are further reduced. However, there are two peaks that have become more pronounced at around 30 degrees which is the same position as the magnitude signal. This again suggests that there is a material variation in the pipe's $Z$ axis.

\section{Phi field magnitude analysis}

The Phi sensor measuring the tangential field relative to the pipe was measured for both magnitude and phase shift from the excitation current. The contour plot of the average field magnitude is shown in figure 4.12. Unlike the $\mathrm{R}$ sensor there is no general curvature along the $Z$ axis for the Phi sensor magnitude. This is in agreement with the assumption that the major cause of this $Z$ axis curvature is the fringe field at the end of the pipe ${ }^{3}$.

\footnotetext{
${ }^{3}$ This affects the Phi sensor much less as seen in the field line plots on figure 2.10 since the field curls in
} 


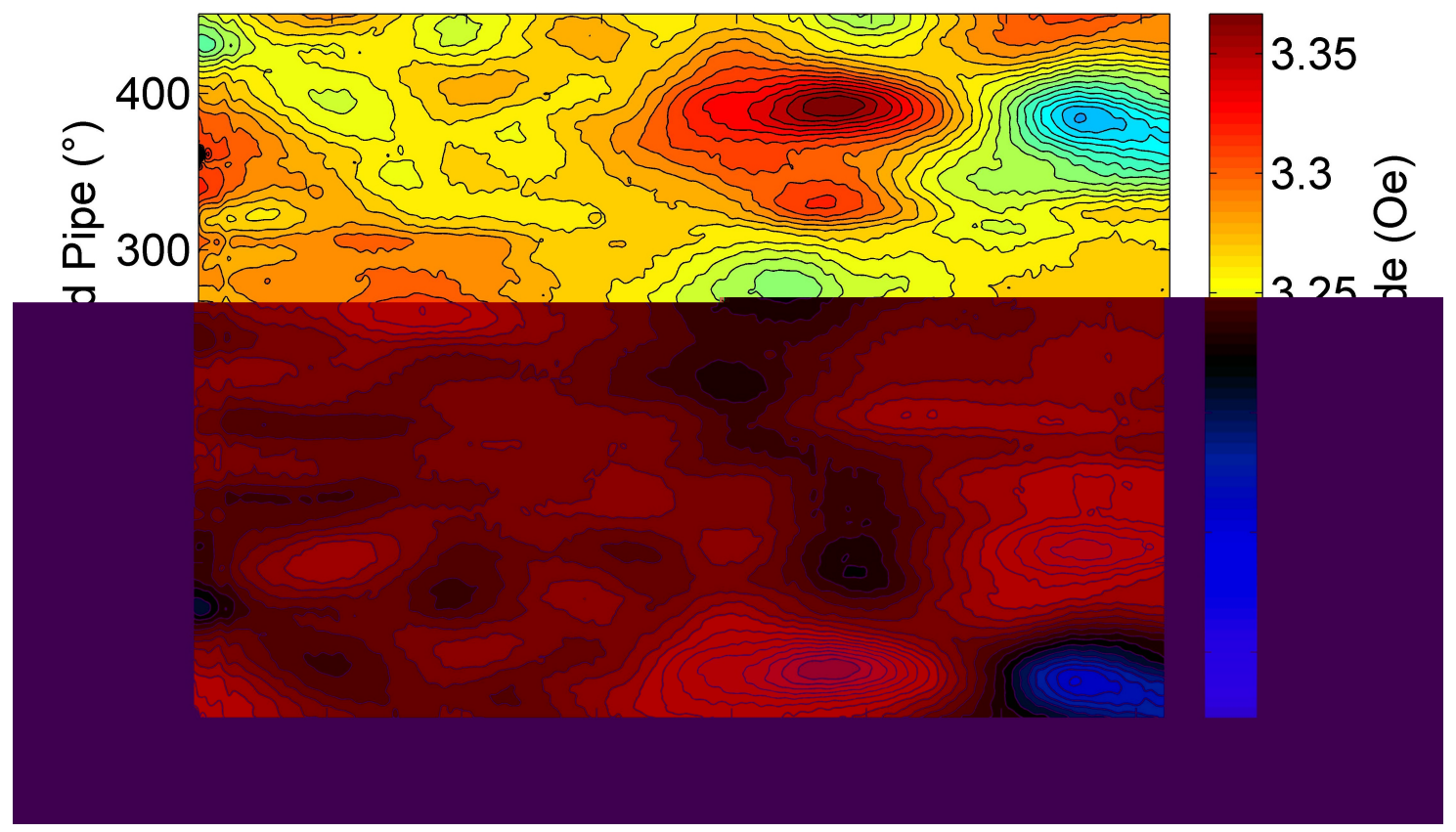

Figure 4.7: R sensor field magnitude over standard pipe with $Z$ and Phi flattening algorithms

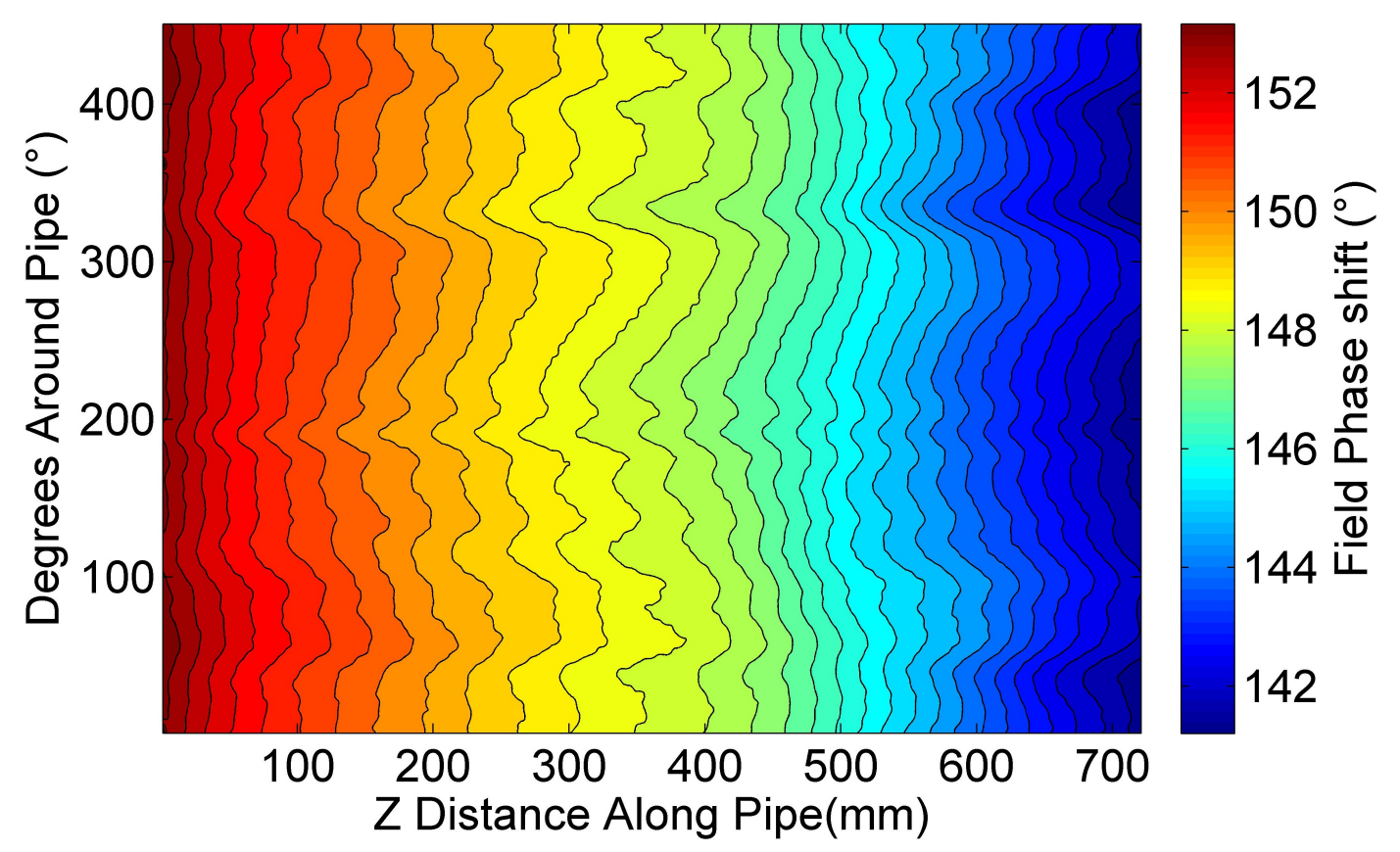

Figure 4.8: $\mathrm{R}$ sensor field phase shift over standard pipe 


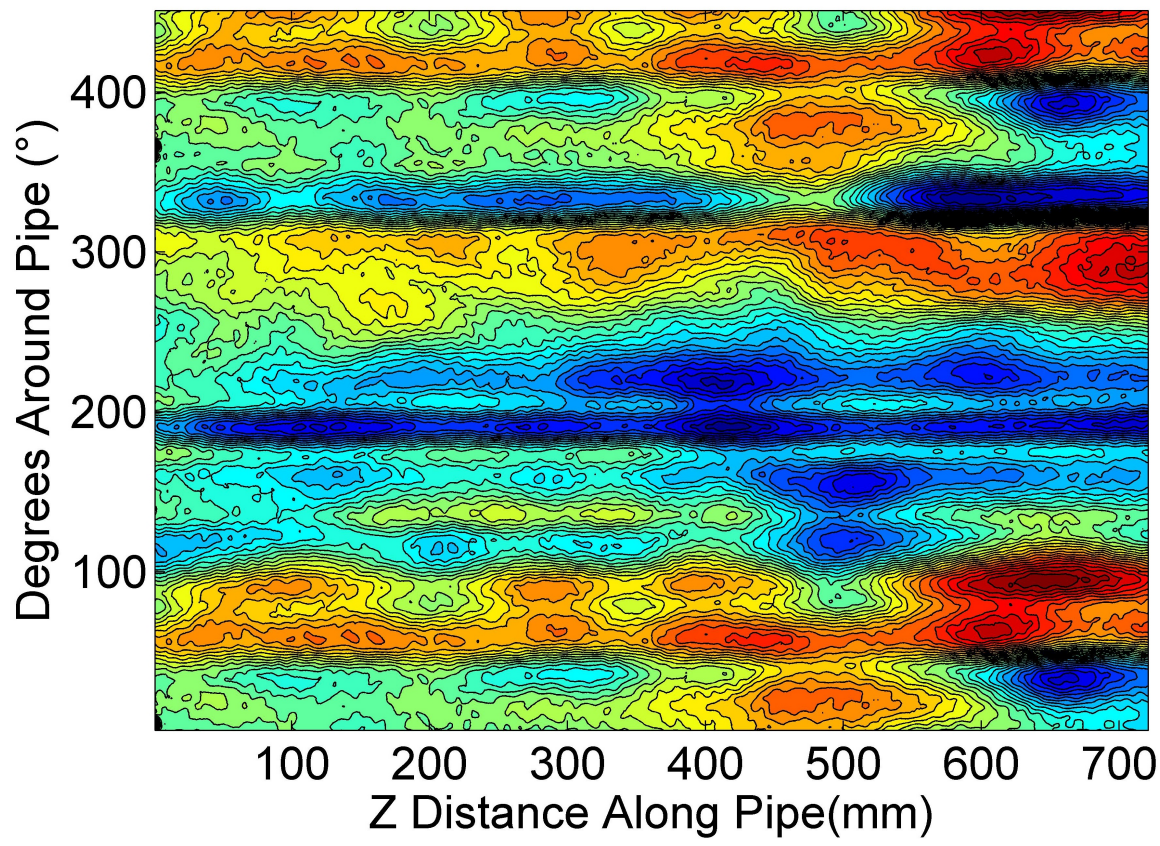

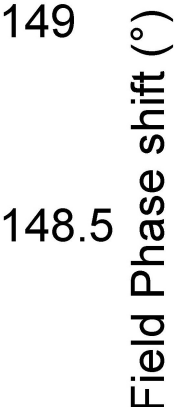

148

Figure 4.9: $\mathrm{R}$ sensor field phase shift over standard pipe with $\mathrm{Z}$ axis correction.

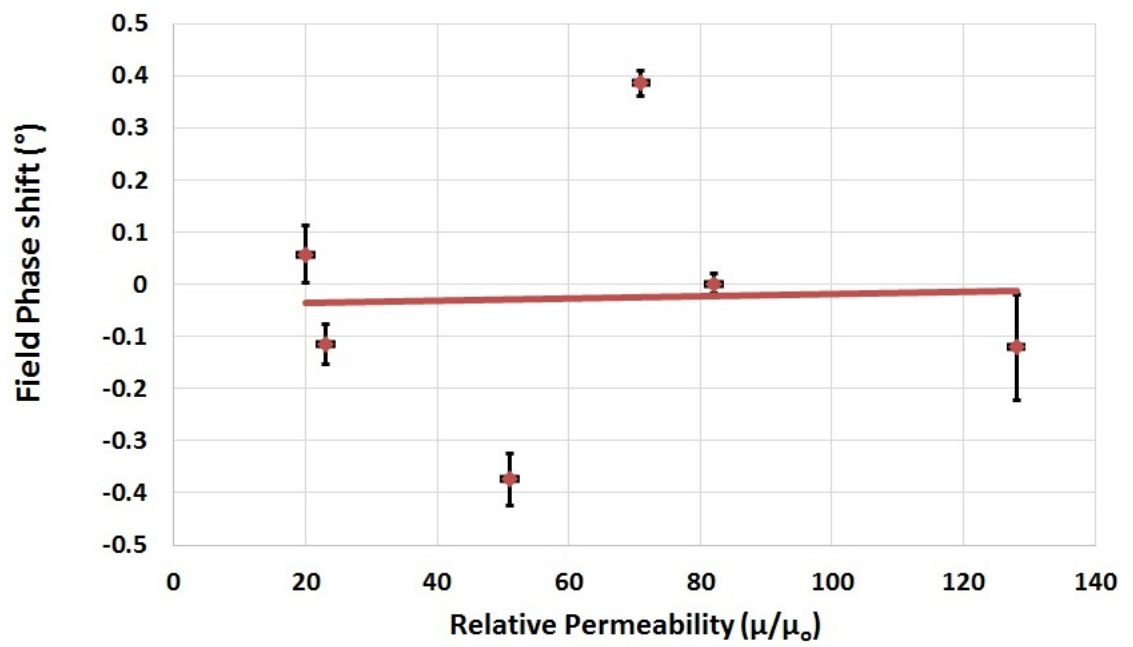

Figure 4.10: $\mathrm{R}$ field phase shift dependence on permeability 


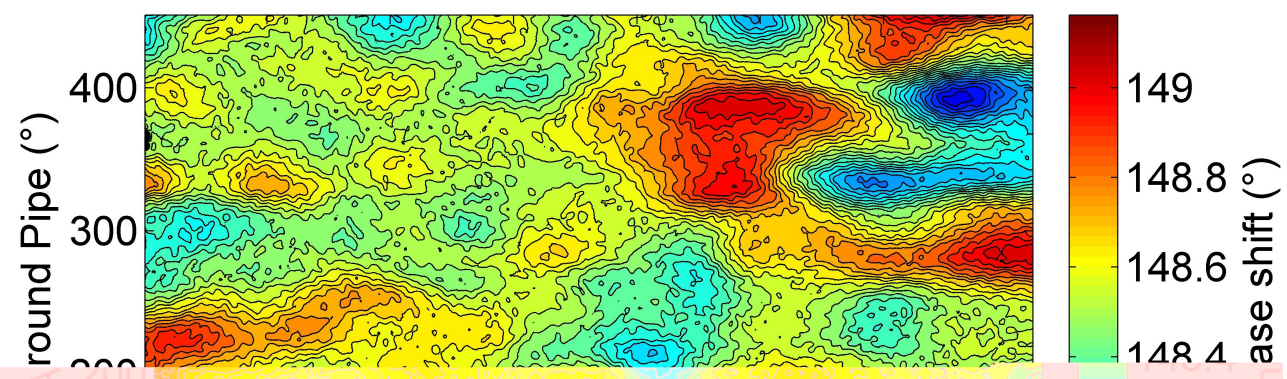

Figure 4.11: $\mathrm{R}$ sensor field phase shift over standard pipe with $\mathrm{Z}$ and $\mathrm{Phi}$ axis correction

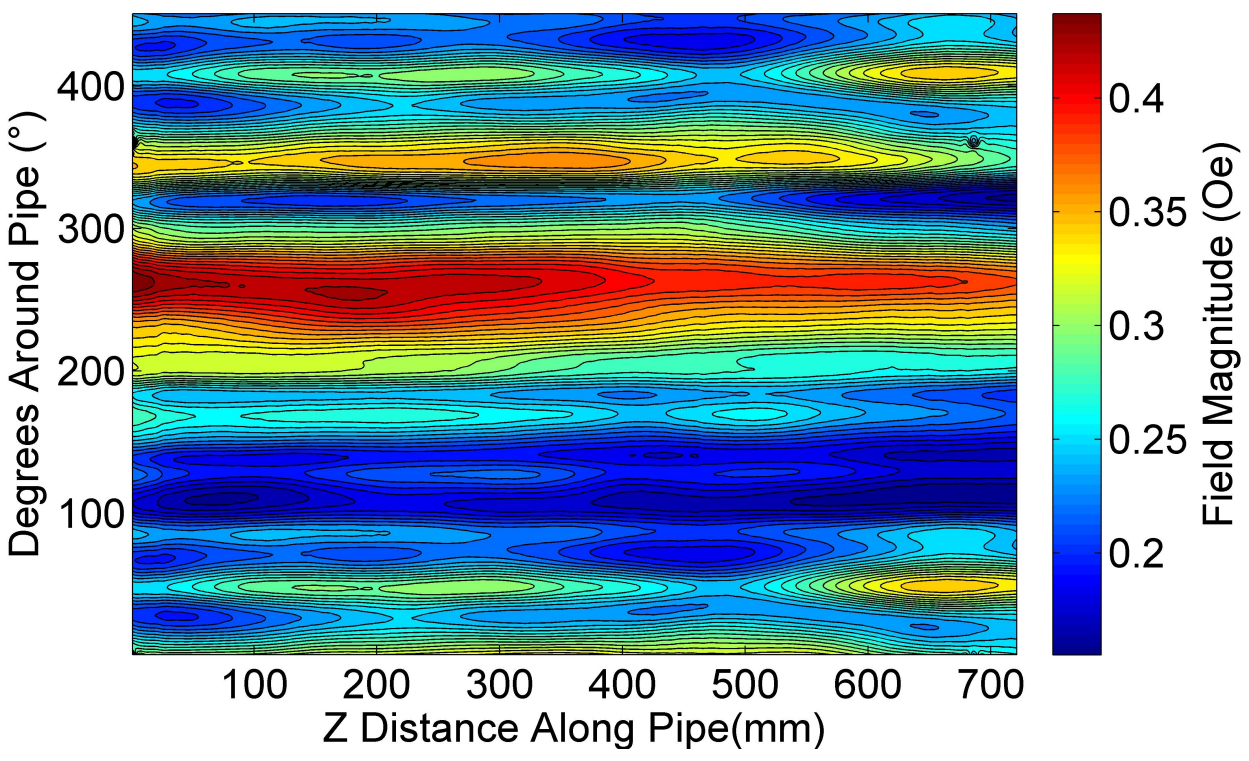

Figure 4.12: Phi sensor field magnitude over standard pipe 


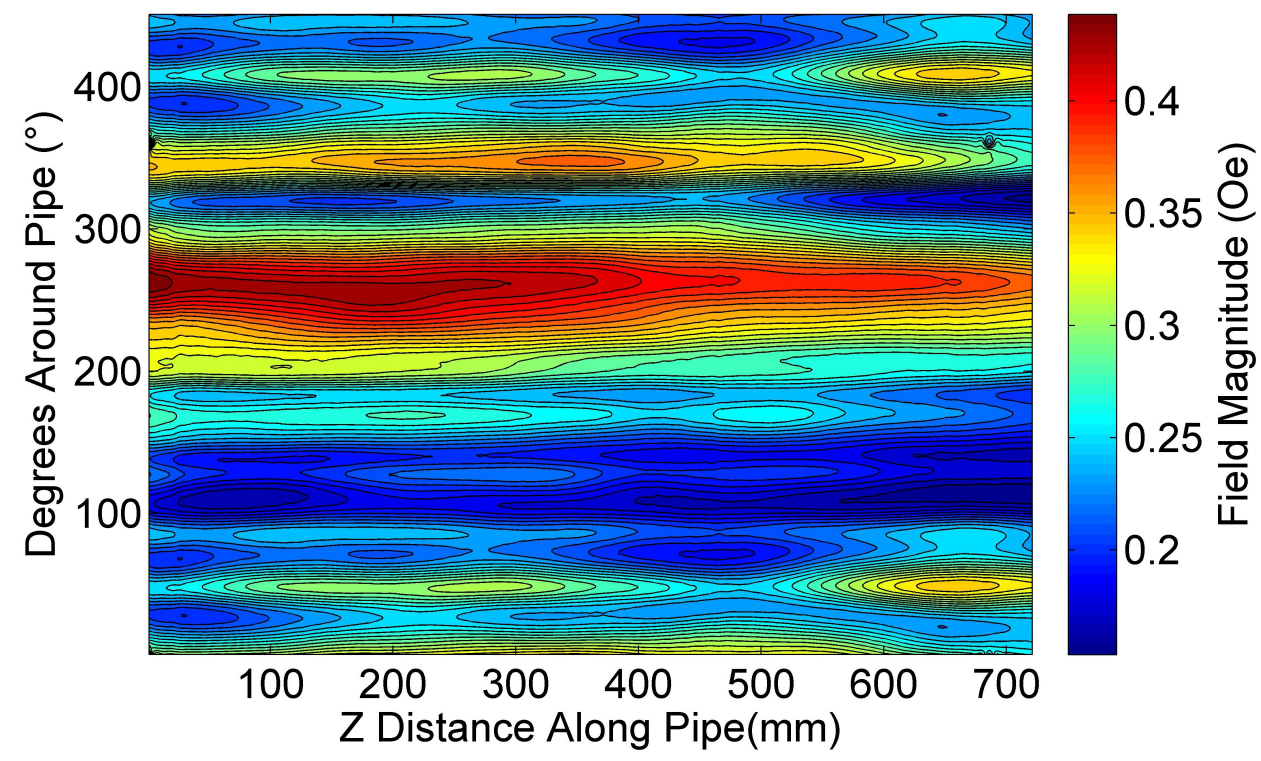

Figure 4.13: Phi sensor field magnitude over standard pipe with current correction

After applying the current correction algorithm to the data, as before, the resulting contour plot is shown in figure 4.13. The contour plots before and after the current correction are compared in figure 4.12 and figure 4.13 Again the expected small decrease in variation is seen.

Applying the $Z$ flattening algorithm to the data is shown in figure 4.15. This reveals that applying the $Z$ axis flattening algorithm has no adverse effects on the noise level of the data, meaning a constant correction method can be applied to all sensor data. The same lines of constant fields are seen along the $Z$ axis as in the $R$ field magnitude.

The magnetic field change with permeability is plotted in figure 4.14 showing the magnetic field's dependence on the permeability of the pipe. Similar to the R field, when two outlier points are ignored, there is a clear dependence on the permeability. However, the outliers are produced in different locations so this again suggests that other parameters changing in the pipe are also influencing the field magnitude.

The last noise source left is the lines of constant field strength along an angular position. This can be corrected with the Phi flattening algorithm and then the result of applying the correction is shown in figure 4.16. Here, like the $\mathrm{R}$ sensor, there is a general reduction in the background noise apart from the feature at around 30 degrees and $600 \mathrm{~mm}$.

\section{Phi Field phase shift analysis}

The raw data of the phase shift of the Phi field is shown in a contour plot in figure 4.17. As with the $\mathrm{R}$ field phase shift a clear linear slope from one end to the other can be seen. As the slope is smaller lines of constant phase shift along the $Z$ axis can be seen.

As with the $\mathrm{R}$ field, the phase shift of the Phi field also does not have a clear linear relationship with current. This means that the current correction algorithm is not effective. Unlike the magnitude signal there is significant change in field as the end of the pipe is

the $\mathrm{R}$ direction 


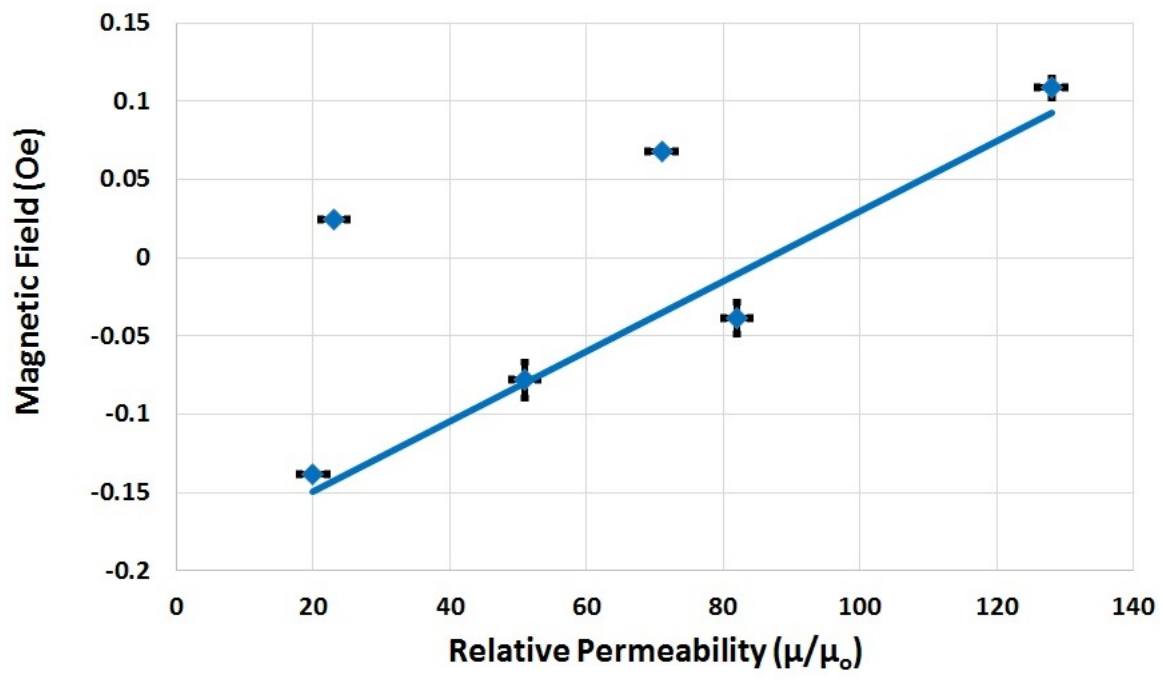

Figure 4.14: Phi field magnitude change with permeability

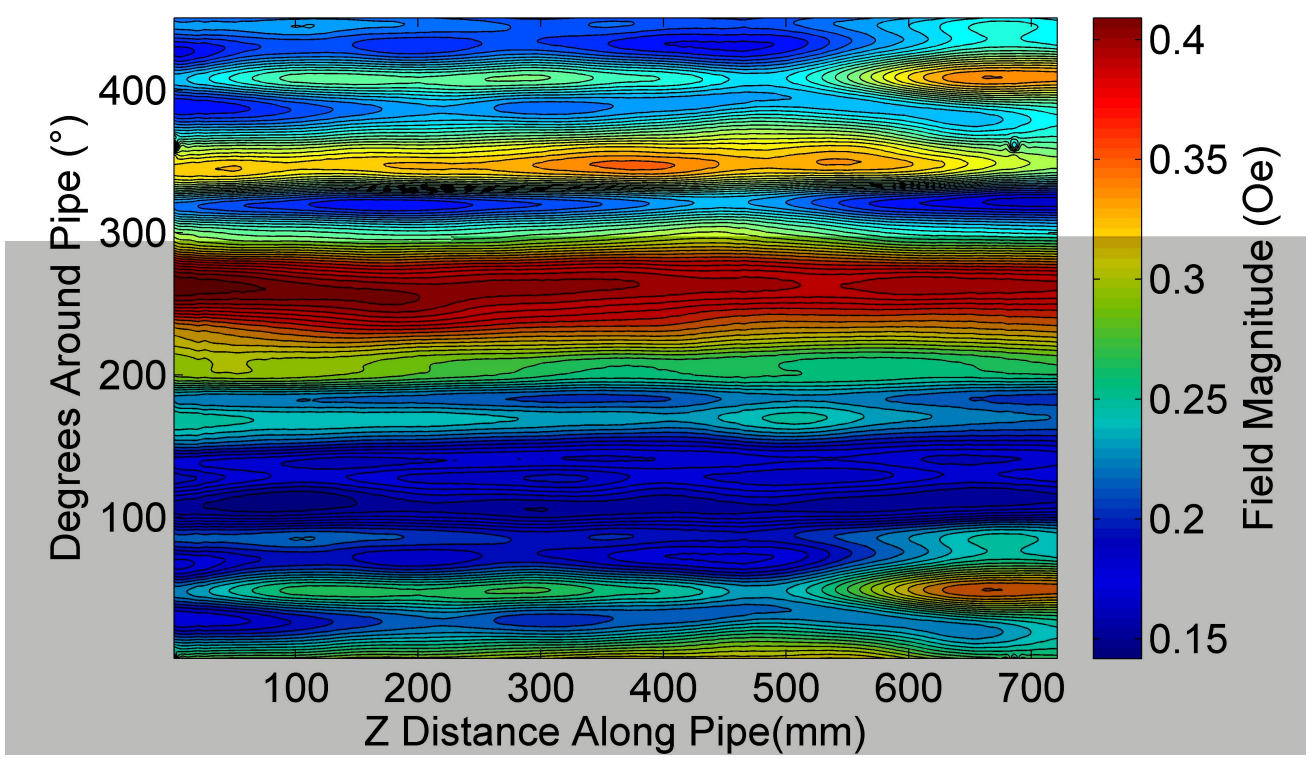

Figure 4.15: Phi sensor field magnitude over standard pipe with $Z$ flattening 


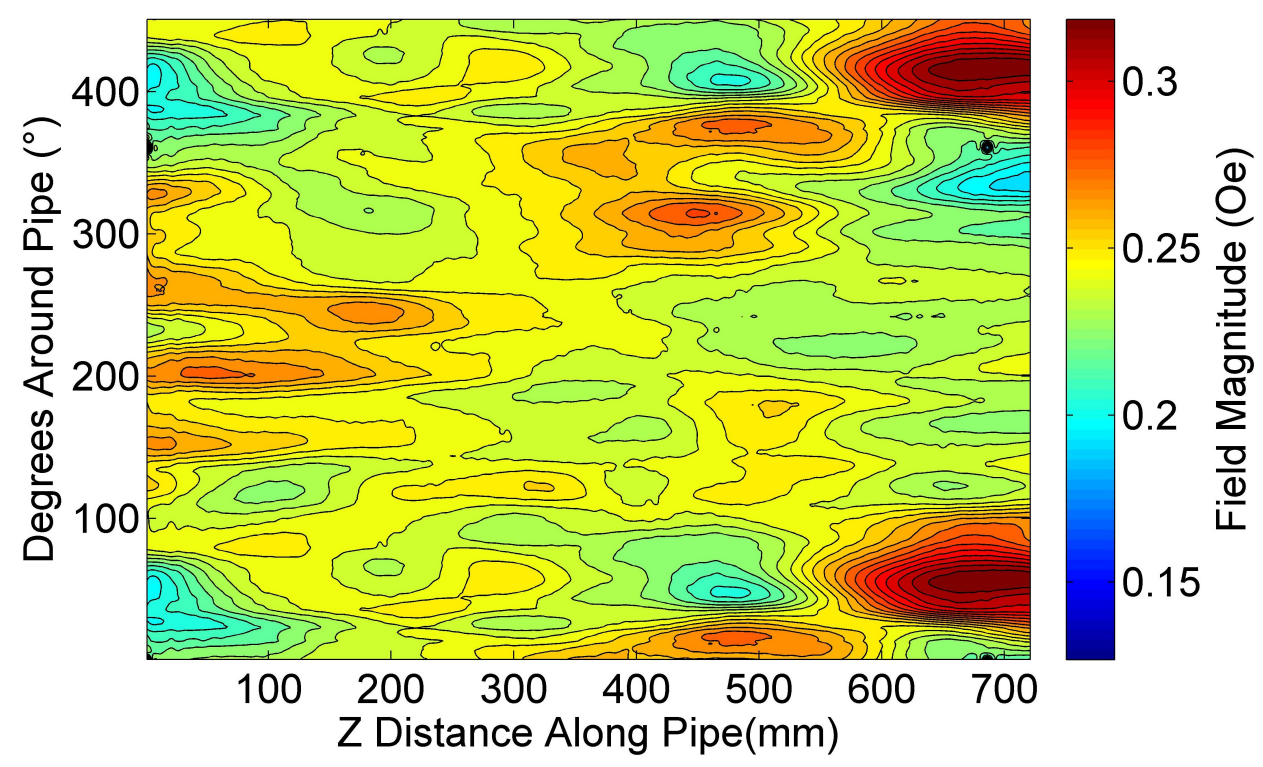

Figure 4.16: Phi sensor field magnitude over standard pipe with $Z$ and Phi flattening

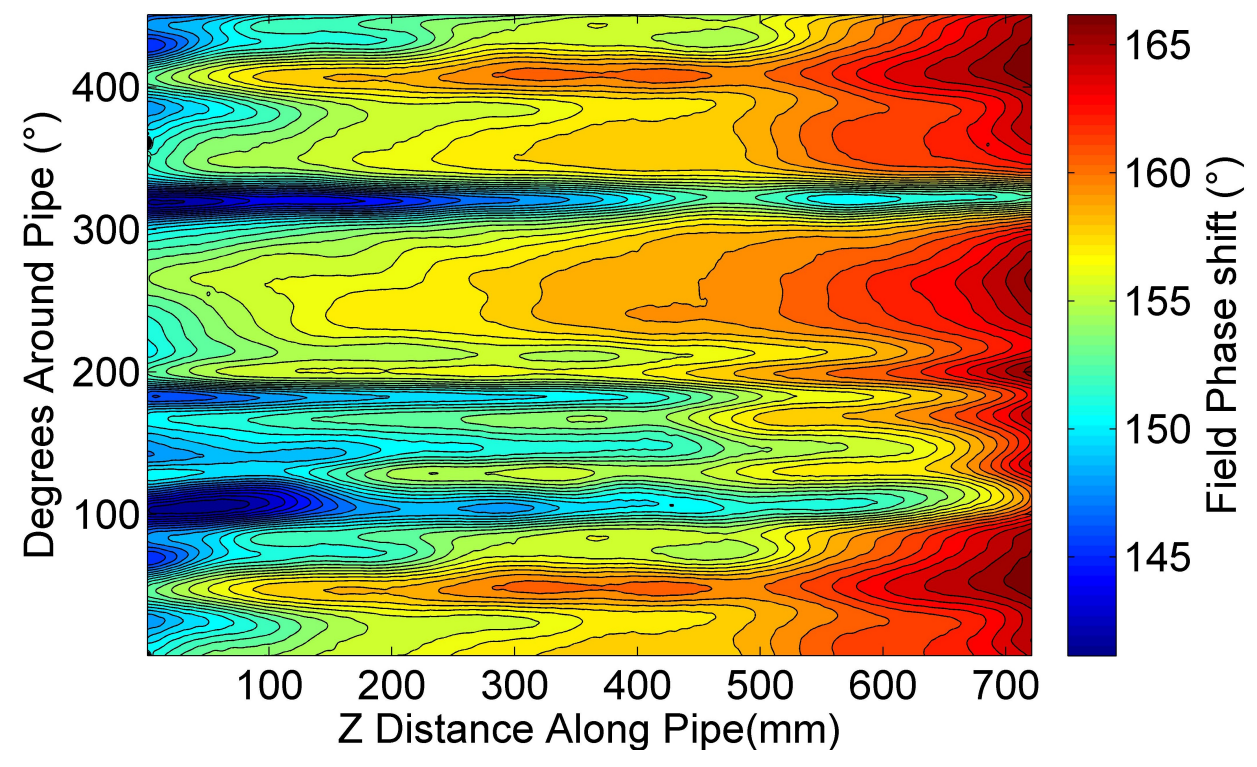

Figure 4.17: Phi sensor field phase shift over standard pipe 


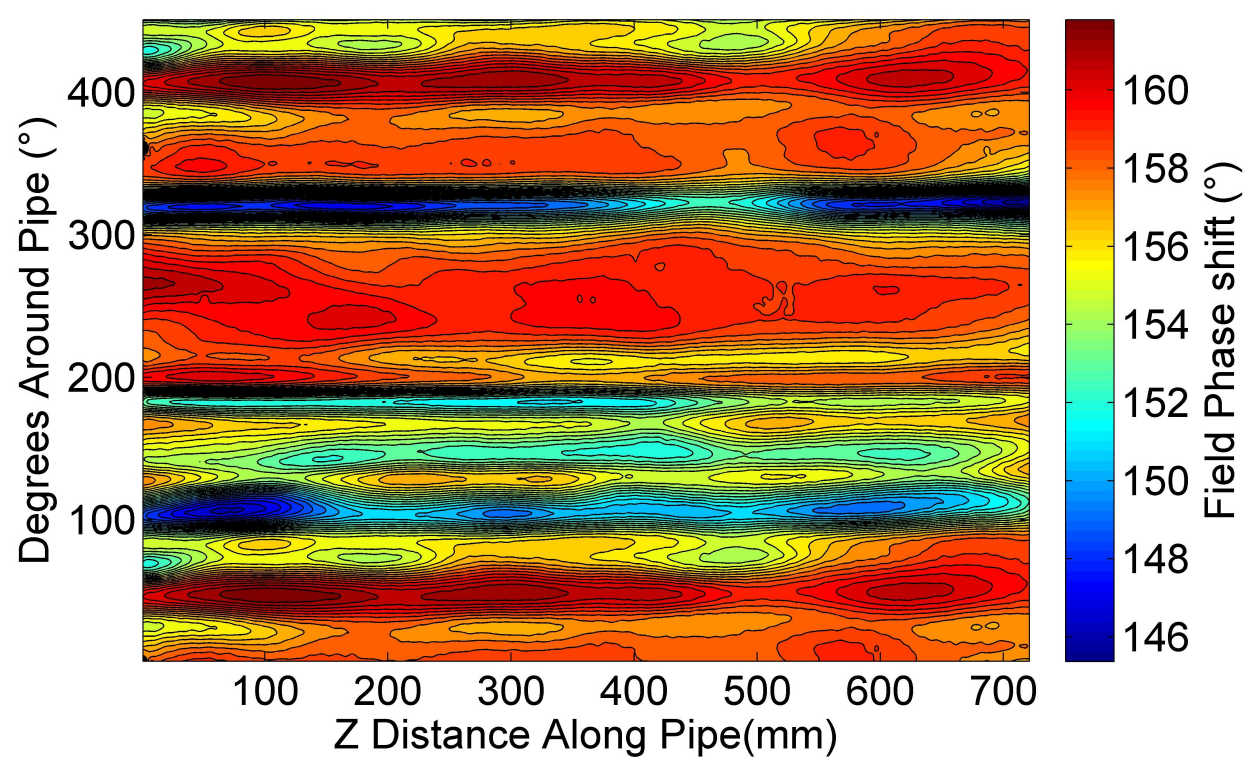

Figure 4.18: Phi sensor field phase shift over standard pipe with $Z$ axis flattening

approached. Therefore the $\mathrm{Z}$ flattening algorithm is applied to the data and the result is shown in figure 4.18.

Once the $\mathrm{Z}$ flattening algorithm has been applied, only lines of constant phase shift at particular angular positions can be seen. Again the correlation with permeability is assessed in figure 4.19 which shows, like the $\mathrm{R}$ field phase shift, there is no clear dependence on the permeability. To correct for the remaining variations, the Phi flattening algorithm is applied to the data and the result is shown in figure 4.20. Once both algorithms have been applied, there is a fairly evenly distributed noise over the whole pipe. This level of variation is the base line noise level that could be achieved with the system. This means any defect signal needs to be larger to be positively identified.

\section{3 summary of repeatability and data correction}

The effects of all correction methods on the $\mathrm{R}$ sensor are summarized in table 4.2. This shows the phase and magnitude standard deviation of all measured points and the percentage reduction in standard deviation from the uncorrected data. In conclusion, the most effective correction method for the $\mathrm{R}$ sensor is the $\mathrm{Z}$ flattening algorithm with the reduction of $73 \%$ for the magnitude and $93 \%$ for the phase. The Phi flattening when used in combination with the $Z$ flattening gives a $50 \%$ improvement over and above the $Z$ flattening. The current correction only shows some improvement, as expected, from the small changes in current but as the $Z$ flattening is far more effective than current correction the most effective correction algorithm is a combination of $Z$ flattening and Phi flattening. This correction algorithm will now be used for the rest of the $\mathrm{R}$ sensor standard pipe data unless otherwise stated.

The effect of the correction methods on the Phi sensor are summarized in table 4.3 which shows the phase and magnitude standard deviation of all the measured points and 


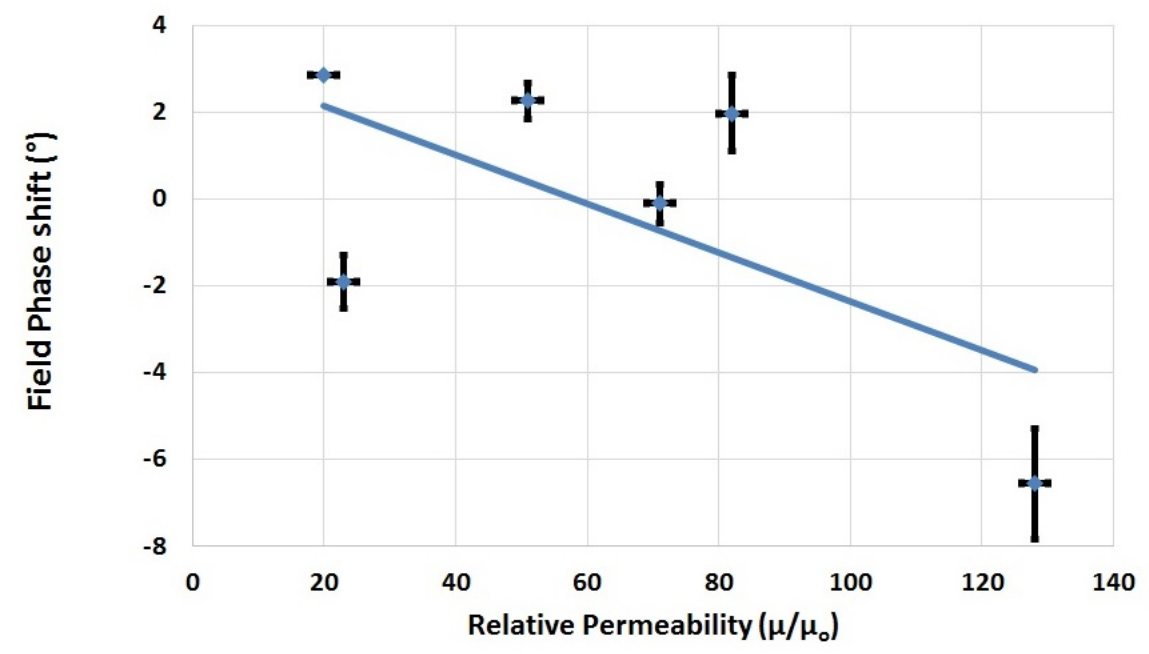

Figure 4.19: Phi field phase shift dependence on permeability

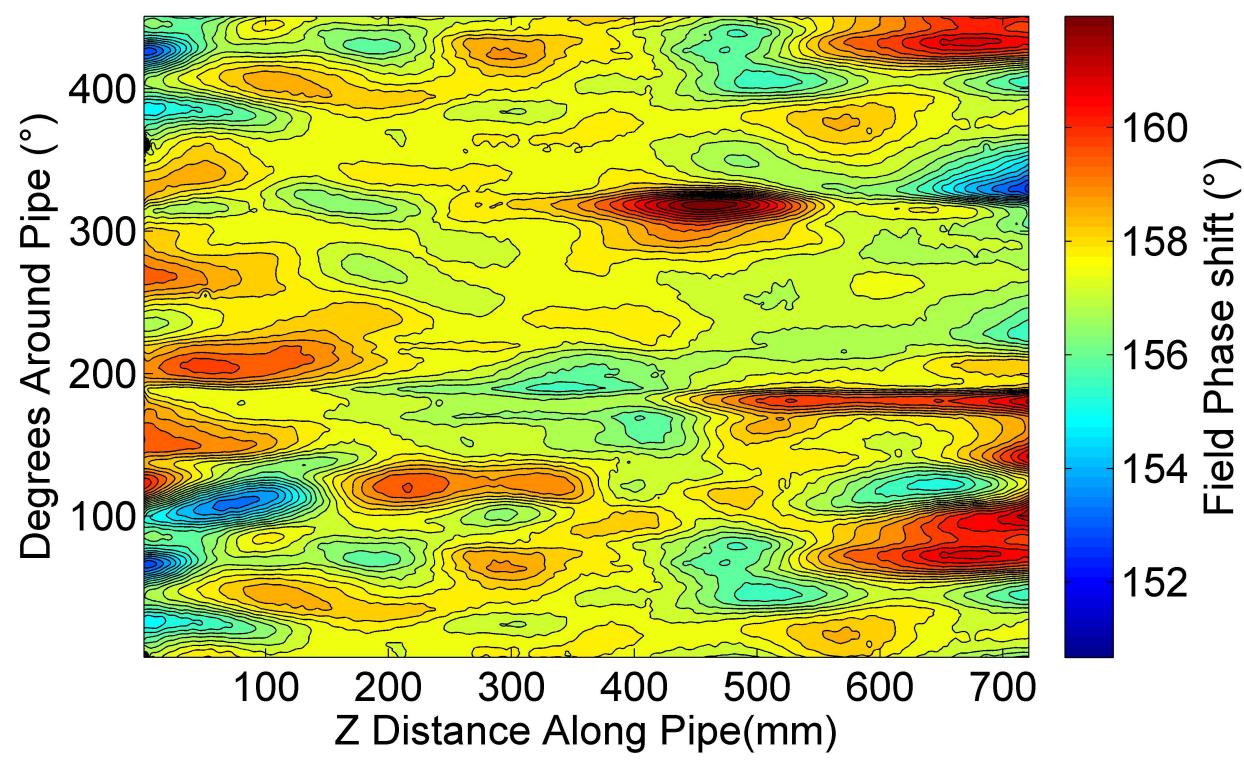

Figure 4.20: Phi sensor field phase shift over standard pipe with Phi and $Z$ axis flattening 
Table 4.2: Effect of correction methods on the standard deviation of the noise of the $R$ sensor

\begin{tabular}{|c|c|c|c|c|}
\hline $\begin{array}{c}\text { Correction } \\
\text { method }\end{array}$ & \multicolumn{2}{|c|}{ R Magnitude } & \multicolumn{2}{c|}{ R Phase } \\
\hline No correction & .220 & $0 \%$ & 5.07 & $0 \%$ \\
\hline $\begin{array}{c}\text { Current } \\
\text { correction }\end{array}$ & .188 & $14 \%$ & 5.10 & $0 \%$ \\
\hline Phi flattening & 0.219 & $3 \%$ & 5.07 & $0 \%$ \\
\hline Z flattening & .0598 & $73 \%$ & .343 & $93 \%$ \\
\hline $\begin{array}{c}\text { Current } \\
\text { correction \& Phi } \\
\text { flattening }\end{array}$ & .181 & $18 \%$ & 5.07 & $0 \%$ \\
\hline $\begin{array}{c}\text { Current } \\
\text { correction \& Z } \\
\text { flattening }\end{array}$ & .0601 & $73 \%$ & .343 & $93 \%$ \\
\hline $\begin{array}{c}\text { Z flattening \& } \\
\text { Phi flattening }\end{array}$ & .0277 & $87 \%$ & .184 & $96 \%$ \\
\hline $\begin{array}{c}\text { Current } \\
\text { correction \& Z } \\
\text { flattening \& Phi } \\
\text { flattening }\end{array}$ & 0.0281 & $87 \%$ & .184 & $96 \%$ \\
\hline
\end{tabular}


Table 4.3: Effect of correction methods on the standard deviation of the noise of the Phi sensor

\begin{tabular}{|c|c|c|c|c|}
\hline $\begin{array}{c}\text { Correction } \\
\text { method }\end{array}$ & \multicolumn{2}{|c|}{ Phi Magnitude } & \multicolumn{2}{c|}{ Phi Phase } \\
\hline No correction & .0625 & $0 \%$ & 4.97 & $0 \%$ \\
\hline $\begin{array}{c}\text { Current } \\
\text { correction }\end{array}$ & .0632 & $-1 \%$ & 4.97 & $0 \%$ \\
\hline Phi flattening & 0.0206 & $67 \%$ & 3.99 & $20 \%$ \\
\hline Z flattening & .0619 & $1 \%$ & 3.09 & $38 \%$ \\
\hline $\begin{array}{c}\text { Current } \\
\text { correction \& Phi } \\
\text { flattening }\end{array}$ & .0215 & $66 \%$ & 3.99 & $20 \%$ \\
\hline $\begin{array}{c}\text { Current \& Z } \\
\text { flattening }\end{array}$ & .0624 & $1 \%$ & 3.09 & $38 \%$ \\
\hline $\begin{array}{c}\text { Z flattening \& } \\
\text { Phi flattening }\end{array}$ & 0.0188 & $70 \%$ & 1.25 & $75 \%$ \\
\hline $\begin{array}{c}\text { Current } \\
\text { correction \& Z } \\
\text { flattening \& Phi } \\
\text { flattening }\end{array}$ & .0189 & $70 \%$ & 1.25 & $75 \%$ \\
\hline
\end{tabular}

the percentage reduction in standard deviation from the uncorrected data. The current correction algorithm has no noticeable effect with changes of only $1 \%$. The Phi flattening algorithm is the most effective algorithm for the magnitude signal, while the $\mathrm{Z}$ flattening algorithm on its own has little effect, but when combined with the Phi flattening algorithm it shows a small improvement. The phase shift signal shows significant improvement for both the Phi and $Z$ flattening algorithms. Again the combination of the two algorithms gives significant improvements. As with the $\mathrm{R}$ sensor the combination of the $Z$ and Phi flattening algorithms will be used for the rest of the data in this chapter unless otherwise stated.

\subsection{Influence of excitation current}

Increasing the current in the excitation sheet increases the magnetic excitation field, so an increase in magnetic field magnitude can be expected (section 2.5.1). This is tested with a full set of scans of the standard pipe for a range of excitation currents from $100 \mathrm{amps}$ to 600 amps. To summarize the general effect of increasing current on signal levels, the median and standard deviation value was calculated for all measured points. The median is plotted against current with the error bars showing one standard deviation range. 


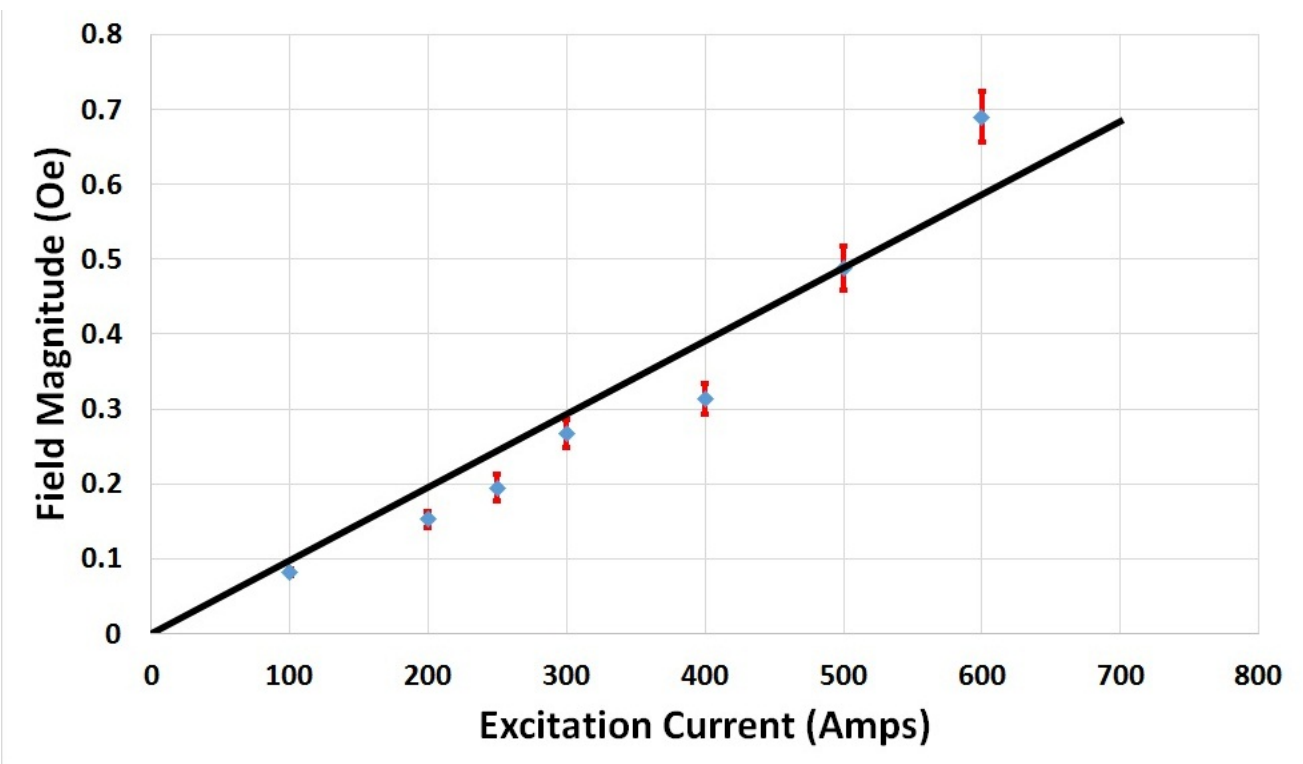

Figure 4.21: Phi sensor median magnitude change with excitation current

\subsubsection{Background level and total noise}

The Phi sensor magnitude is shown in figure 4.21. A linear fit applied to the data shows an increase in field with increasing excitation current with a slope of $0.001 \mathrm{Oe} / \mathrm{A}$. At 500 amps and above the data appears to depart from the linear fit, with an increased slope. The noise also increases with increasing current, with the standard deviation increasing for each current level shown by the error bars.

The $\mathrm{R}$ sensor magnitude is shown in figure 4.22 with a linear fit vs current with a slope of $0.0106 \mathrm{Oe} / \mathrm{A}$. Unlike the Phi sensor the standard deviation is so low that the error bars do not appear on the graph. The variation in the fit line is probably due to slight variations in the position of the sensors relative to the excitation sheet. A rotation of around $0.5 \mathrm{~mm}$ can change the field strength by $10 \%$ as shown in section 3.2.2.

The Phi sensor phase shift is shown in figure 4.23. There are two significant outliers at 250 and 600 amps. These two tests were carried out after the excitation unit and the sensor module were disassembled and reassembled. This suggests that these outliers are due to the excitation unit and the sensor module not being correctly re-assembled. If these two outliers are ignored, a clear linear decay in the phase shift can be seen with a three degrees per 100 amps slope.

The phase shift for the $\mathrm{R}$ sensor is shown in figure 4.24. Again a general downward trend in the phase shift with current is observed, with a value of three degrees over the 500 amps.

In summary, the effect of increasing the excitation current causes the background field magnitude to increase linearly for both $\mathrm{R}$ and Phi sensors. The phase shift appears to be linearly decreasing with increasing excitation current but with very small slope. However, to decide what excitation level is likely to be optimal, the signal noise must be determined.

To measure the noise the standard deviation of the magnitude or phase is calculated for each current level. The $\mathrm{R}$ field magnitude standard deviation is shown in figure 4.25 


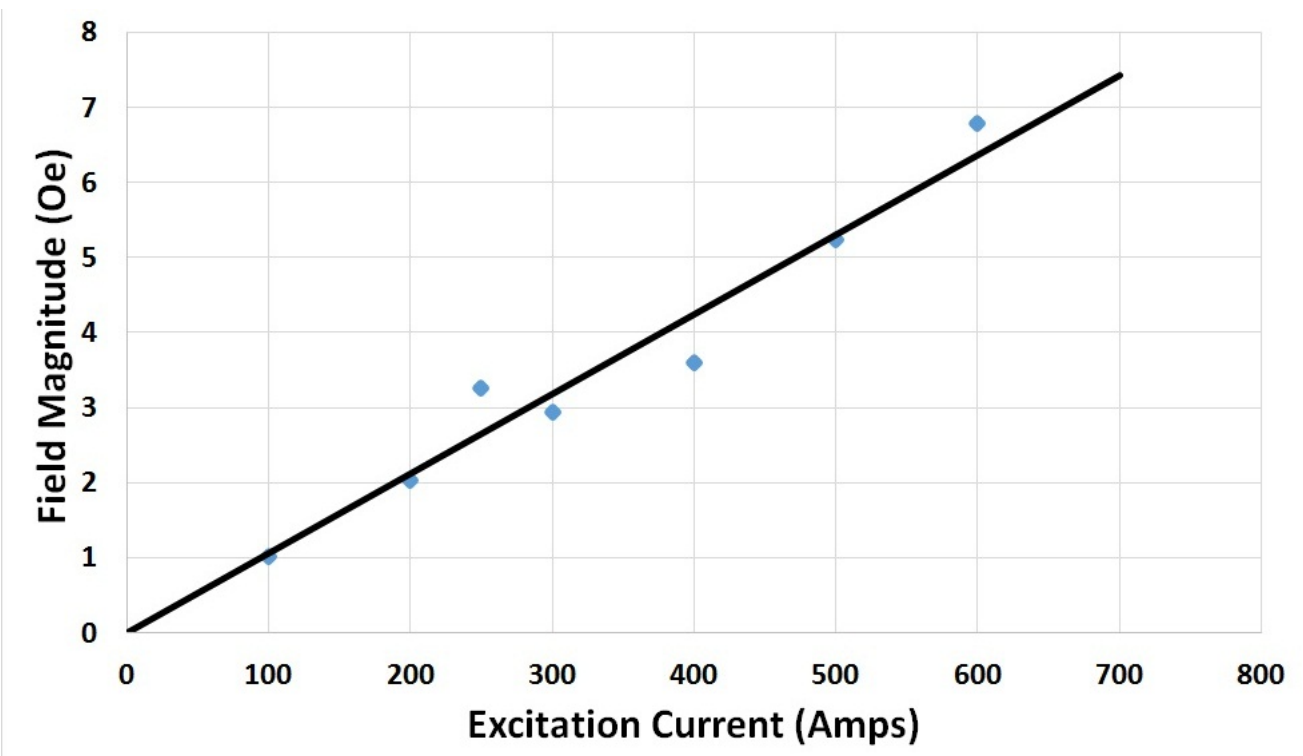

Figure 4.22: $\mathrm{R}$ sensor median magnitude change with excitation current

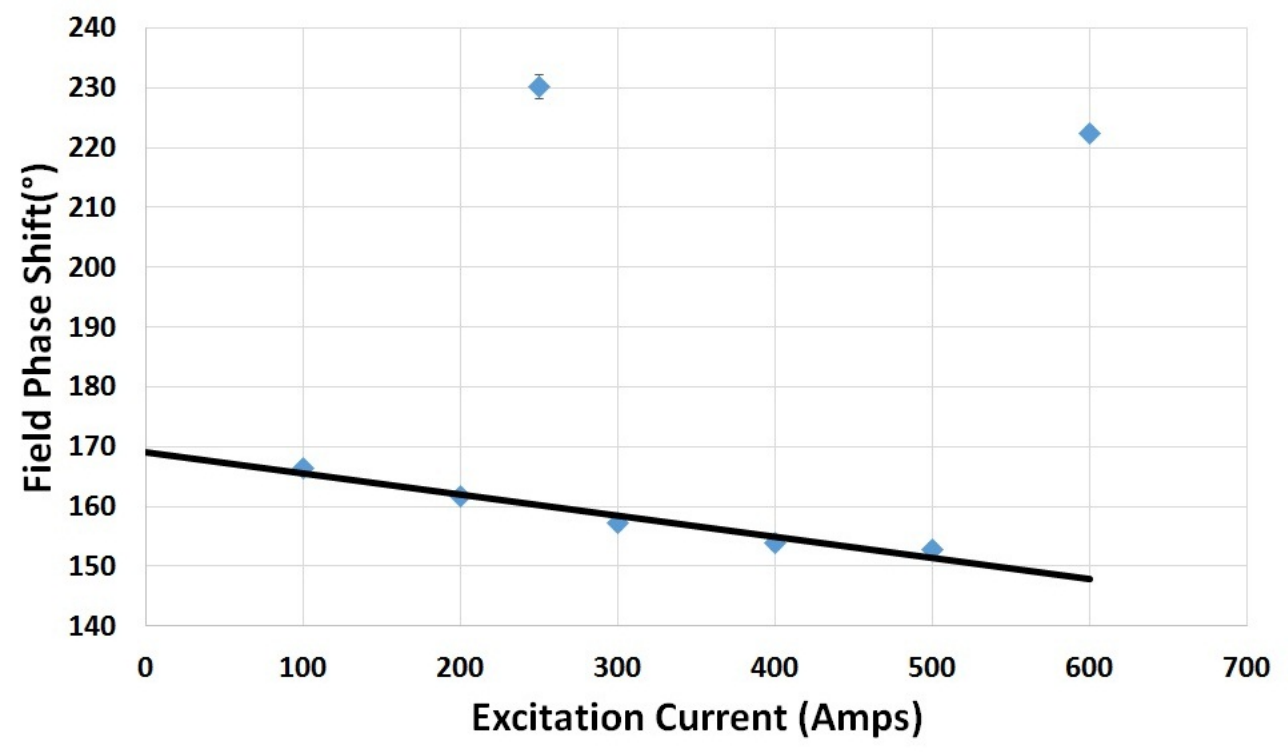

Figure 4.23: Phi sensor median phase shift change with excitation current 


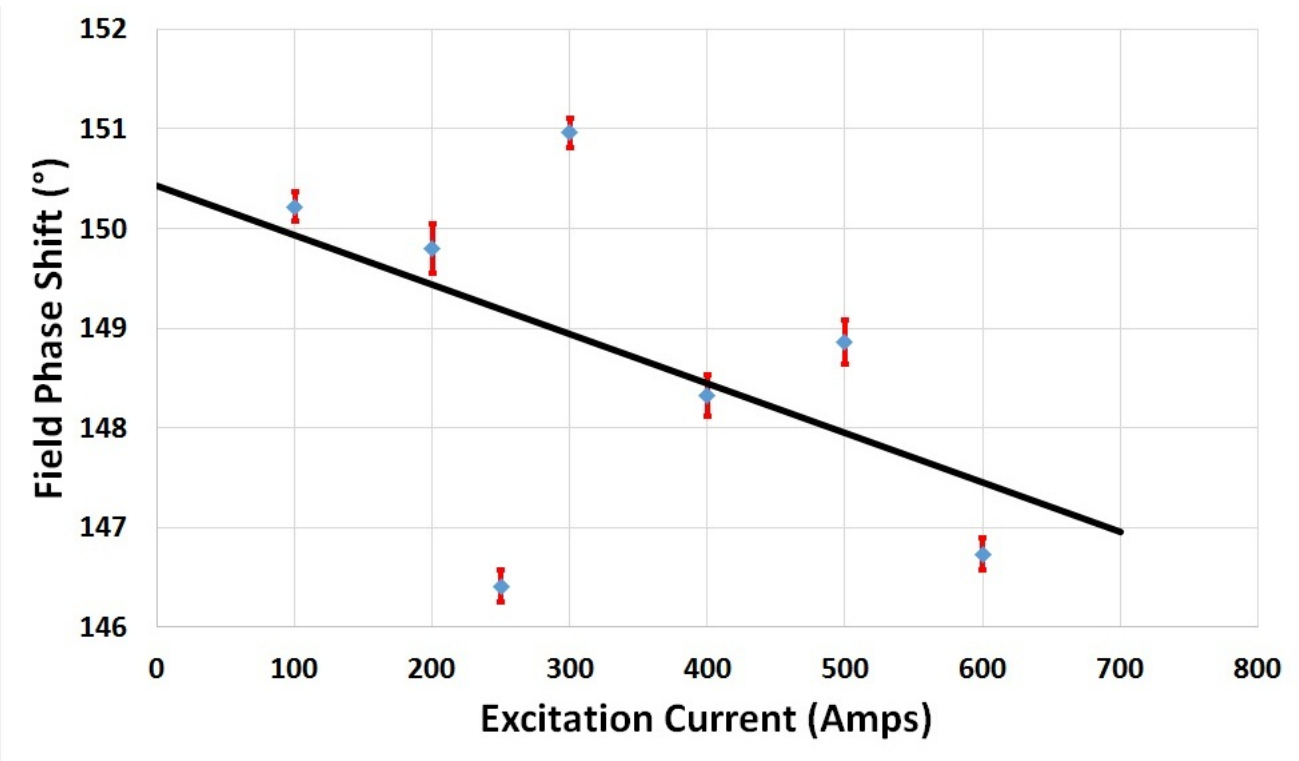

Figure 4.24: $R$ sensor median phase shift change with excitation current

and Phi in figure 4.26. The R field standard deviation has a good linear fit with excitation current. The linear fit crosses just above zero at $0.0012 \mathrm{Oe}$, which is the noise floor of the system, while the slope of $80 \mu \mathrm{Oe} / \mathrm{A}$ shows the rate at which the variations caused by pipe material variations increase with excitation current.

The Phi field standard deviation also shows a good linear fit with excitation current with $60 \mu \mathrm{Oe} / \mathrm{A}$ slope. The linear fit again crosses just above the zero at $0.0004 \mathrm{Oe}$ which shows the noise floor for this sensor. The standard deviation for the Phi sensor is approximately $30 \%$ lower than the $\mathrm{R}$ sensor, however with the background field level reduced by an order of magnitude the SNR is expected to drop too.

The phase shift standard deviation change with excitation current is shown in figure 4.27 for the $\mathrm{R}$ field and in figure 4.28 for the Phi field. The R field shows a very low noise level with just over a quarter of a degree standard deviation and there appears to be no change with excitation current. Variation in standard deviation compared to the absolute value is small.

The Phi field phase shift shows a higher standard deviation than the $\mathrm{R}$ sensor. This shows that variation in the pipe proprieties have a significantly larger effect on the Phi field phase shift compared to the $R$ field with a five times increase in standard deviation.

To understand the relative noise level for the $\mathrm{Phi}$ and $\mathrm{R}$ sensor magnitude, the signal to noise ratio (SNR) is calculated. The SNR is calculated by taking the median field for a given current level and dividing it by the standard deviation of the measurements at that current level. The SNR change for the R sensor is shown in figure 4.29. The black line represents the SNR if the magnitude and standard deviation follow the linear fits shown previously. The predicted SNR should asymptote to the ratio of the slopes at high current. At low current the non-zero standard deviation at zero current causes a rapid decay to zero SNR below 100 amps. There is significant variation around the fit due to the high ratio level between the background and the standard deviation where small changes in the standard 


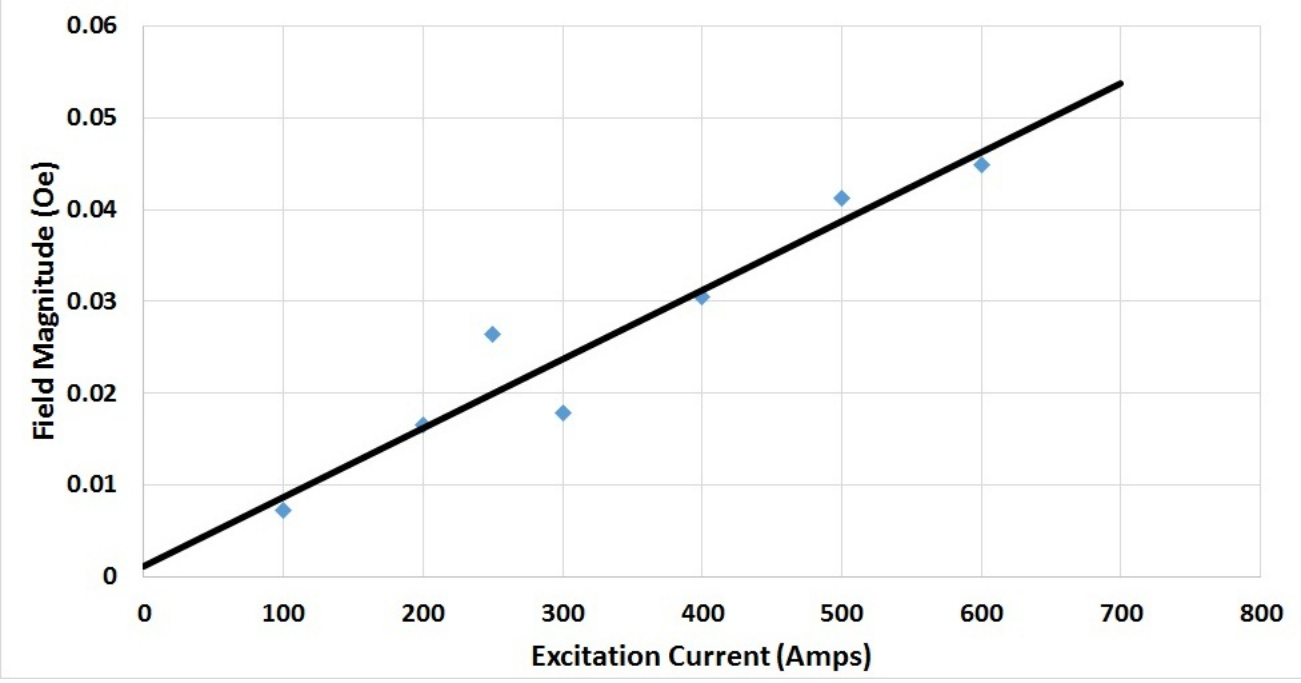

Figure 4.25: $\mathrm{R}$ field magnitude standard deviation change with excitation current

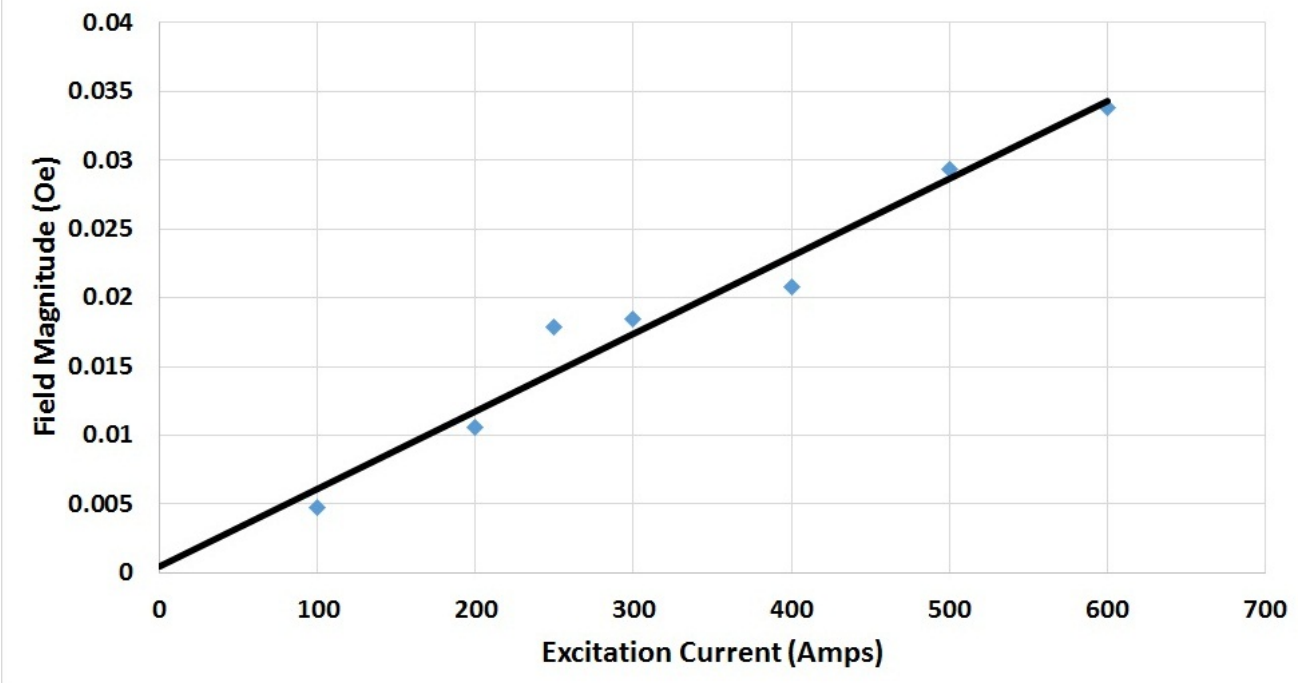

Figure 4.26: Phi field magnitude standard deviation change with excitation current 


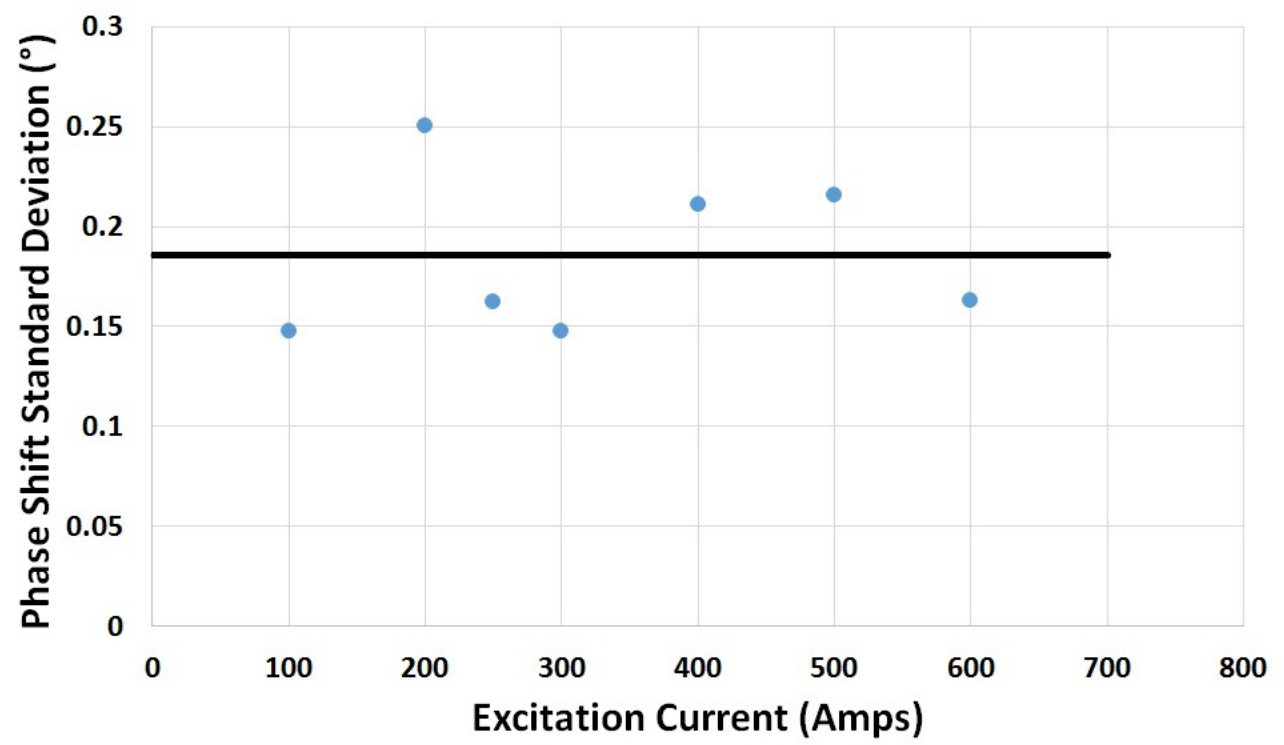

Figure 4.27: $\mathrm{R}$ sensor phase shift standard deviation change with excitation current

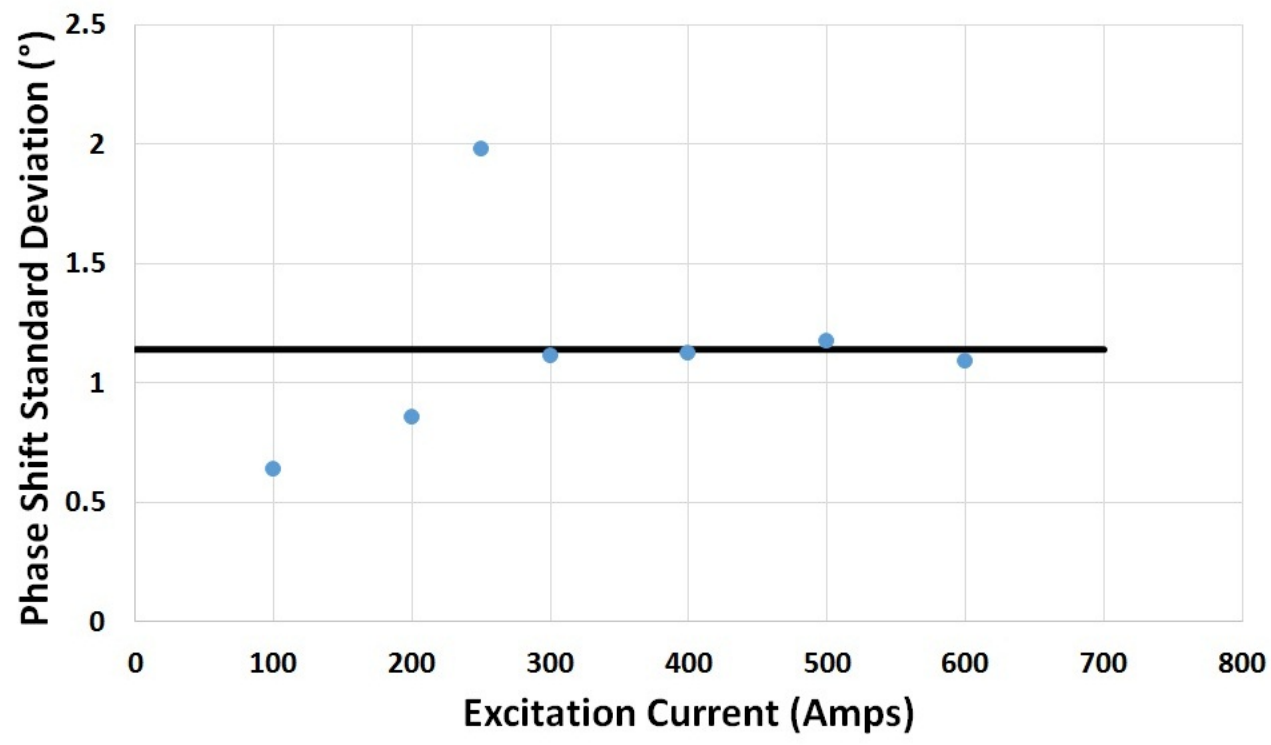

Figure 4.28: Phi sensor phase shift standard deviation change with excitation current 


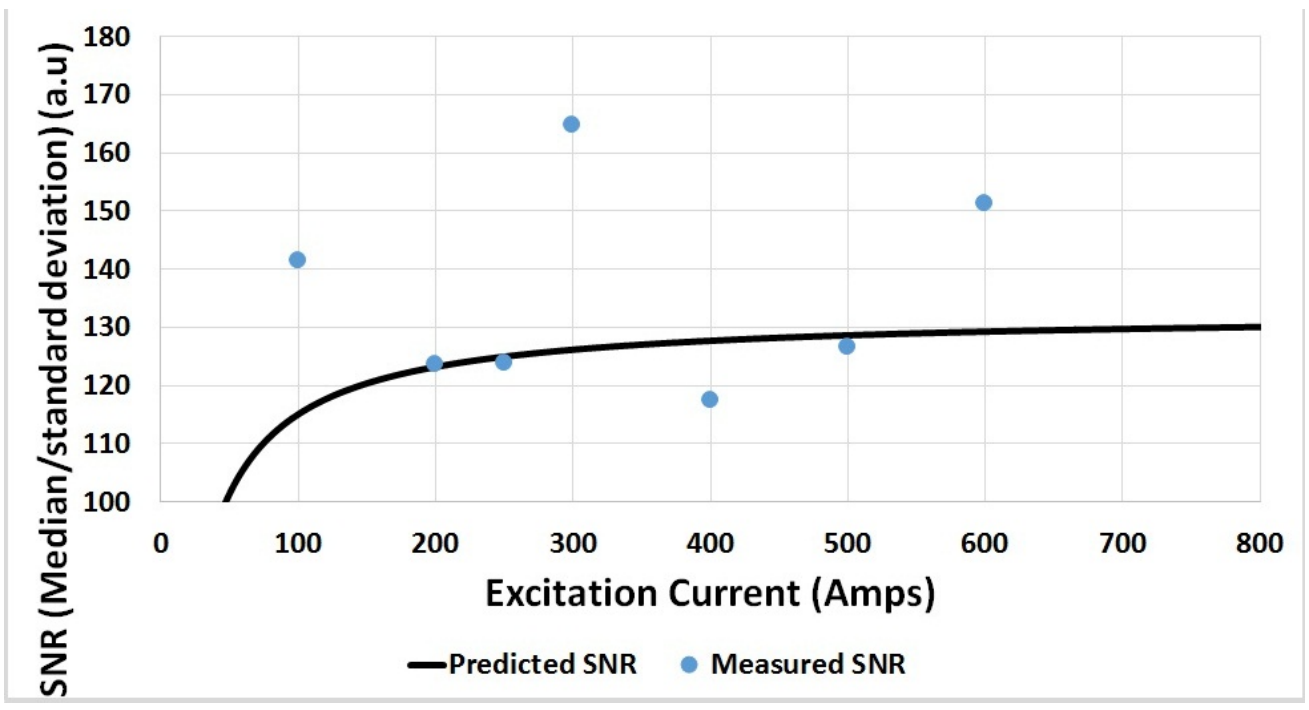

Figure 4.29: R sensor magnitude SNR change with excitation current

deviation produced large changes in the SNR.

The change in SNR with excitation current of Phi sensor magnitude is shown in figure 4.30. Firstly the SNR is much lower, probably due to the lower value for the absolute level of the magnetic field in the Phi direction. Again the black line represents the SNR if the magnitude and standard deviation follow the linear fits shown previously. The variation of the SNR is still large, but much lower than the $R$ field which has a maximum variation of 50 while the Phi field has only 9.

\subsubsection{Effects of excitation current on local variation}

The major source of variation over the pipe is due to variation of the pipe properties such as permeability, conductivity and wall thickness. It is important to identify how these variations are affected by changing excitation current. To assess how the excitation current affects these variations three measures are used. For all measures the median value of all data is calculated and then subtracted from all data points. This shifts the data to be centered around zero and means that variations in the background level which have already been presented can be ignored and only shows how the variations caused by local changes in the properties of pipe change with excitation current.

The first measure shows data without any correction. The median value at each $Z$ position is calculated (figure 4.31) for different excitation currents with the $285 \mathrm{~mm}$ on the $x$ axis representing the middle of the pipe. The second measure applies the $Z$ flattening algorithm to the data and then again calculates the median value at each $Z$ location for all excitation currents. This shows how the variations along the $Z$ axis are affected once the end effect of the pipe is removed. For the third measure the $Z$ flattening algorithm is again applied to the data and then the median value is taken at each Phi location. This is then plotted for each excitation current. This shows how variations along the Phi axis are affected by the excitation current. 


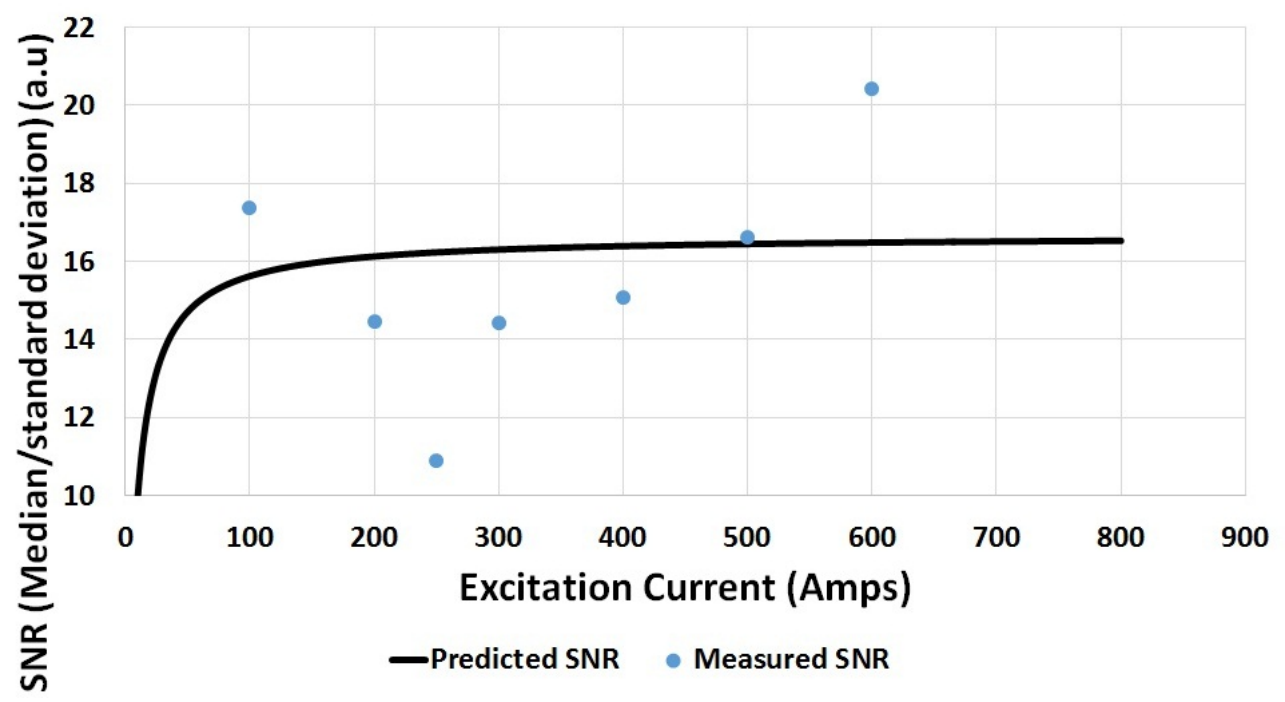

Figure 4.30: Phi sensor magnitude SNR change with excitation current

For the $\mathrm{R}$ sensor magnitude, the first measure with uncorrected data along the $Z$ axis is shown in figure 4.31, the second measure with $Z$ flattened data along the $Z$ axis is shown in figure 4.32, the third measure with $Z$ flattened data along the Phi axis is shown in figure 4.33. The uncorrected data shows that increasing current causes the magnetic field to increase at the end of the pipe. While low currents, e.g 100 amps, show a nearly flat response, a high current e.g $600 \mathrm{amps}$ has no flat section at any point along the pipe.

Applying the $Z$ axis flattening algorithm shows an increase in random variation along the $Z$ axis, but a significantly reduced effect from the end of the pipe. The third measure using the $Z$ axis flattening algorithm looking along the Phi axis shows more peaks and troughs than along the $Z$ axis and a large absolute value. The size of the peaks increases with increasing excitation current.

The phase shift of the $R$ field with uncorrected data along the $Z$ axis is shown in figure 4.34, with the $Z$ flattening algorithm applied along the $Z$ axis in figure 4.35 and $Z$ flattened data along the Phi axis in figure 4.36.

The uncorrected data from the $R$ sensor phase shift along the $Z$ axis in figure 4.34 shows a clear linear dependence for all currents. As the excitation current increases, the slope becomes more linear for the full length of the test area. The $100 \mathrm{Amp}$ data between $250 \mathrm{~mm}$ and $400 \mathrm{~mm}$ suggests that the slope in the phase shift is due to the end effect of the pipe and that increasing excitation current makes the effect spread out further from the end of the pipe. This is similar to magnitude data where increasing excitation current spread the fields further down the pipe.

After applying the $Z$ flattening algorithm to the $R$ sensors phase shift, the data along the $Z$ axis shows noisy variations. The variation due to the excitation current is seen best at $400 \mathrm{~mm}$ in figure 4.35 where the higher excitation current increases the variation of the signal. However, a larger noise level of the data compared to the small changes in signal level is appearing.

After applying the $Z$ flattening algorithm to the $\mathrm{R}$ sensor phase shift data more variation 


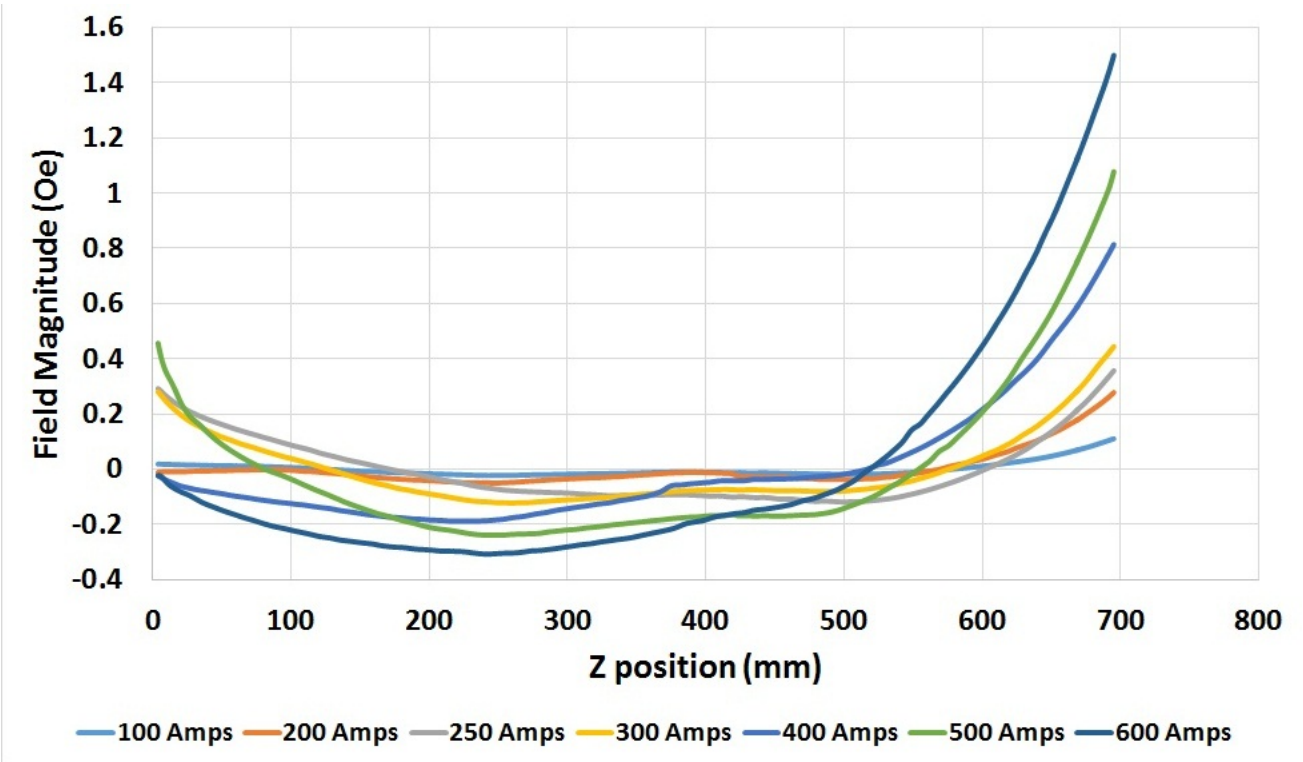

Figure 4.31: $\mathrm{R}$ sensor magnitude median along $\mathrm{Z}$ axis without correction

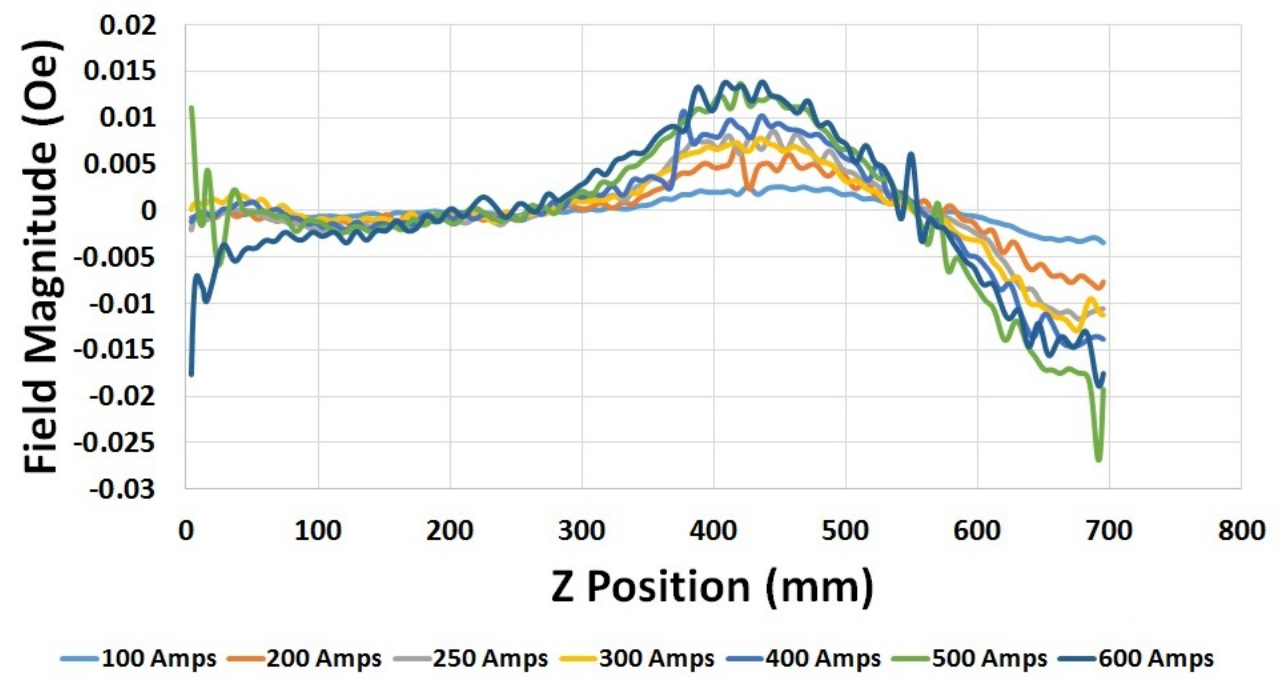

Figure 4.32: $R$ sensor magnitude median along $Z$ axis with $Z$ axis flattening 


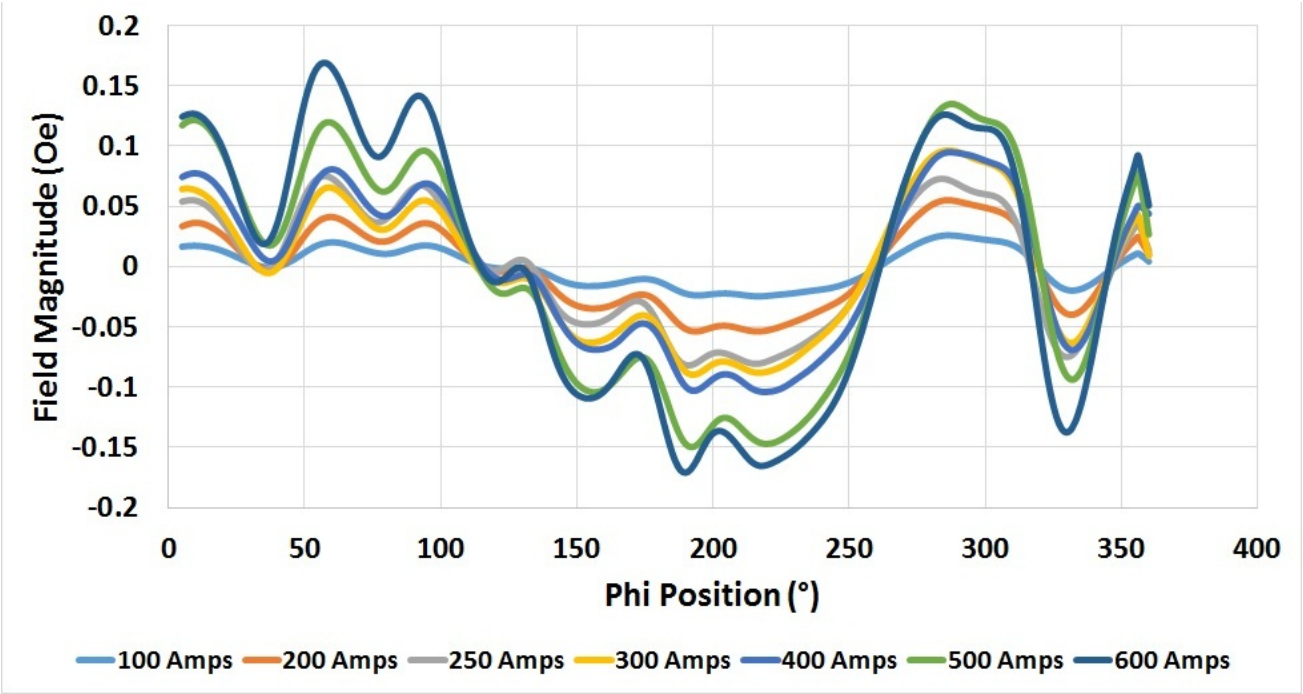

Figure 4.33: $\mathrm{R}$ sensor magnitude median along $\mathrm{Phi}$ axis with $\mathrm{Z}$ axis flattening

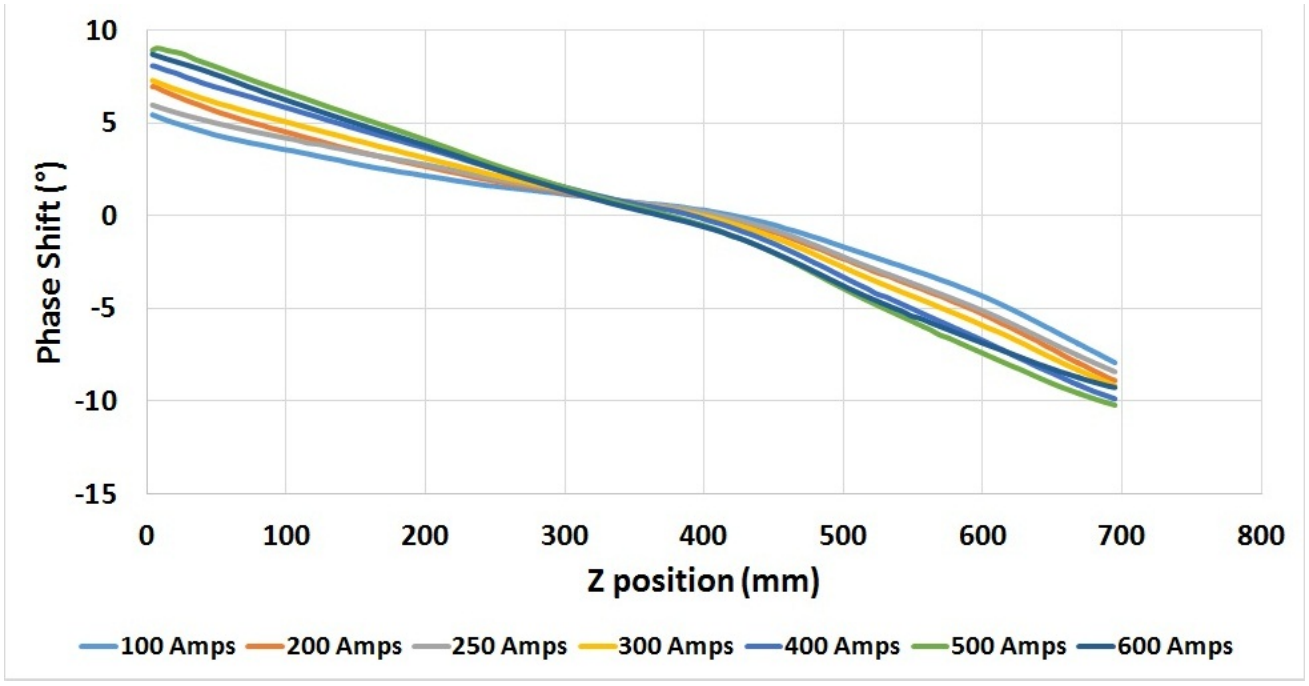

Figure 4.34: $\mathrm{R}$ sensor phase shift median along $Z$ axis 


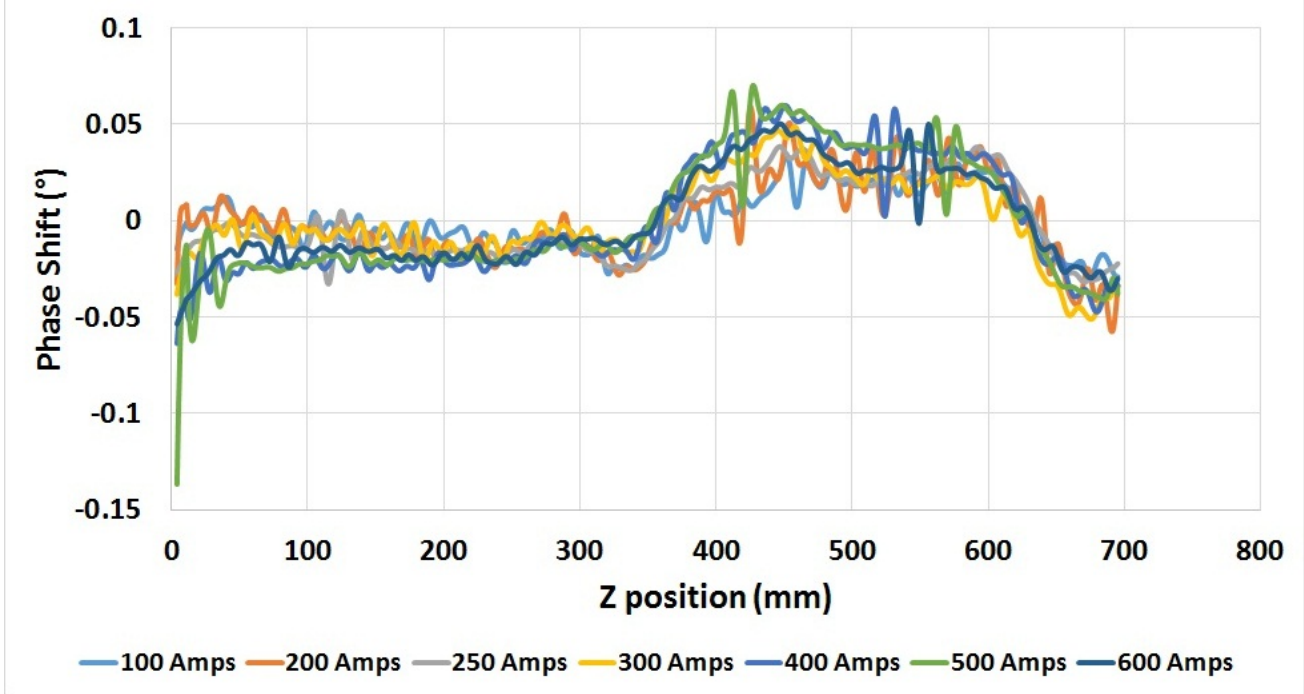

Figure 4.35: $R$ sensor phase shift median along $Z$ axis with $Z$ axis flattening

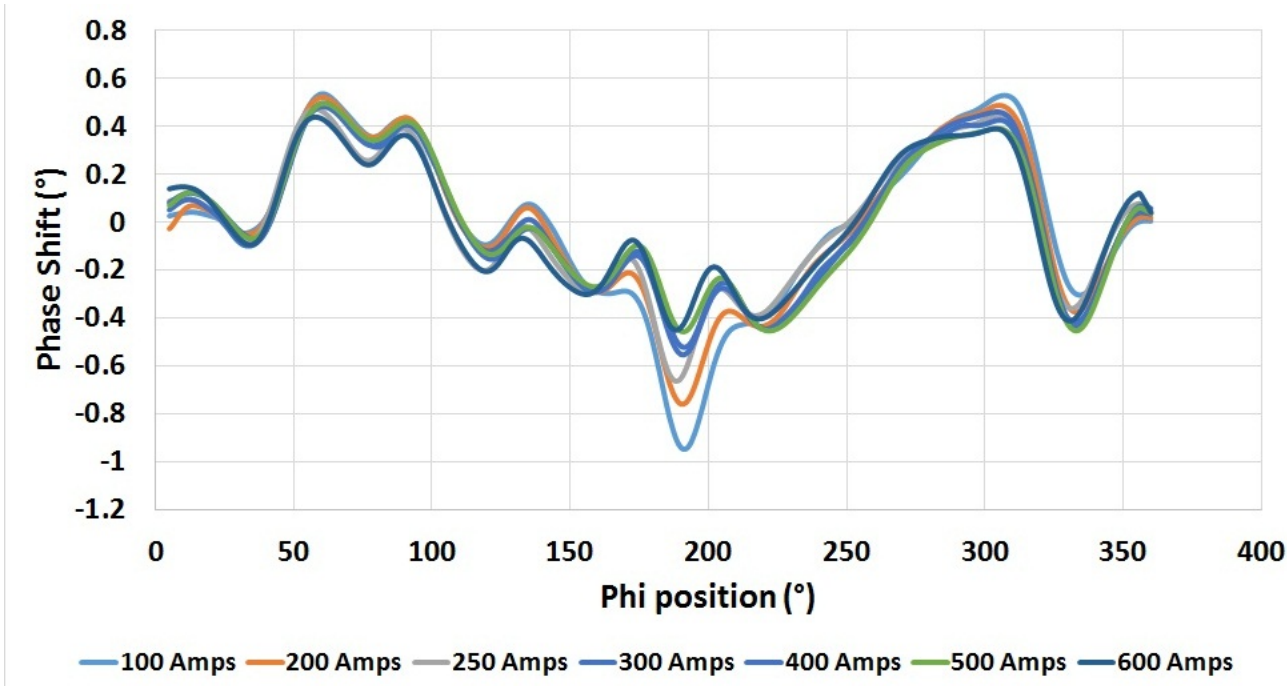

Figure 4.36: $R$ sensor phase shift median along Phi axis with $Z$ axis flattening 


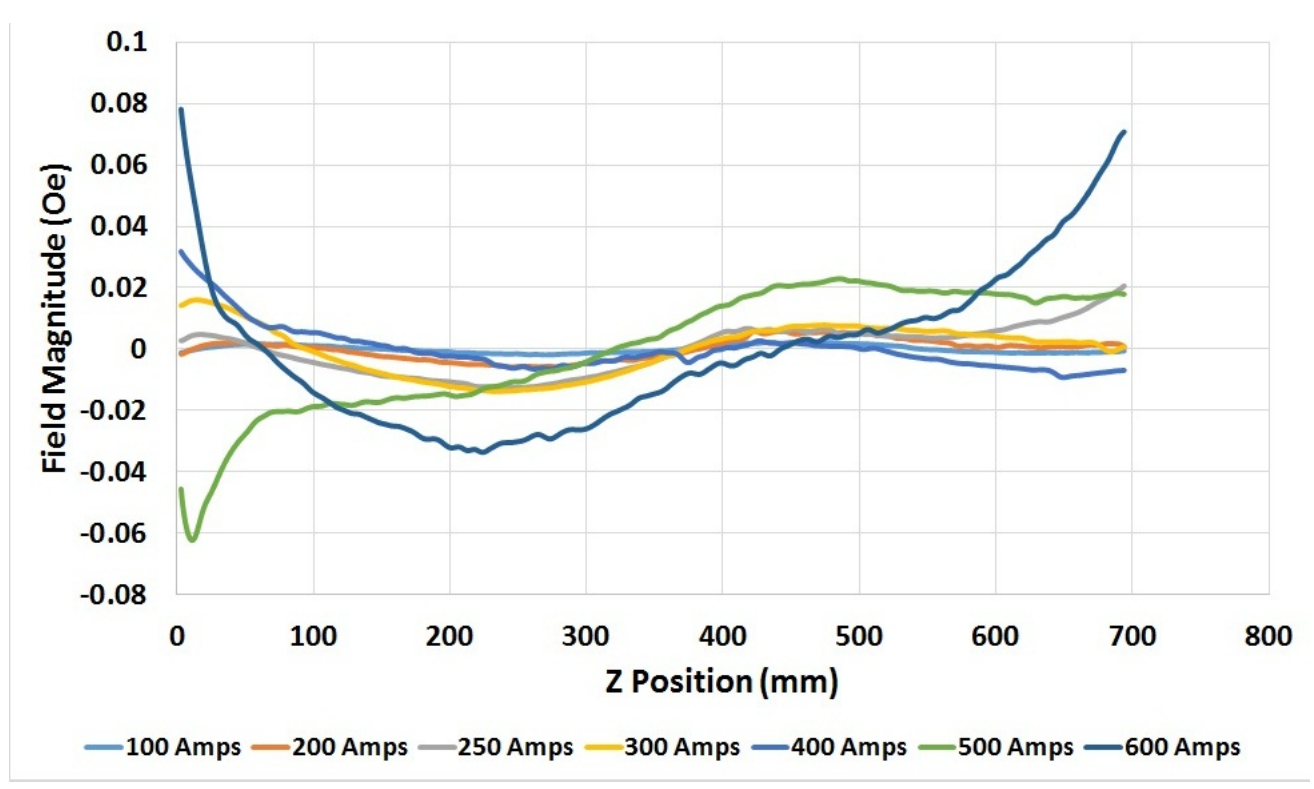

Figure 4.37: Phi sensor field magnitude median along $Z$ axis

than along the $Z$ axis can be seen in figure 4.36. All but the variation at 180 degrees are independent of excitation current. The trough at 180 degrees quickly reduces in size with increasing excitation current until 300 amps excitation current is reached, at which point they become independent of excitation current. This variation is due to the weld seam on the pipe, so a different response at this location can be expected.

The Phi sensor magnitude signal does not show improvement from applying the $Z$ flattening algorithm, so only the uncorrected data along the $Z$ axis and the raw data along the Phi axis are presented in figures 4.37 and 4.38. The uncorrected data along the $Z$ axis for the Phi sensor magnitude shows only small variations with excitation current. This is mainly due to small variations of the pipe parameters along the $Z$ axis, so no correction is required, as there is little effect on the Phi sensor magnitude. The exceptions to this are the higher currents $600 \mathrm{amp}$ and $500 \mathrm{amp}$, to a lesser degree, which show significant increase in field at the pipe end. This is probably due to imperfect mounting of the sensor during these tests, as imperfect mounting means a portion of the $R$ field is measured. A curvature of some degree would be expected in all excitation levels for the Phi sensor magnitude. The uncorrected data along the Phi axis for the Phi sensor magnitude shows a larger variation along the Phi axis than the $Z$ axis and all the peaks in the Phi axis clearly increase with increasing current.

Assessing the Phi field phase shift the first measure with current corrected data along the $Z$ axis is shown in figure figure 4.39, the second measure with current corrected and $Z$ flattened data along the $Z$ axis in figure 4.40 , lastly the third measure with current corrected and $Z$ flattened data along the Phi axis in figure 4.41.

The uncorrected data along the $Z$ axis shows a gradual change in phase shift from the start to the end of the pipe with a slight increase at either end. The $250 \mathrm{amp}$ and 600 amp test shows a significantly larger increase than the rest of the current levels. Again, probably a slight rotation of the Phi sensor in to the $\mathrm{R}$ axis is the reason for this behavior, 


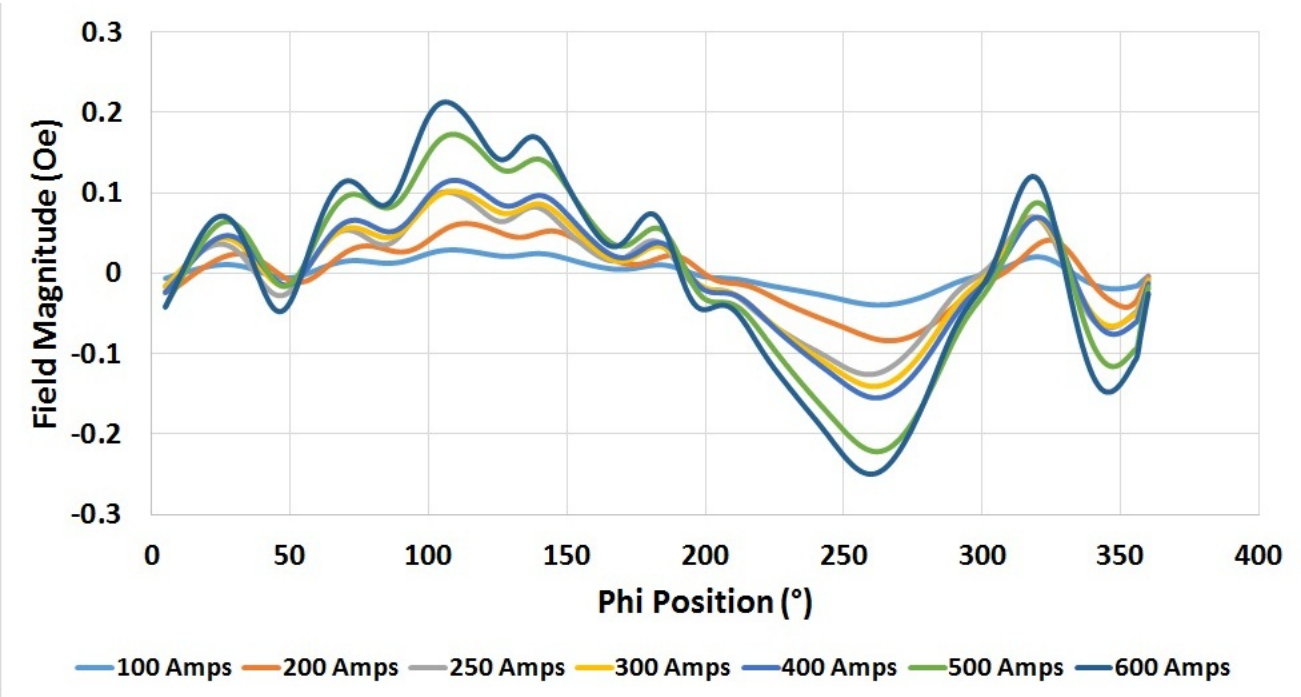

Figure 4.38: Phi sensor field magnitude median along Phi axis

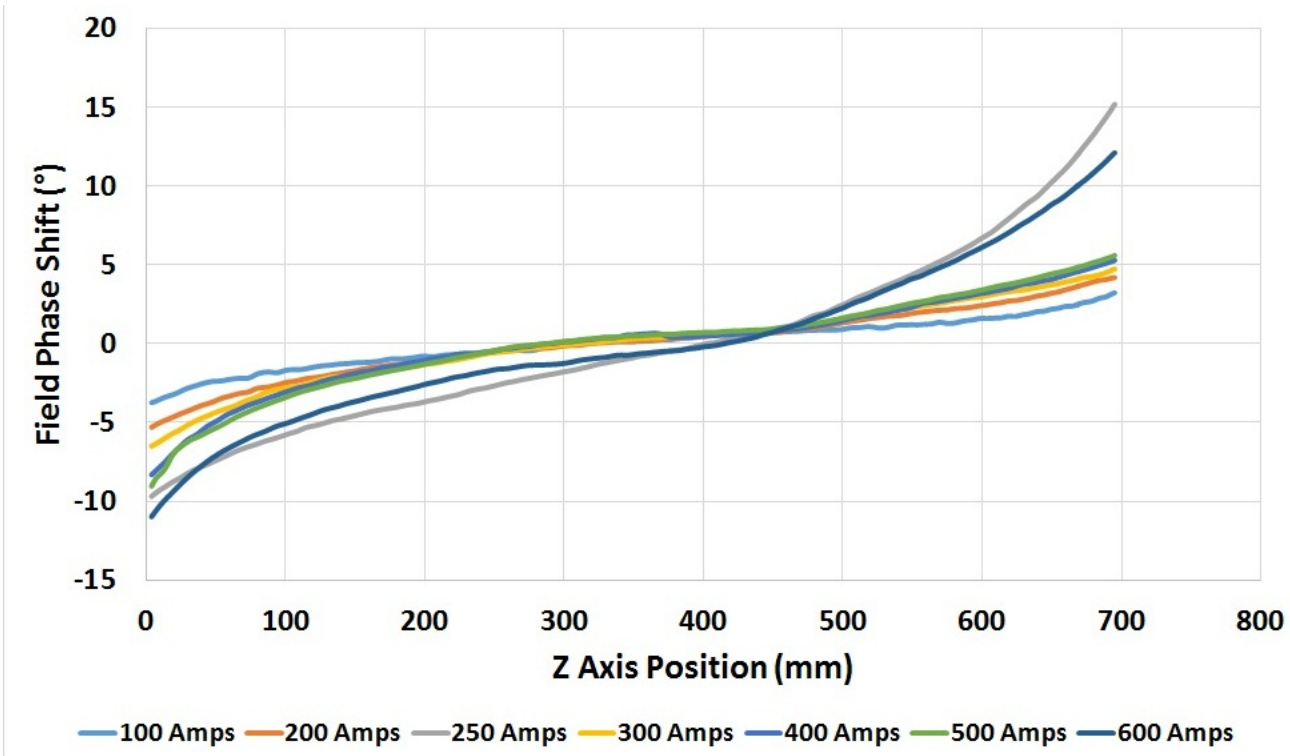

Figure 4.39: Phi sensor phase shift median along $Z$ axis 


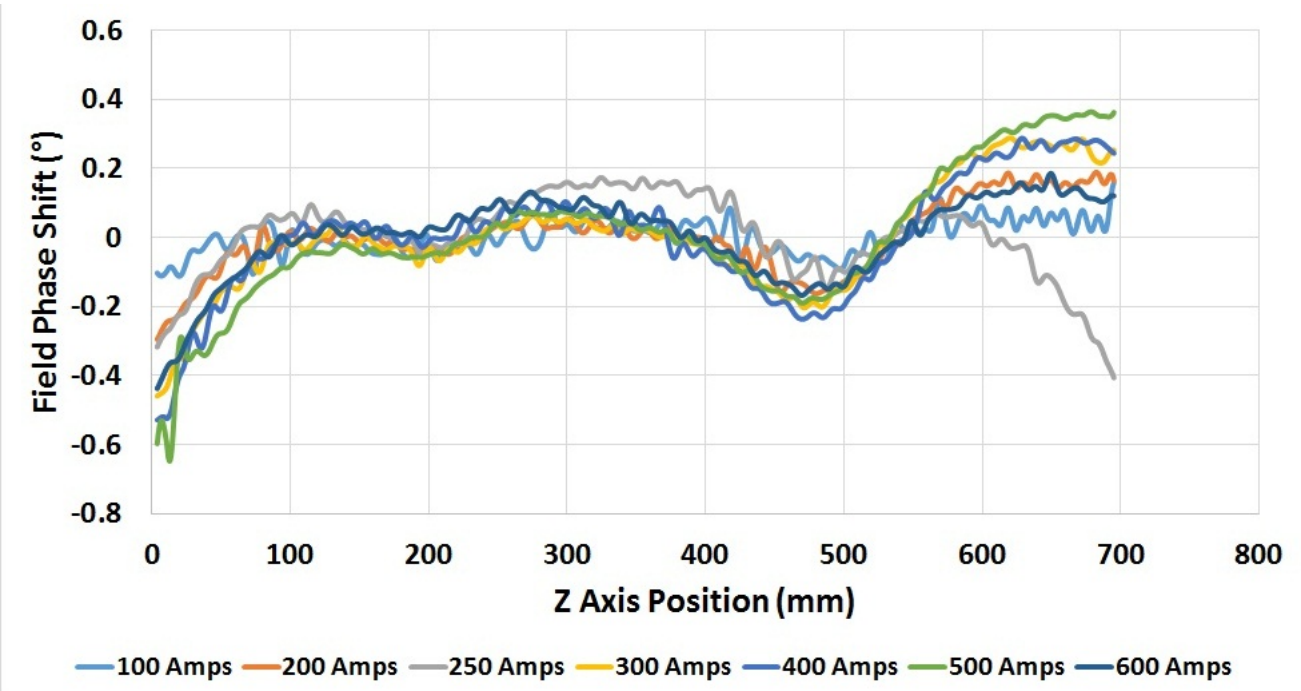

Figure 4.40: Phi sensor phase shift median along $Z$ axis with $Z$ axis flattening

but overall the small change in curvature for all current levels means that the excitation current does not affect the end of pipe feature in the phase data.

After applying the $Z$ flattening algorithm, the phase shift is very low and approaching the noise limits of the system. This again can be seen in rapid fluctuations of the data. All different excitation levels appear to lie on top of each other within the noise limits. They appear to diverge from the $300 \mathrm{~mm}$ point, however there is no correlation with excitation current, but instead this increased variation can be attributed to the flattening algorithm introducing some error. The $Z$ flattened data along the Phi axis, as in all other signals, shows more peaks and troughs than the $Z$ axis. Again the Phi sensor phase shift signals show no clear trend with current. This strengthens the previous conclusion that the excitation current does not affect features measured with the Phi sensor phase shift.

\subsection{Summary of current dependence}

\subsubsection{Magnitude}

The effect of increasing excitation current on the field magnitude is an increase in field strength for both the $\mathrm{R}$ and Phi sensor. The SNR for the field magnitudes reaches $95 \%$ of the maximum value at 280 amps for the $R$ sensor and 130 amps for the Phi sensor. This indicates that the excitation level required for optimal SNR is approximately 300 amps. Past this point it is only beneficial if a different measurement system is used that pushes the noise floor higher and so a higher excitation current would be needed to reach the $95 \%$ of the maximum SNR. 


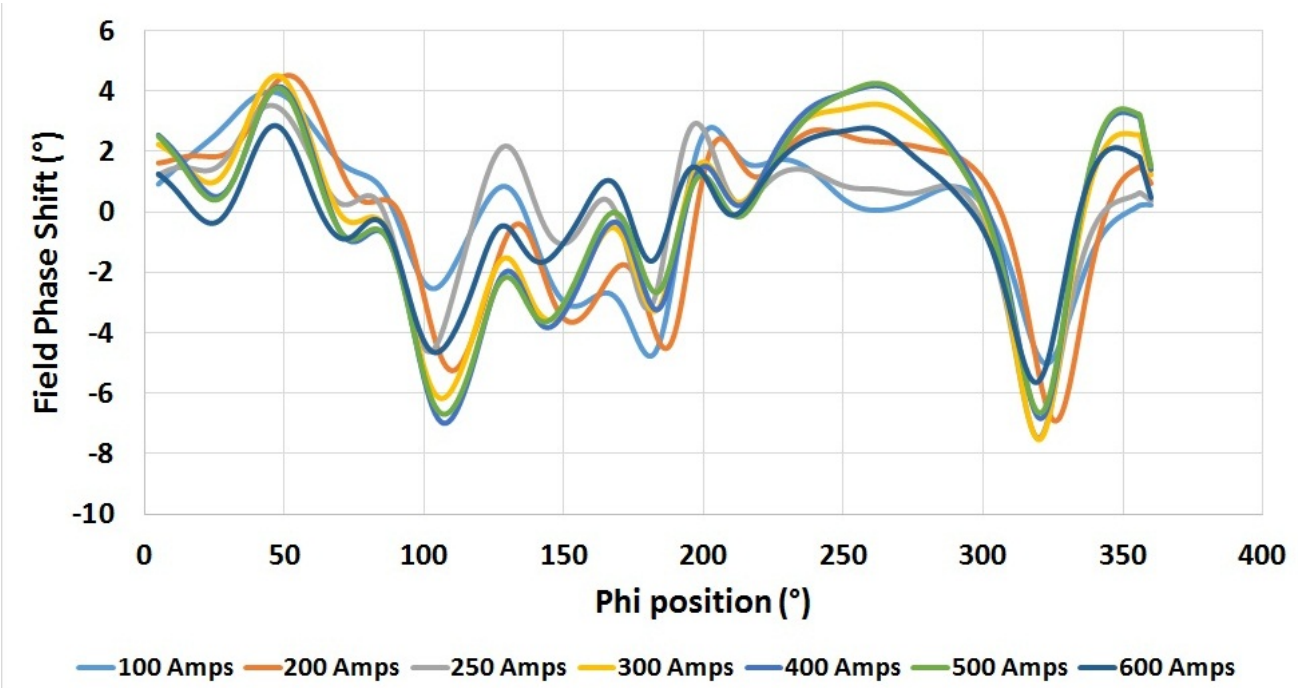

Figure 4.41: Phi sensor phase shift median along Phi axis with $Z$ axis flattening

\subsubsection{Phase shift}

The phase shift of the magnetic fields shows little variation with excitation current for both background and standard deviation. The absolute phase shift is always close to 150 degrees. The toroidal transformer, if ideal, would shift the phase by 180 degrees. The non-ideal resistance and leakage inductance in the primary winding causes some small phase shifts. However, the major source of phase shift is the impedance of the excitation coil. The complex impedance of the excitation coil at $20 \mathrm{~Hz}$ is approximately $2.8 \mathrm{ohms}$ with a 43 degrees phase angle. As the impedance is partly inductive, the excitation current would be expected to lag the excitation voltage. This causes a rotation back to the imaginary axis so that phase shift is approx 137 degrees. The average phase shift for a complete pipe scan is a measure of the phase shift of the excitation field as the majority of the field is produced by the excitation sheet.

Since the phase shift is a product of the impedance of the system, changes with excitation current are small. Part of the linear slope in the phase shift is likely due to a gradual change in impedance due to increasing temperature with increasing current. The slope for the Phi sensor is larger than that of the R sensor because the phase shift of the eddy current circulation around the Phi axis is greater than around the R axis. This means that as eddy currents increase with increased excitation current, the phase angle shifts more rapidly because of the larger phase angle between the excitation current and Phi axis eddy current.

\subsubsection{Effects of excitation current on local variation}

In general variations along the $Z$ axis without correction are larger than variations along the Phi axis. This is due to the effect of a strong change in magnetic field at the end of the pipe. Once this effect has been corrected with the $Z$ flattening algorithm, more variations are seen around the Phi axis. This shows that the underlying cause of the variations must 
be strongly directional since many more peaks and troughs can be identified in the Phi axis than in the $Z$ axis. This is probably due to permeability variations due to the production process. However, there is only a weak correlation of the magnetic field strength with permeability, which suggests another parameter such as conductivity or wall thickness is influencing the field strength.

\begin{abstract}
$Z$ axis
The uncorrected $\mathrm{R}$ sensor magnitude and phase shift show strong effects at the end of the pipe. These end effects spread further along the pipe with increasing current. The Phi sensor data along the $Z$ axis shows this to a much lesser degree. The increasing end effect for the field magnitude is due to increasing excitation current and is a result of the main magnetic field strength increasing, which causes the fringe field to increase. This means that it extends further from the end of the pipe and is detected earlier. This end effect is not seen in the Phi sensor because fringe field lines are not curling into the Phi sensor axis. The effect seen in the phase shift data is due to the impedance of the system decreasing. The impedance drop is due to loss of core material from the inductor formed by the excitation sheet and pipe. As the excitation coil approaches the end, the field lines are pushed out, meaning that more field lines pass through an area of lower permeability. As a result of this the average core permeability effectively drops. There are two reasons that increasing the excitation current may increase the end effects. The first is that as the current increases the flux that needs to be contained within the steel pipe also increases. Because only a fixed amount of flux can be contained within a certain volume of steel, more flux is leaked from the end of the pipe as the current increases. This means the impedance starts to drop sooner than with lower current as the end of the pipe is approached. The second cause is possibly that higher currents mean more power is dissipated in the excitation coil. This means the coil heats up and according to equation 3.3 the resistance increases which produces a change in phase shift.
\end{abstract}

\title{
$Z$ axis flattened
}

The data along the $Z$ axis after the $Z$ flattening algorithm for both $R$ and $P h i$ sensors and their phase and magnitude show small variations. Only the $R$ sensor magnitude shows significant variation with current with one position on the pipe showing increasing variation with current. However, this variation is probably due to an over correction by the $\mathrm{Z}$ flattening algorithm and this would be expected to become more marked as the current increases and larger variations are corrected.

\section{Phi axis}

The data along the Phi axis for the Phi and R sensors and their phase and magnitude show a lot more variation than along the $Z$ axis which is due to changes in permeability of the pipe, as discussed in section 4.2. As the excitation current increases, the magnetic field in the pipe increases. The eddy current circulating is coupled to the permeability of the pipe, but the permeability changes the skin depth according to equation 2.4. This means if the eddy currents are integrated over the total pipe thickness then higher eddy currents can be expected for steel with a lower permeability, which means that higher magnetic fields can be expected. The assumption is that conductivity of the steel is relatively constant as changing conductance would also affect the amount of eddy current flowing. As the eddy current is just a percentage of the excitation current, larger excitation currents result in a 
larger absolute variation in eddy current which is measured as a larger variation of the field.

The variation in the phase shift is also caused by changes in the material properties in the pipe. As the impedance that the eddy currents flow through changes this changing impedance causes a changing phase shift. Like other phase shift measurements there is little change with increasing excitation current. However, one exception is the R sensor at about 180 degrees. The weld is within the vicinity of this (at about 170 degrees) which obviously has a strong effect on how the eddy currents flow. However, the effect of welds is outside the scope of this thesis so it was not investigated further.

\subsection{Influence of excitation frequency}

To determine the effect of excitation frequency on the background signal level and the background noise, tests were carried out at $5 \mathrm{~Hz}, 10 \mathrm{~Hz}, 20 \mathrm{~Hz}, 60 \mathrm{~Hz}, 89 \mathrm{~Hz}$, and 200 $\mathrm{Hz}$, with a nominal $300 \mathrm{amps}$ excitation current. At $200 \mathrm{~Hz}$ the impedance of the system increased to a point where there was not enough power to drive 300 amps excitation current so a nominal 170 amps was used. The $89 \mathrm{~Hz}$ frequency was chosen as it is the upper limit of frequency achievable with 300 amps excitation current. The standard filter cut-off frequency used on the lock-in amplifier was changed from $100 \mathrm{~ms}$ to $1000 \mathrm{~ms}$ for the $5 \mathrm{~Hz}$ and $10 \mathrm{~Hz}$ excitation. This change shifted the cut-off frequency from $10 \mathrm{~Hz}$ down to $1 \mathrm{~Hz}$ which eliminated the effect of aliasing, as discussed in section 3.2.2. As the excitation frequency increases the eddy currents induced into the pipe are expected to drop since the skin depth drops with $\frac{1}{\sqrt{f}}$.

\subsubsection{Background signal level and total noise}

To determine the effect of excitation frequency on the background magnetic field level the median value and standard deviation at each excitation frequency are calculated for each complete dataset after applying the $Z$ flattening, Phi flattening and current correction algorithms. The change of the median value with excitation frequency for the $\mathrm{R}$ sensor magnitude is shown in figure 4.42. The standard deviation was calculated but not plotted since it was not large enough to be seen with values below 0.03 Oersted. The large drop off above $89 \mathrm{~Hz}$ point can be attributed to the excitation current being much lower. Because the excitation current is much lower the current correction that was applied is much larger than at lower frequencies. Since the correction factor used was measured at $20 \mathrm{~Hz}$ there is significant error in the correction factor. This increases the error in the measurements when a large correction is applied.

The change of the median value with excitation frequency for the Phi sensor magnitude is shown in figure 4.43. The standard deviation for a complete pipe scan is shown with red error bars. There is a notch at around $20 \mathrm{~Hz}$ with about a 20\% drop in background field. Like the $\mathrm{R}$ sensor, there is a very fast increase in the field at the lower frequencies, as well as a somewhat slower but still marked increase for higher frequencies. 


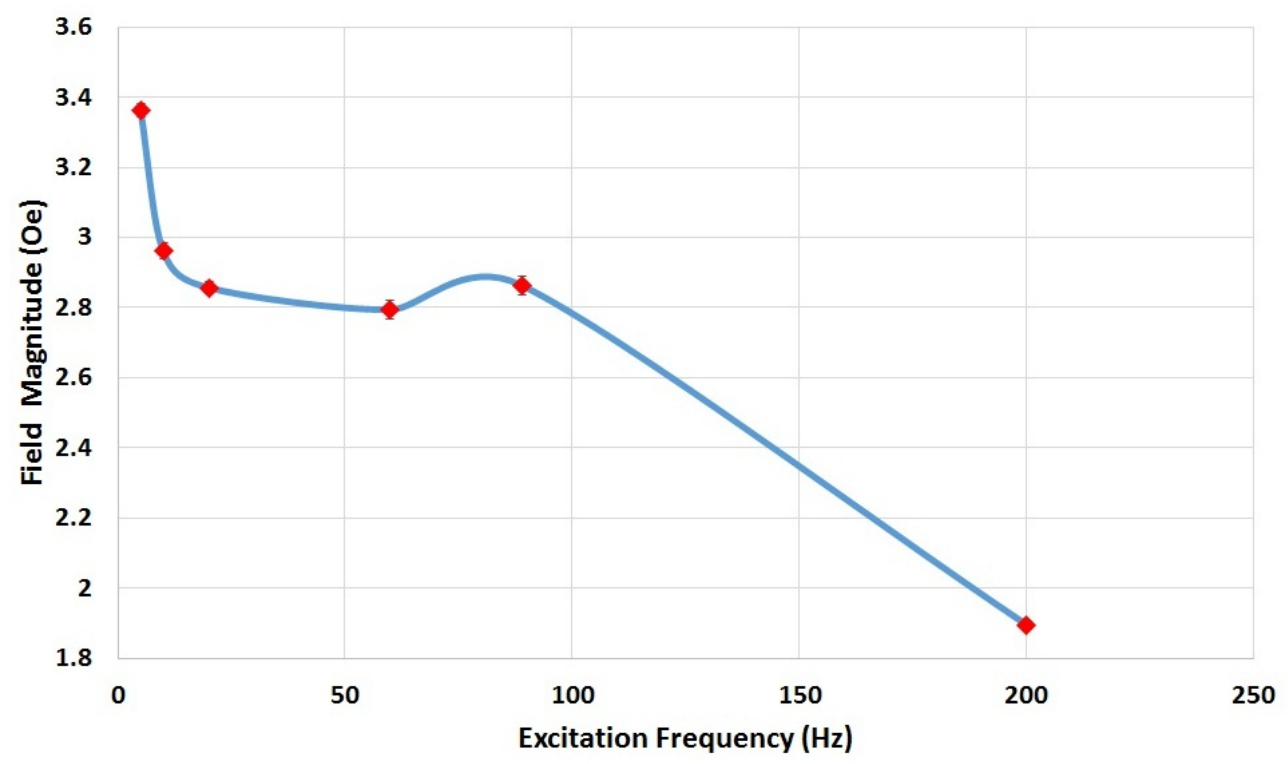

Figure 4.42: $\mathrm{R}$ sensor magnetic field magnitude median vs excitation frequency(the blue line a provides a guide line)

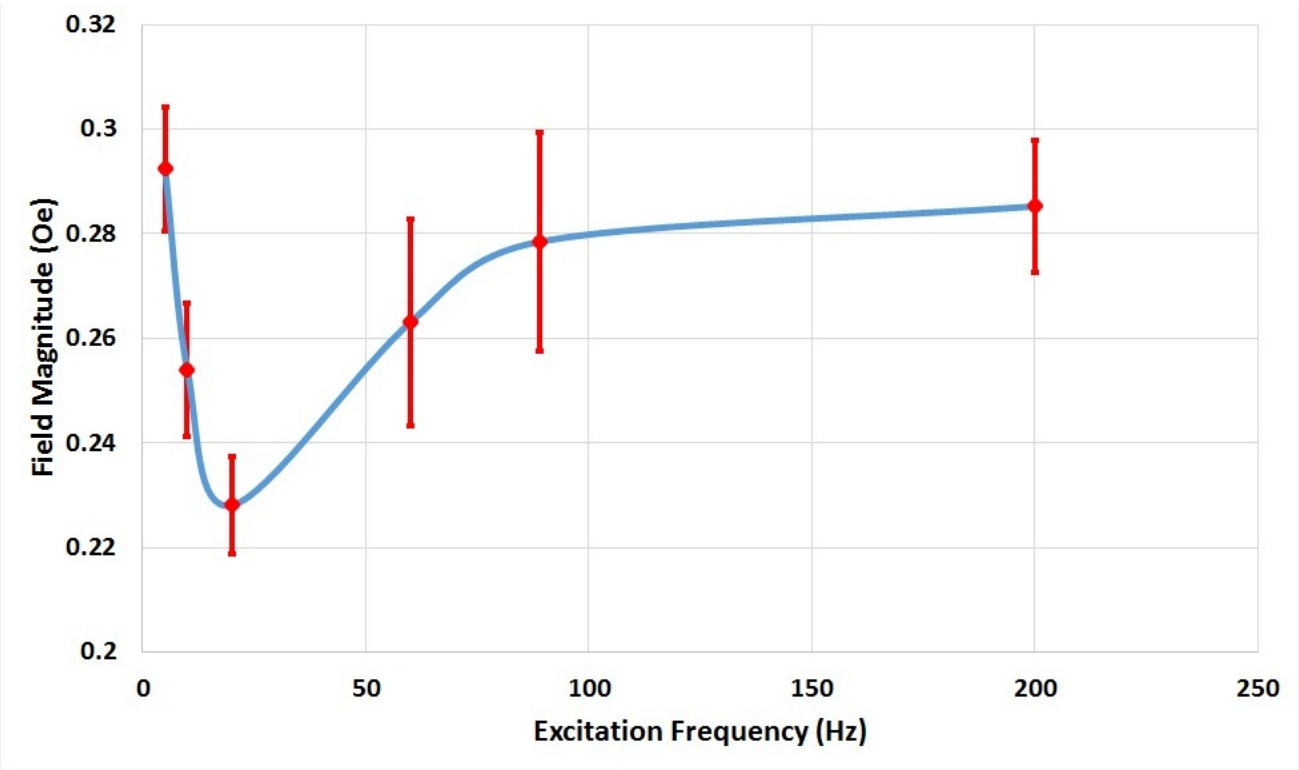

Figure 4.43: Phi sensor magnetic field magnitude median change with excitation frequency(the blue line a provides a guide line) 


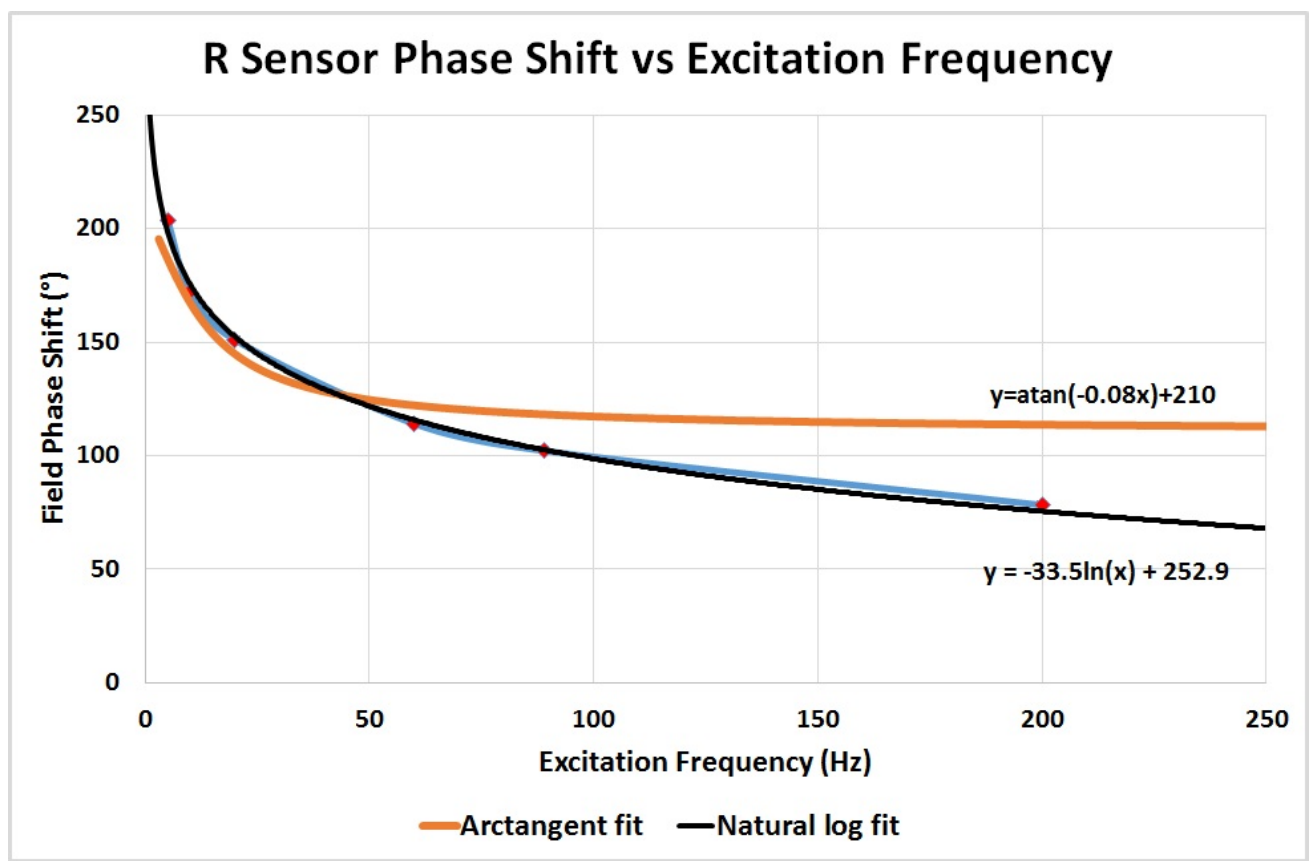

Figure 4.44: $\mathrm{R}$ sensor magnetic field phase shift median change with excitation frequency(the blue line a provides a guide line)

The median change with excitation frequency for the $R$ field phase shift is shown in 4.44. Again the standard deviation has been calculated but is not shown since it is too small to appear. The expected fit would be an arctangent fit(Orange). This is because the impedance is completely resistive at D.C and approaches the imaginary axis when the inductance of the coil dominates at high frequencies. This model would allow for a maximum of 90 degrees range of phase shift. However, 120 degrees change in phase shift can be seen with changing frequency. This means that there must be a capacitive load involved which causes the phase shift at higher frequencies. A logarithmic decay fits the data very well so can be used as a empirical predictive model to determine the phase shift at a given frequency.

The median value change with excitation frequency for the Phi field phase shift is shown in figure 4.45. Again the standard deviation has been calculated, but not shown as it is too small to appear. An arctangent fit and natural log fit is again shown. Compared to the $R$ field the expected arctangent fits well but there is still a greater than 90 degree change in phase shift with frequency suggesting a capacitive load. As with the $R$ field the logarithmic fit provides a good empirical predictive model to predict the expected phase shift for a given excitation frequency.

To characterize the total noise level dependence on excitation frequency a SNR value is calculated as the median value divided by the standard deviation of all data points. For the phase shift just the standard deviation is plotted against excitation frequency since the value has to be between 0 and 360 degrees.

The $R$ sensor magnitude SNR change with excitation frequency is shown in figure 4.46. High values with a marked drop in the 50 to $100 \mathrm{~Hz}$ range can be seen. There appears to 


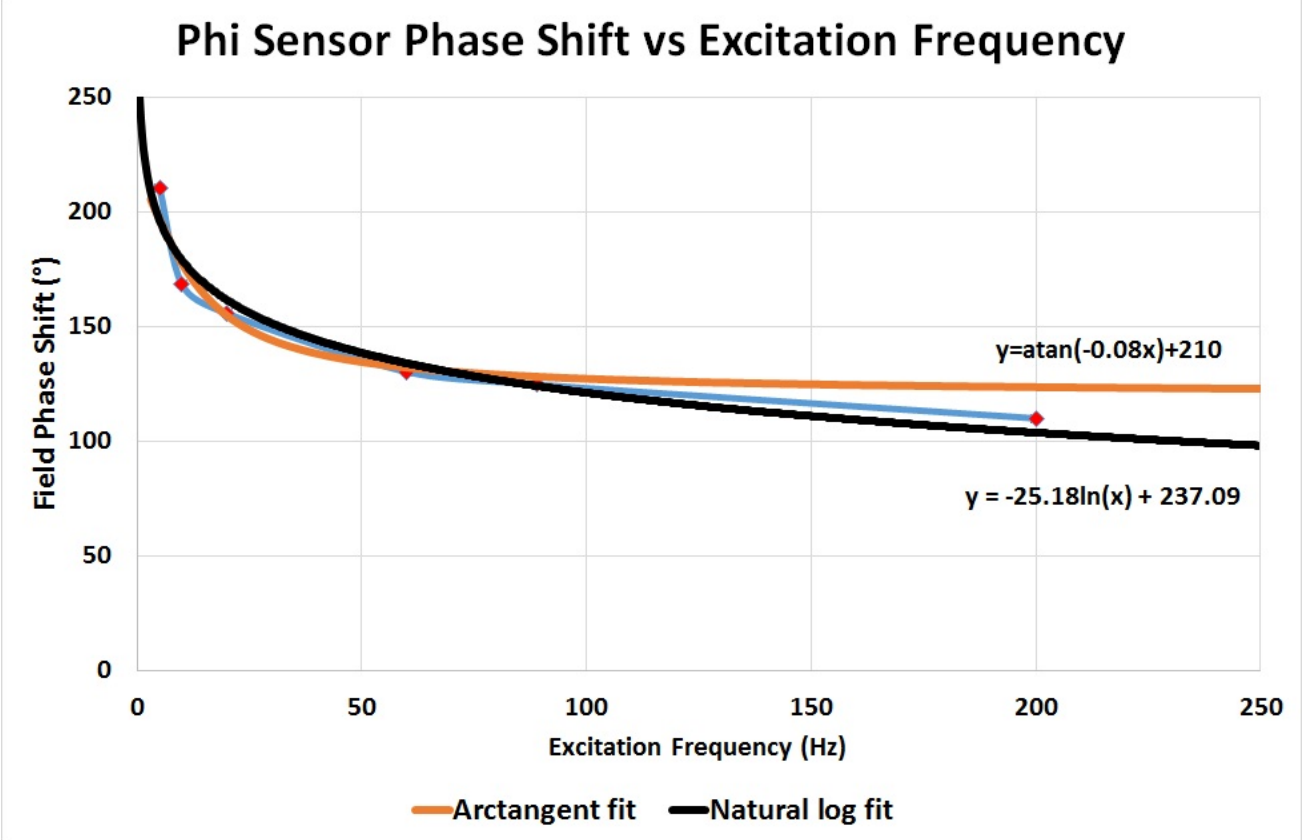

Figure 4.45: Phi sensor magnetic field phase shift median vs excitation frequency(the blue line a provides a guide line)

be significant variation in the measurements due to low standard deviation. This means only a small improvement can be expected by using lower excitation frequencies.

The Phi sensor magnitude SNR change with excitation frequency is shown in figure 4.47. It shows a similar shape as the $\mathrm{R}$ sensor but at a significantly lower level. Again there is a drop for the $10 \mathrm{~Hz}$ data point. This is probably due to a small mechanical change at this frequency that causes a small decrease in SNR. The most likely candidate for this mechanical change is the excitation sheet moving laterally so that the sensor is not exactly centered on the center of the sheet. Again only a small improvement can be expected by using lower excitation frequencies.

The $R$ field phase shift standard deviation is shown in figure 4.48. It shows a low standard deviation for the $R$ sensor phase shift, less than one degree. One outlier at 10 $\mathrm{Hz}$ is caused by the same mechanical change seen in the magnitude. If the $10 \mathrm{~Hz}$ data point is ignored there is a slow but clear increase in standard deviation as the excitation frequency increases.

The Phi field phase shift standard deviation is shown in figure 4.49. As expected, the standard deviation is higher than the $\mathrm{R}$ sensor, due to the lower signal level being measured. Despite the variations in these measurements it appears low excitation frequencies could be slightly advantageous for measuring the Phi sensor phase shift.

\subsubsection{Effects of excitation frequency on local variation}

Variations along the $Z$ and Phi axes are analyzed to determine the effect of excitation frequency. For all measurements the median value of all data is calculated and then 


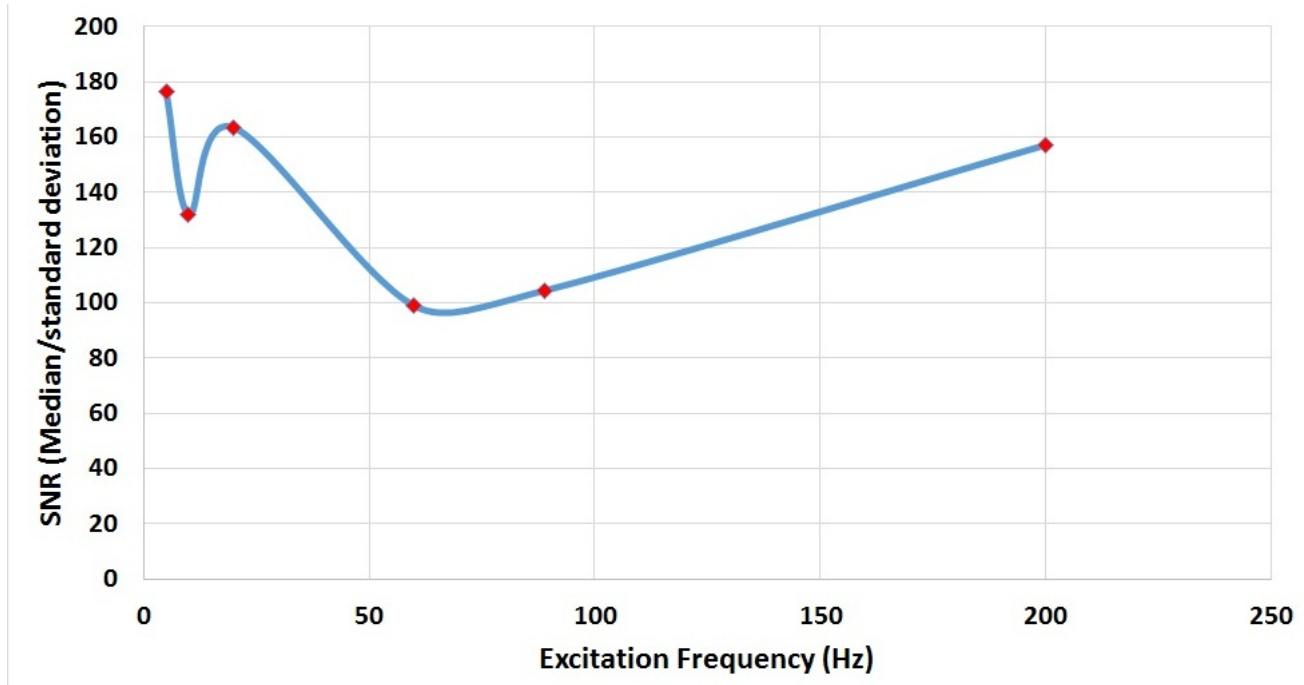

Figure 4.46: $R$ sensor magnitude SNR vs excitation frequency(the blue line a provides a guide line)

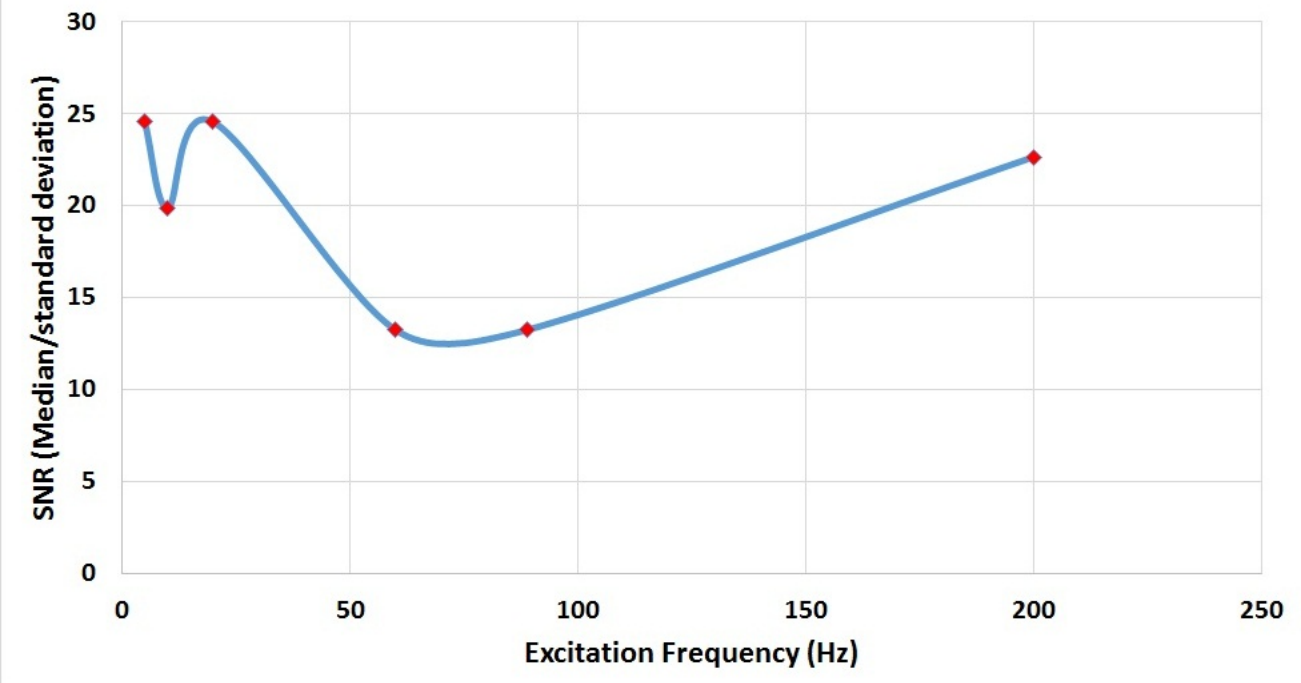

Figure 4.47: Phi sensor magnitude SNR vs excitation frequency(the blue line a provides a guide line) 


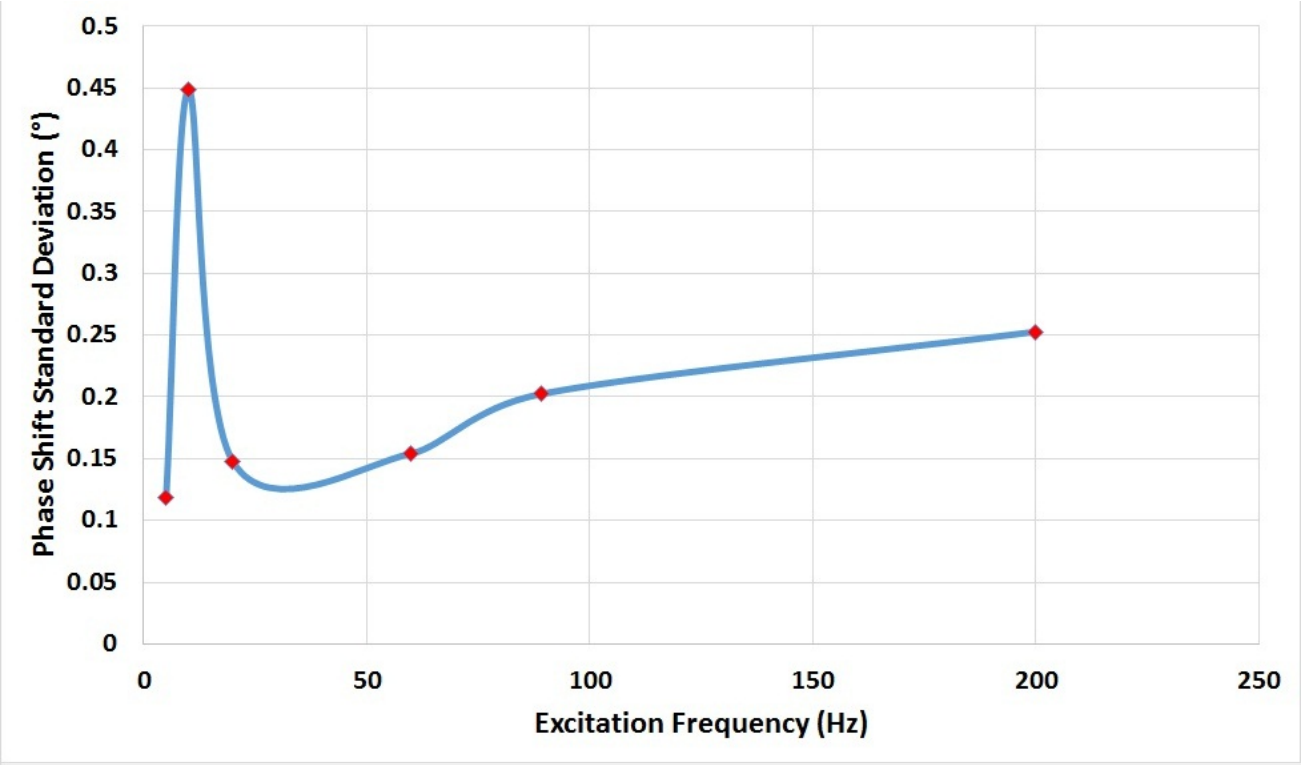

Figure 4.48: $R$ sensor phase shift standard deviation vs excitation frequency(the blue line a provides a guide line)

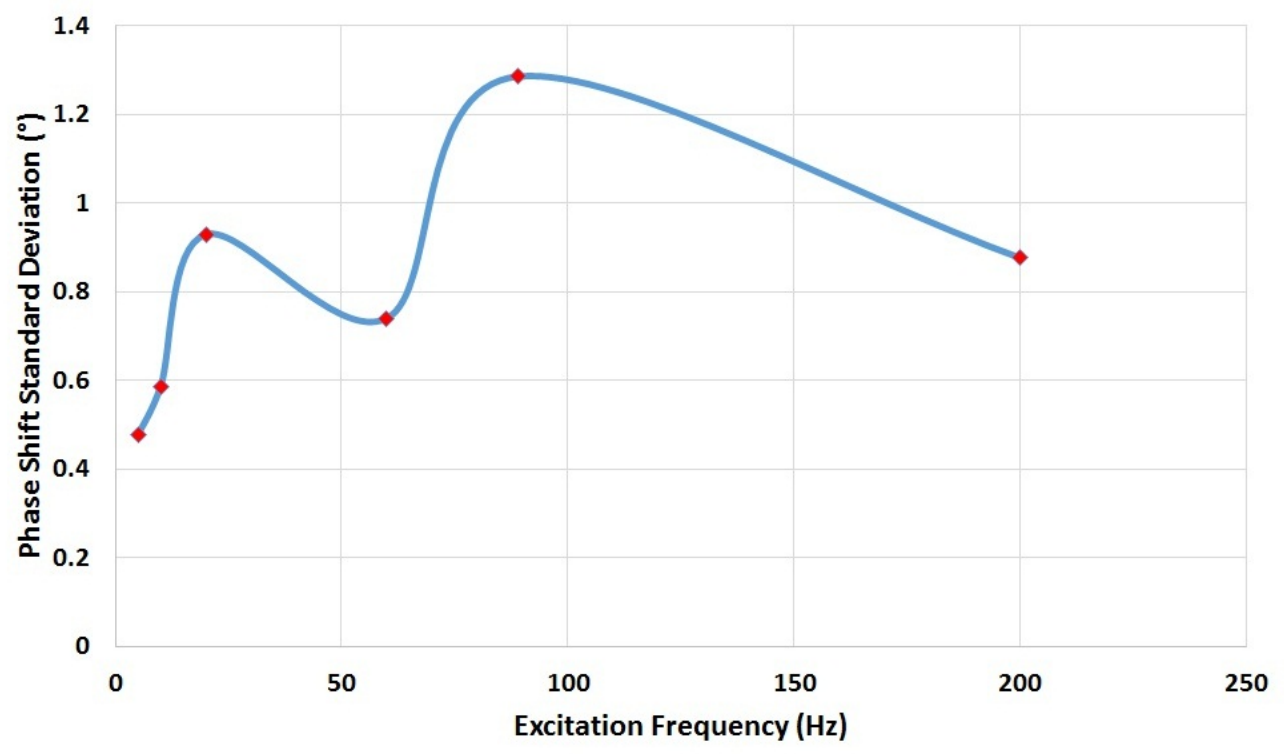

Figure 4.49: Phi sensor phase shift standard deviation vs excitation frequency(the blue line a provides a guide line) 


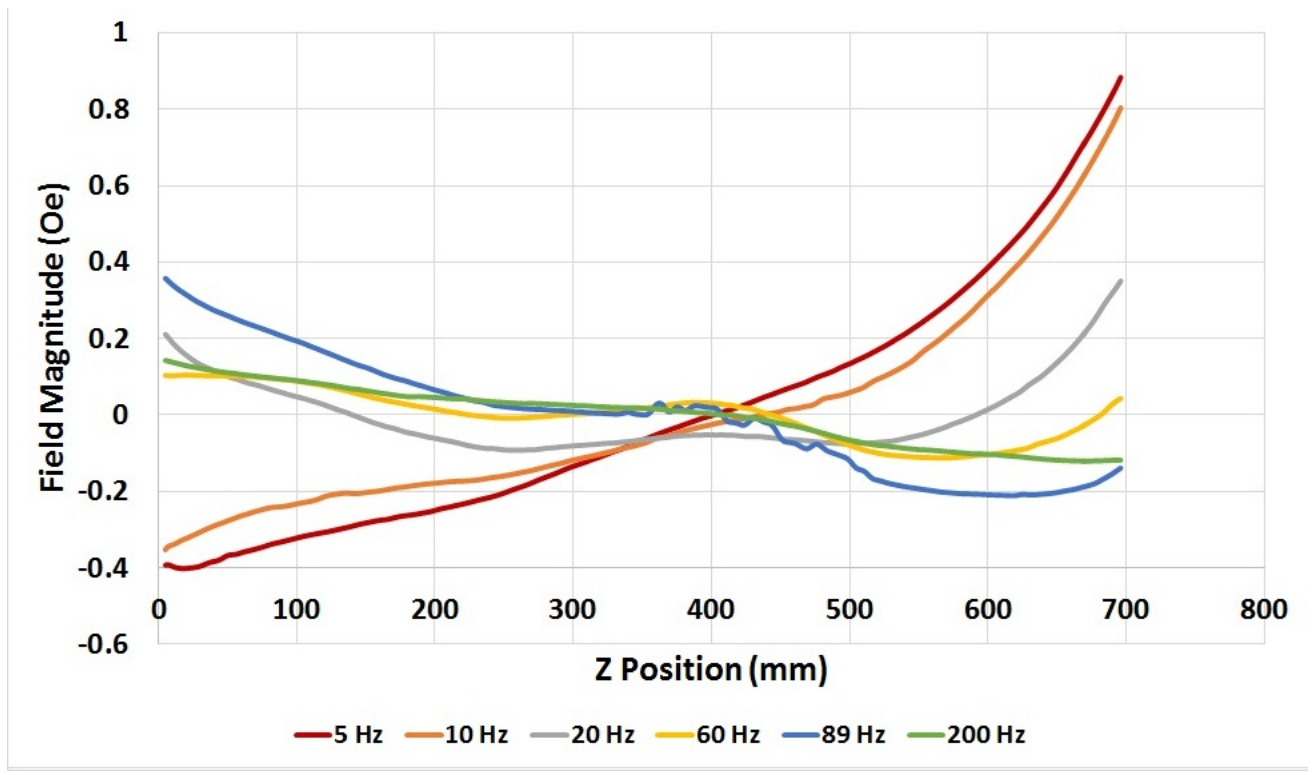

Figure 4.50: $R$ sensor Magnitude Median along $Z$ axis

subtracted from all data points. This shifts the data to be centered around zero and means that variations in the background can be ignored. The analysis shows how the variations in the magnetic field caused by local changes in the properties of pipe change with excitation frequency.

The first measure shows data with current correction and the median value at each $Z$ position is calculated. The second measure applies the $Z$ flattening algorithm to remove the end effect of the pipe. The third measure again applies the $Z$ flattening algorithm and then the median value is taken at each Phi location and then plotted for each excitation frequency. This shows how the variations along the Phi axis are affected by excitation frequency.

The $R$ sensor magnitude with current corrected data along the $Z$ axis is plotted in figure 4.50, the $R$ sensor magnitude with current corrected and $Z$ flattened data along the $Z$ axis is plotted in figure 4.51 and the $R$ sensor magnitude with current corrected and $Z$ flattened data along the Phi axis is plotted in figure 4.52. The unflattened data along the $Z$ axis for the $R$ sensor field magnitude flattens rapidly with increasing excitation frequency with a 1.2 Oe change for the $5 \mathrm{~Hz}$ data and a 0.2 Oe change at $200 \mathrm{~Hz}$. The R field magnitude data along the $Z$ axis after the $Z$ flattening algorithm has been applied shows only small variations at all frequencies apart from $89 \mathrm{~Hz}$ where a burst of noise appears. This can probably be attributed to mechanical variation during the $89 \mathrm{~Hz}$ scan that introduces noise into the data. Without the noise data in the $89 \mathrm{~Hz}$ test the maximum variation is only 20 mOe. The $R$ field magnitude data along the Phi axis has significant spread between different frequency measurements, however no clear frequency dependence can be seen.

Assessing the Phi field magnitude the first measure with current corrected data along the $Z$ axis is shown in figure 4.53, the second measure with current corrected and $Z$ flattened data along the $Z$ axis in figure 4.54, lastly the third measure with current corrected and $Z$ flattened data along the Phi axis in figure 4.55. 


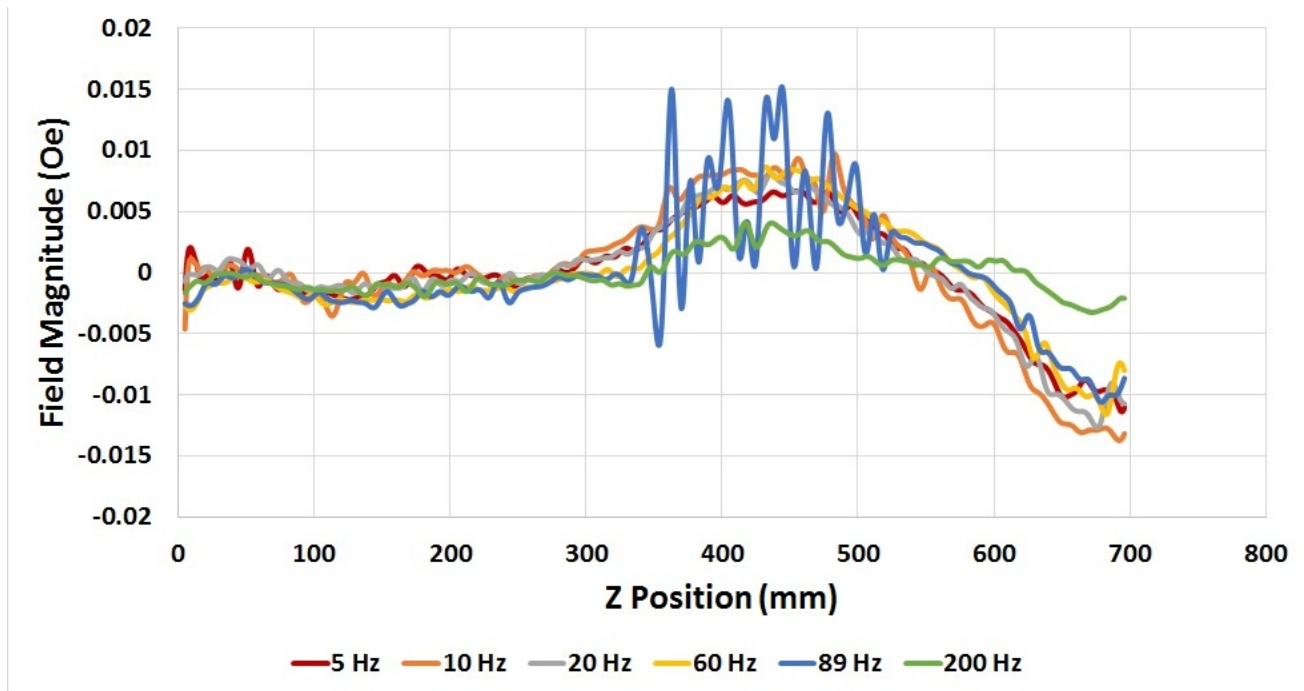

Figure 4.51: $R$ sensor Magnitude Median along $Z$ axis with $Z$ flattening algorithim applied

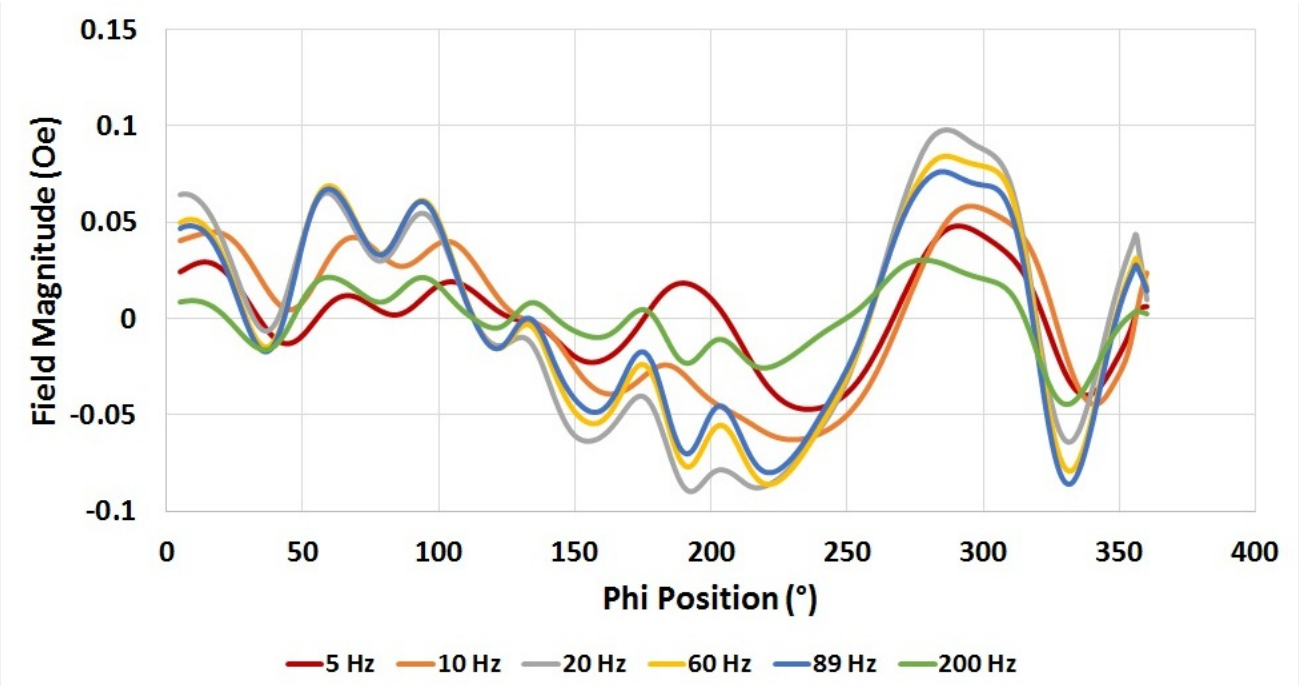

Figure 4.52: $\mathrm{R}$ sensor Magnitude Median along Phi axis with Z flattening algorithm applied 


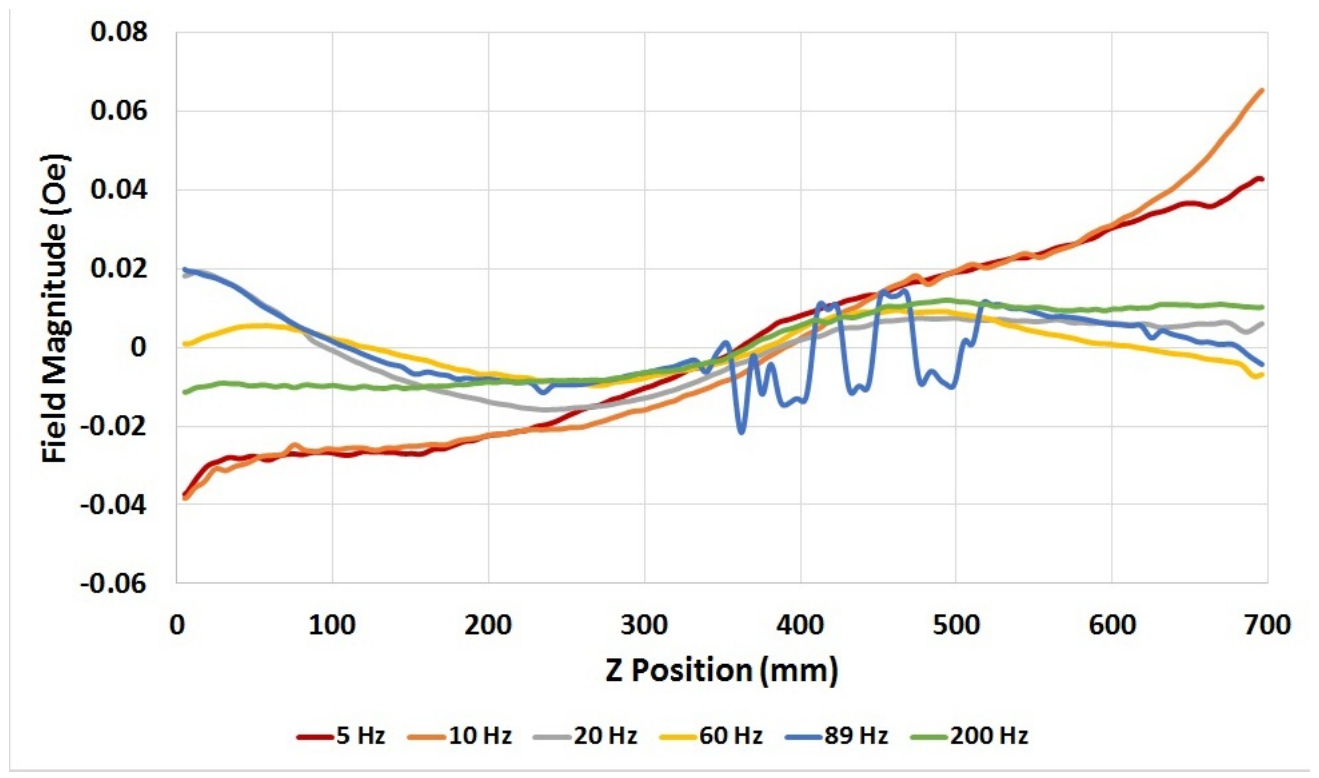

Figure 4.53: Phi sensor magnitude median along $Z$ axis

The slope of the Phi magnetic field magnitude variation along the $Z$ axis rapidly drops with increasing excitation frequency. However, when compared to the $\mathrm{R}$ field variation it is an order of magnitude lower. The same large area of noise is visible on the $89 \mathrm{~Hz}$ data. Once the $Z$ flattening algorithm has been applied, the Phi field magnitude data along the $Z$ axis shows only small variations at all frequencies apart from at $89 \mathrm{~Hz}$. The signals are flat from $100 \mathrm{~mm}$ through to $500 \mathrm{~mm}$ with no more than $2 \mathrm{mOe}$ variation at any frequency. As the end of the pipe is approached some variation appears and the data at different frequencies spreads out but again no clear frequency dependence can be found. Once the $Z$ flattening algorithm has been applied, the Phi field magnitude data along the Phi axis shows significant variation between the frequencies. However, again there is no clear frequency dependence.

Assessing the $R$ field phase shift the first measure with current corrected data along the $Z$ axis is shown in figure figure 4.56, the second measure with current corrected and $Z$ flattened data along the $Z$ axis in figure 4.57 , lastly the third measure with current corrected and $Z$ flattened data along the Phi axis in figure 4.58.

The current corrected $R$ field phase shift signal along the $Z$ axis shows the slope gradually flattens with increasing frequency. The $R$ field phase shift signal with the $Z$ flattening algorithm applied along the $Z$ axis shows a flat response for all frequencies with less than 0.1 degree shift, however a significant signal divergence past $300 \mathrm{~mm}$ can be seen. The step change in the $10 \mathrm{~Hz}$ data could have been due to a movement of the excitation coil in the first part of this test. The 5 and $20 \mathrm{~Hz}$ signals show the smallest variations. The $R$ field phase shift signal with the $Z$ flattening algorithm applied along the Phi axis again shows significant variation and divergence between different frequencies. However, no clear frequency dependence appears.

The same three plots are shown for the Phi phase shift signal. The current corrected data along the $Z$ axis is shown in figure 4.59. The $Z$ flattened data along the $Z$ axis is 


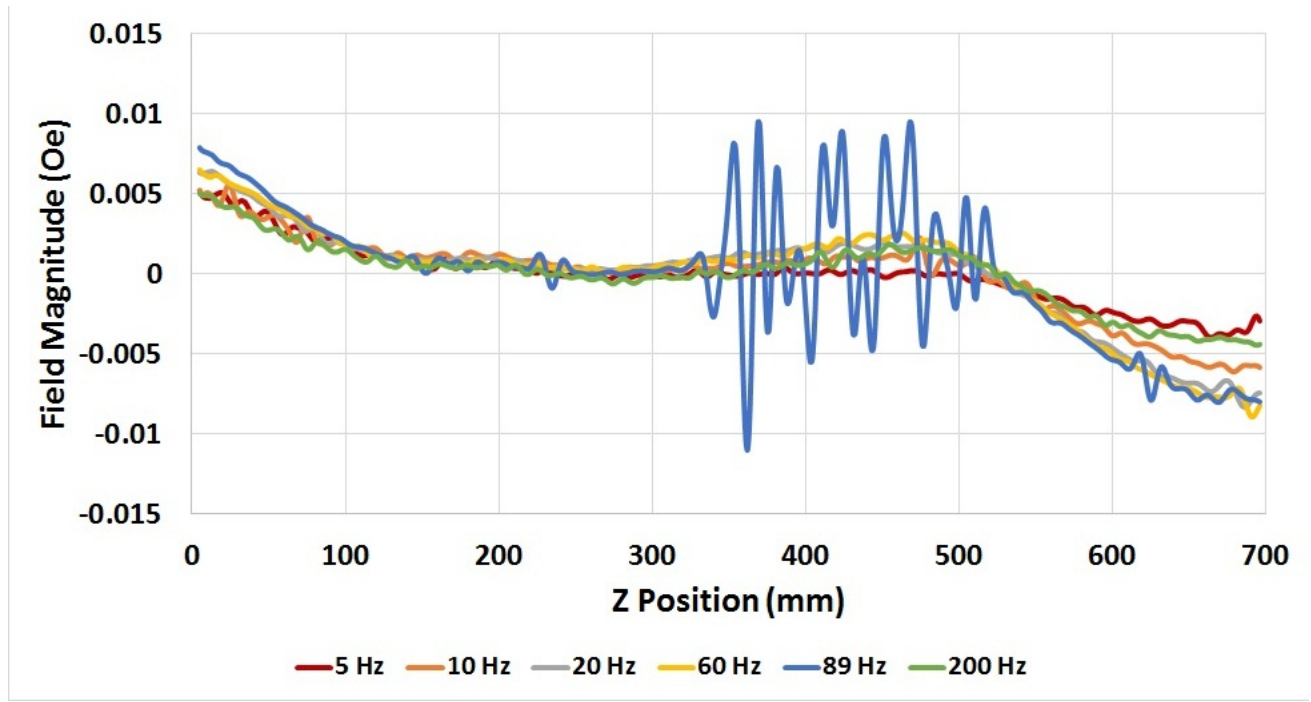

Figure 4.54: Phi sensor magnitude median along $Z$ axis with $Z$ flattening algorithm applied

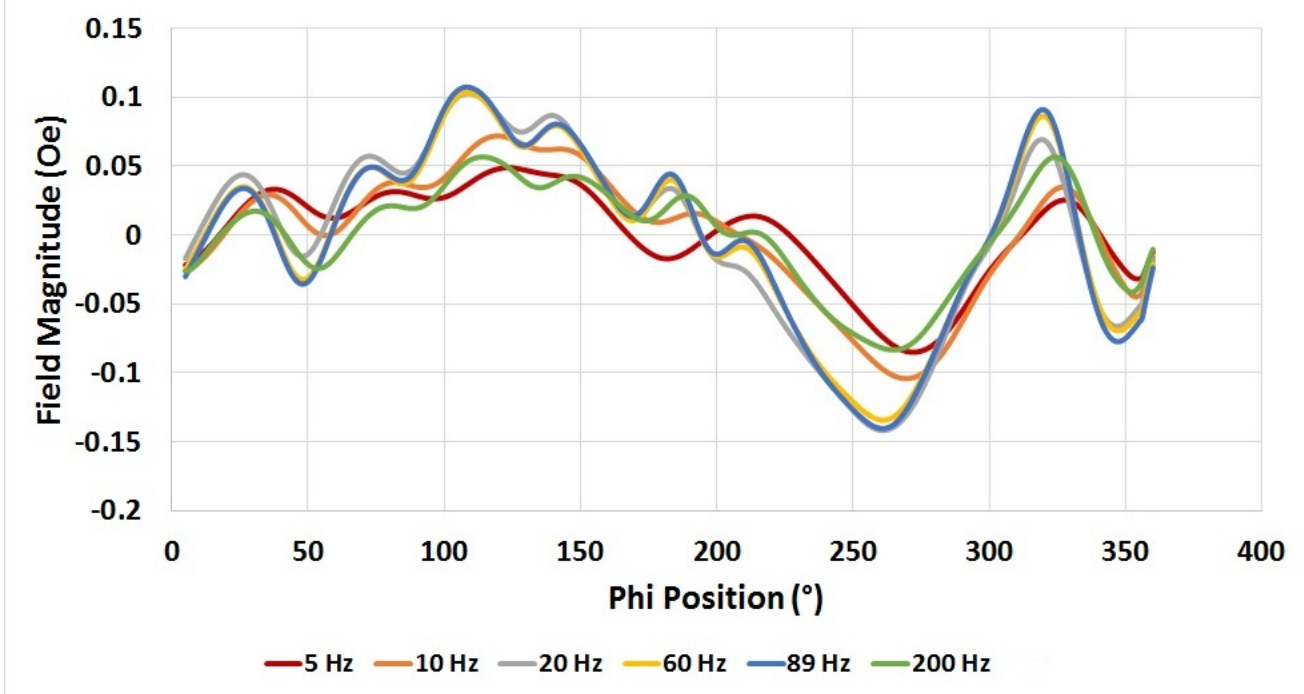

Figure 4.55: Phi sensor magnitude median along Phi axis with $Z$ flattening algorithm applied 


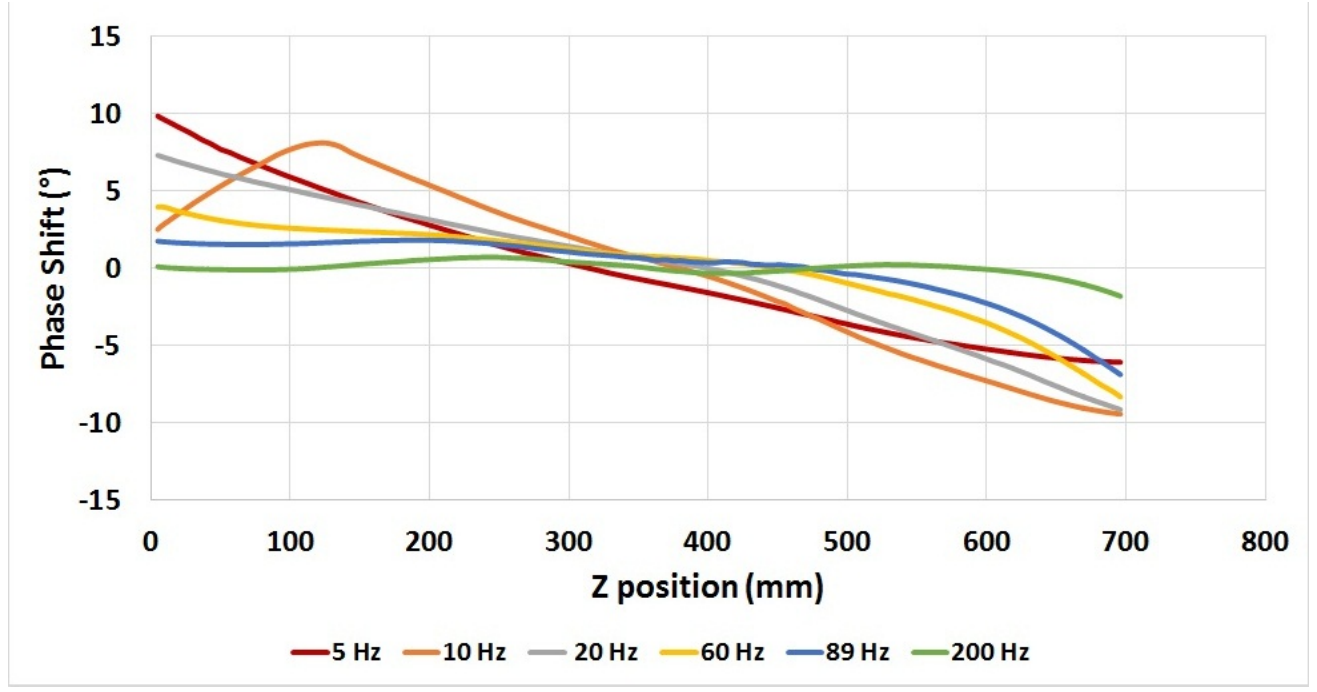

Figure 4.56: $R$ sensor phase shift median along $Z$ axis

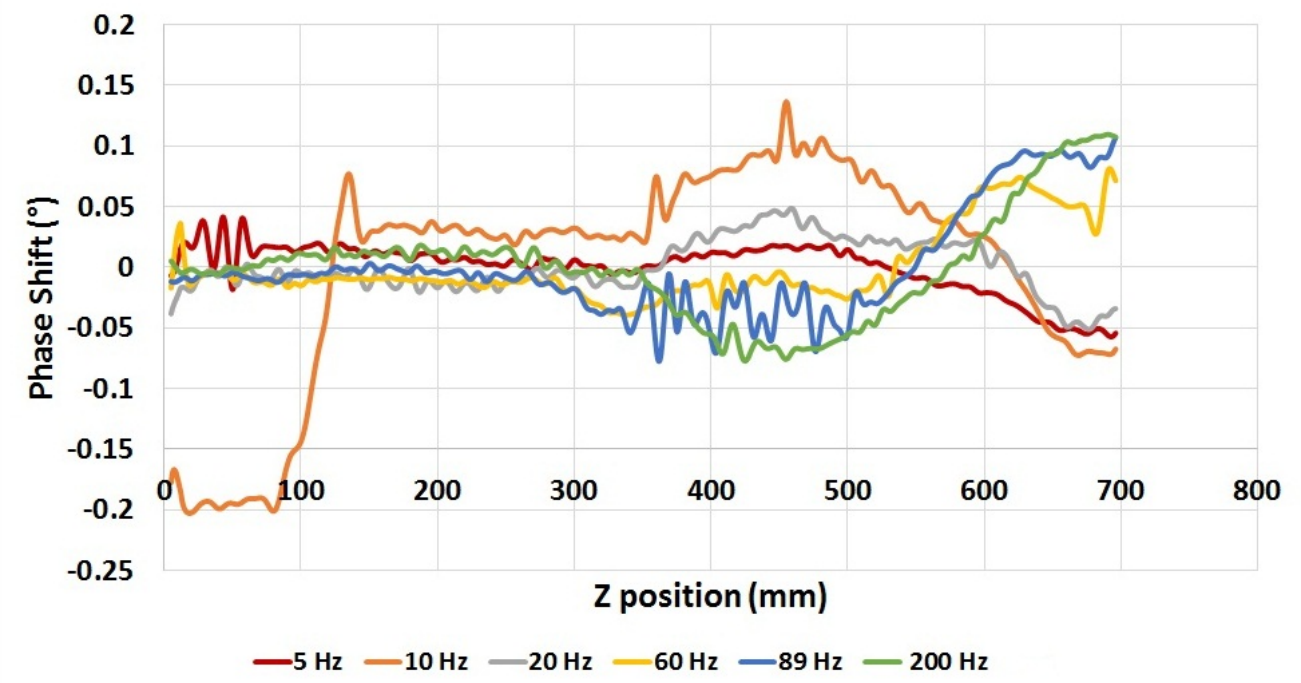

Figure 4.57: $\mathrm{R}$ sensor phase shift median along $\mathrm{Z}$ axis with $\mathrm{Z}$ flattening algorithm applied 


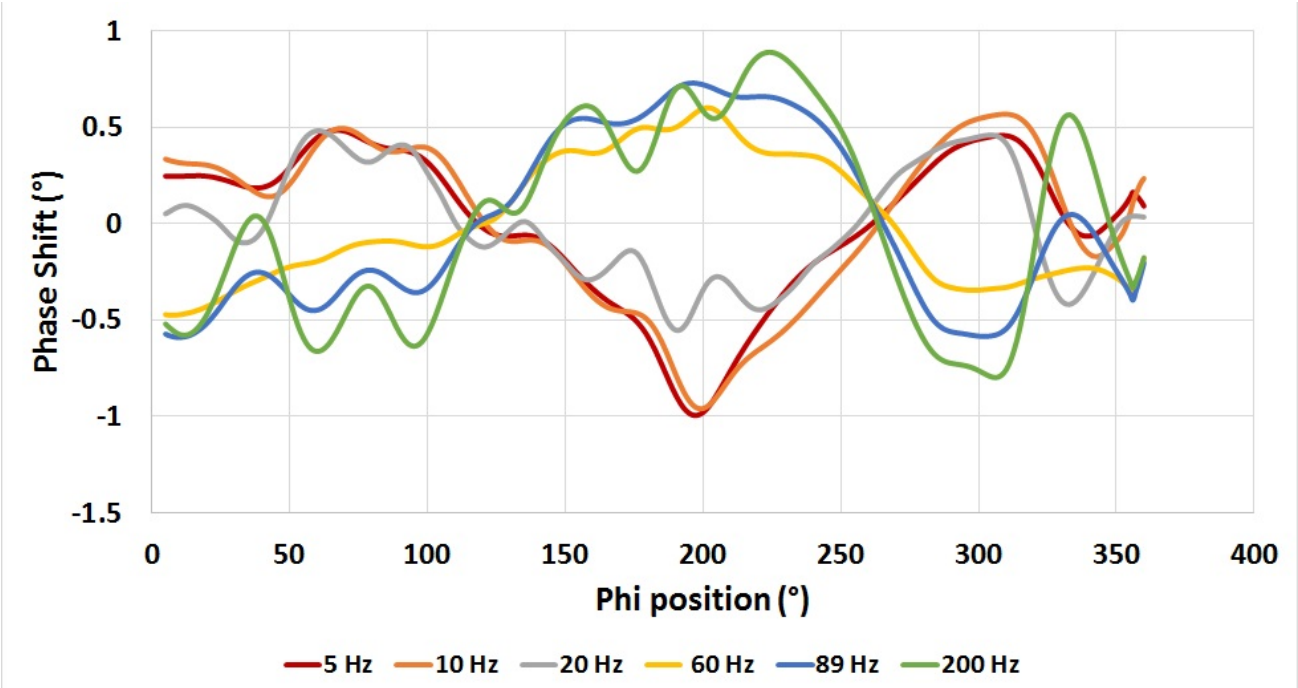

Figure 4.58: $\mathrm{R}$ sensor phase shift median along Phi axis with $\mathrm{Z}$ flattening applied

shown in figure 4.60. The same data along the Phi axis is shown in figure 4.61. The slope of the current corrected Phi field phase shift signal shows a steady decrease with increasing frequency. The mechanical variation in the $89 \mathrm{~Hz}$ signal is again visible. The Phi field phase shift signal after the $Z$ flattening algorithm has been applied along the $Z$ axis shows a very flat section from $100 \mathrm{~mm}$ to $500 \mathrm{~mm}$ with only 0.25 degrees phase shift over all frequencies (ignoring the noise generated by the movement on the $89 \mathrm{~Hz}$ scan). At either end the frequencies diverge, but there is no clear frequency dependence. The Phi field phase shift signal after the $Z$ flattening algorithm has been applied along the Phi axis shows significant divergence between the frequency signal, however there is no clear frequency dependence.

\subsection{Summary of frequency dependence}

\section{Background signal level}

The background level of the magnitude for both sensors, which is determined by the excitation field, was expected to decrease for increasing frequency as discussed in section 2.5.1. There is one exception, which is the Phi field at high frequency which shows some recovery. The source of this deviation from the qualitative model is probably due to a small misalignment of the sensor module. As shown in section 3.2.2 a small rotation of a sensor (especially the Phi sensor) into or out of the main $Z$ excitation field can cause large changes in the measured field. If the sensor was perfectly aligned with the axis, the background field would be zero.

The background level of the phase shift for both sensors fits well to a logarithmic decay with excitation frequency. This fit allows for interpolation of expected phase shift at given excitation frequencies. However, an arctanget fit was expected as the load was assumed to be purely resistive and inductive. This would mean the phase shift would be determined 


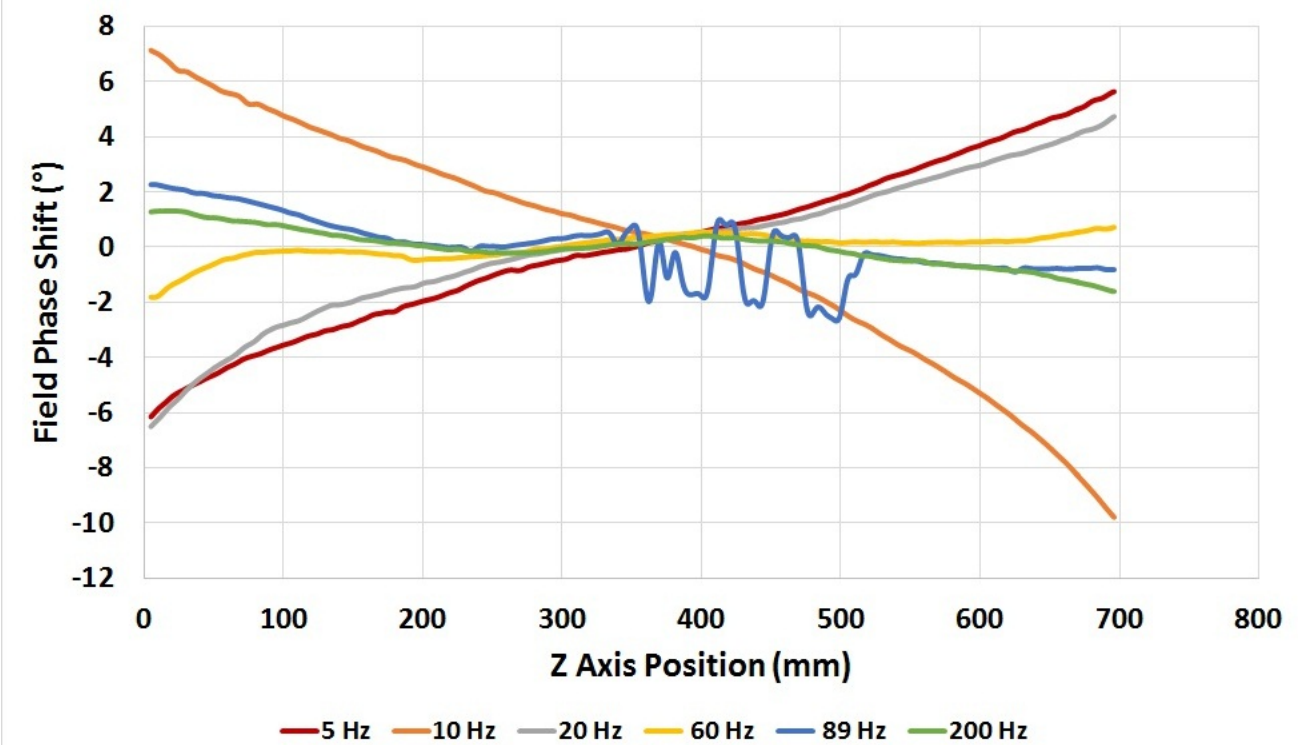

Figure 4.59: Phi sensor Phase shift median along $Z$ axis

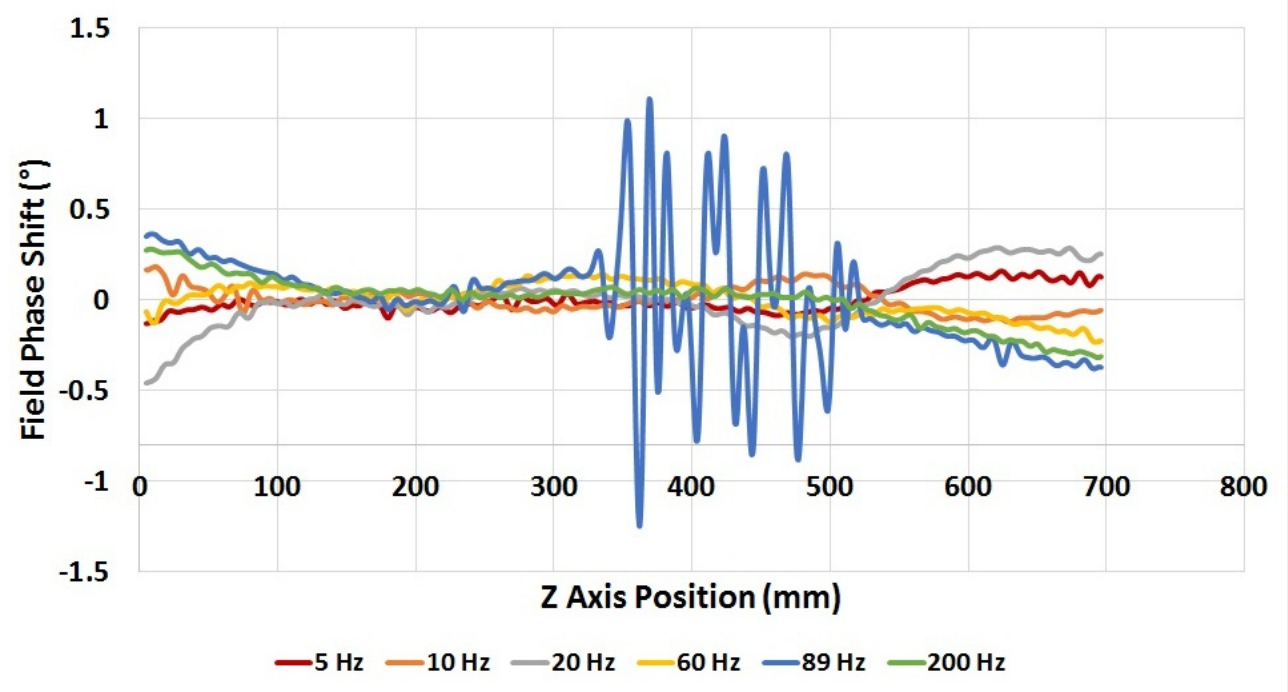

Figure 4.60: Phi sensor phase shift median along $Z$ axis with $Z$ flattening applied 


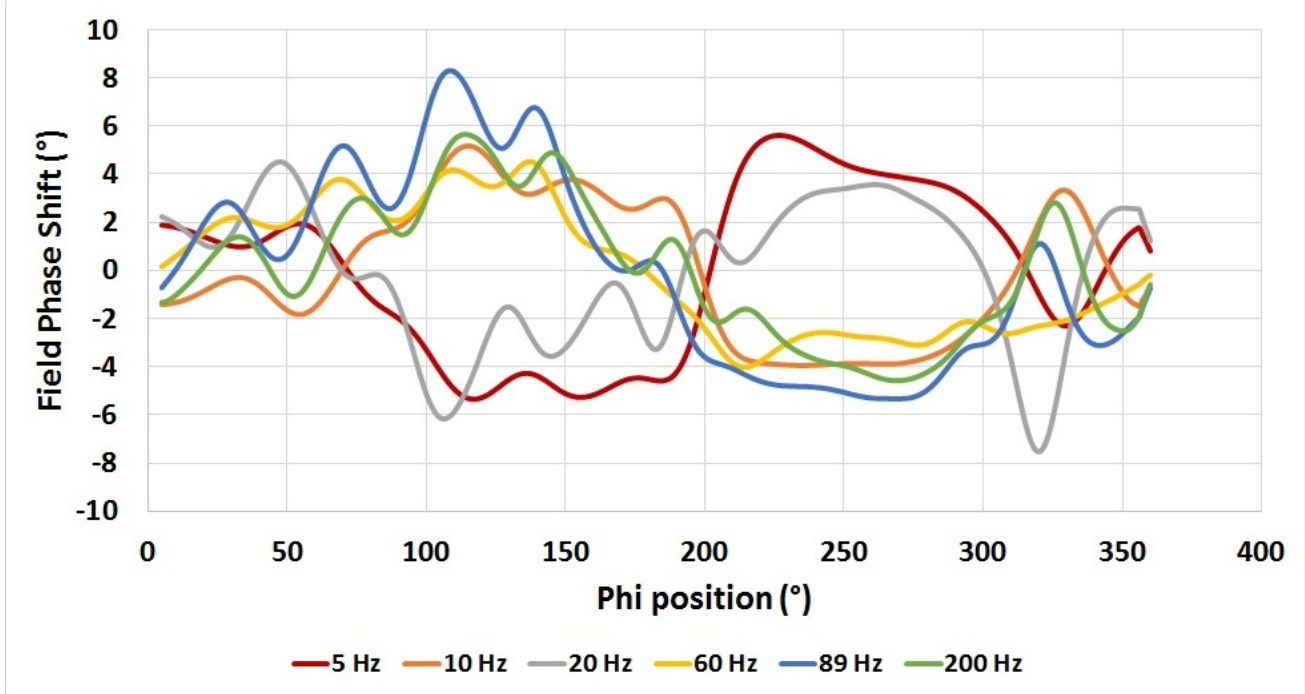

Figure 4.61: Phi sensor phase shift median along Phi axis with Z flattening applied

by the inductive reactance of the excitation coil which, with changing frequency, must follow equation 4.2. While the frequency causes the inductive reactance to increase, it does not affect the resistance. This means the coil's impedance is mostly inductive at high frequency, so a $90^{\circ}$ phase shift is expected from the $180^{\circ}$ phase shift when the load is resistive at DC. This would produce a arctangent curve, however there is a deviation from this model at high frequencies suggesting that a capacitive load is contributing to the phase shift. The capacitive load would mean that the logarithmic fit would fail if extrapolated, because a sharp $180^{\circ}$ phase shift would be expected when the circuit passes through resonance.

$$
X_{L}=2 \pi f L
$$

where

$$
\begin{array}{r}
X_{L}=\text { inductive reactance }(\Omega) \\
f=\text { frequency } \\
L=\text { inductance }(H)
\end{array}
$$

\section{Magnitude SNR}

The SNR of the field magnitude for both sensors shows no clear trend with frequency. However, if the Phi sensor data is normalized to the $R$ sensor (in figure 4.62) a very similar curve appears for both sensors. This suggests that the cause of these variations is again slight movements in the sensor as the data for both sensors is measured simultaneously so a variation in mounting would effect both sensors. The very large variations in SNR for the $\mathrm{R}$ sensor are due to small changes in the standard deviation value, which will cause large changes in the SNR value. It is also important to note that the SNR for the Phi sensor is about seven times smaller than the $\mathrm{R}$ sensor. This suggests that either the $\mathrm{R}$ sensor measures more of the background excitation field so that a large SNR is seen, or the Phi 


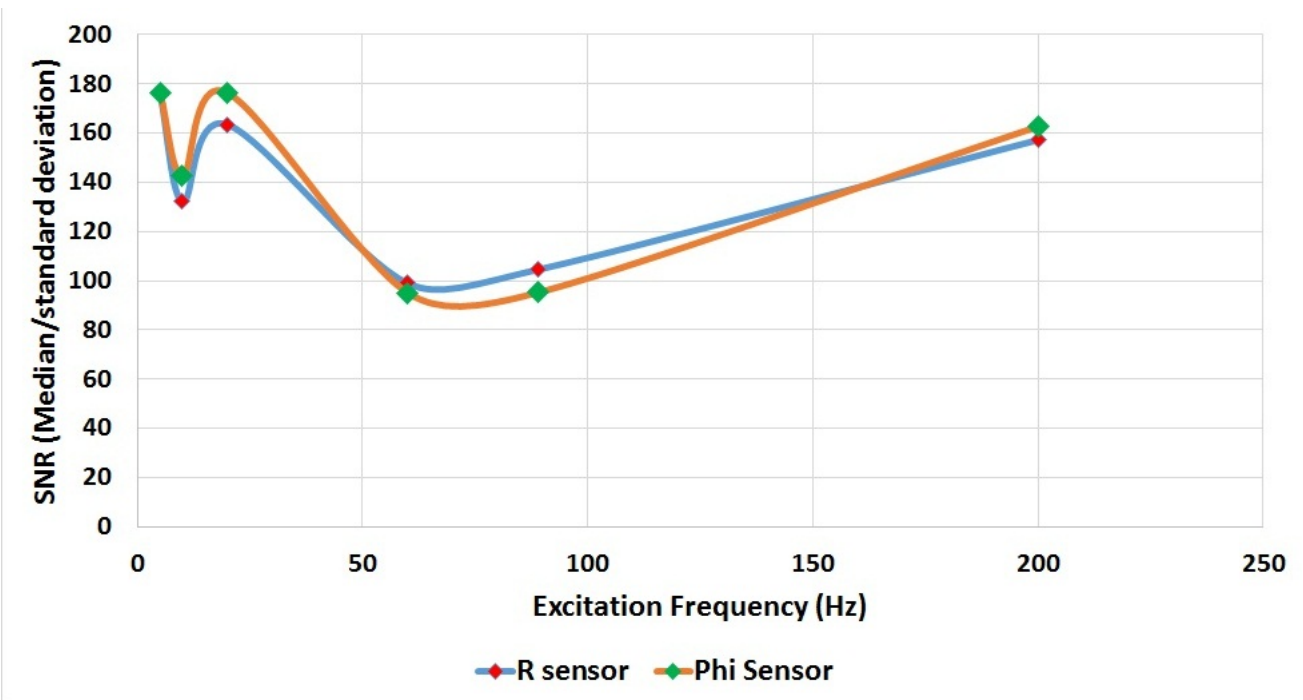

Figure 4.62: R sensor SNR compared to normalized Phi Sensor SNR

sensor is more sensitive to variations caused by the pipe parameters so that a greater standard deviation is produced.

\section{Standard deviation of the phase}

The phase shift standard deviation is very small for the $R$ field with a general increase in standard deviation at higher frequencies. This is due to the increased phase shift of the excitation field relative to the phase angle of the eddy currents. This means the same physical variations in the pipe will produce larger variations in phase shift.

The Phi sensor shows about four times the standard deviation of the R sensor. This is probably because the difference between the phase shift of the excitation field and the eddy current circulating around the Phi axis is greater than the difference between the phase shift of the excitation field and the eddy current circulating around the $R$ axis. This means that the same relative changes would produce larger variations. These larger variations make it impossible to draw any frequency dependence from the Phi sensor phase shift standard deviation.

\section{Effect on local variations}

The uncorrected data along the $Z$ axis for both Phi and $\mathrm{R}$ fields phase shift and magnitude shows a flattening of the rapid increase at the end of the pipe as the frequency increases(seen in figure4.53 and 4.50). This increase in the field along the $Z$ axis is due to the end of the pipe effect. As the frequency increases, the eddy currents are confined to a smaller area [34] this confining of eddy currents is shown in figure 4.63. The figure shows how at low frequency the eddy currents flow in the end of the pipe earlier than at high frequencies. This causes an earlier increase in field at low frequencies when the end of the pipe is approached.

On all the $Z$ axis data, apart from the $R$ field phase shift, there is a section of rapid variation on the $89 \mathrm{~Hz}$ frequency data between $300 \mathrm{~mm}$ and $550 \mathrm{~mm}$. This is due to mechanical movement of the sensors and excitation unit during this measurement. The variation starts to appear on the $R$ field phase shift after flattening, however it is still very small compared to the variations in the other measurements. This indicates the 
Heat Treatment by Induction
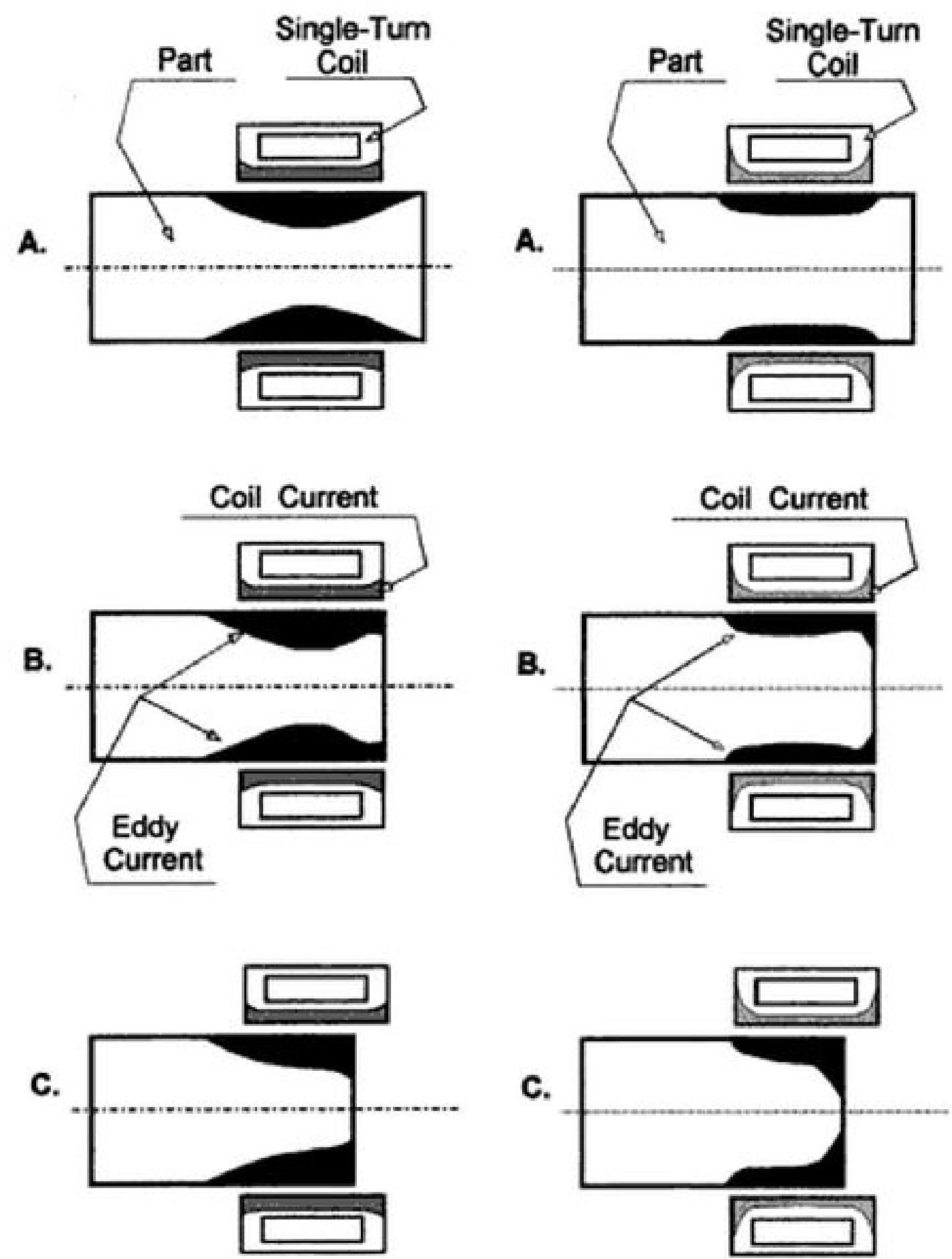

Figure 4.63: Electromagnetic end effect in induction heat treating, low frequency(left) and high frequency (right) figure obtained from [34] 
$R$ field phase shift may be more immune to mechanical variations than the other three measurements.

The data along the $Z$ axis, after the flattening algorithm has been applied has a very low variation. In the center of the pipe no divergence of the frequency signals can be seen. At the ends of the pipe there is some divergence, however there is no clear frequency dependence. This divergence of the signals may be artificially introduced by the $Z$ flattening algorithm. When there is significant deviation from the median value, the larger correction co-efficients are applied amplifying any measurement errors in the area.

The data along the Phi axis for both $\mathrm{R}$ and Phi fields magnitude and phase shift shows significant variation and divergence for different frequencies. However, the excitation frequency does not affect the size of these variations. This suggests there is an unknown parameter changing the field that is being measured. The most likely parameter causing the variation is the relative position of the sensor to the excitation sheet because, despite improvements to the system, there is still approximately 1-2 mm lateral movement possible in the excitation coil.

In summary the results suggest that to get the best SNR, a low excitation frequency should be used and that the rigidity of the excitation and sensor unit is critical to accurate measurement of the defect signals

\subsection{Influence of excitation lift-off}

The excitation lift-off dependence of the system was investigated to determine the effect of increasing the distance of the excitation sheet to the pipe surface on the background signal level and background noise. Tests were carried out with the excitation sheet $25 \mathrm{~mm}$, $29 \mathrm{~mm}, 33 \mathrm{~mm}, 37 \mathrm{~mm}$ and $41 \mathrm{~mm}$ from the pipe surface. The excitation was fixed at 20 $\mathrm{Hz}$ and 300 Amps nominal current. As the excitation sheet was moved, the sensors also needed to be moved further from the pipe. Due to limitations with the design it was not possible to move the sensor module in $4 \mathrm{~mm}$ steps. Instead the sensor was moved in $8 \mathrm{~mm}$ steps so that at $25 \mathrm{~mm}, 33 \mathrm{~mm}$ and $41 \mathrm{~mm}$ there was the same relative position between the sensor and excitation sheet while at $29 \mathrm{~mm}$ and $37 \mathrm{~mm}$ the sensor was 4 $\mathrm{mm}$ further away from the excitation sheet. This range of experimental setups is shown in figure 4.64. From simulation this $4 \mathrm{~mm}$ is expected to reduce the field by $25 \%$ [30], however it is not expected to affect the phase shift as no change in impedance is produced.

\subsubsection{Background signal level and total noise}

To determine the effect of excitation lift-off on the background magnetic field level the median value and standard deviation at each excitation lift-off was calculated after the application of the $Z$ flattening, Phi flattening and current correction algorithms. The change with excitation lift-off for the $R$ sensor magnitude is shown in figure 4.65, indicating an approximately parabolic response. The points at $29 \mathrm{~mm}$ and $37 \mathrm{~mm}$ would be $0.18 \mathrm{Oe}$ and 0.66 Oe higher respectively if the sensors were positioned the same distance to the 


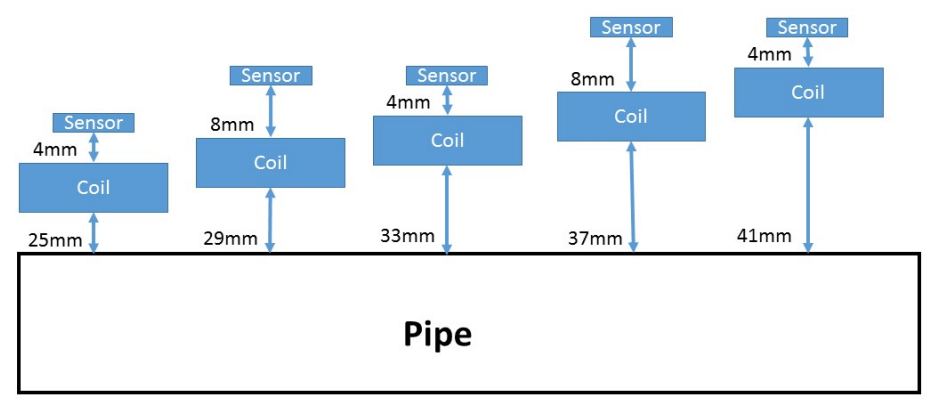

Figure 4.64: Five test configurations used for testing the effect of excitation lift off on the magnetic field

excitation sheet as with the other tests, due to the $25 \%$ reduction stated previously. This would further improve the fit to a parabolic curve.

The $R$ field phase shift change with excitation lift-off is shown in figure 4.66. A measurement error occurred in the $29 \mathrm{~mm}$ lift-off test indicated by the very large error bars which show the plus minus one standard deviation range of a test. With this point ignored a constant response for the excitation lift-off with less than 0.5 degrees change for the whole range can be seen.

The Phi sensor field magnitude change with excitation lift-off is shown in figure 4.67. A similar parabolic shape as the $\mathrm{R}$ field occurs, but an order of magnitude lower signal and with the minimum located at a higher lift-off. The Phi sensor field phase shift change with excitation lift-off is shown in figure 4.68. There appears to be a small increase in phase shift with increasing lift-off, however the standard deviation of the signal is so large at high lift-off levels the results are inconclusive.

The next important measure is the total noise level vs excitation lift-off. For the magnitude signals a SNR value is calculated which is the median value divided by the standard deviation of all data points. Only the standard deviation of the phase shift is plotted with excitation lift-off as, unlike the magnitude, only 360 degrees of phase shift is possible.

The $R$ field SNR change with excitation lift-off is shown in figure 4.69. There is an initial drop in the SNR which is over-estimated due to increased distance between the sensor and excitation sheet. The SNR quickly recovers and surpasses the $25 \mathrm{~mm}$ lift-off value. This higher SNR at higher lift-off is due to less excitation field reaching the pipe which leads to lower eddy currents being induced, thus the noise from material parameters drops away.

The Phi field SNR change with excitation lift-off is shown in figure 4.70. The SNR follows the same curve as the background level because increasing lift-off does not significantly change the standard deviation.

The $\mathrm{R}$ field phase shift standard deviation change with excitation lift-off is shown in figure 4.71. There is a large outlier in the $29 \mathrm{~mm}$ data due to a change during the measurement 


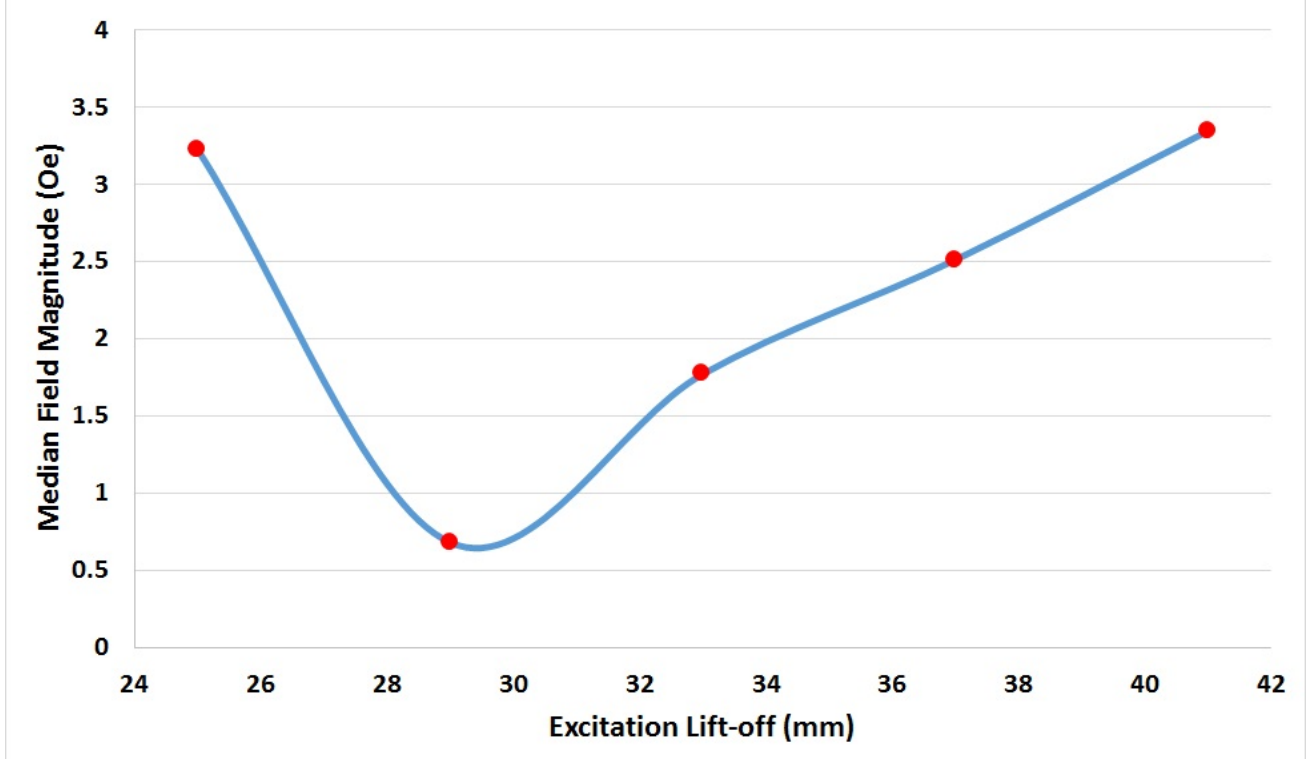

Figure 4.65: $\mathrm{R}$ sensor median field magnitude change with excitation lift-off(the blue line a provides a guide line)

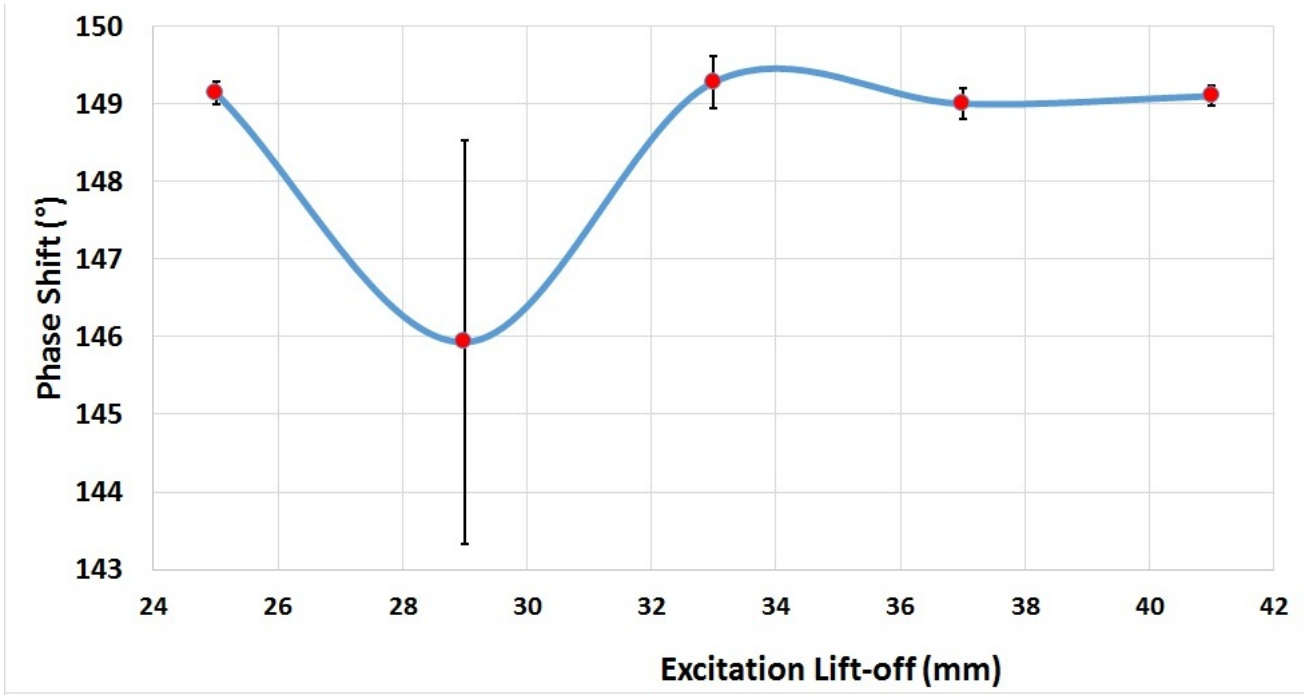

Figure 4.66: $R$ sensor median field phase shift change with excitation lift-off(the blue line a provides a guide line) 


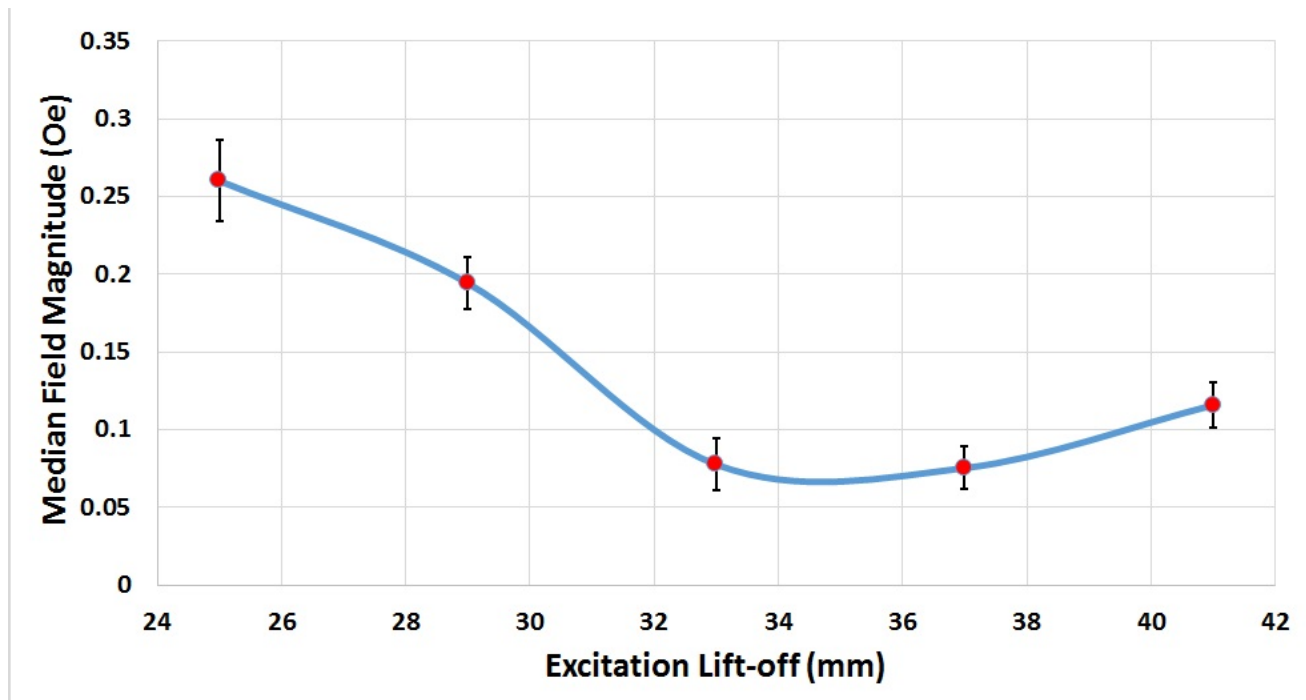

Figure 4.67: Phi sensor median field magnitude change with excitation lift-off (the blue line a provides a guide line)

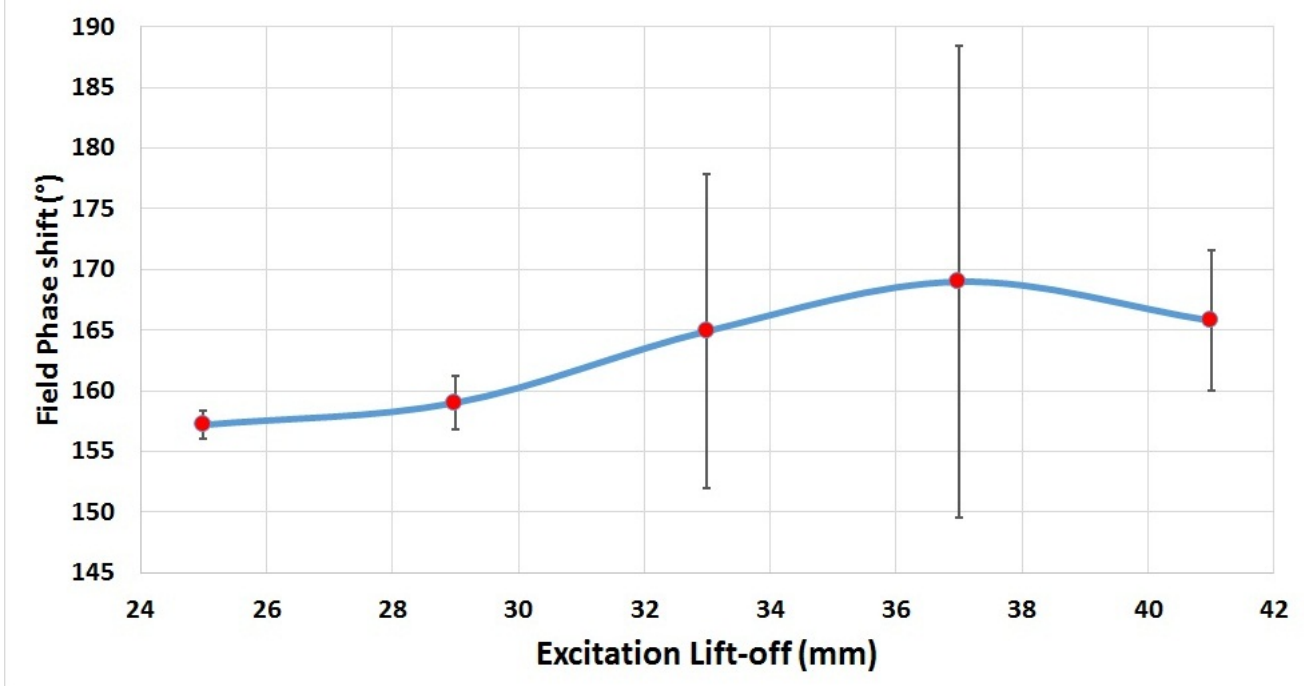

Figure 4.68: Phi sensor median field phase shift change with excitation lift-off (the blue line a provides a guide line) 


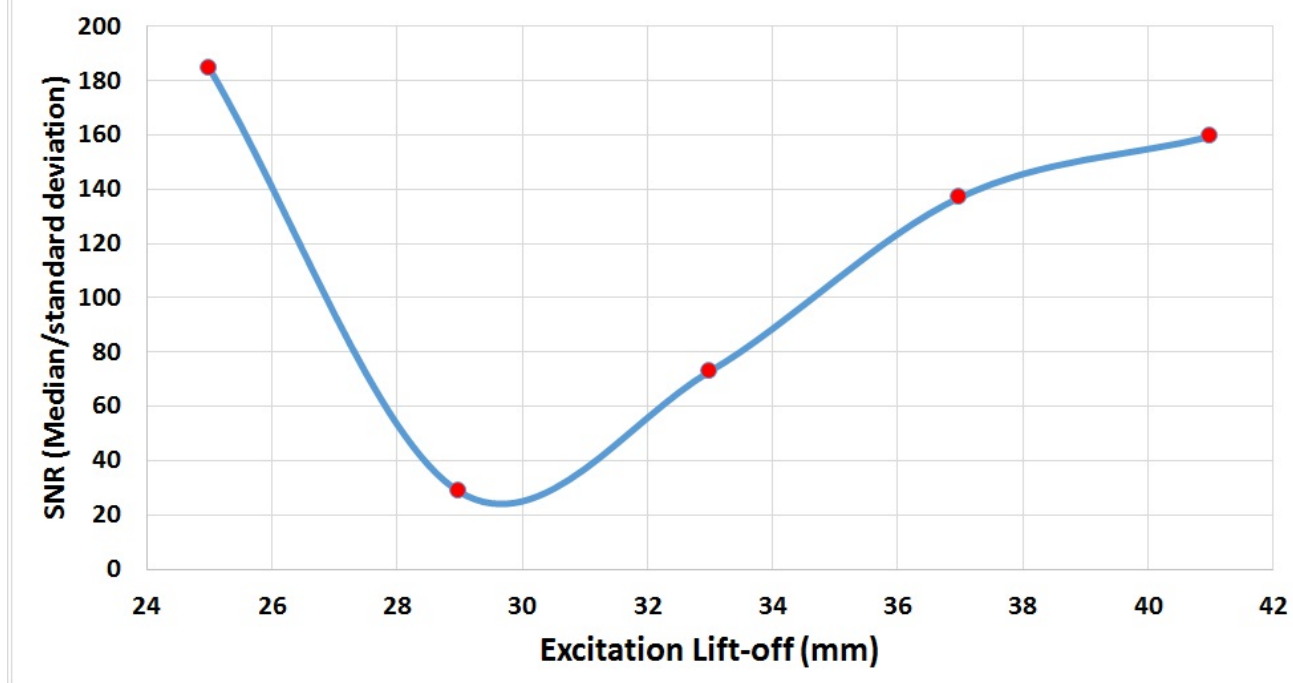

Figure 4.69: $R$ sensor field magnitude SNR change with lift-off (the blue line a provides a guide line)

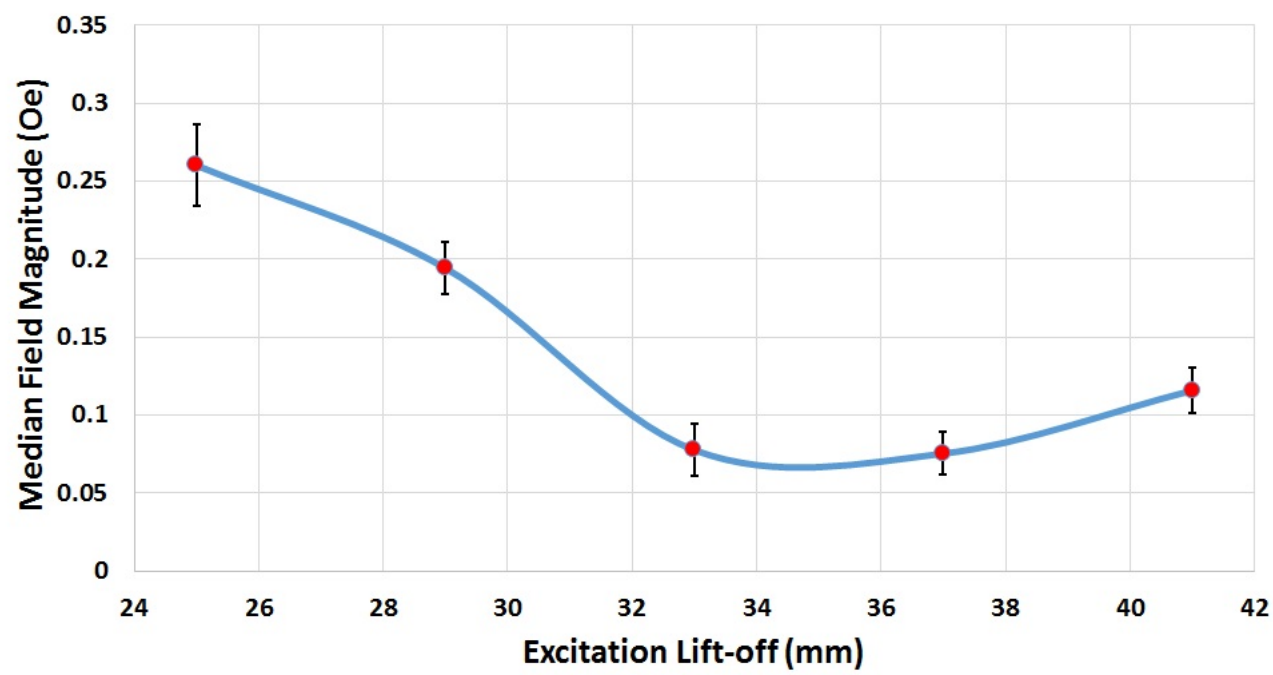

Figure 4.70: Phi sensor field magnitude SNR change with lift-off (the blue line a provides a guide line) 


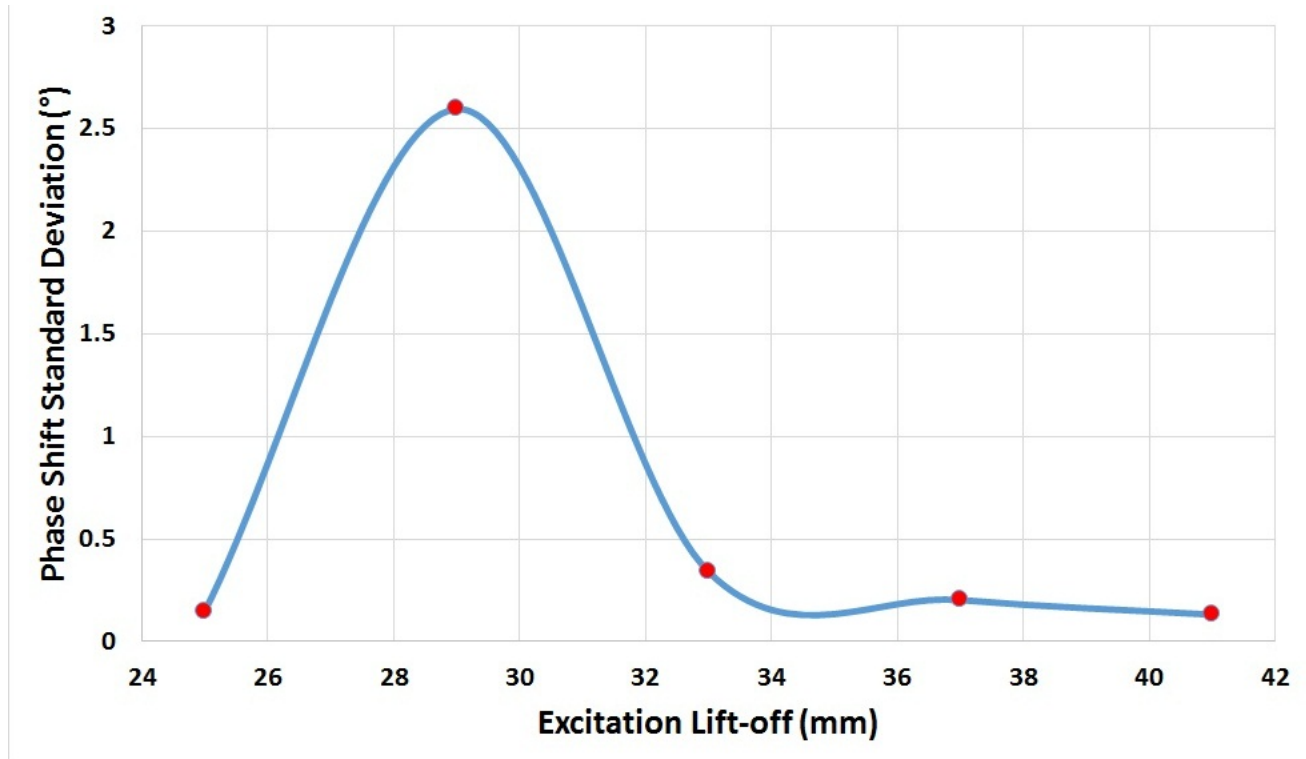

Figure 4.71: $\mathrm{R}$ sensor field phase shift standard deviation change with excitation lift-off (the blue line a provides a guide line)

process. Apart from this a flat response with excitation lift-off can be seen.

The Phi field phase shift standard deviation change with excitation lift-off is shown in figure 4.72. The standard deviation rapidly increases with lift-off until $37 \mathrm{~mm}$ at which point it decreases again. This shows that measurement of the Phi field phase shift at high lift-off would not be possible unless this variation could be reduced. The reduction above $37 \mathrm{~mm}$ is probably due to reducing eddy currents circulating in the pipe, outweighing the effect of increased variation produced at higher lift-off.

\subsubsection{Effects of excitation lift-off on local variation}

For all measures the median value of the data is calculated and then subtracted from all the data points. This shifts the data to be centered around zero and means that the variations in the background level which have already been presented can be ignored. This shows how the variation caused by local changes in the material properties of the pipe change with excitation lift-off.

The first measure shows data without any correction. The median value at each $Z$ position is calculated and this data is presented in figure 4.73 for each excitation lift-off level. The second measure applies the $Z$ flattening algorithm to the data and then again finds the median value at each $Z$ location for all the excitation lift-off values. This shows how the variation along the $Z$ axis is affected once the end effect of the pipe is removed. The third measure again applies the $Z$ flattening algorithm to the data and then the median value is taken at each Phi location. This is plotted for each excitation lift-off value to show how variations along the Phi axis are affected by the excitation lift-off.

Assessing the $\mathrm{R}$ sensor magnitude, the first measure with current corrected data along the $Z$ axis is shown in figure 4.73 , the second measure with current corrected and $Z$ 


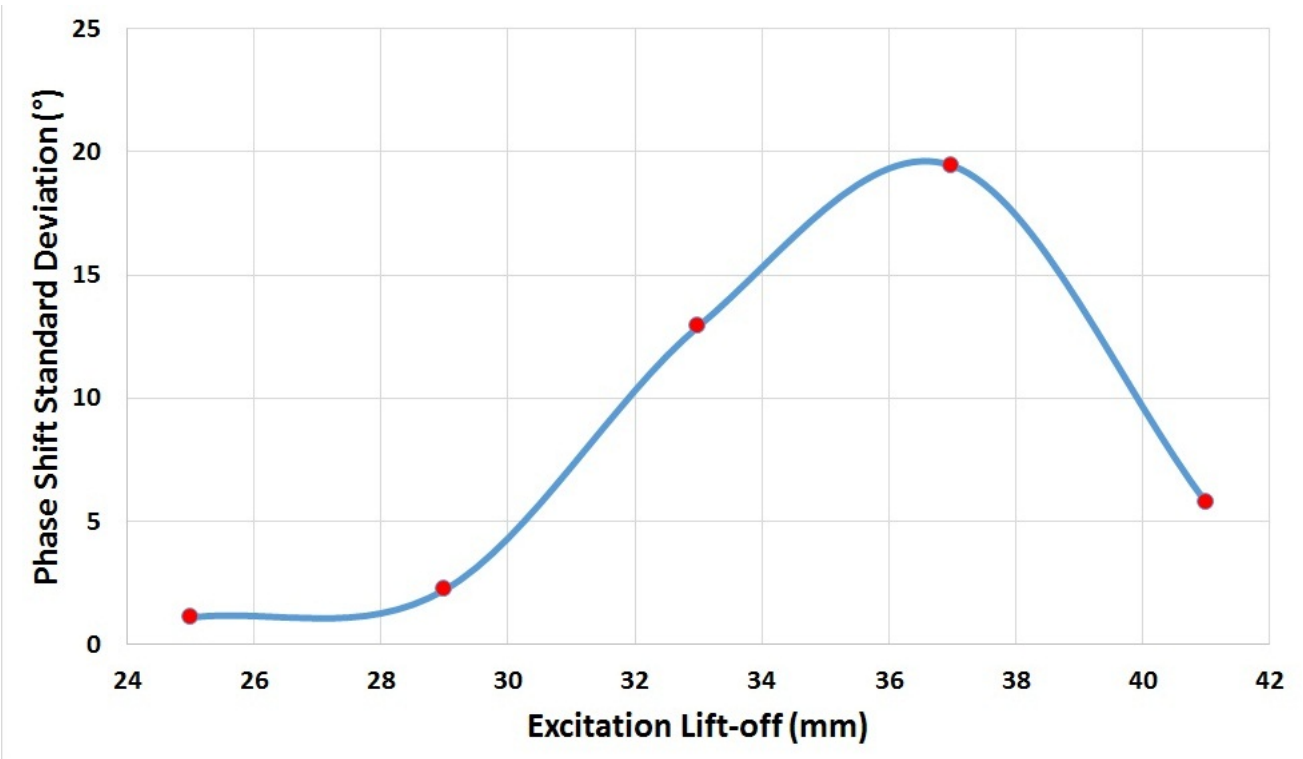

Figure 4.72: Phi sensor field phase shift standard deviation change with excitation lift-off (the blue line a provides a guide line)

flattened data along the $Z$ axis in figure 4.74, lastly the third measure with current corrected and $Z$ flattened data along the Phi axis in figure 4.75 .

Figure 4.73 shows a rapid increase towards the end of the pipe for all lift-offs. There is some divergence between the signals, but no clear lift-off dependence. The two data sets with an extra $4 \mathrm{~mm}$ lift-off for the sensor module are similar, while the other three measurements are grouped together. Figure 4.74 shows very little variation and almost no divergence giving no lift-off dependence. Figure 4.75 shows, as before, larger variation along the Phi axis than along the $Z$ axis. Also dependence on lift-off can be seen with the variations decreasing with increasing lift-off. This is expected, as the increased lift-off means lower excitation field at the pipe surface causing lower eddy currents which means less effect from pipe material variations.

The same three measures are applied for the Phi field magnitude signal. The current corrected data along the $Z$ axis is shown in figure 4.76, the current corrected and $Z$ flattened data along the $Z$ axis in figure 4.77 and the same data along the Phi axis in figure 4.78.

Figure 4.76 shows significant variation and divergence of the signals. However, there is no clear dependence with the excitation lift-off. Figure 4.77 shows some small variation near the end of the pipe but no divergence between the signals at different lift-off levels meaning there appears to be no lift-off dependence. Figure 4.78 shows large variation and divergence between the different excitation lift-off levels. This divergence shows that the variations on the Phi axis are lift-off dependent with the variation reducing in size as the lift-off increases.

The same three measures were again applied for the $R$ field magnitude phase shift. The current correct data along the $Z$ axis is shown in figure 4.79. The current correct and $Z$ flattened data along the $Z$ axis is shown in figure 4.80. The same data along the Phi axis 


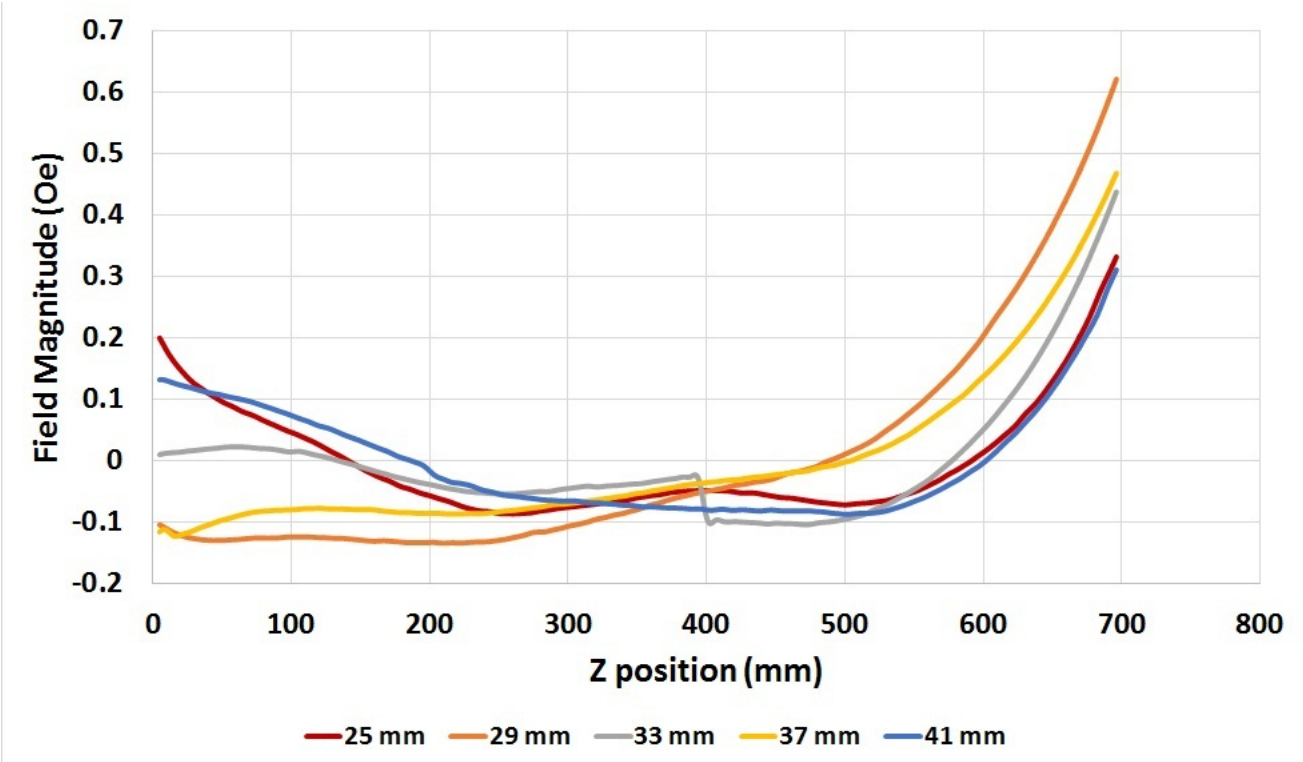

Figure 4.73: $R$ sensor median magnitude along the $Z$ axis

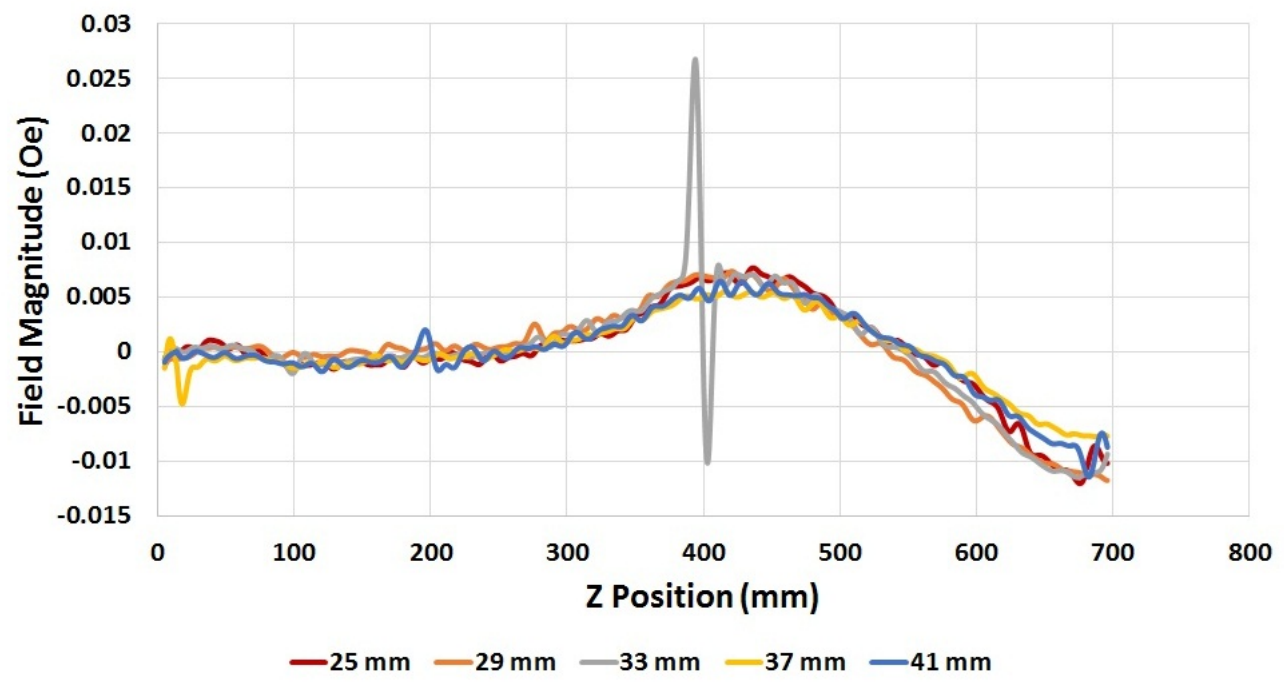

Figure 4.74: $\mathrm{R}$ sensor median magnitude along the $\mathrm{Z}$ axis with $\mathrm{Z}$ flattening applied 


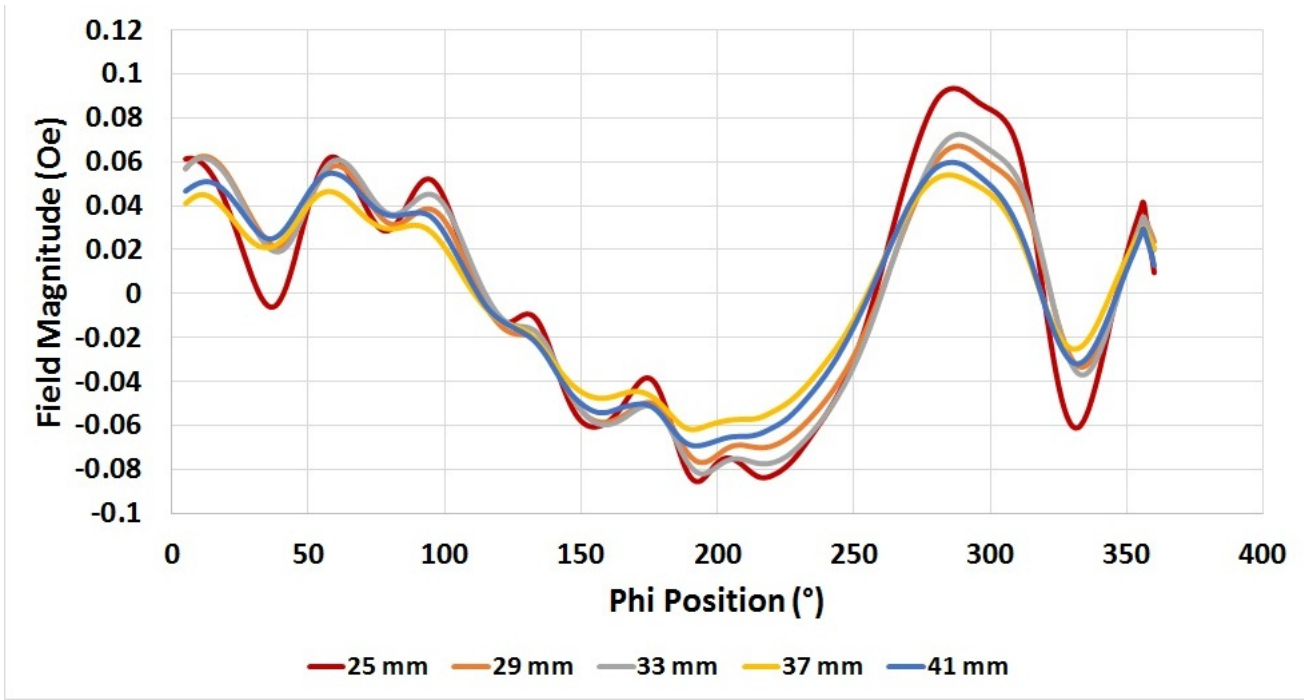

Figure 4.75: $\mathrm{R}$ sensor median magnitude along Phi axis with $\mathrm{Z}$ flattening applied

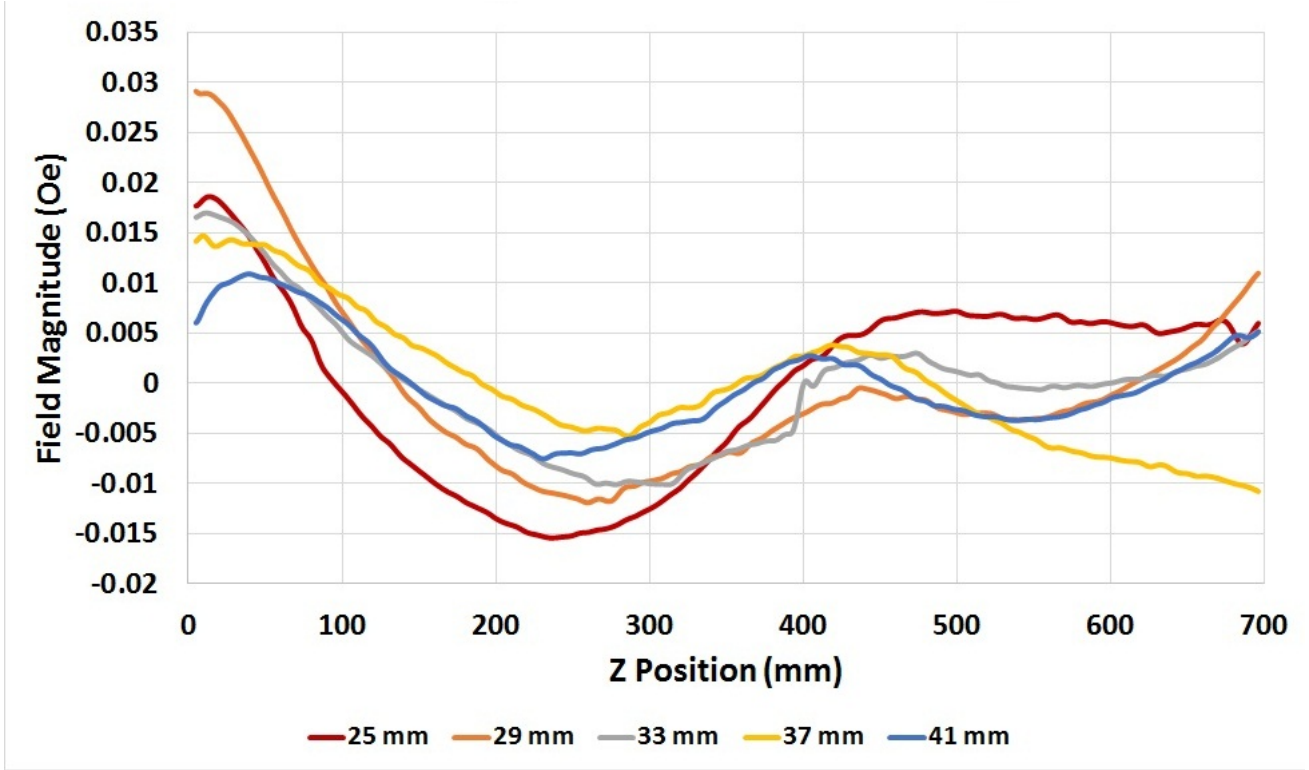

Figure 4.76: Phi sensor median magnitude along the $Z$ axis 


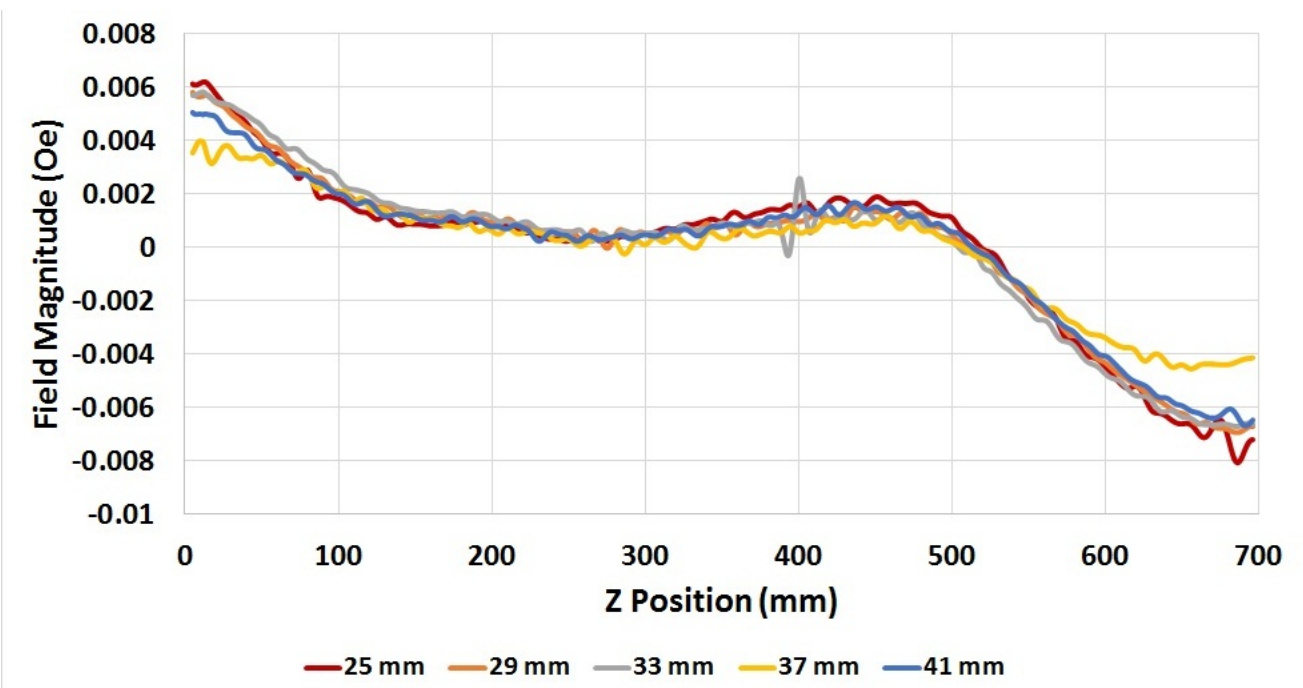

Figure 4.77: Phi sensor median magnitude along the $Z$ axis with $Z$ flattening applied

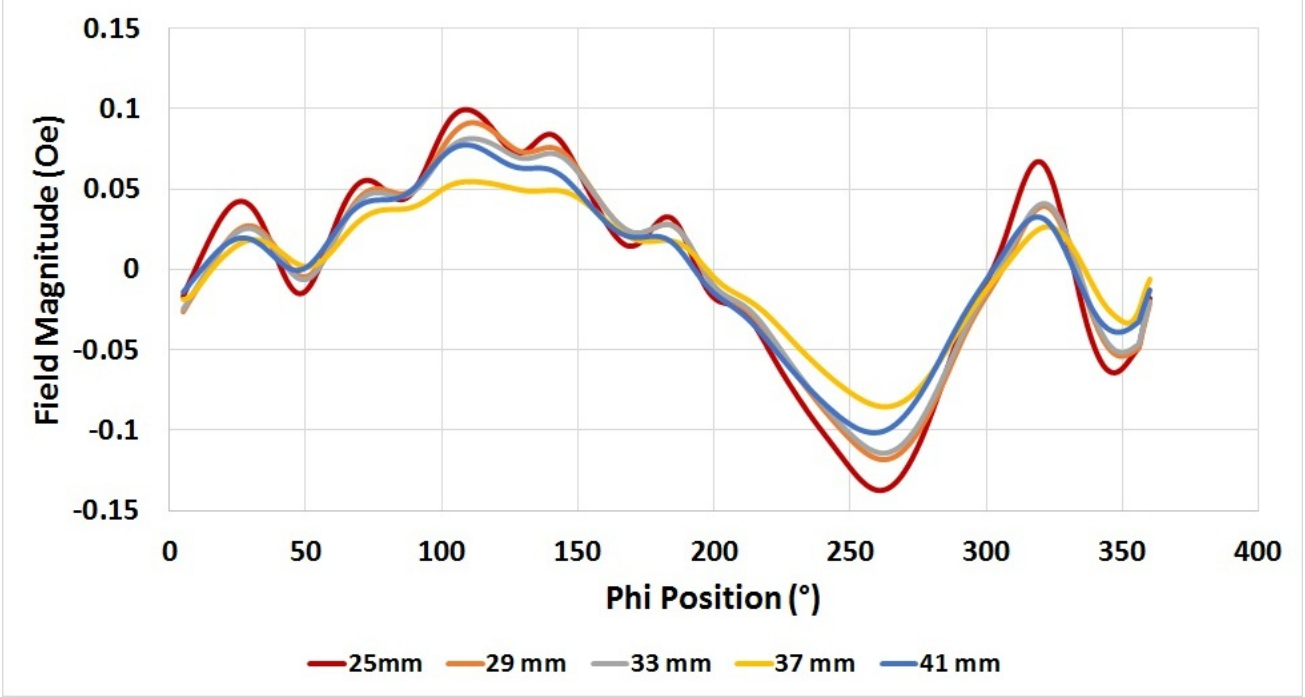

Figure 4.78: Phi sensor median magnitude along Phi axis with Z flattening applied 


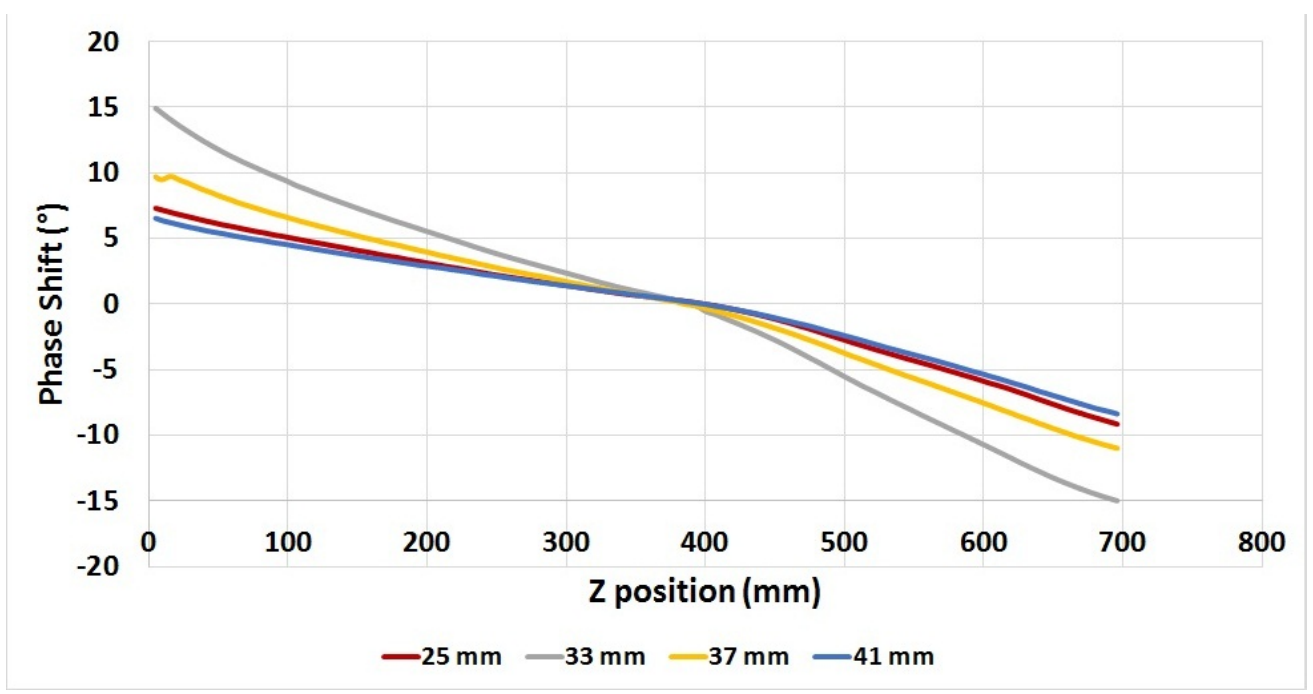

Figure 4.79: $R$ sensor median phase shift along $Z$ axis with $29 \mathrm{~mm}$ data removed

is shown in figure 4.81 . The $29 \mathrm{~mm}$ data has been removed from all of these plots as a movement in the sensor caused a phase reversal partway through the test.

Figure 4.79 shows a linear trend which changes slope between tests, but no lift-off dependence can be observed. Figure 4.80 shows a very flat response with less than 0.1 degree change for all lift-off levels. Figure 4.81 shows significant divergence between the signals but, like the data along the $Z$ axis, there is no lift-off dependence.

The same three measures were again applied for the Phi field phase shift signal. The current correct data along the $Z$ axis is shown in figure 4.82. The current correct and $Z$ flattened data along the $Z$ axis is shown in figure 4.83. The same data along the Phi axis is shown in figure 4.84 .

Figure 4.82 shows a flat response at low lift-off, however at the higher lift-off levels there appears to be a phase reversal occurring in the phase shift. Figure 4.83 shows the same flat response for the lower excitation lift-off however at higher lift-off large noise is introduced due to the flattening algorithm not handling the rapid change in the phase shift. Figure 4.84 again shows the large variation for the higher lift-off levels.

\subsection{Summary of excitation lift-off dependence}

\section{Background signal level}

The effect of increasing the excitation lift-off on the field magnitudes shows a quite different response between $\mathrm{Phi}$ and $\mathrm{R}$ fields. The $\mathrm{R}$ field initially drops but recovers at a higher lift-off while the Phi field decays with increasing lift-off. The expected result is an increase in field with increasing lift-off is shown in section 2.5.1. For the $R$ field, if the $25 \mathrm{~mm}$ lift-off data is ignored, this increasing field with increasing lift-off can be observed as expected. The Phi field however appears to have a decay with increasing lift-off. This does not fit with the model previously presented.

This decay with increasing lift-off could be due to the fact that, if the setup is symmetrical, 


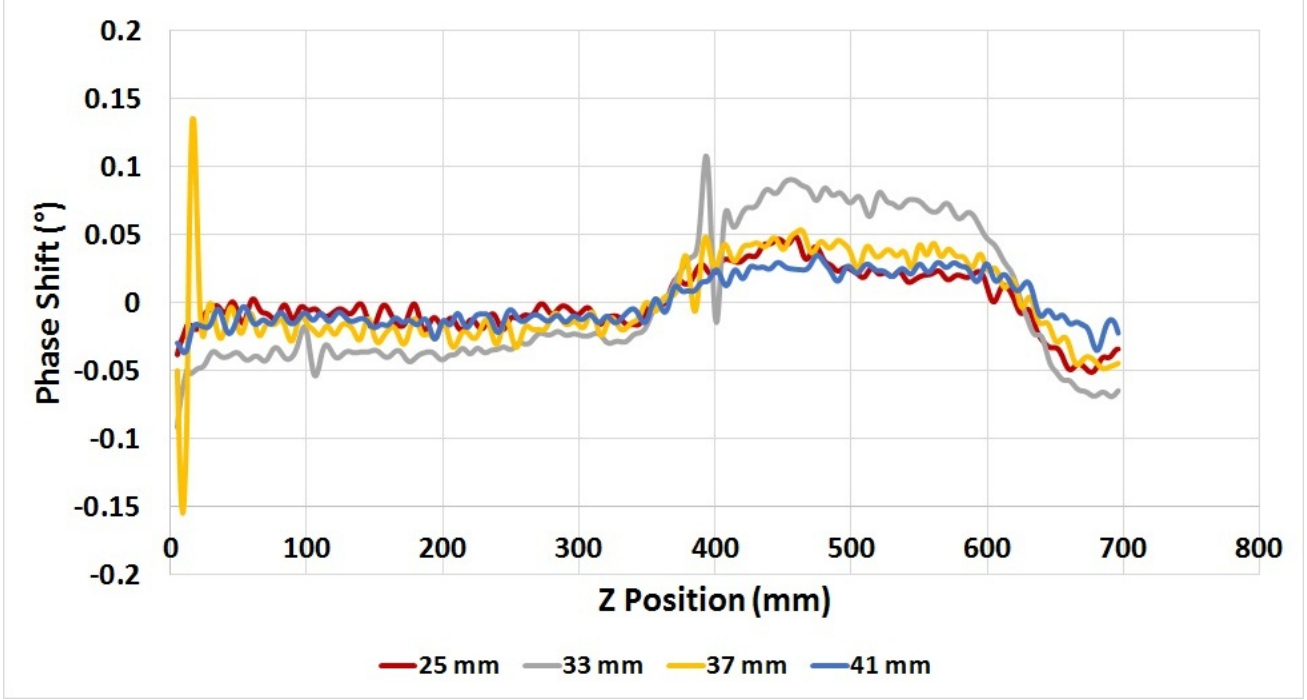

Figure 4.80: $R$ sensor median phase shift along the $Z$ axis with $Z$ flattening applied and $29 \mathrm{~mm}$ data removed

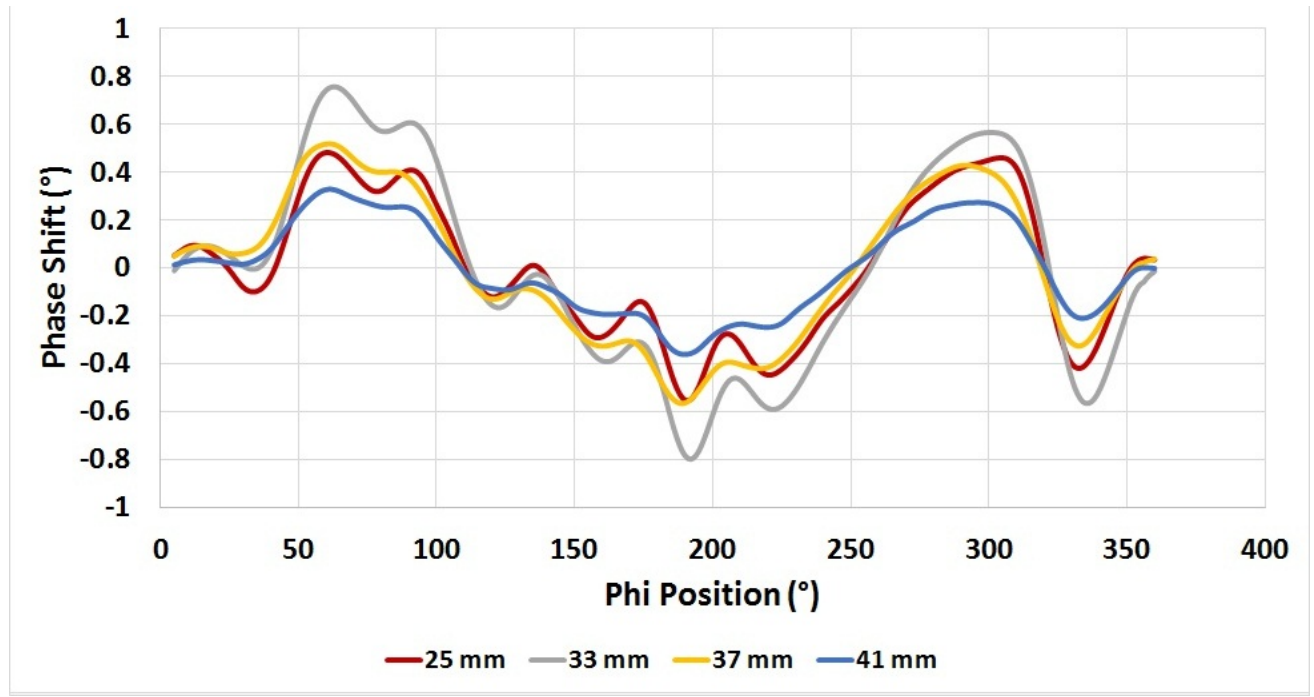

Figure 4.81: $\mathrm{R}$ sensor median phase shift along Phi axis with $\mathrm{Z}$ flattening applied and 29 $\mathrm{mm}$ data removed 


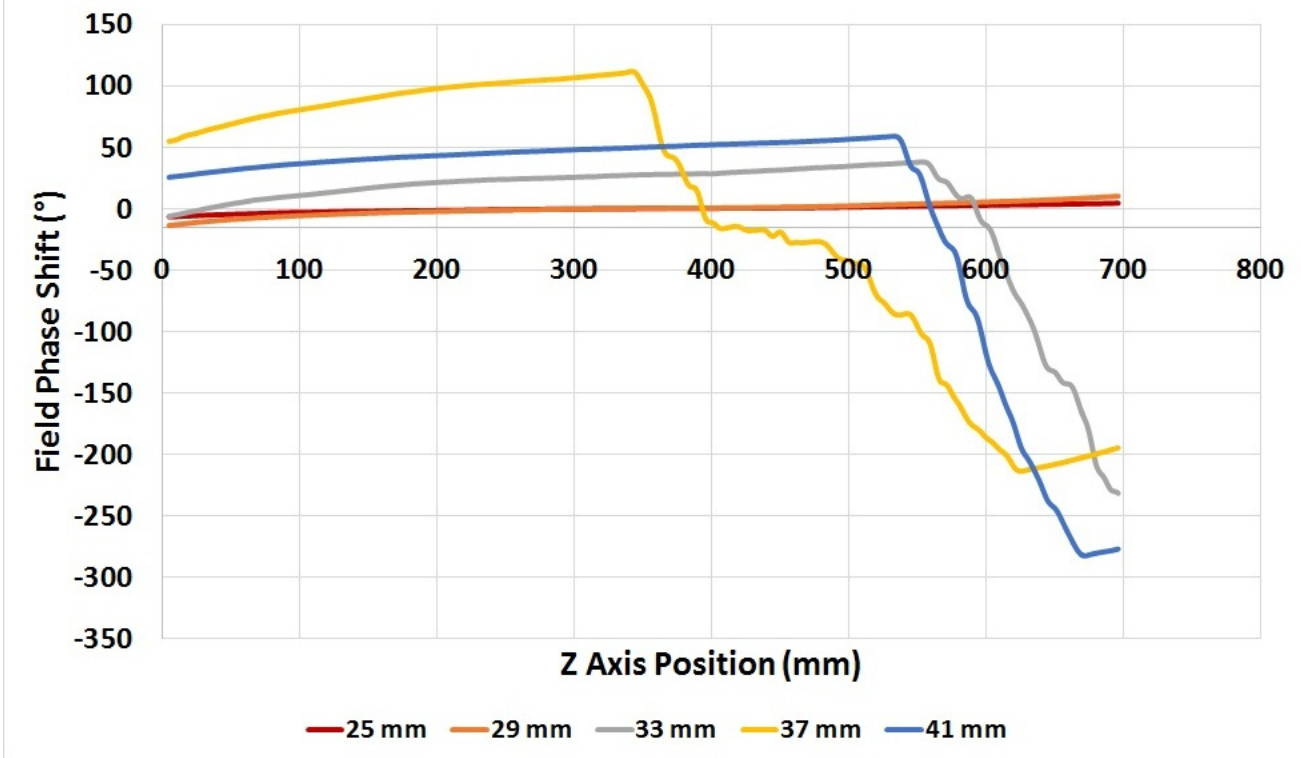

Figure 4.82: Phi sensor median phase shift along the $Z$ axis

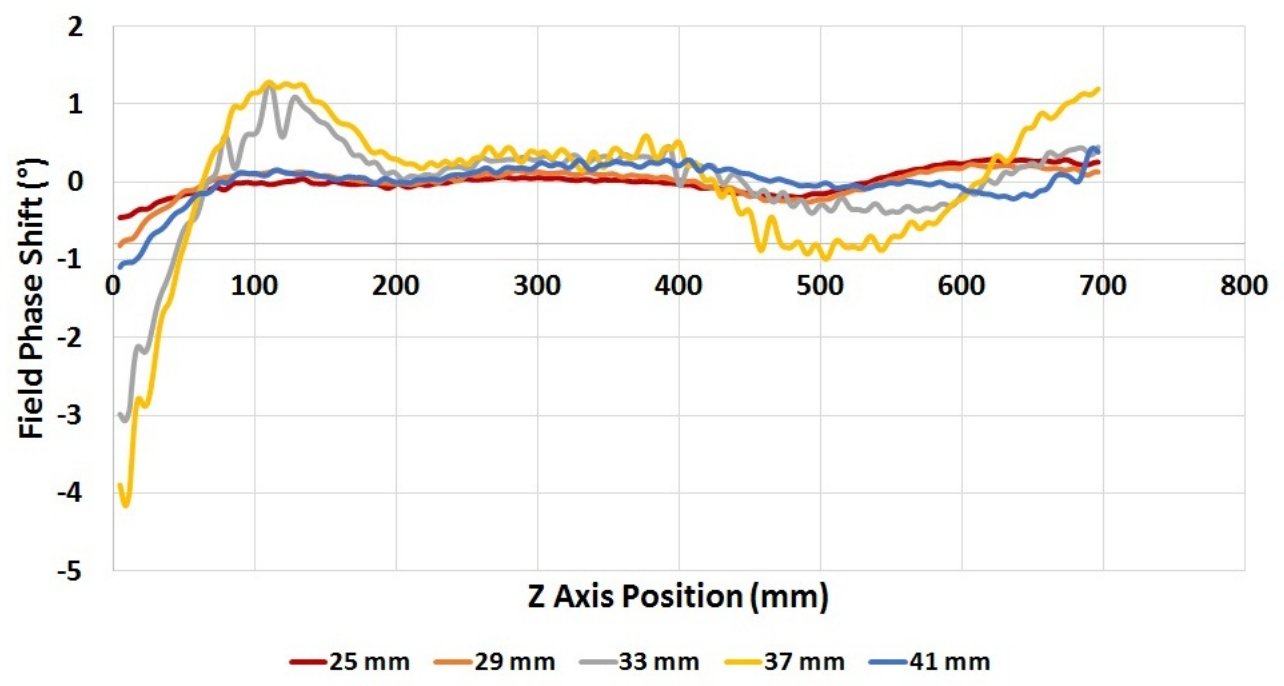

Figure 4.83: Phi sensor median phase shift along the $Z$ axis with $Z$ flattening applied 


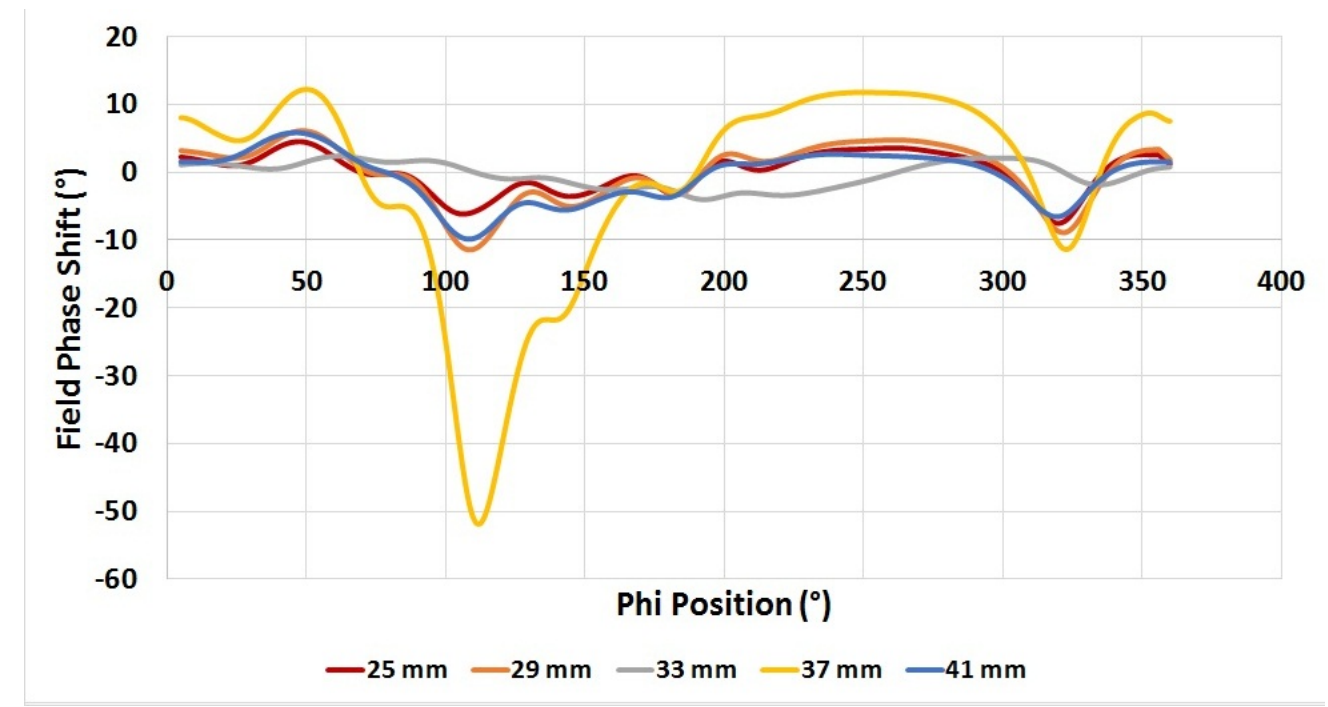

Figure 4.84: Phi sensor median phase shift along Phi axis with Z flattening applied

no Phi field is generated by the excitation coil . However, if the pipe and excitation coil are not co-centric Phi field would then be generated. As the lift-off increases the field generated by this mis-alignment would be expected to drop.

The phase shift signal for the $R$ and Phi field is constant with changing lift-off. The $R$ field, apart from the scan at $29 \mathrm{~mm}$ lift-off, shows less than 0.1 degrees change. The spike at $29 \mathrm{~mm}$ shows a very large standard deviation which is probably due to the excitation unit shifting during the test. The Phi sensor shows a high variation at the higher lift-off levels which is due to step changes in signal that occur as the end of the pipe is approached.

\section{SNR of magnitude}

The SNR for the Phi and R field magnitude signals shows similar behavior to the median background field. This shows that the standard deviation is relatively constant with changing excitation lift-off. The $\mathrm{R}$ field shows that a higher lift-off will increase the SNR faster than the median magnitude. This is because, if the lift-off increases, the variation in the pipe has less effect on the magnetic field. The Phi sensor has this effect counteracted by the dropping background field level.

\section{Standard deviation of phase}

The standard deviation change for the $R$ field phase shift shows a noise point at $29 \mathrm{~mm}$ which is due to variations that occurred during the test. However, the rest of the data points show near constant value with excitation lift-off. The Phi field standard deviation is dominated at the higher lift-off by large changes occurring in the scan. This shows that this sensor is particularly sensitive to its placement as small changes in position can result in a large change in the signal.

\section{Effect on local variations}

The variation along the $Z$ axis for the $R$ sensor magnitude shows that the distance of the sensor from the excitation sheet affects the amplitude of the variation more than the distance from the excitation sheet to the pipe. This is shown with the data at $29 \mathrm{~mm}$ and 
$37 \mathrm{~mm}$ lift-off grouped together and the $25 \mathrm{~mm}, 33 \mathrm{~mm}$ and $41 \mathrm{~mm}$ grouped together. Once the $\mathrm{Z}$ flattening algorithm is applied the only noticeable variation is a small step change in the $33 \mathrm{~mm}$ data which creates a large spike compared to the background noise level. This shows that very small shifts in position are enough to change the measured field significantly. The variation of the $R$ field magnitude around the Phi axis shows some divergence between the lift-off levels with the $25 \mathrm{~mm}$ lift-off data showing the largest variations and the $41 \mathrm{~mm}$ the smallest variations. This is because, as the excitation is moved away, less eddy current is induced and less of the resulting field is returned to the sensor. As a result the constant component from the excitation field starts to dominate.

The uncorrected $P h i$ field magnitude variation along the $Z$ axis shows no clear pattern despite large variations between the tests. The flattened data shows little variation with just the $37 \mathrm{~mm}$ data diverging a small amount. The Phi field magnitude variation around the Phi axis shows less divergence between the signals than for the $\mathrm{R}$ field. There appears to be a general trend that the variation decreases with increasing lift-off for the same reason as the $\mathrm{R}$ sensor, because lower eddy currents in the pipe create less variation in magnetic field.

The $R$ field phase shift along the $Z$ axis shows clear changes with excitation lift-off, however no lift-off dependence is apparent which is either due to a lack of data or other excitation parameters affecting the phase shift. Once the $Z$ flattening algorithm has been applied there is very little variation with less than 0.2 degree change over the whole scan area. The $\mathrm{R}$ field phase shift along the Phi axis shows, like the $Z$ axis, clear changes with lift-off but no lift-off dependence.

The Phi field phase shift along the $Z$ axis shows phase reversal, at higher lift-off levels, as the sensor is moved down the pipe. This indicates that as the lift-off increases the Phi field phase shift measurement is near a threshold where very small changes can have large effects on the phase shift. Because of these phase reversals the $Z$ flattened data along the $Z$ and $P h i$ axis does not add any further information on the sensitivity of local variations to lift-off levels.

\subsection{Effect of the Aluminum shield}

Pipes in processing plants often have a weather shielding which is normally made of aluminum. The shielding provides protection from the weather as well as holding the thermal insulation in place. This shield needs to be left in place during testing so that the weather proofing is not compromised. The standard pipe used in the previous test was wrapped in $25 \mathrm{~mm}$ mineral wool insulation. Then $0.5 \mathrm{~mm}$ aluminum sheet was wrapped around the insulation with a $300 \mathrm{~mm}$ overlap joint riveted along the seam with aluminum rivets. This wrapped pipe is shown in figure 4.85 with a close up of the joint in figure 4.86.

As the aluminum is more conductive than the steel and closer to the excitation source a much higher eddy current density is expected. The proportion of the total eddy current flowing in the aluminum is small at low frequency, compared to the steel, as the aluminum is thin compared to the steel. However, at high frequency the proportion increases as the skin effect reduces the eddy current flowing in the steel, this effect of frequency on the eddy currents is shown in figure 4.87 . 


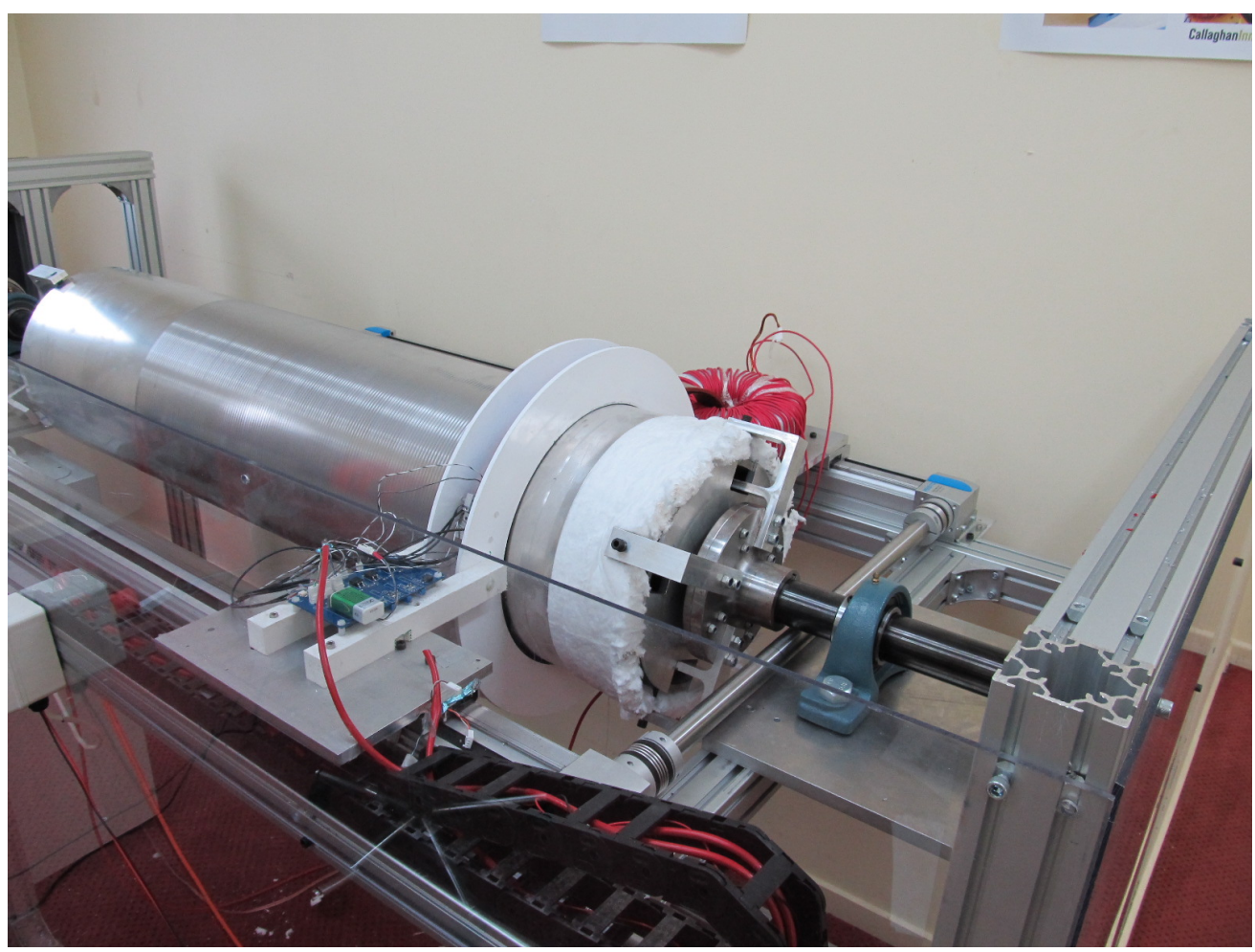

Figure 4.85: Pipe wrapped in aluminum shield

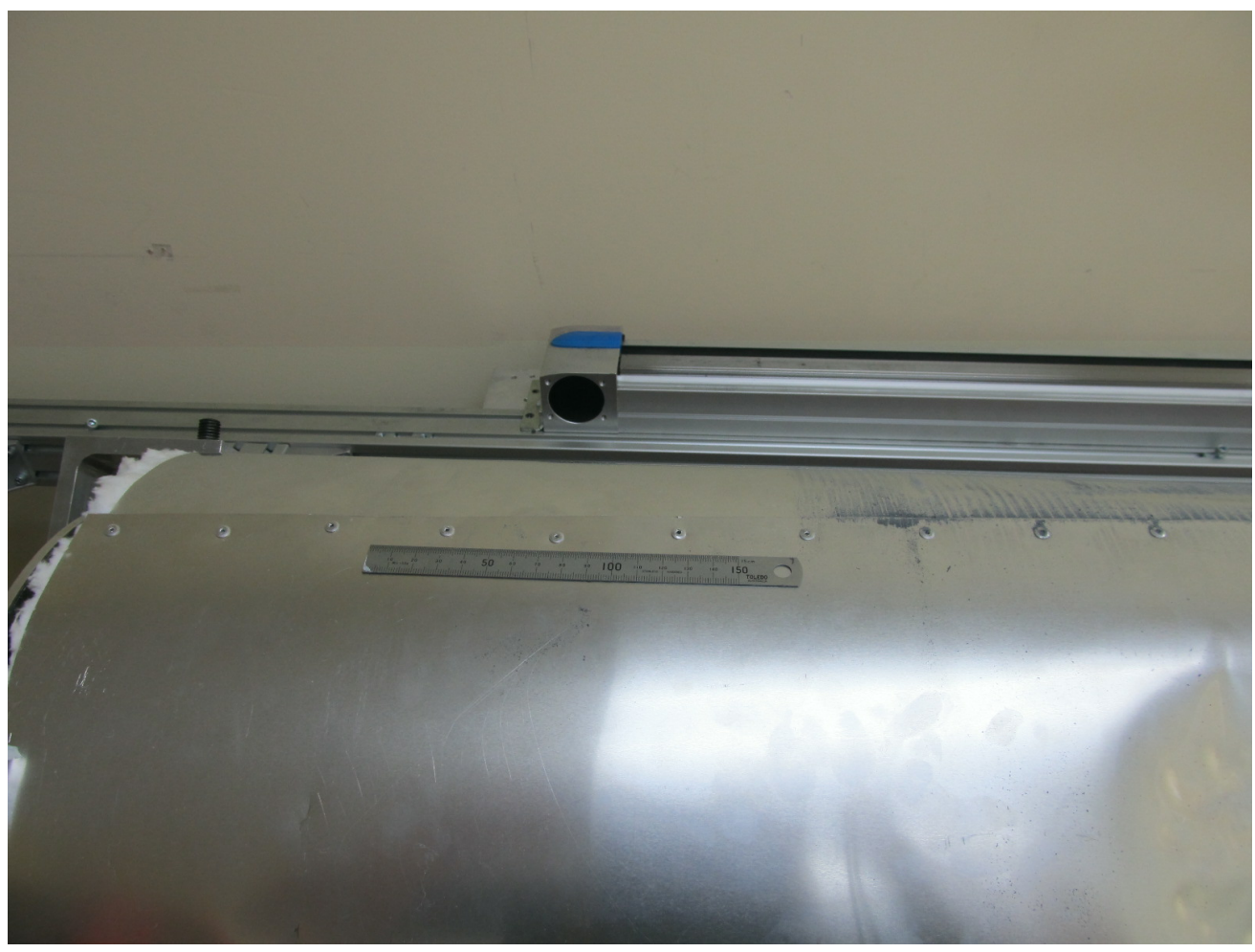

Figure 4.86: Photo of riveted joint in aluminum shield 


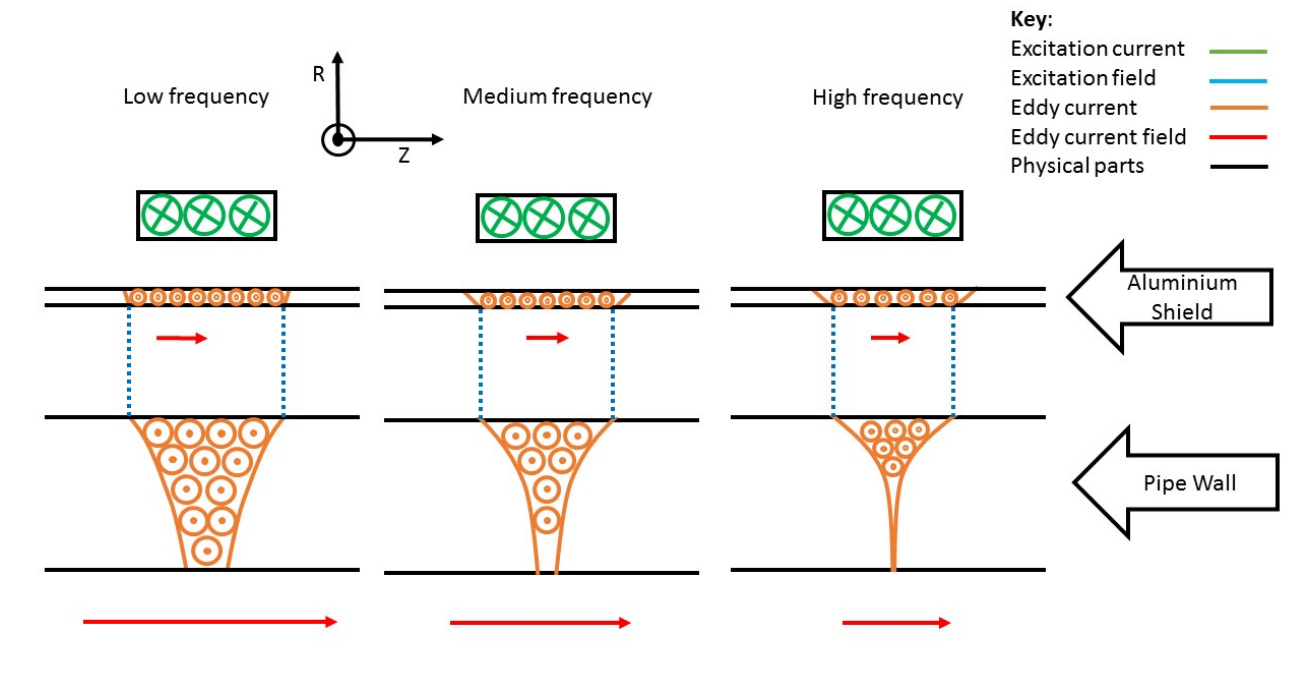

Figure 4.87: Effect of excitation frequency on eddy current in shielded pipe

A full test of the aluminum shielded pipe was carried out with the excitation frequency set at $20 \mathrm{~Hz}$ and the current at $300 \mathrm{amps}$. The contour plot for the $\mathrm{R}$ field magnitude of the insulated pipe is shown in figure 4.88. When compared to figure 4.89 , some extra features appear along the 50 degree line. These come from the outside edge of the aluminum shield where it is riveted to the inside. At this point the eddy currents induced in the shield are required to make a rapid change in direction which, in turn, causes changes in the magnetic field. At some points, with good electrical contact and smooth seams, only little deviation in the eddy current occurs, as shown in the $400 \mathrm{~mm}$ to $700 \mathrm{~mm}$ section. However, if there is poor electrical connection to the rest of the aluminum shield the currents are significantly rerouted, as is the case for the $0 \mathrm{~mm}$ to $400 \mathrm{~mm}$ section.

\subsubsection{Effect of excitation frequency on features introduced by the shielding}

To assess whether a multi-frequency algorithm can be developed to remove the effect of an aluminum shield, a set of tests was done at a range of frequencies from $5 \mathrm{~Hz}$ to $200 \mathrm{~Hz}$. Contour plots for Phi and $\mathrm{R}$ field magnitude and phase shift have been plotted. The $\mathrm{R}$ field magnitude at $5 \mathrm{~Hz}$ is shown in figure 4.90 and at $200 \mathrm{~Hz}$ in figure 4.91 . At $5 \mathrm{~Hz}$ there is a distinct negative and positive peak pair with 0.4 Oe peak to peak size. At $200 \mathrm{~Hz}$ excitation frequency there is a clear line at 50 degrees with very large variations compared to the background and with much steeper peaks than at $5 \mathrm{~Hz}$.

The contour plot of the $\mathrm{R}$ field phase shift with $5 \mathrm{~Hz}$ excitation is shown in figure 4.92 and at $200 \mathrm{~Hz}$ in figure 4.93. The $\mathrm{R}$ field phase shift change at $5 \mathrm{~Hz}$ shows sharper peaks than the magnitude signal at $5 \mathrm{~Hz}$. At $200 \mathrm{~Hz}$ there is an increase in the magnitude of the peaks. However, the difference between the $5 \mathrm{~Hz}$ and $200 \mathrm{~Hz}$ is small compared to the difference seen in the field magnitude with changing frequency, as shown in figure 4.90 


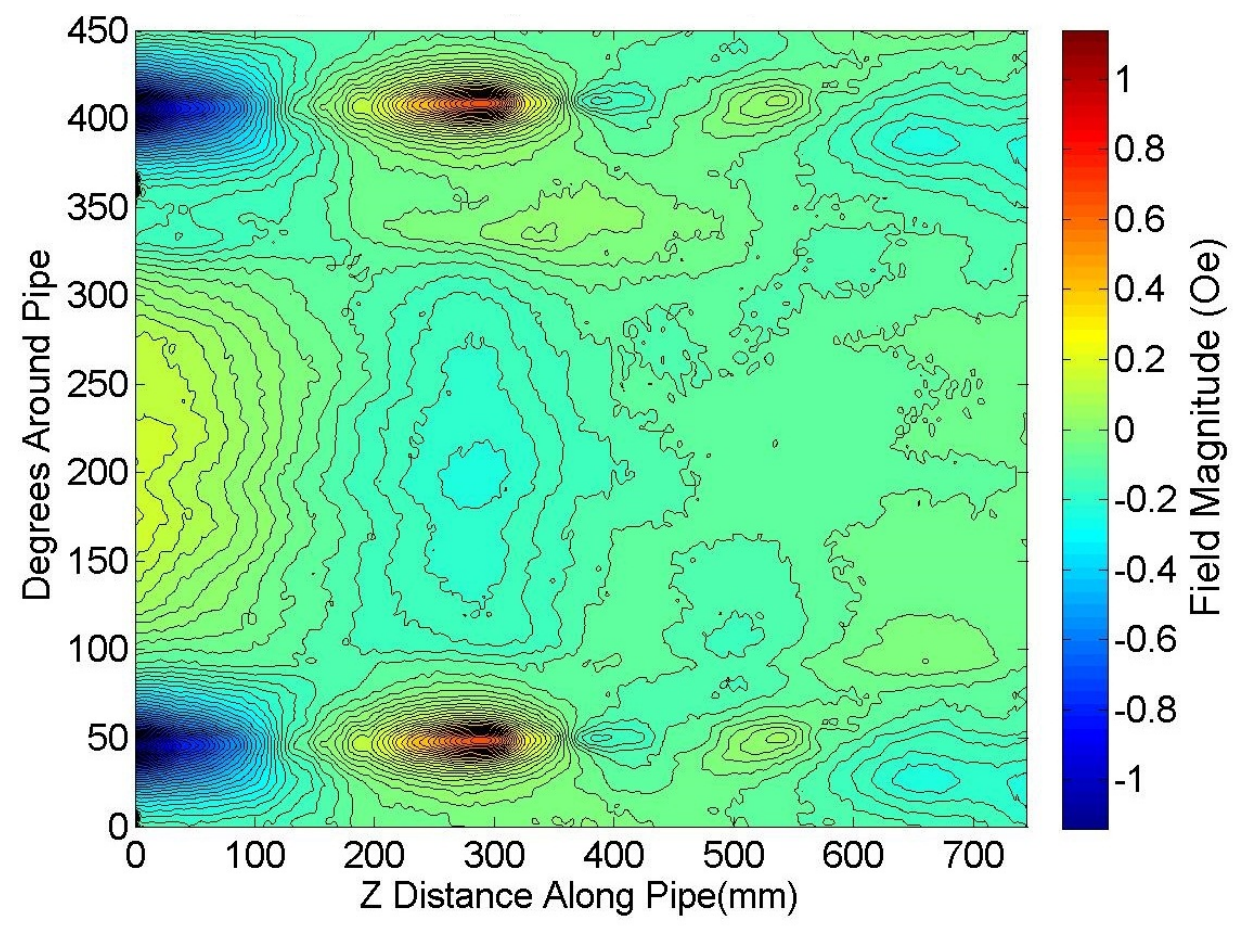

Figure 4.88: $\mathrm{R}$ field magnitude over standard pipe with aluminum shielding with $20 \mathrm{~Hz}$ excitation

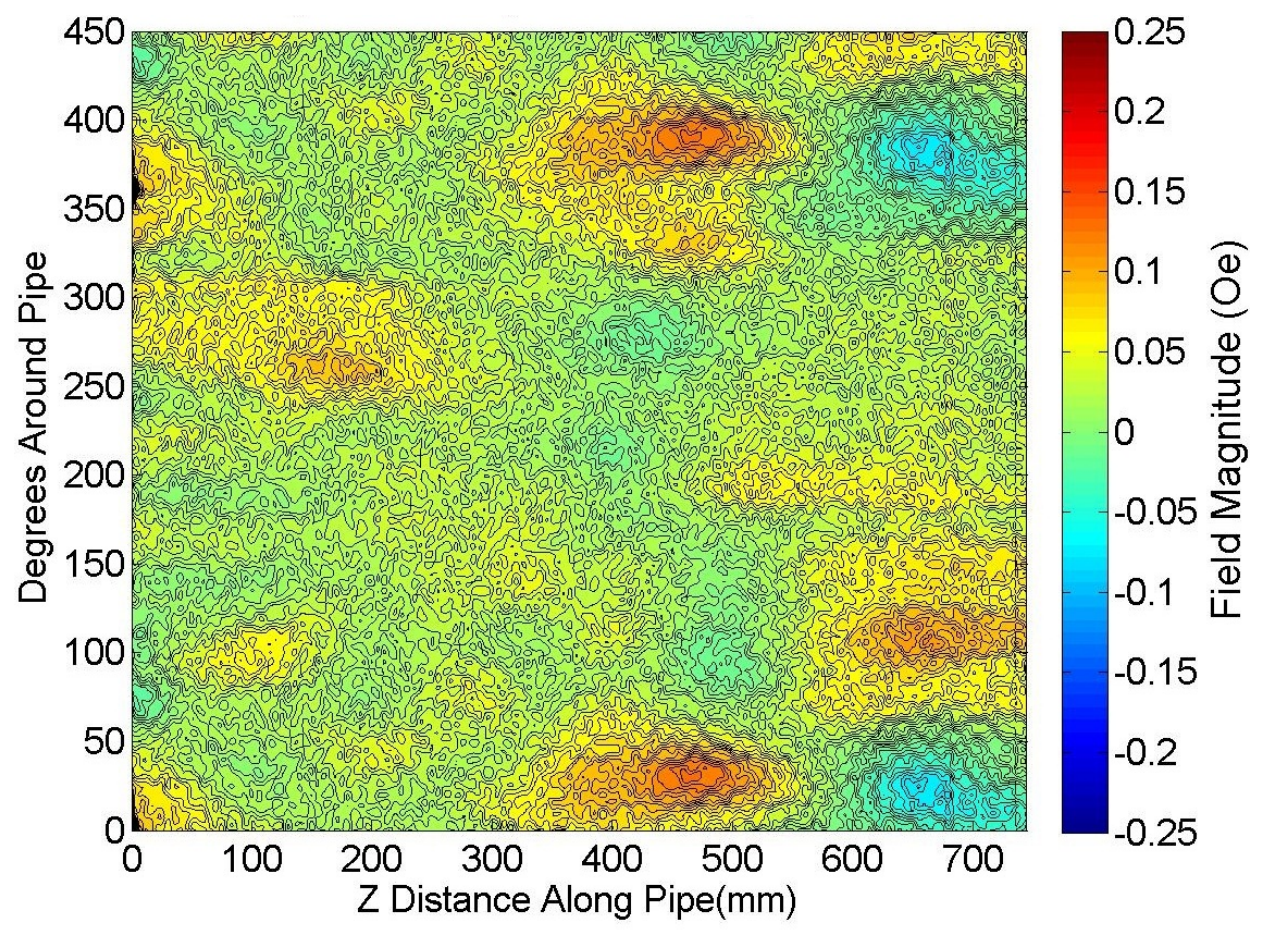

Figure 4.89: $\mathrm{R}$ field magnitude, over unshielded pipe with $20 \mathrm{~Hz}$ excitation 


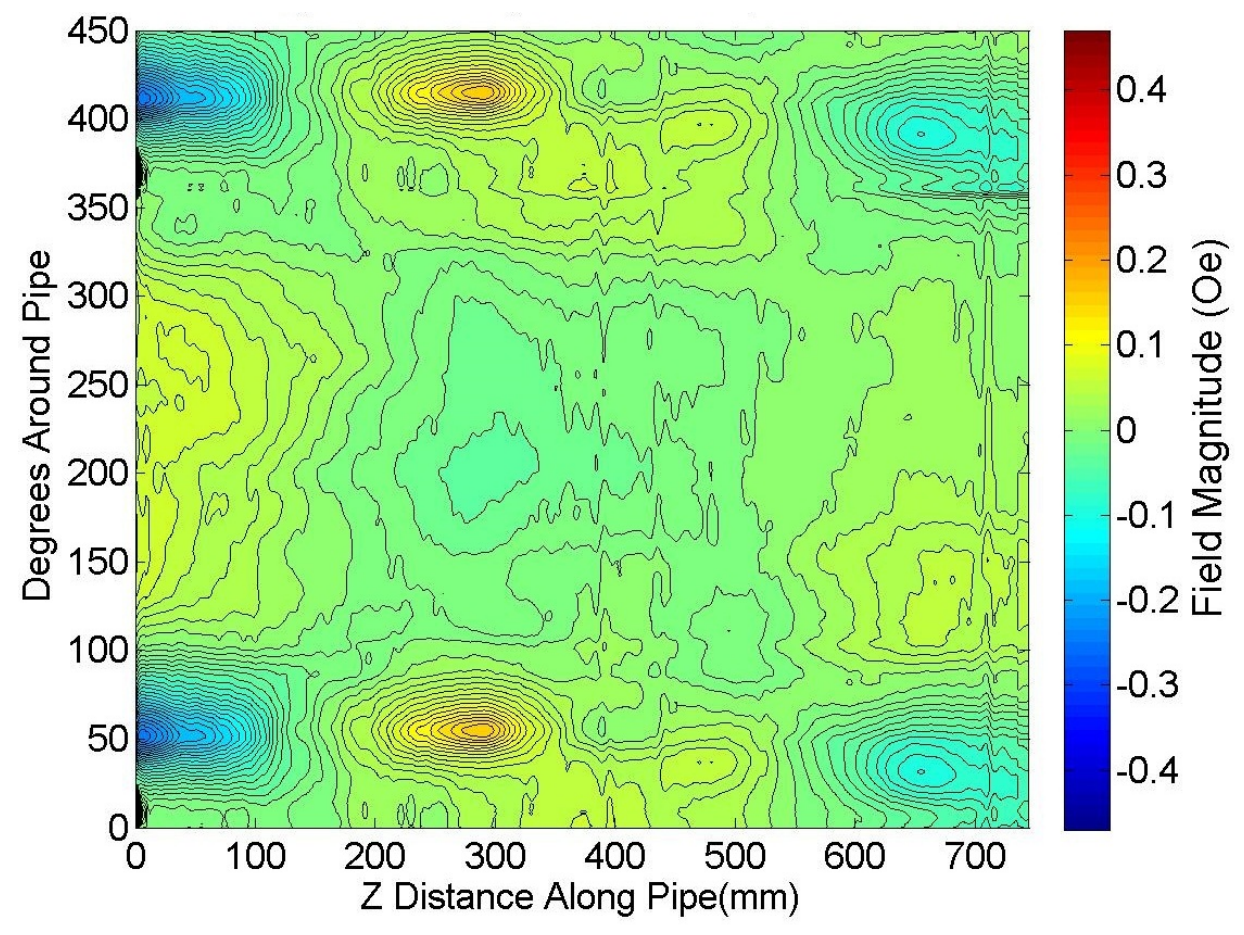

Figure 4.90: $\mathrm{R}$ field magnitude change over insulated pipe with $5 \mathrm{~Hz}$ excitation frequency

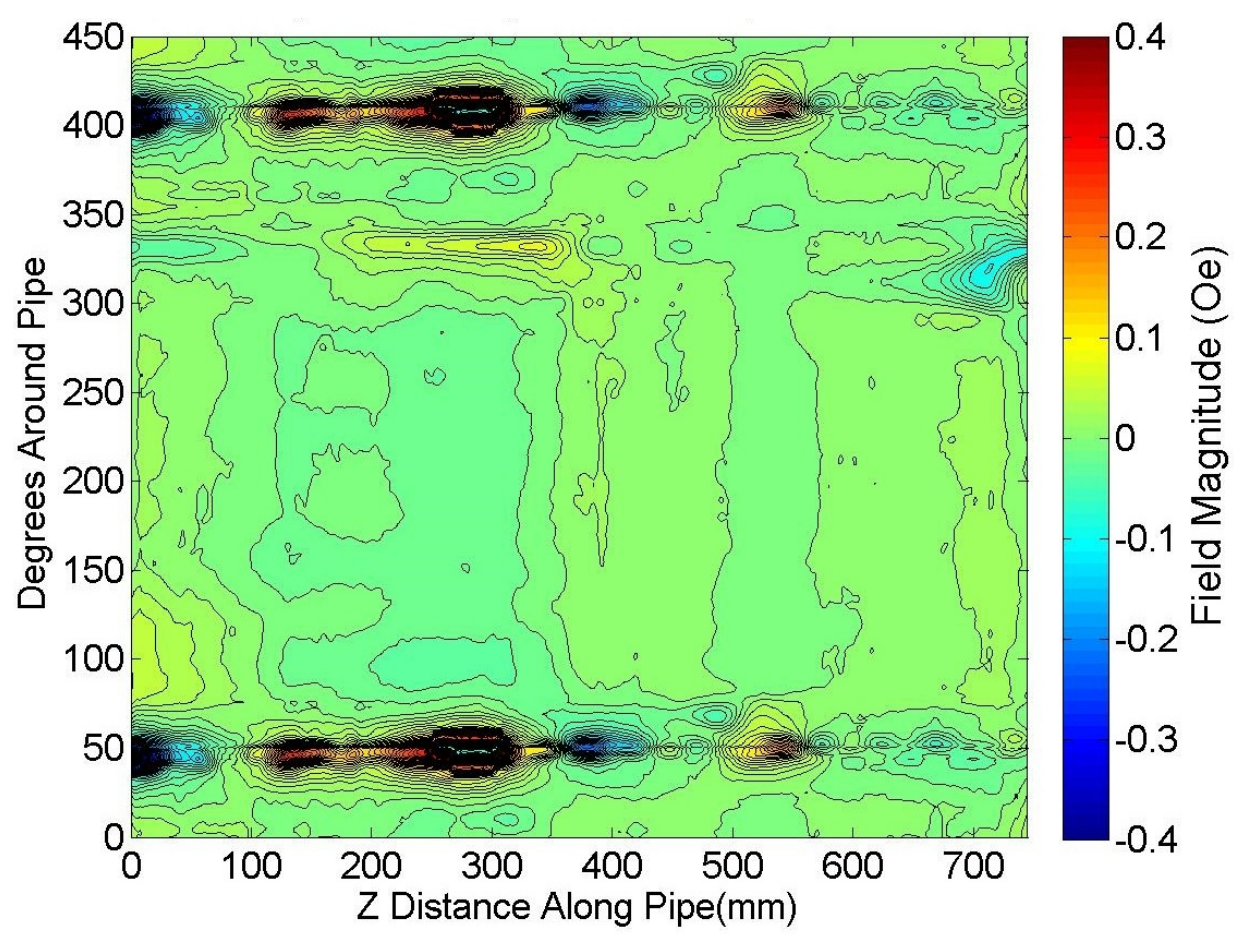

Figure 4.91: R field magnitude change over insulated pipe with $200 \mathrm{~Hz}$ excitation frequency 


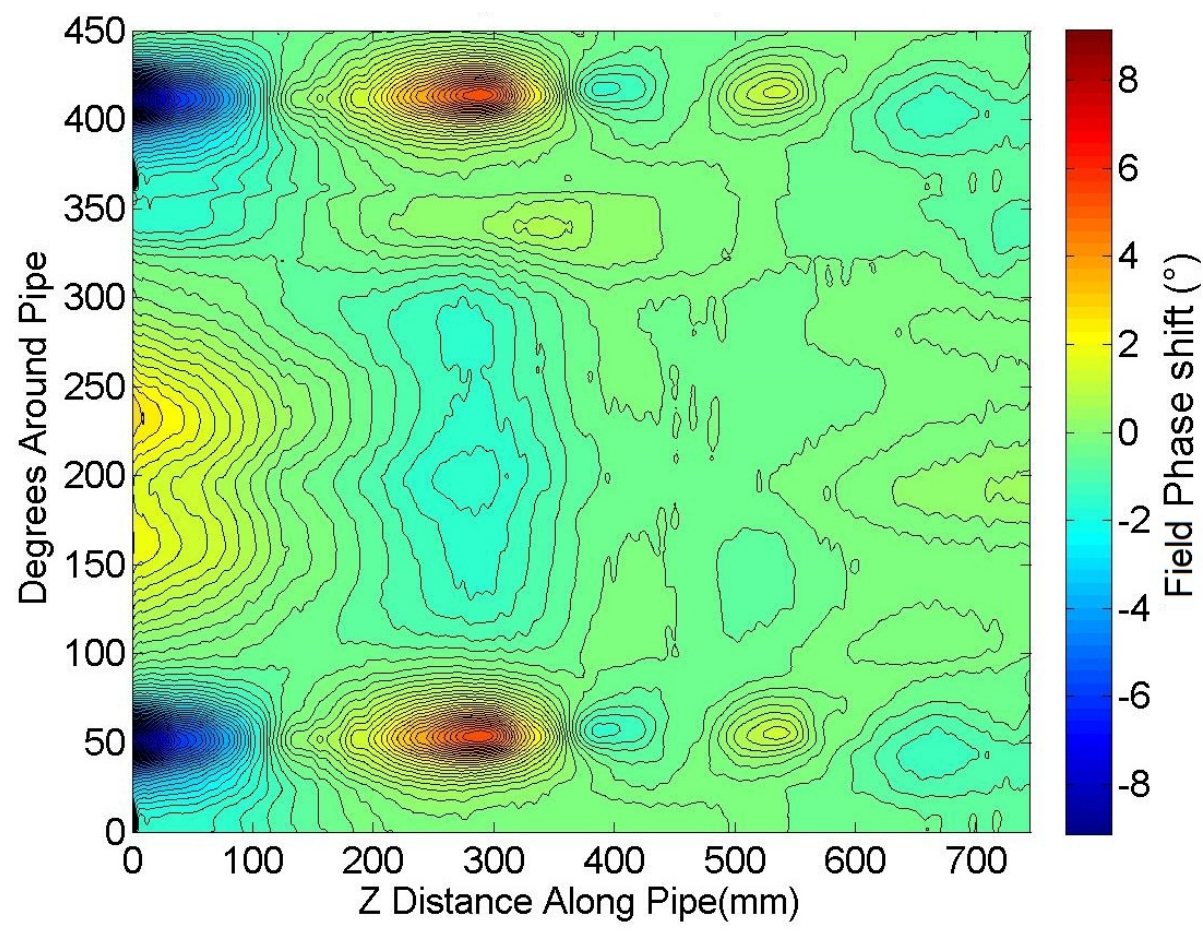

Figure 4.92: $\mathrm{R}$ field Phase shift change over insulated pipe with $5 \mathrm{~Hz}$ excitation frequency

and figure 4.91.

The contour plot for the Phi field magnitude at $5 \mathrm{~Hz}$ is shown in figure 4.94 and at 200 $\mathrm{Hz}$ in figure 4.95. The Phi magnitude shows pairs of positive and negative peaks at the same $Z$ location as each of the peaks in the $R$ field. Like the $R$ field magnitude, when the frequency is increased to $200 \mathrm{~Hz}$ the size of the peaks increases. New pairs of peaks can been seen at 340 degrees, which is the location of the buried edge of the aluminum shield.

The contour plot of the Phi field phase shift with $5 \mathrm{~Hz}$ excitation is shown in figure 4.96 and for $200 \mathrm{~Hz}$ excitation in figure 4.97. The $5 \mathrm{~Hz}$ plot shows the peaks in the same locations as the Phi field magnitude but larger compared to the background variation. When the frequency is increased to $200 \mathrm{~Hz}$ the peaks become larger and sharper.

\section{Background signal level and total noise}

The results from tests with the aluminum shield were processed in the same way as the results from the standard pipe. The background signal levels were assessed at each excitation frequency for each $R$ and Phi field magnitude and phase shift. The $R$ field magnitude is shown in figure 4.98 compared with the unshielded pipe. The aluminum shield reduced the magnetic field by about $25 \%$.

The background change in $\mathrm{R}$ sensor phase shift with frequency for shielded and unshielded pipe is shown in figure 4.99. This shows that there is no effect from the aluminum shield. The data at each measurement frequency is almost the same at all points with the same logarithmic curve fitting all the data.

The Phi sensor magnitude change with frequency for the shielded pipe is plotted in 


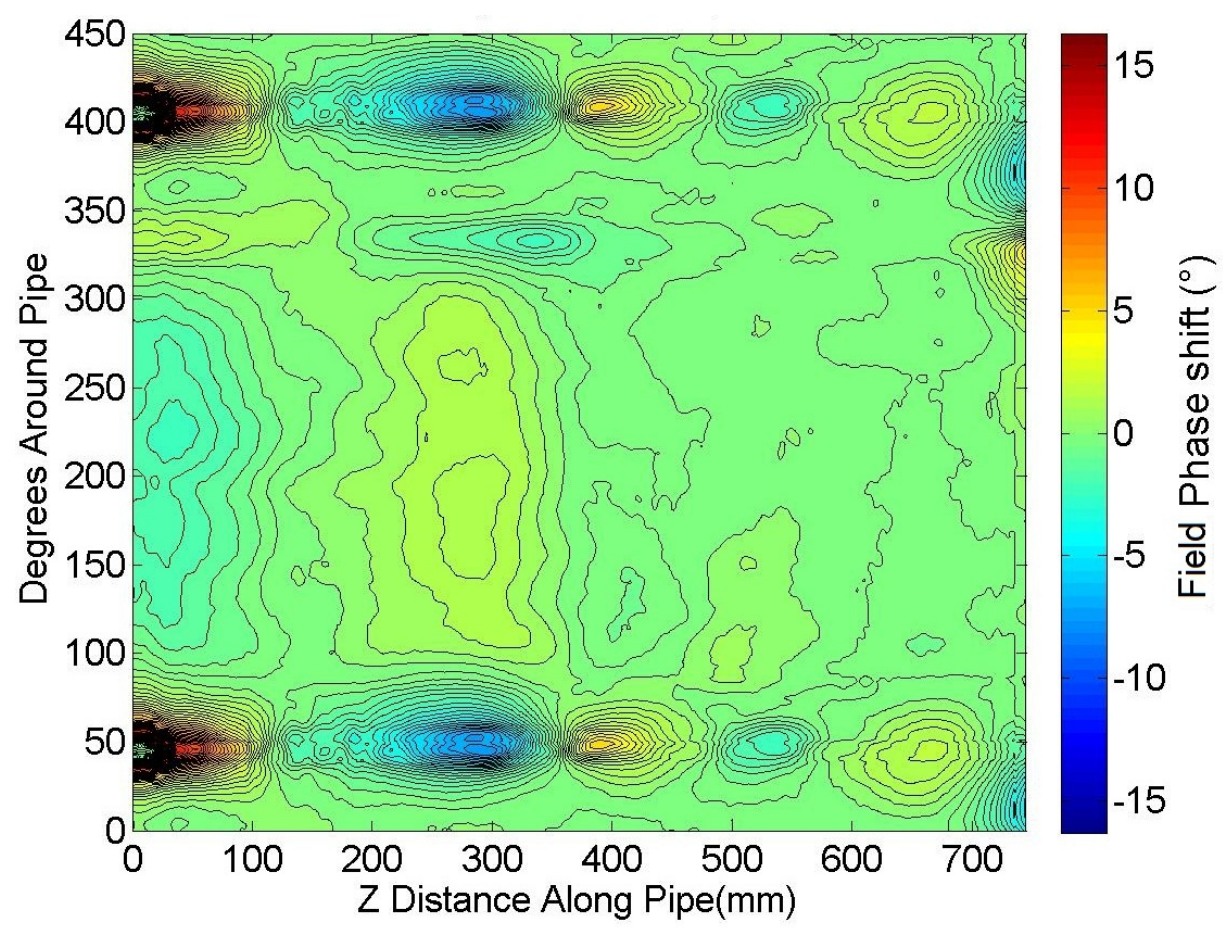

Figure 4.93: R field Phase shift change over insulated pipe with $200 \mathrm{~Hz}$ excitation frequency

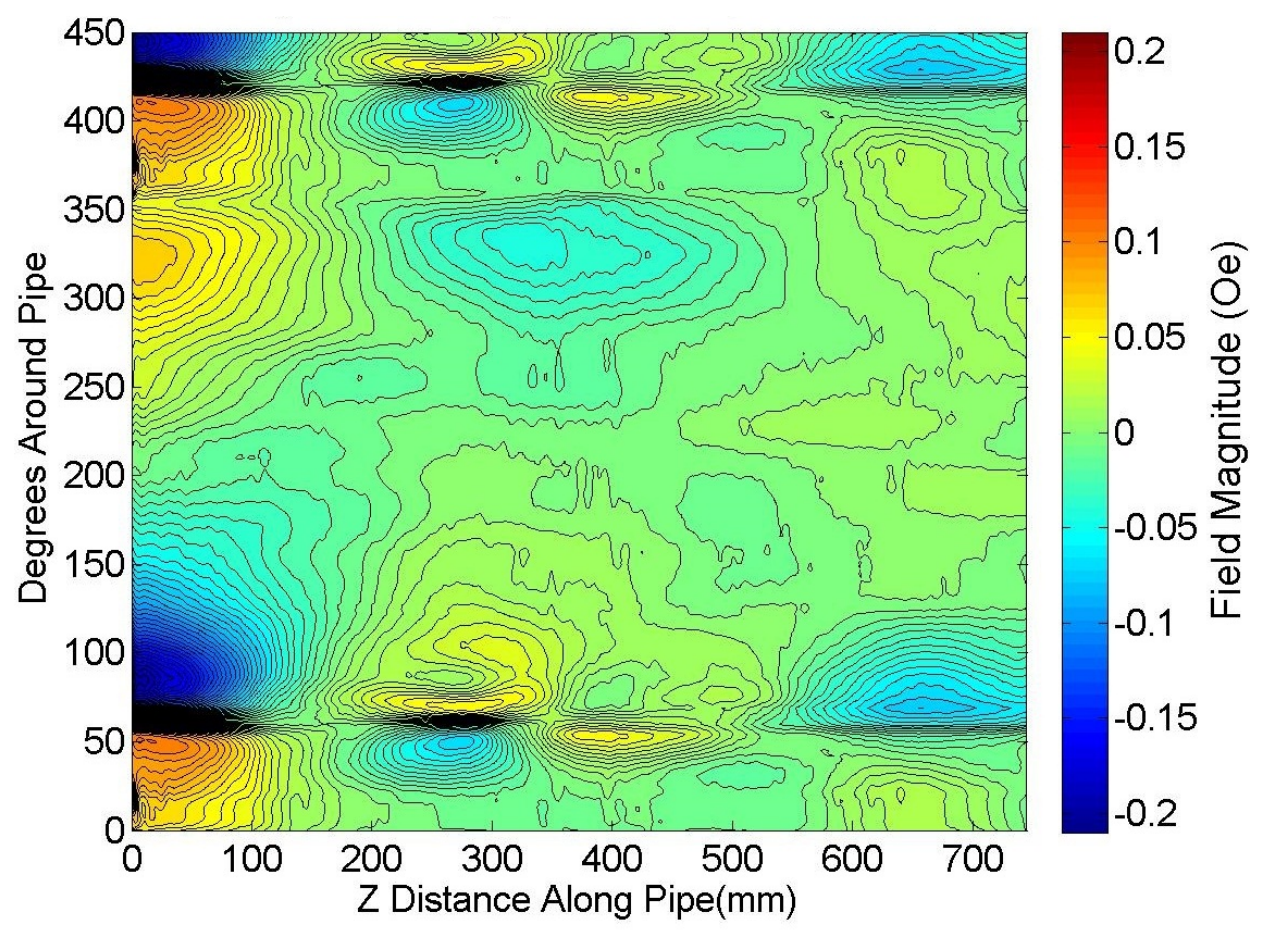

Figure 4.94: Phi field magnitude change over insulated pipe with $5 \mathrm{~Hz}$ excitation frequency 


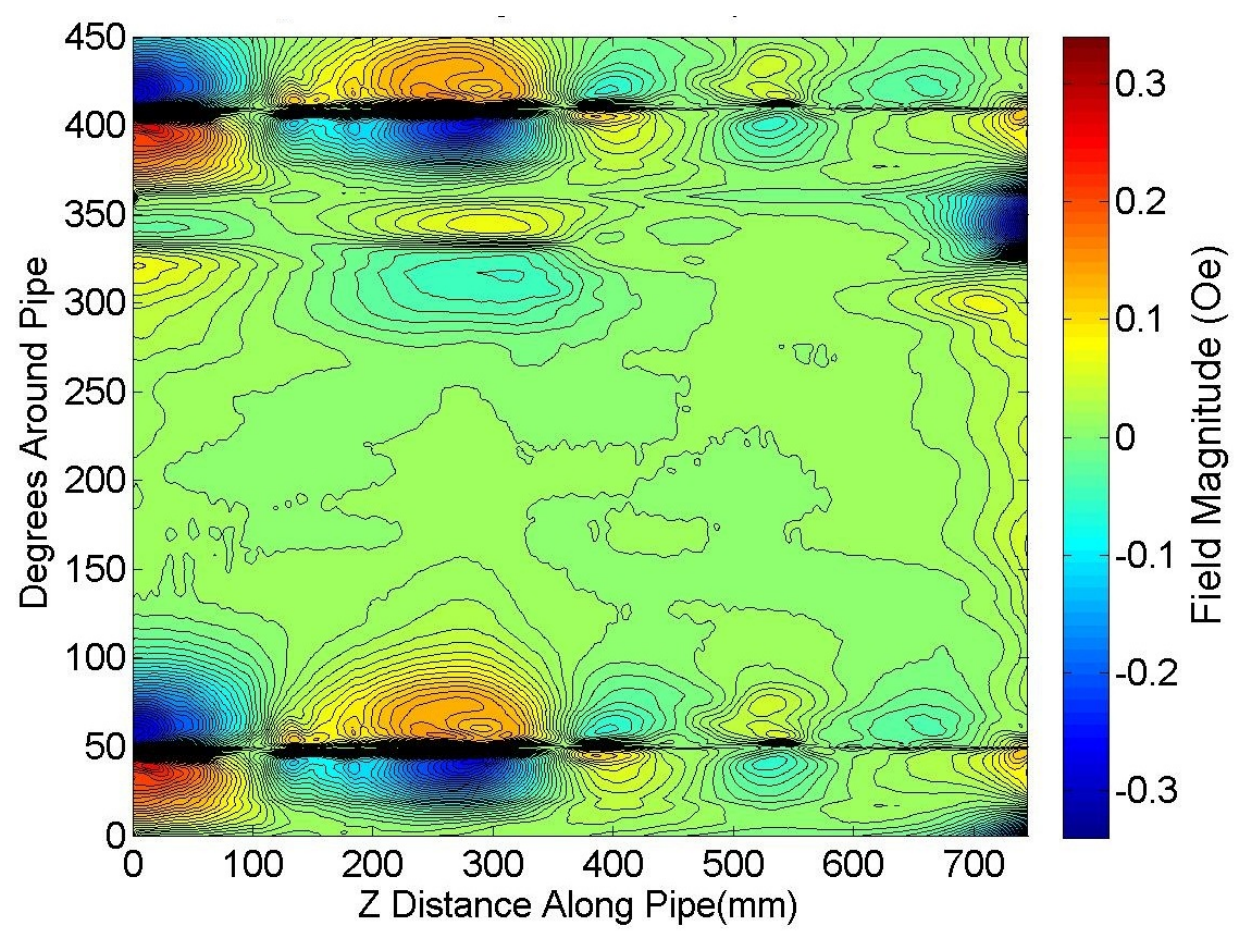

Figure 4.95: Phi field magnitude change over insulated pipe with $200 \mathrm{~Hz}$ excitation frequency

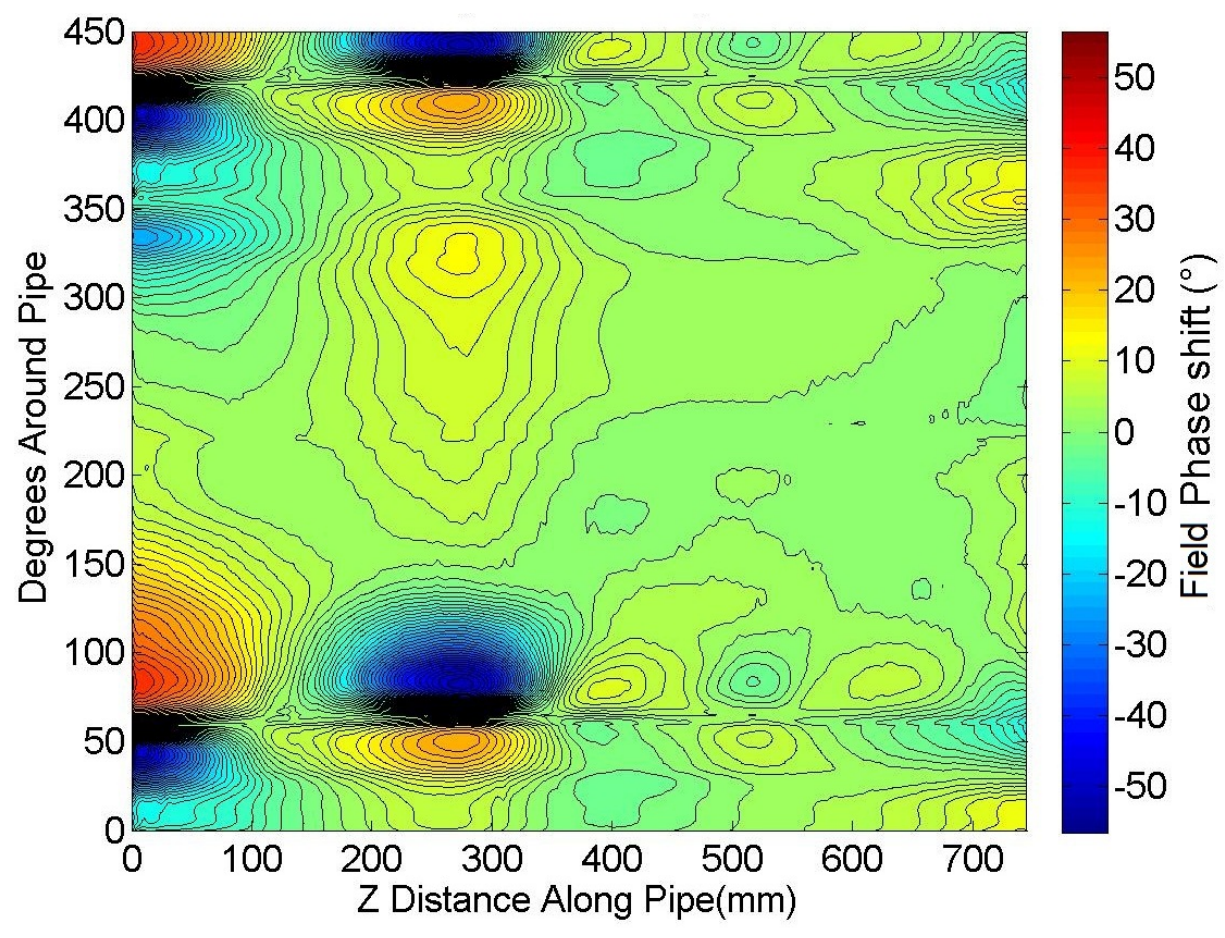

Figure 4.96: Phi field phase shift change over insulated pipe with $5 \mathrm{~Hz}$ excitation frequency 


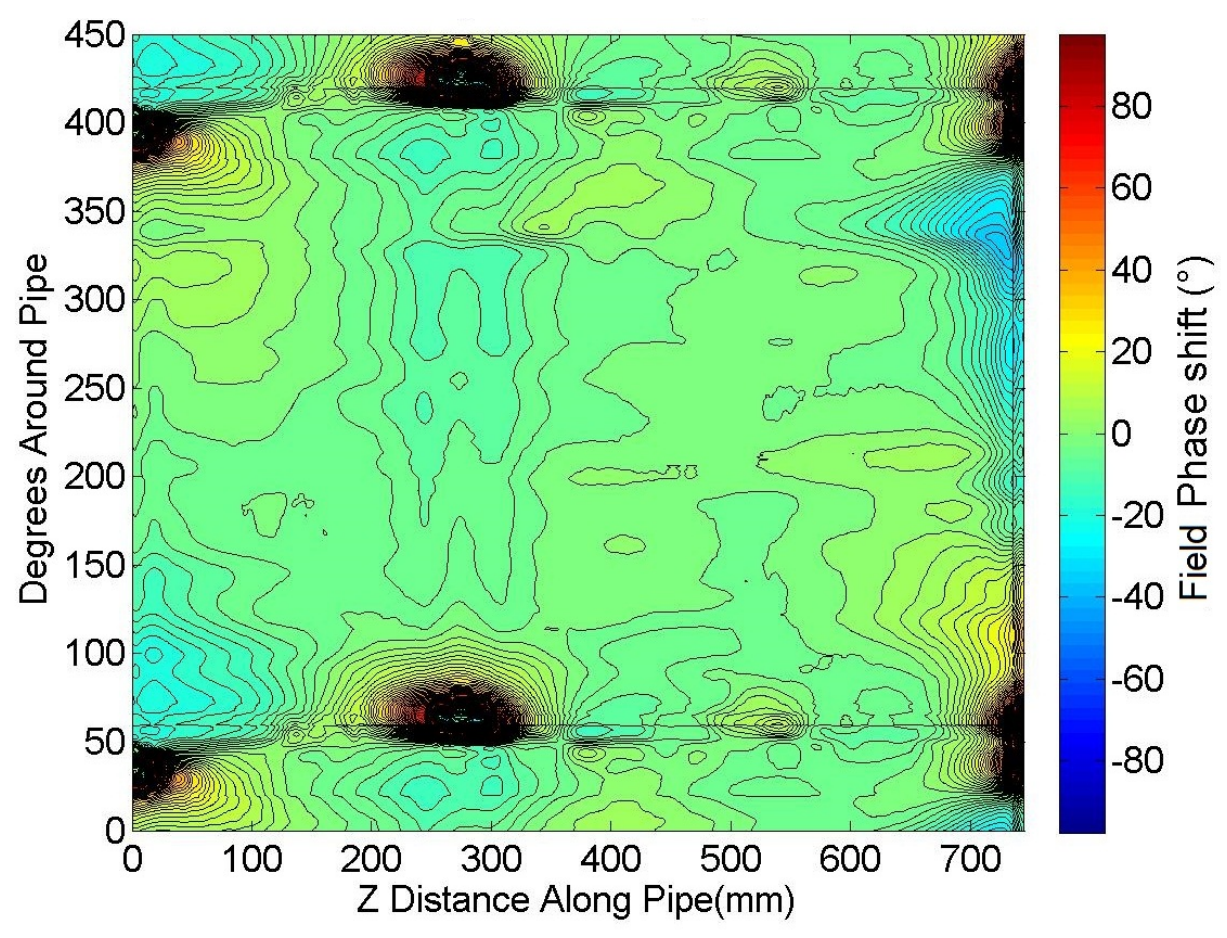

Figure 4.97: Phi field phase shift change over insulated pipe with $200 \mathrm{~Hz}$ excitation frequency

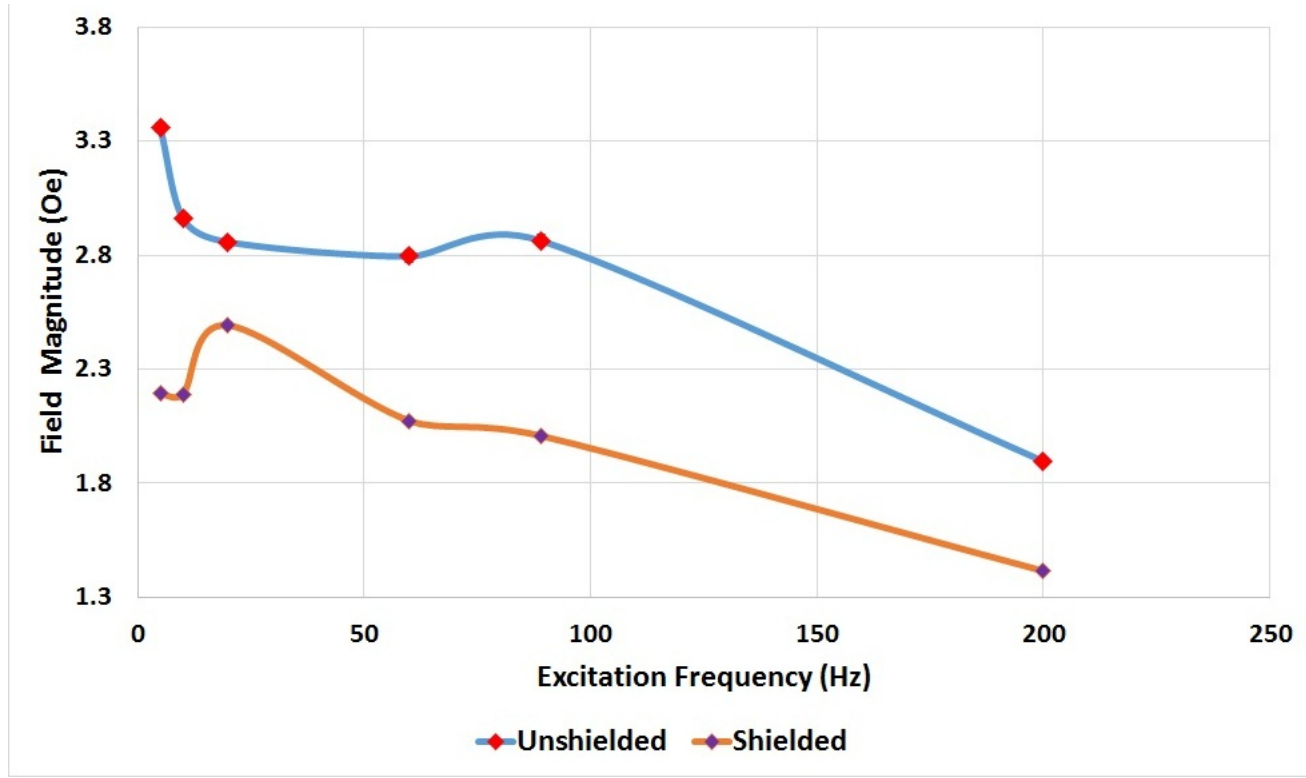

Figure 4.98: Comparison of $\mathrm{R}$ sensor median magnitude with changing excitation frequency for shielded and unshielded pipe(the blue and orange lines a provides a guide for the eye) 


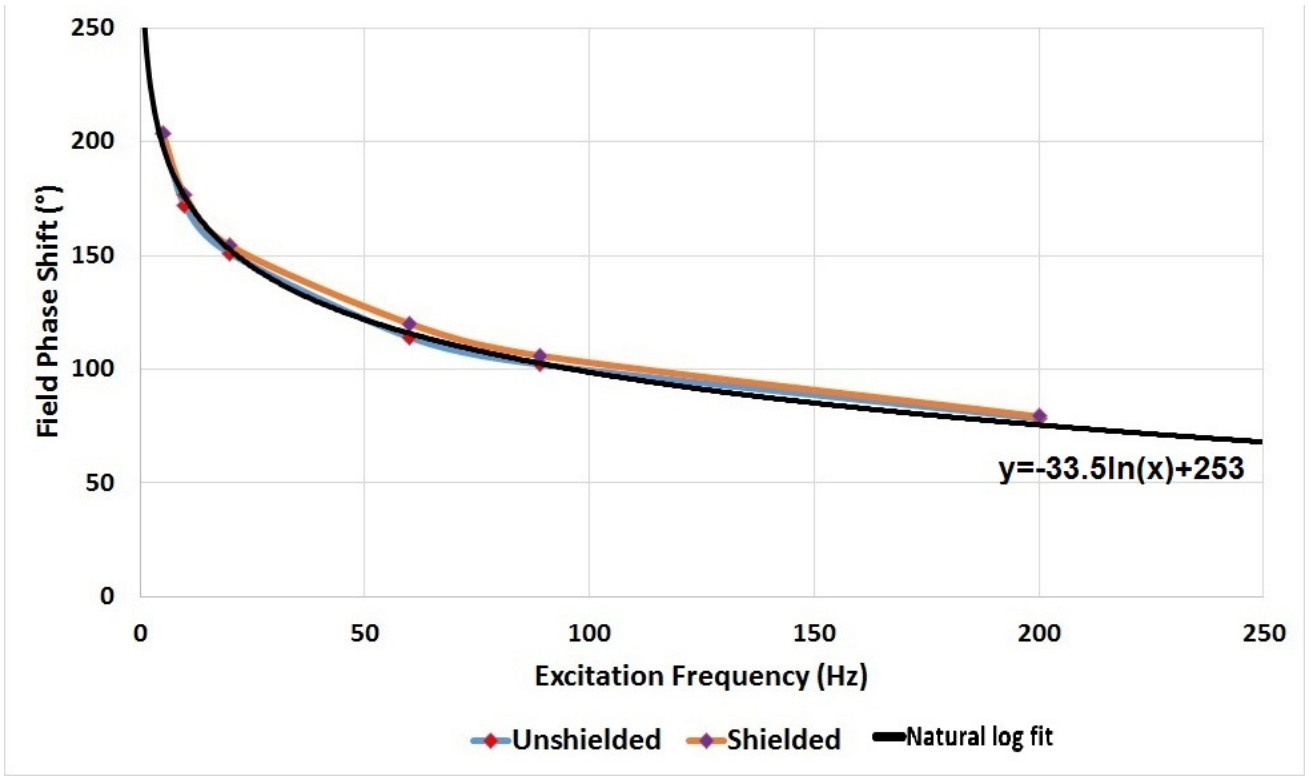

Figure 4.99: Comparison of $\mathrm{R}$ sensor median phase shift with changing excitation frequency for shielded and unshielded pipe

figure 4.100. At high excitation frequency the data has the same (approx $25 \%$ ) reduction in field as the $R$ field magnitude, however at low frequencies there is significant variation with the unshielded pipe showing less field at $20 \mathrm{~Hz}$ than the shielded pipe. As commented before, the Phi sensor is more sensitive to misalignments due to the small signal being measured. This small signal is due to the Phi sensor being easier to align than the $R$ sensor as its mounting allows for some adjustment to improve alignment. This results in less of the background field being measured.

The Phi sensor phase shift background change with excitation frequency is compared with the shielded and unshielded pipe as shown in figure 4.101. Here, like the R sensor phase shift, there is no noticeable effect from the introduction of an aluminum shield. The phase shift is still the same at each excitation frequency with the same logarithmic decay.

To understand how the aluminum shield affects the noise of each measurement, the SNR for the magnitudes is plotted and compared with the unshielded data in figure 4.102 for the $\mathrm{R}$ sensor and in figure 4.103 for the Phi sensor. Both the $\mathrm{R}$ and Phi sensor magnitude SNRs are reduced with an aluminum shield. There is a clear and rapid increase in SNR as the frequency drops below $20 \mathrm{~Hz}$.

To look at the noise change for phase shift over unshielded and shielded pipe at increasing frequencies, the standard deviation is plotted in figure 4.104 for the $\mathrm{R}$ sensor and figure 4.105 for the Phi sensor. The R field phase shift has a significant increase in standard deviation with the aluminum shield. This still appears to have a general upwards trend with increasing frequency. The Phi field phase shift shows a massive standard deviation from $20 \mathrm{~Hz}$ to $89 \mathrm{~Hz}$ suggesting there is a phase reversal occurring multiple times during these tests. 


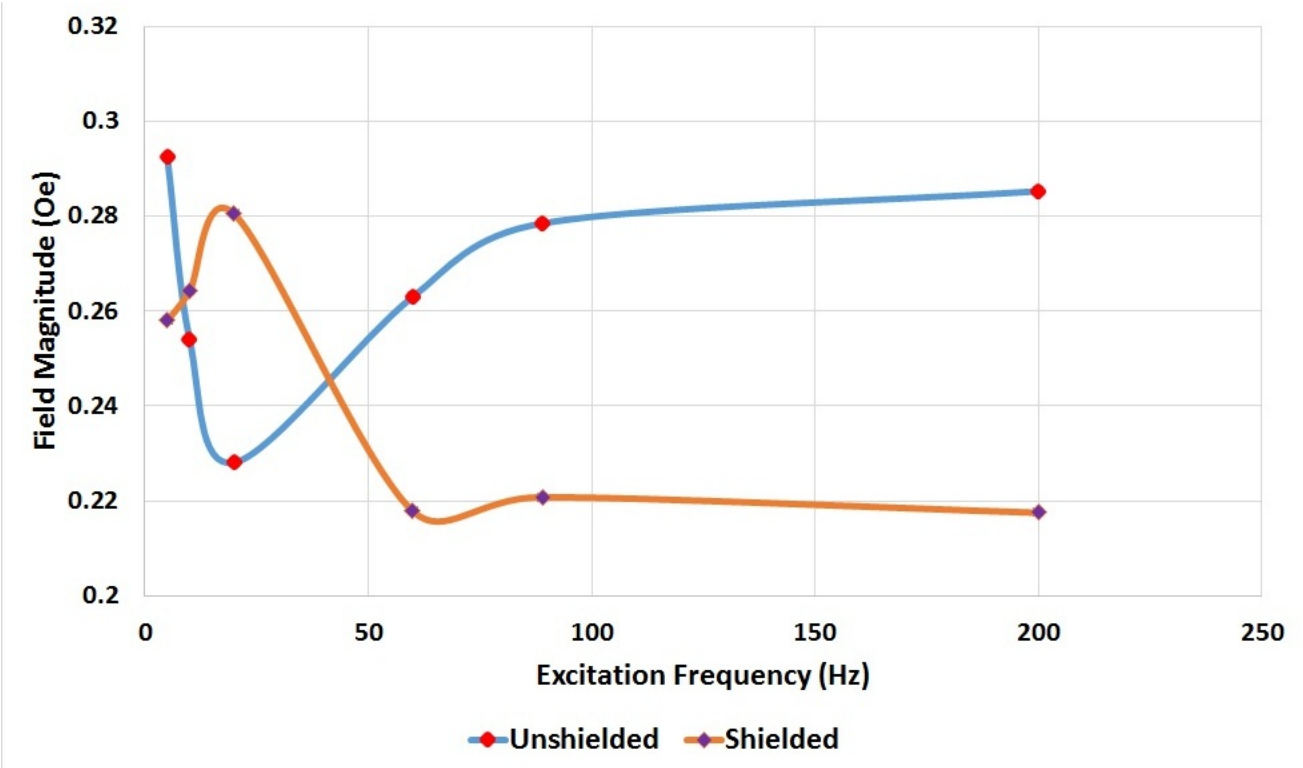

Figure 4.100: Comparison of Phi sensor median magnitude change with frequency with and without aluminum shield(the blue and orange lines a provides a guide for the eye)

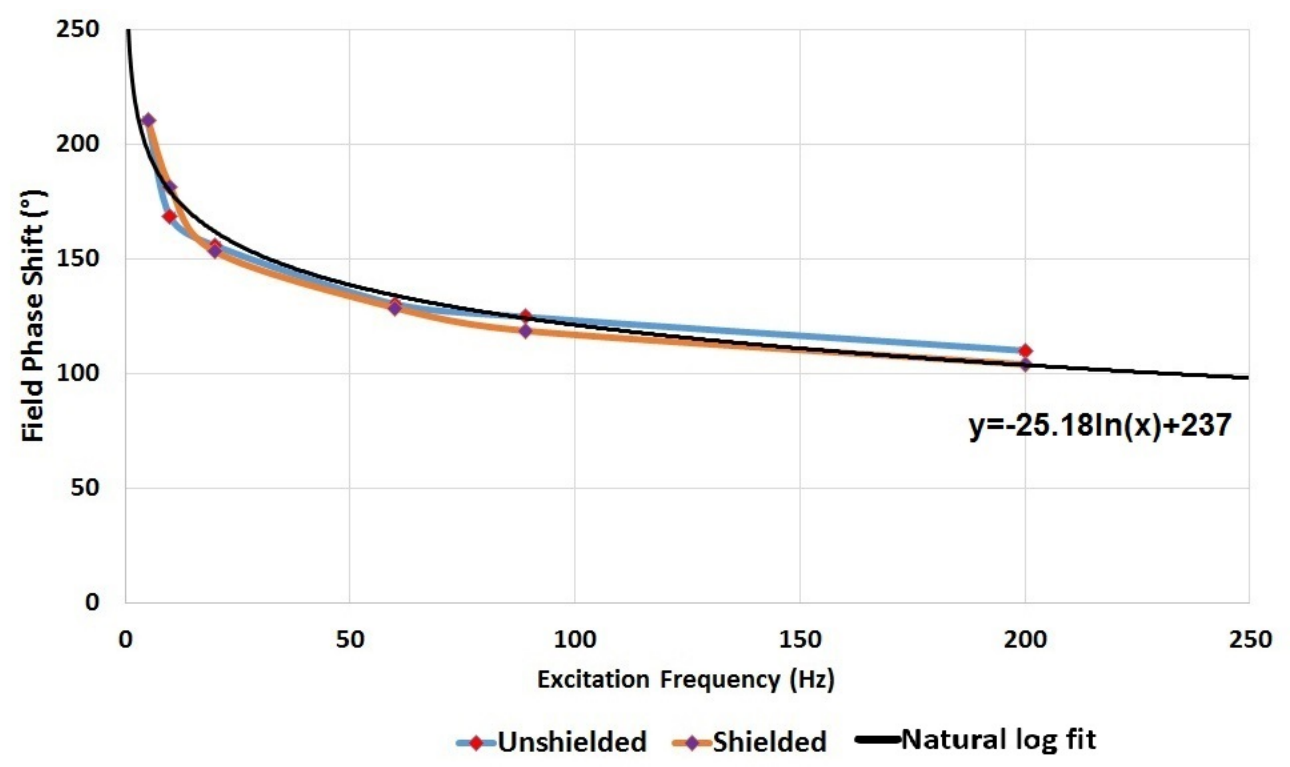

Figure 4.101: Comparison of the Phi sensor median phase shift change with excitation frequency with shielded and unshielded pipe(the blue and orange lines a provides a guide for the eye) 


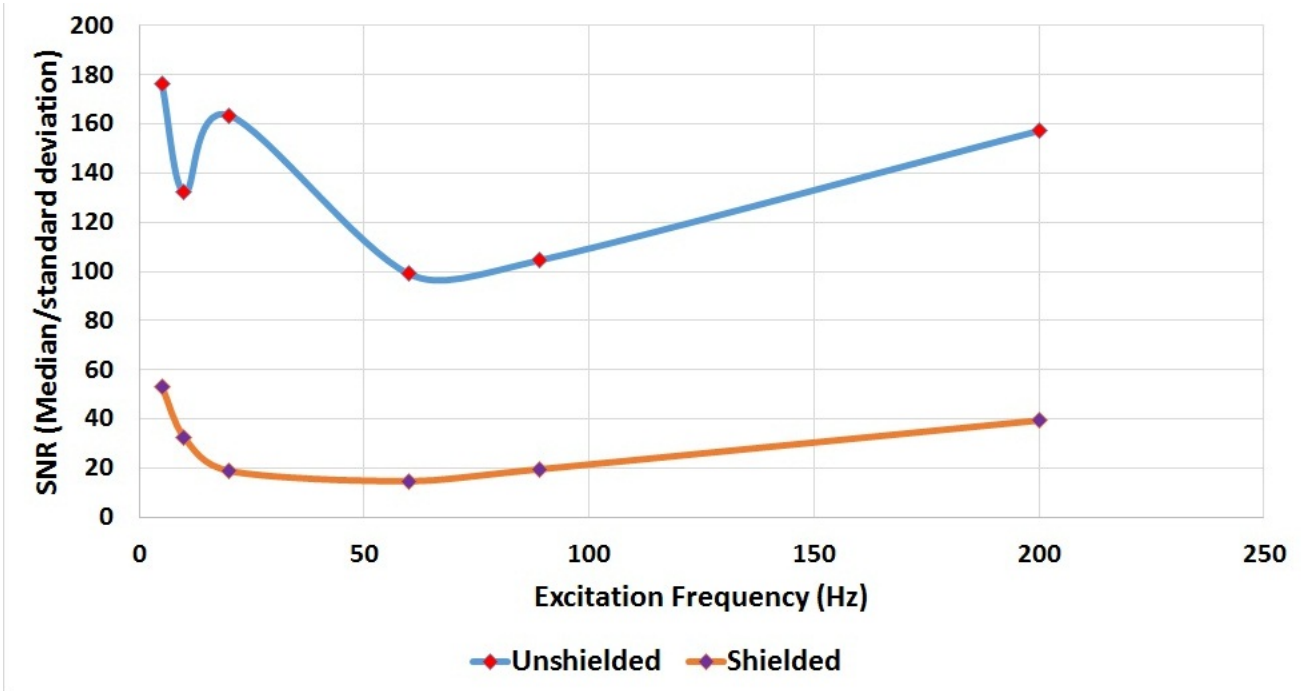

Figure 4.102: $R$ sensor SNR change with excitation frequency comparison between shielded and unshielded pipe(the blue and orange lines a provides a guide for the eye)

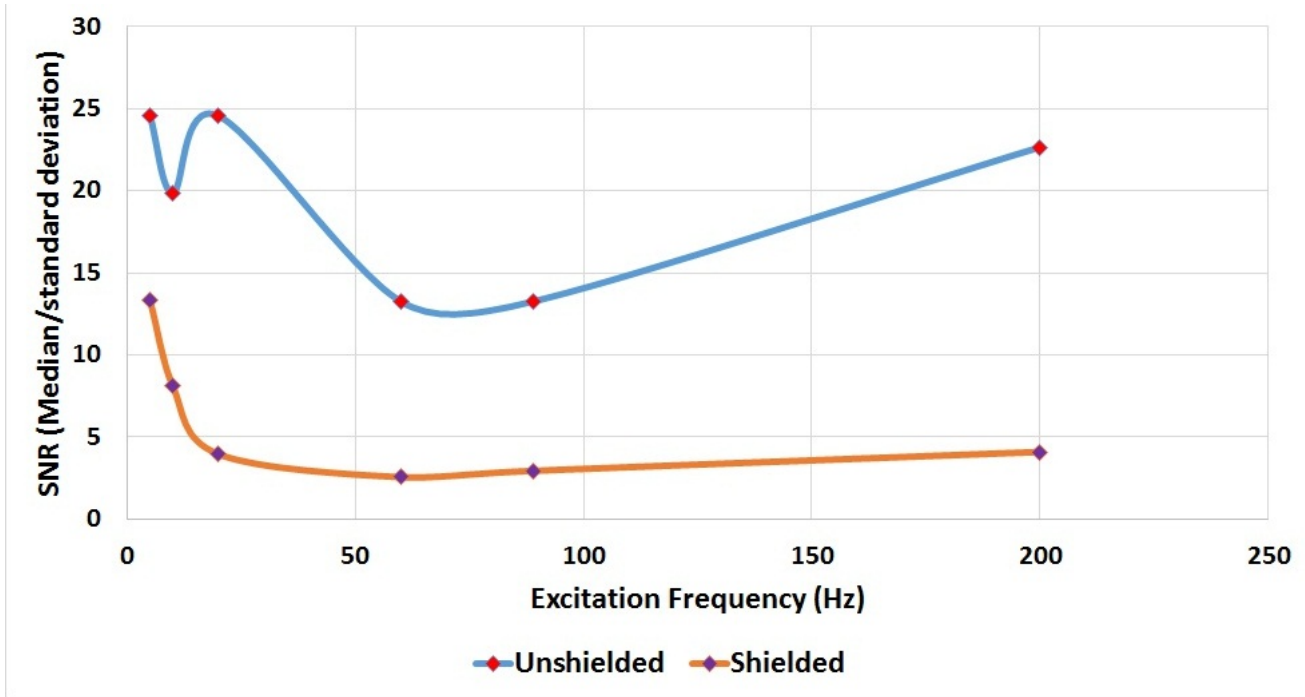

Figure 4.103: Phi sensor SNR change with excitation frequency comparison between shielded and unshielded pipe(the blue and orange lines a provides a guide for the eye) 


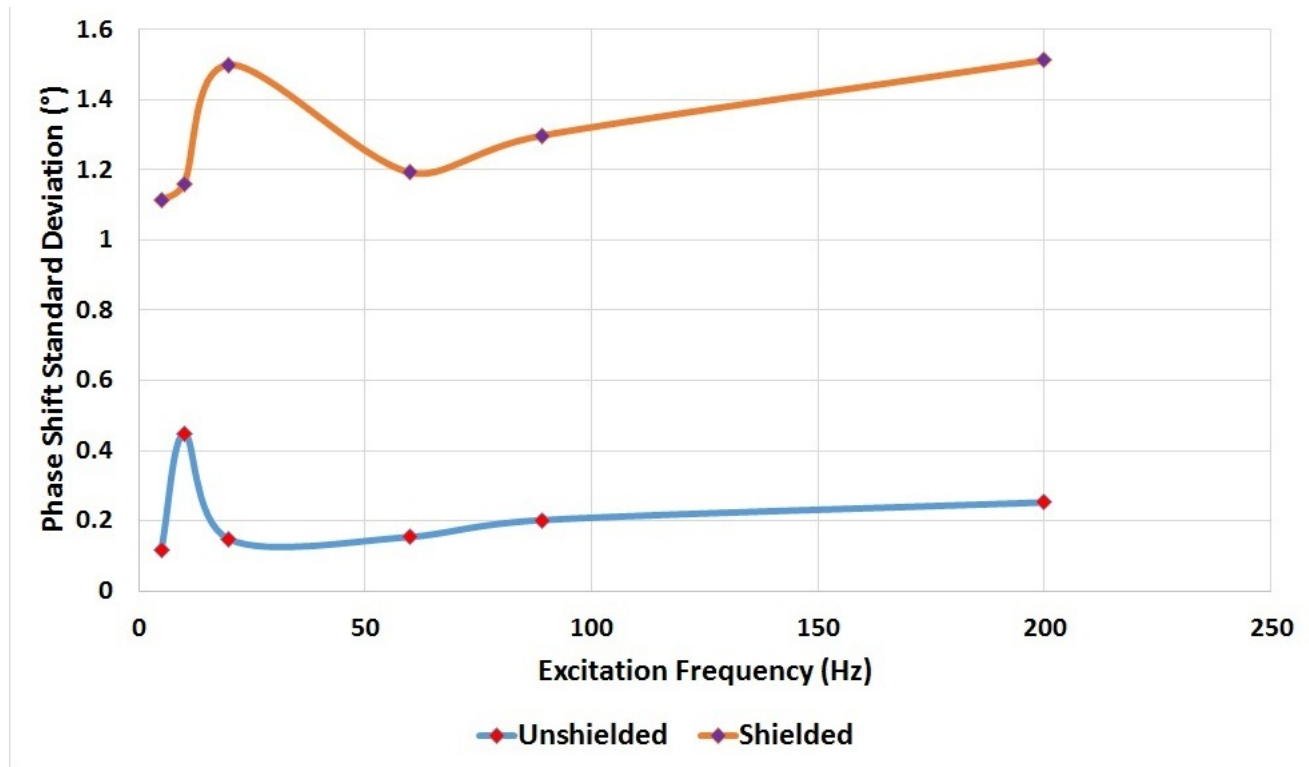

Figure 4.104: $R$ sensor phase shift standard deviation change with frequency over shielded and unshielded pipe(the blue and orange lines a provides a guide for the eye)

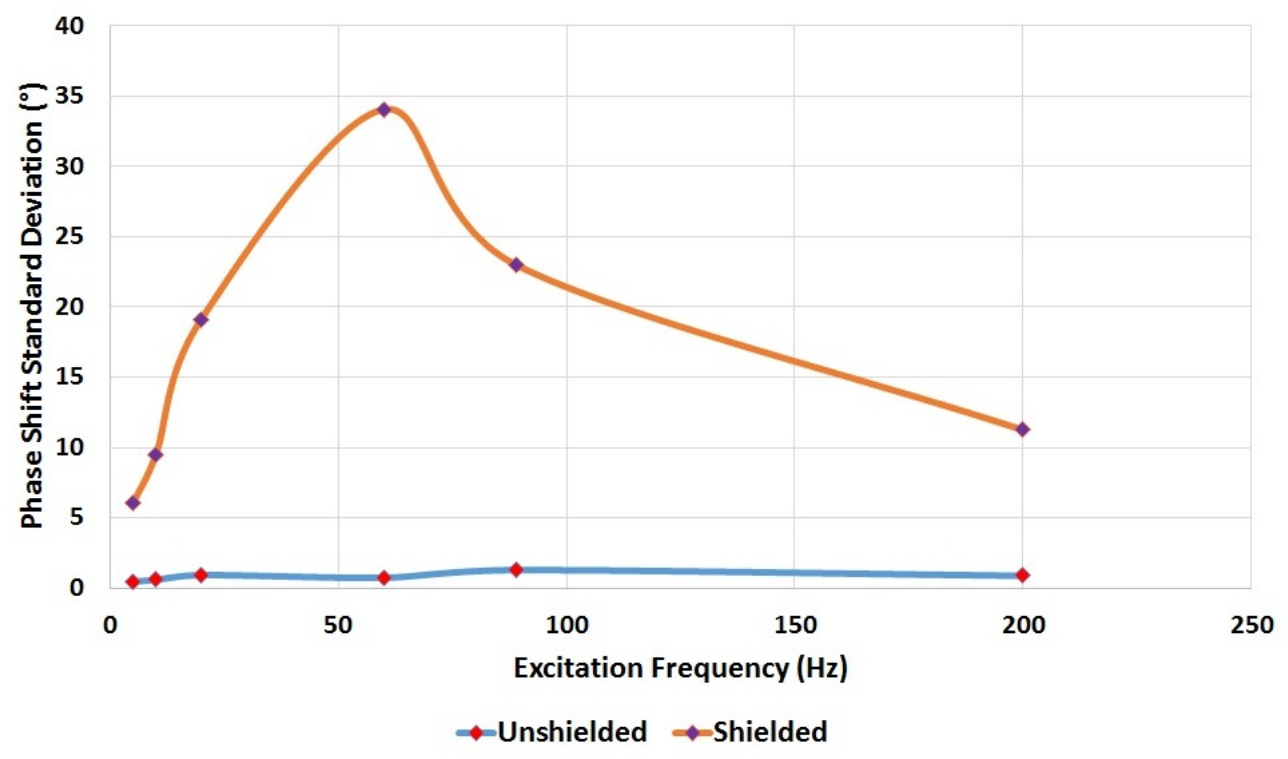

Figure 4.105: Phi sensor phase shift standard deviation change with frequency over shielded and unshielded pipe(the blue and orange lines a provides a guide for the eye) 


\subsection{Summary of effects of aluminum shield}

\section{Aluminum shield features}

Figure 4.87 shows a qualitative model of how the eddy current in the steel and aluminum shield would be expected to change with excitation frequency. The majority of the eddy current still flows in the steel pipe wall until high frequencies are reached. This is because the aluminum shield is very thin compared to the pipe wall. The eddy currents flowing in the aluminum shield are fairly constant across all frequencies measured because at 200 $\mathrm{Hz}$ the skin depth is 12 times the thickness of the aluminum. This means that the decay in current density from the skin effect is negligible for the aluminum, resulting in little change in current in the aluminum across the frequencies of interest.

The contour plots of the $\mathrm{R}$ and Phi field magnitude and phase shift at $5 \mathrm{~Hz}$ and 200 $\mathrm{Hz}$ shows that $\mathrm{R}$ field magnitude at $5 \mathrm{~Hz}$ has the best immunity to effects caused by the aluminum shield. This is expected, as the skin depth for aluminum shield at $5 \mathrm{~Hz}$ is $36 \mathrm{~mm}$ (equation 2.4) whereas at $200 \mathrm{~Hz}$ the skin depth is $6 \mathrm{~mm}$. This means the ratio between the eddy current in the steel pipe and the eddy current in the aluminum shield is maximized. This can be seen in figure 4.87 by comparing, at each frequency, the area in which the eddy current flows in the steel pipe to the area in the aluminum shield. This means that a variation caused by distortion of the eddy currents in the aluminum shield will only have a small effect on the magnetic field.

The contour plots of the $\mathrm{R}$ field magnitude and phase shows pairs of positive and negative peaks at points along the joint between the top and bottom layers of the aluminum shield. The origin of these peaks are areas with poor conductivity due to the connection between the two aluminum sheets warping or having a low clamping force. These areas can be thought of as defects, as the eddy current is distorted to flow around the areas of high resistance. As predicted in the qualitative model in section 2.5.2, redirection of eddy currents produces a pair of positive and negative peaks in the $\mathrm{R}$ field.

The contour plots of the Phi field magnitude and phase show a pair of positive and negative peaks centered at each of the peak locations in the $R$ field. This is the same pattern predicted for a defect in the quantitative model in section 2.5.2. At higher frequencies there are some peaks appearing at the buried end of the joint. As the frequency increases the skin depth of the aluminum decreases, so a greater portion of the eddy currents flow in the top layer of the aluminum joint until the joint ends and the current is forced to transition to the bottom layer.

\section{Background signal level}

The general effect of adding an aluminum shield to the $\mathrm{R}$ and Phi field magnitude was to reduce the background field by around $25 \%$. However, the Phi field reduction is not as clear as that of the $\mathrm{R}$ field, since not all values showed reduction. The reduction in field is due to eddy currents flowing in the aluminum shield, creating a field which opposes the excitation field as shown in figure 4.87. This is in addition to the opposing field created by eddy currents in the pipe. This reduction of the main field means that the component of the main field measured, due to mis-alignment, also drops and this is the major contributor to the field measurements. 
The phase shift of the background shows no change with the addition of a aluminum shield, as the background phase shift is a measure of the phase shift of the excitation field. The phase shift depends on the impedance of the excitation coil, as described in section 4.7 and the addition of aluminum with a permeability of approximately 1 has no effect on the impedance of the coil.

\section{Magnitude SNR}

The SNR for both the R and Phi field magnitude shows a significant decrease with the addition of an aluminum shield. This is expected as the eddy current in the aluminum shield, when disturbed, produces a new strong source of noise because it is much closer to the sensor. With the addition of the aluminum shield there is a clear rapid increase with low excitation frequencies for both $\mathrm{R}$ and Phi fields. This is a result of the large skin depth at low frequency, which means that there is significantly more eddy current flowing in the pipe compared to the aluminum shield. The exponential nature of the skin depth means that there is a rapid increase in SNR at the low frequencies.

\section{Phase shift standard deviation}

As expected, the $\mathrm{R}$ field phase shift standard deviation increases with the addition of an aluminum shield for the same reason the magnitude SNR drops. However, the Phi field phase shift standard deviation has a very large increase. The contour plot of the $60 \mathrm{~Hz}$ data, where the biggest standard deviation is found, is shown in figure 4.106. Peaks produced by the aluminum shield are a maximum of 50 degrees at $5 \mathrm{~Hz}$ and 80 degrees at $200 \mathrm{~Hz}$. However, at $60 \mathrm{~Hz}$ a phase reversal occurs in these peaks with 180 degree phase shift. As a result of these peaks there is a significant increase in the standard deviation.

\subsection{Effects of aluminum dents}

The aluminum shielding on the pipe is thin so it can become dented easily in an industry setting. This denting is expected to change the magnetic field as the eddy currents in the aluminum shield are forced to change direction. To understand how denting may affect the magnetic field, dents were introduced into the pipe. A summary of these dents is provided in table 4.4 and photo of dent 6 is shown in figure 4.108 and of dent 4 in figure 4.107.

\subsubsection{Effect of excitation frequency on features introduced by dents in shielding}

The same tests that were done on the pipe with a smooth aluminum shield are repeated for the pipe with a dented aluminum shield. Figure 4.109 shows a contour plot of the $\mathrm{R}$ field magnitude with $200 \mathrm{~Hz}$ excitation current. This shows the greatest effect of the aluminum dents on the magnetic field. When compared to figure 4.91, it can be seen that pairs of positive and negative peaks produced by the dents have a similar magnitude as the peaks generated by the shield joint. However, these peaks are much broader. 


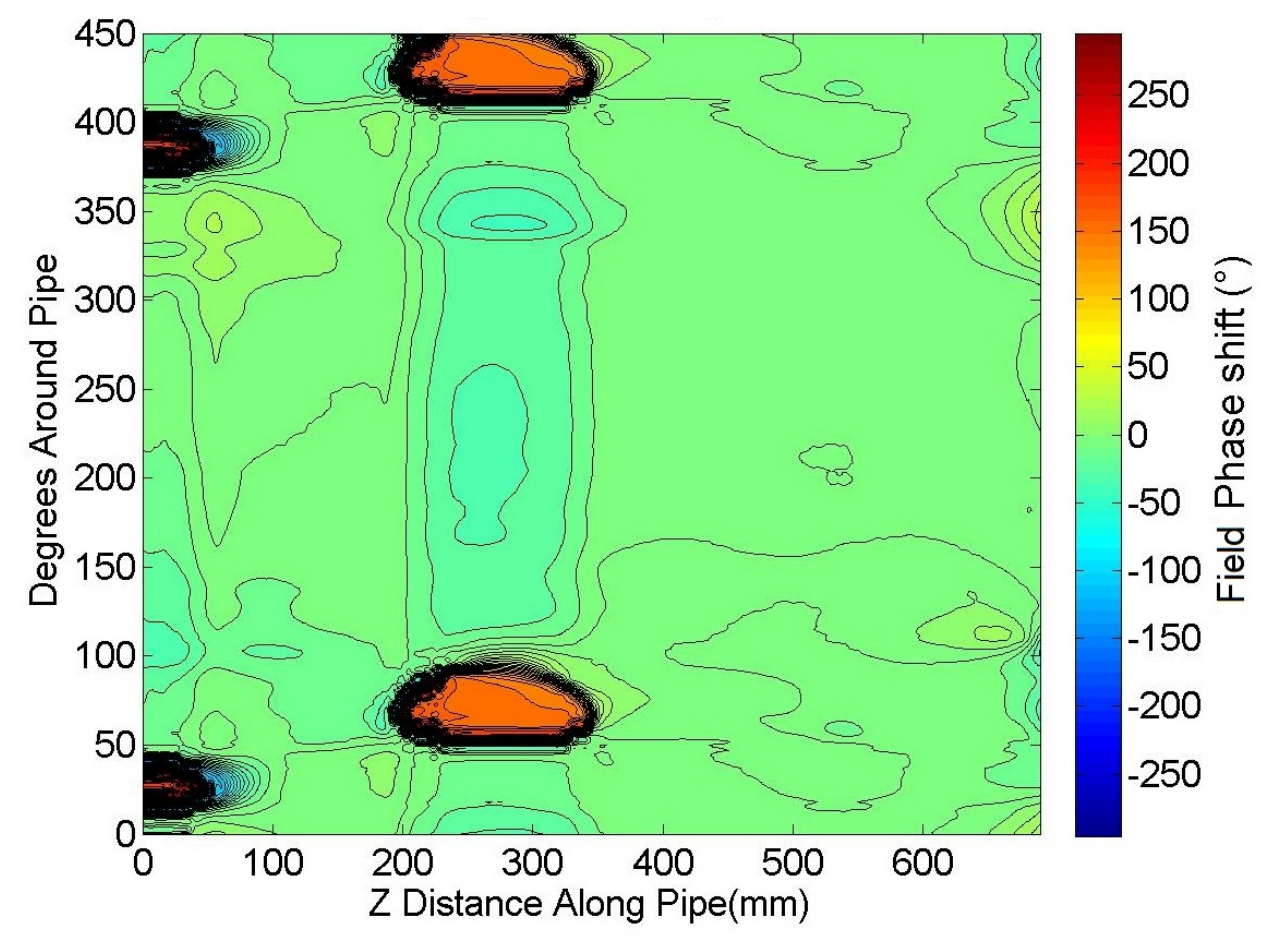

Figure 4.106: Phi field phase shift with $60 \mathrm{~Hz}$ excitation current over insulated pipe

Table 4.4: Description of dents in aluminum shield

\begin{tabular}{|c|c|c|c|c|c|c|}
\hline $\begin{array}{c}\text { Dent } \\
\text { number }\end{array}$ & Shape & $\begin{array}{l}Z \text { edge } \\
1(\mathrm{~mm})\end{array}$ & $\begin{array}{l}Z \text { edge } \\
2(\mathrm{~mm})\end{array}$ & $\begin{array}{l}\text { Phi } \\
\text { edge } 1 \\
\left(^{\circ}\right)\end{array}$ & $\begin{array}{l}\text { Phi } \\
\text { edge } 2 \\
\left(^{\circ}\right)\end{array}$ & $\begin{array}{c}\text { Depth } \\
(\mathrm{mm})\end{array}$ \\
\hline 1 & Ellipsoid & 60 & 260 & 330 & 20 & 4.5 \\
\hline 2 & Circular & 400 & 500 & 55 & 110 & 5 \\
\hline 3 & Circular & -60 & 80 & 85 & 140 & 10.5 \\
\hline 4 & Ellipsoid & 250 & 420 & 130 & 180 & 2.5 \\
\hline 5 & $\begin{array}{l}\text { Narrow on Phi } \\
\text { axis }\end{array}$ & 340 & 560 & 220 & 250 & 7 \\
\hline 6 & $\begin{array}{c}\text { Narrow rotated } \\
45 \text { degree from } \\
\text { Z axis }\end{array}$ & 115 & -10 & 210 & 250 & 5.5 \\
\hline
\end{tabular}




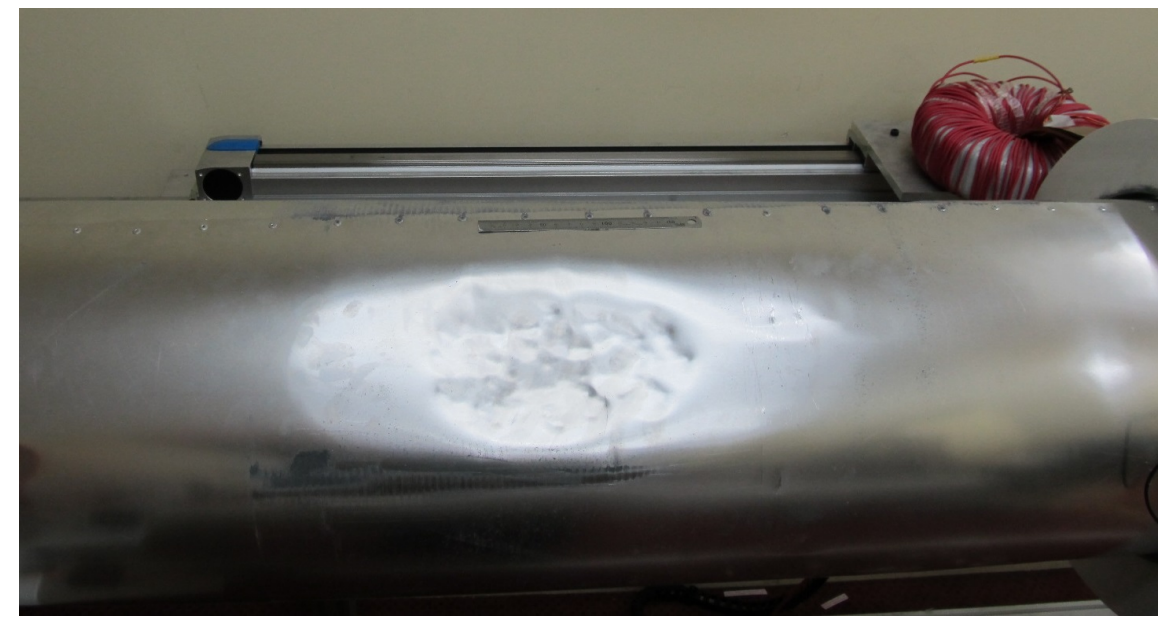

Figure 4.107: Photo of dent 4 in aluminum shield

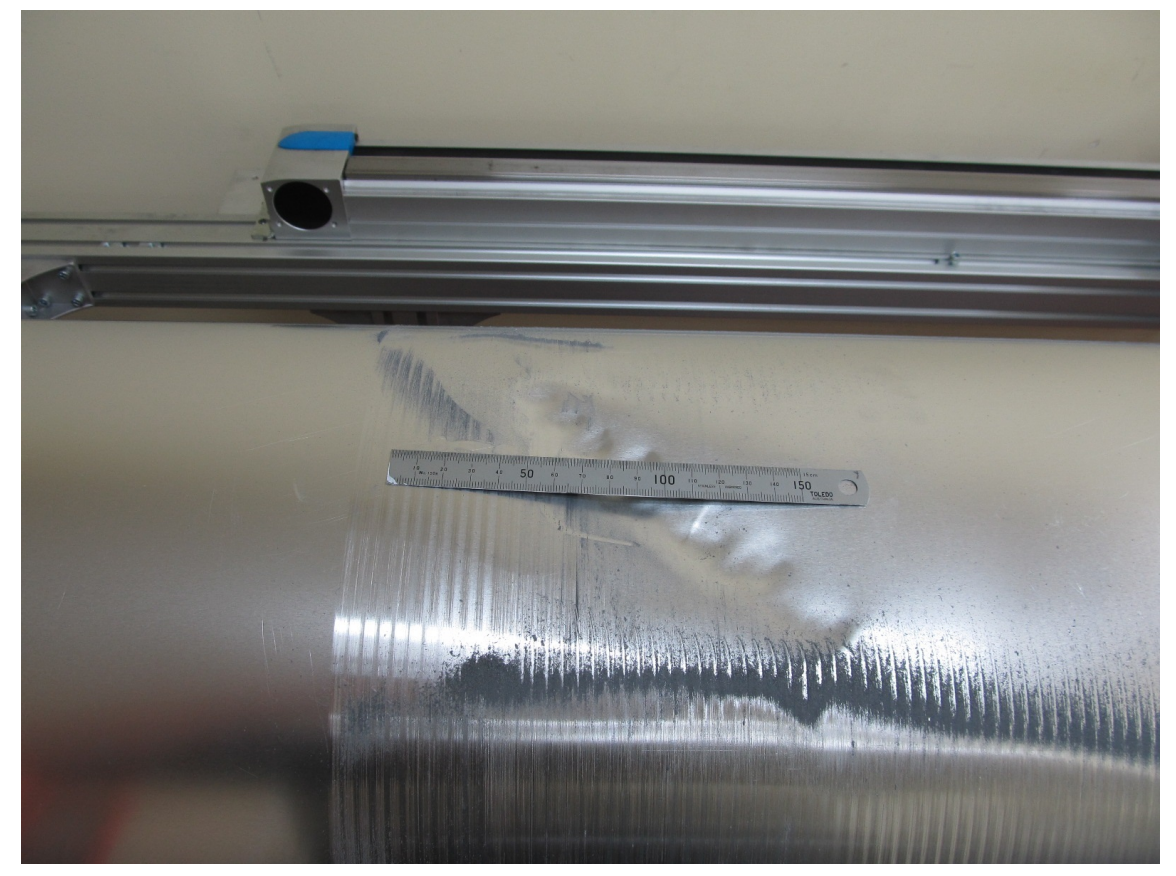

Figure 4.108: Photo of dent 6 in aluminum shield 


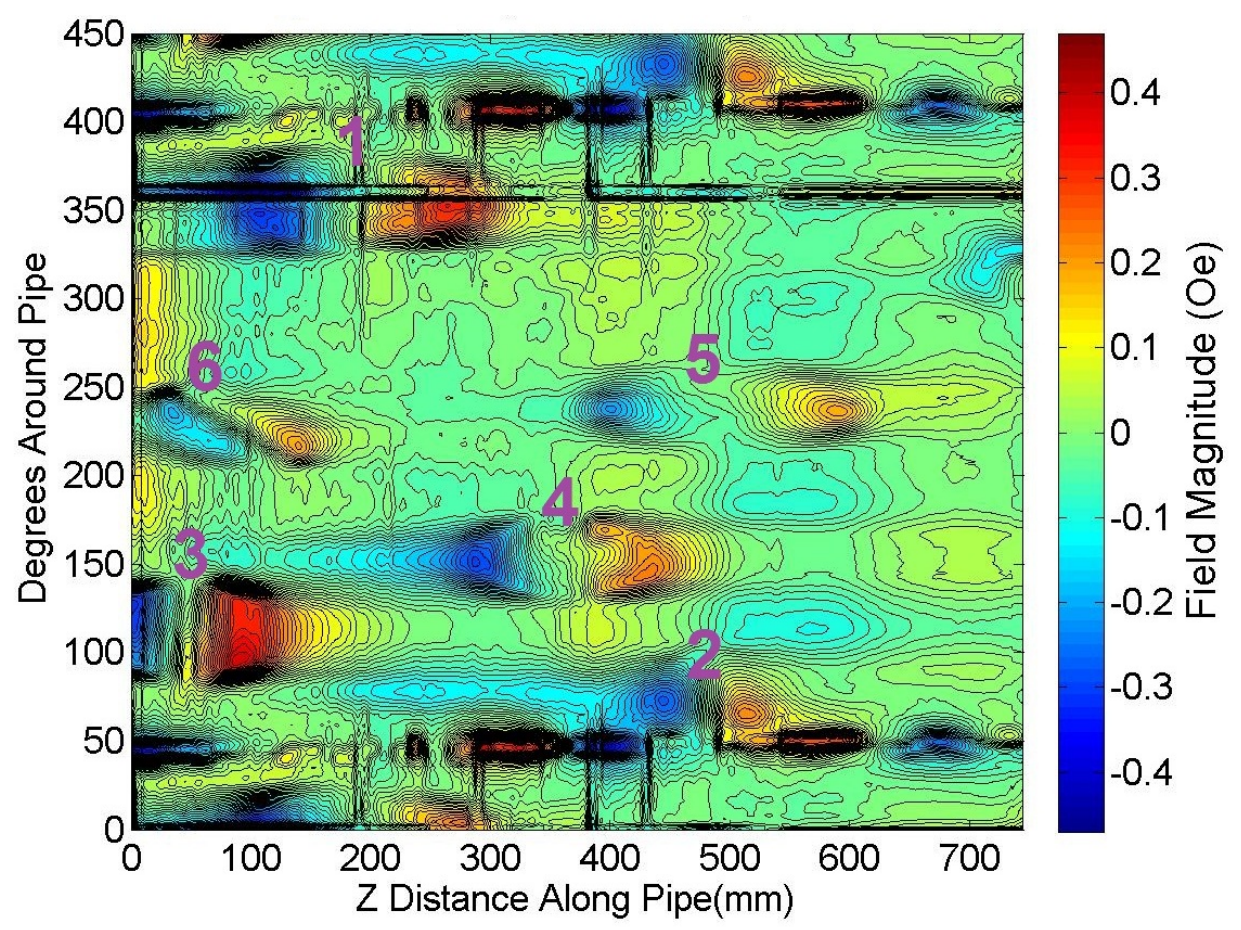

Figure 4.109: $R$ field magnitude over dented aluminum shield wrapped pipe with $200 \mathrm{~Hz}$ excitation current

How these peaks, created by the dented aluminum, changed with excitation frequency is assessed. Also assessed was whether a multi-frequency correction algorithm could be used to remove their effects. Contour plots at $5 \mathrm{~Hz}$ and at $200 \mathrm{~Hz}$ for the $\mathrm{R}$ and Phi sensor phase and magnitude were drawn. The $\mathrm{R}$ field magnitude at $5 \mathrm{~Hz}$ is shown in figure 4.110 and at $200 \mathrm{~Hz}$ in figure 4.109

The $5 \mathrm{~Hz}$ data shows a noisy background and no clear defect signals from the dented aluminum while the $200 \mathrm{~Hz}$ data shows clear peaks at each aluminum dent location. The contour plot of the Phi field magnitude at $5 \mathrm{~Hz}$ is shown in figure 4.111 and at $200 \mathrm{~Hz}$ in figure 4.112. Like the $\mathrm{R}$ field magnitude, the $5 \mathrm{~Hz}$ data shows no defect signals from the dented aluminum. The $200 \mathrm{~Hz}$ data shows clear defect signals for most of the dents, however they are not as large as the $\mathrm{R}$ field magnitude signals.

The contour plot of the $\mathrm{R}$ field phase shift at $5 \mathrm{~Hz}$ is shown in figure 4.113 and at 200 $\mathrm{Hz}$ in figure 4.114. At both frequencies little effect is seen from denting the aluminum, only some weak defect signals appearing in both frequencies.

The contour plot of the Phi field phase shift at $5 \mathrm{~Hz}$ is shown in figure 4.115 and at 200 $\mathrm{Hz}$ in figure 4.116. The phase shift at $5 \mathrm{~Hz}$ excitation current is dominated by the peaks along the joint in the shield with just one weak defect signal starting to appear. However, at $200 \mathrm{~Hz}$ excitation frequency there are some clear defect signals appearing which almost overlap with each other as the dents are close together.

To compare tests with dented shield to tests without shield and with dent free shield, the median value at each frequency was calculated and then plotted with the results from the unshielded pipe and the dent free shielded pipe. The $\mathrm{R}$ field magnitude medians are 


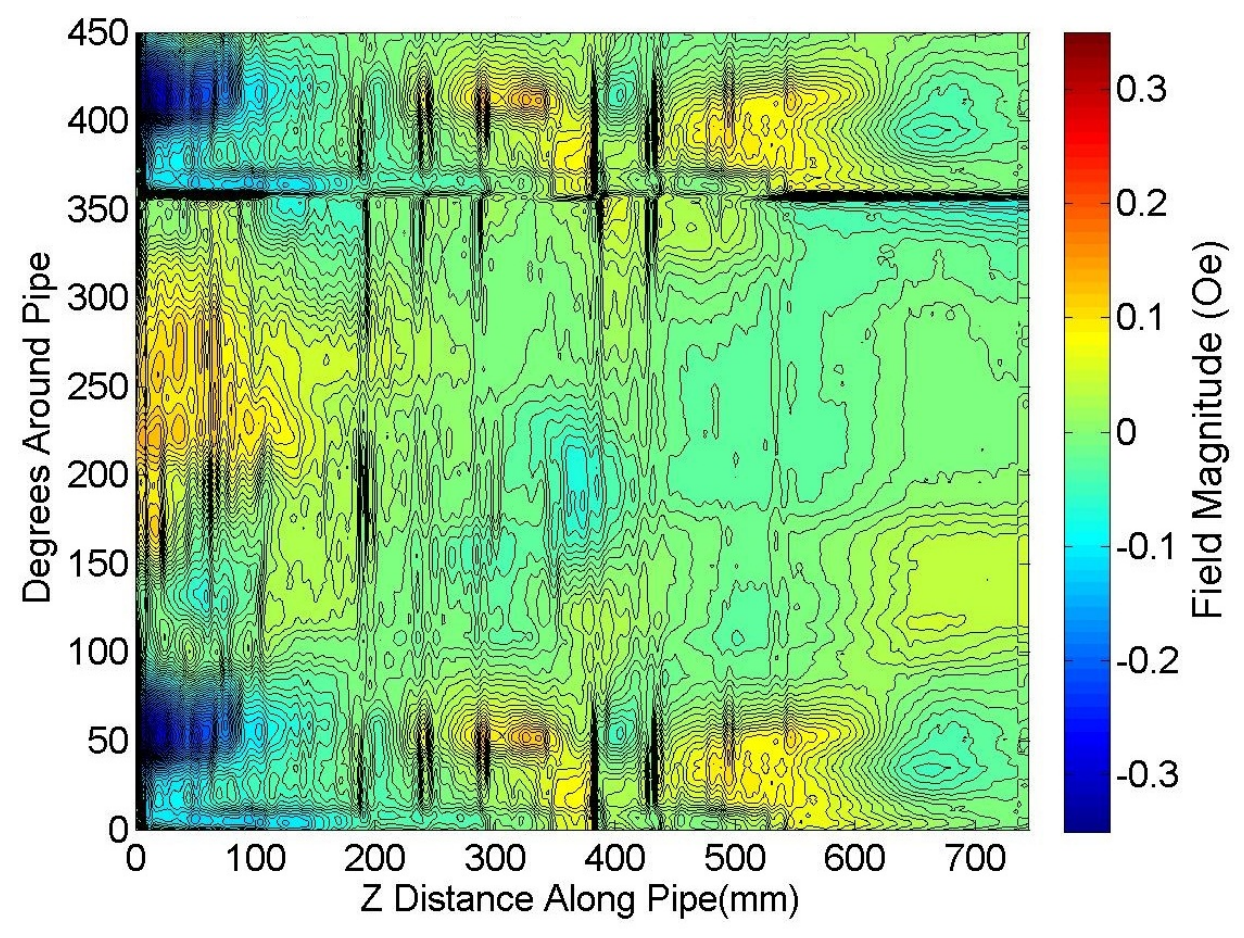

Figure 4.110: $R$ field magnitude over dented aluminum shield wrapped pipe with $5 \mathrm{~Hz}$ excitation current

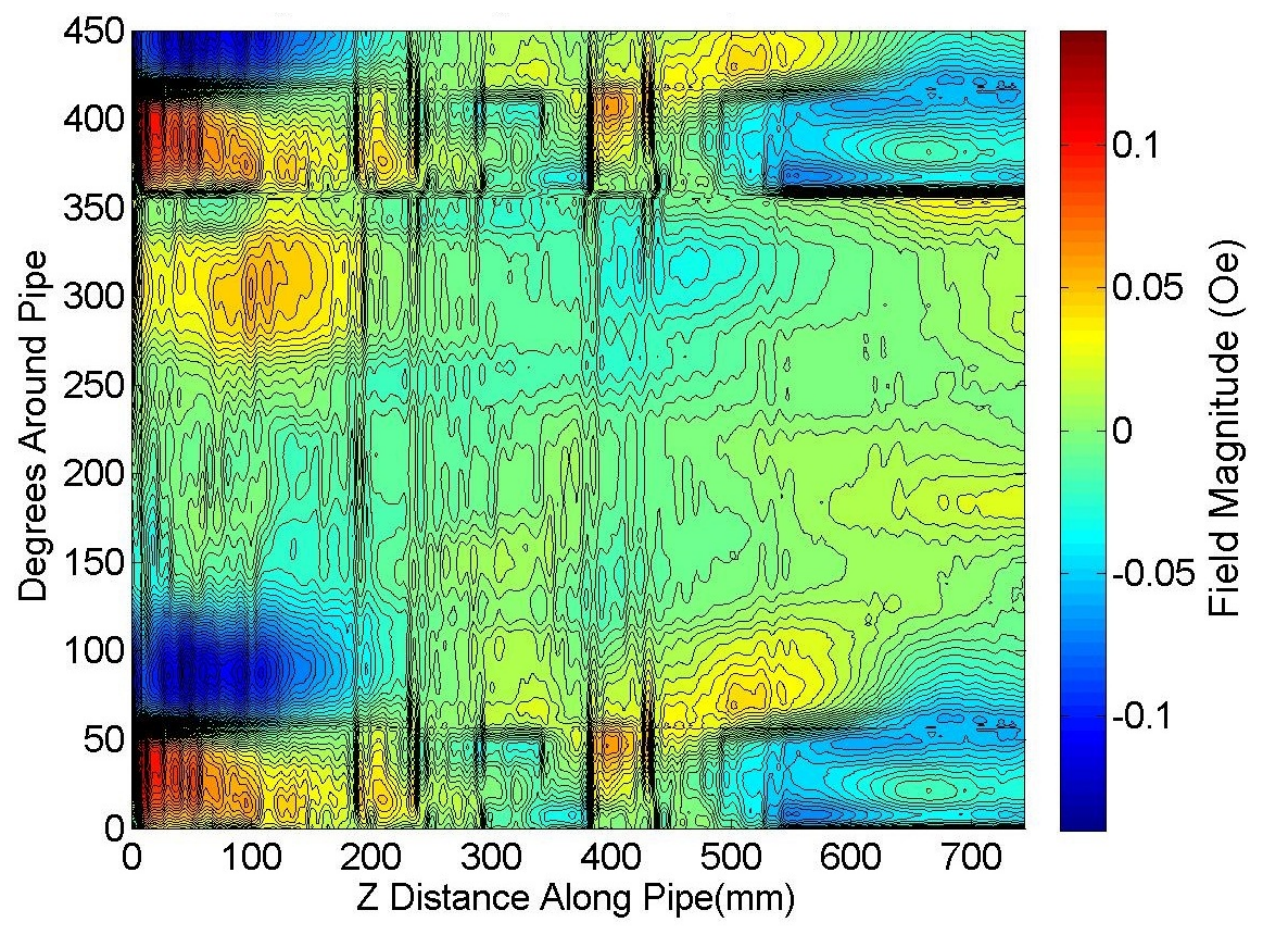

Figure 4.111: Phi field magnitude over dented aluminum shield wrapped pipe with $5 \mathrm{~Hz}$ excitation current 


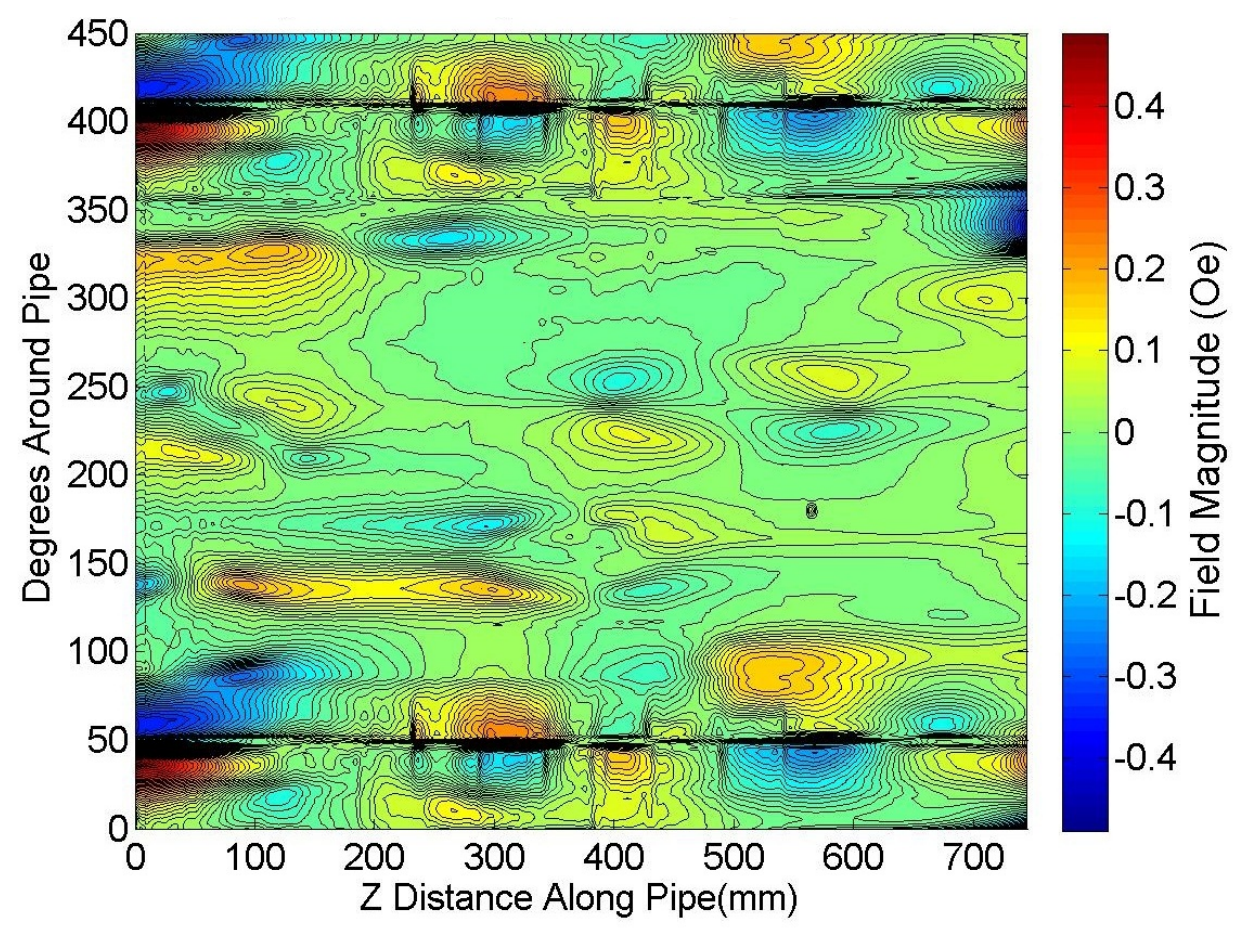

Figure 4.112: Phi field magnitude over dented aluminum shield wrapped pipe with $200 \mathrm{~Hz}$ excitation current

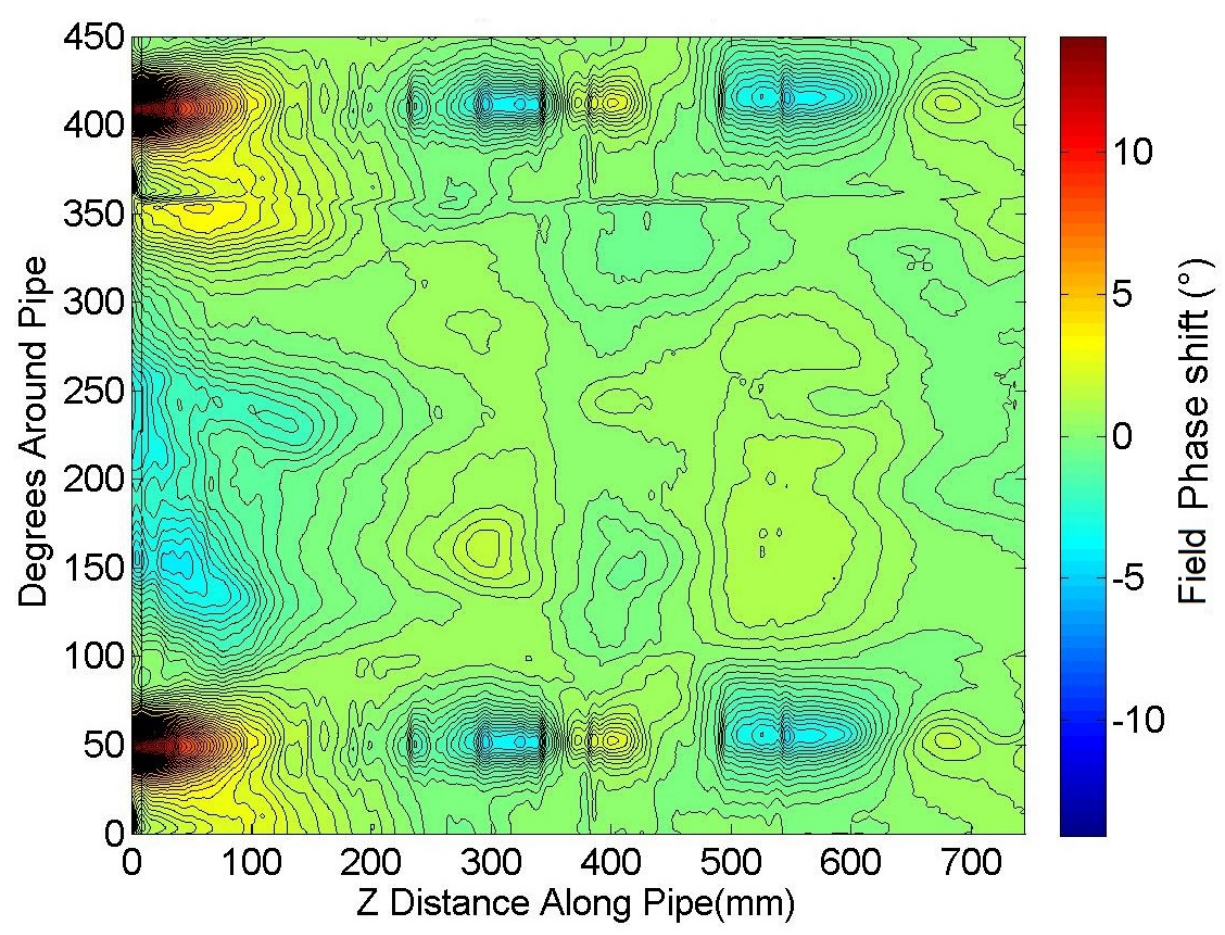

Figure 4.113: $\mathrm{R}$ field phase shift over dented aluminum shield wrapped pipe with $5 \mathrm{~Hz}$ excitation current 


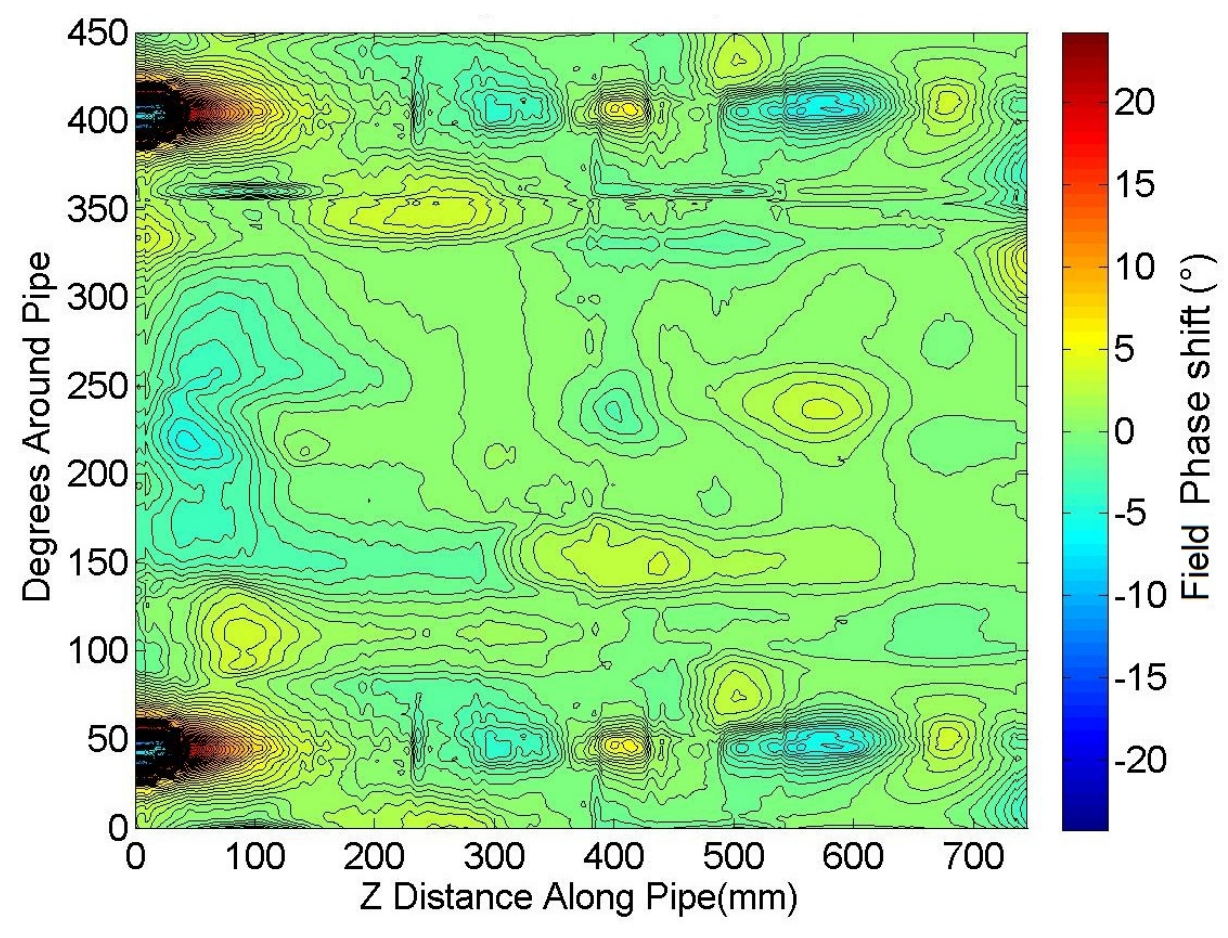

Figure 4.114: R field phase shift over dented aluminum shield wrapped pipe with $200 \mathrm{~Hz}$ excitation current

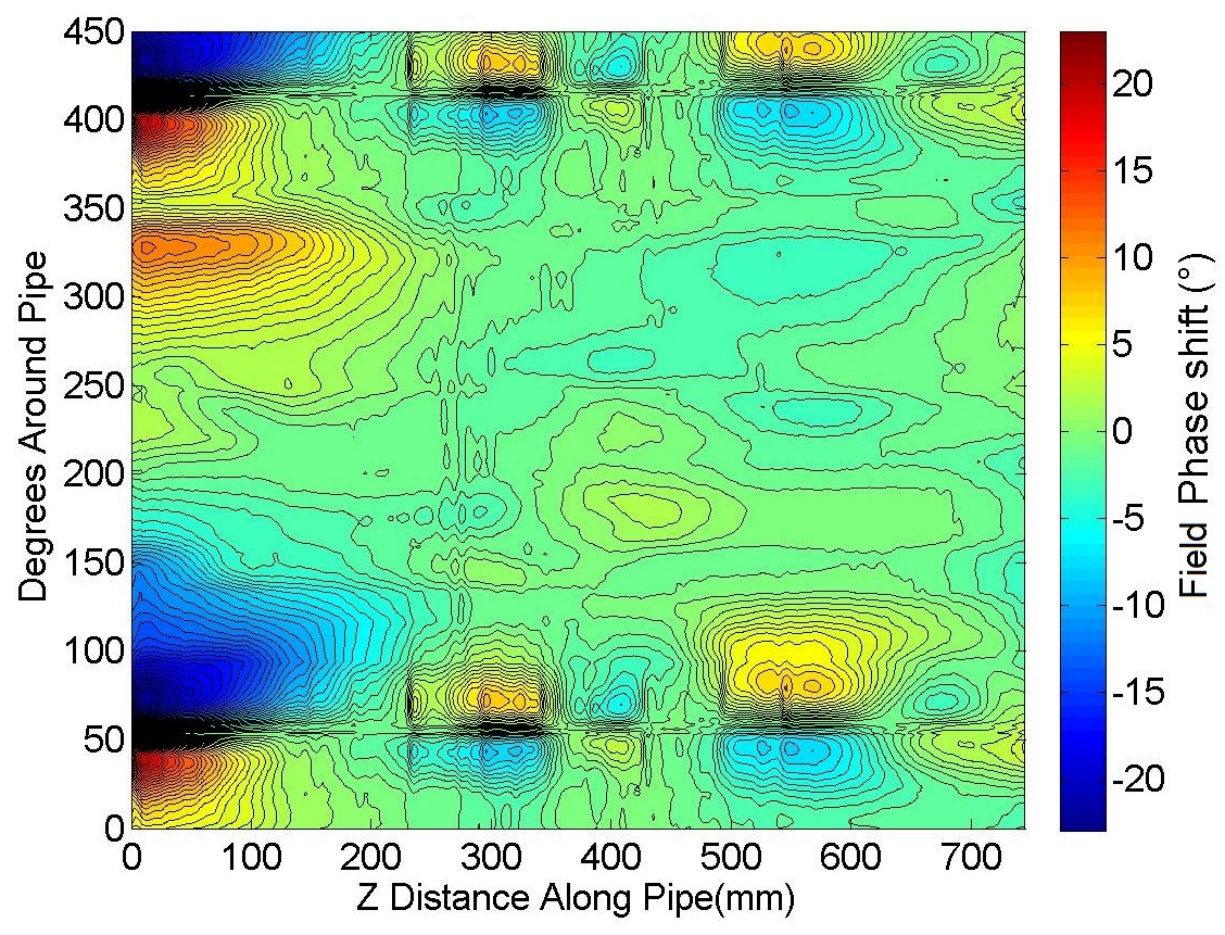

Figure 4.115: Phi field phase shift over dented aluminum shield wrapped pipe with $5 \mathrm{~Hz}$ excitation current 


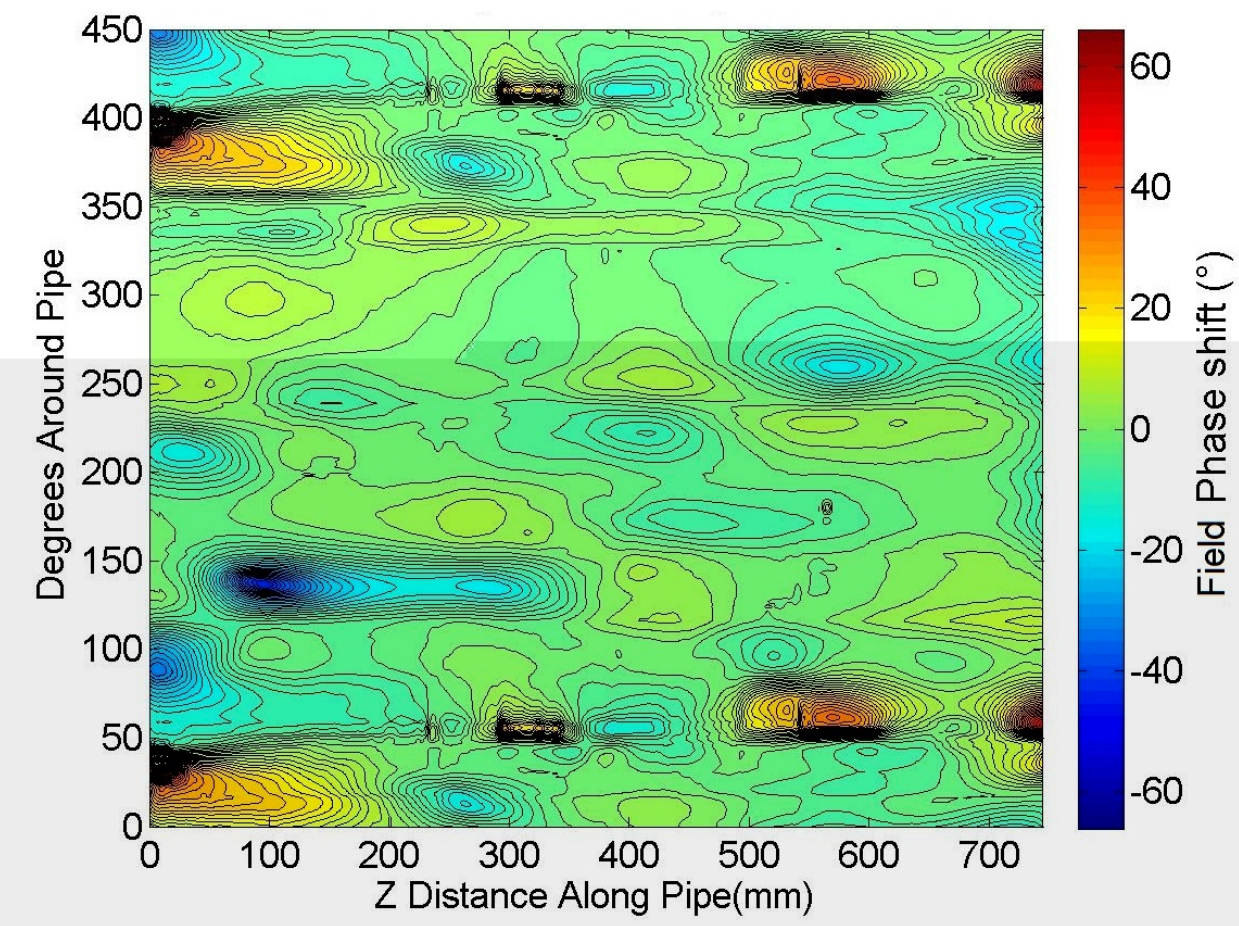

Figure 4.116: Phi field phase shift over dented aluminum shield wrapped pipe with $200 \mathrm{~Hz}$ excitation current

shown in figure 4.117. Again the same upwards trend with reducing excitation frequency with a similar level of variation can be found.

The Phi field magnitude medians are shown in figure 4.118. An increase in field for the Phi field can be seen. This is due to the Phi sensor being far more sensitive to its placement. The process of producing the aluminum dents must have moved the sensor module slightly, causing it to measure more of the excitation field.

The $\mathrm{R}$ field phase shift medians are shown in figure 4.119 and the Phi field in figure 4.120. As with adding an aluminum shield, denting the shield has no effect on the phase shift as it does not change the impedance of the excitation sheet.

To determine how the dents in the aluminum shield affect the noise of each measurement, the SNR for the magnitudes is plotted in figure 4.121 for the R sensor and in figure 4.122 for the Phi sensor. This shows that denting the aluminum shield has no effect on the noise level of the magnitudes with the SNR for the shielded and unshielded pipe essentially the same.

To assess how the phase shift noise changes with frequency over the dented shield, the standard deviation is plotted in figure 4.123 for the R sensor and in figure 4.124 for the Phi sensor. The dents have increased the standard deviation of the $R$ field phase shift at the high frequencies where they have a significant effect. However, at low frequencies, the denting has not increased the standard deviation. The standard deviation of the Phi field phase shift shows similar values at all frequencies apart from at $60 \mathrm{~Hz}$ where the large peak at $60 \mathrm{~Hz}$ does not appear. 


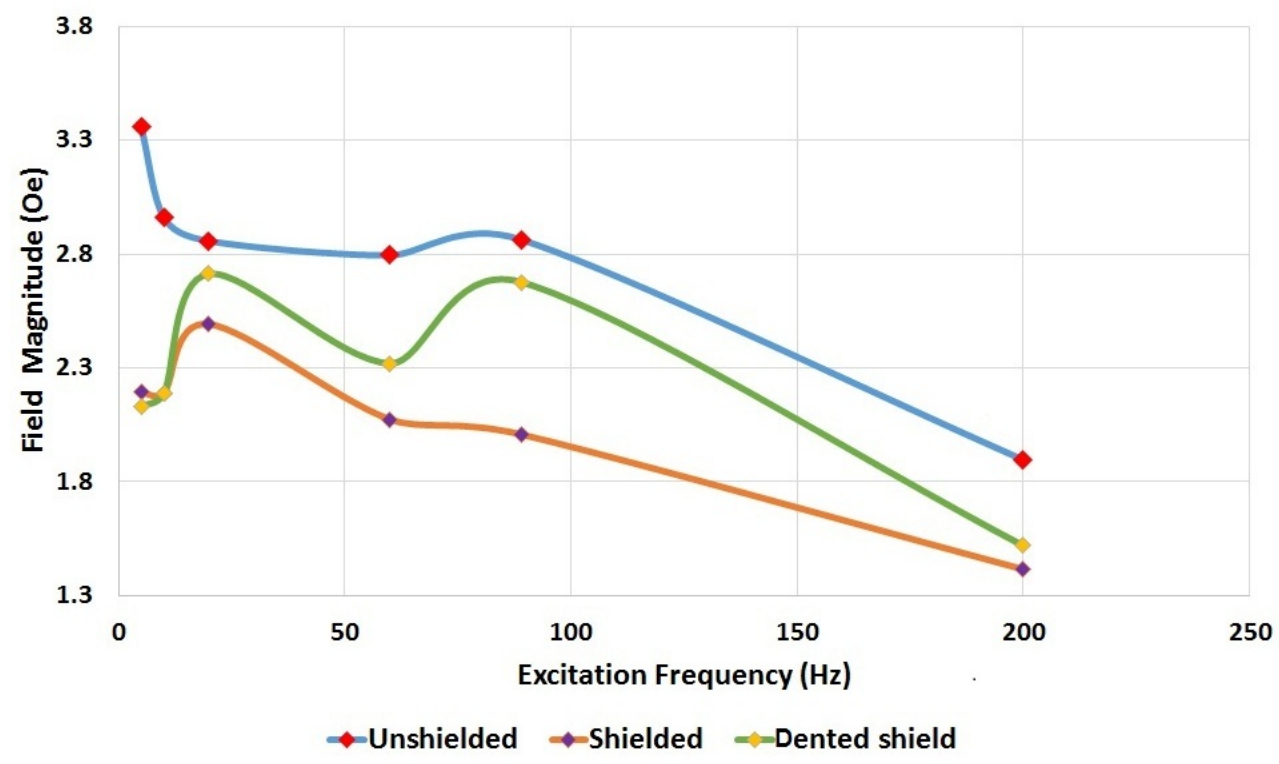

Figure 4.117: $\mathrm{R}$ field magnitude median change with excitation frequency over dented aluminum

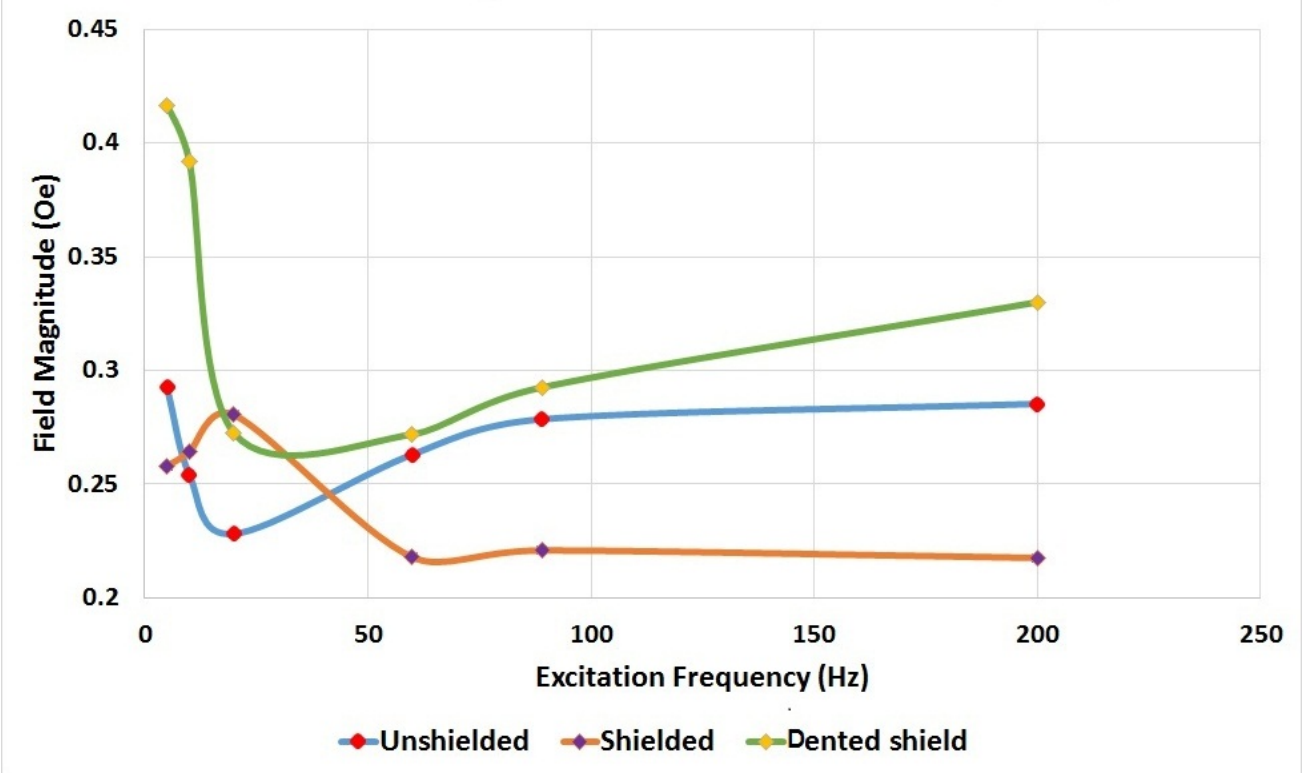

Figure 4.118: Phi field magnitude median change with excitation frequency over dented aluminum 


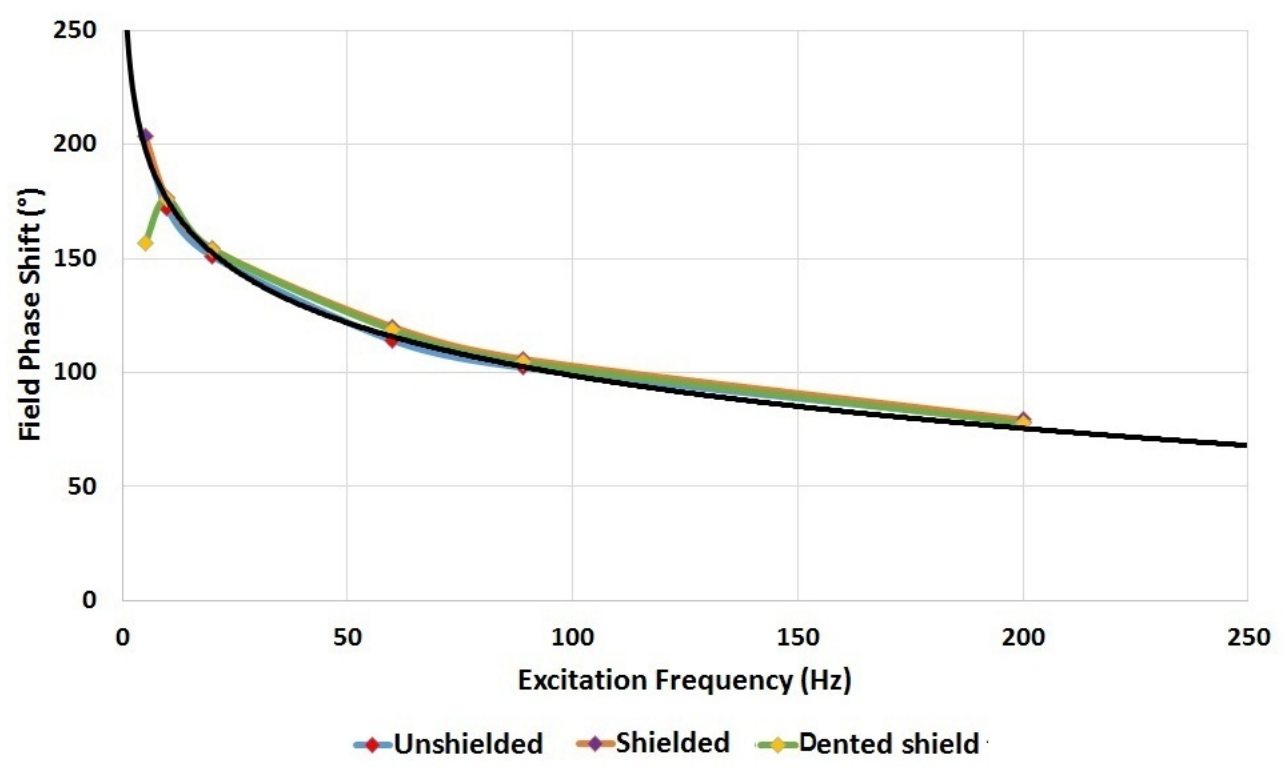

Figure 4.119: $\mathrm{R}$ field phase shift median change with excitation frequency over dented aluminum

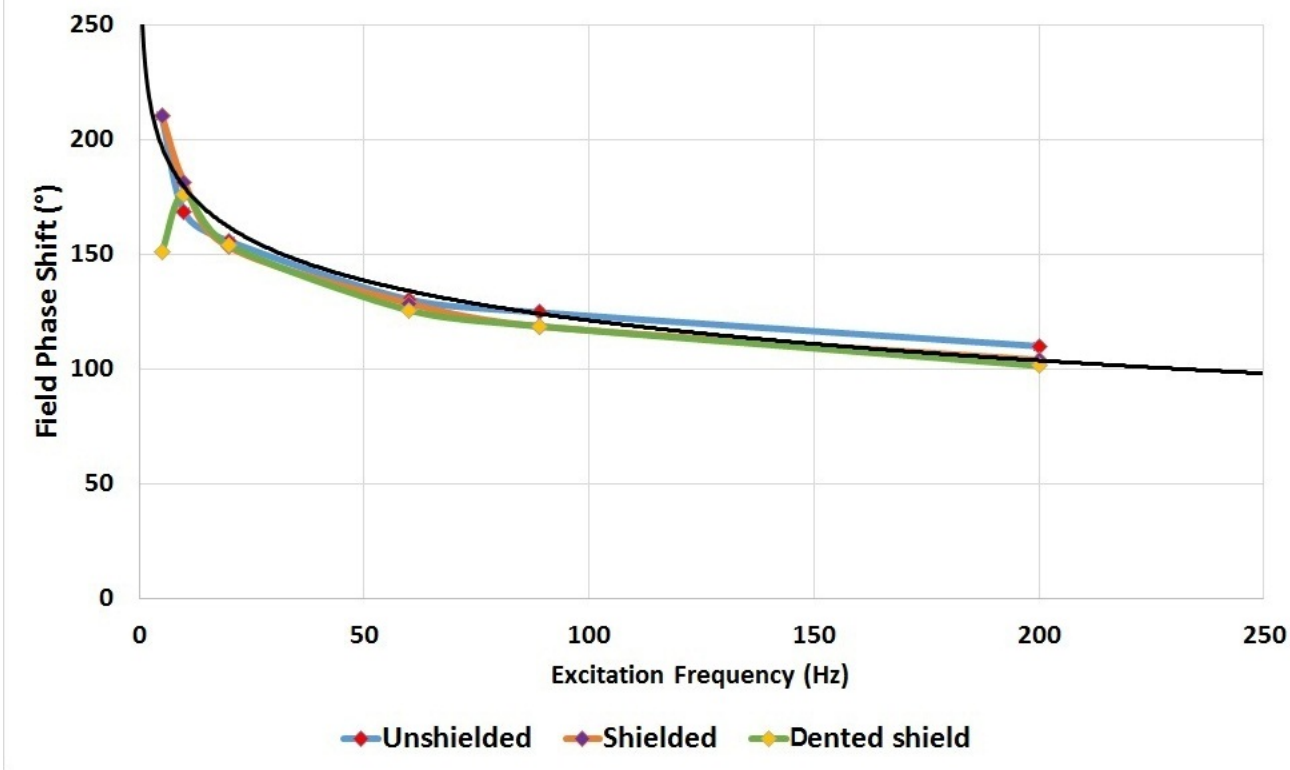

Figure 4.120: Phi field phase shift median change with excitation frequency over dented aluminum 


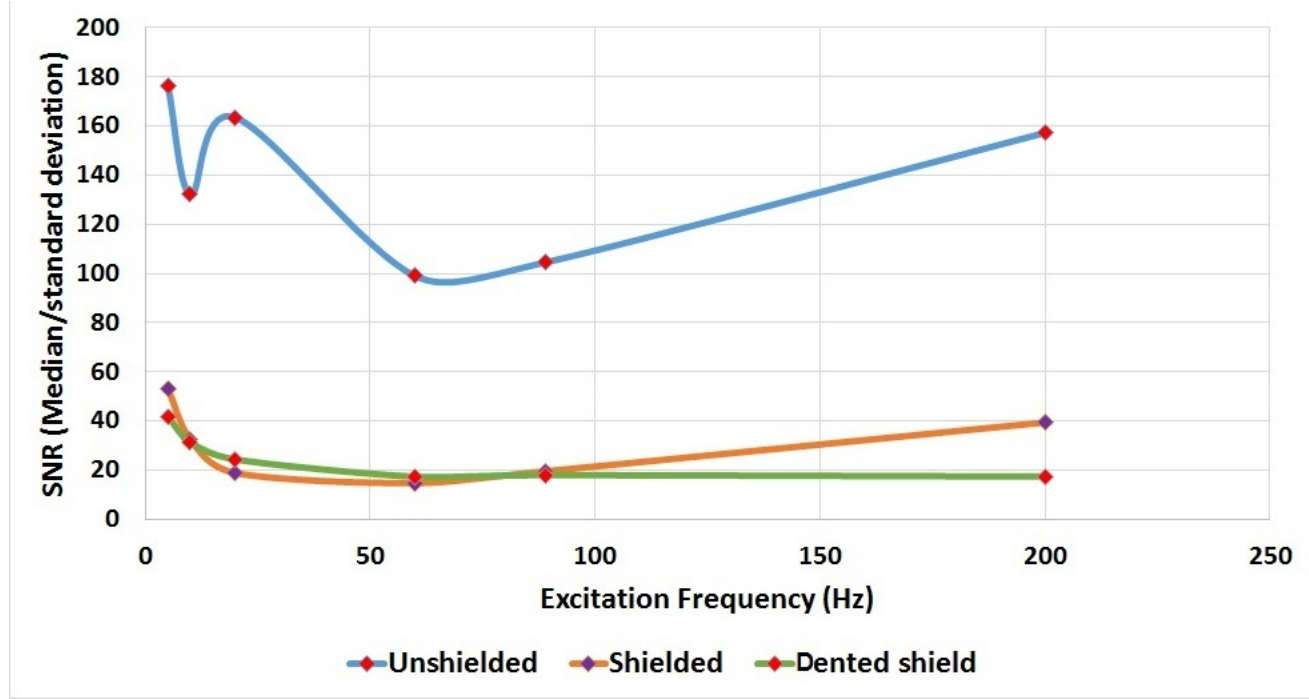

Figure 4.121: R field magnitude SNR change with excitation frequency

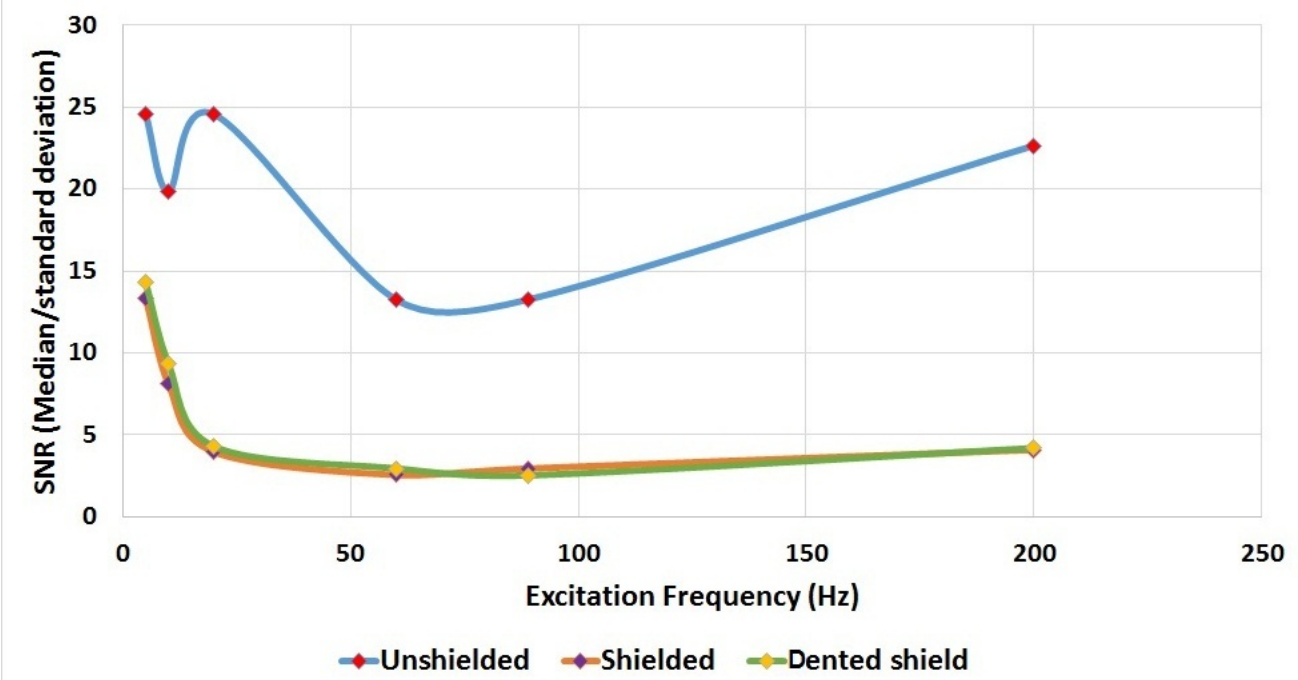

Figure 4.122: Phi field magnitude SNR change with excitation frequency 


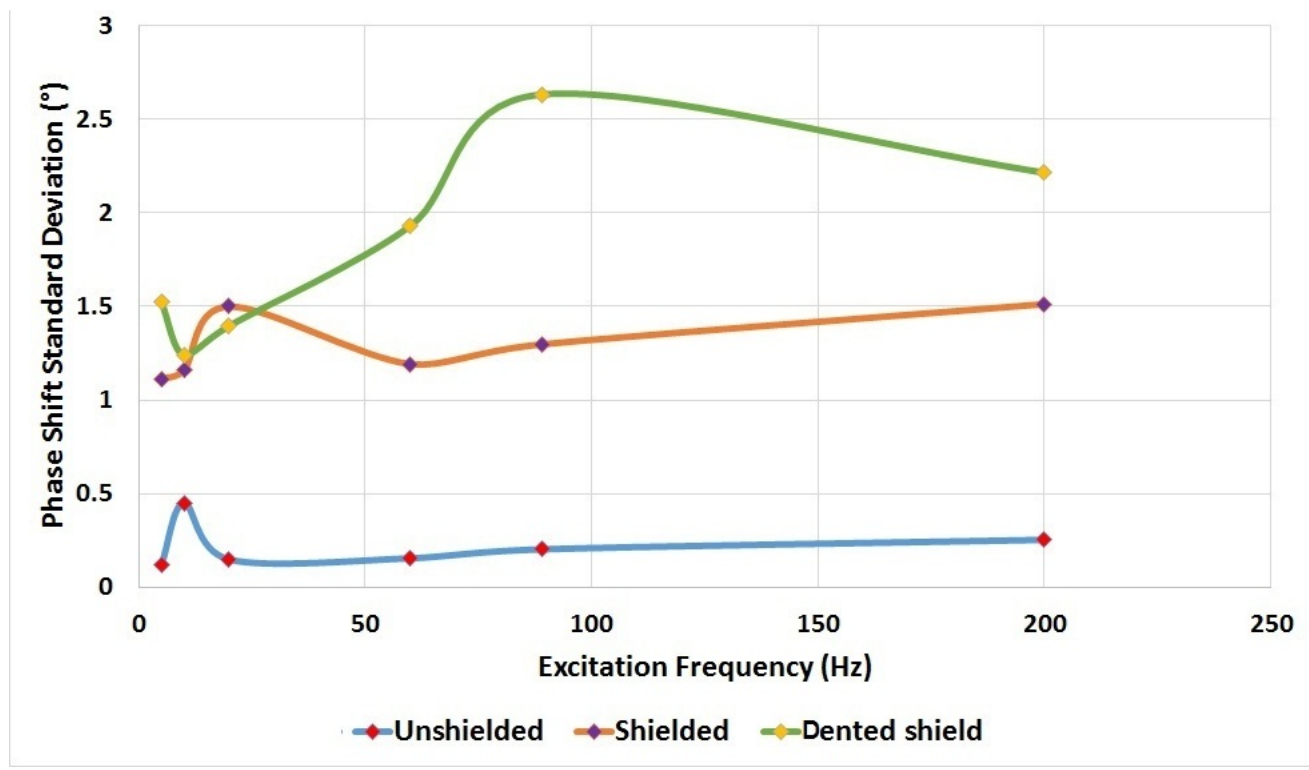

Figure 4.123: $\mathrm{R}$ field phase shift standard deviation change with excitation frequency

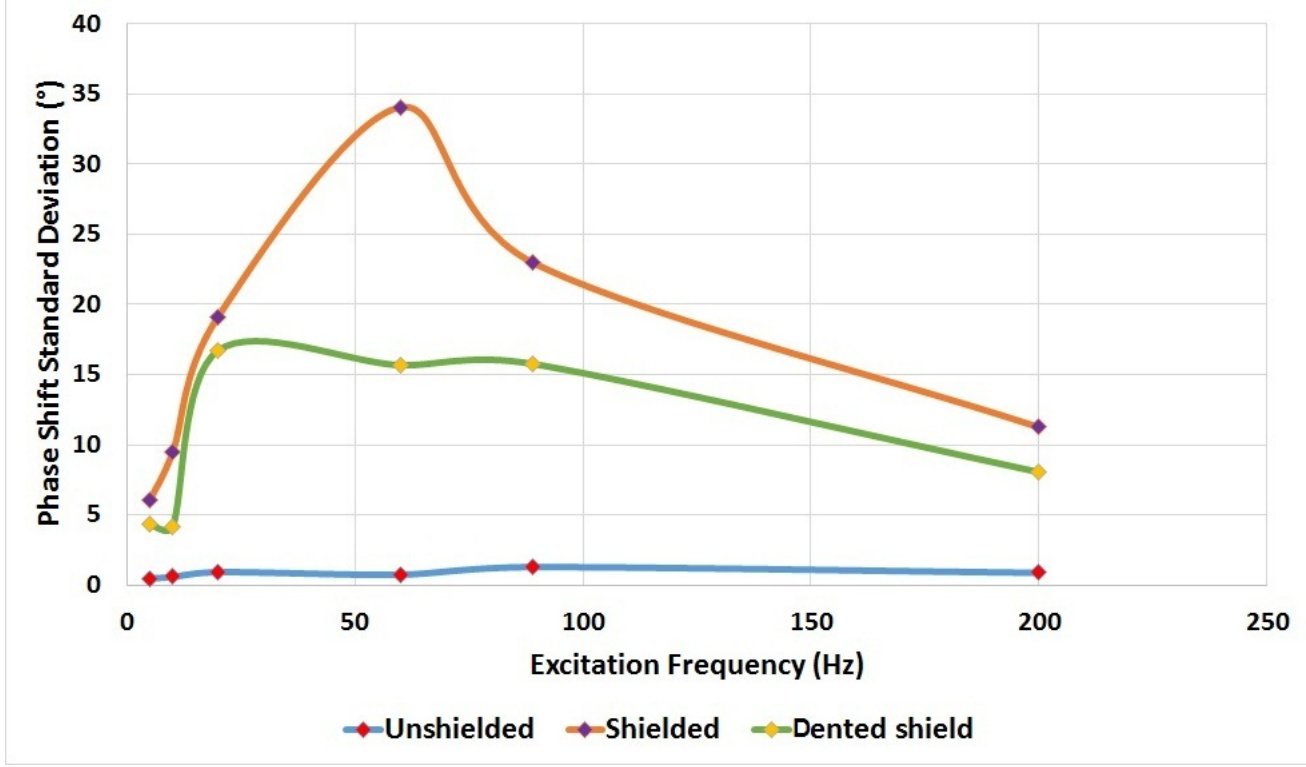

Figure 4.124: Phi field phase shift standard deviation change with excitation frequency 


\subsection{Summary of effects of aluminum dents}

\section{Signals from dents in the aluminum shield}

The effect of introducing dents into the aluminum shield for an excitation with low frequency was minimal. Figure 4.87 shows that at low frequencies the majority of the eddy currents circulate in the steel pipe, so any distortions of the eddy currents in the aluminum shield will have a minimal effect on the measured fields.

The effect at high frequency was a signal with the same pattern that was predicted for defects in the steel pipe. This is expected as the eddy currents are distorted by dents in the same way that they are distorted by a defect in the steel. This frequency dependence of these peaks in the magnetic field means that a multi-frequency algorithm can be used. This would involve using a low frequency measurement, which would mostly test the pipe, and using a high frequency measurement to predict and remove the effect of shield dents on the low frequency measurements. This approach appears to be feasible.

\section{Background level}

The background level of the $R$ field magnitude is approximately the same as in the test with undented shield, with the same general increase in field with decreasing excitation frequency. The Phi field magnitude again shows greater variation in percentage than the $R$ field. However, the absolute variation is still smaller than $\mathrm{R}$ field, showing that this variation can still be explained by movements of the sensor module between tests.

As expected, the phase shift for both the $\mathrm{R}$ and Phi field shows no change with the addition of dents. This is because the background is determined by the impedance of the excitation sheet which is not affected by the geometry of a low permeability material within the coil.

\section{SNR of magnitude}

Dents in the aluminum shield had no effect on the SNR of magnitude for both the R and Phi fields. This shows that the major improvements will come from measuring and removing the effects of the joint in the aluminum, not the dents in the shield.

\section{Standard deviation of phase shift}

Dents in the aluminum shield have had no effect for excitation frequency below $20 \mathrm{~Hz}$. This shows, like the SNR, that if a low frequency excitation is used the increase in noise from aluminum dents will be minimal. This low frequency requirement fits well with the parameters required for field magnitude measurements because, as shown previously, low frequencies are required to identify defects in the field magnitude plots. 


\section{Chapter 5}

\section{Experimental Results: Measurement regular defects}

To investigate detection of defects in pipes a set of measurements was carried out on a $1.3 \mathrm{~m}$ steel pipe with 13 regular defects machined into it. A $700 \mathrm{~mm}$ section in the middle of the pipe was tested as shown in figure 5.1. The way the defect parameters influenced the magnetic field was investigated. Then the effect of changing the excitation current and frequency was measured to determine the optimum excitation parameters. Then a range of excitation lift-off levels were tested to determine the maximum lift-off level before defect signals became unidentifiable.

\subsection{Defect parameters}

Three different styles of regular defects were manufactured to simulate real corrosion defects. Real corrosion generally starts at the point where moisture reaches the pipe. It then grows outwards in all directions, generally creating a shallow dish shaped loss of material. When material loss reaches $25 \%$ of the pipe wall thickness it becomes a concern.

The segment defect shown in figure 5.2 has a segment section of the pipe removed and can be defined by the maximum depth and the $Z$ length. The box defect shown in figure 5.3 has a box section removed from the pipe and can be defined by the Phi length and the $Z$ length and the minimum depth. The through hole defect shown in figure 5.4 removes the entire wall of the pipe for a box section and can be defined by the Phi length and $Z$ length.

The segment defect was chosen because it was an easy defect shape to manufacture that incorporated two edges with less than 90 degree corners. The box defect gave a defined minimum depth throughout the defect. The through hole defect gave a very large defect signal and it allowed an easy initial identification of the defect pattern. A completed list of the defects manufactured is listed in table 5.1. The defects have a range of depths and volumes in order to investigate the relationships between the signals measured and the physical dimensions of the defects. 


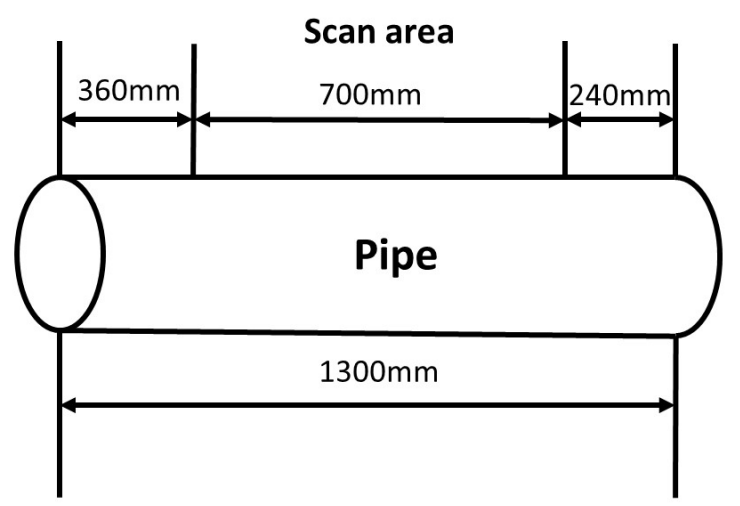

Figure 5.1: $700 \mathrm{~mm}$ scan area
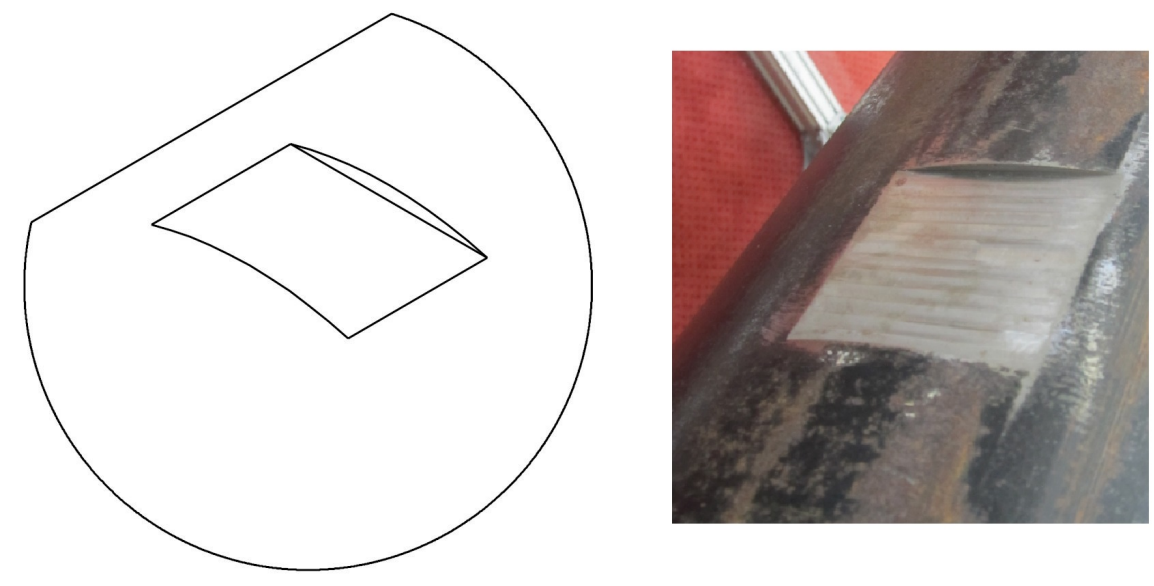

Figure 5.2: Segment defect
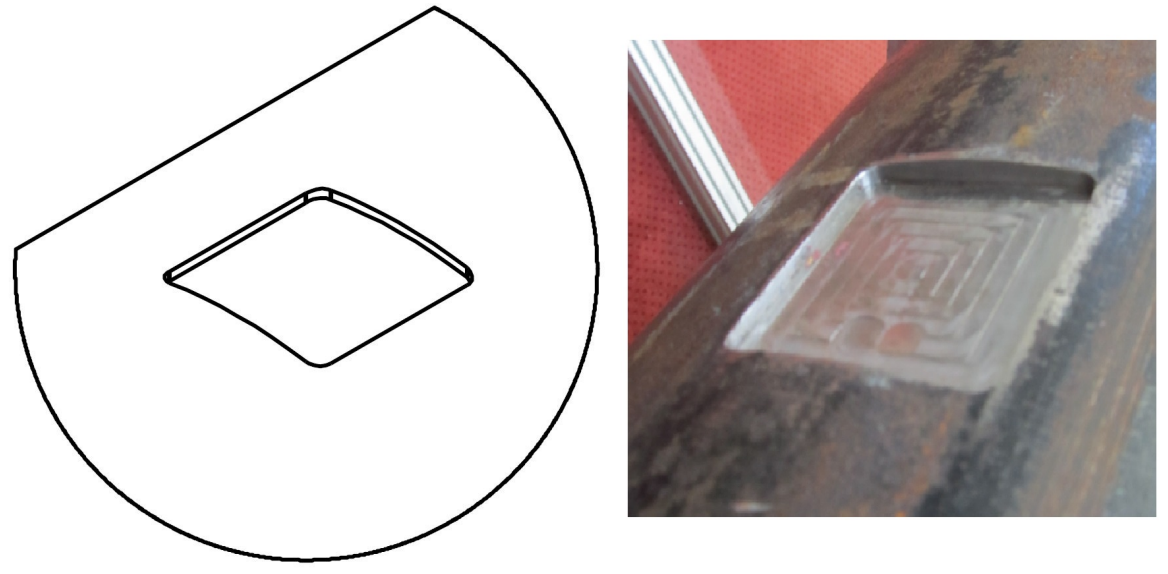

Figure 5.3: Box defect 


\begin{tabular}{|c|c|c|c|c|c|}
\hline Defect & Type & $\begin{array}{c}\text { Volume } \\
\left(\mathrm{mm}^{3}\right) \\
\text { (approx) }\end{array}$ & $\begin{array}{c}\text { Defect Z } \\
\text { length }(\mathrm{mm})\end{array}$ & $\begin{array}{l}\text { Defect Phi } \\
\text { length }\left(^{\circ}\right)\end{array}$ & $\begin{array}{c}\text { Average } \\
\text { defect depth } \\
(\mathrm{mm})\end{array}$ \\
\hline 1 & box & 30000 & 70 & 29 & 6.1 \\
\hline 2 & box & 13500 & 70 & 14.5 & 5.5 \\
\hline 3 & $\begin{array}{l}\text { through } \\
\text { hole }\end{array}$ & 23750 & 50 & 21 & 9.5 \\
\hline 4 & segment & 20000 & 100 & 29 & 2.9 \\
\hline 5 & segment & 6000 & 100 & 20 & 1.2 \\
\hline 6 & segment & 30800 & 50 & 41 & 6.3 \\
\hline 7 & $\begin{array}{l}\text { through } \\
\text { hole }\end{array}$ & 23275 & 70 & 16 & 9.5 \\
\hline 8 & box & 7000 & 70 & 16 & 2.9 \\
\hline 9 & box & 15000 & 50 & 21 & 6 \\
\hline 10 & segment & 22000 & 50 & 35 & 5.2 \\
\hline 11 & segment & 12000 & 50 & 30 & 3.4 \\
\hline 12 & segment & 4400 & 50 & 21 & 1.7 \\
\hline 13 & box & 13000 & 50 & 21 & 5.2 \\
\hline
\end{tabular}

Table 5.1: Regular defect sizes 

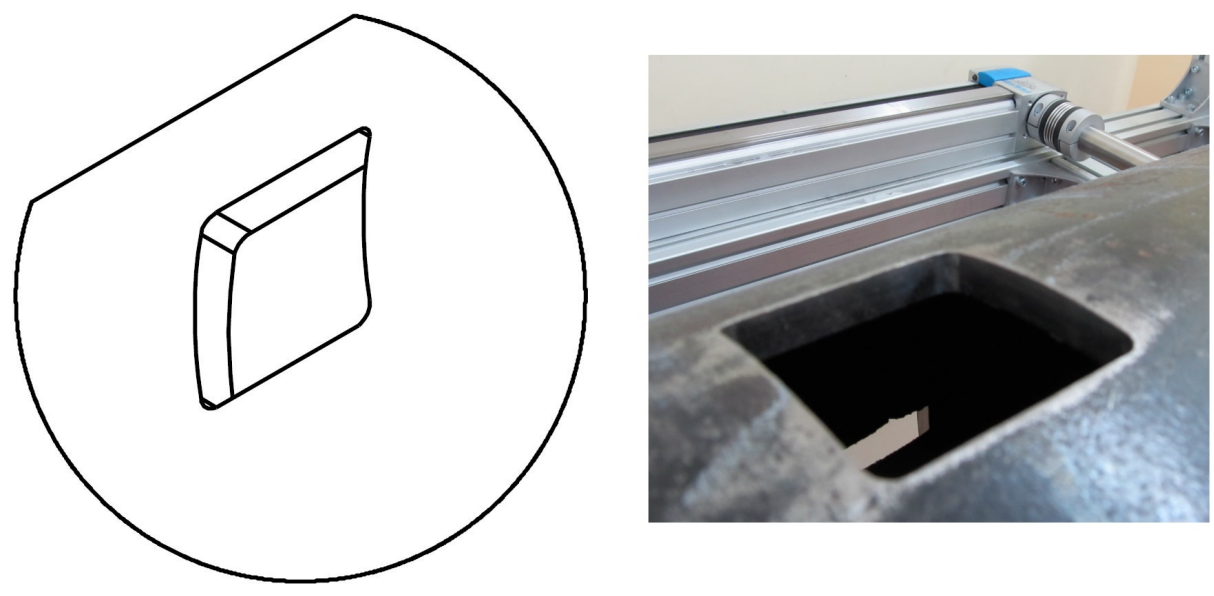

Figure 5.4: Through hole defect

\subsection{Results}

The response of the $\mathrm{R}$ and Phi magnetic field magnitude and phase shift to defects in the pipe was determined. Then the effect of the defect size on the magnitude of the response was evaluated. The remaining results are split into three sections, each one addressing the influence of one excitation parameter on the defect signals.

For each measure a range of contour plots, at key levels, is shown of the entire test area in order to give a view of the changes each parameter caused. A closer view of defects 3 , 9 and 12 is then presented. There was a focus on these three defects because they were each of a different basic shape and different average wall loss. The peak to peak signal for defects 3, 9 and 12 was calculated to determine the effect of each parameter. Defects 9 and 12 are of particular interest as these are realistic defect sizes for an operational pipe[8] while defect 3 provides very large signals for reference. By using the peak to peak values of the defect signals and the standard deviation values from the plain pipe measurements, a SNR value according to equation 5.1 is calculated.

$$
S N R=\frac{\text { peak to peak signal }}{\text { standard deviation of the plain pipe data }}
$$

The SNR values are plotted to determine the limit of detection for each of the defects in regards to the excitation parameters. After each results section, a summary section discusses likely causes of the results and what it means for detecting defects within the pipe.

\subsubsection{Influence of defect shape on defect signals}

The data presented here has been corrected to a nominal $300 \mathrm{~A}$ excitation current, then shifted to center around zero. The $Z$ and Phi flattening algorithms were then applied, as discussed in section 4.2. Throughout this chapter contour plots are used extensively. Figure 5.5 shows how a three dimensional view on the top is translated to a two dimensional plot on the bottom. 
$\mathrm{R}$ sensor magnetic field magnitude defect 3
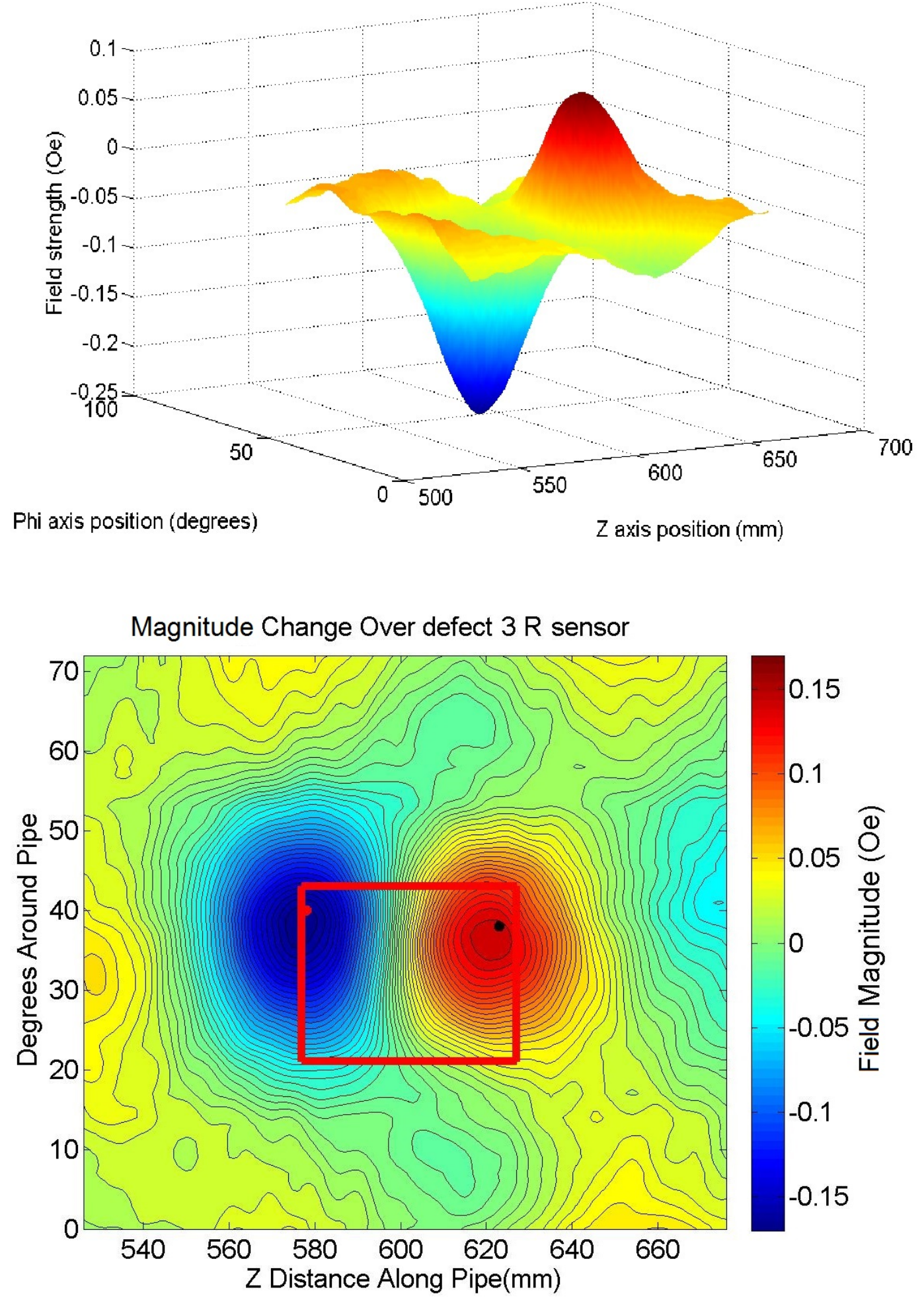

Figure 5.5: Magnetic field over defect 3 in 3D plot(top) and contour plot(bottom) 


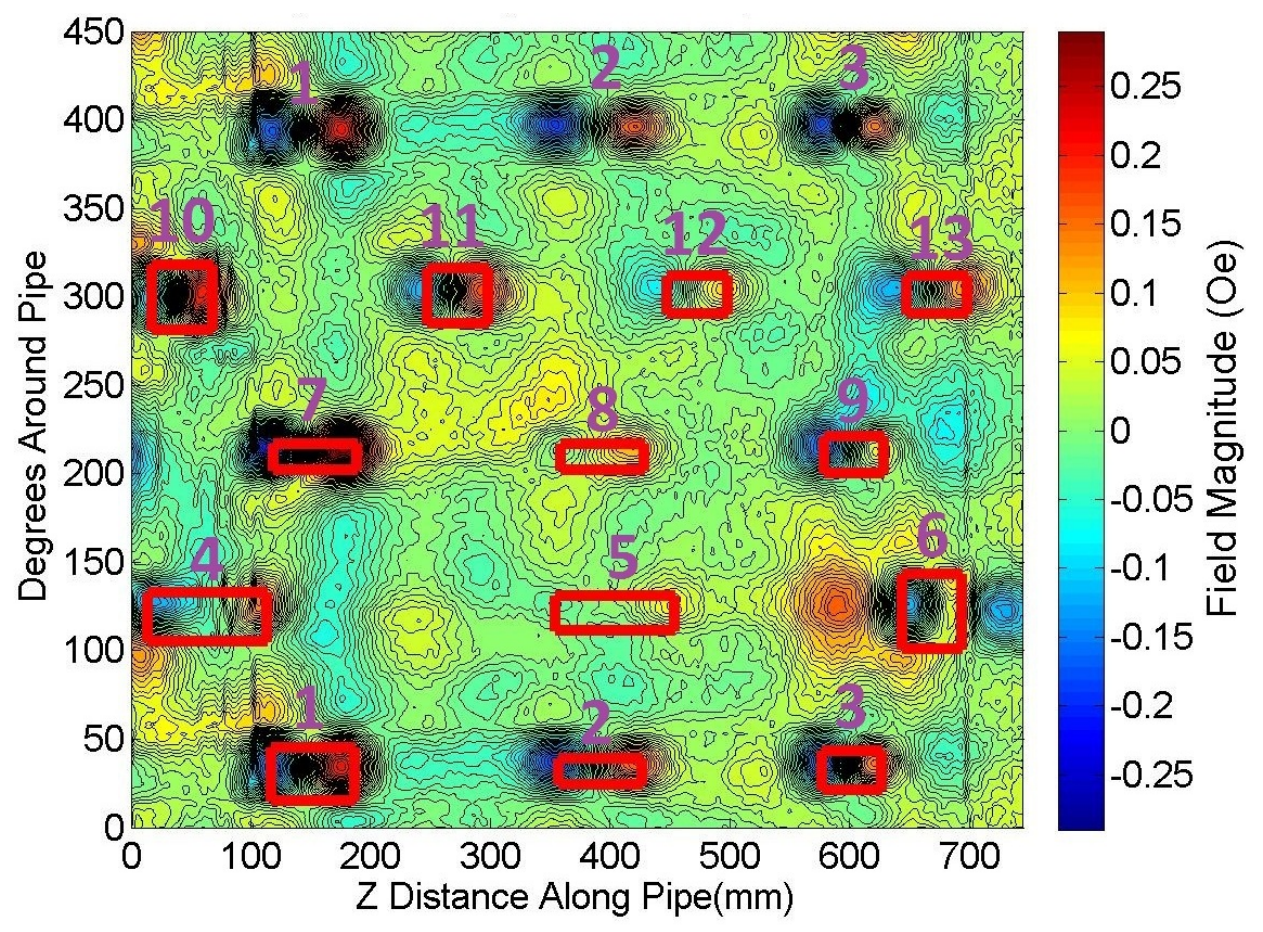

Figure 5.6: $\mathrm{R}$ field Magnitude for whole scan area with regular defects with $\mathrm{Z}$ flattening, current correction and Phi flattening algorithms applied

Throughout these results red boxes are plotted on the contour plots to show the approximate location of the defects. The Gaussian peaks are often displaced by 5 to 10 degrees from their correct position and this is due to a combination of effects. There are off-set changes with excitation frequency as lower excitation frequency reports the peaks further from their correct location. In addition, measurement errors in the location of the defect create a fixed off-set from the correct location.

On the contour plots of one defect, such as figure 5.7, small red and black dots can be seen. These show the location of the peaks found by a peak location algorithm to assist the measurement of peak to peak signal of defects.

\section{The influence of defect shape on $\mathbf{R}$ field magnitude defect signals}

A contour map of a whole $750 \mathrm{~mm}$ scan area of the pipe surface is shown in figure 5.6. It can be seen that each defect has an associated positive and negative Gaussian peak centered at the $Z$ edges of the defect, as explained in section 2.5.2. However, three exceptions can be seen between 100 and 150 degrees. Defects 4 and 5 have the expected Gaussian peaks distorted by nearby variations in the magnetic field. Defect 6 shows three Gaussian peaks appearing. This is the defect with the largest volume loss so it indicates that defect signals change once the defect reaches certain dimensions.

A more detailed plot of each of the different types of defects is shown: in figure 5.7 for the $50 \times 50 \mathrm{~mm}$ segment (defect 12), in figure 5.8 for the $50 \times 50 \times 5 \mathrm{~mm}$ box defect (defect 9) and in figure 5.9 for the $50 \times 50 \mathrm{~mm}$ through hole defect (defect 3). All three defect signals have a similar shape. The main difference is the amplitude of the Gaussian peaks 


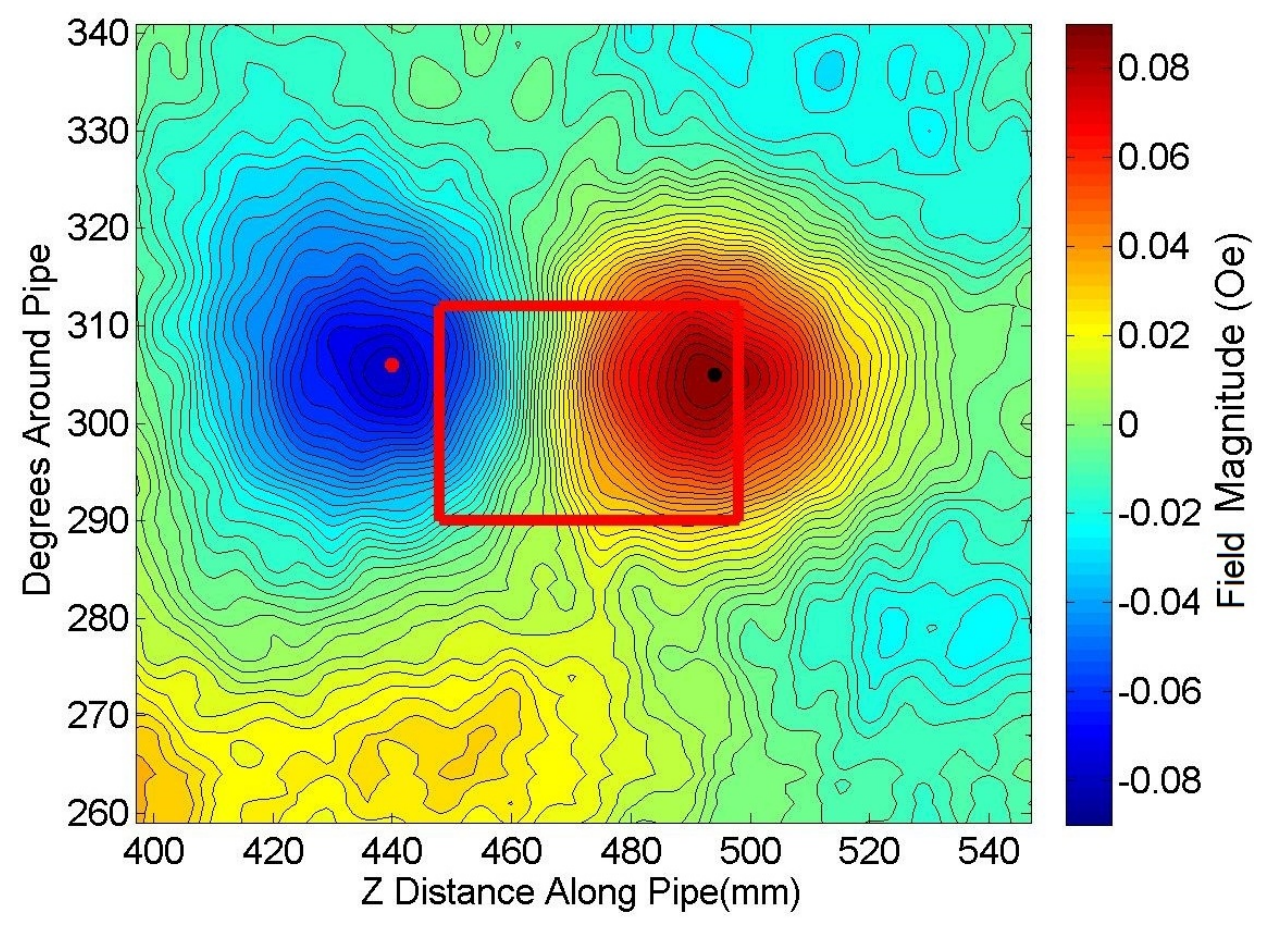

Figure 5.7: $R$ field magnitude over $50 \times 50 \mathrm{~mm}$ segment defect

with defect 12 having the smallest amplitude and defect 3 the largest amplitude.

To determine the influence of defect depth and volume, the peak to peak signal value is calculated for each defect, with an identifiable maximum and minimum peak. This is plotted vs the defect volume in figure 5.10 as well as the average depth in figure 5.11. A linear fit has been applied to both plots. The linear fit ignores two outlier points which are in blue. One point represents the peak to peak signal of defect 6 which, as shown previously, splits into three peaks so a different relationship would be expected. The other represents defect 7 which is a through hole defect which is long and thin. This extreme nature of the defect being deep and narrow may causes a distortion from standard defect signal similar to defect 6 causing a variation from the standard linear fit.

Although the peak to peak signal fit to the defect volume is good when the outliers are removed, significant variation of the linear fit occurs. This suggests the defect volume plays a part in the size of the signal amplitude but there are also additional factors which have a significant effect.

The peak to peak signal fitted to the average wall loss of the defect shows a similar level of variation as the defect volume. This is expected since there is a linear relationship between the defect volume and the average depth. This means the data quality is not good enough to determine if the defect depth or volume has the strongest influence on the signal peak to peak level for the $\mathrm{R}$ field magnitude.

\section{The influence of defect shape on $\mathbf{R}$ field phase shift defect signals}

The $R$ field phase shift contour map of the whole scan area is shown in figure 5.12. It shows the same basic behavior as the field magnitude with a Gaussian peak centered 


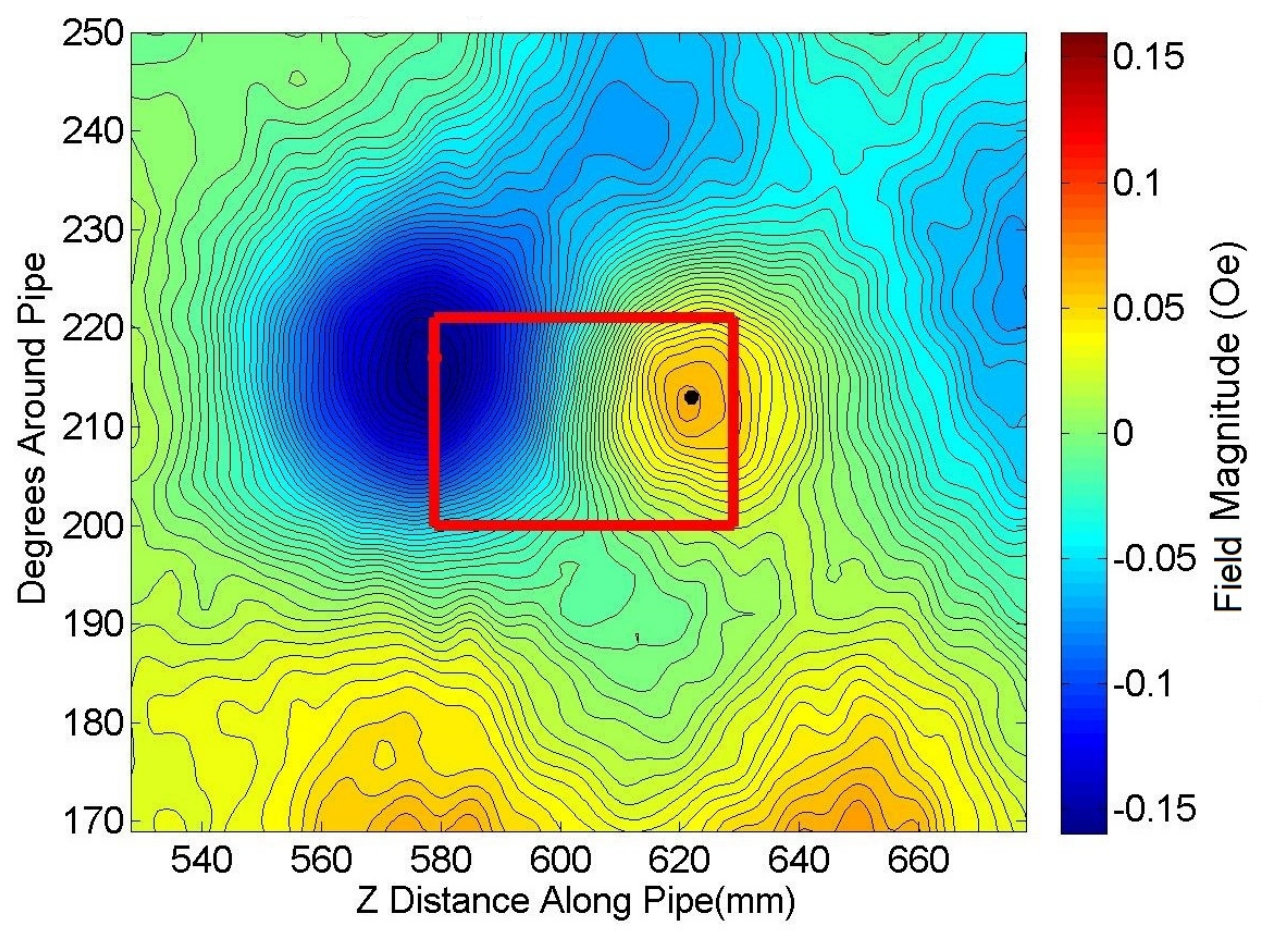

Figure 5.8: $\mathrm{R}$ field magnitude over $50 \times 50 \times 5 \mathrm{~mm}$ box defect

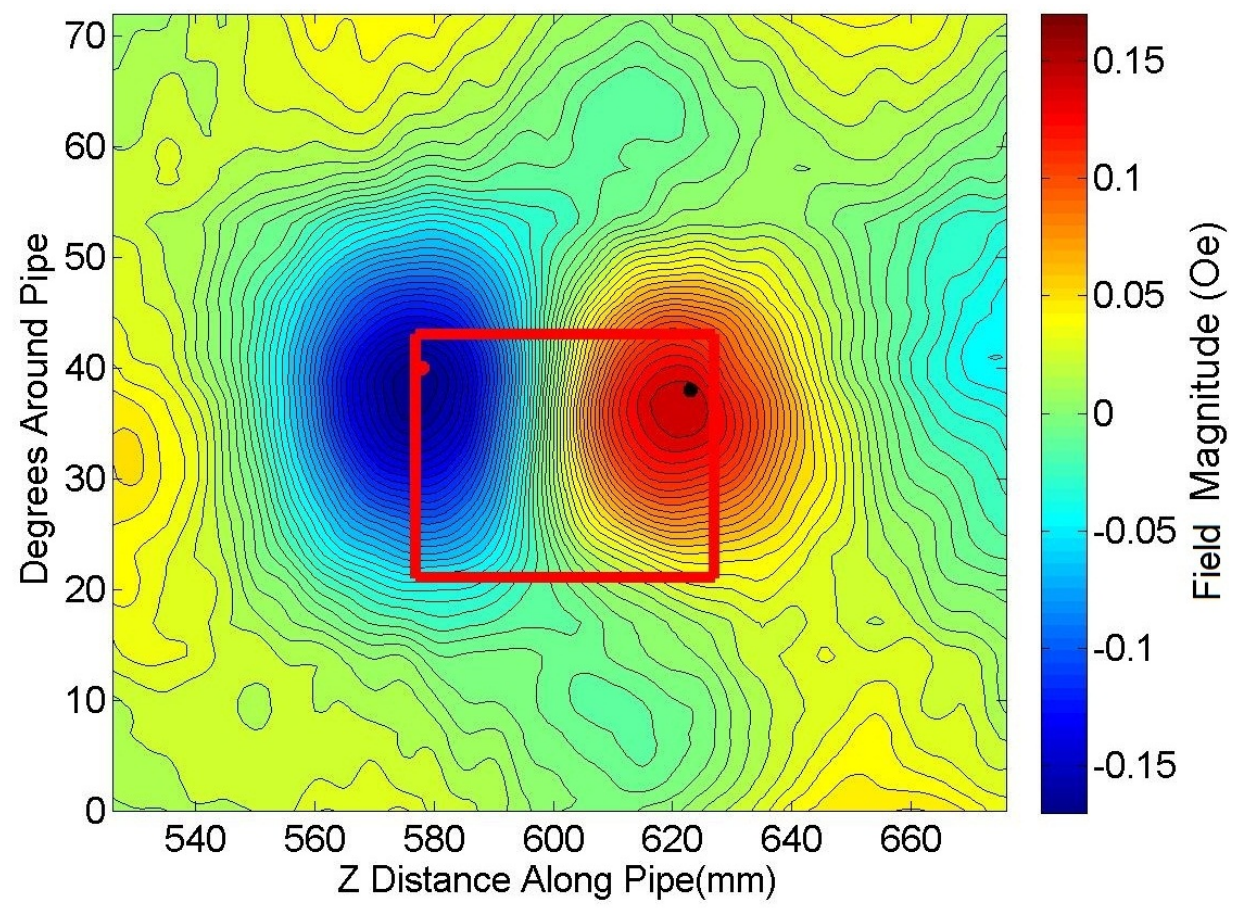

Figure 5.9: R field magnitude over $50 \times 50 \mathrm{~mm}$ through hole defect 


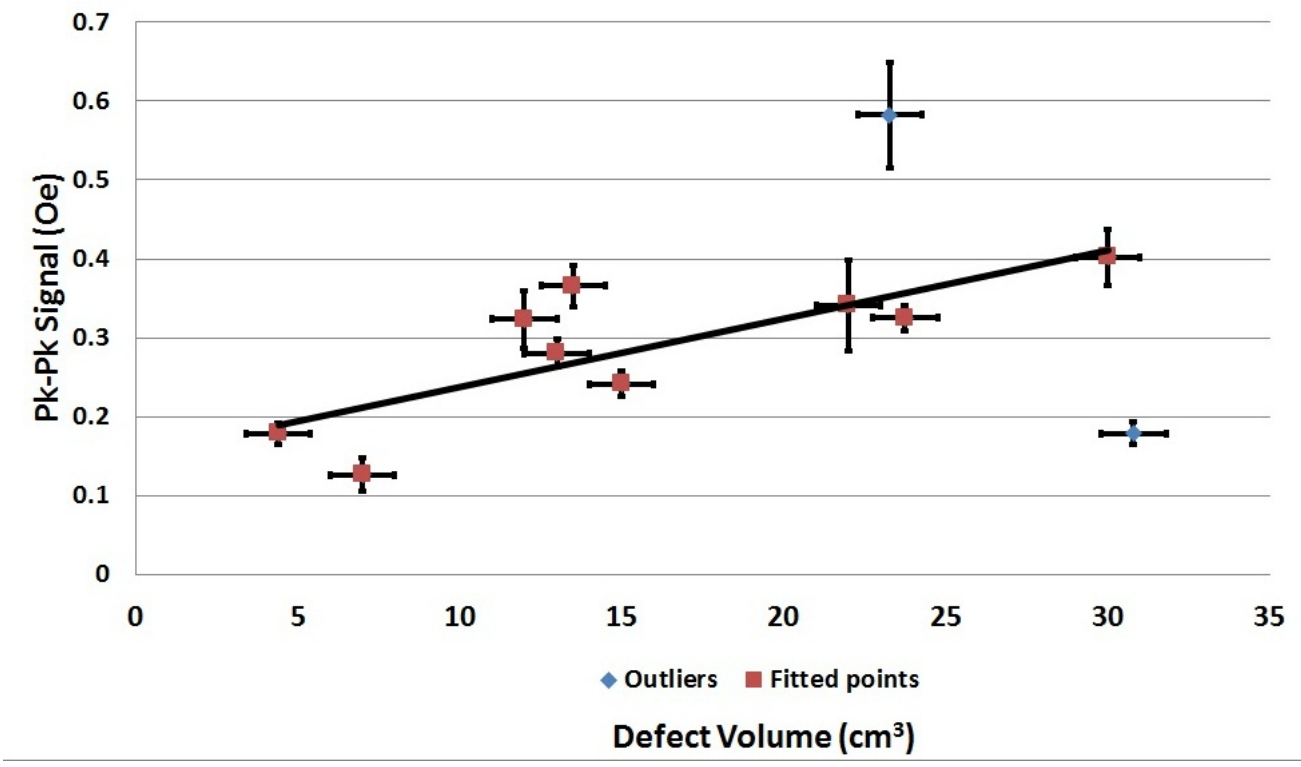

Figure 5.10: $\mathrm{R}$ field magnitude peak to peak defect signal vs defect volume

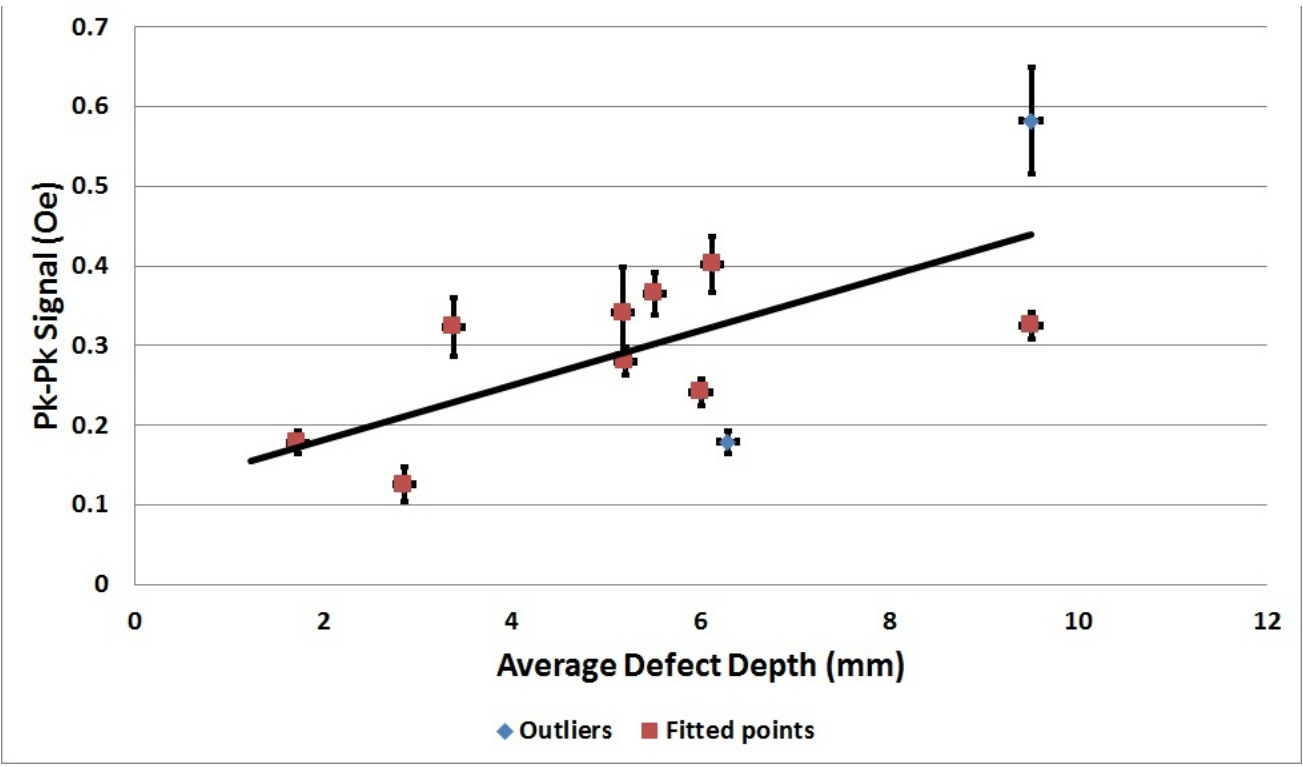

Figure 5.11: R field magnitude peak to peak defect signal vs average defect depth 


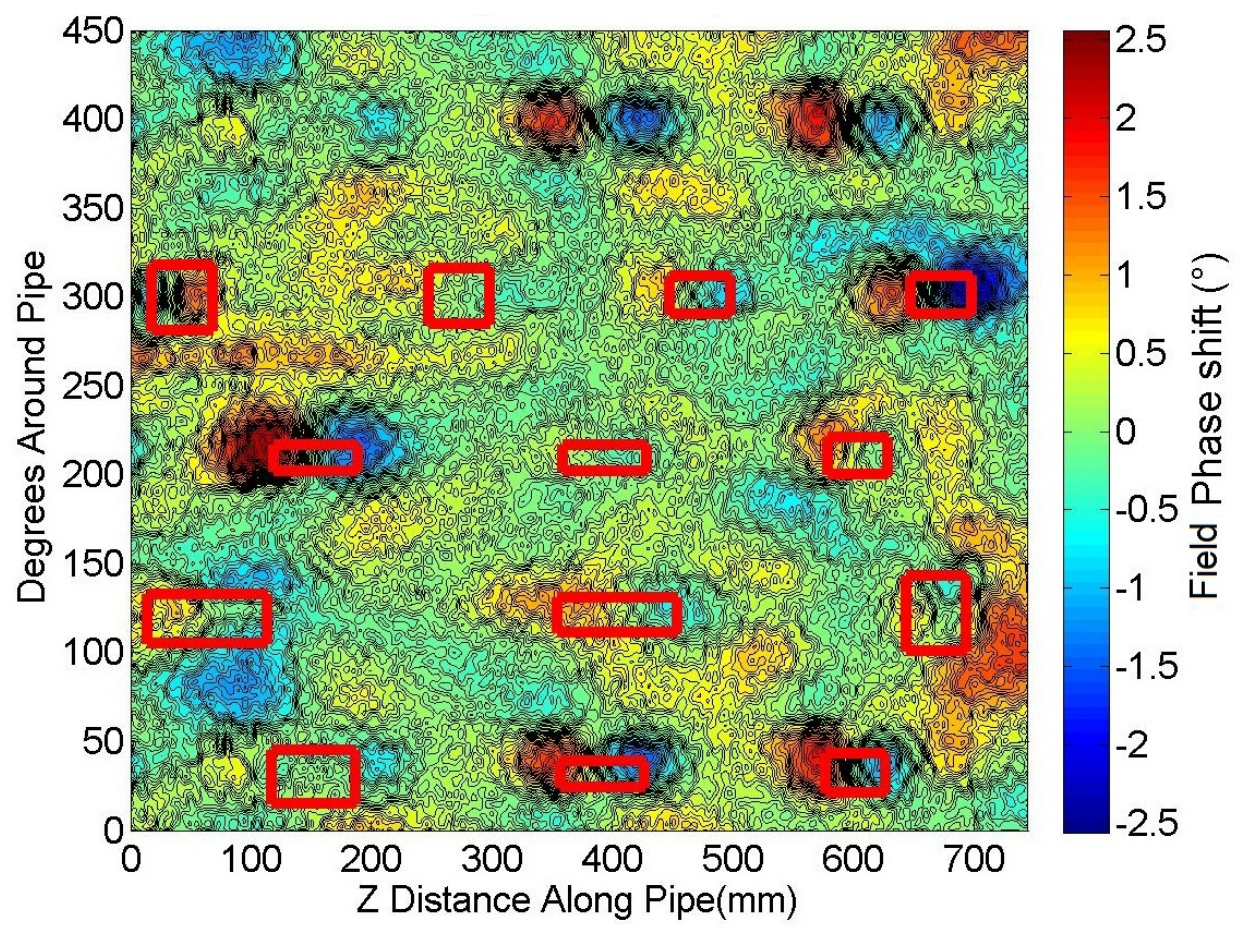

Figure 5.12: $R$ field phase shift for whole scan area with regular defects, with $Z$ flattening, current correction and Phi flattening algorithms applied

on each $\mathrm{Z}$ edge of the defect. However, the size compared to the background variation is much smaller than for the field magnitude.

A more detailed view of three defects is shown in figure 5.13(50 $\times 50$ segment defect 12), figure 5.14 ( $50 \times 50 \times 5 \mathrm{~mm}$ box defect 9$)$ and figure $5.15(50 \times 50 \mathrm{~mm}$ through hole defect 3).

The segment defect is not visible when the whole pipe is displayed due to its small size compared to other features. However, the positive and negative peaks are clearly identifiable when the small area is plotted in greater detail. These peaks are not sharp when compared to the $\mathrm{R}$ field magnitude for the same defect, which could make it hard for an automated system to identify it as a defect.

The box defect is also not visible when the whole pipe is displayed, but it becomes apparent when plotted in greater detail. Again the peaks are very noisy and a nearby area of similar positive and negative amplitude appears which would hamper automated identification.

The through hole defect shows the biggest effect for the $\mathrm{R}$ field phase shift. The peaks become clearly identifiable, but are still significantly less sharp than field magnitude signals for any of the defects.

The peak to peak signal is plotted against the defect volume in figure 5.16 and the average defect depth in figure 5.17. Unlike the $\mathrm{R}$ field magnitude, the $\mathrm{R}$ field phase shift does not show a clearly identifiable defect signal for all defects. The defects without identifiable peaks are plotted with zero peak to peak signal. A red line on each plot shows the average value of the measured defects. 


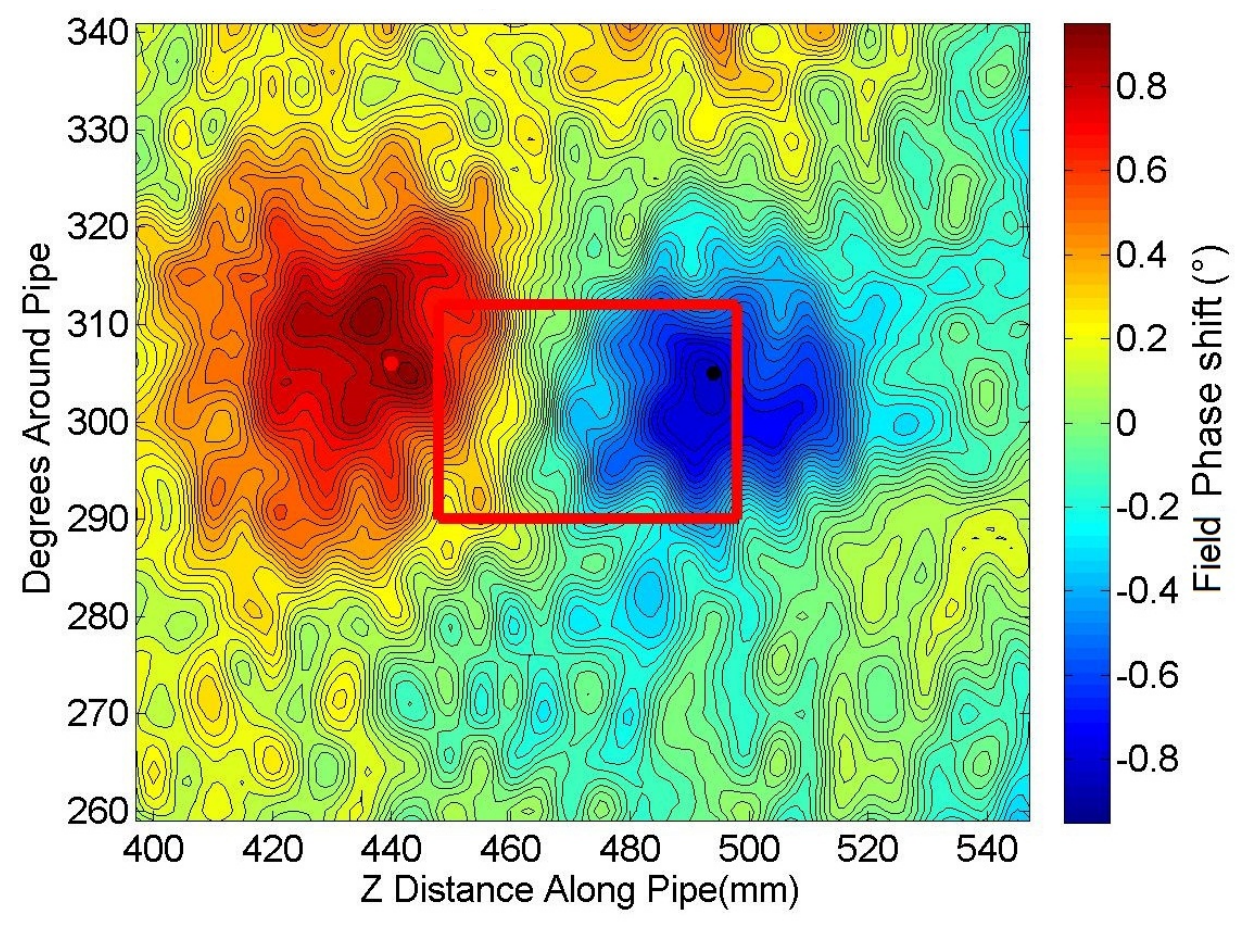

Figure 5.13: $\mathrm{R}$ field phase shift $50 \times 50$ segment defect

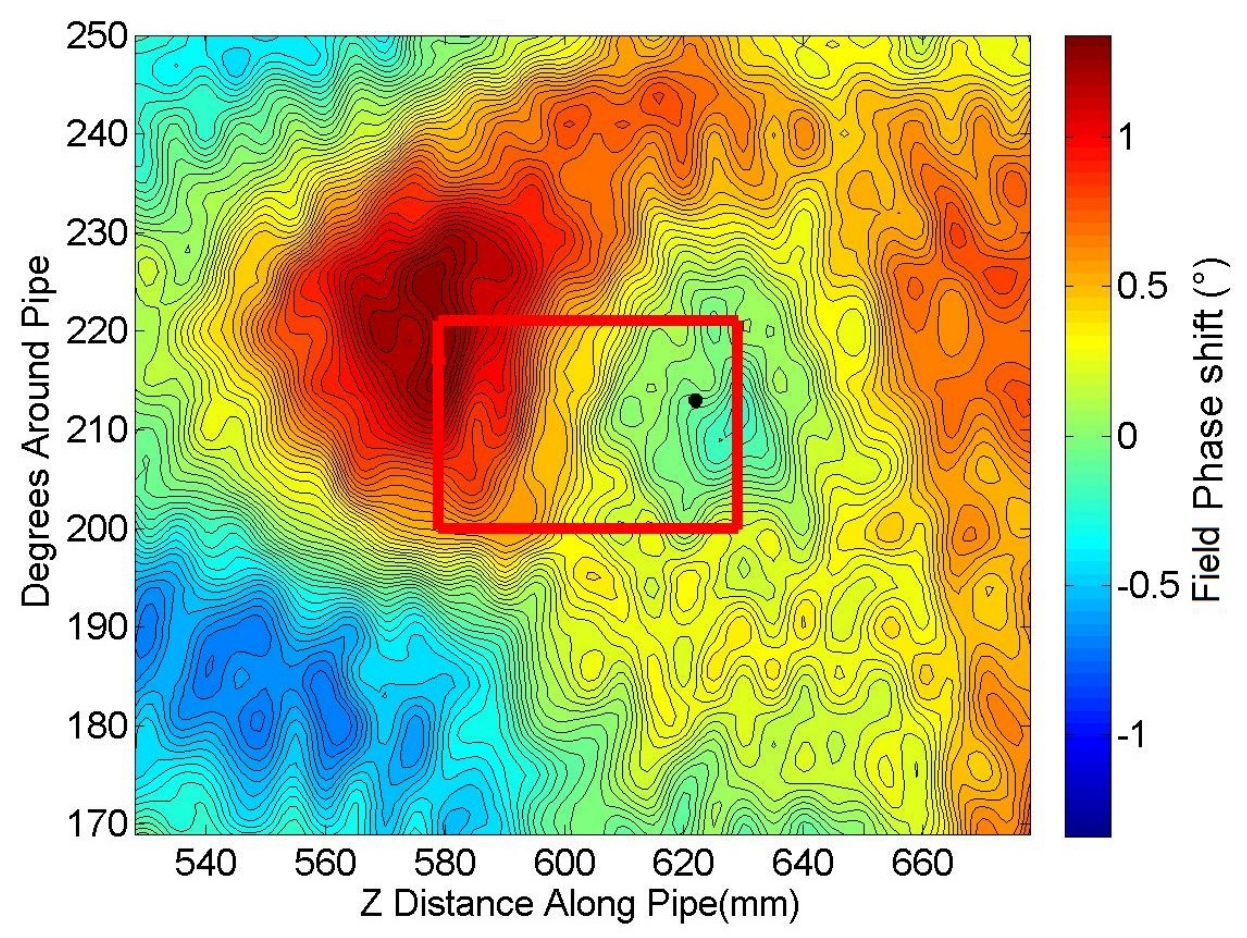

Figure 5.14: $R$ field phase shift $50 \times 50 \times 5 \mathrm{~mm}$ box defect 


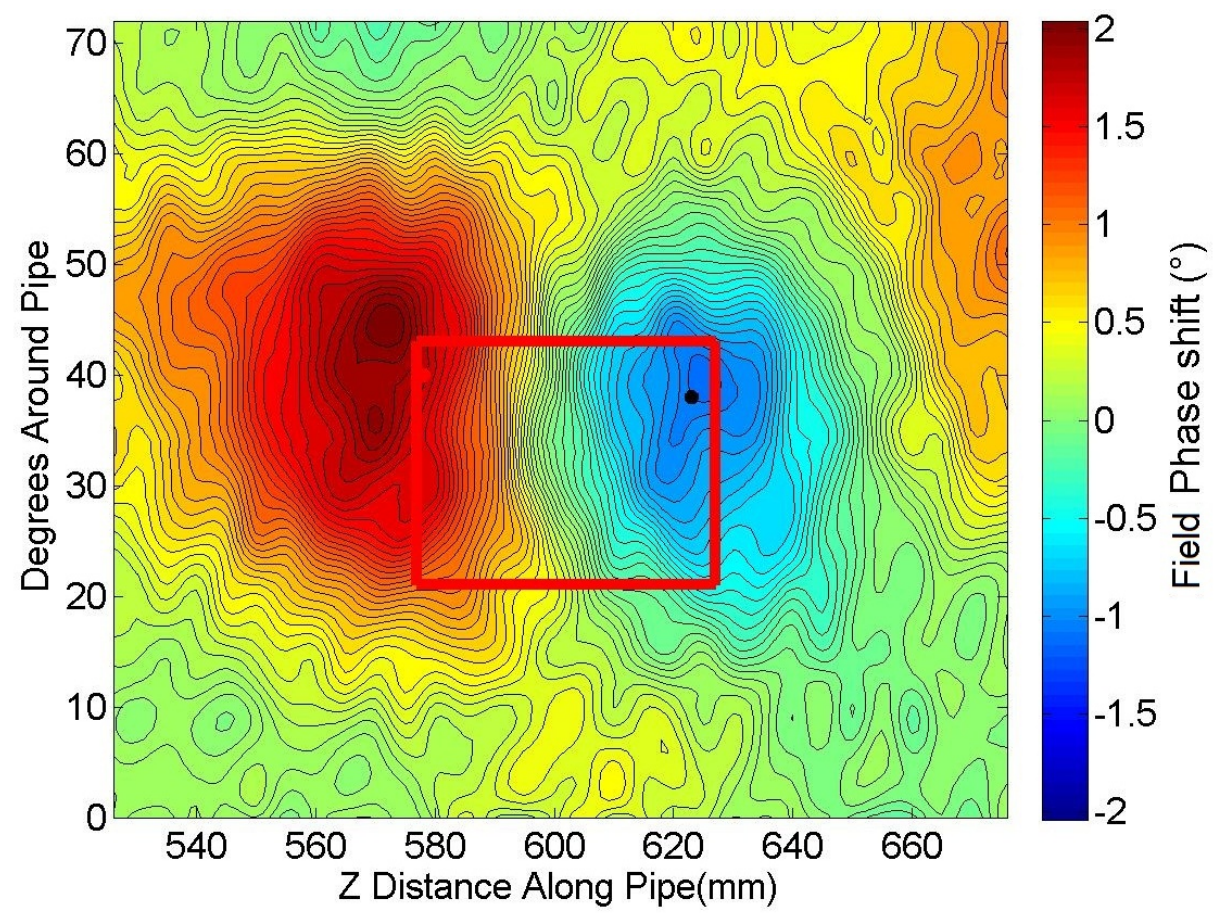

Figure 5.15: R field phase shift $50 \times 50 \mathrm{~mm}$ through hole defect

The peak to peak signal shows no clear trend vs volume or depth and shows significant variation around the mean value.

The influence of defect shape on Phi field magnitude defect signals

The Phi field magnitude contour map for the scan area is shown in figure 5.18. Unlike the $R$ field there are two positive peaks and two negative peaks, each centered approximately at the corner of the defect, as explained in section 2.5.2. Like the $\mathrm{R}$ field magnitude, the defect at 120 degrees and $700 \mathrm{~mm}$ shows a different signal shape with only two peaks. However, the two other defects at 120 degrees are more prominent than in the R field magnitude data.

The $50 \times 50 \mathrm{~mm}$ segment is plotted in more detail in figure 5.19 , the $50 \times 50 \times 5 \mathrm{~mm}$ box defect in figure 5.20 and the $50 \times 50 \mathrm{~mm}$ through hole defect in figure 5.21 .

The segment defect shows the expected two positive and two negative peaks approximately located on the corners of the defect. These signals are clearly identifiable with just one of the peaks partially obscured by a nearby variation in the field. The box defect shows a significantly higher peak to peak variation than the segment defect and all the peaks are clearly identifiable despite large changes in the background field. Like the $R$ field magnitude, the through hole defect shows the biggest peak to peak signal, which is expected as the entire wall of the pipe is removed.

The correlation of the defect volume with the peak to peak signal is shown in figure 5.22 and for the average depth in figure 5.23. The Phi field magnitude peak to peak signal change with defect volume has a good agreement with a linear fit. The Phi field magnitude peak to peak signal change with average defect depth also follows a linear fit but with larger variations. 


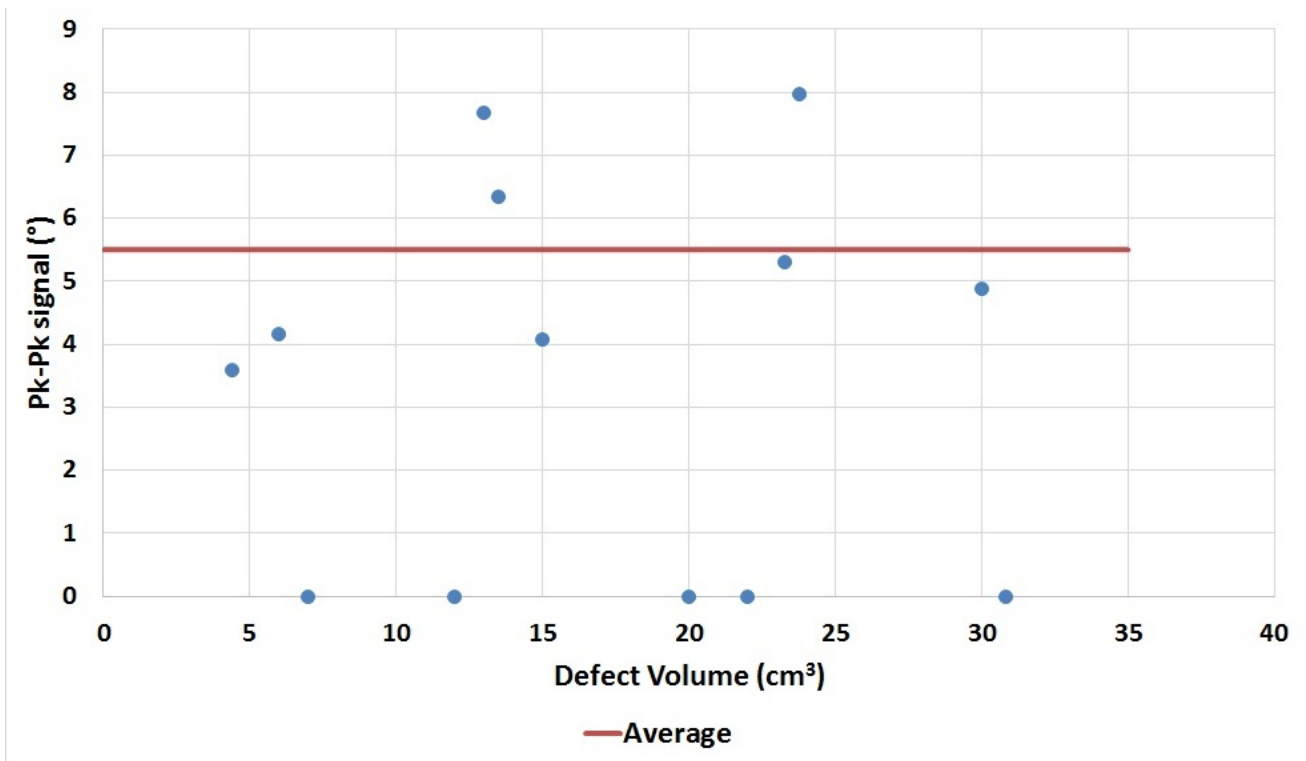

Figure 5.16: R field phase shift peak to peak signal vs defect volume

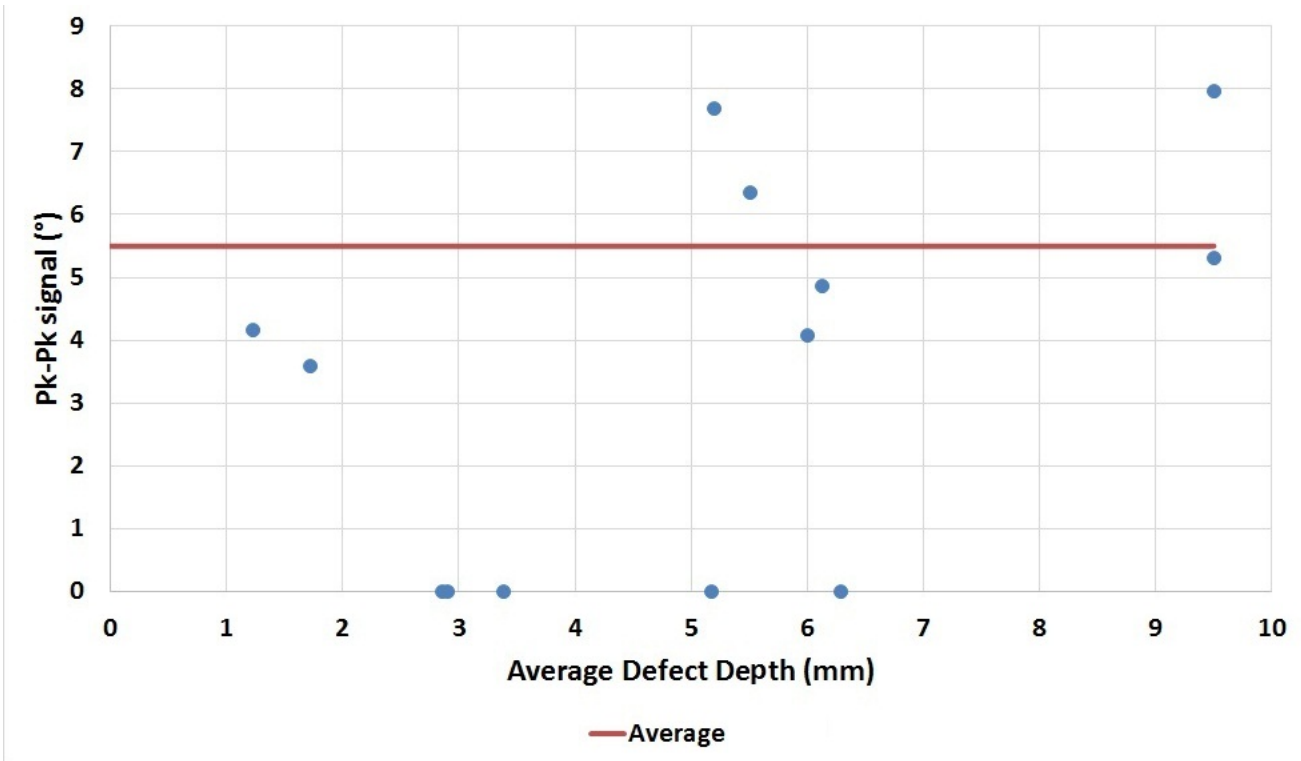

Figure 5.17: $\mathrm{R}$ field phase shift peak to peak signal compared to average defect depth 


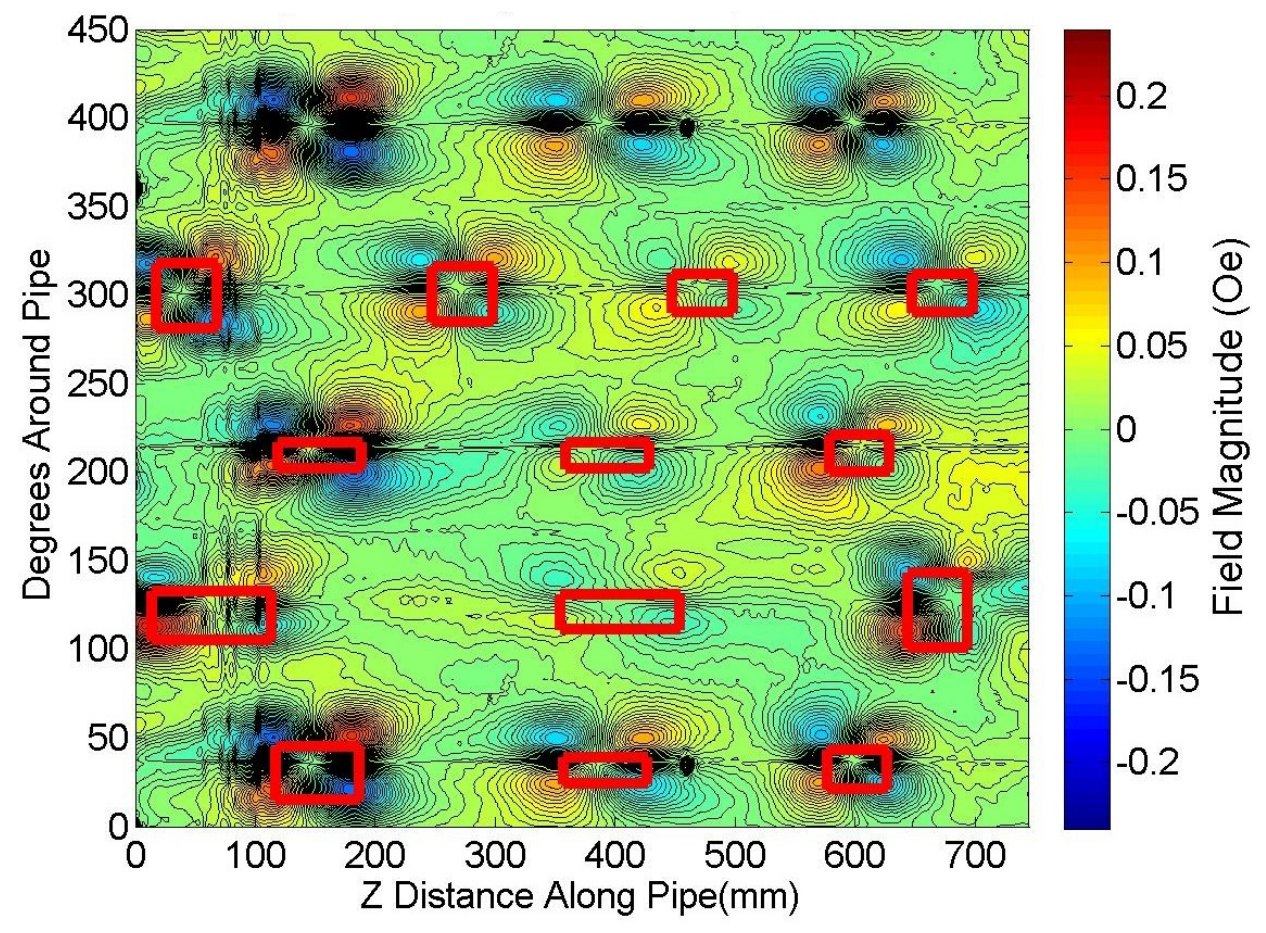

Figure 5.18: Phi field magnitude for the whole scan area with regular defects with $Z$ flattening, current correction and Phi flattening algorithms applied

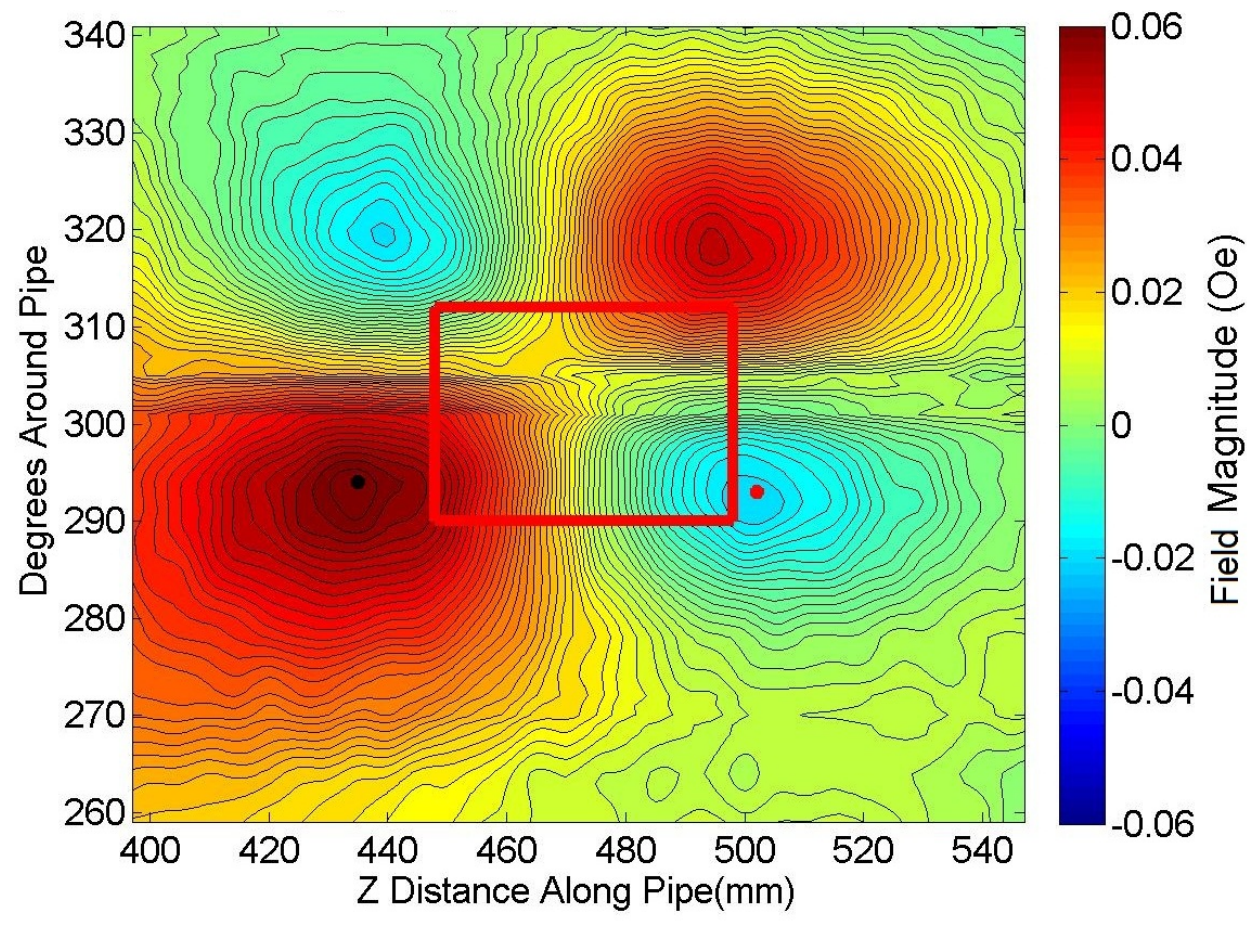

Figure 5.19: Phi field magnitude $50 \times 50 \mathrm{~mm}$ segment defect 


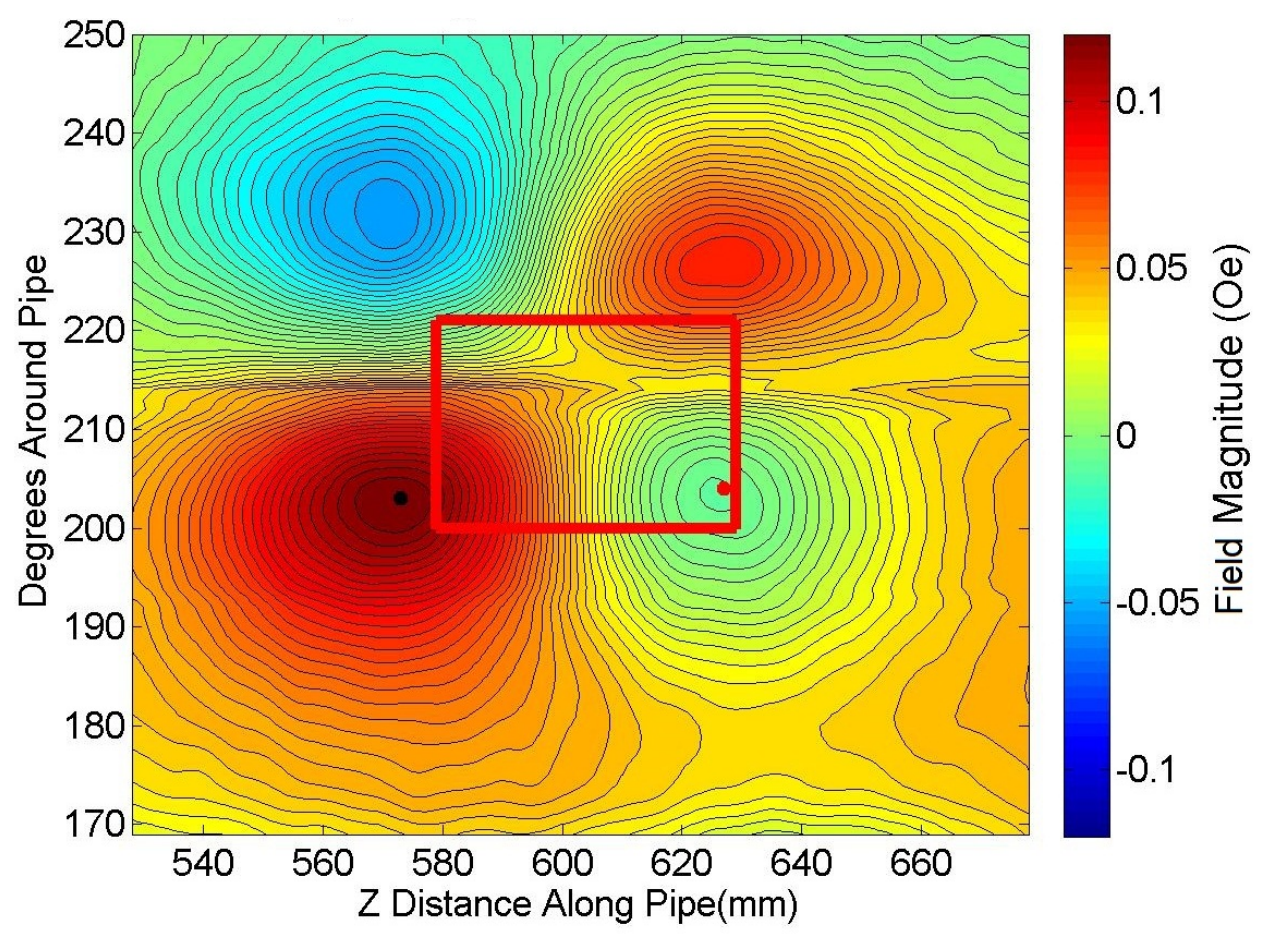

Figure 5.20: Phi field magnitude $50 \times 50 \times 5 \mathrm{~mm}$ box defect

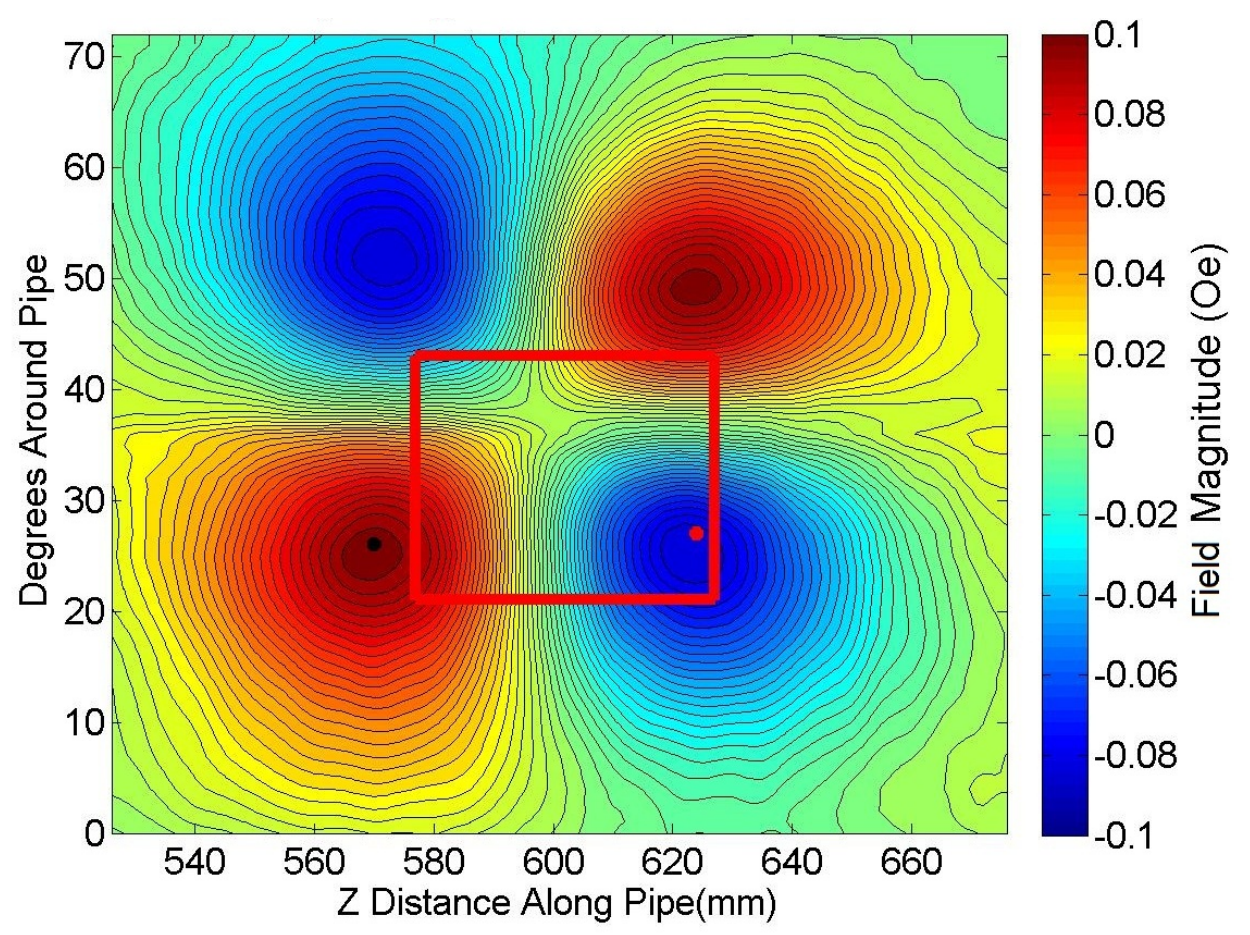

Figure 5.21: Phi field magnitude $50 \times 50 \mathrm{~mm}$ through hole defect 




Figure 5.22: Phi field magnitude defect signal peak to peak change with defect volume

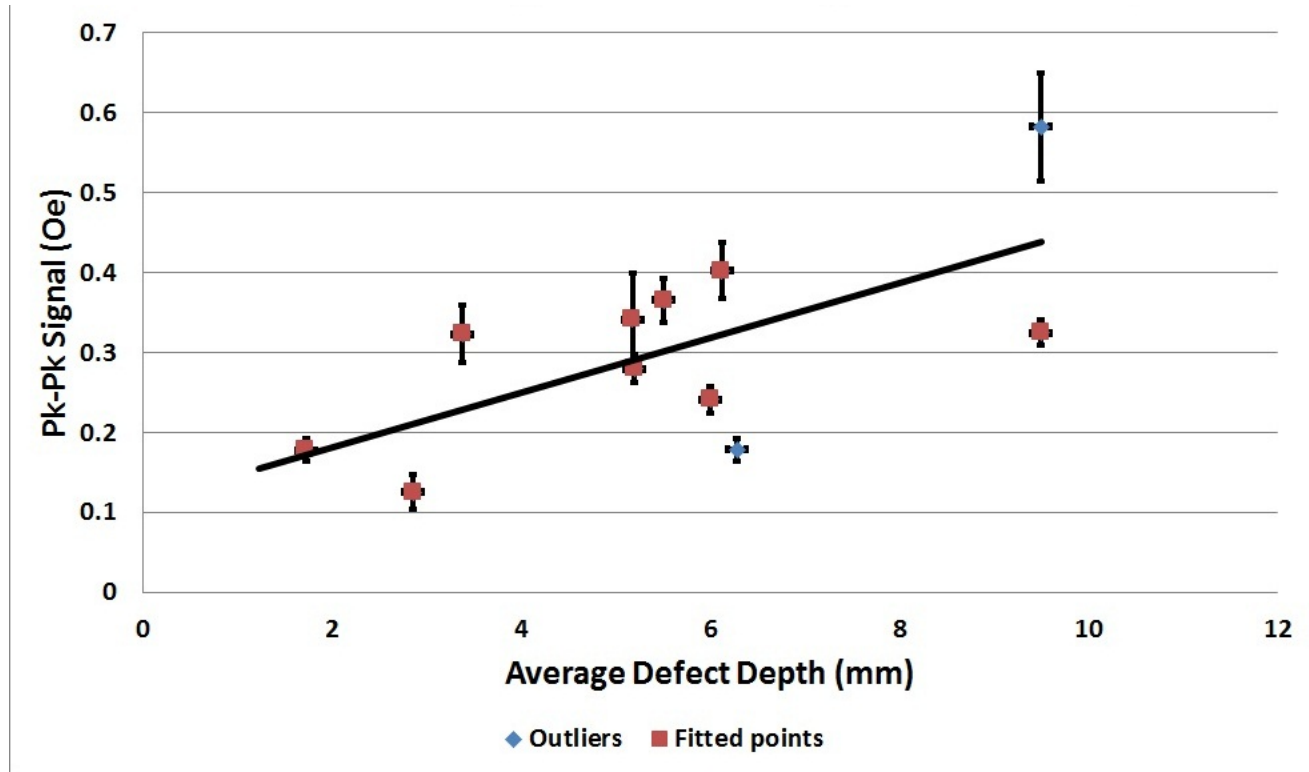

Figure 5.23: Phi field magnitude defect signal peak to peak change with average defect depth 


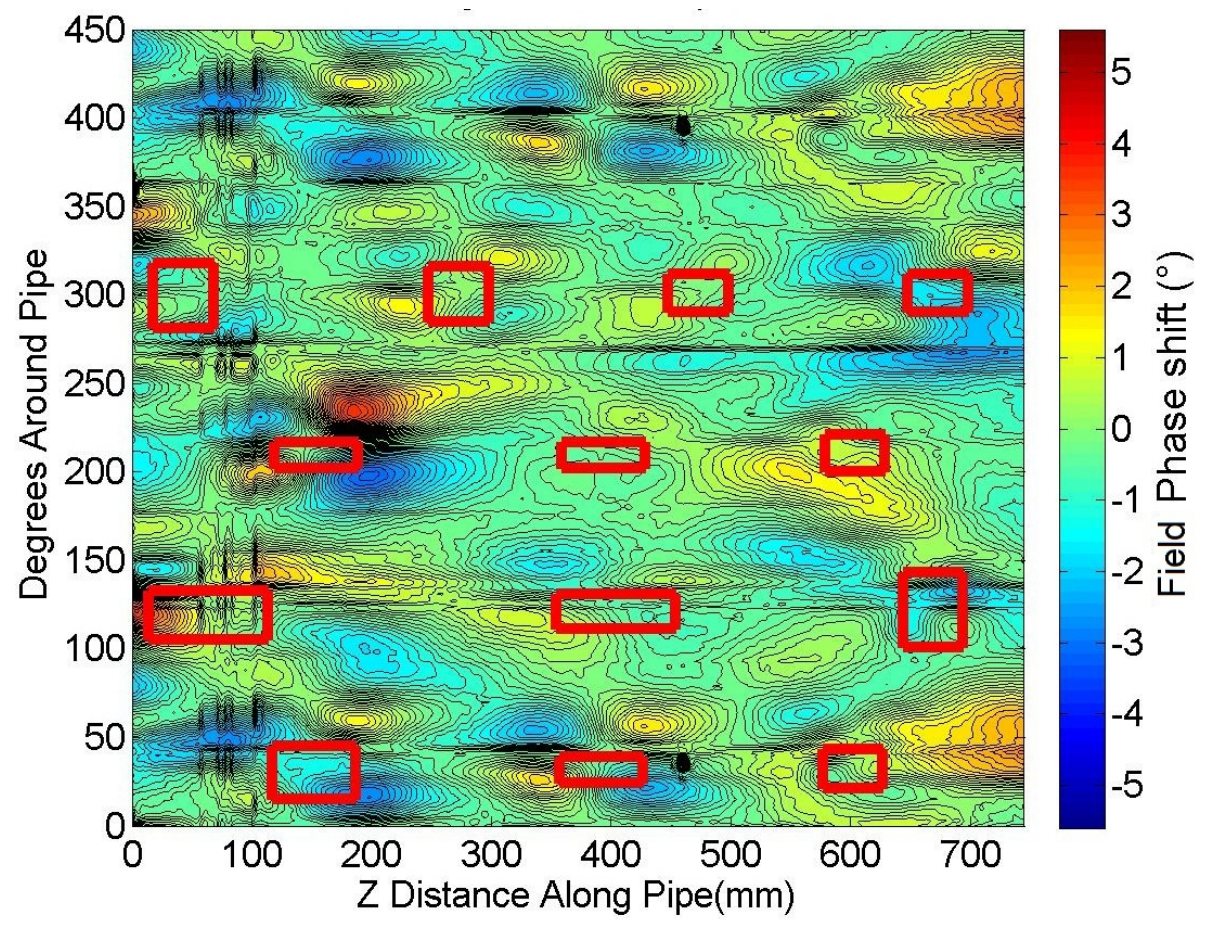

Figure 5.24: Phi field phase shift whole pipe with regular defects with Z flattening, current correction and Phi flattening algorithms applied

The influence of defect shape on Phi field Phase shift defect signals The Phi field phase shift full contour map is shown in figure 5.24. The Phi field phase change over the whole pipe shows the same four peaks centered approximately on each corner of the defects. Like the R field, the phase signal is weaker than the field magnitude with only the biggest signals showing a clear strong phase shift signal.

The more detailed contour plots of each different defect are shown for the $50 \times 50 \mathrm{~mm}$ segment defect in figure 5.25, the $50 \times 50 \times 5 \mathrm{~mm}$ box defect in figure 5.26 and the $50 \times 50$ $\mathrm{mm}$ through hole defect in figure 5.27.

The Phi field phase shift over the segment defect shows the clear two positive and two negative peaks, however they differ from a purely Gaussian shape and are just on the limit of detection. The Phi field phase shift over the box defect has a large variation nearby that obscures the defect signal. The Phi field phase shift over the through hole defect also has a large variation nearby which strongly disturbs the defect signal. This shows that local variations have a big influence on whether defect signals can be identified.

The peak to peak signal is plotted vs the defect volume in figure 5.28 and the defect average depth in figure 5.29. Unlike the Phi field magnitude, the Phi field phase shift does not show a clearly identifiable defect signal for all defects. The defects without any peaks identified are plotted with zero peak to peak signal. Two outlier points defect 1 and 6 are removed. They represent the two largest defects and have been removed before applying a linear fit. Unlike the $\mathrm{R}$ field phase shift there appears to be a linear upwards trend with both volume and average defect depth. 


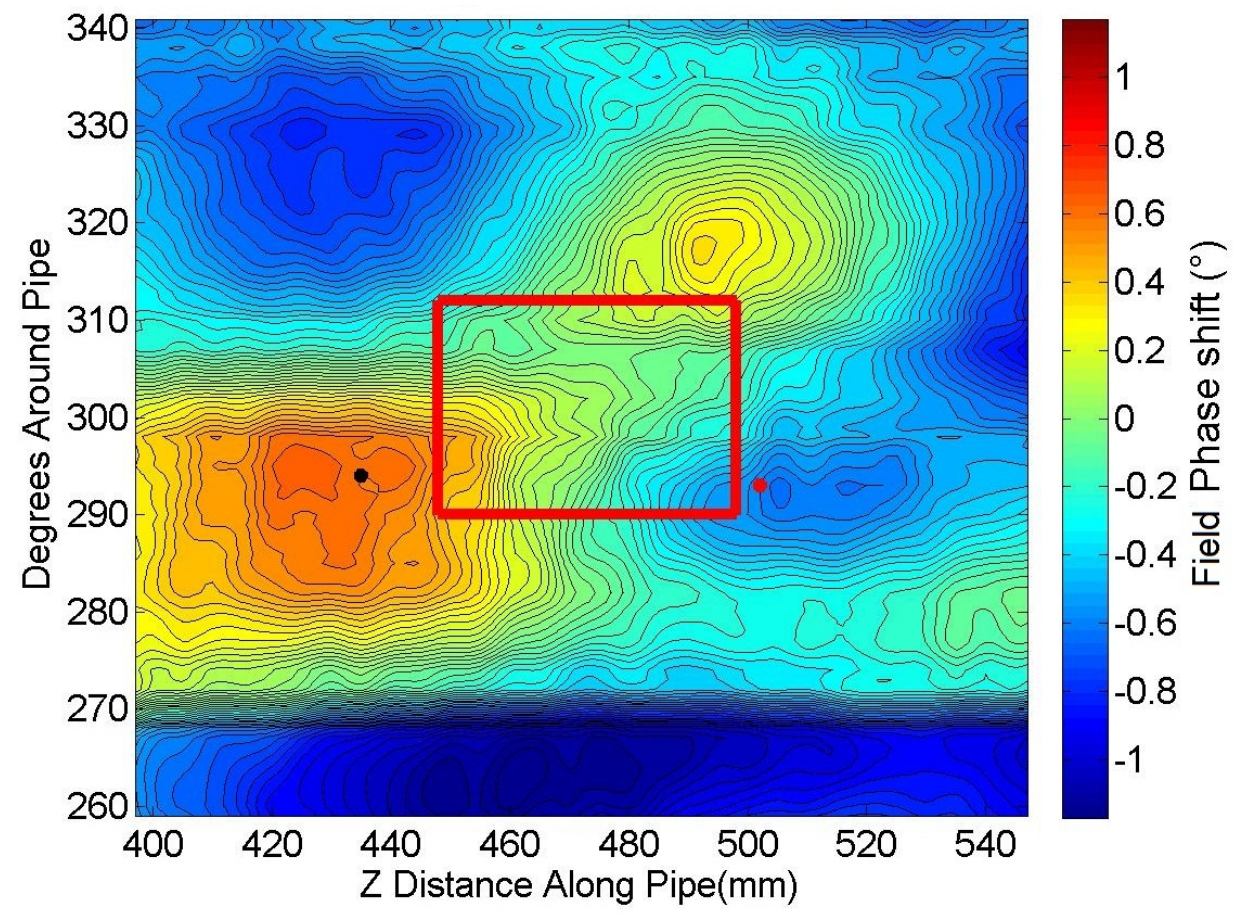

Figure 5.25: Phi field phase shift $50 \times 50 \mathrm{~mm}$ segment defect

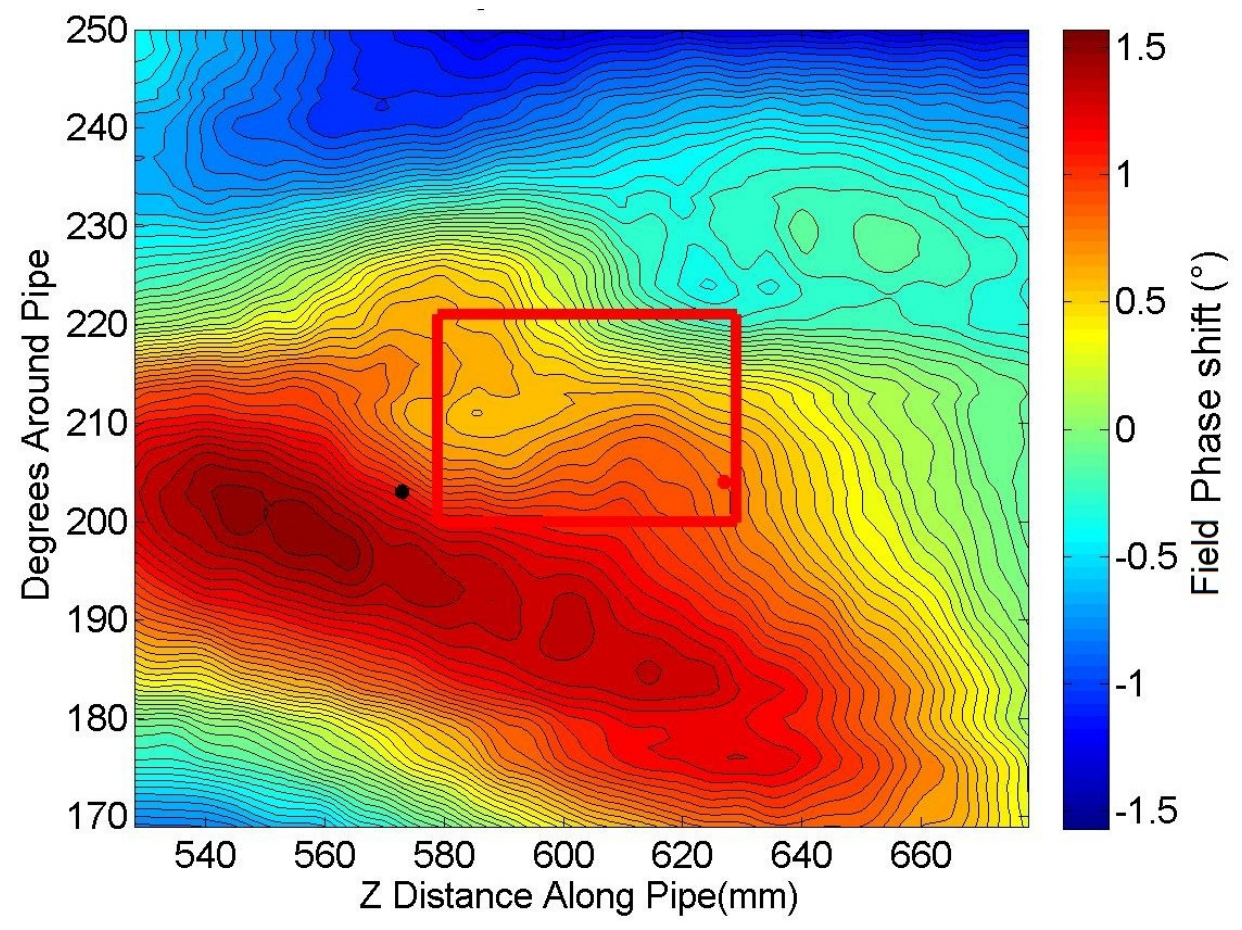

Figure 5.26: Phi field phase shift over $50 \times 50 \times 5 \mathrm{~mm}$ box defect 


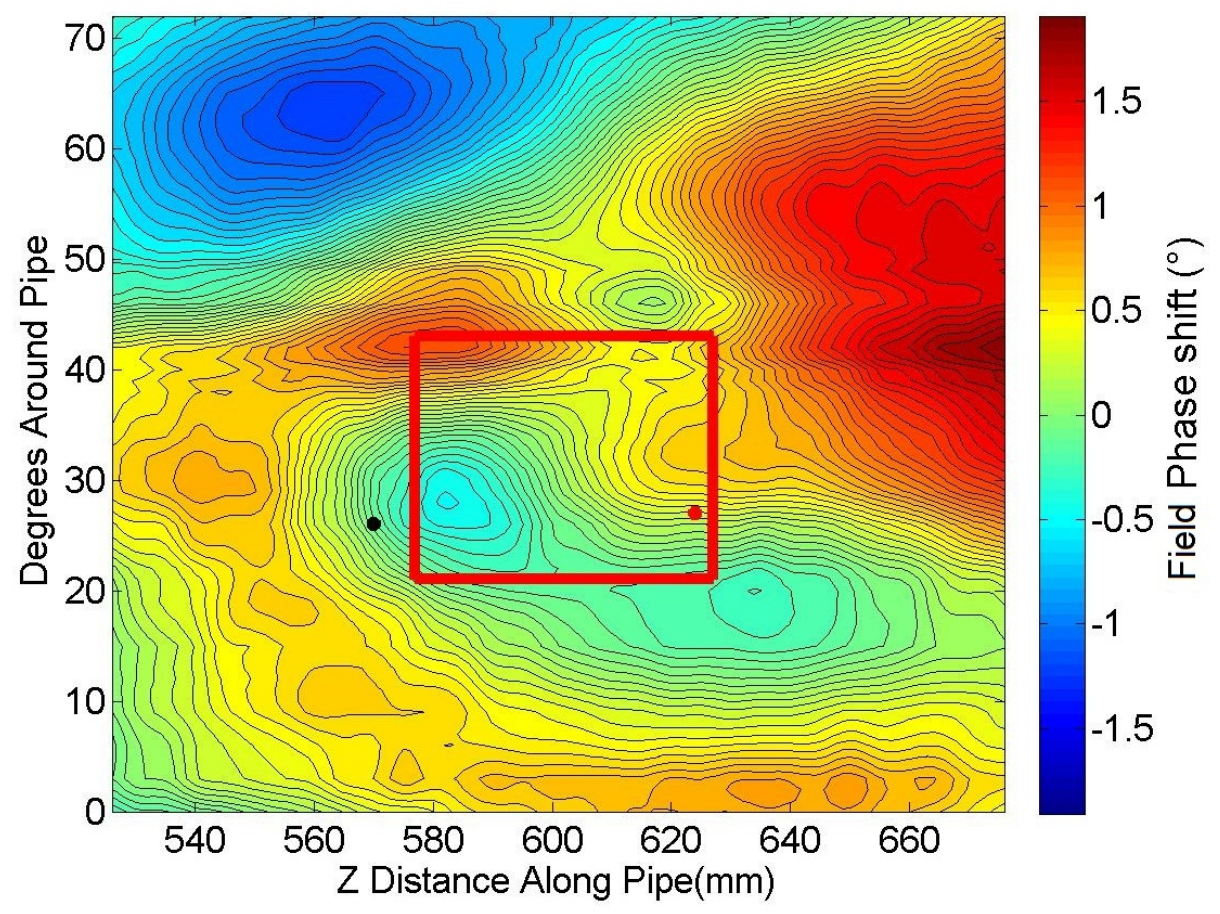

Figure 5.27: Phi field phase shift over $50 \times 50 \mathrm{~mm}$ through hole defect

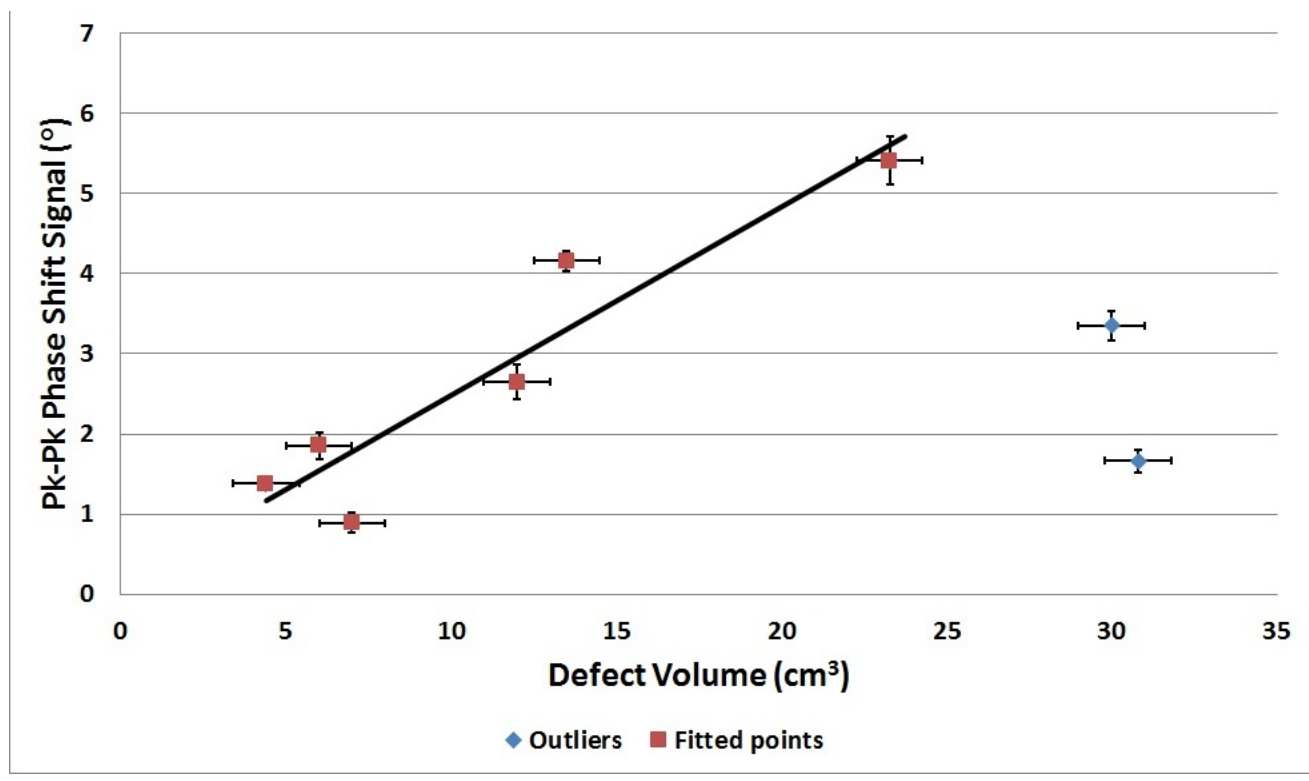

Figure 5.28: Phi field phase shift peak to peak signal change with defect volume 


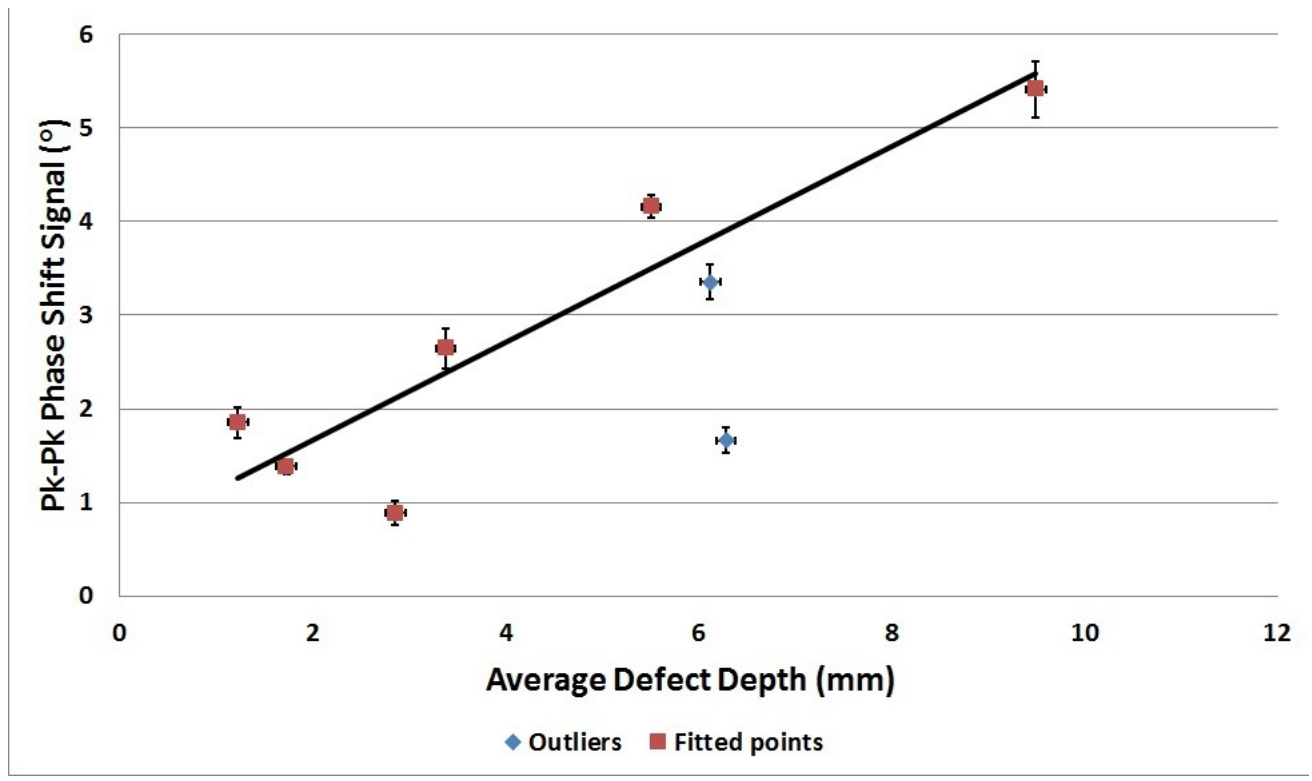

Figure 5.29: Phi field phase shift peak to peak signal change with average defect depth

\subsubsection{Discussion: Influence of defect shape on defect signals}

The results showed that the $R$ field magnitude and phase signals show a pair of positive and negative Gaussian peaks, each centered at one of the $Z$ edges of the defect. The Phi field magnitude and phase signals show two pairs of positive and negative Gaussian peaks, each centered on a corner of the defect with the positive peaks diagonally opposite each other. The peaks occur due to the way in which the eddy currents in the pipe are distorted. This distortion is described in section 2.5.2.

The phase shift signals show the same shape as the field magnitudes. As the eddy currents around the Phi or R axis become larger or smaller, seen in the field magnitude as positive or negative peaks, the phase shift of the magnetic field rotates towards or away from the phase shift of the eddy current. The direction of the rotation depends on whether the field magnitude increases or decreases. Therefore, magnitude and phase shift peaks for each axis occur in the same locations.

The Phi field magnitude peak to peak signal is $30 \%$ smaller than the $\mathrm{R}$ field, indicating that the level of distortion of eddy currents around the Phi axis is lower than around the $R$ axis.

It was also found that the defect signal peak to peak size is largely dependent on the defect volume. As the volume of the defect increases, the level of distortion of the eddy currents is increased, as they are forced to deviate further from their original path. However, as can be seen in the fits, the defect volume does not fully explain the peak to peak amplitude. This means the shape of the defect will probably have an effect on the size of the peak to peak signals as the shape of the defect determines how the eddy currents are redistributed. 


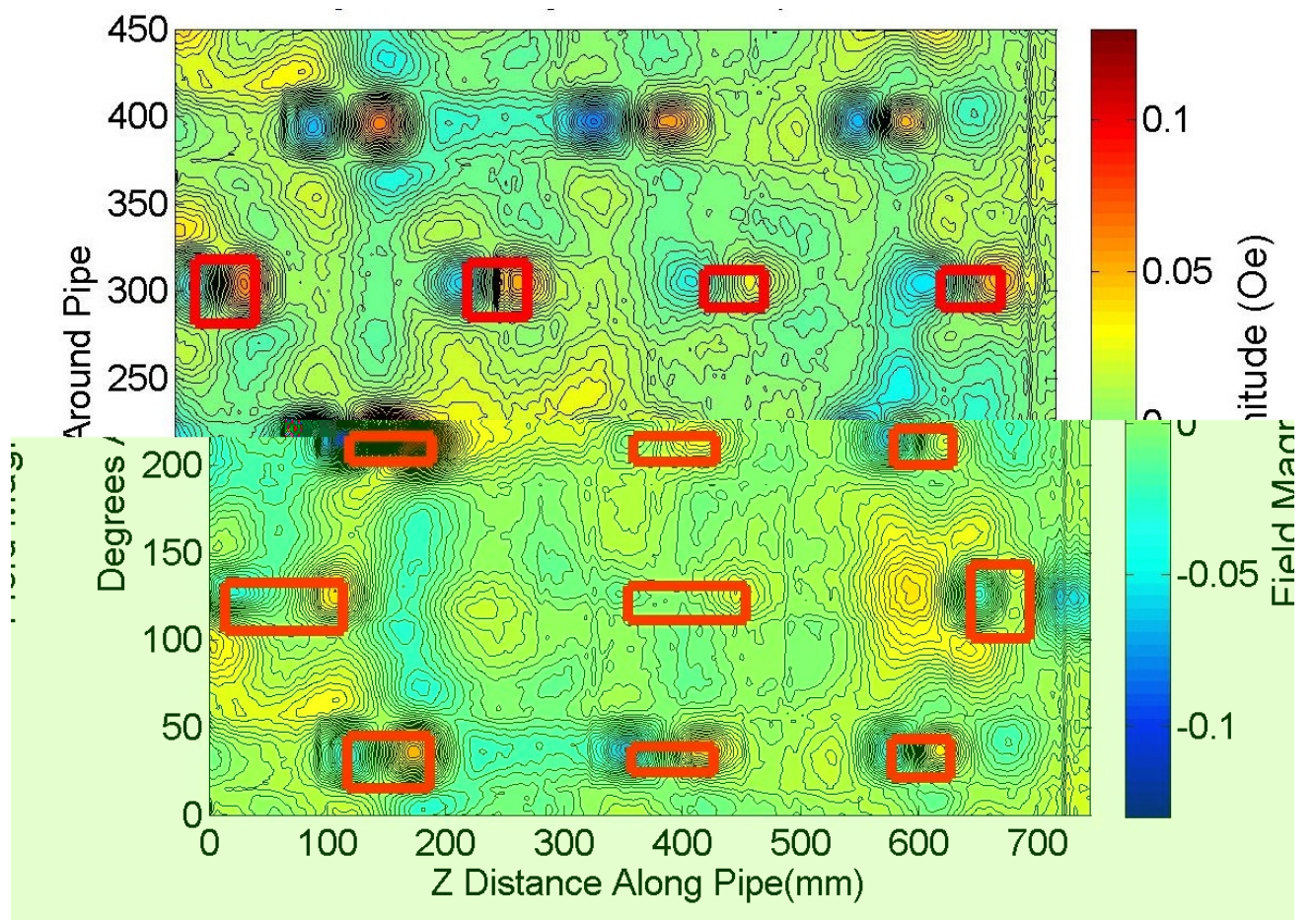

Figure 5.30: R field magnitude, regular defects, $100 \mathrm{~A}$ excitation current

\subsubsection{Influence of excitation current on defect signals}

The influence of increasing excitation current on the peak to peak signal of the defects was investigated to determine the optimum excitation current. The same range of excitation currents was used as was used for experiments with the standard pipe measurement in section 4.4 (100 A to $600 \mathrm{~A})$.

\section{Influence of excitation current on $\mathbf{R}$ field magnitude defect signals}

To compare the general effect of increasing the excitation current, figure 5.30 (100 A excitation current) is compared with figure $5.31(500 \mathrm{~A})$. The $\mathrm{R}$ field magnitude at $100 \mathrm{~A}$ excitation current shows clearly identifiable defect signals for all but one defect. This shows that the same level of identification can be achieved at lower excitation current. The $R$ field magnitude at $500 \mathrm{~A}$ excitation current shows almost identical behavior as the field at $100 \mathrm{~A}$ excitation current but it has five times larger absolute signals.

The peak to peak values are plotted against the excitation current in figure 5.32 for defects 3,9 and 12 . The linear fit of the standard deviation with changing excitation current for the plain pipe found in section 4.4 is multiplied by six to show the three standard deviation point of the background noise for comparison. Figure 5.32 shows that there is a good linear relationship for the peak to peak signal with excitation current for all three defect signals. The three standard deviation line also shows that all three defect signals are well above three standard deviations from the mean field.

To quantify the SNR change with excitation current, the SNR is calculated for each excitation current for each of the three defects $(3,9,12)$ and plotted in figure 5.33 . The fit 


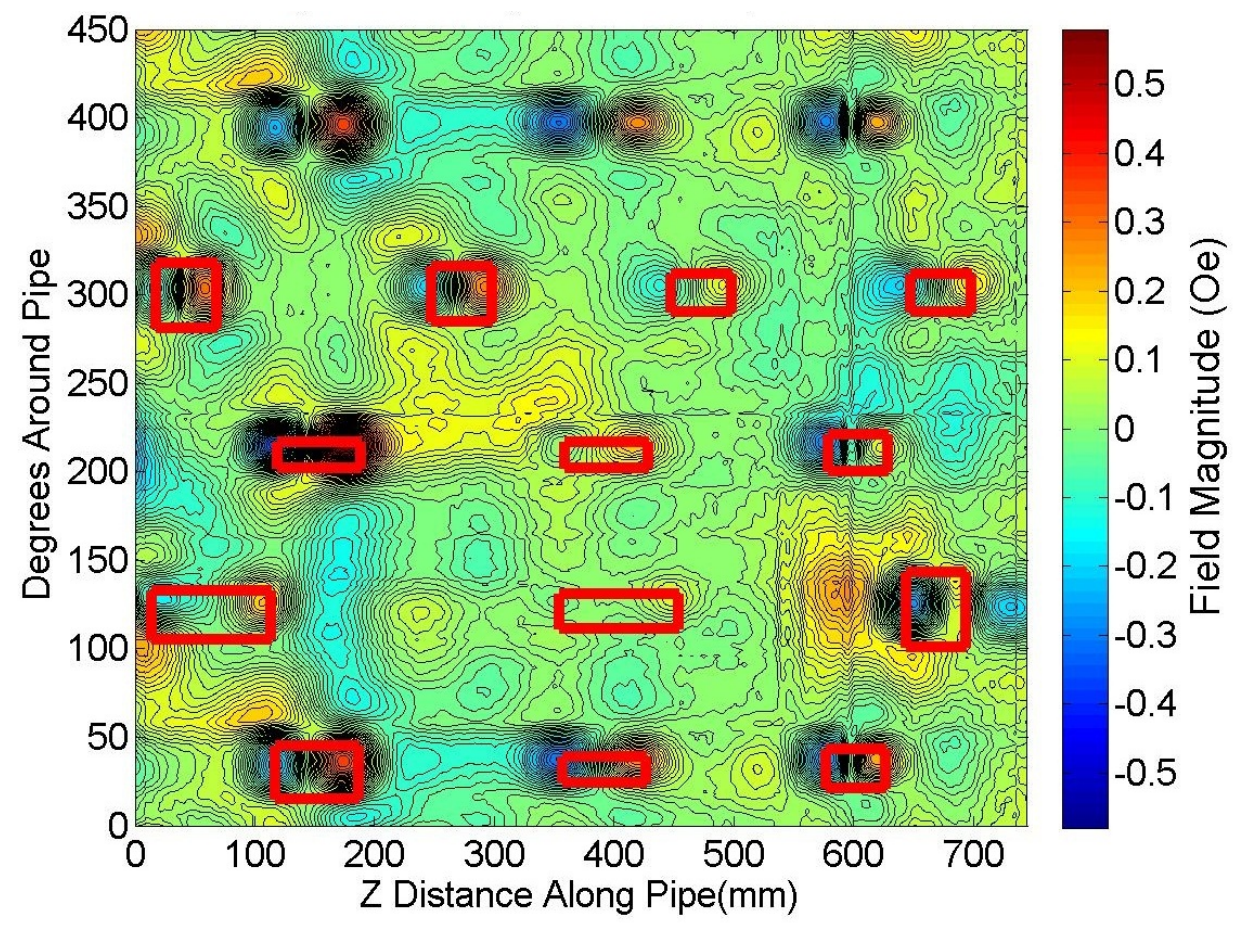

Figure 5.31: $\mathrm{R}$ field magnitude, regular defects, $500 \mathrm{~A}$ excitation current

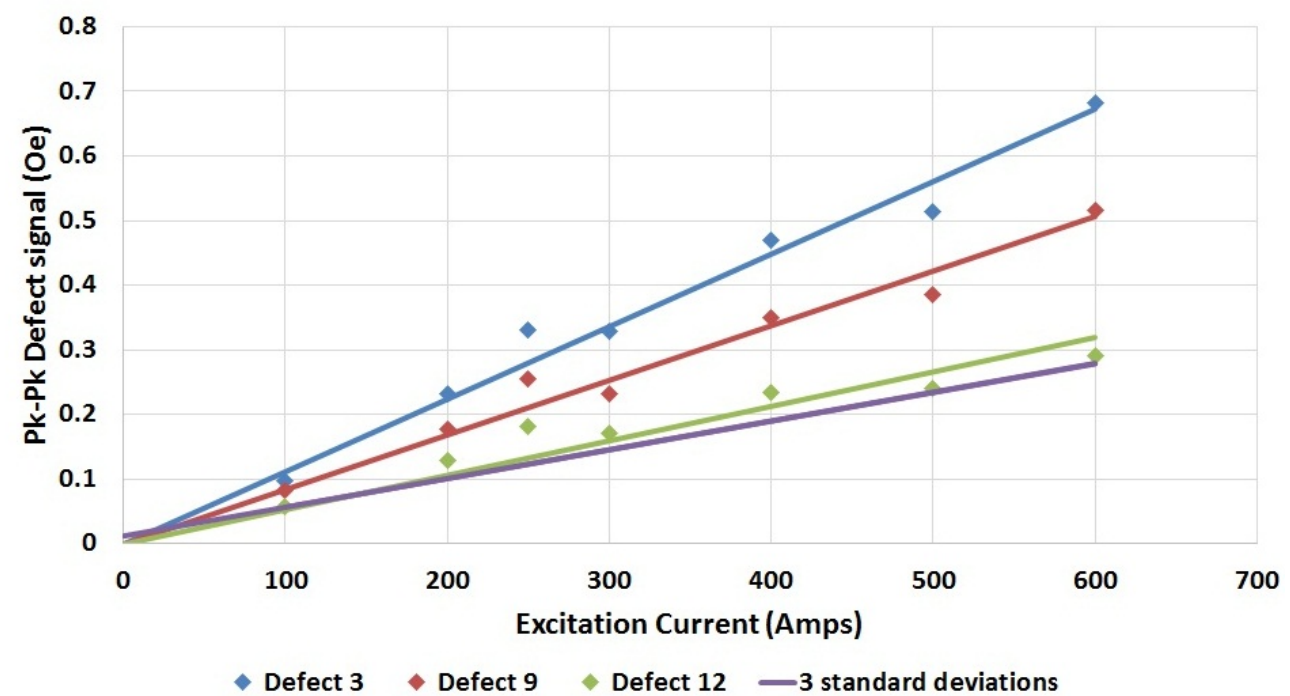

Figure 5.32: $\mathrm{R}$ field magnitude peak to peak change with excitation current 


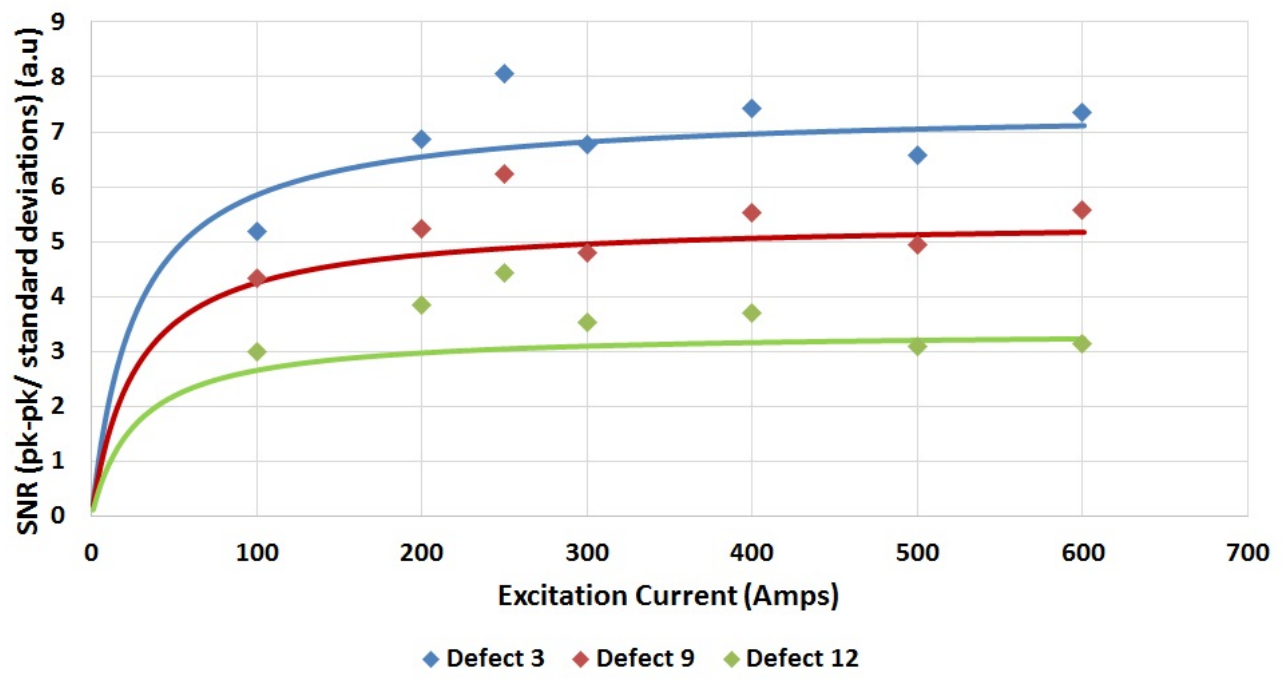

Figure 5.33: R field magnitude defect signals SNR change with excitation current (predicted lines and measured points)

lines are calculated from the linear fits obtained for the peak to peak signal change with current and the plain pipe standard deviation change with current. The predicted rapid decay to zero under 100 A must occur because, by definition, the SNR must be zero at zero current.

The R field SNR plot shows that excitation current needed to maximize the SNR for a given defect depends on the size of the defect, with defect 12, the smallest defect, reaching $95 \%$ of its maximum value at a lower current than defect 3 , the largest defect. For this range of defects there is no advantage in increasing the excitation current above $300 \mathrm{~A}$, but using excitation current below 200 A means that SNR will start to rapidly decay.

\section{Influence of excitation current on Phi field magnitude on defect signals}

The full pipe contour is shown at $100 \mathrm{~A}$ excitation current in figure 5.34 and at $500 \mathrm{~A}$ in figure 5.35. The Phi field at $100 \mathrm{~A}$ excitation current shows clearly identifiable peaks for all defects, however the largest defect shows a distortion. Compared to the $\mathrm{R}$ field sensor, these signals are easy to identify despite a considerably smaller signal. The Phi field at 500 A excitation current again shows clearly identifiable peaks for all the defects, with similar distortion for the whole pipe surface. Again, like the R sensor, the field level is approximately five times larger due to the higher current.

The peak to peak values of the same three defects are again calculated and plotted in figure 5.36 with the three standard deviation line from the plain pipe data also plotted for comparison. Figure 5.36 shows good linear behavior for each defect. However, the $50 \mathrm{x}$ $50 \mathrm{~mm}$ segment is only at the 3 standard deviation level of the plain pipe noise unlike in the $\mathrm{R}$ field where this defect is at approximately 3.5 standard deviations.

To determine the excitation level required to get the best possible defect signal from the Phi field sensor, the SNR of each defect is calculated and plotted in figure 5.37. The fit lines are calculated from the linear fits obtained for the peak to peak signal change with current and the plain pipe standard deviation change with current. The SNR for Phi field 


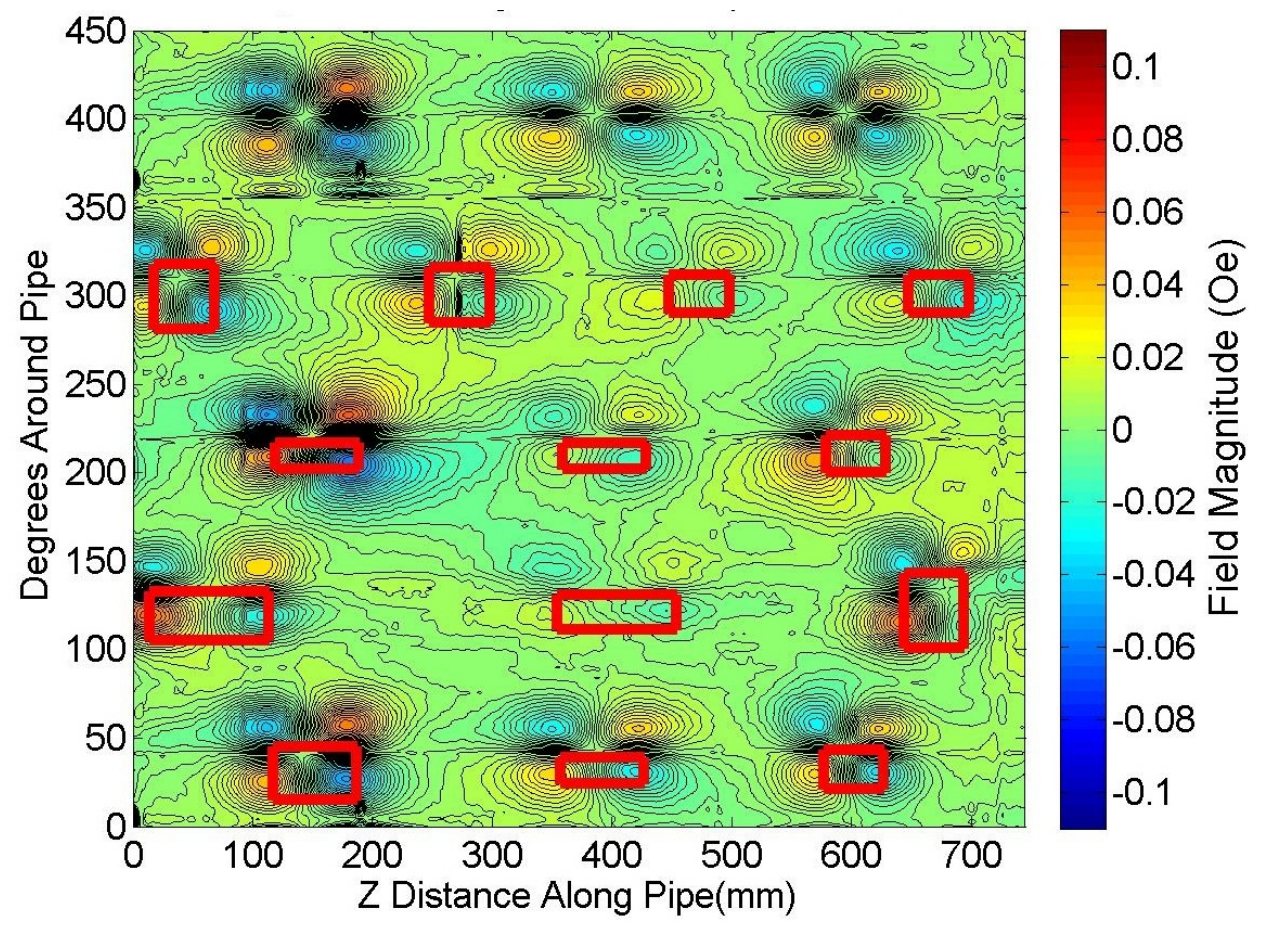

Figure 5.34: Phi field magnitude, full scan area, $100 \mathrm{~A}$ excitation current

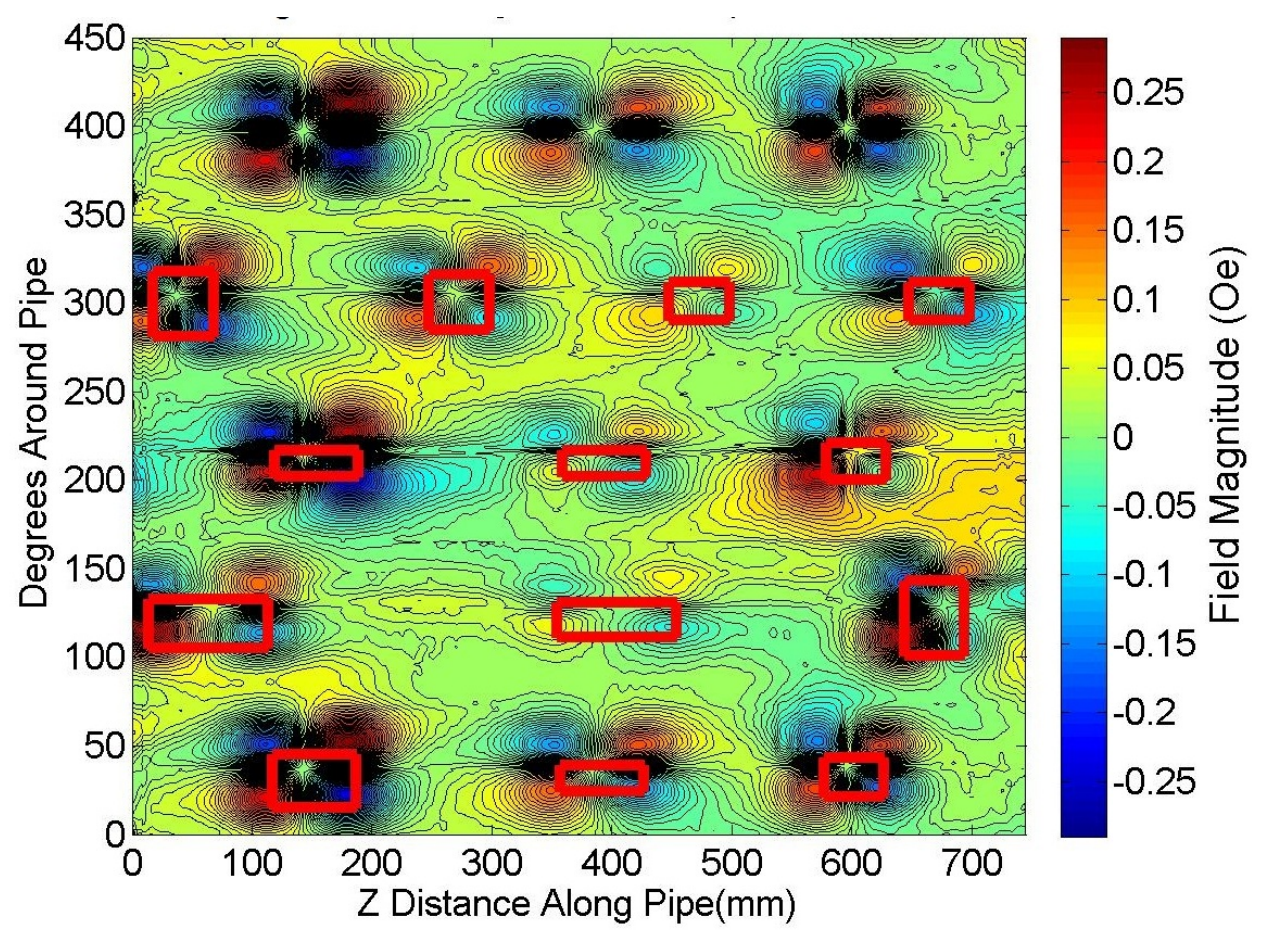

Figure 5.35: Phi field magnitude, full scan area, $500 \mathrm{~A}$ excitation current 


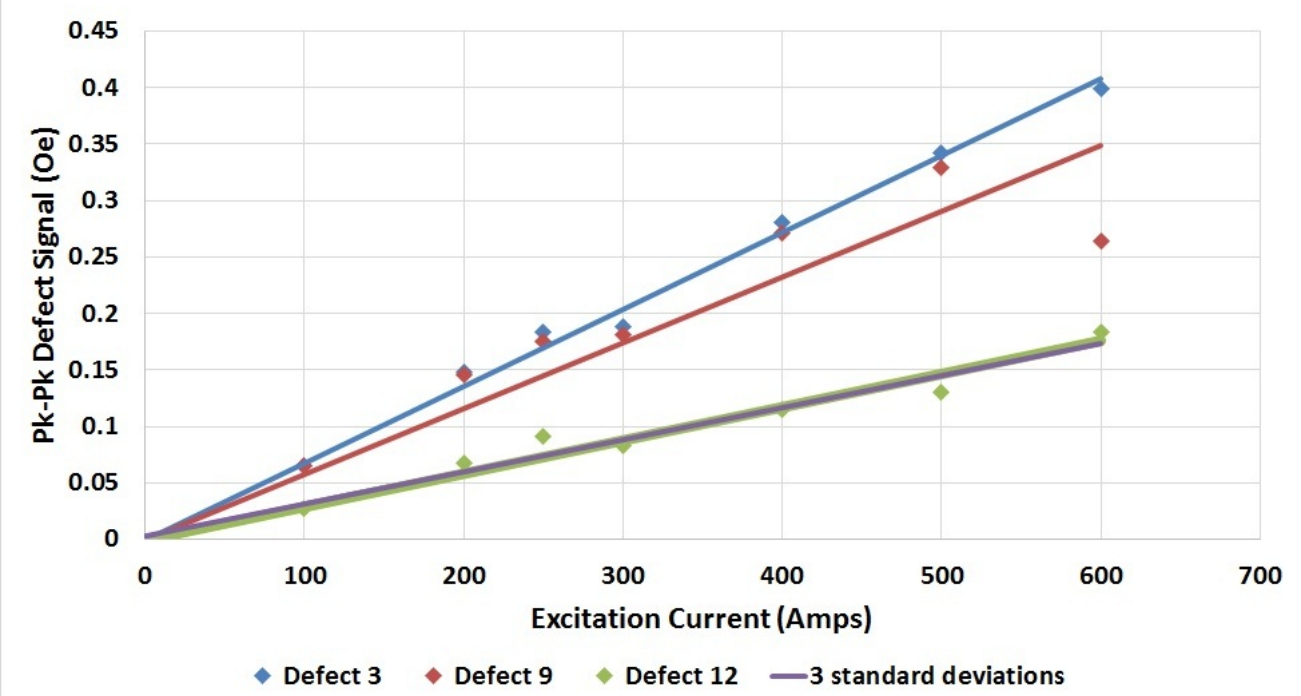

Figure 5.36: Phi field magnitude peak to peak signal change with excitation current

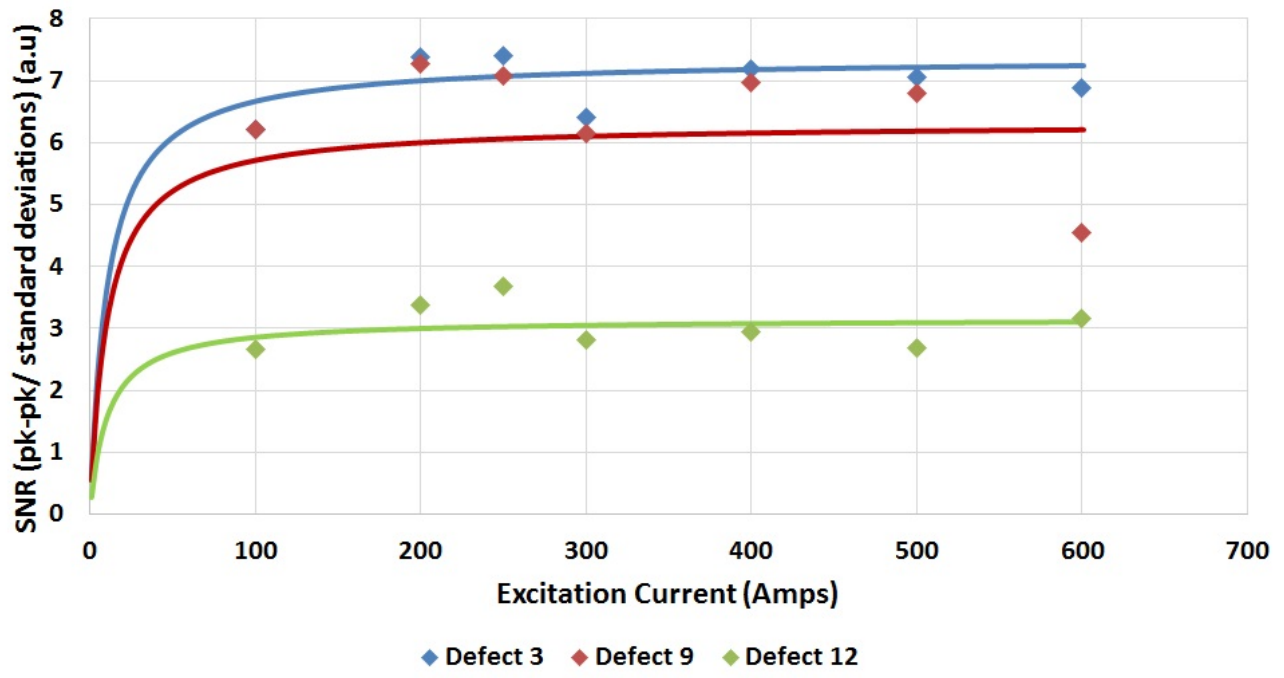

Figure 5.37: Phi field magnitude SNR change with excitation current(predicted lines and measured points) 


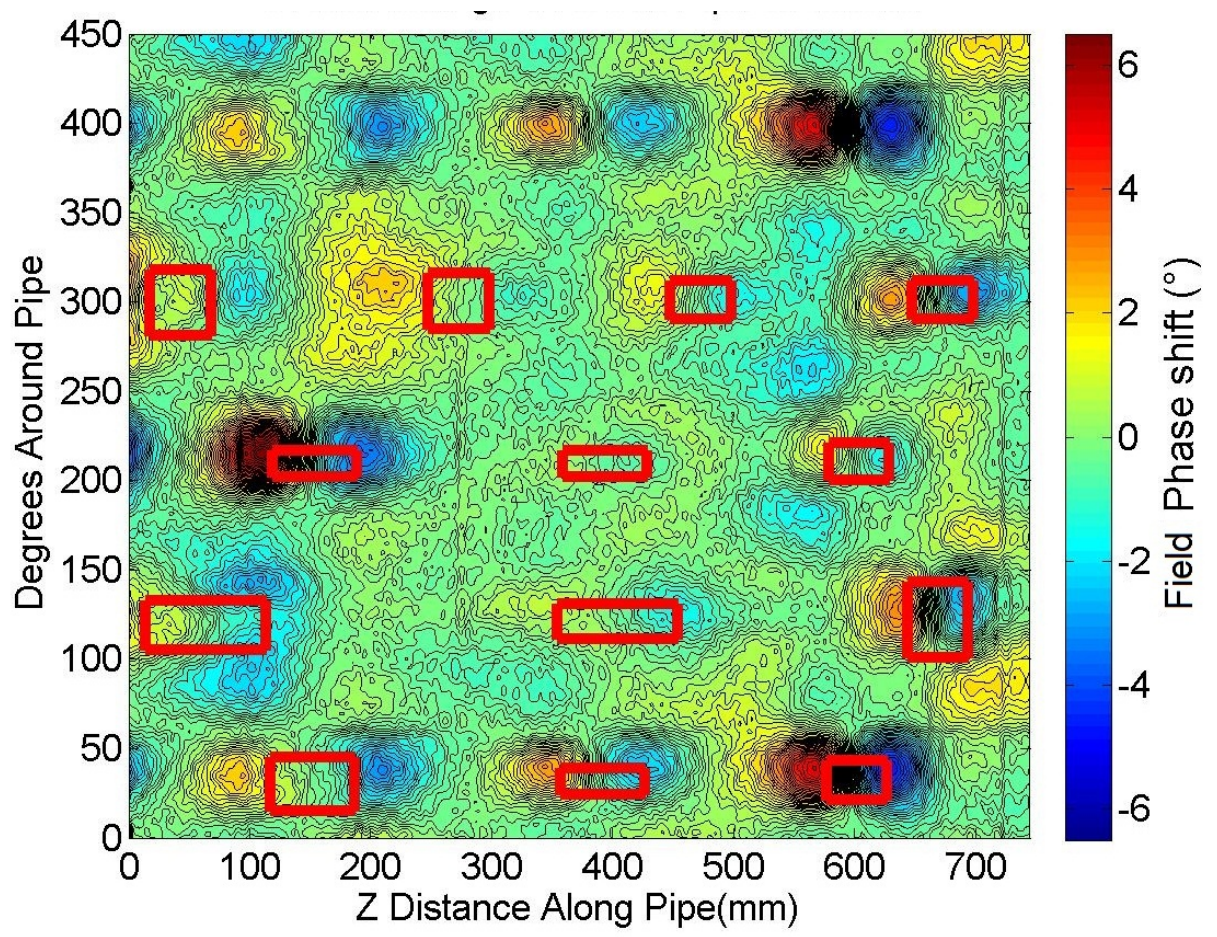

Figure 5.38: $\mathrm{R}$ field phase shift, full scan area, $100 \mathrm{~A}$ excitation current

magnitude shows that a lower current is required to maximize the SNR with approximately 250 A excitation current giving a maximum response. The SNR value for the Phi field is similar to the R field for the $50 \times 50 \mathrm{~mm}$ segment defect and the $50 \times 50 \mathrm{~mm}$ through hole defect while the $50 \times 50 \times 5 \mathrm{~mm}$ box defect shows a significantly higher SNR. This means the magnitude change in both the Phi and R fields is a good parameter to identify defects.

\section{Influence of excitation current on $\mathbf{R}$ field phase shift defect signals}

The $R$ field phase shift contour plots are shown in figure 5.38 for $100 \mathrm{~A}$ and in figure 5.39 for $500 \mathrm{~A}$. At $100 \mathrm{~A}$ excitation the defect signals can be clearly identified for most defects. The background level has larger variations, showing a significant amount of measurement jitter. At 500 A excitation current noise spikes appear in the data for the larger defects which makes smaller defects hard to identify when the whole pipe is viewed. However, the defect signal size has increased slightly from the $100 \mathrm{~A}$ scan.

The peak to peak value for the same three defects is again calculated and plotted in figure 5.40 with the 3 standard deviation line from the plain pipe data for comparison. The $R$ field phase shift defect signal shows no clear trend with excitation current. The large values around $500 \mathrm{~A}$ and $100 \mathrm{~A}$ suggest there are significant advantages to adjusting the excitation current to achieve higher peak to peak amplitudes which may at occur at both high and low currents.

The $R$ field phase shift SNR vs excitation current in figure 5.41 shows that a low excitation current is just as good as a high excitation current, with the SNR for the defects at 100 A equal to or better than SNR at any other excitation current. However, the large variation in data suggests that another parameter is affecting the phase shift. 


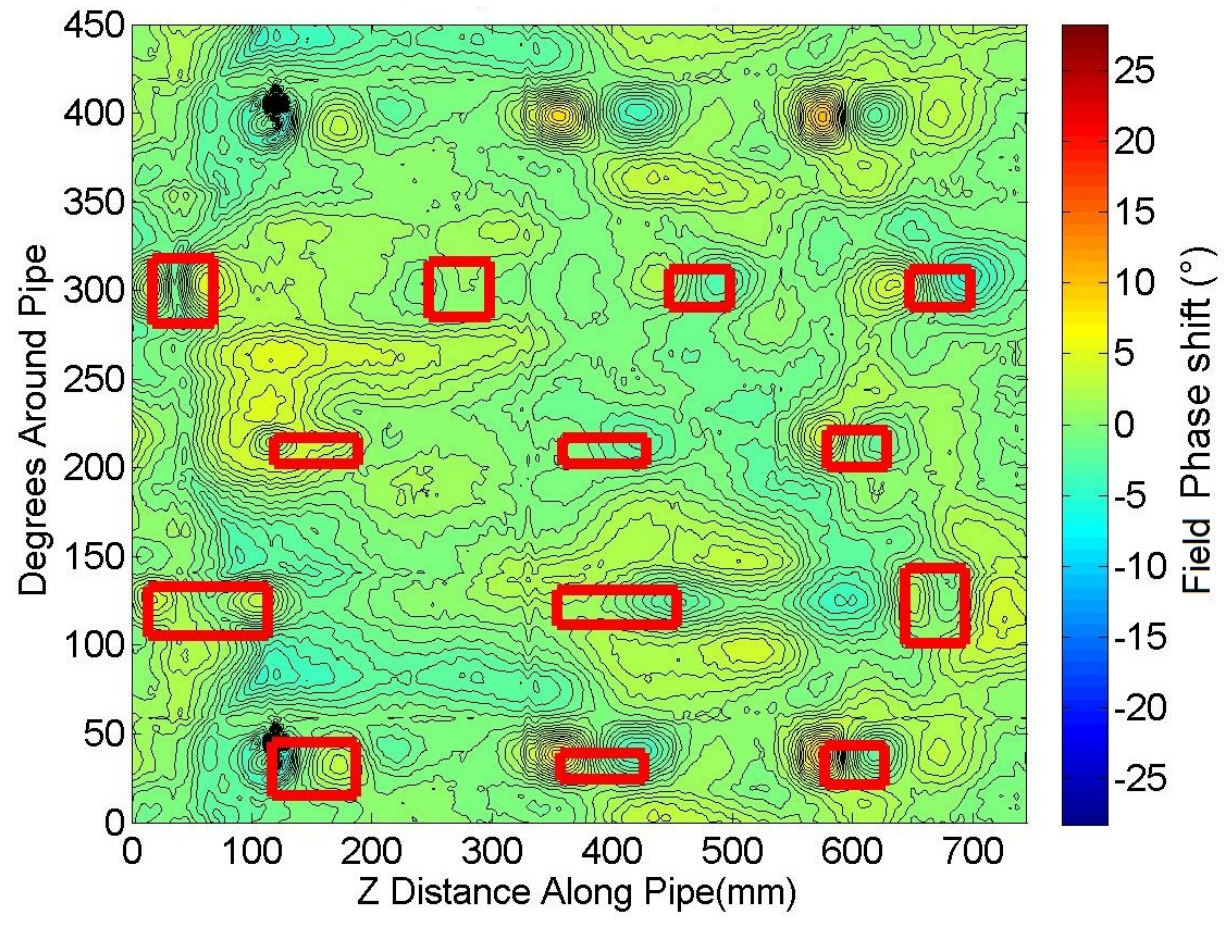

Figure 5.39: $\mathrm{R}$ field phase shift, full scan area, $500 \mathrm{~A}$ excitation current

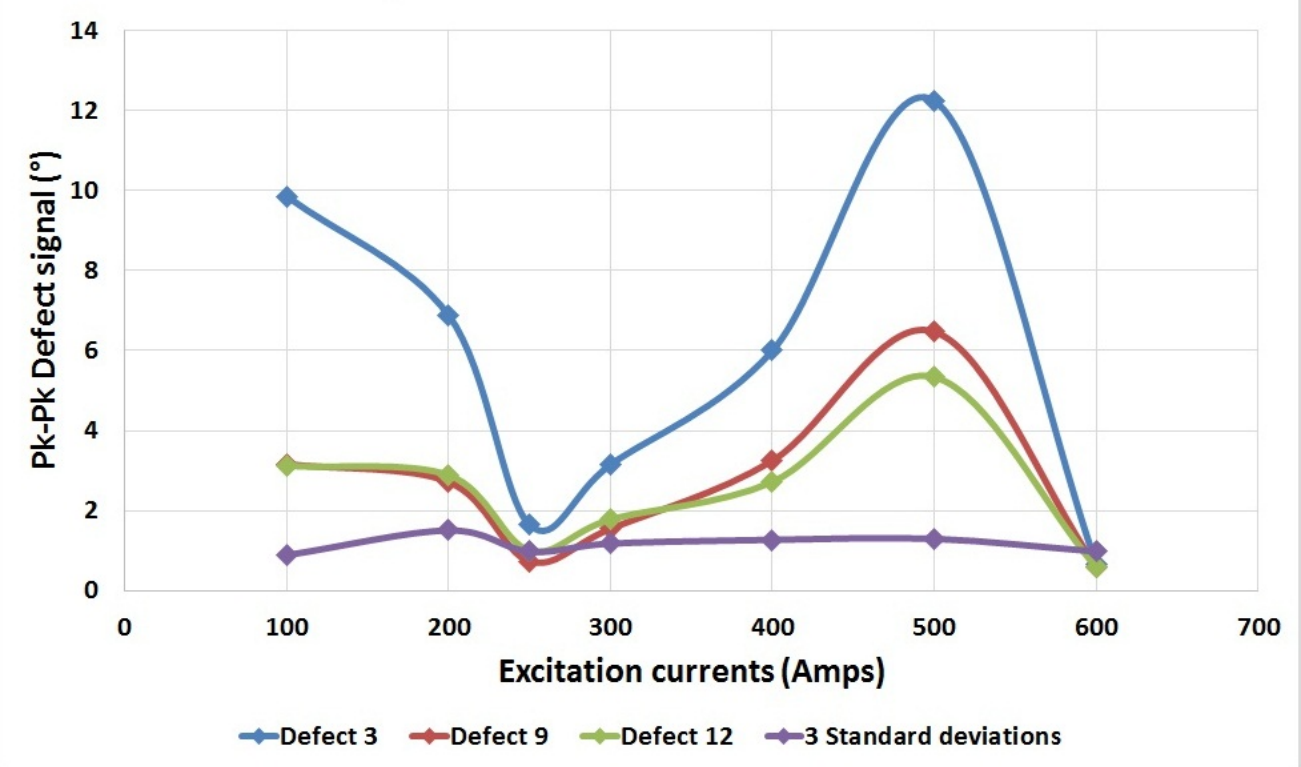

Figure 5.40: $\mathrm{R}$ field phase shift peak to peak change with excitation current(Points from each of the defects have been connected with a guide line) 


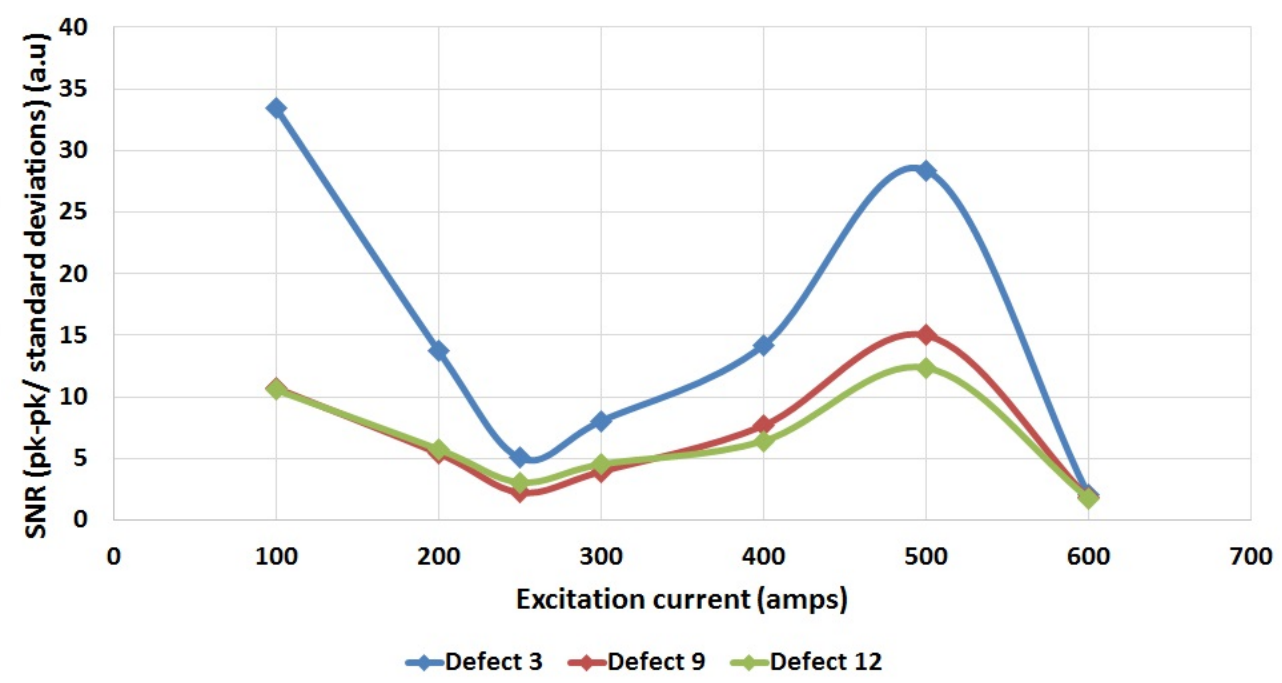

Figure 5.41: R field phase shift SNR change with excitation current(Points from each of the defects have been connected with a guide line)

\section{Influence of excitation current on Phi field phase shift defect signals}

The Phi field phase shift plots for the scan area are shown in figure 5.42 for $100 \mathrm{~A}$ excitation current and figure 5.43 for $500 \mathrm{~A}$ excitation current. The Phi field phase shift defect signals at $100 \mathrm{~A}$ excitation current can be identified for most defects but a few of the small defect signals disappear into the background variations.

Apart from the largest defects, at $500 \mathrm{~A}$ excitation current the defect signals tend to be unclear, with the peak to peak signal level reduced compared to $100 \mathrm{~A}$.

The peak to peak value for the same three defects is again calculated and plotted in figure 5.44 with the 3 standard deviation line from the plain pipe data for comparison. The Phi field phase shift defect signal peak to peak change with excitation current shows a drop at higher currents for all defects. The largest defect (defect 3) shows the most signification decrease with excitation current.

To determine the excitation to get the best possible defect signal from the Phi field sensor the SNR of each defect is calculated and plotted in figure 5.45. The phase shift SNR response is constant with excitation current apart from the 100 amp data. This means there is no advantage gained by increasing the excitation current.

\subsubsection{Discussion: Excitation current vs defect signals}

\section{Field magnitude}

The $R$ and Phi field magnitude defect signals both increase linearly with excitation current and with increasing defect volume. This is expected, because larger excitation currents generate larger eddy currents so more current is distorted when a defect is present.

The SNR values for the $R$ and Phi field magnitude defect signals have been predicted using the linear fits of the peak to peak signal change with excitation current and the plain 


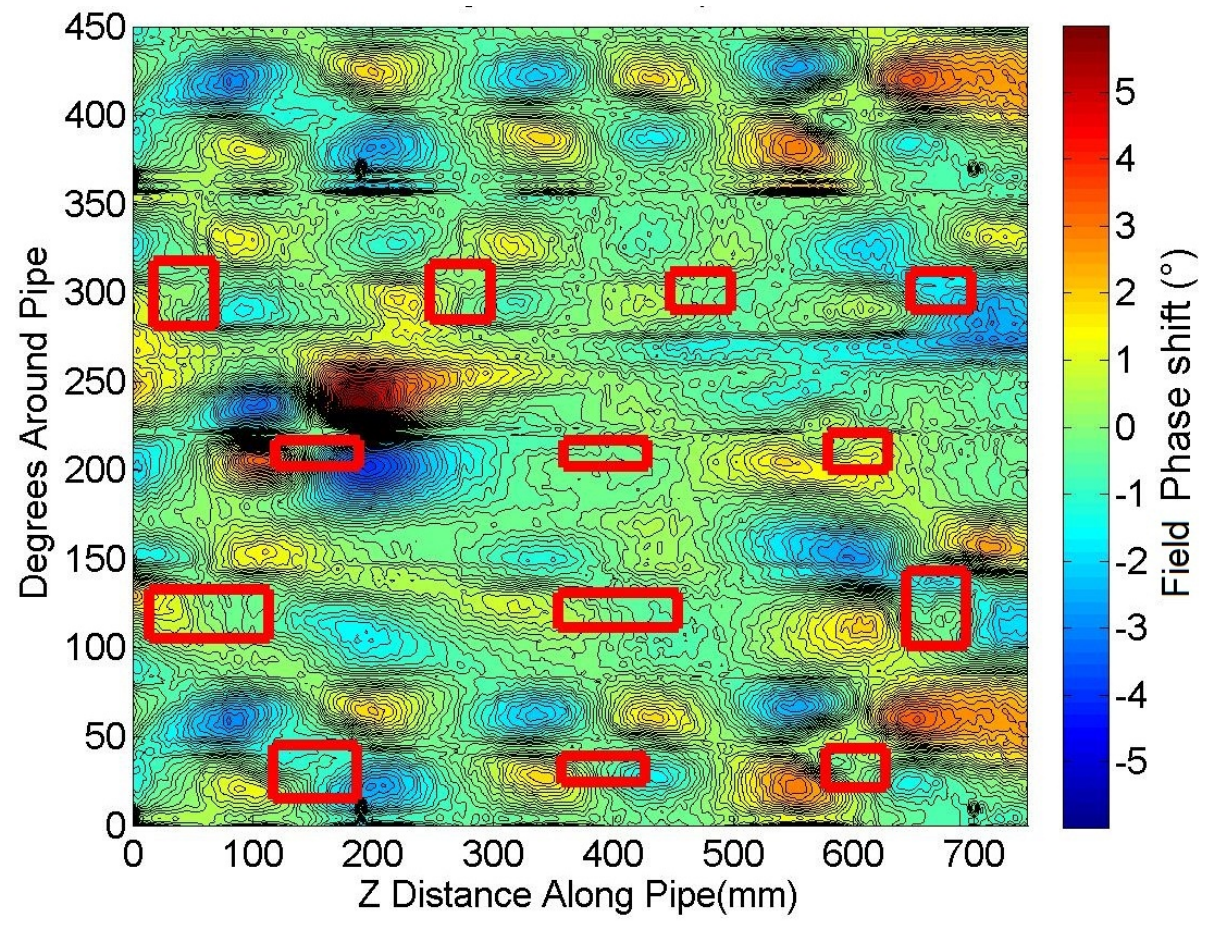

Figure 5.42: Phi field phase shift, full scan area, $100 \mathrm{~A}$ excitation current

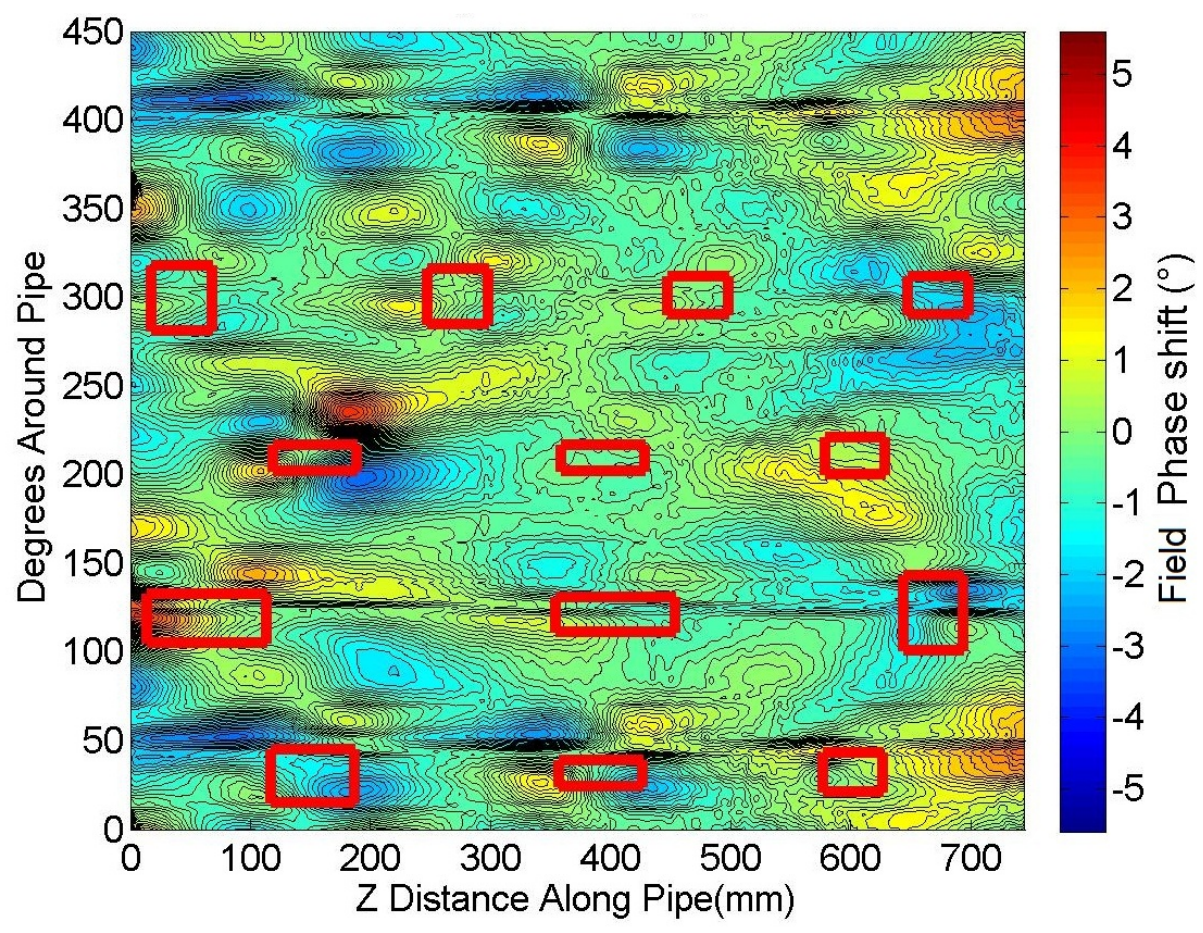

Figure 5.43: Phi field phase shift, full scan area, scan at $500 \mathrm{~A}$ excitation current 


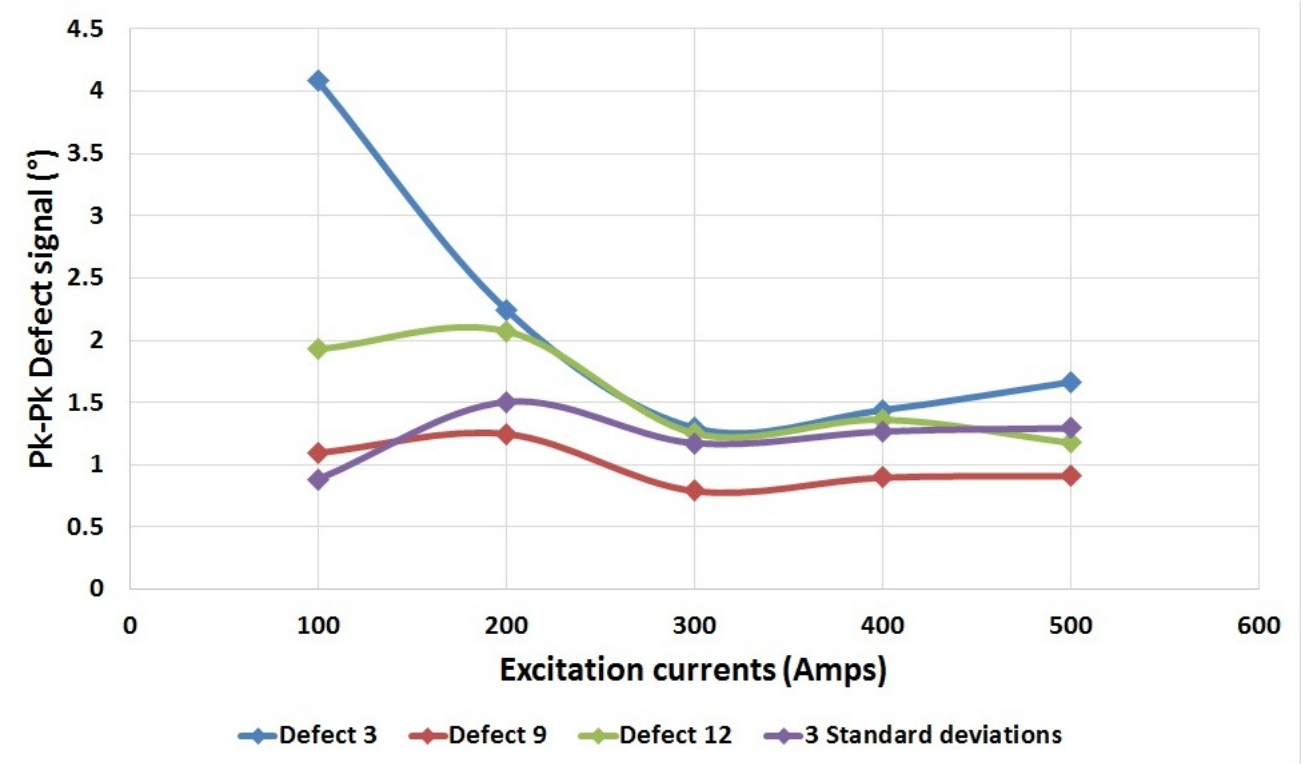

Figure 5.44: Phi field phase shift peak to peak change with excitation current(Points from each of the defects have been connected with a guide line)

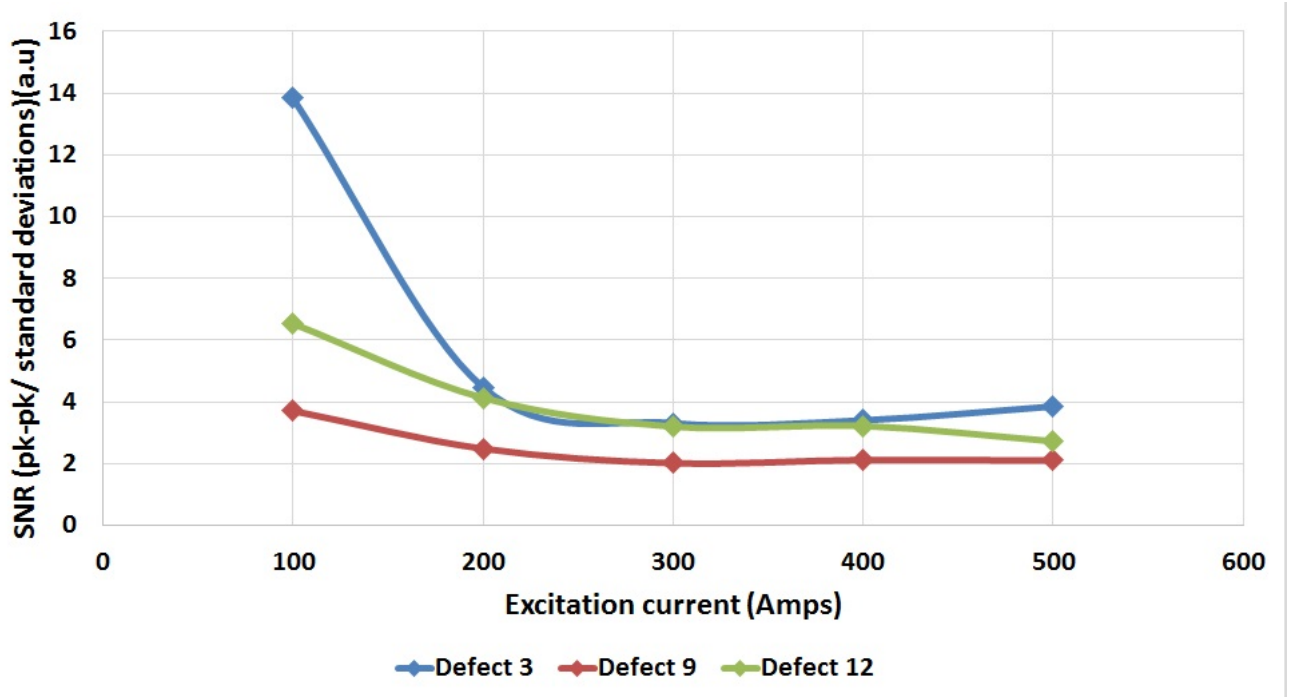

Figure 5.45: Phi field phase shift SNR change with excitation current(Points from each of the defects have been connected with a guide line) 
pipe standard deviation change with excitation current. The predicted values fit well with the actual measured values. The predicted values show that the excitation current needed for the best possible SNR is approximately $300 \mathrm{~A}$. The predicted SNR value asymptotes towards the maximum SNR, which is the ratio of the slope of the defect signal and the slope of the plain pipe noise. The point at which the SNR reaches $95 \%$ of the theoretical maximum is, in practice, the maximum that can be achieved due to natural variation in measurements. The current required to reach the $95 \%$ mark is determined by the level of noise in the measurement system when zero excitation current is applied.

\section{Field phase shift}

The $R$ field phase shift shows a strong variation with changing excitation current with both high and low excitation current producing large defect signals. This shows that there is probably an unknown parameter affecting the phase shift. The large peak to peak signals measured at $100 \mathrm{~A}$ excitation currents shows that a defect could easily be identified at low excitation currents.

The Phi field phase shift defect signal peak to peak magnitude shows little change with excitation current. The SNR data suggests that at a low excitation current the phase shift is becoming easier to identify and, like the $R$ field phase shift, there is no advantage to increasing the excitation current.

\subsubsection{Influence of excitation frequency on defect signals}

To determine the relationship between excitation frequency and defect signals tests were done at $5 \mathrm{~Hz}, 10 \mathrm{~Hz}, 20 \mathrm{~Hz}, 60 \mathrm{~Hz}, 89 \mathrm{~Hz}$, at a nominal current of $300 \mathrm{~A}$, while for $200 \mathrm{~Hz}$ a nominal current of $170 \mathrm{~A}$ was used. Selected results will be displayed and discussed in this following section.

\section{Influence of excitation frequency on $\mathbf{R}$ field Magnitude defect signals}

The contour plots of the $\mathrm{R}$ field magnitude at $5 \mathrm{~Hz}$ is shown in figure 5.46 , at $20 \mathrm{~Hz}$ in figure 5.47 and at $200 \mathrm{~Hz}$ in figure 5.48. The R field at $5 \mathrm{~Hz}$ excitation frequency clearly identifies all the defect signals apart from defect 5 . The Gaussian peaks have also shifted in the Phi axis from the center of the $Z$ edge of the defect. This is caused by a lag in the measurements as discussed in section 5.2.1.

The $\mathrm{R}$ field at $20 \mathrm{~Hz}$ excitation frequency clearly identifies all the defect signals apart from defect 5 . The defect signal magnitude is almost twice the size of the defect signal at 5 $\mathrm{Hz}$. Some extra features that appeared near the defects in the $5 \mathrm{~Hz}$ data are not visible. However, because the defects are close to each other, it is difficult to establish whether they are a result of the defects or of increasing background variation.

The $\mathrm{R}$ field at $200 \mathrm{~Hz}$ excitation frequency shows a few of the larger defects, but the signals are almost an order of magnitude lower than at $20 \mathrm{~Hz}$. However, the background field is also relatively constant which makes it possible to identify defect signals at a very low level.

The peak to peak value for defects 3,9 and 12 is calculated for each excitation frequency and plotted in figure 5.49. There is no clear relationship for the data. A purple line has 


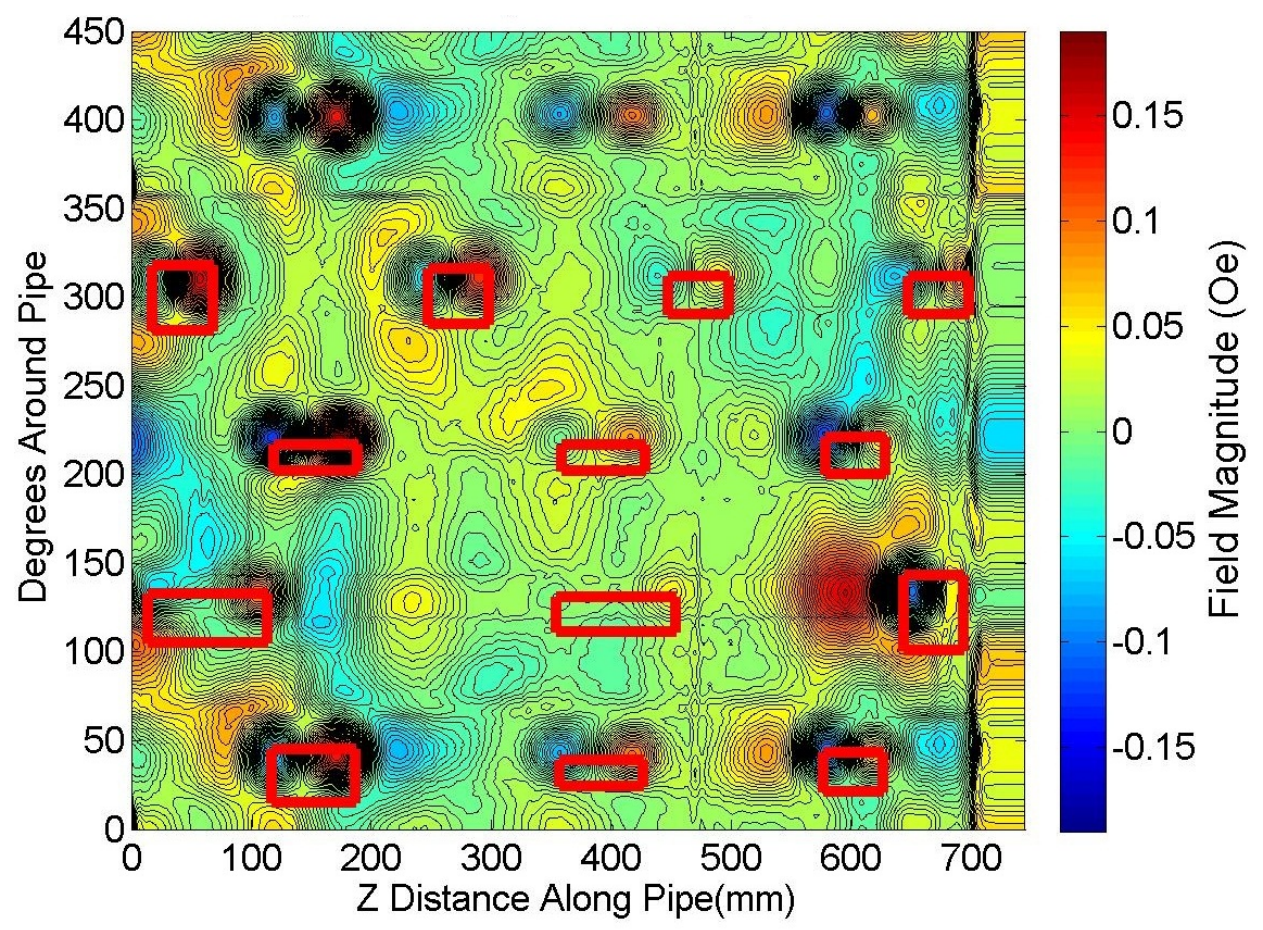

Figure 5.46: $\mathrm{R}$ field magnitude, full scan area, $5 \mathrm{~Hz}$ excitation frequency

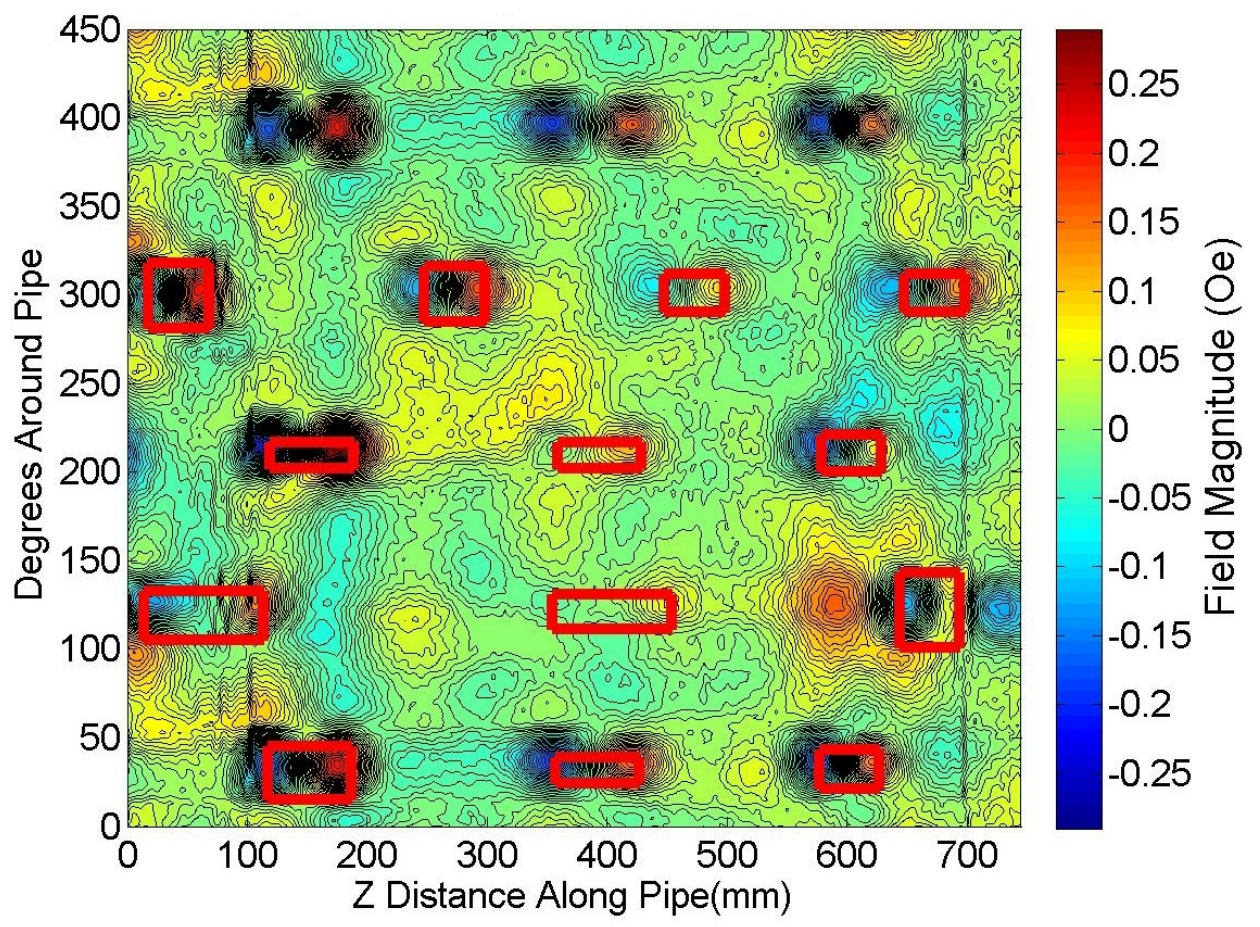

Figure 5.47: $\mathrm{R}$ field magnitude, full scan area, $20 \mathrm{~Hz}$ excitation frequency 


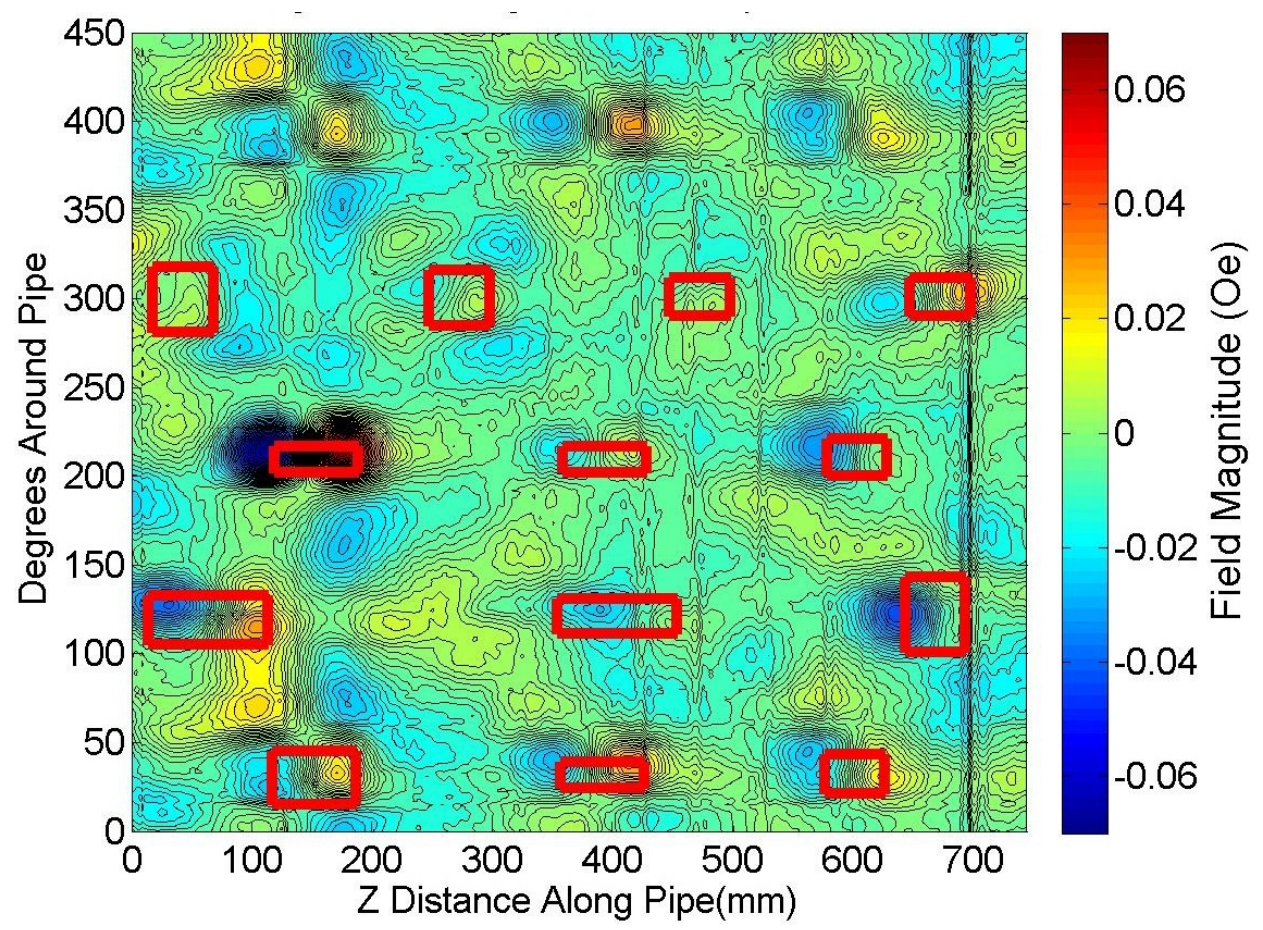

Figure 5.48: $\mathrm{R}$ field magnitude, full scan area, $200 \mathrm{~Hz}$ excitation frequency

been plotted to show the three standard deviation level of the plain pipe data from section 4.6.

An optimal excitation frequency appears between $60 \mathrm{~Hz}$ and $20 \mathrm{~Hz}$ showing the largest response. For these frequencies the defect signals are well above the 3 standard deviation level.

The standard deviation measured at each frequency on the plain pipe is used to calculate the SNR for each of the three defects and is shown in figure 5.50. The SNR shows a large increase for all defects at around $20 \mathrm{~Hz}$ where the SNR is at its maximum.

\section{Influence of excitation frequency on Phi field Magnitude defect signals}

The Phi field magnitude for the full scan area is shown in figure 5.51 for $5 \mathrm{~Hz}$, figure 5.52 for $20 \mathrm{~Hz}$ and figure 5.53 for $200 \mathrm{~Hz}$.

The Phi field magnitude at $5 \mathrm{~Hz}$ shows Gaussian peaks near each corner of the defect. As mentioned in the start of section 5.2.1 and discussed in section 3.4, the Gaussian peak misalignment with the predicted location is frequency dependent. This means when the measurements are taken with $5 \mathrm{~Hz}$, the 5 to 10 degrees of Phi axis shift of the peaks is expected.

The Phi field magnitude at $20 \mathrm{~Hz}$ excitation shows clearly identifiable defect signals centered on the defect corners and an increase in the peak to peak amplitude compared with $5 \mathrm{~Hz}$. The Phi field magnitude at $200 \mathrm{~Hz}$ excitation shows clear peaks on most of the defects in contrast to the $\mathrm{R}$ field magnitude at $200 \mathrm{~Hz}$. However, the peak to peak amplitude is now $50 \%$ less than the value at $20 \mathrm{~Hz}$.

The peak to peak amplitudes are calculated for defects 3,9 and 12 and plotted in figure 


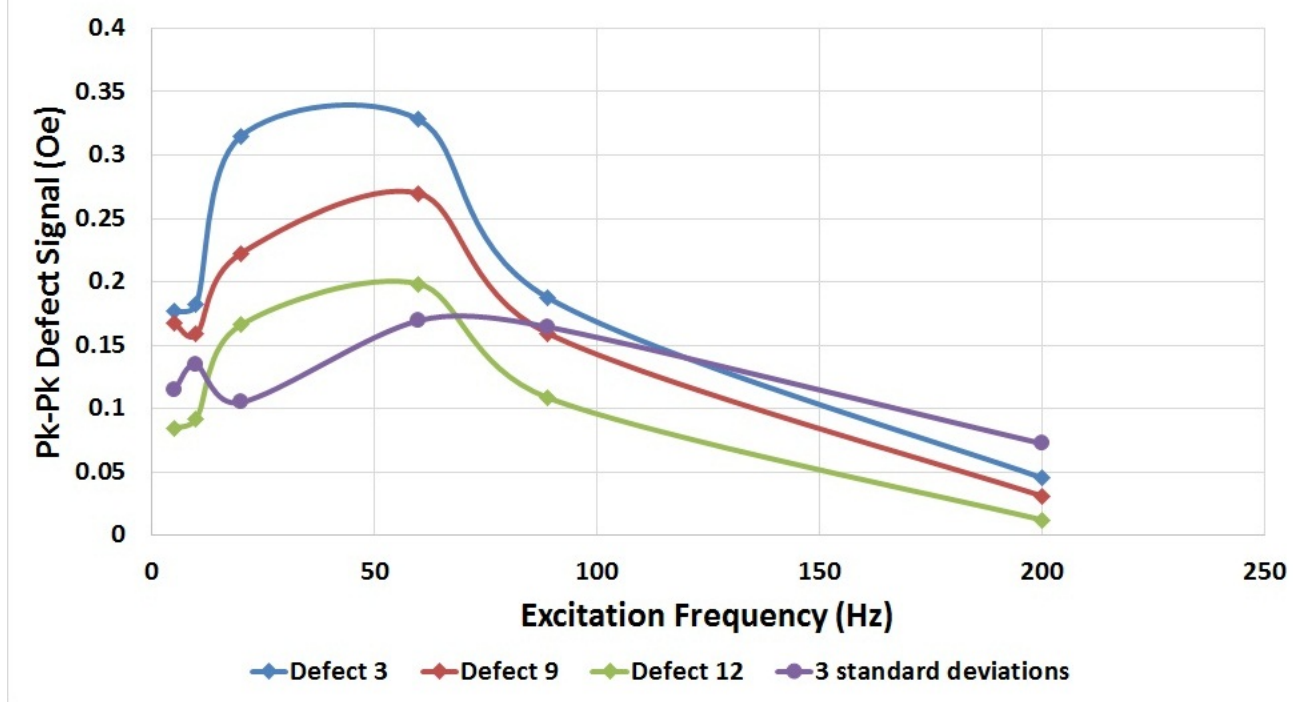

Figure 5.49: $R$ field magnitude defect signal peak to peak change with excitation frequency (Points from each of the defects have been connected with a guide line)

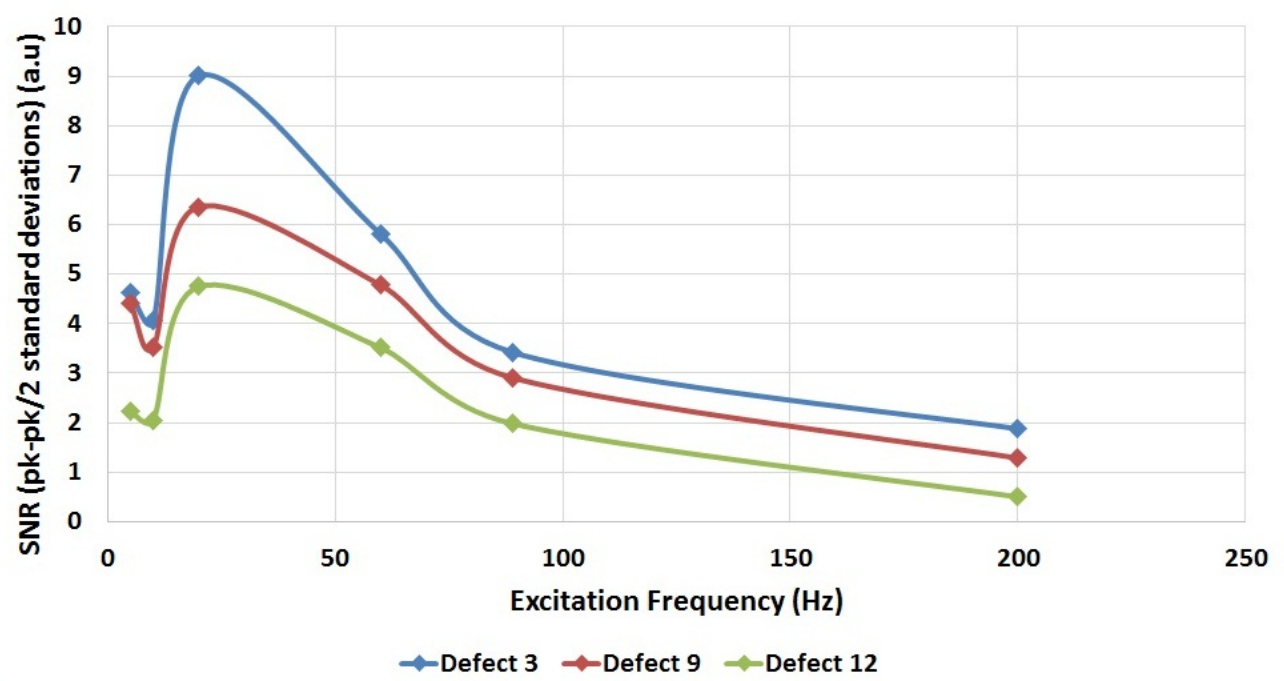

Figure 5.50: $R$ field SNR change with excitation frequency (Points from each of the defects have been connected with a guide line) 


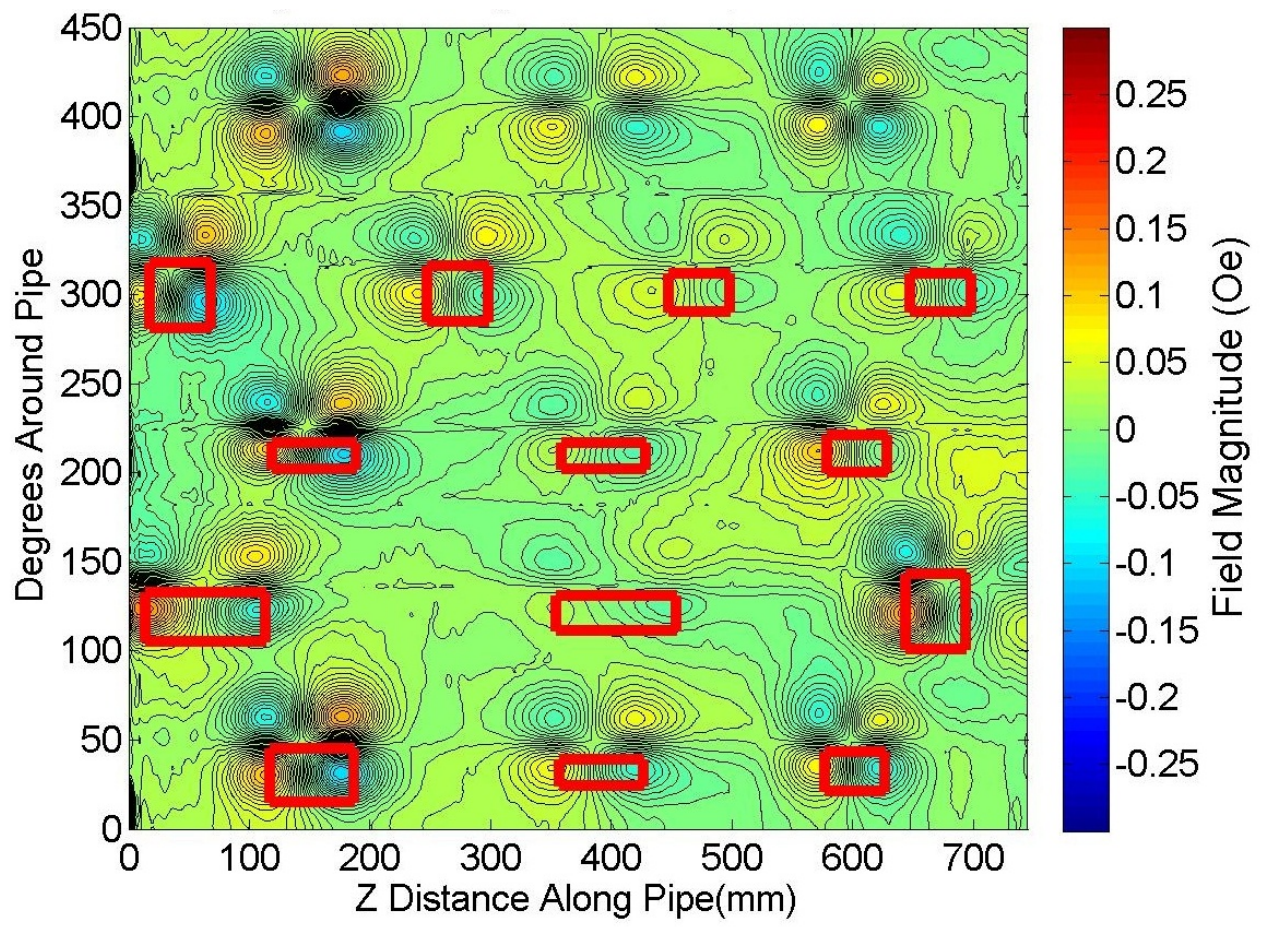

Figure 5.51: Phi field magnitude change, full scan area, at $5 \mathrm{~Hz}$ excitation frequency

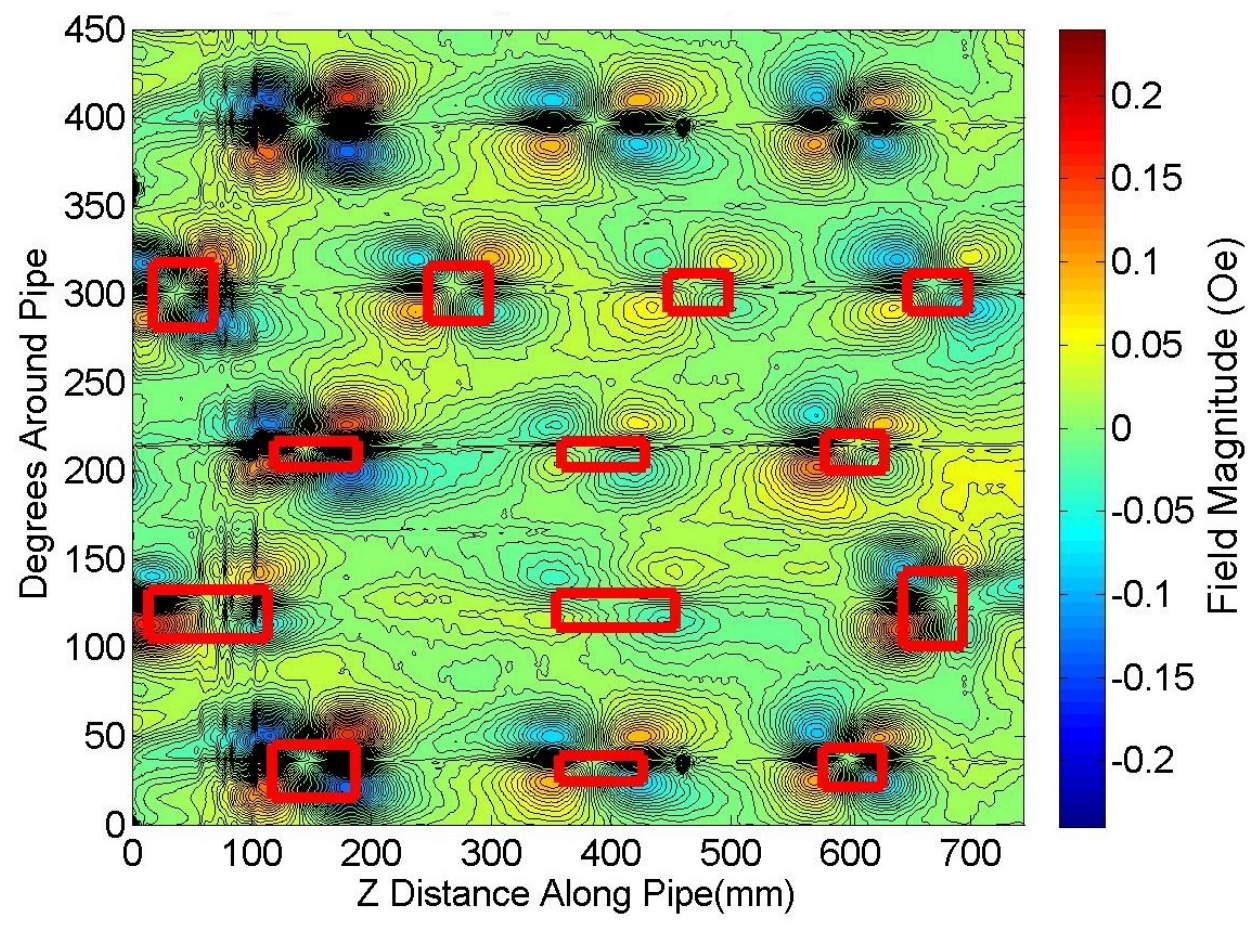

Figure 5.52: Phi field magnitude, full scan area, $20 \mathrm{~Hz}$ excitation frequency 


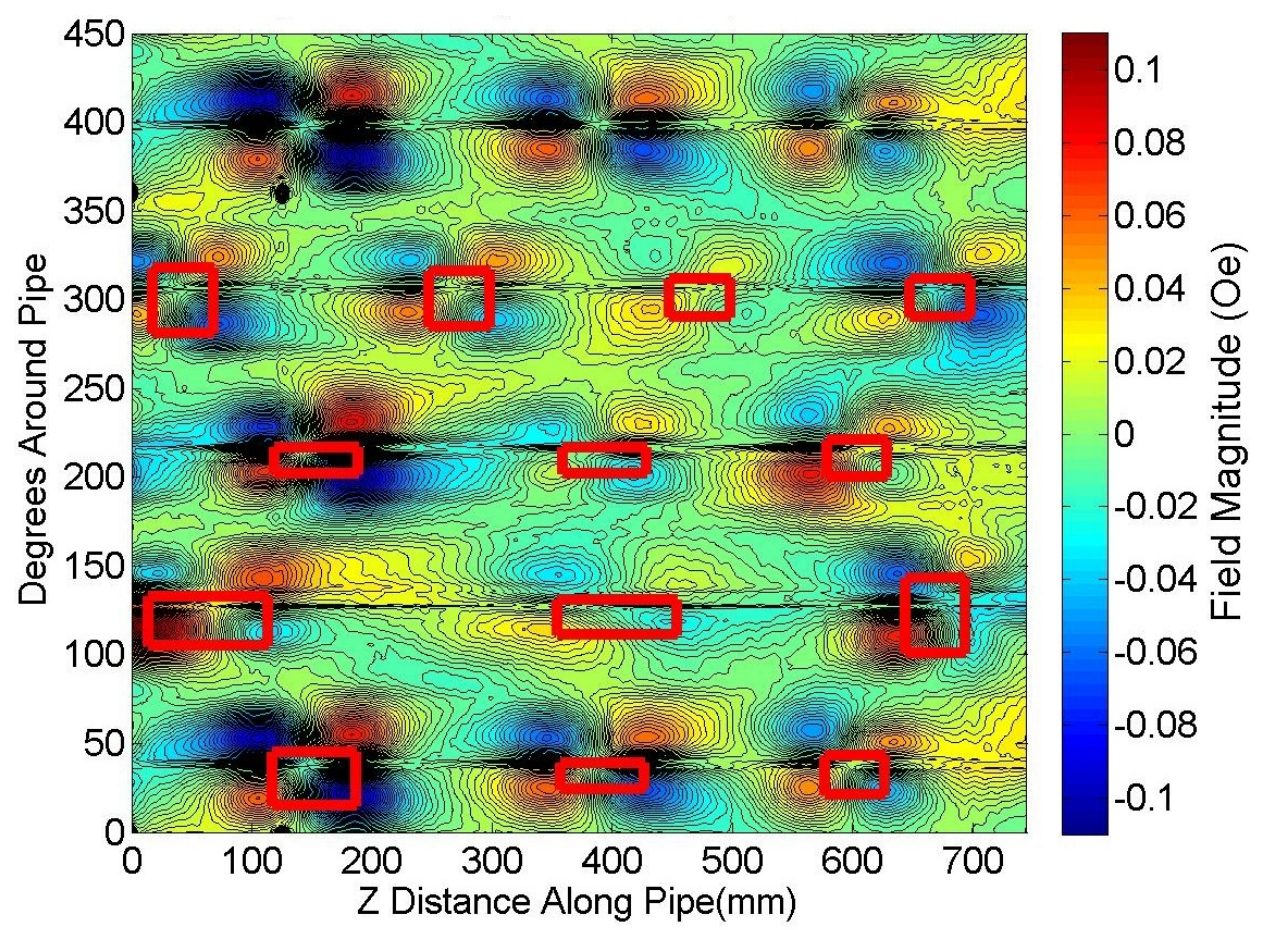

Figure 5.53: Phi field magnitude, full scan area, at $200 \mathrm{~Hz}$ excitation frequency

5.54. As with the $\mathrm{R}$ field there is no clear relationship. The three standard deviation line from the plain pipe data is again plotted. The defect signal peak to peak change with excitation frequency shows, like the $R$ field, a maximum response between 20 and 60 $\mathrm{Hz}$. The three standard deviation line shows that the segment defect would be hard to detect apart from close to $20 \mathrm{~Hz}$ excitation frequency. The other two defects show similar magnitudes despite having different volumes.

As before, the SNR values are calculated at each frequency with the plain pipe standard deviation values and are plotted in figure 5.55. The Phi field SNR change with excitation frequency has a similar response as the $R$ field with a sharp peak at $20 \mathrm{~Hz}$ meaning these defects would be very easy to identify.

\section{Influence of excitation frequency on $\mathbf{R}$ field Phase Shift defect signals}

The full pipe plot of the $\mathrm{R}$ field phase shift is shown in figure 5.56 for $5 \mathrm{~Hz}$, figure 5.57 for $20 \mathrm{~Hz}$ and figure 5.58 for $200 \mathrm{~Hz}$.

The $\mathrm{R}$ field phase shift with $5 \mathrm{~Hz}$ excitation shows clear signals for most of the defects however some of the larger defects obscure the smaller defects making them difficult to identify. The $\mathrm{R}$ field phase shift with $20 \mathrm{~Hz}$ excitation shows an overall phase shift of about half that of the $5 \mathrm{~Hz}$ data. The noise has increased significantly making some of the smaller defects seen at $5 \mathrm{~Hz}$ unidentifiable.

The $\mathrm{R}$ field phase shift with $200 \mathrm{~Hz}$ excitation shows the overall phase shift has come back to the same level as the $5 \mathrm{~Hz}$ data. However, the peaks have become significantly more prominent, making visual identification of the defects easier. The shallow long defect 5 at 100 degrees and $400 \mathrm{~mm}$ still does not produce a strong defect signal. 


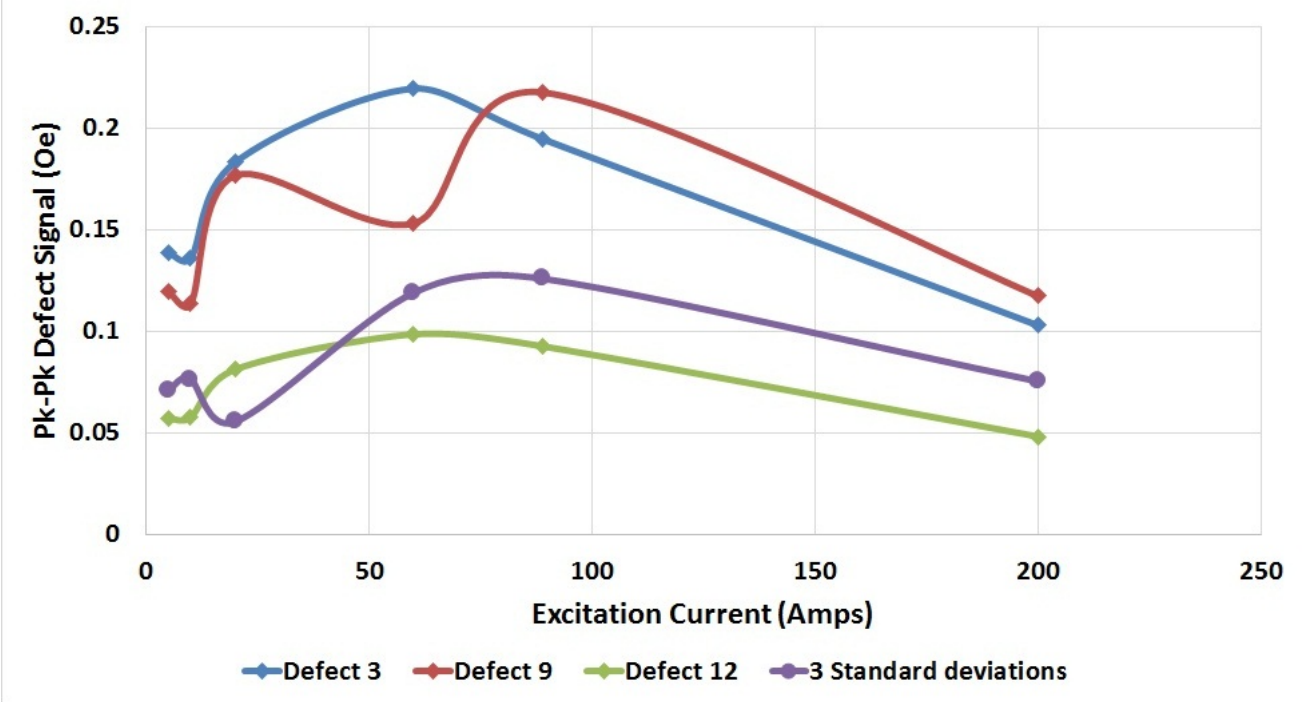

Figure 5.54: Phi field magnitude defect signal peak to peak vs excitation frequency (Points from each of the defects have been connected with a guide line)

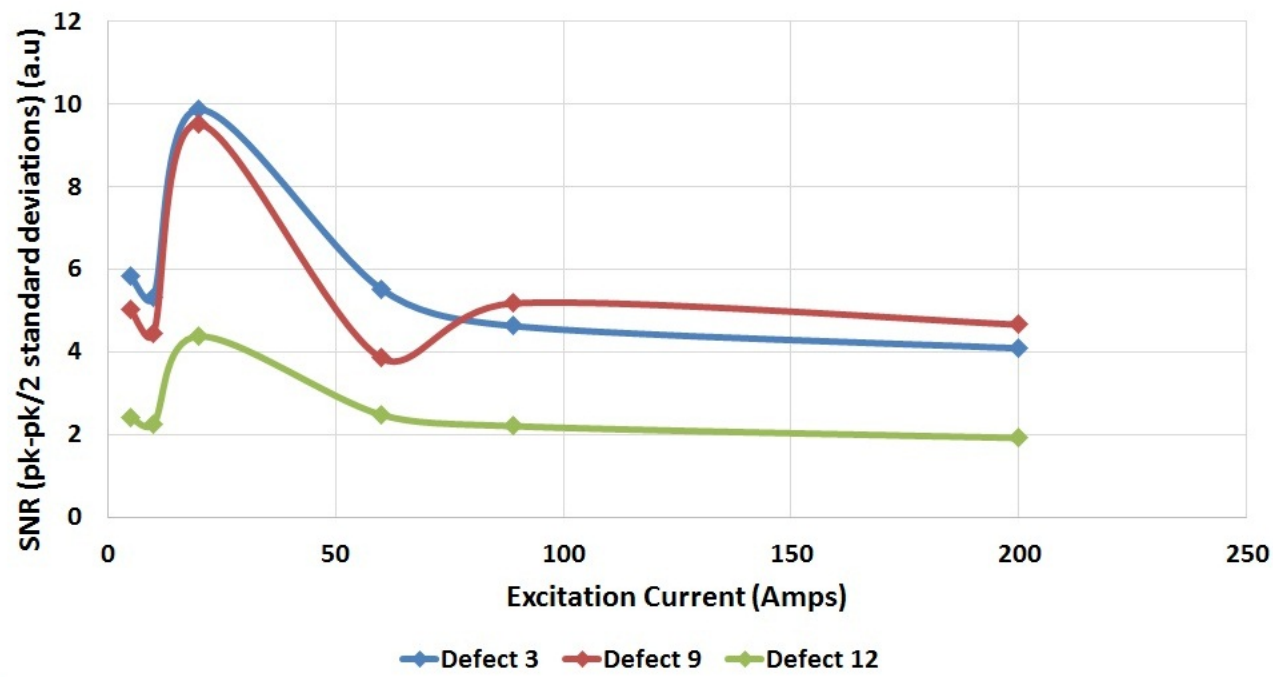

Figure 5.55: Phi field defect signal SNR vs excitation frequency (Points from each of the defects have been connected with a guide line) 


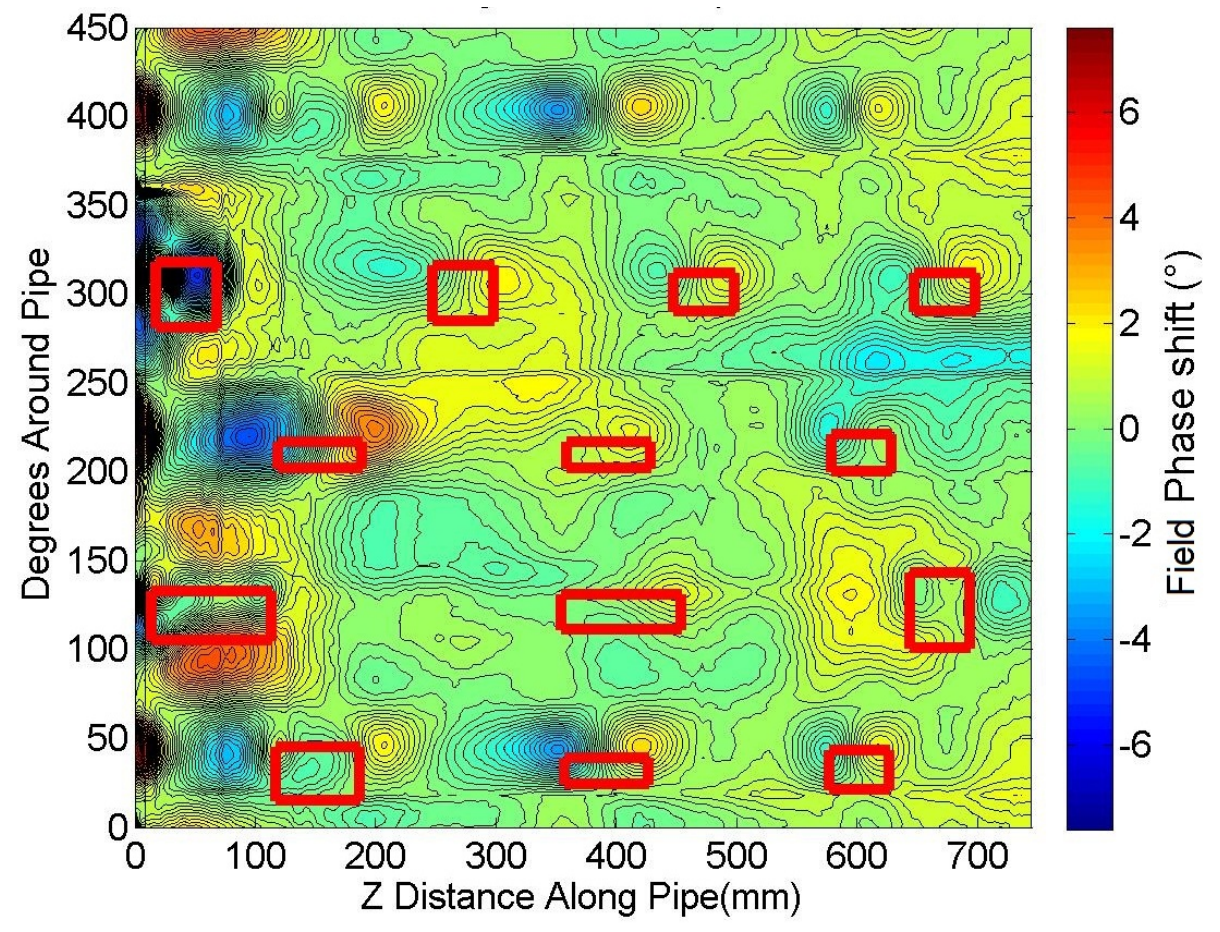

Figure 5.56: $\mathrm{R}$ field phase shift, full scan area, $5 \mathrm{~Hz}$ excitation frequency

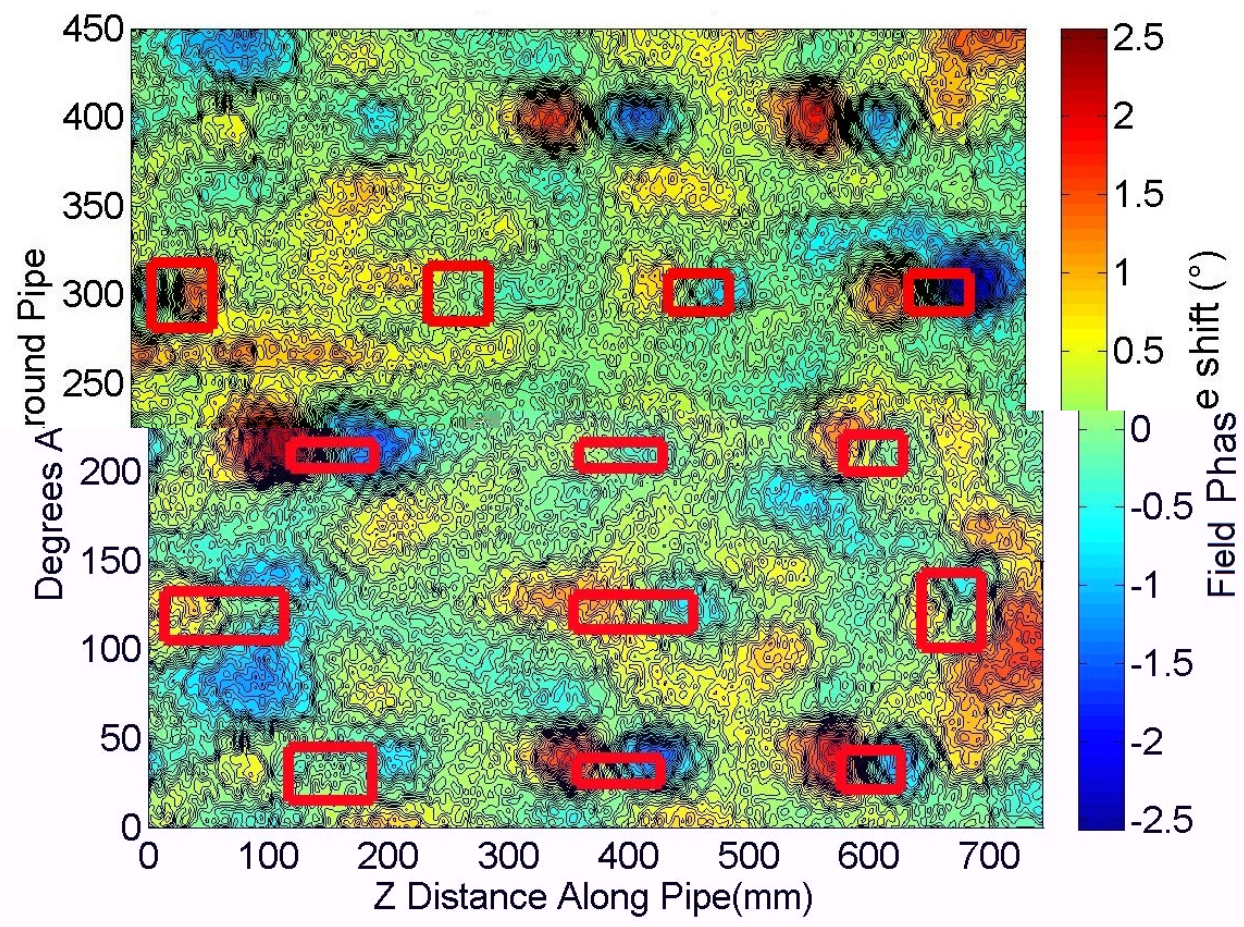

Figure 5.57: $\mathrm{R}$ field phase shift, full scan area, $20 \mathrm{~Hz}$ excitation frequency 


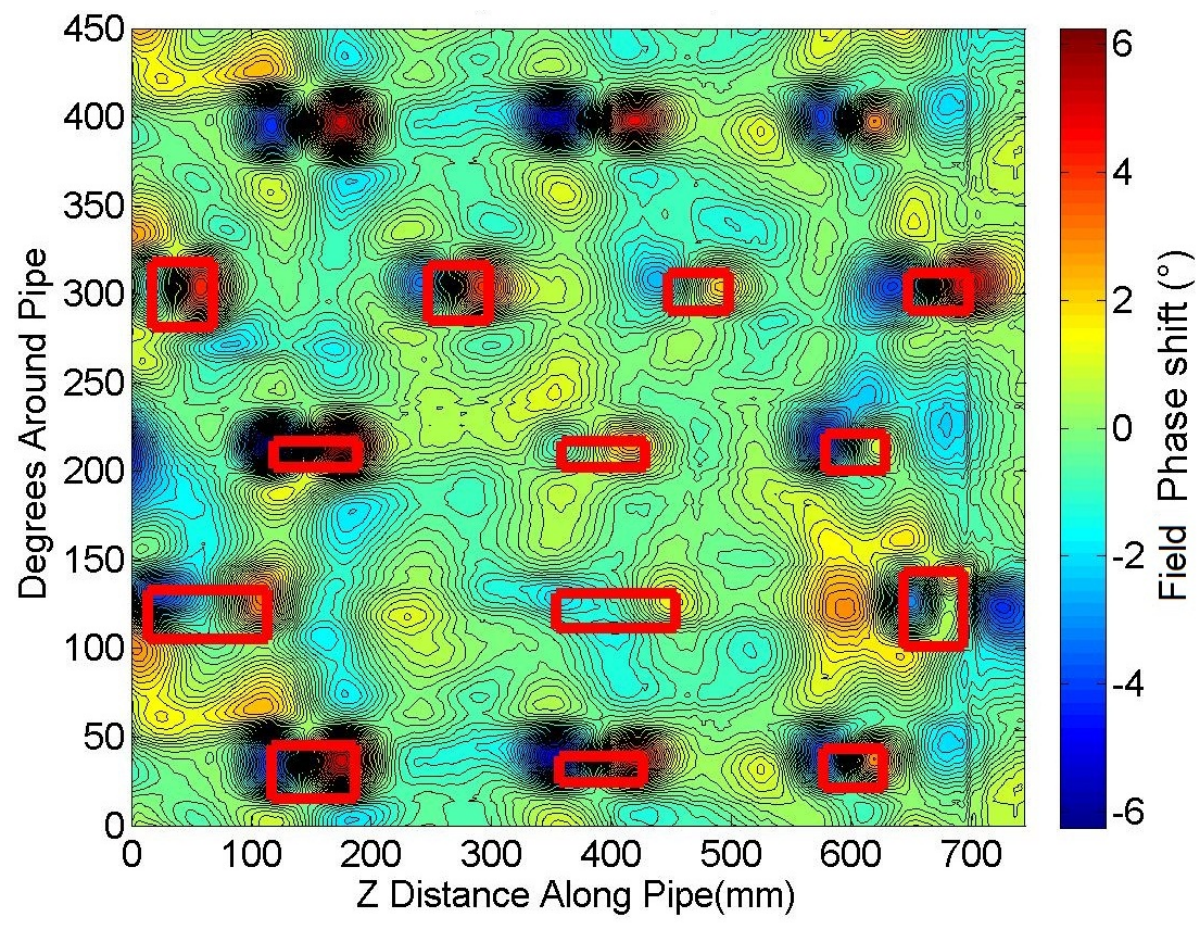

Figure 5.58: $\mathrm{R}$ field phase shift, full scan area, $200 \mathrm{~Hz}$ excitation frequency

The peak to peak values for defects 3, 9 and 12 are plotted in figure 5.54. As with the $\mathrm{R}$ field, there is no clear relationship for the data points. The three standard deviation line from the plain pipe data is overlaid.

The $R$ field phase shift signal change with excitation frequency is large for frequencies above $60 \mathrm{~Hz}$, with the $89 \mathrm{~Hz}$ test producing the largest phase shift, and only very small signals occurring between $20 \mathrm{~Hz}$ to $60 \mathrm{~Hz}$ with an increase at the lower frequency. However, this could just be natural variation.

The SNR values at each frequency are shown in figure 5.66. The $R$ field phase shift SNR change with frequency shows a similar shape as the peak to peak signals. However, the increasing SNR at the lower frequency is more pronounced with a clear minimum at 60 $\mathrm{Hz}$.

\section{Influence of excitation frequency on Phi field Phase shift defect signals}

The full pipe contour plots are shown in figure 5.61 for $5 \mathrm{~Hz}$, figure 5.62 for $20 \mathrm{~Hz}$ and figure 5.63 for $200 \mathrm{~Hz}$.

The Phi field phase shift at $5 \mathrm{~Hz}$ excitation frequency shows clear defect signals for only four of the largest defects. This is due to larger background variations.

The Phi field phase shift at $20 \mathrm{~Hz}$ excitation frequency shows the same four defect signals and a small reduction in background variation. Some of the other defect peaks appear, but not enough to clearly identify them as defects.

The Phi field phase shift at $200 \mathrm{~Hz}$ excitation frequency shows a much higher phase shift with more than double the phase shift seen at $20 \mathrm{~Hz}$. The defect signals are also more pronounced making defect identification possible of all but defect 5 . 


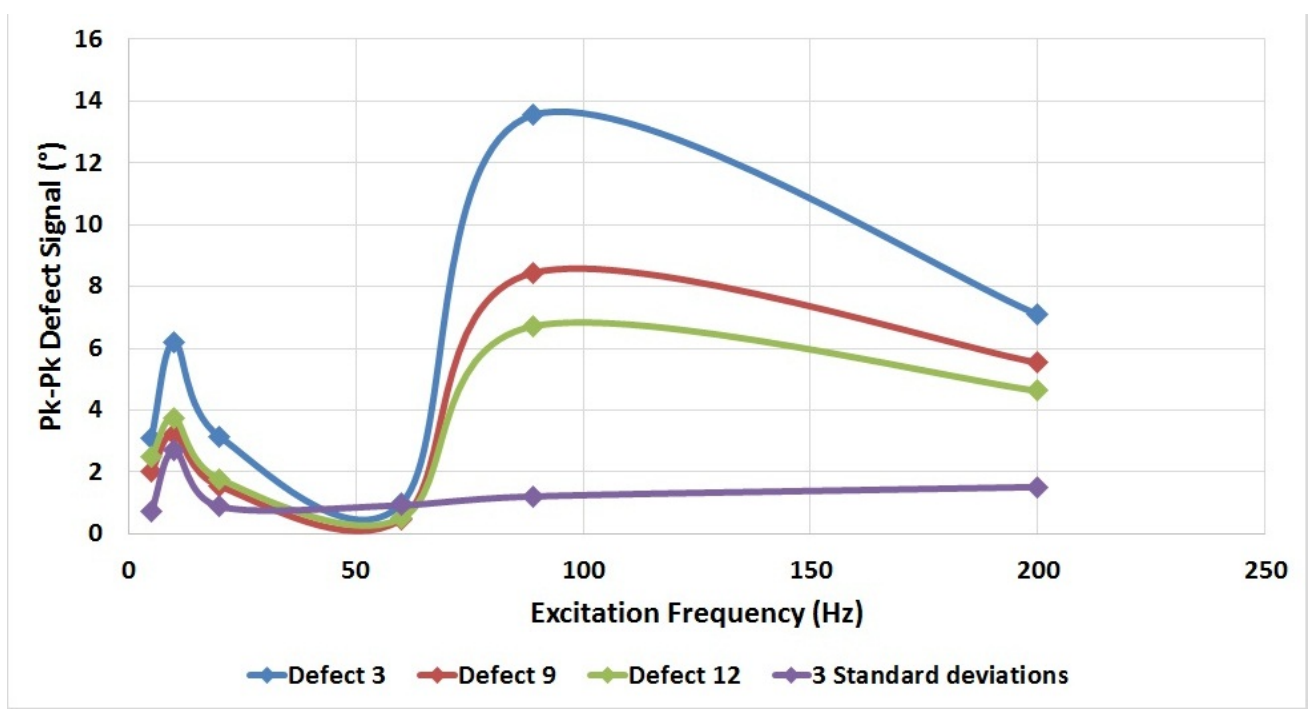

Figure 5.59: $\mathrm{R}$ field phase shift defect signal peak to peak vs excitation frequency (Points from each of the defects have been connected with a guide line)

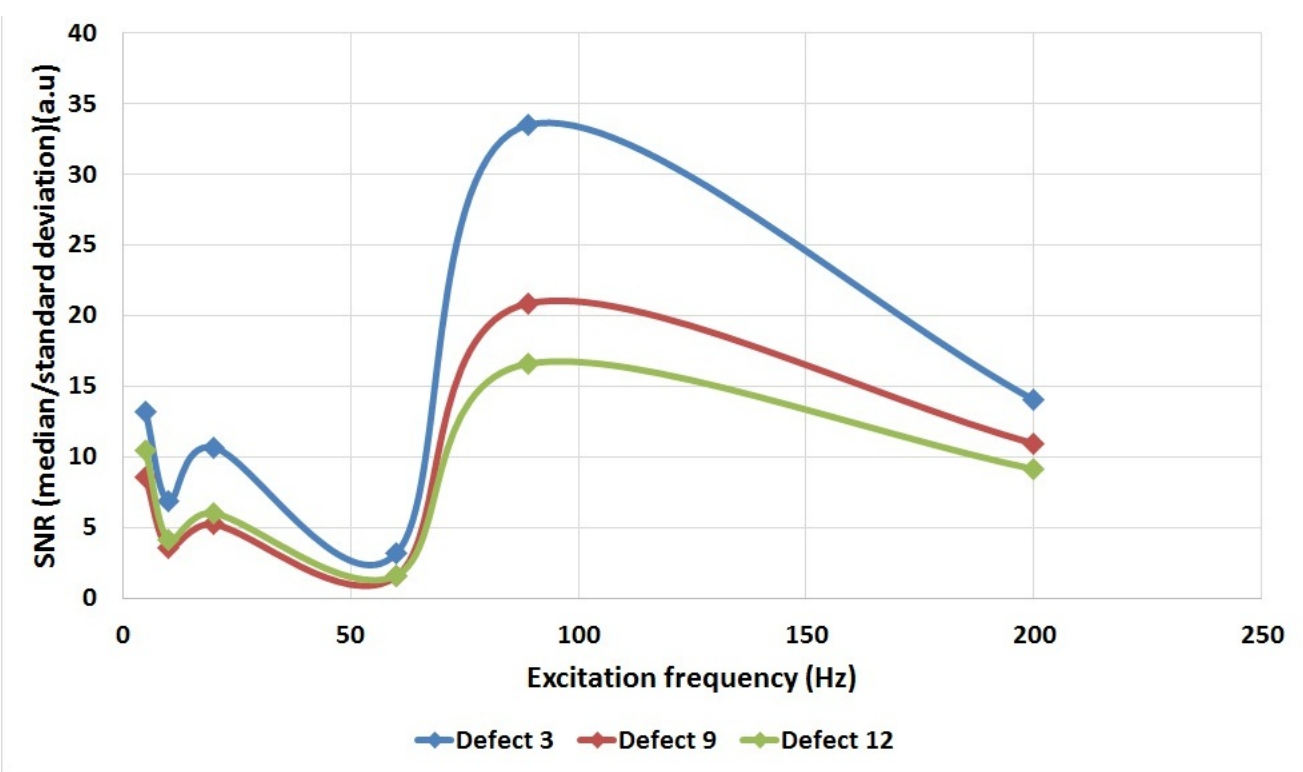

Figure 5.60: R field phase shift defect signal SNR change with excitation frequency (Points from each of the defects have been connected with a guide line) 


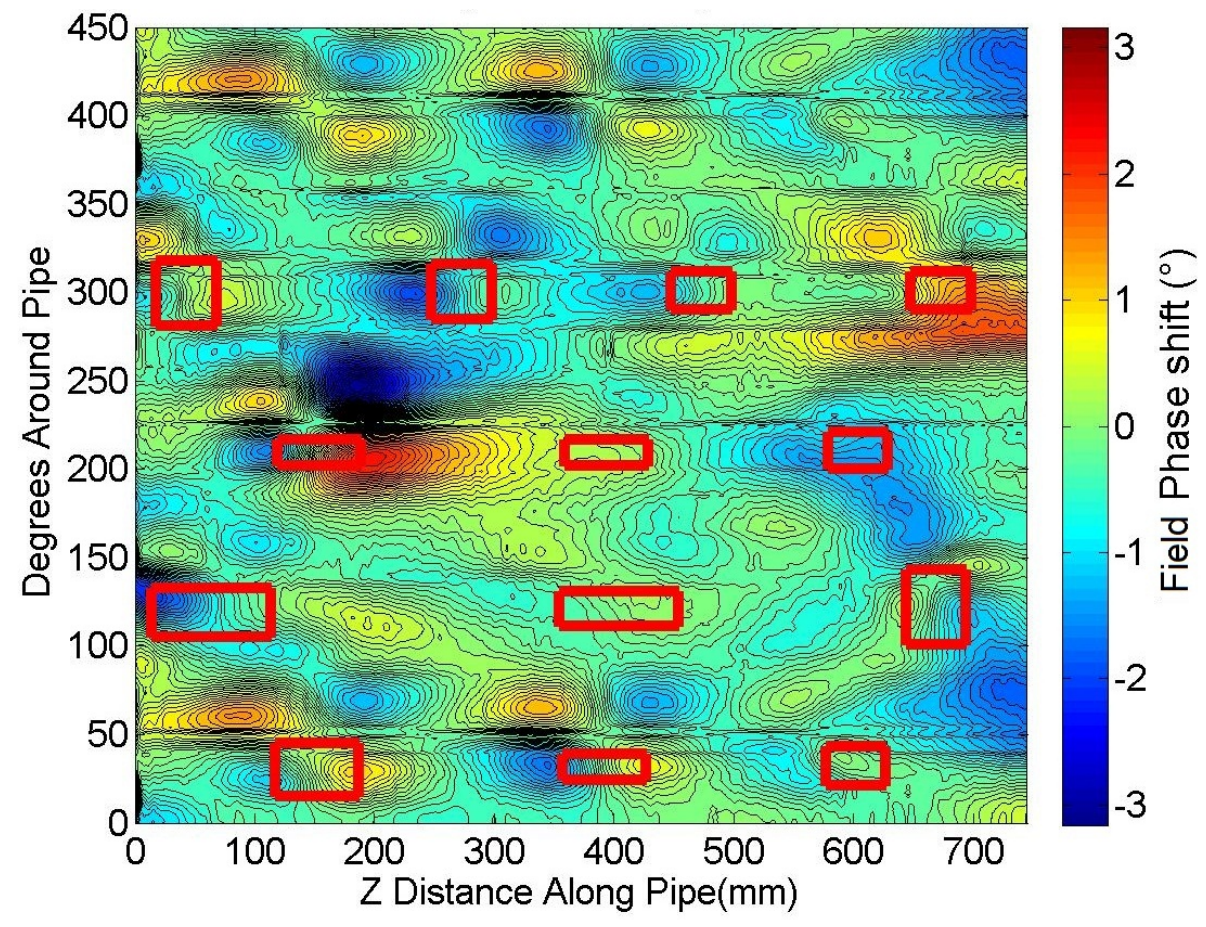

Figure 5.61: Phi field phase shift, whole scan area, $5 \mathrm{~Hz}$ excitation frequency

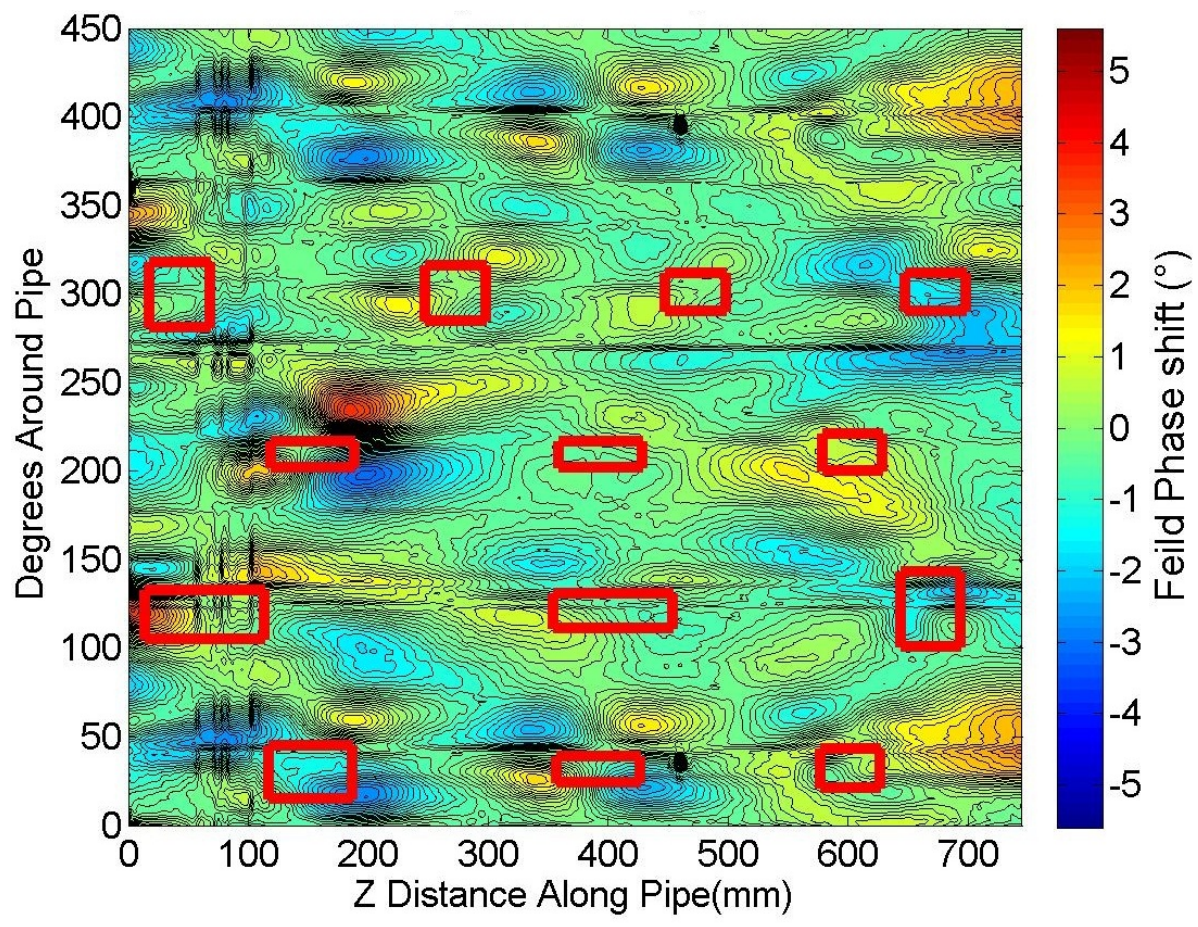

Figure 5.62: Phi field phase shift, whole scan area, $20 \mathrm{~Hz}$ excitation frequency 


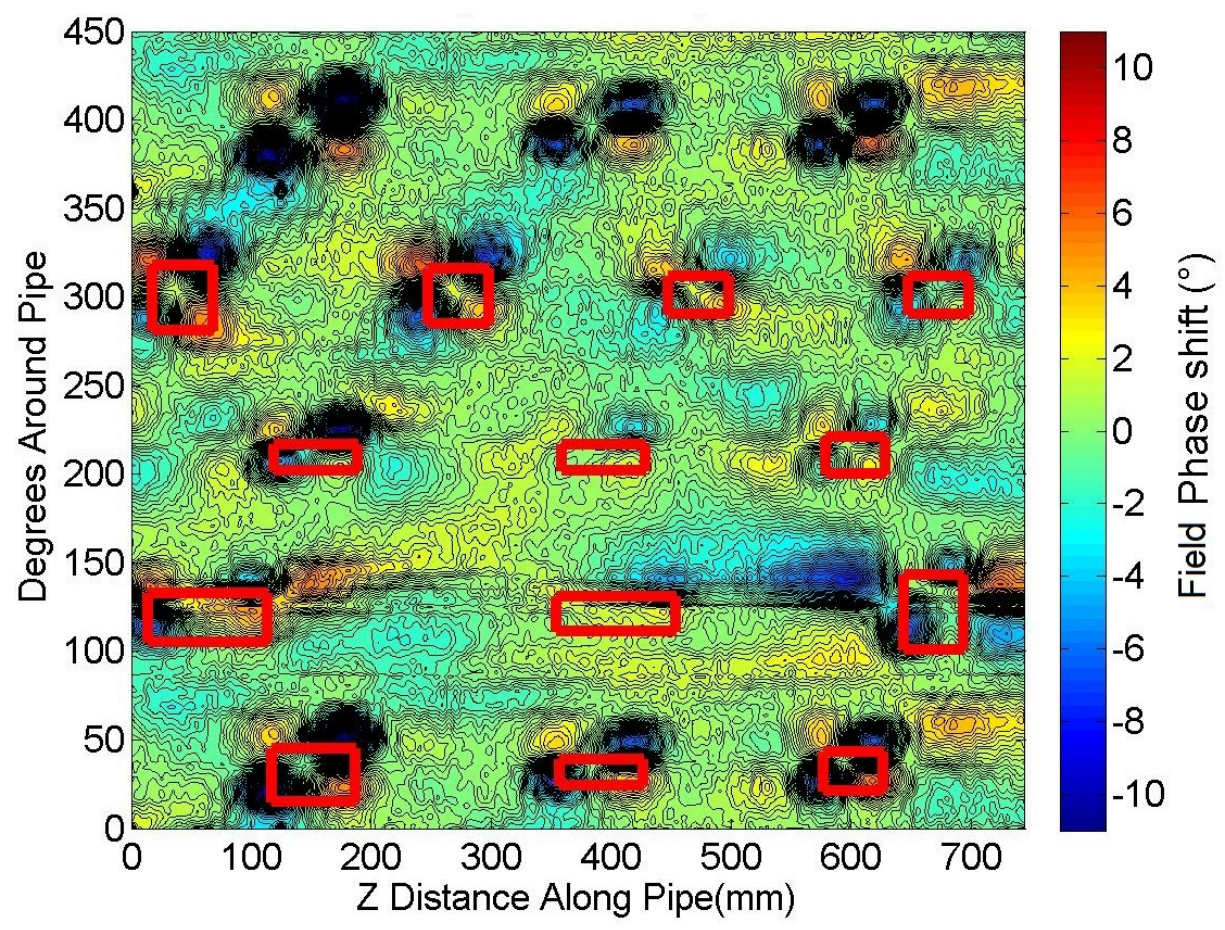

Figure 5.63: Phi field phase shift, whole scan area, $200 \mathrm{~Hz}$ excitation frequency

The peak to peak values for defects 3, 9 and 12 are plotted in figure 5.64. As with the $\mathrm{R}$ field, there is no clear relationship for the data points. The three standard deviation line from the plain pipe data is overlaid. There are large peaks in the defect signals at $60 \mathrm{~Hz}$ that may be anomalies readings. A plot with the $60 \mathrm{~Hz}$ data removed is shown in figure 5.65 .

This plot shows a very clear increase with excitation frequency for all defects. This means higher excitation frequencies would produce better defect signals for the Phi field phase shift, but the aluminum cladding would probably absorb most of the excitation.

The SNR values are calculated at each frequency with the plain pipe standard deviation values and then plotted in figure 5.66. As before, the data at $60 \mathrm{~Hz}$ excitation frequency is too large to see the effects for other excitation frequencies in figure 5.66. So the $60 \mathrm{~Hz}$ data is removed and then plotted again in figure 5.67. This plot shows the same clear increase for higher frequency. However, if the aluminum cladding is assumed to limit the excitation frequency to $100 \mathrm{~Hz}$ then there is no advantage from higher excitation frequencies.

\subsubsection{Discussion: Influence of excitation frequency on defect sig- nals}

Field magnitude

Both $\mathrm{R}$ and $\mathrm{Phi}$ field magnitudes show a similar response to the excitation frequency, with Phi field magnitude having approximately 30 to 50 percent lower absolute signal. They both show maximum peak values in a narrow frequency band of $20 \mathrm{~Hz}$ to $89 \mathrm{~Hz}$. When the SNR is calculated the band narrows and is around $20 \mathrm{~Hz}$ to $40 \mathrm{~Hz}$. 


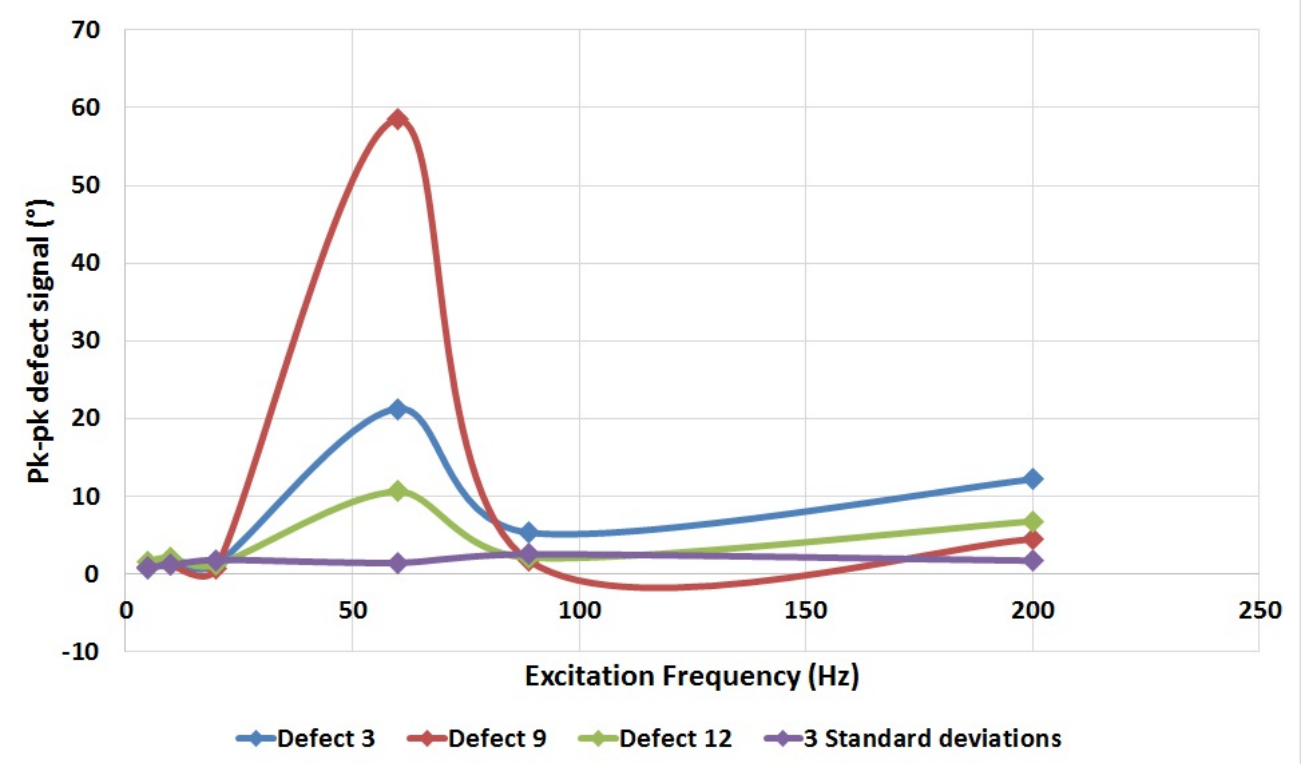

Figure 5.64: Phi field phase shift defect signal peak to peak vs excitation frequency(Points from each defect have been drawn as a guide)

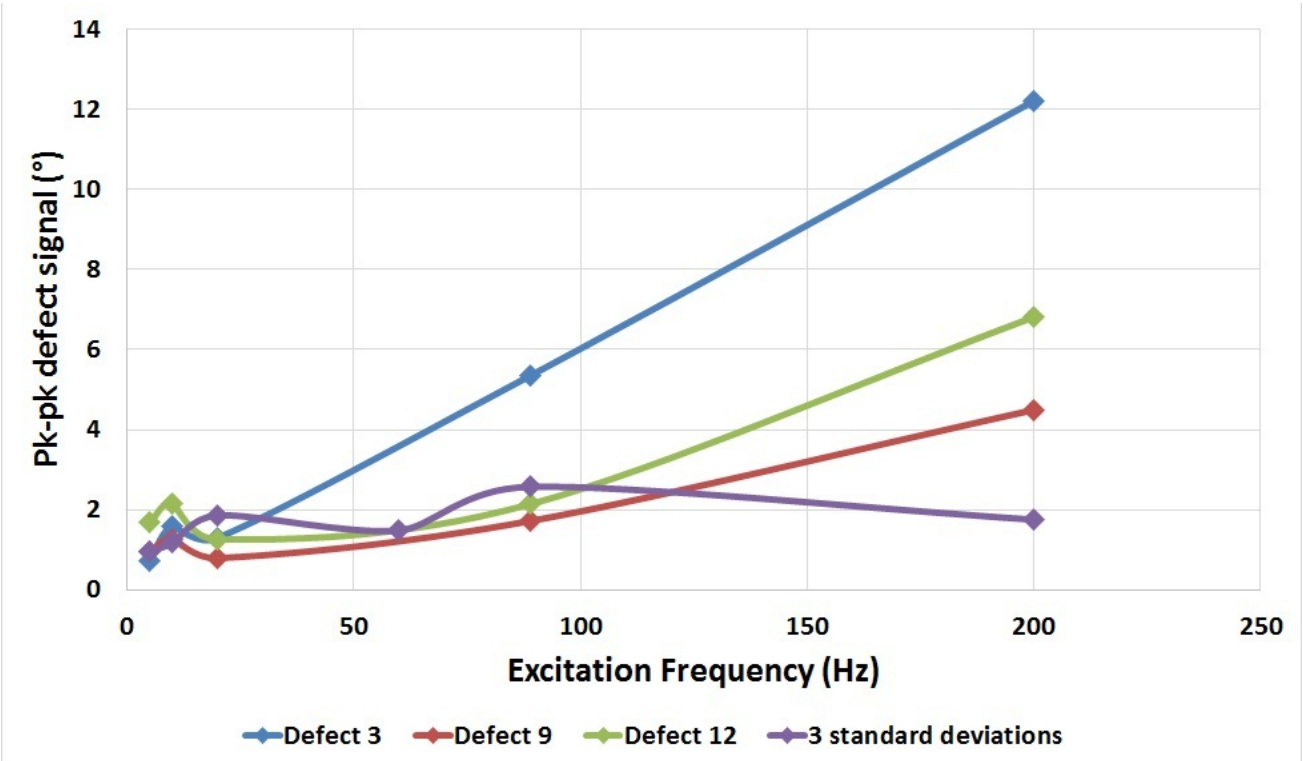

Figure 5.65: Phi field phase shift defect signal peak to peak vs excitation frequency with $60 \mathrm{~Hz}$ data removed(Points from each defect have been drawn as a guide) 


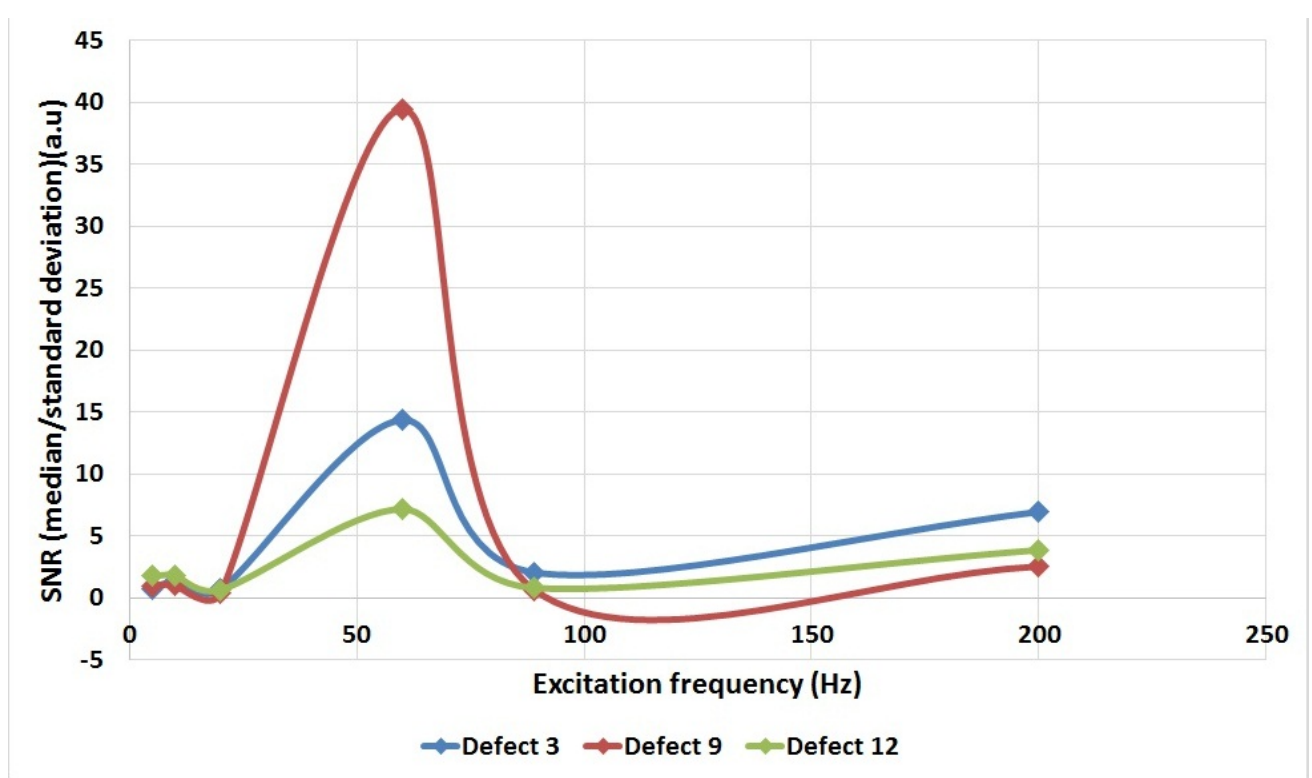

Figure 5.66: Phi Phase defect signal SNR vs excitation frequency(Points from each defect have been drawn as a guide)

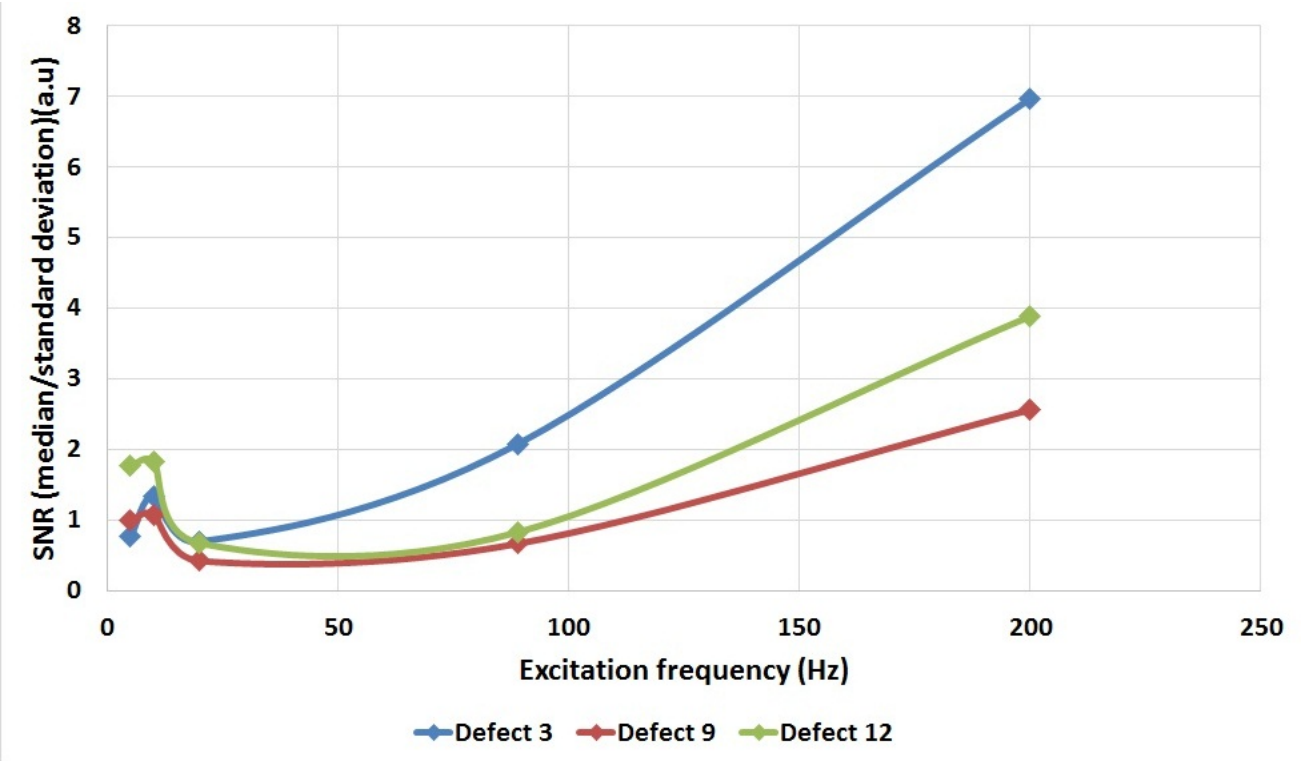

Figure 5.67: Phi Phase defect signal SNR vs excitation frequency $60 \mathrm{~Hz}$ data removed(Points from each defect have been drawn as a guide) 


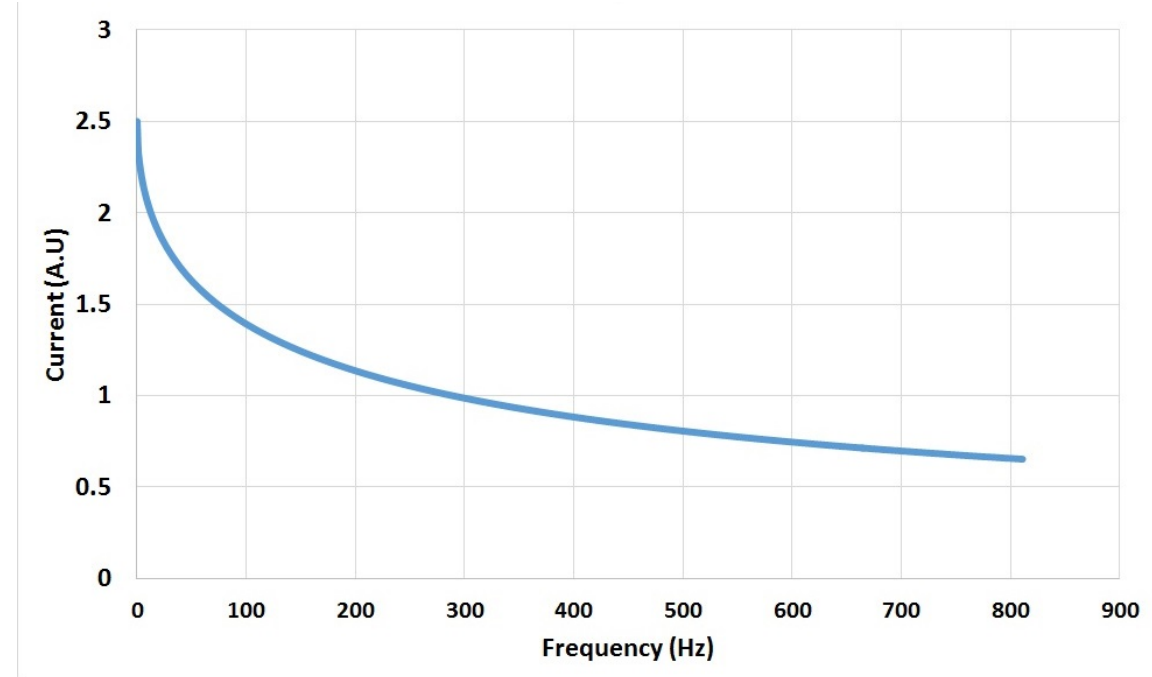

Figure 5.68: Eddy current distorted by $2.5 \mathrm{~mm}$ deep defect change with frequency

The eddy current distortion, caused by a defect, can be estimated by assuming that all eddy currents that pass through a defect would be distorted. To calculate this for a box defect, equation 5.2 [12] is integrated from 0 to the depth of the defect. The result is an exponential decay with frequency as shown in figure 5.68 .

$$
J=J_{S} e^{\frac{-d}{\sigma}}
$$

where

$$
\begin{aligned}
J & =\text { eddy current density } \\
J_{S} & =\text { surface current density } \\
d & =\text { depth } \\
\sigma & =\text { skin depth }
\end{aligned}
$$

The defect signal peak to peak change with excitation frequency would be expected to follow this exponential increase with decreasing frequency. However, at frequencies below $20 \mathrm{~Hz}$ there is a sudden drop in field which must be due to a boundary condition for this model being reached. One possibility would be a significant field breaking through the inside wall of the pipe.

The SNR would be expected to follow a curve defined by the derived equation 5.3 (without the $\gamma f$ term) with changing frequency changing the skin depth by equation 2.4.

$$
S N R=\frac{\int_{0}^{\text {defectdepth }} e^{\frac{-x}{\sigma}} \mathrm{d} x}{\int_{0}^{\text {wallthicknees }} e^{\frac{-x}{\sigma}} \mathrm{d} x+\gamma f}
$$




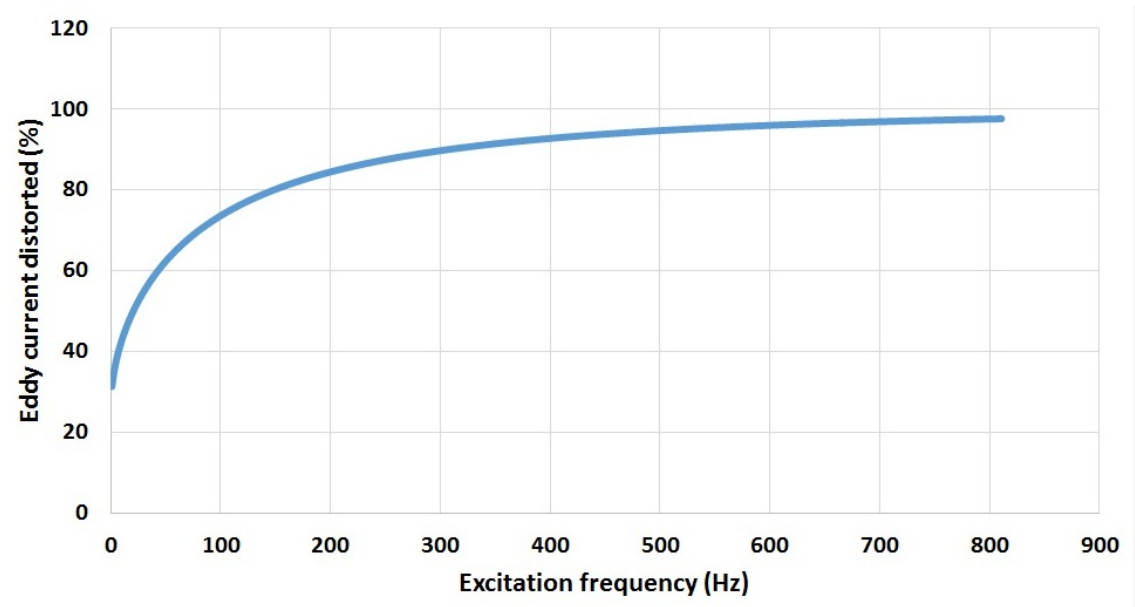

Figure 5.69: Percentage of eddy current distorted by $25 \%$ wall loss defect change with excitation frequency

where

$$
\begin{aligned}
x & =\text { depth } \\
\sigma & =\text { skin depth } \\
\gamma & =\text { background noise frequency dependent coef ficient } \\
f & =\text { frequency }
\end{aligned}
$$

This gives the percentage of the total eddy current being distorted, producing a curve seen in figure 5.69 which approaches $100 \%$ distortion at high frequencies. This model assumes that there is no variation in field caused by eddy currents flowing alongside the defect. However, the standard pipe data shows significant variations in the current flowing alongside the defect, these variations gradually increase with increasing excitation frequency. This is seen in the standard deviation change with frequency in figure 5.70. As a result of this, a frequency dependent value is added to equation 5.3 as the $\gamma f$ term to predict the SNR change with frequency. This is plotted in figure 5.71 and shows a rapid increase at low frequencies which gradually reduces at high frequencies. This same basic shape is seen in the R and Phi field magnitude SNR change with frequency in figure 5.50 and figure 5.55, however the peaks are observed at a significantly lower frequency than predicted.

\section{Field phase shift}

The Phi field phase shift vs excitation frequency increases with frequency seen in figure 5.65. As the angle of lag of the eddy current is determined by the inductive reactance of the eddy current path and this inductive reactance increases with frequency, then the phase shift would also be expected to increase with frequency.

The $R$ field phase shift vs excitation frequency shows a high variability. This means that the expected gradual increase with frequency cannot be seen. The source of this variation is again likely due to position shifts of the sensor module. However, there are not enough data points to conclusively prove this theory. 


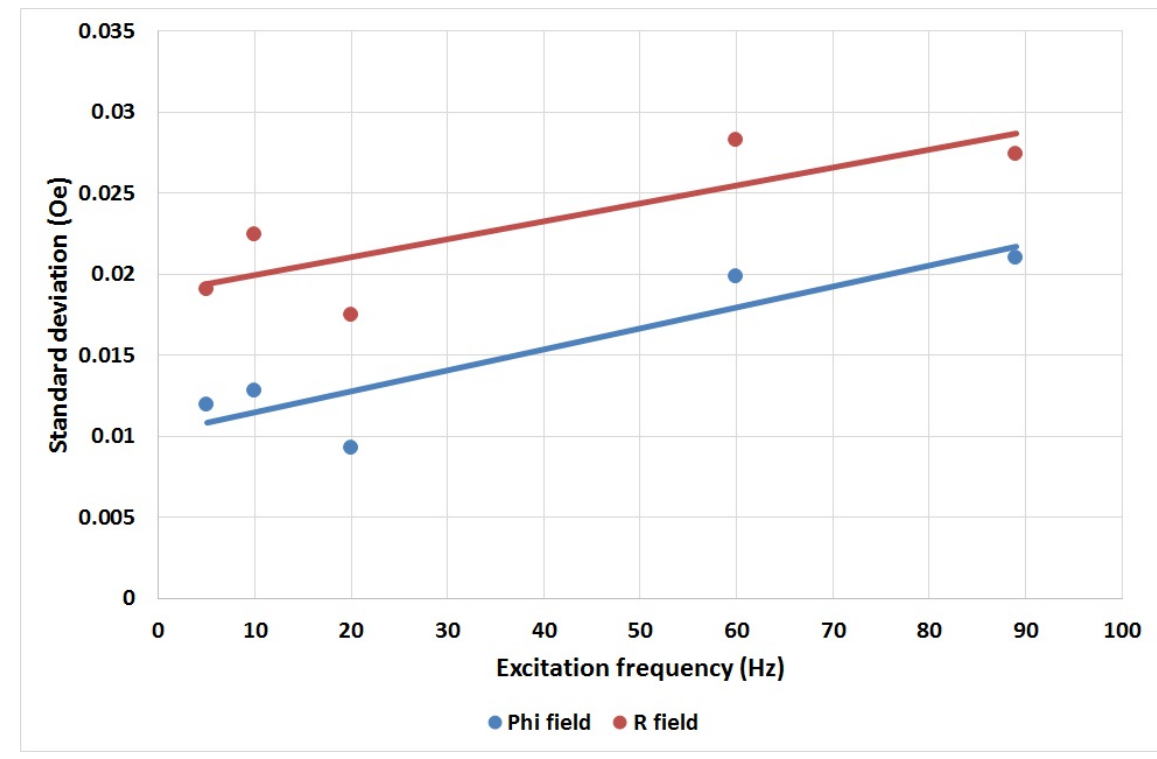

Figure 5.70: Standard pipe magnetic field standard deviation vs excitation frequency

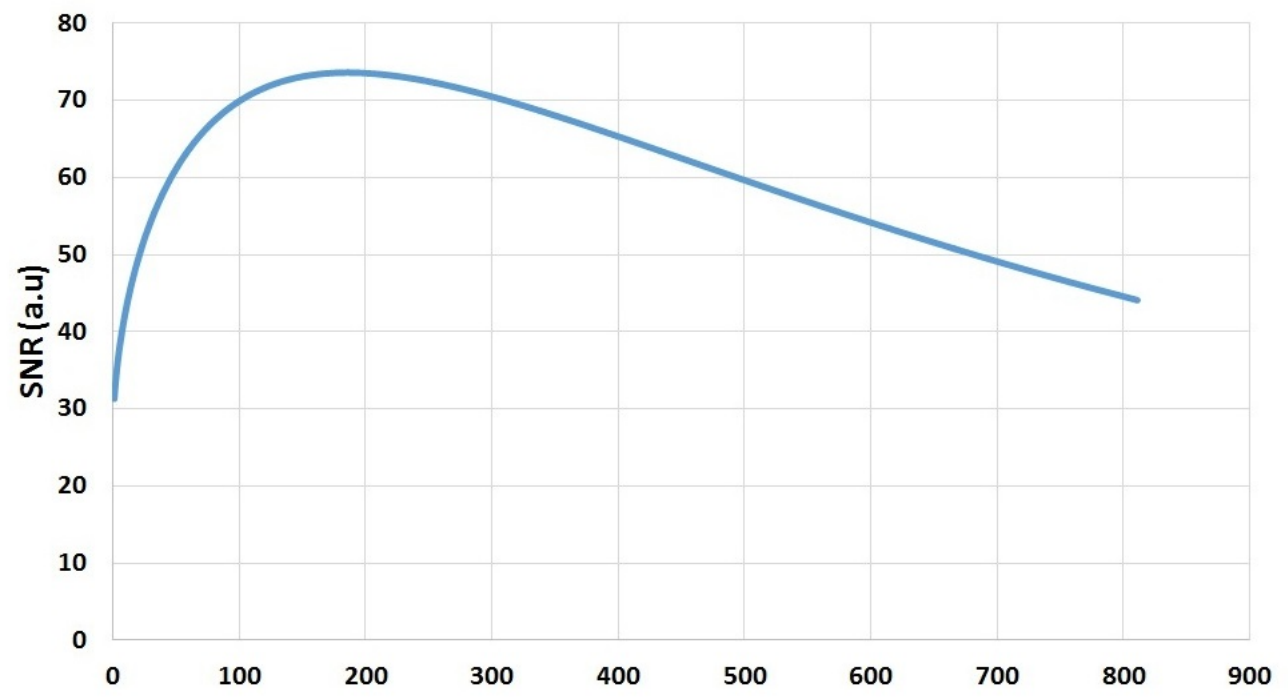

Figure 5.71: Predicted change in SNR with frequency if frequency dependent noise component is added 


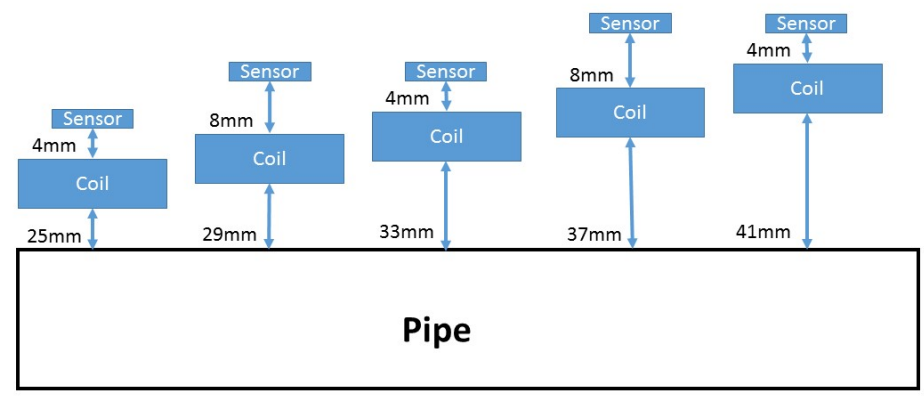

Figure 5.72: The five test configuration of excitation coil and sensor

\subsubsection{Influence of excitation lift-off on defect signals}

To determine the relationship between excitation lift-off and defect signals tests were done at $25 \mathrm{~mm}, 29 \mathrm{~mm}, 33 \mathrm{~mm}, 37 \mathrm{~mm}$ and $41 \mathrm{~mm}$, at a nominal current of $300 \mathrm{~A}$ and $20 \mathrm{~Hz}$. As in section 4.8 the excitation lift-off is the distance from the pipe surface to the excitation unit. The sensor is moved in $8 \mathrm{~mm}$ steps so that there is the same relative distance from the excitation coil to the sensor at $25 \mathrm{~mm}, 33 \mathrm{~mm}$ and $41 \mathrm{~mm}$, while at $29 \mathrm{~mm}$ and 37 $\mathrm{mm}$ the sensor is $4 \mathrm{~mm}$ further from the excitation coil, causing an expected $25 \%$ drop in magnetic field. This five test configurations are shown in figure ${ }^{* *}$. Selected results will be displayed and discussed in this following section

\section{Influence of excitation lift-off on $\mathbf{R}$ field Magnitude defect signals}

The $R$ field magnitude contour plot for $25 \mathrm{~mm}$ lift-off is shown in figure 5.73 , for $33 \mathrm{~mm}$ lift-off in figure 5.74 and for $41 \mathrm{~mm}$ lift-off in figure 5.75 .

The $R$ field magnitude at $25 \mathrm{~mm}$ shows clearly identifiable defect signals for all defects except defects 4,5 and 6 . The $R$ field magnitude at $33 \mathrm{~mm}$ shows clearly identifiable defect signals for the same defects as $25 \mathrm{~mm}$ lift-off. However, a $20 \%$ drop in the peak to peak signal as well as the background variation has been found. The $\mathrm{R}$ field magnitude at $41 \mathrm{~mm}$ shows the same defect signals as the other lift-off levels. There are some large noise spikes at the start and end of the pipe and a further reduction in the absolute defect signals.

To quantify the effect of changing the excitation lift-off on the peak to peak magnitude, the peak to peak magnitude for defects 3,9 and 12 is calculated at each excitation lift-off level and plotted in figure 5.76. Linear fits are applied to each of the defects with the 37 $\mathrm{mm}$ point for defect 12 ignored. A purple line has been overlaid to show the three standard deviation level of the plain pipe data from section 4.8. The excitation lift-off shows a clear linear decay in signal strength with increasing lift-off. The linear decay of the standard deviation of the plain pipe field is significantly less than the defect signals which means defects become harder to identify at high lift-off levels. 


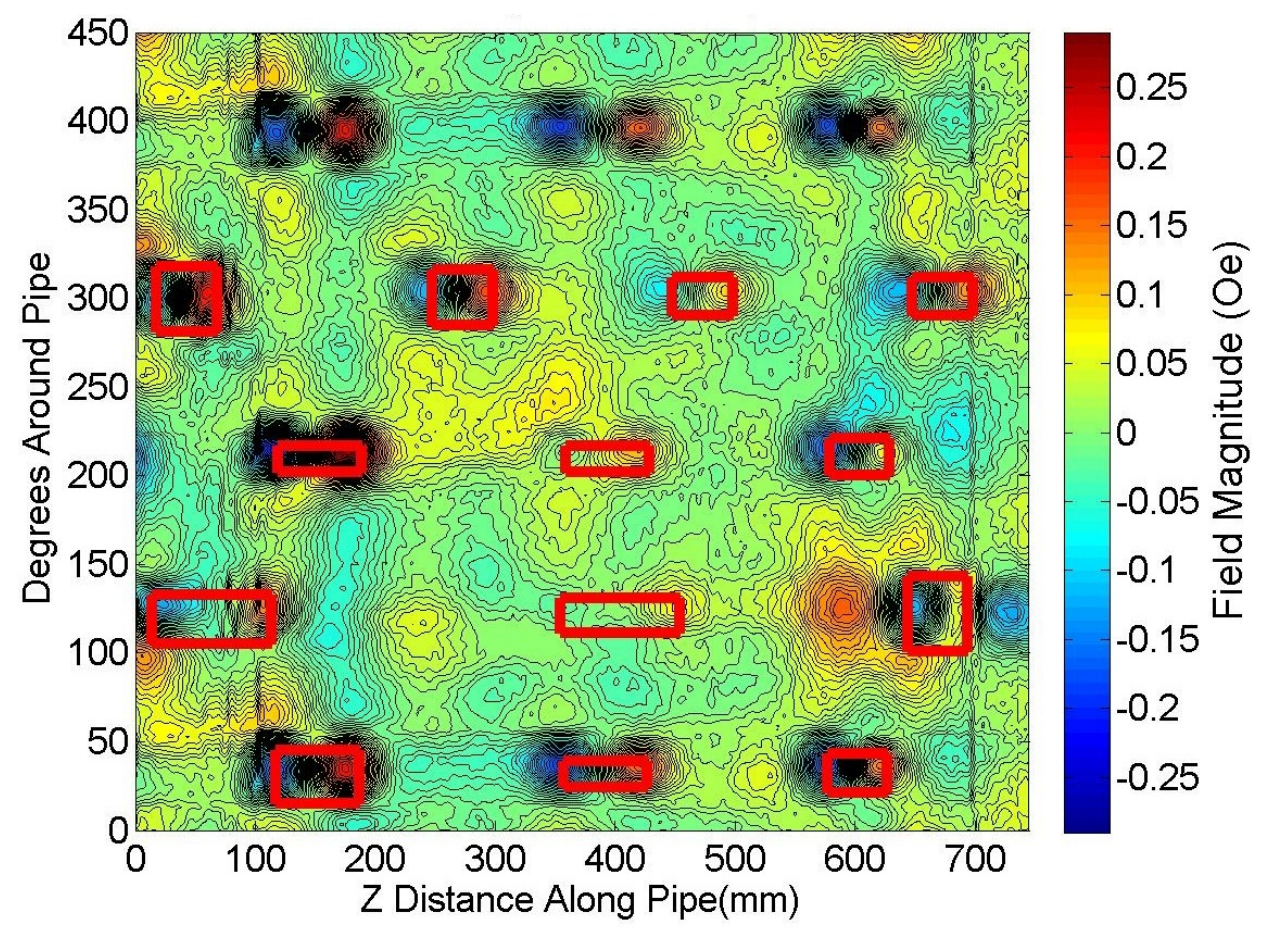

Figure 5.73: $\mathrm{R}$ field magnitude, whole scan area, $25 \mathrm{~mm}$ excitation lift-off

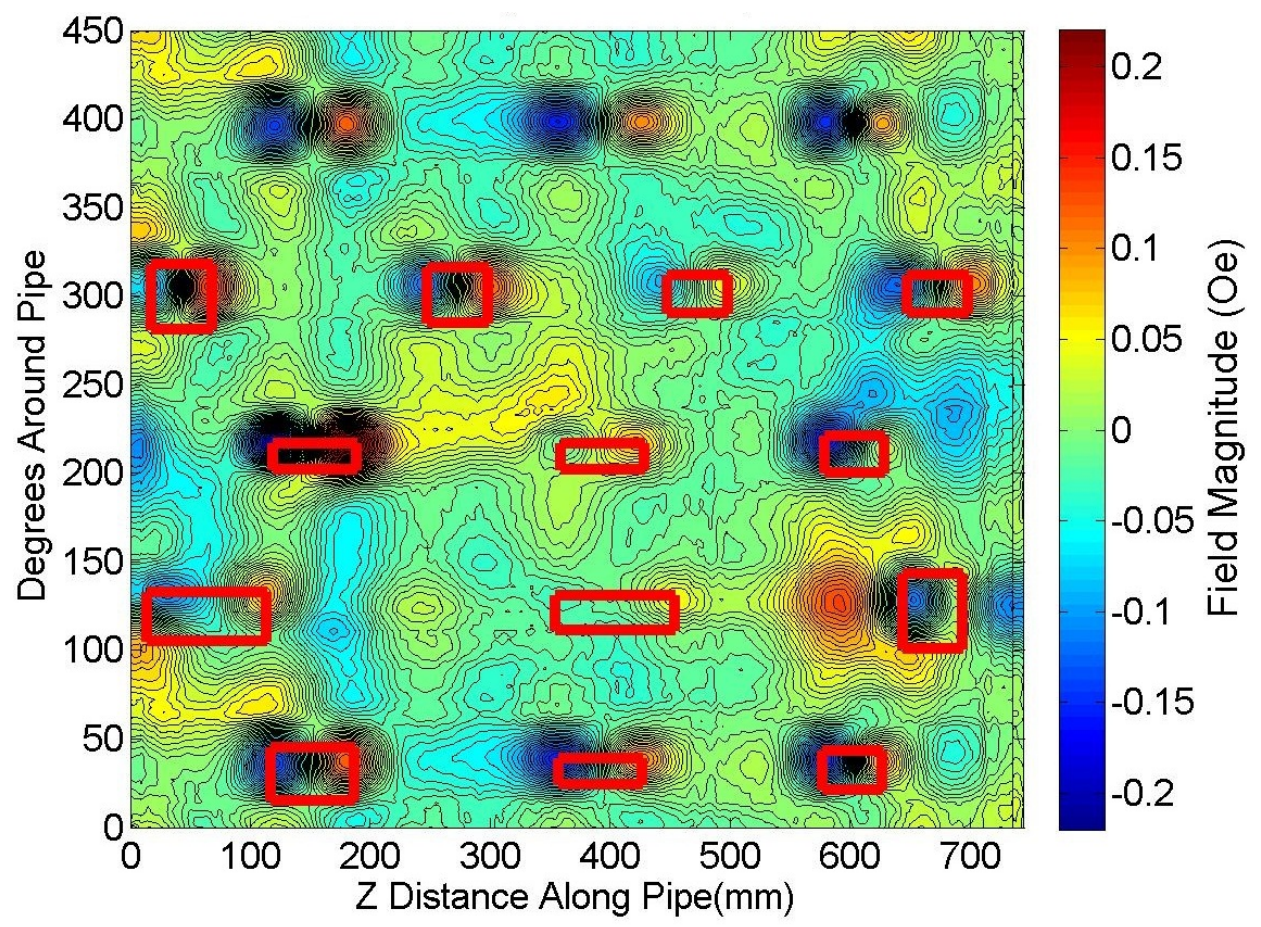

Figure 5.74: $\mathrm{R}$ field magnitude, whole scan area, $33 \mathrm{~mm}$ excitation lift-off 


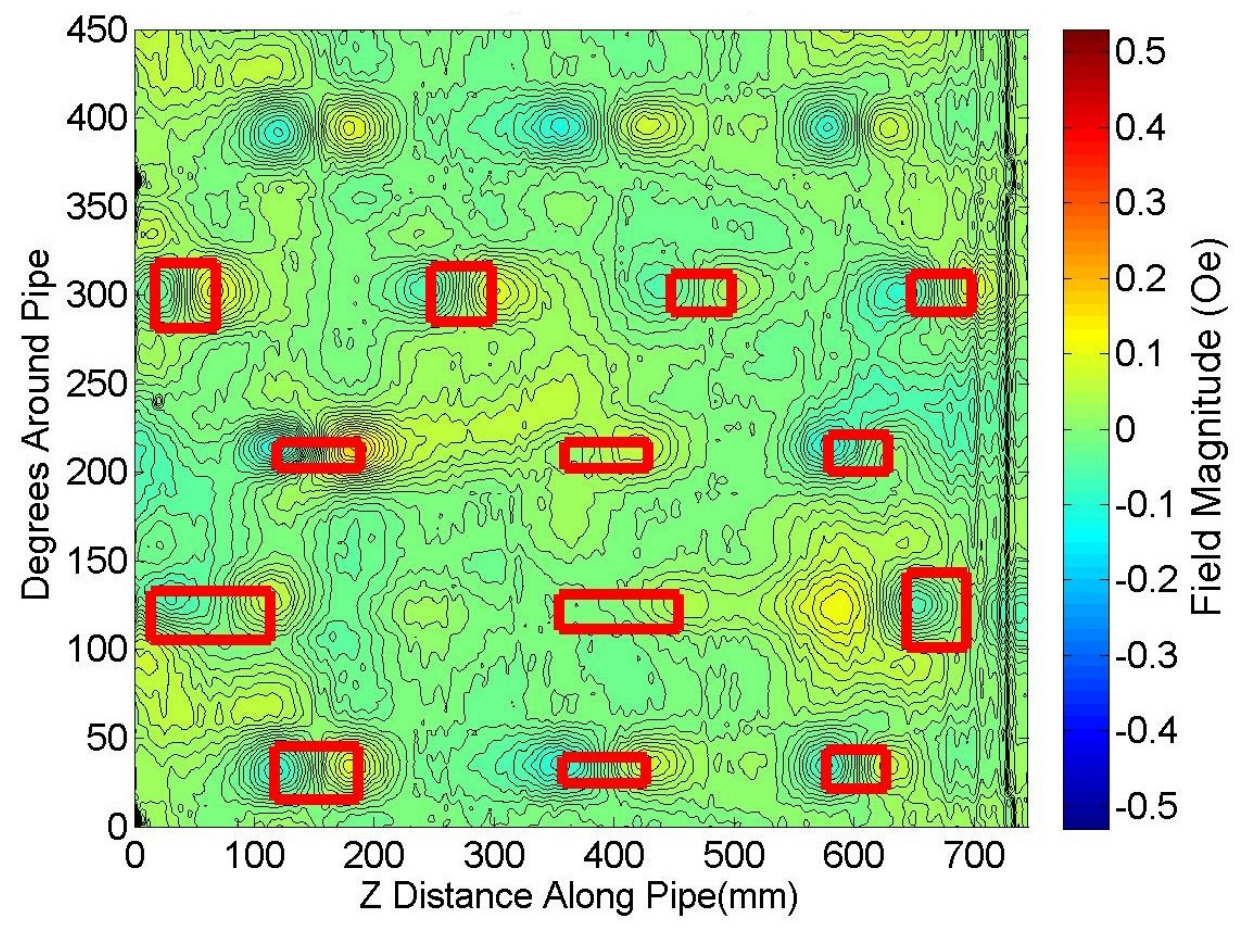

Figure 5.75: $\mathrm{R}$ field magnitude, whole scan area, $41 \mathrm{~mm}$ excitation lift-off

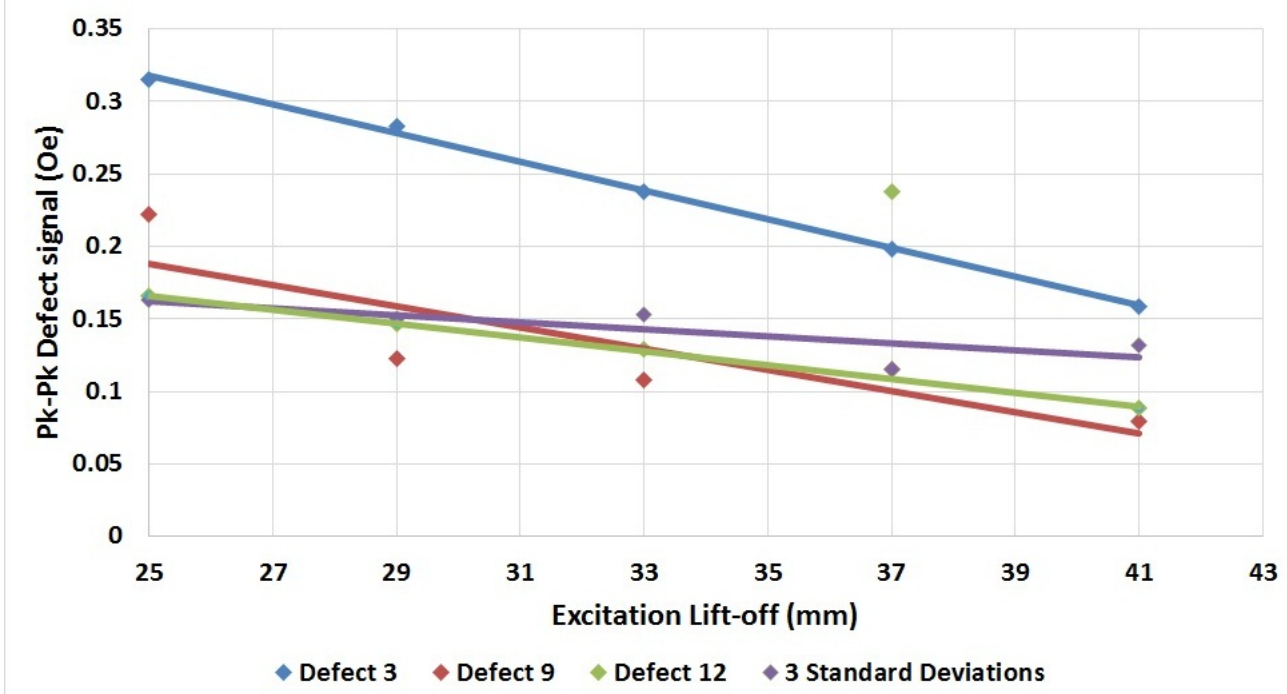

Figure 5.76: $R$ field magnitude defect signal peak to peak $R$ field vs excitation lift-off 


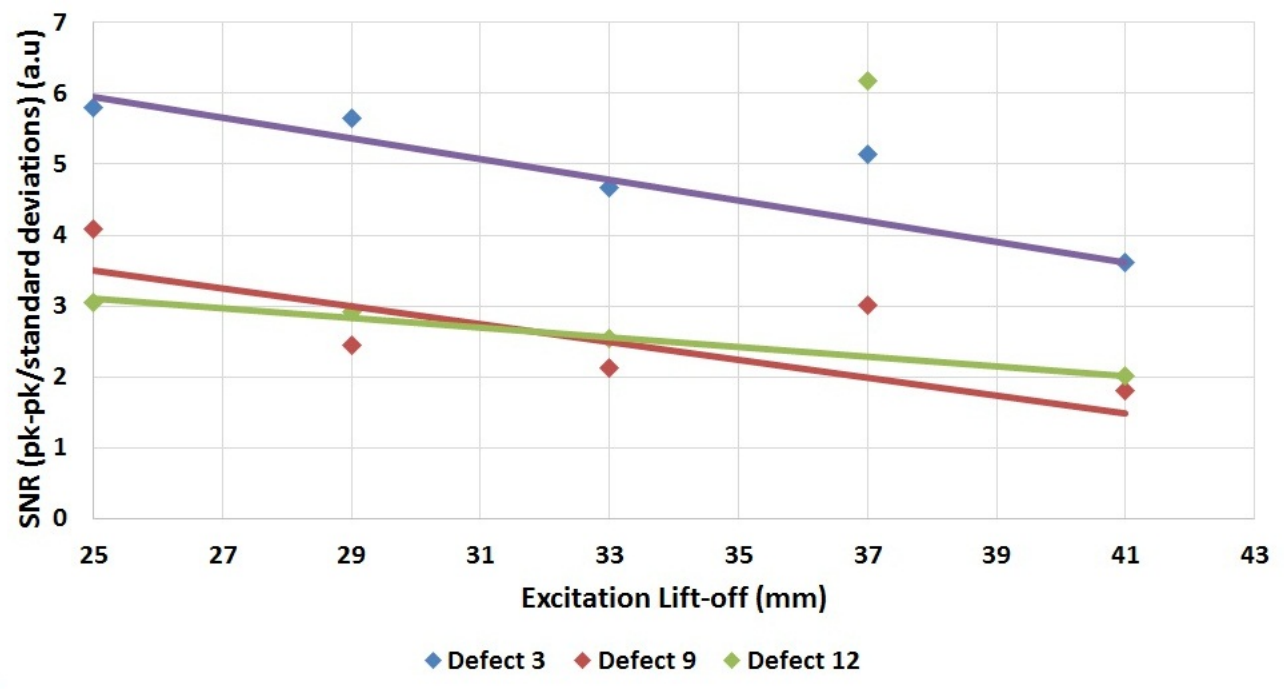

Figure 5.77: R field magnitude defect signal SNR vs excitation lift-off

The standard deviation measured at each lift-off level on the plain pipe is used to calculate the SNR for defects 3,9 and 12 and is plotted in figure 5.77. It becomes more apparent that the test with $37 \mathrm{~mm}$ excitation lift-off is an outlier and with this point removed, a linear fit is applied to the remaining data. The linear fits show that the large through-hole defect has a significantly larger SNR compared to the box defect and segment defect, both of which have very similar SNR levels. The box defect and segment defect are of particular interest as these are realistic defect sizes for an operational pipe [8]. The decay in SNR for these defects would predict an SNR 1 at $57 \mathrm{~mm}$ lift-off at which point the defect would be expected to disappear.

\section{Influence of excitation lift-off on Phi field Magnitude defect signals}

The effect of the excitation lift-off on the Phi field magnitude is plotted for $25 \mathrm{~mm}$ lift-off in figure 5.78 , for $33 \mathrm{~mm}$ lift-off in figure 5.79 , and for $41 \mathrm{~mm}$ in figure 5.80 .

The Phi field magnitude change at $25 \mathrm{~mm}$ excitation lift-off identifies all but defect 6 which has a significant distortion from the expected pattern due to its large volume. The Phi field magnitude change at $33 \mathrm{~mm}$ excitation lift-off shows a close to $50 \%$ drop in absolute defect signals. However, there are still clear defect signals in the same locations as at $25 \mathrm{~mm}$ lift-off due to a smaller background. The Phi field magnitude change at 41 $\mathrm{mm}$ excitation lift-off shows an approximately $40 \%$ drop in signal level, compared to 33 $\mathrm{mm}$ lift-off. The same defects are still clearly identifiable which indicates the Phi sensor is capable of identifying defects with large lift-off values.

The peak to peak amplitudes are calculated for the defects 3,9 and 12 and plotted in figure 5.81. The Phi field magnitude data does not show the same large outlier at $37 \mathrm{~mm}$ excitation lift-off. However, the overall variation is much larger and does not fit well to a linear trend. The peak to peak magnitude is about half of the $R$ field magnitude so this increase in variation is expected.

As before, the SNR values are calculated at each excitation lift-off level and plotted in figure 5.82. The segment defect shows a clear linear decay with lift-off. The larger defect 3 


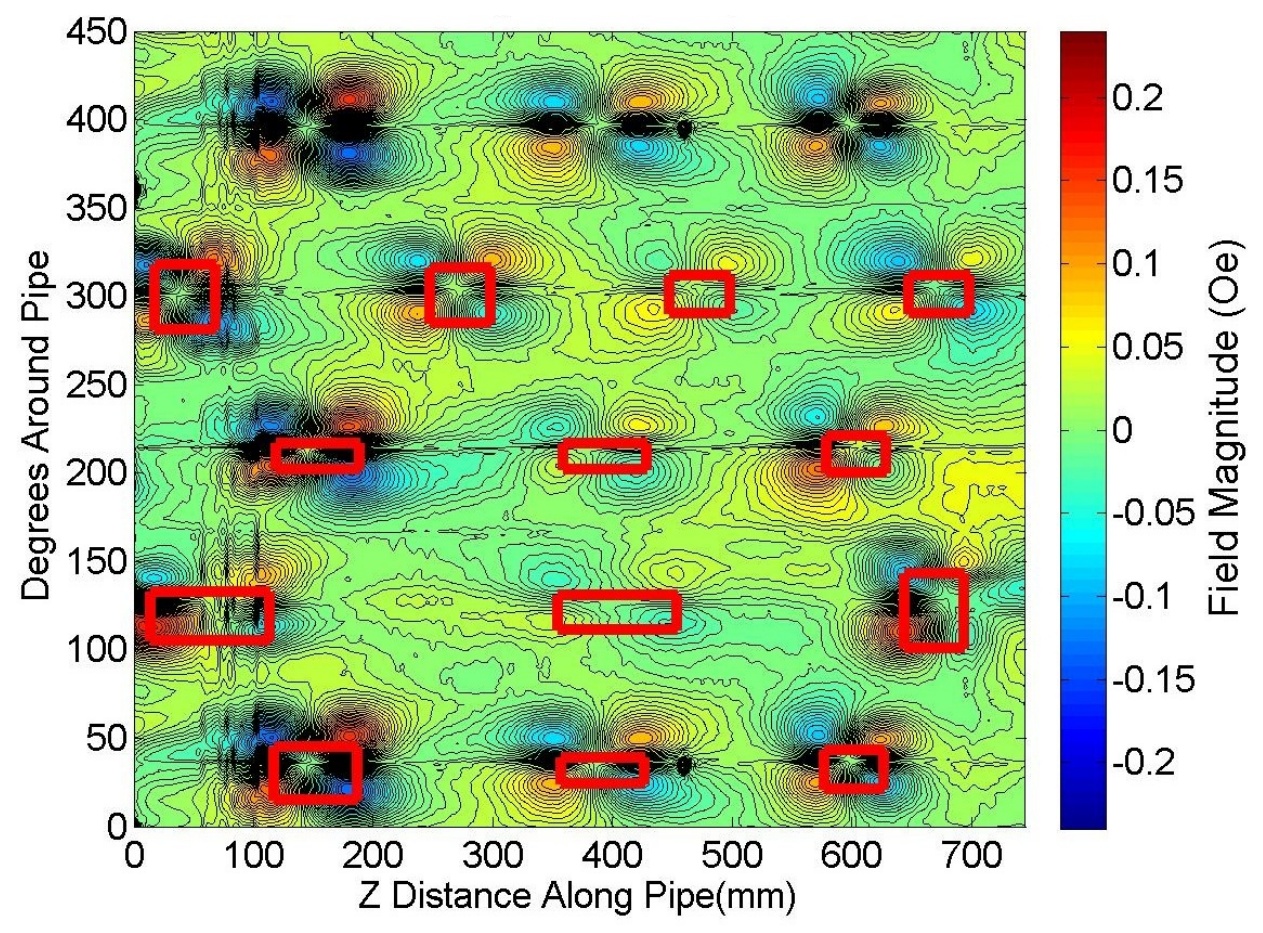

Figure 5.78: Phi field magnitude, whole scan area, $25 \mathrm{~mm}$ excitation lift-off

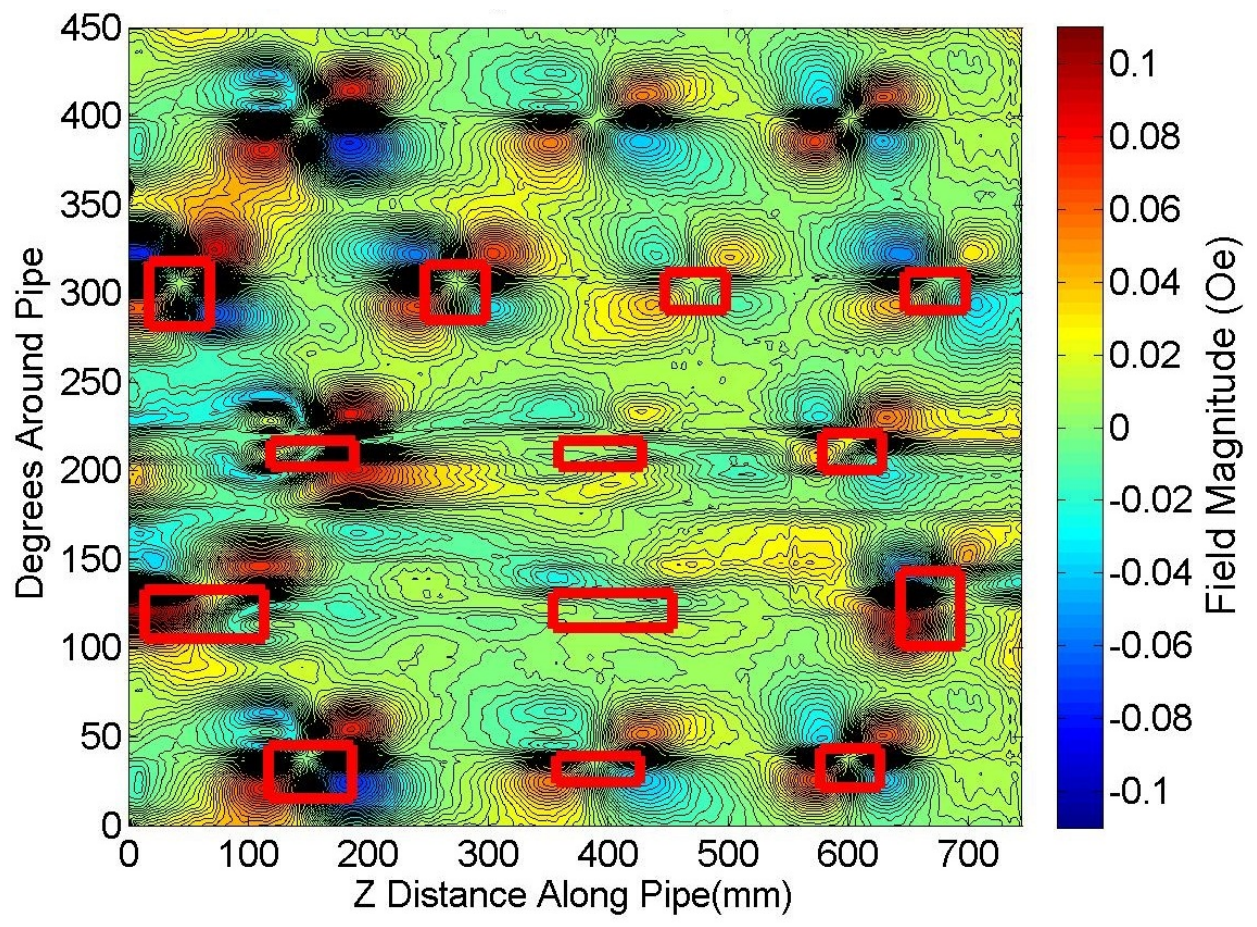

Figure 5.79: Phi field magnitude, whole scan area, $33 \mathrm{~mm}$ excitation lift-off 


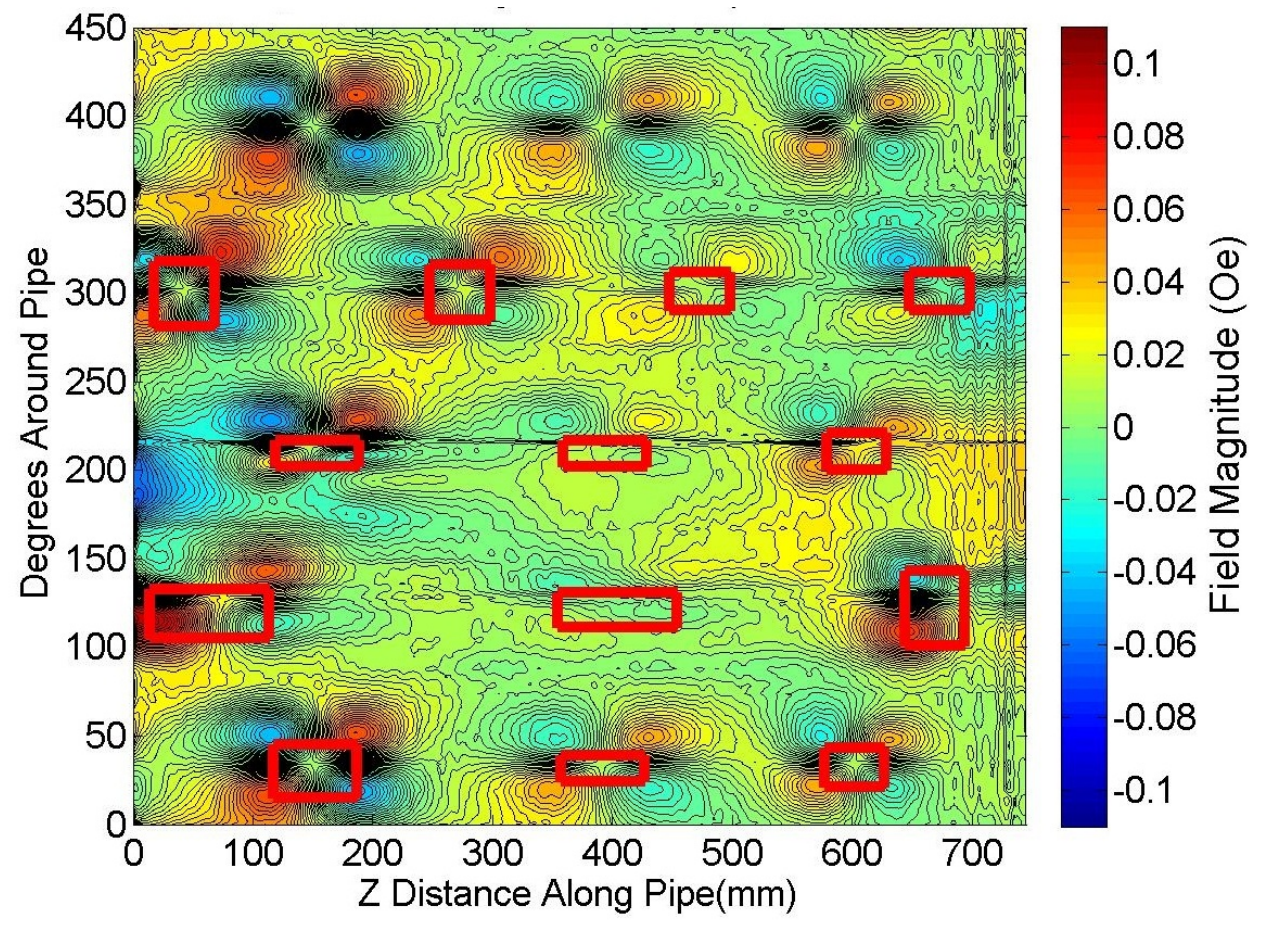

Figure 5.80: Phi field magnitude, whole scan area, $41 \mathrm{~mm}$ excitation lift-off

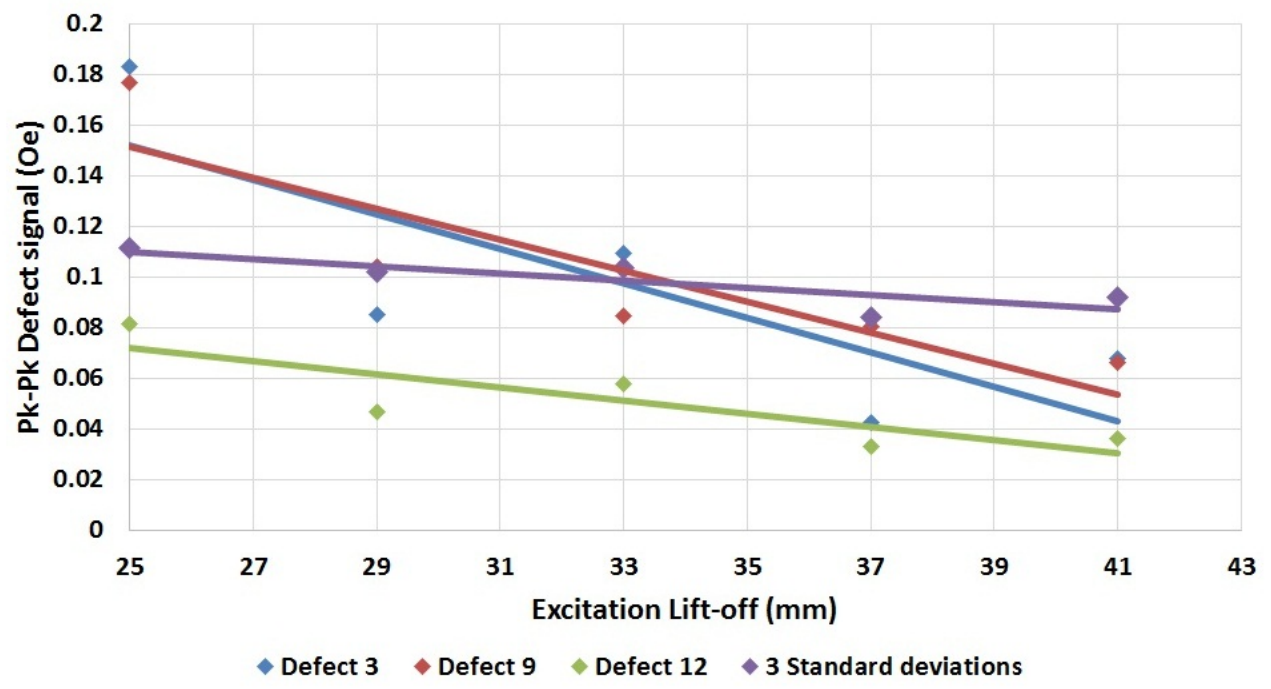

Figure 5.81: Phi field magnitude defect signal peak to peak change with excitation lift-off 


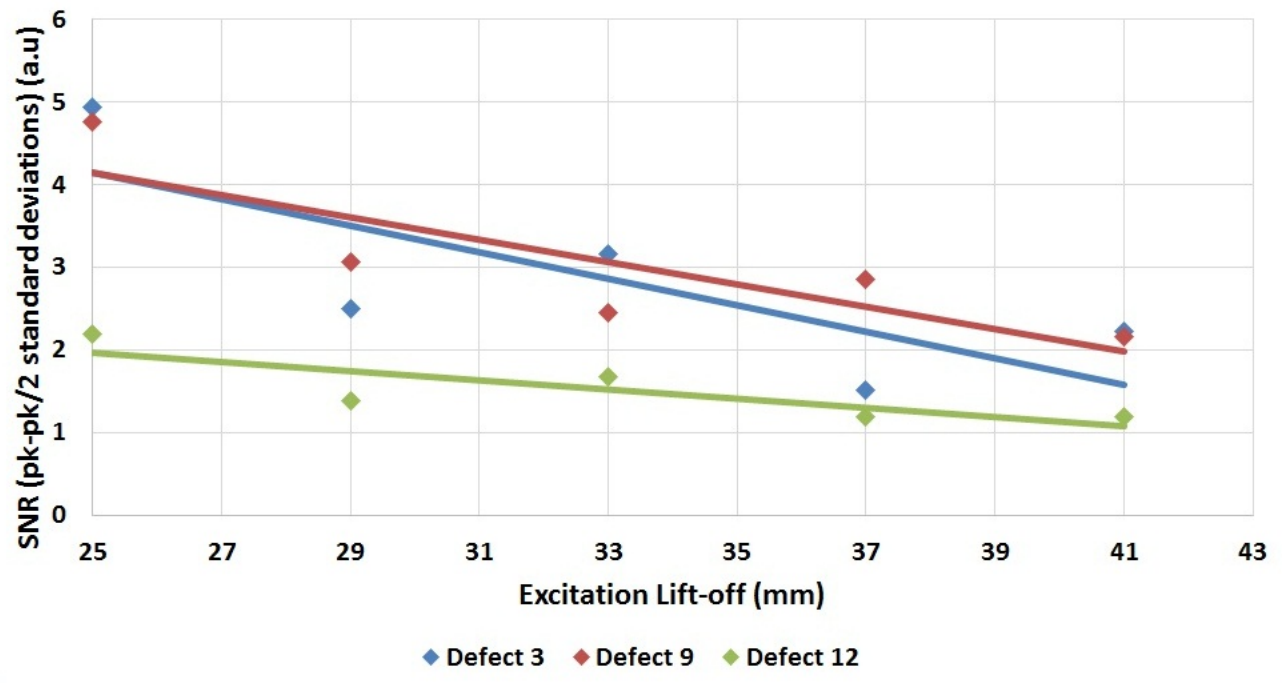

Figure 5.82: Phi field magnitude defect signal SNR change with excitation lift-off

shows larger decay with increasing lift-off but more variation than the $R$ field magnitude. The segment defect shows an SNR of 1 at $41 \mathrm{~mm}$ excitation lift-off. However this defect signal can still be identified in figure 5.80 at 300 degrees and $470 \mathrm{~mm}$. This shows that a SNR of 1 is a reasonable level to visually identify a defect signal.

\section{Influence of excitation lift-off on $\mathbf{R}$ field Phase Shift defect signals}

The effect of the excitation lift-off on the $R$ field phase shift is plotted at $25 \mathrm{~mm}$ lift-off in figure 5.83, at $33 \mathrm{~mm}$ lift-off in figure 5.84, and at $41 \mathrm{~mm}$ lift-off in figure 5.85 .

The $R$ field phase shift at $25 \mathrm{~mm}$ excitation lift-off shows defect signals for most defects however the background noise level is high. The $R$ field phase shift at $33 \mathrm{~mm}$ excitation lift-off shows a more than $50 \%$ drop in absolute signal level with some signals disappearing into the measurement noise. The $R$ field phase shift at $41 \mathrm{~mm}$ excitation lift-off shows a further drop in signal level with only a few of the largest defects showing clear signals.

The peak to peak values are calculated for defects 3,9 and 12 and are plotted in figure 5.86. The 3 standard deviation line from the plain pipe data is overlaid. The standard deviation of the plain pipe has a large spike at $29 \mathrm{~mm}$ excitation lift-off so this point is not shown in the plot. All the defects show an initial drop in signal strength at $29 \mathrm{~mm}$ then a relatively constant response for increasing excitation lift-off.

The SNR values are calculated at each excitation lift-off level and plotted in figure 5.87. The SNR shows an initial drop with excitation lift-off exacerbated by a sudden increase in the plain pipe standard deviation. From $33 \mathrm{~mm}$ to $41 \mathrm{~mm}$ lift-off the SNR is consistently around 1 to 2 for all defects, which suggests larger lift-off measurements could be done without a reduction in SNR.

\section{Influence of excitation lift-off on Phi field phase shift defect signals}

The effect of the excitation lift-off field on the Phi field phase shift is plotted at $25 \mathrm{~mm}$ lift-off in figure 5.88, at $33 \mathrm{~mm}$ lift-off in figure 5.89, and at $41 \mathrm{~mm}$ lift-off in figure 5.90 .

The Phi field phase shift at $25 \mathrm{~mm}$ excitation lift-off shows defects 1,2,7,11. The level 


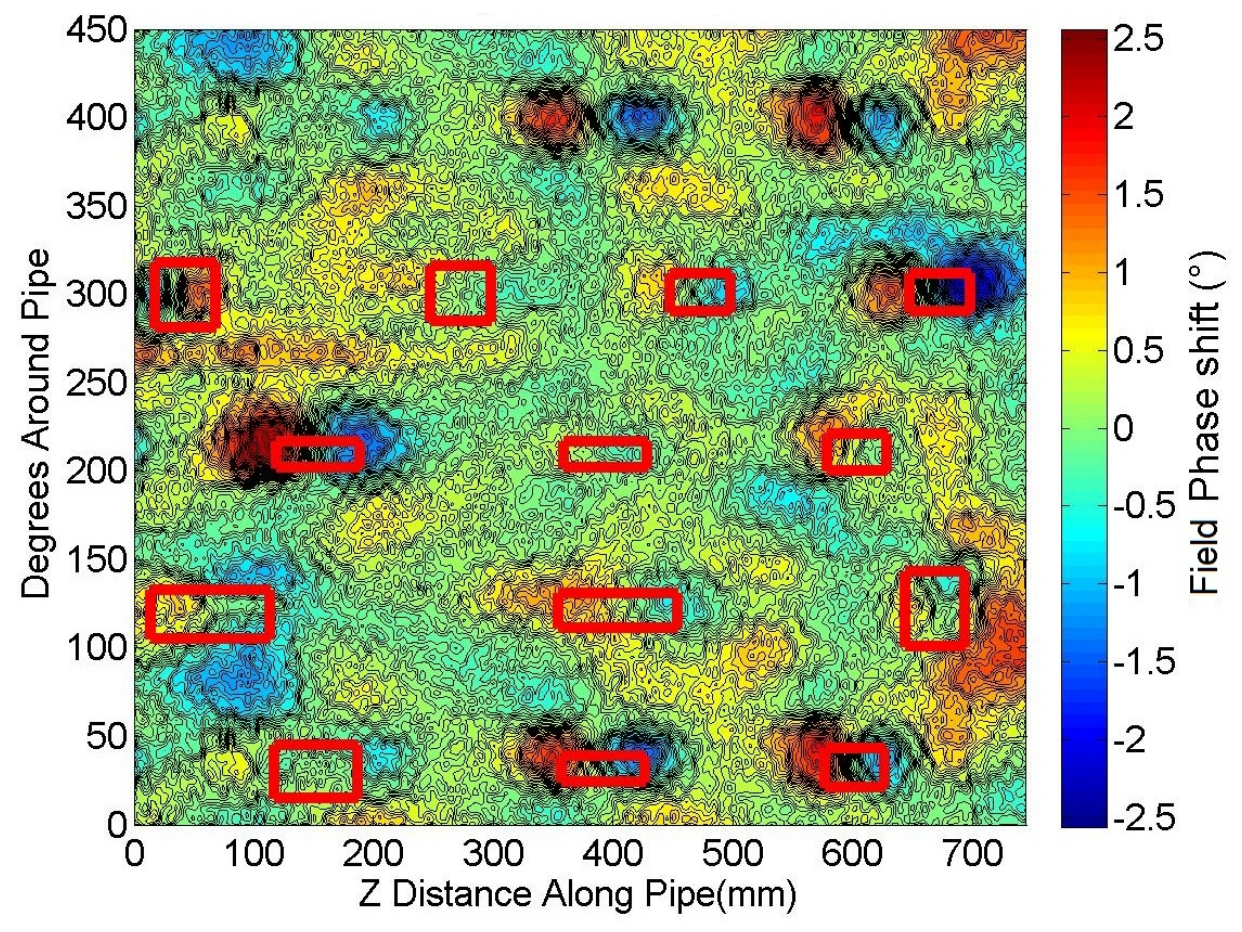

Figure 5.83: $\mathrm{R}$ field phase shift, whole scan area, with $25 \mathrm{~mm}$ excitation lift-off

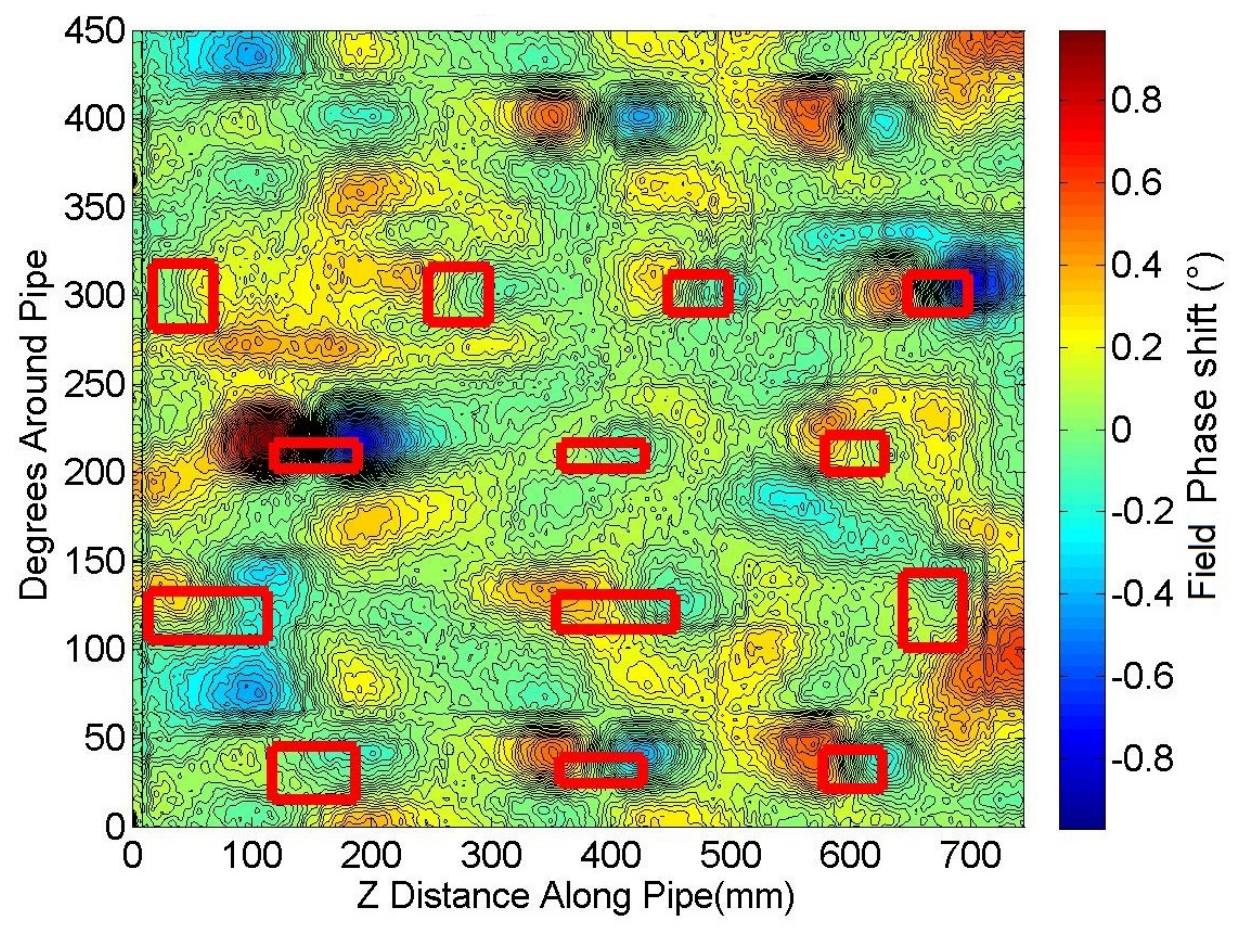

Figure 5.84: $\mathrm{R}$ field phase shift, whole scan area, with $33 \mathrm{~mm}$ excitation lift-off 


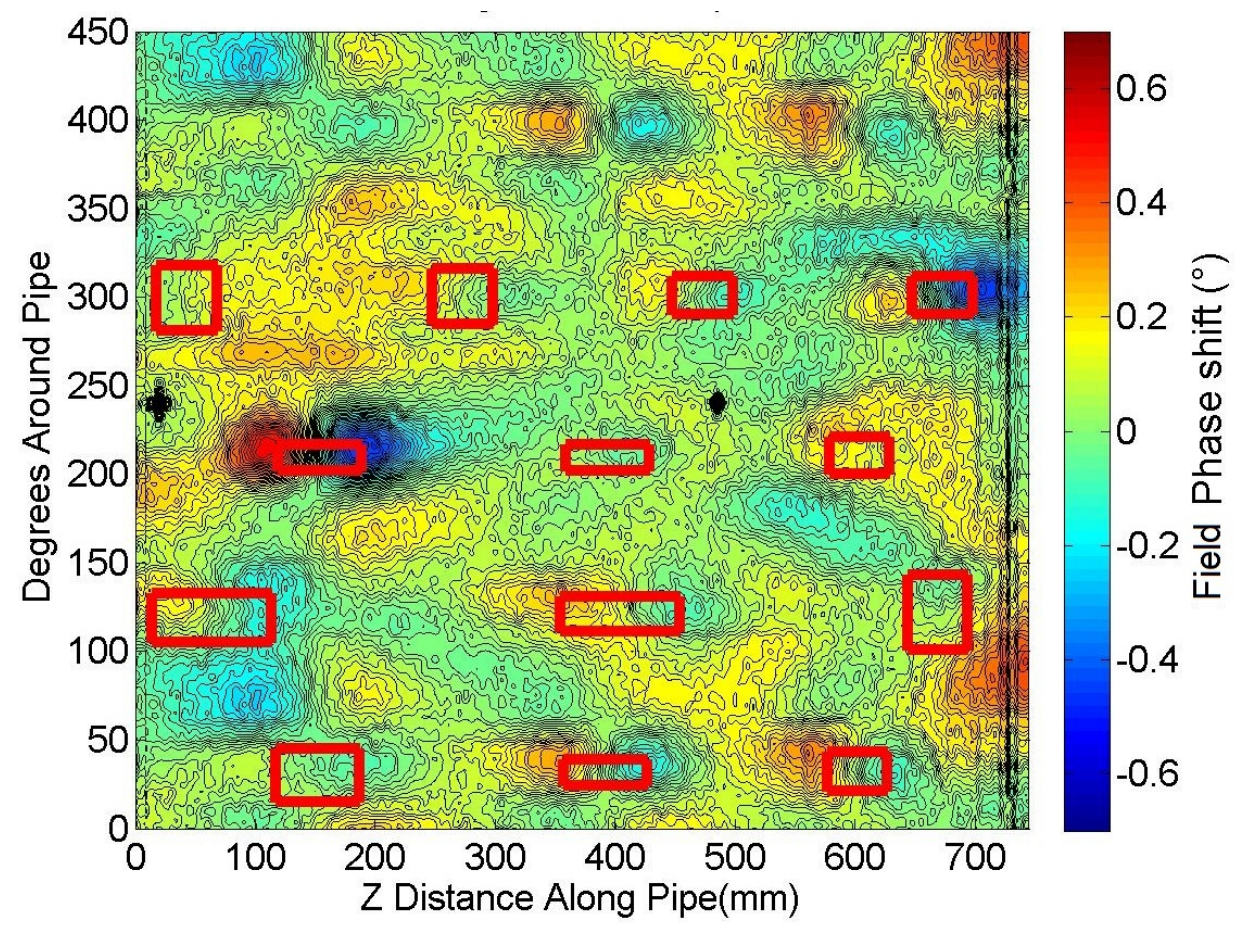

Figure 5.85: $\mathrm{R}$ field phase shift, whole scan area, with $41 \mathrm{~mm}$ excitation lift-off

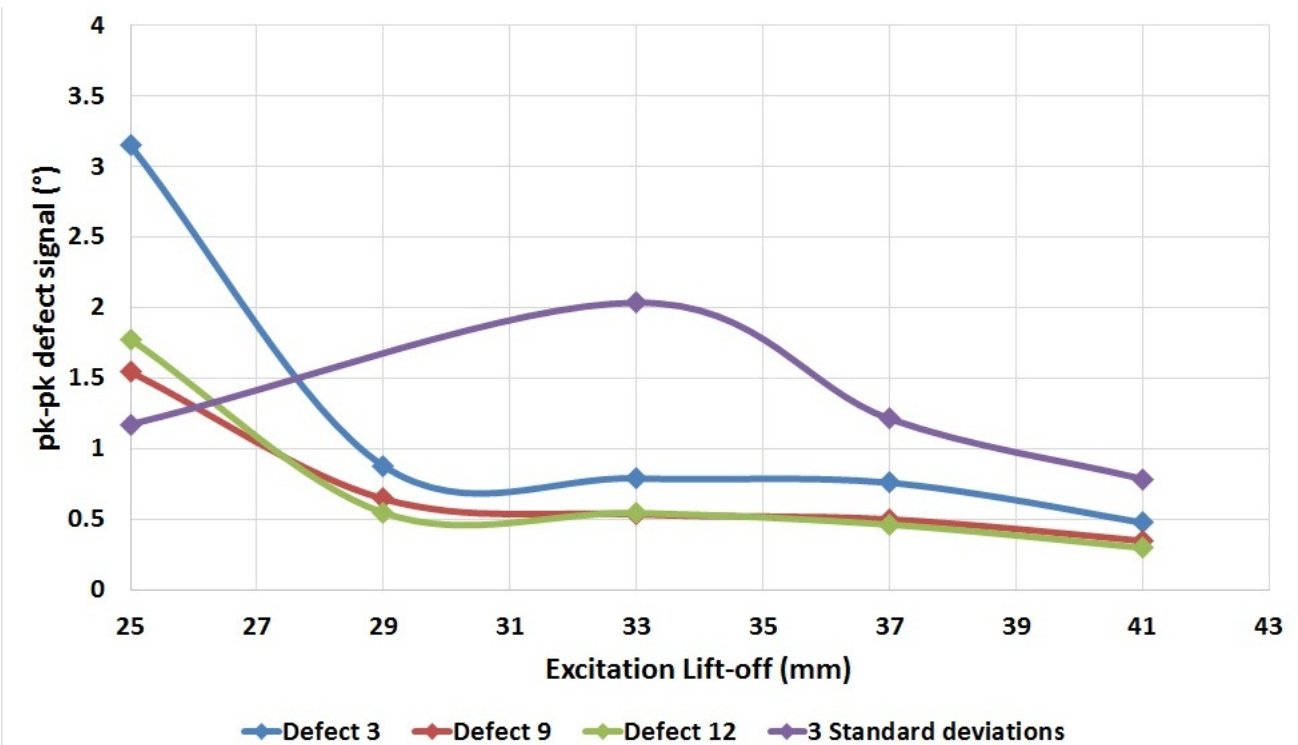

Figure 5.86: $R$ field phase shift defect signal peak to peak change with excitation liftoff(Points from each defect have been drawn as a guide) 


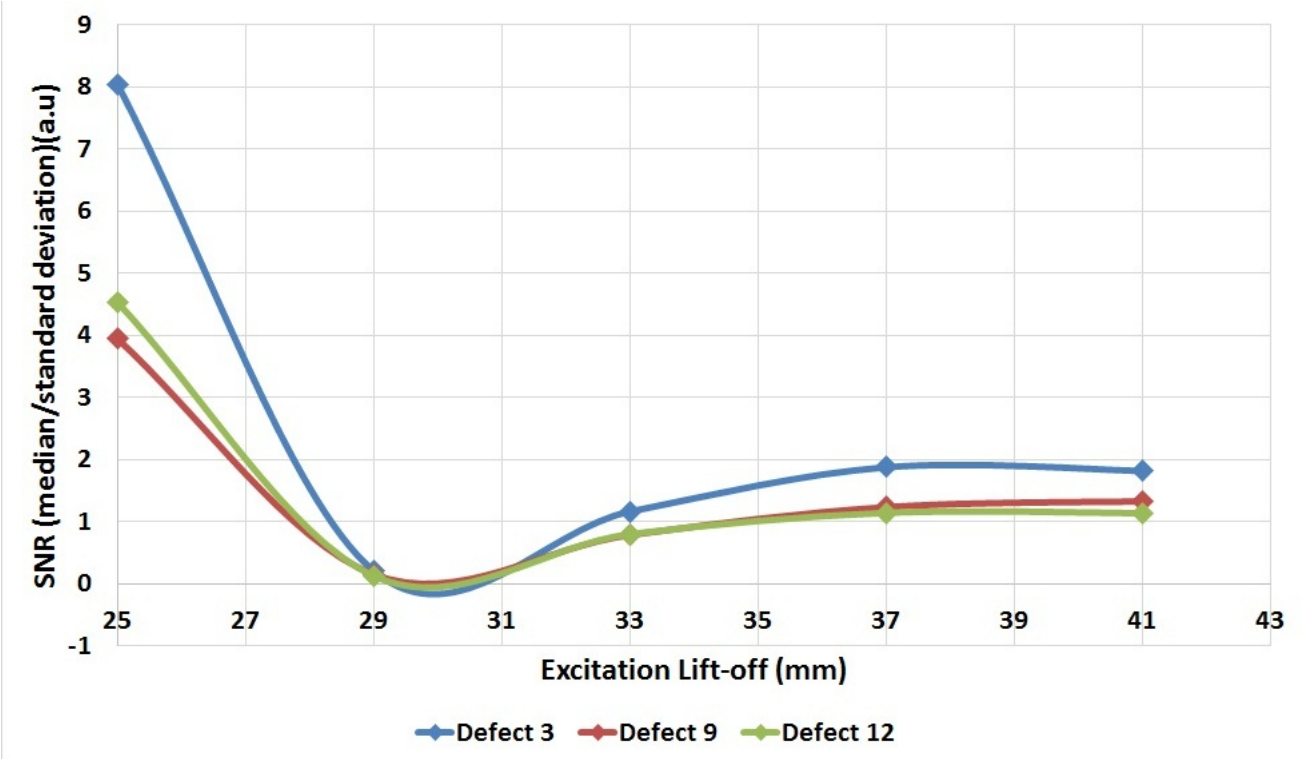

Figure 5.87: $R$ field phase shift defect signal SNR change with excitation lift-off(Points from each defect have been drawn as a guide)

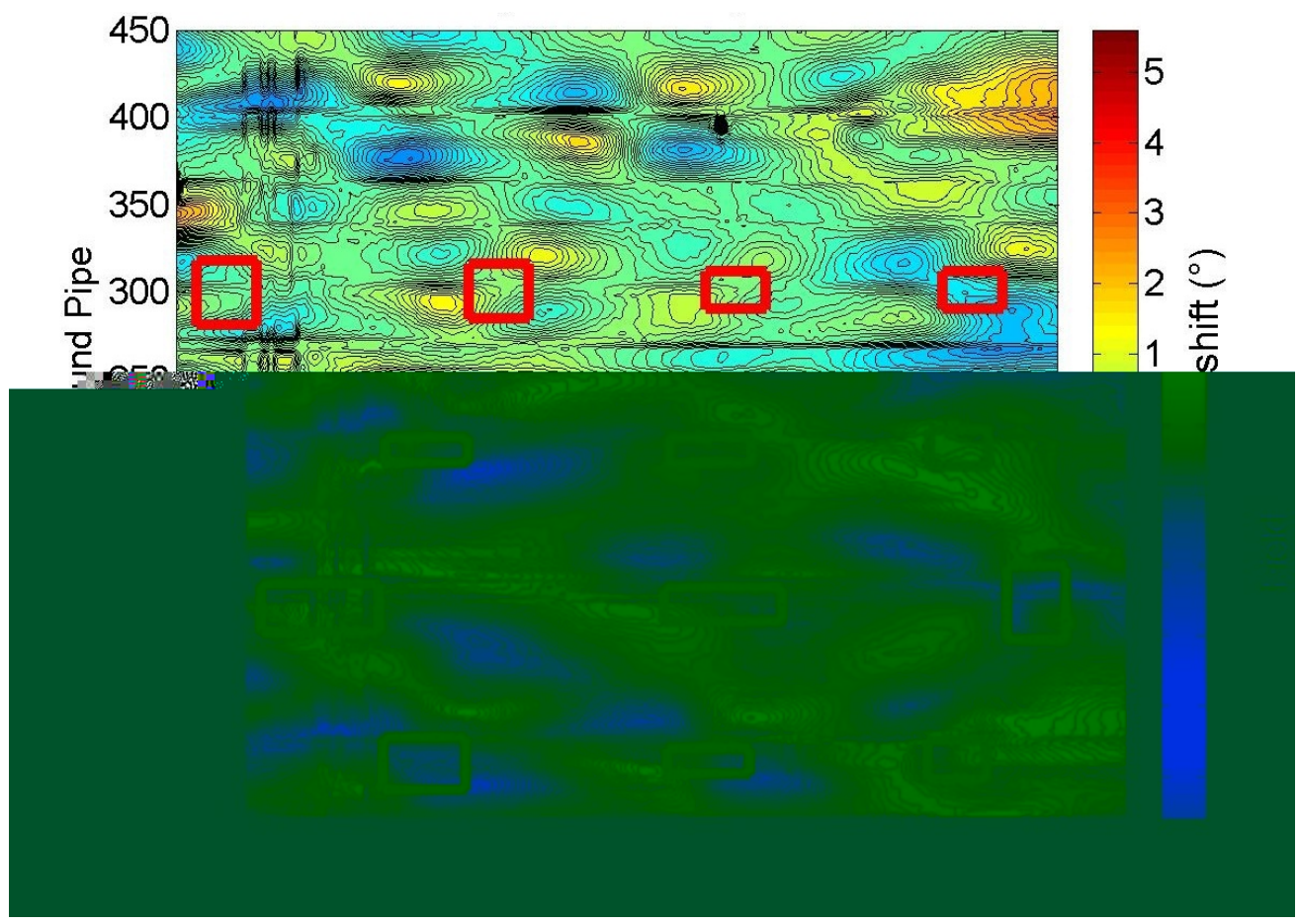

Figure 5.88: Phi field phase shift, whole scan area, with $25 \mathrm{~mm}$ excitation lift-off 


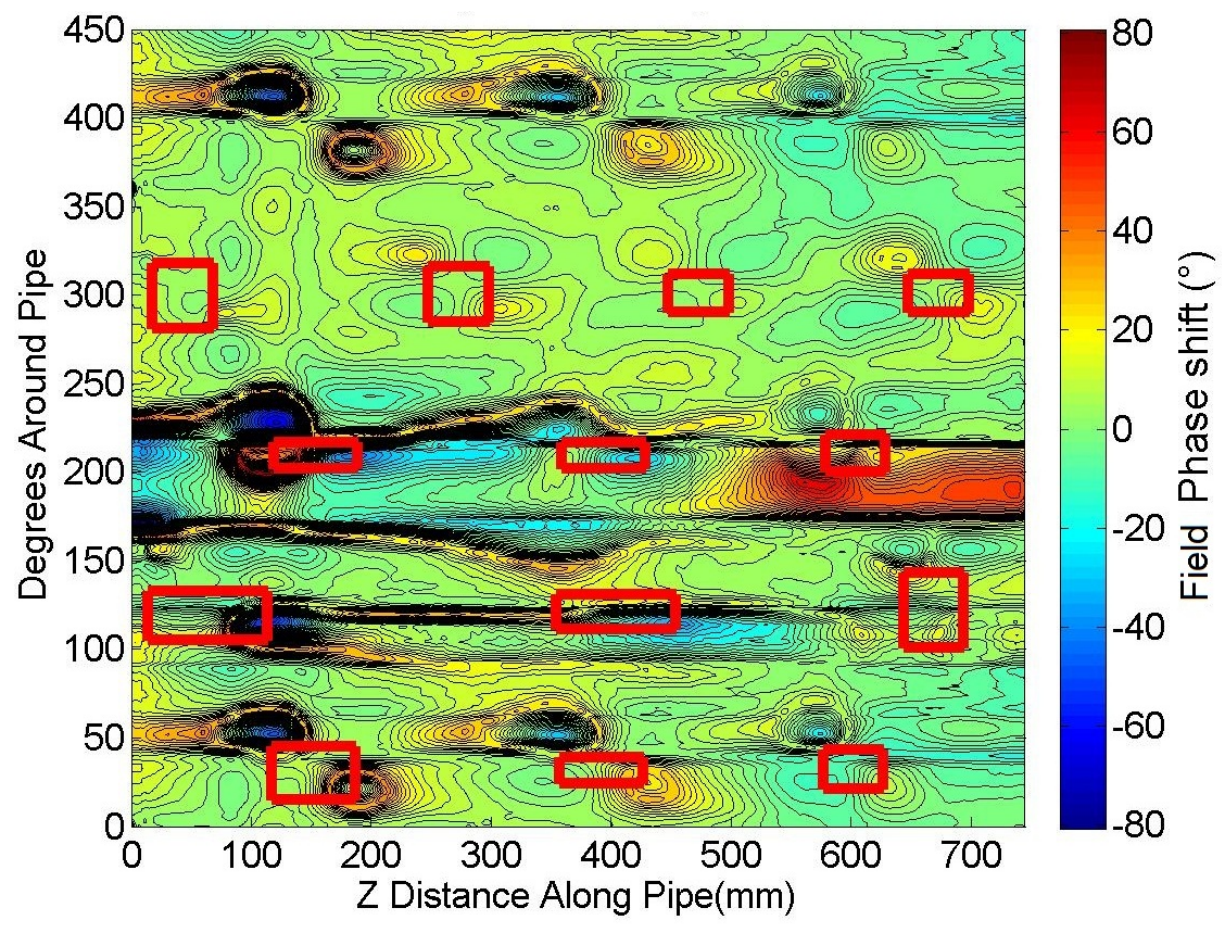

Figure 5.89: Phi field phase shift, whole scan area, with $33 \mathrm{~mm}$ excitation lift-off

of background variation makes all other defects hard to identify. The Phi field phase shift at $33 \mathrm{~mm}$ excitation lift-off shows a very large variation with defect peaks inverting, going to positive 80 degree phase shift and then back to -80 degrees phase shift within the same peak. A good example of this is the peak at 50 degrees and $100 \mathrm{~mm}$. These very large defect signals would make defect identification very easy due to an increased SNR. However, there is also an increase in background variation which may be linked to the defects as some areas have a relatively flat background.

The Phi field phase shift at $41 \mathrm{~mm}$ excitation lift-off shows a reduction in phase shifts seen at $33 \mathrm{~mm}$, however a large noise feature has appeared at 200 degrees. This may have been further exacerbated by the flattening algorithm as the algorithm does not handle variations that do not extend the total length of one axis.

The peak to peak values are calculated for defects 3,9 and 12 and plotted in figure 5.91. There is no clear relationship with lift-off. A massive variation can be seen in the phase shift defect signals with excitation lift-off. This suggests that there is a parameter that is not held constant throughout the tests. Again, this parameter is most likely the positioning of the sensors. This also suggests that there is a particular sensor placement in a small window where very large defect signals can be observed. If these measurements could be made repeatable, they would result in the best SNR achieved so far for high excitation lift-off levels.

The SNR values are calculated at each excitation lift-off level and plotted in figure 5.92. The SNR response with excitation lift-off is dominated by the data at $29 \mathrm{~mm}$ lift-off with very large defect signals and no increase in the standard deviation of the standard pipe data. 


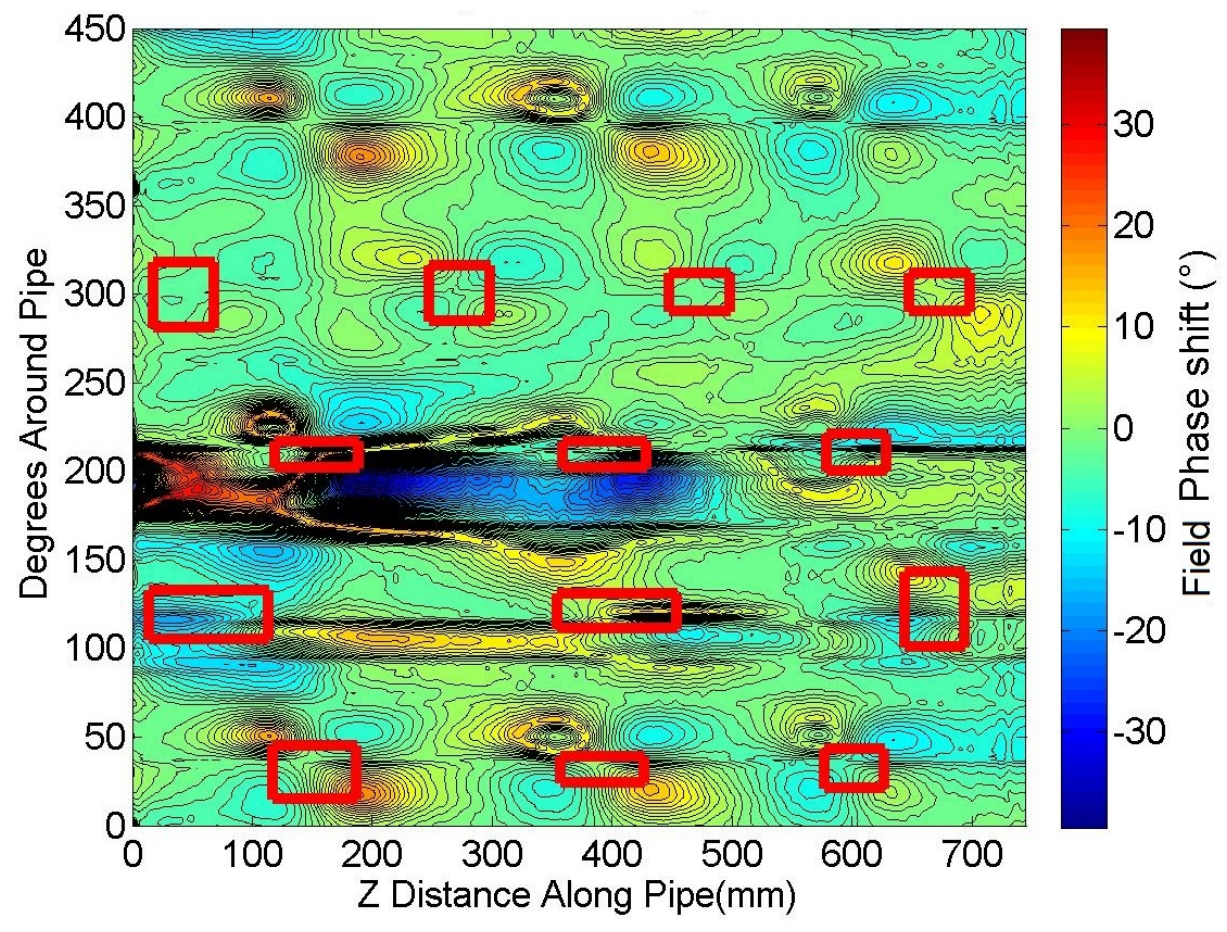

Figure 5.90: Phi field phase shift, whole scan area, with $41 \mathrm{~mm}$ excitation lift-off

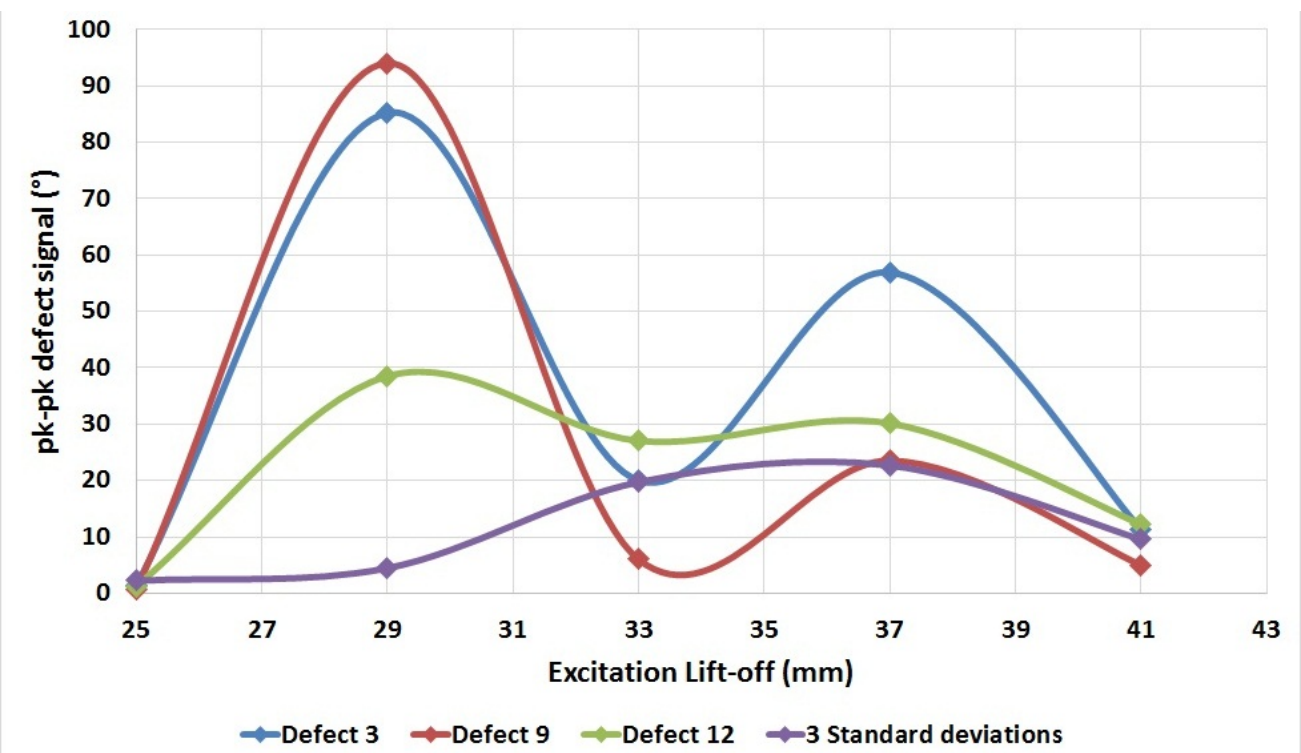

Figure 5.91: Phi field phase shift defect signal peak to peak change with excitation liftoff(Points from each defect have been drawn as a guide) 


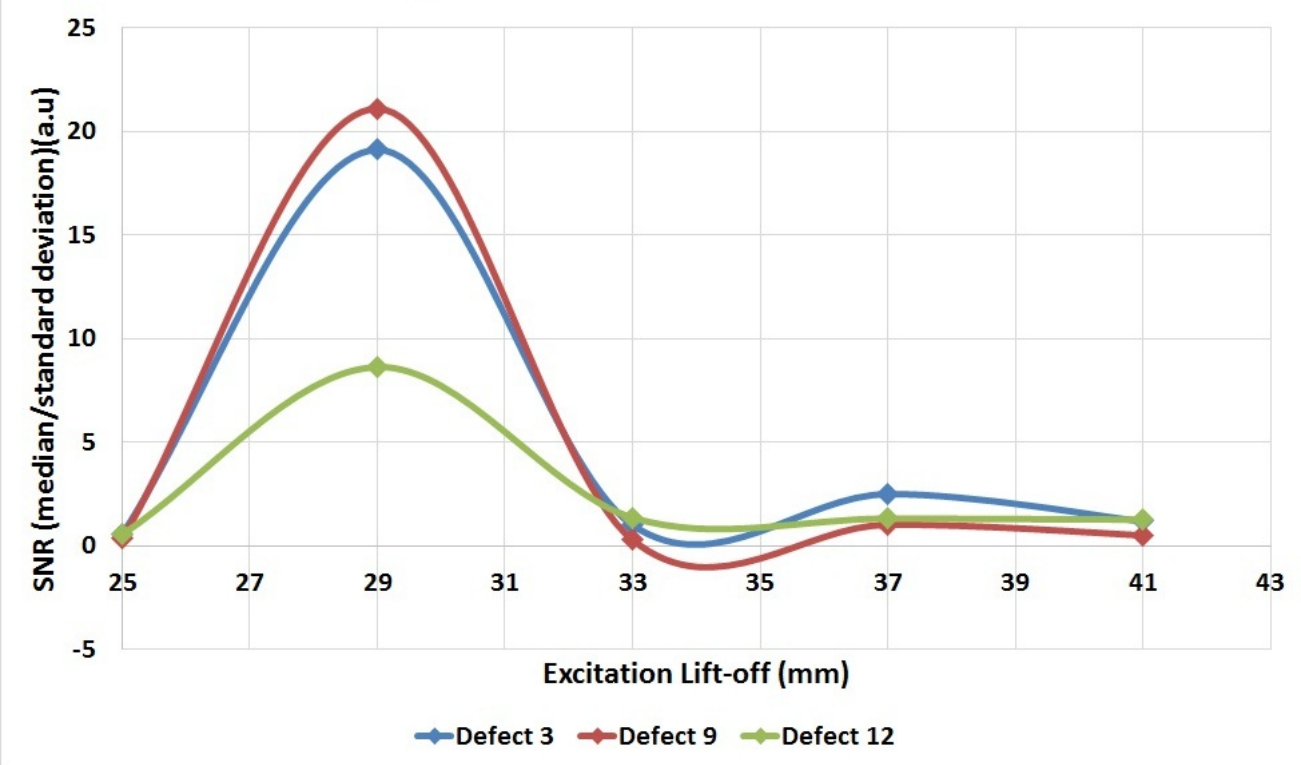

Figure 5.92: Phi field phase shift defect signal SNR change with excitation lift-off(Points from each defect have been drawn as a guide)

\subsubsection{Discussion: Influence of the excitation lift-off on defect signals}

\section{Field magnitude}

The Phi and R field magnitude shows a clear linear relationship with excitation lift-off for magnitude and SNR. The defect signal peak to peak amplitude drops with increasing lift-off due to dropping excitation field at the pipe surface so lower eddy currents are induced.

Increasing the lift-off has essentially the same effect on the defect signals as decreasing the excitation current. The SNR change with excitation lift-off for the $R$ and Phi field magnitude shows a linear decay with excitation lift-off. The limit of detection of a defect is approximately an SNR equal to 1. Figure 5.93 extends the linear fits from figure 5.77 to predict when a SNR of 1 is reached for each defect. This shows that all defects would be identifiable up to $45 \mathrm{~mm}$ lift-off and defect 3 and 12 out to $55 \mathrm{~mm}$ lift-off. This linear fit model fails when the SNR reaches zero as, by definition, the SNR cannot be negative.

\section{Field phase shift}

The field phase shift change with excitation lift-off appears to be very volatile, especially the Phi field. A gradual decay would be expected for the same reasons as the magnitude, as less eddy current circulating around the defect means lower phase shift. However, the phase shift measurements are very sensitive to the sensor placement. If the sensor placement was stabilized, the large variation seen could provide excellent data for defect identification at large lift-off levels. 


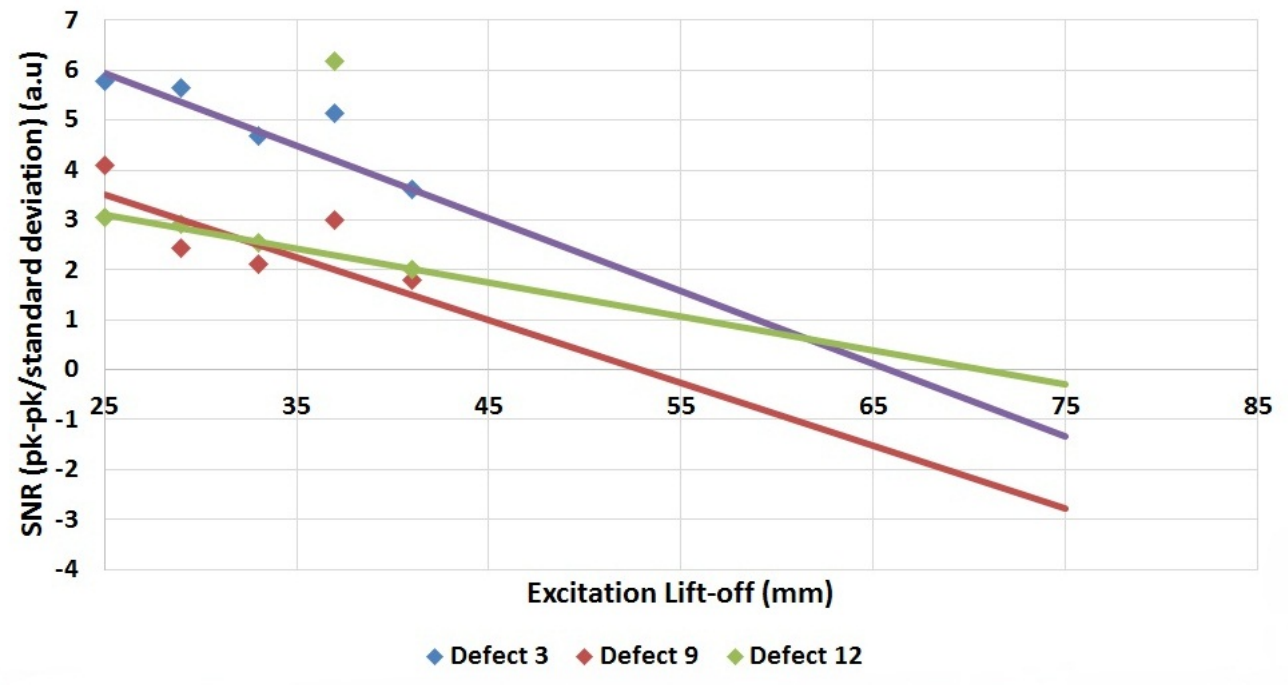

Figure 5.93: Predicted R field magnitude defect signal SNR change with lift-off out to 75 $\mathrm{mm}$ 


\section{Chapter 6}

\section{Summary and conclusions}

A prototype test rig including a sensor module and excitation unit was designed and built. The test rig enabled measurements of defects in steel pipes under aluminum weather cladding and up to $41 \mathrm{~mm}$ insulation with low frequency current excitation and a 3-axis GMR sensor measurement unit. The test rig allowed for flexible adjustment of excitation current, frequency and lift-off. It was capable of measuring a complete $0.7 \mathrm{~m}$ of pipe in 4 hrs without any intervention.

Measurements of a defect-free pipe to characterize the typical magnetic field profile were done. These tests showed that there was a strong variation in the magnetic field along the axis around the pipe (Phi axis). These variations are probably due to variation in the permeability and conductivity of the pipe inherent to the manufacturing process. Along the $Z$ axis of the pipe a strong increase in the field was observed as the end of the pipe was approached. This was caused by a combination of effects. Firstly the excitation current increased as the end of the pipe was approached due to reduced impedance. Secondly the excitation field was distorted into the direction radial to the pipe (R direction). A set of three algorithms was developed to remove these effects for further evaluation, thus decreasing the standard deviation of the field by a least $70 \%$.

A range of excitation parameters (frequency, current, lift-off) were then tested and their influence for sensors in Phi and R direction determined. This data, in combination with tests of a pipe with manufactured defects, was used to determine optimum excitation parameters.

In the next step, tests were conducted on a defect free pipe with $0.5 \mathrm{~mm}$ aluminum weather cladding wrapped around $25 \mathrm{~mm}$ insulation. The largest source of variation in the field was the riveted joint of the aluminum shield while large dents in the aluminum had only a small effect on the magnetic field at low frequency. This means the aluminum shield will not lead to a significant increase in measurement noise for tests with less than $20 \mathrm{~Hz}$ excitation frequency. Furthermore, the influence from the aluminum cladding has a different frequency dependency than defects in the pipe due to a larger penetration depth for aluminum. This means that the effect of the aluminum shield can be removed with a multi-frequency measurement and an appropriate algorithm.

Next, a range of tests were done with a pipe with manufactured defects. These tests showed that defects can be identified in the $\mathrm{R}$ direction magnitude and phase shift as a pair of positive and negative peaks each centered on the $Z$ edges of the defect. This gives 
an indication of the length of the defect. In the Phi direction magnitude and phase shift the defects can be identified as a group of 4 peaks in a square with positive and negative peaks diagonally opposite each other. This allows for a measurement of the surface area of the defect. This pattern of peaks is in agreement with the qualitative model developed.

The peak to peak amplitude of defect signals in the Phi and R direction depends on the defect volume, with larger volumes producing larger peaks.

A high variability of phase shift with changing excitation parameters indicates an unidentified parameter which is not being held constant from test to test. The most probable source of this variation is movement of the sensor module in relation to the excitation sheet between tests. This means the phase shift is very sensitive to movements of the sensor module and that the position of the sensor must be stabilized in order to achieve high sensitivity to defects. Further investigations are required to determine if this is possible.

The maximum signal to noise (SNR) for defect signals in Phi and R field magnitude occurs if the excitation current is above 300 amps. At 300 amps the SNR is $95 \%$ of its theoretical maximum. The current required to reach this $95 \%$ threshold is dependent on the standard deviation of the field at zero excitation current. This means if the field is measured less accurately, higher excitation currents are required to reach the same SNR value.

The peak-to-peak amplitude of the defect signal was expected to increase with decreasing excitation frequency. However, measurements showed there was a rapid decline below $20 \mathrm{~Hz}$. This was further emphasized by the SNR of both the Phi and R sensors which showed a sharp peak at around $20 \mathrm{~Hz}$. This sharp peak shows that an optimal SNR occurs within a narrow band as at lower frequencies larger variations in the background reduce the SNR and at higher frequencies less eddy current is distorted by the defect thus reducing the SNR.

Tests at different excitation lift-off levels showed that defects larger than $4400 \mathrm{~mm}^{3}$ can be easily identified up to a maximum lift-off of $45 \mathrm{~mm}$. Defect signals are still present at higher lift-off levels and are probably measurable, but to positively identify them will require further data manipulation.

Two possible methods have been identified that could determine the difference between variation in the pipe parameters and defects. The first method is a test at a range of frequencies to determine if the peak was a defect. A peak in the amplitude would be expected at an optimal frequency for a defect, while a parameter variation will show a linear decrease with frequency. The second method is to test at a range of excitation currents. A peak from a defect would be expected to decay at a faster rate with increasing current than a peak from a parameter variation.

In summary, this project has demonstrated that using GMR sensors for low frequency eddy current testing is a viable technology to identify defects within steel pipes with insulation up to $50 \mathrm{~mm}$ and aluminum weather cladding. The next step to develop the technology further would be a prototype suitable for field testing. It would need to incorporate an array of sensor modules to be able to operate at a useful rate in the field. It would be important to fix the sensor rigidly to the excitation unit to produce repeatable results. Algorithms would have to be developed to remove the effect of the aluminum shield. To identify defects at lift-off higher than $50 \mathrm{~mm}$, tests would have to use a range of frequency and excitation 
currents. Algorithms developed to identify peaks could further increase the probability of identification of a defect. The effect of excitation frequency and current should be examined with better resolution to determine the effect on defect signals. To be able to determine the shape and size of the defect accurately, further measurements should be done to establish the dependence of peak size and shape vs defect shape and depth. If phase shift measurement variations could be reduced, then defect identification at lift-off greater than $50 \mathrm{~mm}$ could be achieved. 
Appendix A

NVE AA004 data sheet extract 


\section{AA and AB-Series Analog Sensors}

NVE's AA and AB-Series analog GMR sensors offer unique and unparalleled magnetic sensing capabilities. These sensors are characterized by high sensitivity to applied magnetic fields, excellent temperature stability, low power consumption, and small size. These characteristics make them suitable for use in a wide variety of applications from rugged industrial and automotive position, speed, and current sensors, to low-voltage, battery-powered sensors for use in hand-held instrumentation and implantable medical devices. The unmatched versatility of these basic magnetic sensors makes them an excellent choice for a wide range of analog sensing applications.

The AA-Series sensors use NVE's patented GMR materials and on-chip flux concentrators to provide a directionally sensitive output signal. These sensors are sensitive in one direction in the plane of the IC, with a cosine-scaled falloff in sensitivity as the sensor is rotated away from the sensitive direction. Also, these devices provide the same output for magnetic fields in the positive or negative direction along the axis of sensitivity (omnipolar output). All sensors are designed in a Wheatstone bridge configuration to provide temperature compensation. Two packages are offered, an SOIC8 and an MSOP8. These sensors are also available in die form on a special-order basis.

There are three families of NVE's basic AA-Series sensors: the standard AA-Series, the AAH-Series, and the AAL-Series. Each of these sensor families uses a different GMR material, with its own characteristics. The comparison table below summarizes the different characteristics of the GMR materials:

\begin{tabular}{|l|l|l|l|}
\hline Parameter & AA Series & AAH Series & AAL Series \\
\hline Sensitivity to Applied Fields & High & Very High & High \\
\hline Field Range of Operation & High & Low & Medium \\
\hline Hysteresis & Medium & High & Low \\
\hline Temperature Range & High & Very High & Very High \\
\hline
\end{tabular}

The AB-Series sensors are differential sensor devices, or gradiometers, which take advantage of the high output characteristics of NVE's GMR materials. Two families of AB sensors are offered, the standard AB-Series and the ABH-Series. They have operational characteristics similar to the AA and AAH sensors described in the table above but with the bipolar linear output characteristics of a differential sensor.

Within these different sensor families, customers can find an excellent match to their analog sensor requirements. 


\section{Quick Reference: $A A$ and $A B-S e r i e s$}

For comparison and product selection purposes, the following table lists all available AA and ABSeries analog sensors, with some of their key characteristics:

\section{Magnetometers:}

\begin{tabular}{|c|c|c|c|c|c|c|c|c|c|}
\hline \multirow[t]{2}{*}{$\begin{array}{l}\text { Part } \\
\text { Number }\end{array}$} & \multicolumn{2}{|c|}{$\begin{array}{l}\text { Linear } \\
\text { Range } \\
\left(\left|\mathrm{Oe}^{1}\right|\right) \\
\end{array}$} & \multicolumn{2}{|c|}{$\begin{array}{l}\text { Sensitivity } \\
\left(m \mathrm{~m} / \mathrm{V}-\mathrm{Oe}^{1}\right)\end{array}$} & \multirow[t]{2}{*}{$\begin{array}{c}\text { Maximum } \\
\text { Non- } \\
\text { linearity } \\
\left(\% \text { Uni. }^{2}\right) \\
\end{array}$} & \multirow[t]{2}{*}{$\begin{array}{c}\text { Maximum } \\
\text { Hyster- } \\
\text { esis } \\
\left(\% \text { Uni. }^{2}\right) \\
\end{array}$} & \multirow[t]{2}{*}{$\begin{array}{c}\text { Maximum } \\
\text { Operating } \\
\text { Temp } \\
\left({ }^{\circ} \mathrm{C}\right) \\
\end{array}$} & \multirow[t]{2}{*}{$\begin{array}{l}\text { Typical } \\
\text { Resis- } \\
\text { tance } \\
\text { (Ohms) }\end{array}$} & \multirow[t]{2}{*}{ Package } \\
\hline & Min & Max & Min & Max & & & & & \\
\hline AA002-02 & 1.5 & 10.5 & 3.0 & 4.2 & 2 & 4 & 125 & $5 \mathrm{~K}$ & SOIC8 \\
\hline AA003-02 & 2.0 & 14 & 2 & 3.2 & 2 & 4 & 125 & $5 \mathrm{~K}$ & SOIC8 \\
\hline AA004-00 & 5.0 & 35 & 0.9 & 1.3 & 2 & 4 & 125 & $5 \mathrm{~K}$ & MSOP8 \\
\hline AA004-02 & 5.0 & 35 & 0.9 & 1.3 & 2 & 4 & 125 & $5 \mathrm{~K}$ & SOIC8 \\
\hline AA005-02 & 10.0 & 70 & 0.45 & 0.65 & 2 & 4 & 125 & $5 \mathrm{~K}$ & SOIC8 \\
\hline AA006-00 & 5.0 & 35 & 0.9 & 1.3 & 2 & 4 & 125 & $30 \mathrm{~K}$ & MSOP8 \\
\hline AA006-02 & 5.0 & 35 & 0.9 & 1.3 & 2 & 4 & 125 & $30 \mathrm{~K}$ & SOIC8 \\
\hline AAH002-02 & 0.6 & 3.0 & 11.0 & 18.0 & 6 & 15 & 150 & $2 \mathrm{~K}$ & SOIC8 \\
\hline $\mathrm{AAH} 004-00$ & 1.5 & 7.5 & 3.2 & 4.8 & 4 & 15 & 150 & $2 \mathrm{~K}$ & MSOP8 \\
\hline AAL002-02 & 1.5 & 10.5 & 3.0 & 4.2 & 2 & 2 & 150 & $5.5 \mathrm{~K}$ & SOIC8 \\
\hline
\end{tabular}

\section{Gradiometers:}

\begin{tabular}{|l|c|c|c|c|c|c|c|c|}
\hline $\begin{array}{l}\text { Part } \\
\text { Number }\end{array}$ & $\begin{array}{c}\text { Linear } \\
\text { Range } \\
\left(\left|\mathbf{O e}^{1}\right|\right)\end{array}$ & $\begin{array}{c}\text { Resistor } \\
\text { Spacing } \\
(\mathbf{m m})\end{array}$ & $\begin{array}{c}\text { Maximum } \\
\text { Non- } \\
\text { linearity } \\
\left(\% \text { Uni. }{ }^{2}\right)\end{array}$ & $\begin{array}{c}\text { Maximum } \\
\text { Hyster- } \\
\text { esis } \\
\left(\% \text { Uni. } .^{2}\right)\end{array}$ & $\begin{array}{c}\text { Maximum } \\
\text { Operating } \\
\text { Temp } \\
\left({ }^{\circ} \mathbf{C}\right)\end{array}$ & $\begin{array}{c}\text { Typical } \\
\text { Resis- } \\
\text { tance } \\
(\text { Ohms })\end{array}$ & Package \\
\hline Min & Max & & & & & & \\
\hline AB001-02 & 20 & 200 & 0.5 & 2 & 4 & 125 & $2.5 \mathrm{~K}$ & SOIC8 \\
\hline ABH001-00 & 20 & 200 & 0.5 & 2 & 4 & 125 & $2.5 \mathrm{~K}$ & MSOP8 \\
\hline
\end{tabular}

\section{Notes:}

1. Oersted $(\mathrm{Oe})=1$ Gauss in air.

2. Unipolar operation means exposure to magnetic fields of one polarity, for example 0 to +30 Gauss, or -2 to -50 Gauss.

Bipolar operation (for example, -5 to +10 Gauss) will increase nonlinearity and hysteresis 


\section{AA Sensors}

\section{Features:}

- Excellent Sensitivity to Applied Magnetic Fields

- Wheatstone Bridge Analog Output

- Operating Temperature to $125^{\circ} \mathrm{C}$ Continuous

- Wide Linear Range of Operation

- Near-Zero Voltage Operation

- $\quad \mathrm{DC}$ to $>1 \mathrm{MHz}$ Frequency Response

- Small, Low-Profile Surface Mount Packages

\section{Applications:}

- General Motion, Speed, and Position Sensing

- Low Power, Low Voltage Applications

- Low Field Sensing for Magnetic Media Detection

- Current Sensing

\section{Description:}

The basic AA-Series GMR sensors are general-purpose magnetometers for use in a wide variety of applications. They exhibit excellent linearity, a large output signal with applied magnetic fields, stable and linear temperature characteristics, and a purely ratiometric output.

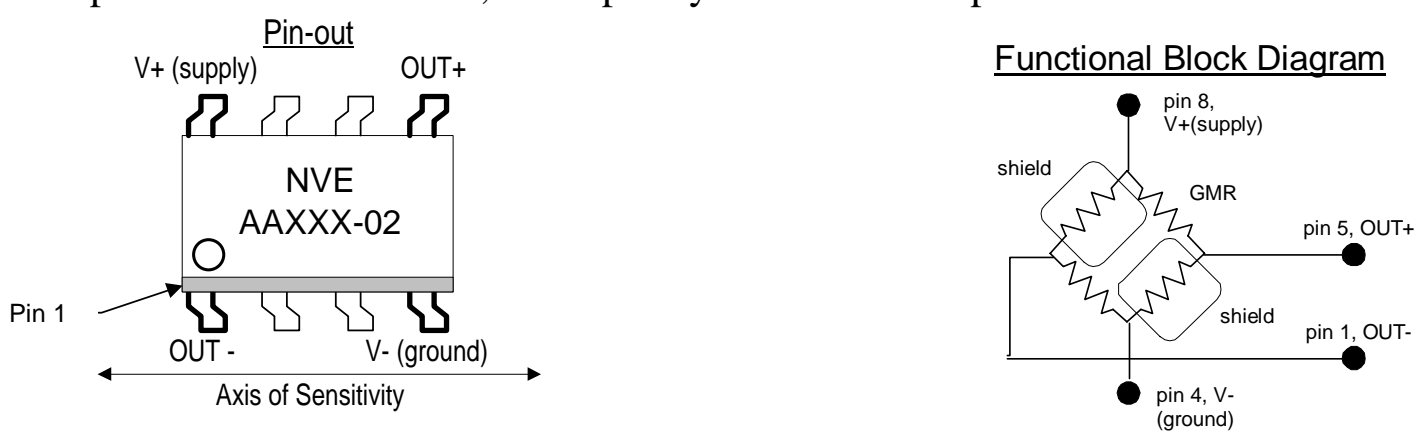

\section{Magnetic Characteristics:}

\begin{tabular}{|c|c|c|c|c|c|c|c|c|}
\hline \multirow[t]{2}{*}{$\begin{array}{l}\text { Part } \\
\text { Number }\end{array}$} & \multirow[t]{2}{*}{$\begin{array}{l}\text { Saturation } \\
\text { Field }\left(\mathrm{Oe}^{1}\right)\end{array}$} & \multicolumn{2}{|c|}{$\begin{array}{c}\text { Linear } \\
\text { Range } \\
\left(\left|\mathrm{Oe}^{1}\right|\right) \\
\end{array}$} & \multicolumn{2}{|c|}{$\begin{array}{l}\text { Sensitivity } \\
\left(m \mathrm{~m} / \mathrm{V}-\mathrm{Oe}^{1}\right)\end{array}$} & \multirow[t]{2}{*}{$\begin{array}{c}\text { Resistance } \\
\text { (Ohms) }\end{array}$} & \multirow{2}{*}{ Package $^{2}$} & \multirow[t]{2}{*}{$\begin{array}{c}\text { Die Size }^{3} \\
\quad(\mu \mathrm{m})\end{array}$} \\
\hline & & Min & Max & Min & Max & & & \\
\hline AA002-02 & 15 & 1.5 & 10.5 & 3.0 & 4.2 & $5 \mathrm{~K} \pm 20 \%$ & SOIC8 & $436 \times 3370$ \\
\hline AA003-02 & 20 & 2.0 & 14 & 2 & 3.2 & $5 \mathrm{~K} \pm 20 \%$ & SOIC8 & $436 \times 3370$ \\
\hline AA004-00 & 50 & 5 & 35 & 0.9 & 1.3 & $5 \mathrm{~K} \pm 20 \%$ & MSOP8 & $411 \times 1458$ \\
\hline AA004-02 & 50 & 5 & 35 & 0.9 & 1.3 & $5 \mathrm{~K} \pm 20 \%$ & SOIC8 & $411 \times 1458$ \\
\hline AA005-02 & 100 & 10 & 70 & 0.45 & 0.65 & $5 \mathrm{~K} \pm 20 \%$ & SOIC8 & $411 \times 1458$ \\
\hline AA006-00 & 50 & 5 & 35 & 0.9 & 1.3 & $30 \mathrm{~K} \pm 20 \%$ & MSOP8 & $836 \times 1986$ \\
\hline AA006-02 & 50 & 5 & 35 & 0.9 & 1.3 & $30 \mathrm{~K} \pm 20 \%$ & SOIC8 & $836 \times 1986$ \\
\hline
\end{tabular}

-14 - 


\section{General Characteristics:}

\begin{tabular}{|l|c|c|c|c|}
\hline Parameter & Min & Typical & Max & Unit \\
\hline Input Voltage Range & $<^{4}$ & & $24^{4}$ & Volts \\
\hline Operating Frequency & $\mathrm{DC}$ & & $>1$ & $\mathrm{MHz}$ \\
\hline Operating Temperature Range & -50 & & 125 & ${ }^{\circ} \mathrm{C}$ \\
\hline Bridge Electrical Offset & -4 & & +4 & $\mathrm{mV} / \mathrm{V}$ \\
\hline Signal Output at Max. Field & & 60 & & $\mathrm{mV} / \mathrm{V}$ \\
\hline Nonlinearity & & & 2 & $\%(\text { unipolar })^{5}$ \\
\hline Hysteresis & & & 4 & $\%(\text { unipolar })^{5}$ \\
\hline TCR & & +0.14 & & $\% /{ }^{\circ} \mathrm{C}^{6}$ \\
\hline TCOI & & +0.03 & & $\% /{ }^{6}$ \\
\hline TCOV & & -0.1 & & $\% / \mathrm{C}^{6}$ \\
\hline Off Axis Characteristic & & $\operatorname{Cos} \beta^{7}$ & & \\
\hline ESD Tolerance & & 400 & & $\mathrm{~V}$ pin-to-pin HBM \\
\hline
\end{tabular}

\section{Notes:}

1. 1 Oersted $(\mathrm{Oe})=1$ Gauss in air.

2. See the Appendix for package dimensions and tolerances.

3. Sensors can be provided in die form by special request.

4. GMR AA-Series sensors are pure ratiometric devices meaning that they will operate properly at extremely low supply voltages. The output signal will be proportional to the supply voltage. Maximum voltage range is limited by the power dissipation in the package and the maximum operating temperature of the sensor.

5. Unipolar operation means exposure to magnetic fields of one polarity, e.g., 0 to 30 Gauss, or 2 to -50 Gauss, but not -20 to +30 Gauss (bipolar operation). Bipolar operation will increase nonlinearity and hysteresis.

6. TCR is resistance change with temperature with no applied field. TCOI is the output change with temperature using a constant current source to power the sensor. TCOV is the output change with temperature using a constant voltage source to power the sensor. See the graphs below.

7. Beta $(\beta)$ is any angle deviation from the sensitive axis.
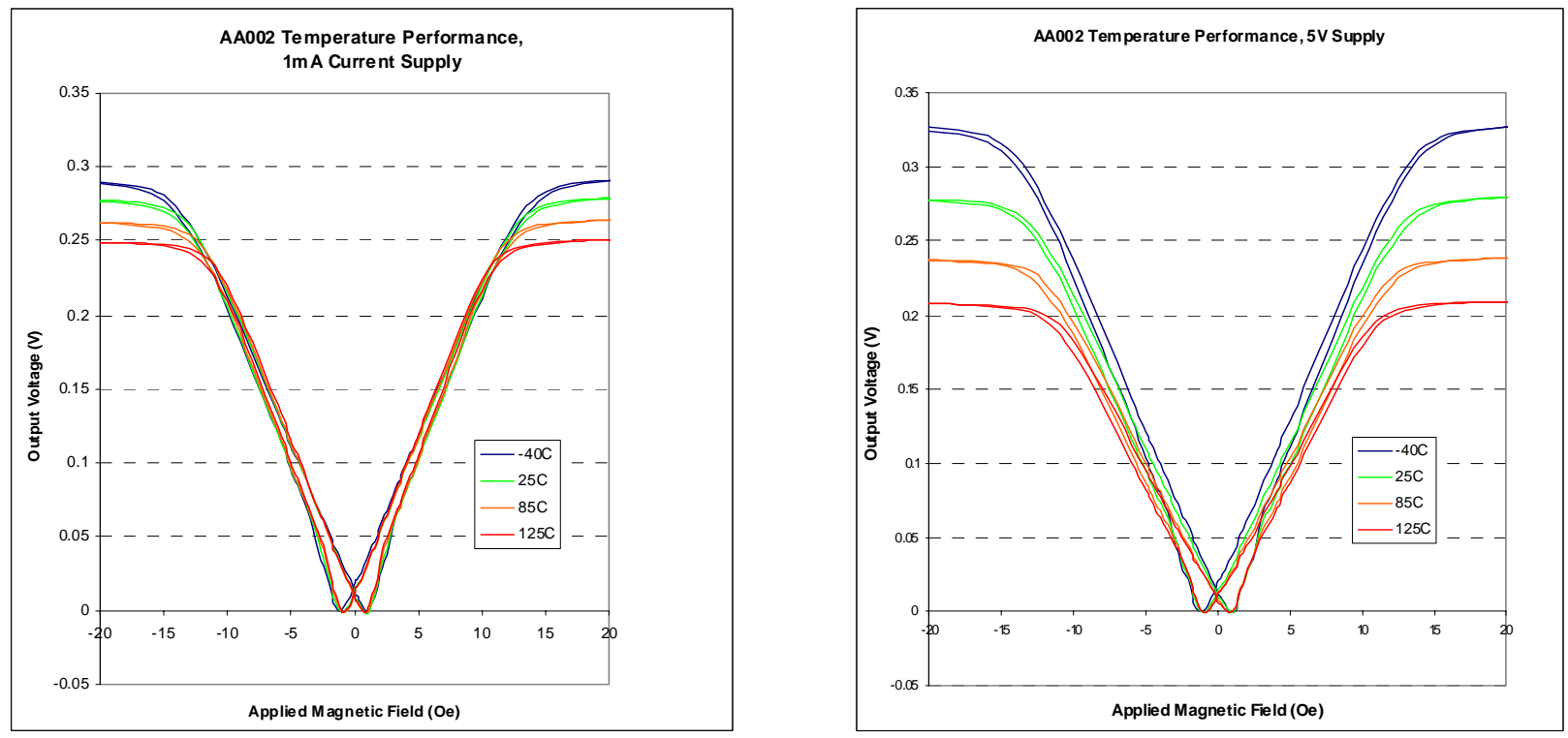

$-15-$ 


\section{Bibliography}

[1] (2014, Sep) Corrosion costs and preventive strategies in the united states. National Association of Corrosion Engineers. [Online]. Available:

http://www.nace.org/uploadedFiles/Publications/ccsupp.pdf

[2] (2014, Oct) Bp completes commissioning of whiting refinery units. BP. [Online]. Available: http://www.bp.com/en/global/corporate/press/press-releases/ bp-completes-commissioning-of-whiting-refinery-units.html

[3] (2014, Oct) Ultrawave Irt guided wave system for in-service pipe inspection. Olympus. [Online]. Available:

http://www.olympus-ims.com/en/.downloads/download/?file=285214432\&fl=en_US

[4] A. Washabaugh, S. Haque, D. Jablonski, and N. J. Goldfine, "Eddy current sensor arrays for pipeline inspection with and without coatings," in Proc. 8th International Pipeline Conference, Calgary, Alberta, Canada, Sep 2010. [Online]. Available: http: //proceedings.asmedigitalcollection.asme.org/proceeding.aspx?articleid=1614382

[5] S. Denenberg, D. Straney, T. Dunford, Y. Sheiretov, S. Haque, B. Manning, J. Kott, A. Washabaugh, and N. Goldfine, "Advancements in imagin corrosion under insulation (cui) for piping and vessels," presented at the ASNT Annual Conference, Las Vegas, Nevada, Nov 2013. [Online]. Available: http: //www.jenteksensors.com/media/2013\%20ASNT\%20Fall_JENTEK_CUI_Final.pdf

[6] N. Goldfine, T. Dunford, A. Washabugh, S. Haque, and S. Denenberg, "Mwm-array electromagnetic techniques for crack sizing, weld assessment, wall loss / thickness measuremen, and mechanical damage profilometry," JENTEK Sensors, Inc, Tech. Rep., 2012. [Online]. Available: http://www.jenteksensors.com/media/ TR_2012_05_02\%200il\%20and\%20Gas\%20Technology\%20Overview.pdf

[7] A. Washabaugh, "Inspection of pipelines using high-resolution mwm and mr-mwm-arrays," presented at the API Pipeline Conference, Loews Coronado Bay, San Diego, CA, April 2013. [Online]. Available: http://www.jenteksensors.com/media/ JENTEK\%20API\%20Pipeline\%20Conf\%20SanDiego\%202013.pdf

[8] K. Stevens and D. Drablle, "Private commucation," 2014.

[9] G. L. Workman and P. O. Moore, Eds., Nondestructive testing overview, 3rd ed., ser. Nondestructive testing handbook. American society for nondestructive testing, Inc, 2012, vol. 10. 
[10] P. J. Shull, Ed., Nondestructive Evaluation Theory, Techniques, and Applications. Marcel Dekker, Inc, 2002.

[11] S. Tumanski, Ed., Handbook of Magnetic Measurements. CRC Press Taylor and Francis Group, 2011, ch. 2 Fundamentals of Magnetic Measurements.

[12] S. S. Udpa, Electromagnetic Testing, 3rd ed., ser. Nondestructive testing handbook. American society for nondestructive testing, Inc, 2004, vol. 5, ch. 5 Probes for Electromagnetic Testing.

[13] T. Bratland, M. J. Caruso, R. W. Schneider, and C. H. Smith, "A new perspective on magnetic field sensing," Honeywell, Tech. Rep., 1998. [Online]. Available: https://aerospace.honeywell.com/ /media/Images/Plymouth\%20Website\%20PDFs/ Magnetic\%20Sensors/Technical\%20Articles/

A_New_Perspective_on_Magnetic_Field_Sensing.ashx

[14] S. Tumanski, Ed., Handbook of Magnetic Measurements. CRC Press Taylor and Francis Group, 2011, ch. 4 magnetic sensors.

[15] J. Daughton, "Spin-dependent sensors," Proceedings of the IEEE, vol. 91, no. 5, pp. 681-686, May 2003.

[16] (2014, Sep) Application notes for gmr sensors. NVe corporation. [Online]. Available: http://www.nve.com/Downloads/apps.pdf

[17] (2014, Sep) Gmr sensor catalog. NVE corporation. [Online]. Available: http://www.nve.com/Downloads/catalog.pdf

[18] N. Goldfine, D. Clark, and H. Eckhardt, "Meandering winding test circuit," U.S Patent US5 793 206, Aug. 11, 1998. [Online]. Available:

http://www.google.com/patents/US5793206

[19] S. Denenberg, T. Dunford, N. Goldfine, and Y. Sheiretov, "Method and apparatus for inspection of corrosion and other defects through insulation," U.S Patent US20130124109 A1, 2013. [Online]. Available:

https://www.google.com/patents/US20130124109

[20] S. S. Udpa, Electromagnetic Testing, 3rd ed., ser. Nondestructive testing handbook. American society for nondestructive testing, Inc, 2004, vol. 5, ch. 9 Magnetic Flux Leakage Testing.

[21] M. Kaack, T. Orth, G. Fischer, W.Weingarten, S. A.Koka, and N.Arzt, "Application of gmr sensors for the industrial inspection of seamless steel pipes," in Proc. 11th Symposium on Magnetoresistive Sensors and Magnetic Systems, Wetzlar, Germany, Mar 2011.

[22] G. Fischer, S. Gwildies, M. Kaack, A. Graff, A. Koka, and S. Nitsche, "Device for nondestructive testing of pipes," U.S Patent 20100219818 A1, Sep. 2, 2010. [Online]. Available: http://www.google.com/patents/US20100219818 
[23] W. Du, H. Nguyen, A. Dutt, and K. Scallion, "Design of a gmr sensor array system for robotic pipe inspection," in Proc. Sensors, 2010 IEEE, Nov 2010, pp. 2551-2554.

[24] L. Chen, P.-W. Que, and T. Jin, "A giant-magnetoresistance sensor for magnetic-flux-leakage nondestructive testing of a pipeline," Russian Journal of Nondestructive Testing, vol. 41, pp. 462-465, 2005.

[25] J. P. Hansen, "Using eddy current for corrosion inspection," Inspection Trends, Fall 2007.

[26] F. Vacher, C. Gilles-Pascaud, J. Decitre, C. Fermon, and M. Pannetier, "Non destructive testing with gmr magnetic sensor arrays," in Proc. European Conference on Non-Destructive Testing, September 2006.

[27] C. J. Carpenter. (2014, Sep) Magnetism. AccessScience. McGraw-Hill Education. [Online]. Available: http://www.accessscience.com/content/magnetism/398800

[28] S. S. Udpa, Electromagnetic Testing, 3rd ed., ser. Nondestructive testing handbook. American society for nondestructive testing, Inc, 2004, vol. 5, ch. 4 modeling of electromagnetic testing.

[29] P. N. Murgatroyd and N. J. Walker, "Frequency-dependent inductance and resistance of foil conductor loops," Proceedings of the IEEE, vol. 124, no. 5, pp. 493-496, 1977.

[30] A. Hunze, "Private commucation, fem simulations," 2014.

[31] D. Halliday, R. Resnick, and J. Walker, Eds., Fundamentals of physics, 7th ed., ch. 29 Magnetic Fields Due to Currents.

[32] "Copper wire tables 3rd edition," Circular of the Bureau of Standards.

[33] (2014, Oct) Convective heat transfer. Thermopedia. [Online]. Available: http://www.thermopedia.com/content/660/

[34] B. Rudnev, D. Loveless, R. Cook, and M. Black, Handbook of induction heating. Marcel Dekker, 2003. 